\title{
ALEXANDER SCHIFF
}

\section{Informationsintermediäre}

Internet und Gesellschaft

Mohr Siebeck 


\title{
Internet und Gesellschaft
}

Schriften des Alexander von Humboldt Institut für Internet und Gesellschaft

\author{
Herausgegeben von \\ Jeanette Hofmann, Matthias C. Kettemann, \\ Björn Scheuermann, Thomas Schildhauer \\ und Wolfgang Schulz
}

23

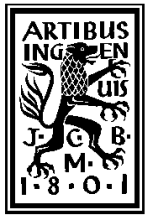





\section{Alexander Schiff \\ Informationsintermediäre}

Verantwortung und Haftung

Mohr Siebeck 
Alexander Schiff, geboren 1988; Studium der Rechtswissenschaft an der Universität Freiburg; 2013 Erste juristische Prüfung; LL.M.-Studium an der London School of Economics and Political Science; Referendariat am Kammergericht Berlin; 2016 Zweite juristische Staatsprüfung; Wissenschaftlicher Mitarbeiter am BMBF-geförderten Verbundprojekt Weizenbaum-Institut für die vernetzte Gesellschaft, Anstellung beim Verbundpartner Humboldt-Universität zu Berlin; seit 2020 Referent im Bundesministerium der Justiz und für Verbraucherschutz; 2021 Promotion.

ISBN 978-3-16-160188-0 / eISBN 978-3-16-160189-7

DOI 10.1628/978-3-16-160189-7

ISSN 2199-0344 / eISSN 2569-4081 (Internet und Gesellschaft)

Die Deutsche Nationalbibliothek verzeichnet diese Publikation in der Deutschen Nationalbibliographie; detaillierte bibliographische Daten sind über $h t t p: / / d n b . d n b . d e$ abrufbar.

(C) 2021 Mohr Siebeck Tübingen. www.mohrsiebeck.com

Das Werk einschließlich aller seiner Teile ist urheberrechtlich geschützt. Jede Verwertung außerhalb der engen Grenzen des Urheberrechtsgesetzes ist ohne Zustimmung des Verlags unzulässig und strafbar. Das gilt insbesondere für die Verbreitung, Vervielfältigung, Übersetzung und die Einspeicherung und Verarbeitung in elektronischen Systemen.

Das Buch wurde von Gulde Druck in Tübingen auf alterungsbeständiges Werkdruckpapier gedruckt und von der Buchbinderei Spinner in Ottersweier gebunden.

Printed in Germany. 
Für Joel Mischa 



\section{Vorwort}

Diese Abhandlung unternimmt den Versuch einer Bestandsaufnahme und Kritik des unionalen sowie des deutschen Rechts der Haftung von Diensteanbietern für nutzergenerierte Inhalte und stellt diese Rechtsmaterie in den Kontext der Entstehung und Anwendung vergleichbarer Vorschriften in den USA. Die Diskussionen um diese Regelungskomplexe haben auf beiden Seiten des Atlantiks in letzter Zeit eine Dynamik angenommen, die zu Beginn dieses Vorhabens kaum absehbar war, wiewohl der richtige regulative Umgang mit den Intermediären in der Europäischen Union sowie gerade hierzulande schon seit langem kontrovers diskutiert wird. Diese Dynamik zwang mich, für den Abschluss dieses Buches einen klaren Schnitt vorzunehmen, dem die Berücksichtigung der Reformbemühungen um das Netzwerkdurchsetzungsgesetz, der deutschen Umsetzung der Richtlinie über das Urheberrecht im Binnenmarkt sowie des geplanten „Digital Services Act“ zum Opfer fielen. Insbesondere mit Blick auf den zuletzt genannten, im Dezember 2020 vorgelegten Verordnungsentwurf der Europäischen Kommission hege ich dennoch die Hoffnung, mit einer kritischen Analyse der E-Commerce-Richtlinie einen kleinen Beitrag zu ihrer überfälligen Reform zu leisten.

Die Juristische Fakultät der Humboldt-Universität zu Berlin hat diese Studie im November 2020 als Dissertation angenommen. Rechtsprechung und Literatur sind bis dahin berücksichtigt. Naturgemäß haben sich um dieses Vorhaben und die Bedingungen seiner Realisierung viele Menschen verdient gemacht. Herr Professor Dr. Martin Eifert begleitete den Entstehungsprozess von Beginn an mit im besten Sinne kritischen und ermutigenden Anregungen, wirkte stets auf eine fundierte sowie zugleich pragmatische Vorgehensweise hin und trug so wesentlich zum erfolgreichen Abschluss meines Promotionsvorhabens bei, für dessen Betreuung ich ihm herzlich danke. Für die rasche Erstellung des Zweitgutachtens danke ich Herrn Professor Dr. Herbert Zech.

Entstanden ist dieses Buch während meiner Tätigkeit beim durch das Bundesministerium für Bildung und Forschung geförderten Verbundprojekt „Weizenbaum-Institut für die vernetzte Gesellschaft“, dessen Entstehung ich als wissenschaftlicher Mitarbeiter beim Verbundpartner Humboldt-Universität zu Berlin fast von Beginn an begleiten durfte. Verbunden durch den Anspruch, ausschließlich mit öffentlicher Förderung interdisziplinäre und am Gemeinwohl orientierte Grundlagenforschung zu den Implikationen der 
Digitalisierung zu betreiben, hat sich eine Vielzahl interessierter und engagierter Wissenschaftlerinnen und Wissenschaftler in Berlin versammelt und so ein inspirierendes Umfeld geschaffen, von dem meine Forschungsvorhaben sehr profitiert haben. Für die vielen Einblicke, Einsichten und Erfahrungen, die mir diese Tätigkeit ermöglicht hat, danke ich pars pro toto den Herren Professoren Dr. Axel Metzger und Dr. Herbert Zech.

Für die kritische Lektüre des Manuskripts danke ich Dr. Julius Goldmann. Wichtige Anregungen, die mich nicht nur bei der Konkretisierung des Promotionsthemas vorangebracht haben, verdanke ich Paul Nemitz. Mein Dank gilt ferner dem Projektträger des Weizenbaum-Instituts für die großzügige und unbürokratisch gewährte Förderung der Drucklegung sowie dem Herausgeberkreis für die Aufnahme in die Schriftenreihe „Internet und Gesellschaft".

Meine Eltern haben mich mit ihrer liebevollen Unterstützung in jeglicher Hinsicht zu dem Menschen gemacht, der ich heute bin. Ebenfalls keinen geringen Anteil hieran hatte mein Bruder. Ihnen danke ich von Herzen für alles. Dr. Mailies Wirminghaus danke ich schließlich für die kaum ermessliche Aufmunterung und Unterstützung in allen Phasen meines Promotionsvorhabens sowie für das größte Geschenk überhaupt, dem dieses Buch gewidmet ist. 


\section{Inhaltsverzeichnis}

Vorwort $\ldots \ldots \ldots \ldots \ldots \ldots \ldots \ldots \ldots \ldots \ldots \ldots \ldots \ldots \ldots, \quad$ VII

Abkürzungsverzeichnis $\ldots \ldots \ldots \ldots \ldots \ldots \ldots \ldots \ldots \ldots \ldots$ XXI

Einführung $\ldots \ldots \ldots \ldots \ldots \ldots \ldots \ldots \ldots \ldots \ldots \ldots \ldots \ldots \ldots \ldots \ldots, 1$

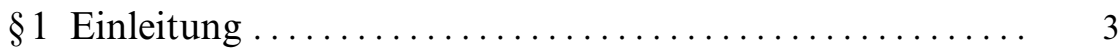

A. Haftung für Inhalte Dritter als Kernfrage des Verhältnisses von Internet und Regulierung ........................ 3

B. Gegenstand ............................ 7

C. Ziel ..................................... 7

$\S 2$ Gang der Untersuchung $\ldots \ldots \ldots \ldots \ldots \ldots \ldots \ldots \ldots$

Kapitel 1: Entwicklung des Rechts der Haftungsprivilegierungen $\ldots \ldots \ldots \ldots \ldots \ldots \ldots \ldots \ldots, 11$

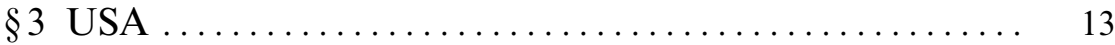

A. $\$ 230$ Communications Decency Act ................. 13

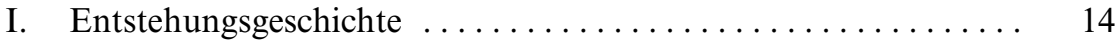

II. Auslegung von $\S 230 \mathrm{CDA}$ durch die Gerichte ........... 17

1. Keine Differenzierung zwischen „Publisher“ und „Distributor“ führt zur Immunisierung auch bei Kenntnis . .......... 17

2. Das „Information Content Provider"-Kriterium und die Anforderungen an eine haftungsbegründende „material contribution" ............................ 20

3. Negierung des ,Treatment"-Kriteriums . ............ 22

4. Engführung des Ausnahmenkatalogs ............... 25

III. Verfassungsrechtlicher Kontext des $\S 230$ CDA . . . . . . . . . . . 25

IV. Neuere Entwicklungen . . . . . . . . . . . . . . . . . . 27

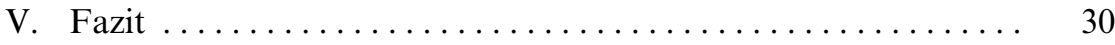


B. $\S 512$ Digital Millennium Copyright Act $\ldots \ldots \ldots \ldots \ldots \ldots \ldots, 32$

I. Entstehungsgeschichte ...................... 33

II. Handhabung des Notice-and-Takedown-Verfahrens nach $\S 512$ DMCA .................................... 35

1. Allgemeines ............................. 35

2. Speicherung, at the direction of a user" $\ldots \ldots \ldots \ldots \ldots .36$

3. Die sog. ,threshold requirements“ ................ 37

a) „Repeat Infringer Policy“ und „Standard Technical Measures“" §512(i) DMCA .................. 37

b) Keine tatsächliche Kenntnis oder Missachtung von „Red Flags“. .............................. 39

c) Kein ,right and ability to control“ ............ 40

4. Anforderungen und Konsequenzen einer „notice“......... 41

III. Kritik .................................. 42

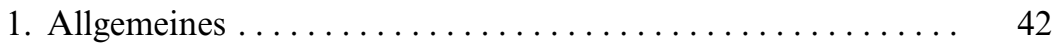

2. Merkmale des „Safe Harbor" im Einzelnen ........... 46

a) Keine „Red Flags“ in Sicht? ................ 46

b) Die Macht der Diensteanbieter vs. ,right and ability to control" ............................... 47

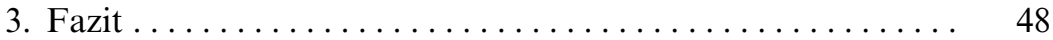

$\S 4$ Europäische Union \& Deutschland $\ldots \ldots \ldots \ldots \ldots \ldots \ldots \quad 51$

A. Rechtslage in Deutschland vor Erlass des TDG .......... 51

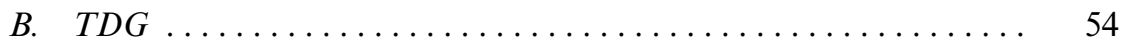

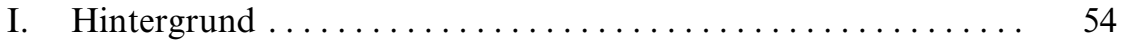

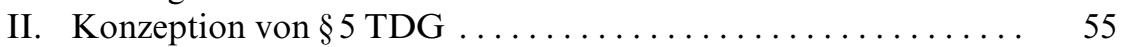

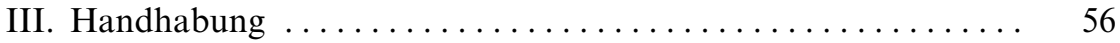

1. „Filterfunktion“, Darlegungs- und Beweislast .......... 56

2. Bereithalten eines fremden Inhalts $\ldots \ldots \ldots \ldots \ldots \ldots \ldots$

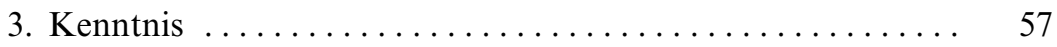

4. Technische Möglichkeit und Zumutbarkeit der Verhinderung der Nutzung von Inhalten $\ldots \ldots \ldots \ldots \ldots \ldots \ldots \ldots$

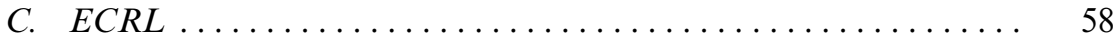

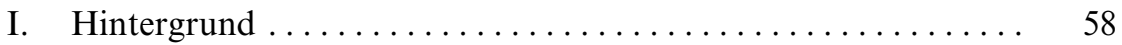

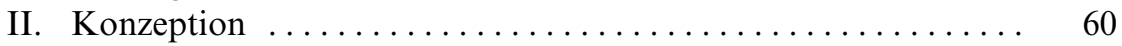

1. Allgemeines, Systematik .................... 60

2. Persönlicher Anwendungsbereich der Art. 12 ff. ......... 60

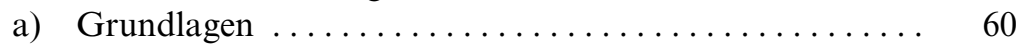

b) Suchmaschinen als Sonderfall ................. 61

3. Die Rolle des Diensteanbieters im System der Art. 12 ff. .... 63 
III. Privilegierung des Hostings (Art. 14) $\ldots \ldots \ldots \ldots \ldots \ldots \ldots .65$

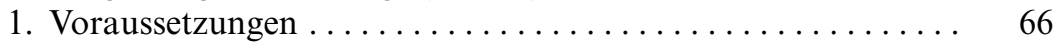

a) Speicherung von Informationen $\ldots \ldots \ldots \ldots \ldots \ldots 66$

b) Durch einen Nutzer eingegebene Informationen ....... 66

c) Speicherung im Auftrag des Nutzers ........... 67

d) Keine Kenntnis von der rechtswidrigen Information (Art. 14 Abs. 1 lit. a Var. 1) .................. 68

e) Kein Bewusstsein über Tatsachen und Umstände, aus denen die Rechtsverletzung offensichtlich wird (Art. 14 Abs. 1 lit. a Var. 2) ..................... 72

f) Unverzügliche Entfernung oder Sperrung nach Kenntnisbzw. Bewusstseinserlangung (Art. 14 Abs. 1 lit. b) .... 73

2. Rechtsfolgen, erfasste Regelungsbereiche ............ 73

IV. Verbot der allgemeinen Überwachungspflicht (Art. 15) . . . . . . 75

1. Bedeutung .............................. 75

2. Reichweite .......................... 76

a) Allgemeine vs. spezifische Überwachungspflichten ..... 76

aa) Unzulässigkeit von Filtersystemen ............ 77

bb) Automatisierte Techniken und Mittel zur Nachforschung ..................... 78

cc) Verhältnismäßigkeit von Verhinderungspflichten .... 79

b) Zwischenfazit ........................... 80

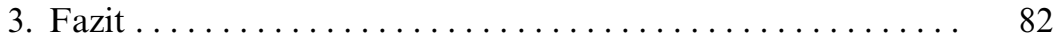

D. Die Inanspruchnahme von Diensteanbietern im deutschen Recht 83

I. Haftung für eigene Inhalte . . . . . . . . . . . . . . . . . . 84

1. Tatsächlich eigene Inhalte ...................... 85

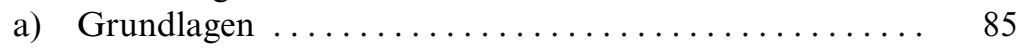

b) „Autocomplete“ ....................... 87

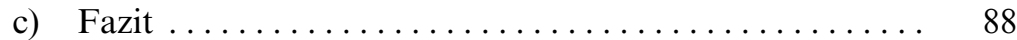

2. Die Kategorie des Zueigenmachens fremder Inhalte als Bruchstelle im Verhältnis zum Unionsrecht ............. 88

a) Grundlagen .............................. 88

b) Verhältnis zum Unionsrecht $\ldots \ldots \ldots \ldots \ldots \ldots \ldots .91$

II. Haftung für fremde Inhalte: Das Privileg des $\S 10$ TMG ..... 93

1. Allgemeines . . . . . . . . . . . . . . . . . . . . . 93

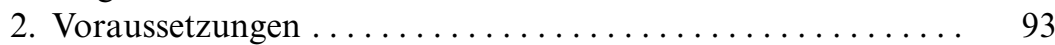

a) Abspeichern einer fremden Information für einen Nutzer . . . . . . . . . . . . . . . . . . . . . . . . . . . 93

b) Keine Kenntnis ........................... 94

aa) Positive Kenntnis eines konkreten Inhalts . . . . . . . 94

bb) Kenntnis der Rechtswidrigkeit . . . . . . . . . . . . . . . . 94 
cc) Kenntniserlangung durch automatisierte Systeme? . . .

c) Unverzügliche Entfernung oder Sperrung nach Kenntniserlangung $\ldots \ldots \ldots \ldots \ldots \ldots \ldots$

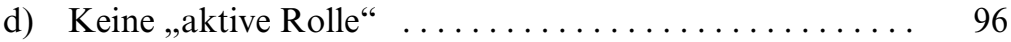

e) Grenze der Haftung: allgemeine Überwachungspflichten (§7 Abs. $2 \mathrm{TMG}) \ldots \ldots \ldots \ldots \ldots \ldots \ldots \ldots \ldots \ldots$

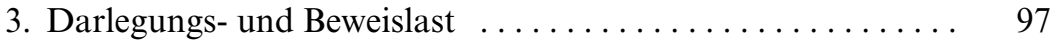

E. Kritische Annäherung in institutioneller Perspektive . . . . . . . . 97

I. Probleme eines materiell horizontalen Ansatzes . . . . . . . . . . 98

II. Herausforderungen der unionsrechtlichen Harmonisierung

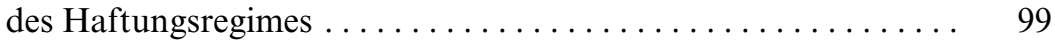

1. Im Gesetz angelegte Probleme ................. 99

2. Die Rolle der Rechtsprechung ................. 100

3. Rechtspolitische Aktivitäten des Gesetzgebers .......... 101

III. Die Herausforderung föderal verteilter Gesetzgebungskompetenzen ............. 102

$\S 5$ Gemeinsamkeiten und Unterschiede in übergeordneter Perspektive . . . . . . . . . . . . . . . . 105

A. Die Grundsatzentscheidungen für eine Haftungsprivilegierung . . . 105

B. Innovationssicherung als Leitidee . . . . . . . . . . . . . . . . 107

C. Vergleich der Anreizwirkungen ... . . . . . . . . . . . . . . 109

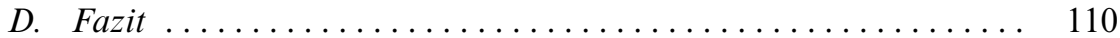

Kapitel 2: Phänomenologische Annäherungen an die Intermediäre und die Problematik ihrer Konzeption im geltenden Recht

$\S 6$ Funktionale Zuordnung der Intermediäre . . . . . . . . . 115

A. Schichtenbasierte Modelle des Internets . . . . . . . . . . . . 115

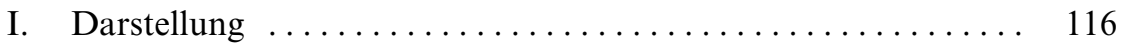

II. Bedeutung für die Thematik dieser Untersuchung . . . . . . . 117

B. Funktionen der Intermediäre . . . . . . . . . . . . . . . . . . . . . . 119

I. Kommunikationsdienste ....................... 120

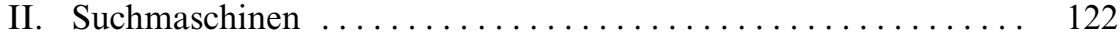

III. Vergleichende Betrachtungen . . . . . . . . . . . . . . . . . . 124

1. Unterschiede zwischen Kommunikationsdiensten und Suchmaschinen 
2. Wesentliche Gemeinsamkeiten ................. 125

a) Dominanz weniger Akteure . . . . . . . . . . . . 126

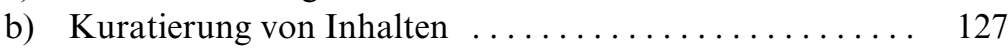

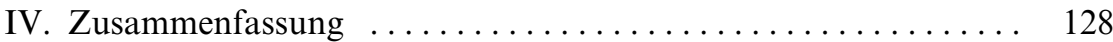

$\S 7$ Begriffliche Zuordnung als Element des rechtlichen

Zugriffs auf Intermediäre . ..................... 131

A. Plattformen ............................... 132

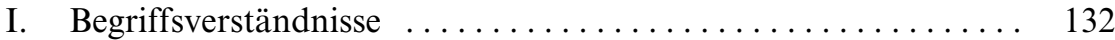

1. Allgemeine Verständnis . ....................... 132

2. Rechtliche Rezeption des Plattformbegriffs $\ldots \ldots \ldots \ldots \ldots 135$

II. Überzeugungskraft ............................. 137

B. Soziale Netzwerke .............................. 139

I. Begriffsverständnisse .......................... 140

1. Allgemeines Verständnis . ...................... 140

2. Rechtliche Rezeption ....................... 141

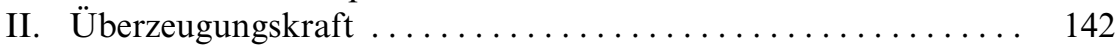

C. Diensteanbieter ................................. 143

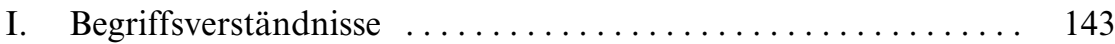

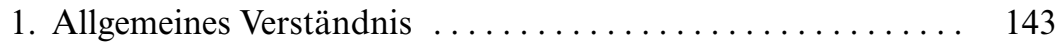

2. Rechtliche Rezeption ........................ 143

II. Überzeugungskraft ........................... 144

D. Vorzugswürdiger Begriff: (Informations-) Intermediäre ........ 145

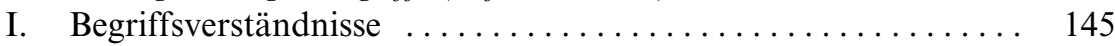

1. Allgemeines Verständnis $\ldots \ldots \ldots \ldots \ldots \ldots \ldots \ldots \ldots \ldots \ldots \ldots$

2. Rechtliche Rezeption ....................... 147

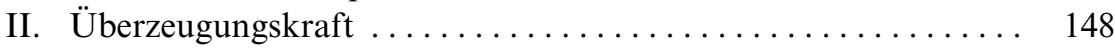

E. Fazit ...................................... 149

$\S 8$ Strukturelle Defizite bei der Zuweisung von

Verantwortung an die Intermediäre für Rechtsverletzungen 151

A. Host-Providing als funktional ungenügende Kategorie zur

Beschreibung intermediärer Dienste .................. 152

I. De lege lata vermitteltes normatives Leitbild intermediärer Dienste ......................... 152

1. USA ................................. 152

2. EU und Deutschland ..................... 153

II. Verfügungsmacht der Intermediäre über gehostete Inhalte $\ldots$. 154

1. Zugangskontrolle .......................... 154 
2. Gestaltungsmacht bei der Kommunikationsordnung ...... 156

a) Design des kommunikativen Umfelds ........... 156

b) Filterung, Sortierung und Personalisierung von Inhalten . . . . . . . . . . . . . . . . . . . 157

c) Reaktive Zugriffe auf einzelne Inhalte . . . . . . . . . . . 159

d) Zwischenfazit ......................... 160

III. Speicherung von Informationen als unangemessenes Merkmal für die Tätigkeit der Intermediäre . . . . . . . . . . . . . . . .

1. Fokus auf der technischen Lokalisierung „gespeicherter“" Informationen ........................... 16

2. Normativ unterstellte Unabhängigkeit der Dienstenutzer ... 163

B. Passivität/Neutralität als unangemessenes Leitbild der haftungsrechtlichen Zuordnung der Intermediäre

I. Rechtssystematische Zweifel am Begründungsansatz des EuGH

II. Normative Brüche bei der Anwendung des Kriteriums der Neutralität ................................. 166

1. Inkohärenz der Rechtsprechung ................... 166

a) Normative Maßstäbe innerhalb der ECRL ......... 166

b) Widersprüchliche Wertungen zwischen ECRL und Datenschutzrecht

2. Inkohärenz mit Blick auf wirtschaftliche Ausrichtung der Diensteanbieter ..........................

III. Neutralität als prinzipiell unplausibler Maßstab . . . . . . . . . . 169

1. Neutrale Intermediäre? . . . . . . . . . . . . . . . . . . . . . . . . 169

2. Neutrale Technologie? ....................... 171

C. Unklare Zuordnung von Suchmaschinendiensten im System der Haftungsprivilegien ..........................

I. Gestaltungsmacht bei der Sortierung und Verfügbarmachung eines bedeutsamen Wissensbestandes ..................

1. Die Rolle von Suchmaschinendiensten bei der Verfügbarkeit von Wissen
a) Abhängigkeit des Wissensbestandes von dessen Auffindbarkeit
b) Organisation der Suchmaschinendienste

2. Einwirkungspotenziale der Suchmaschinenbetreiber ........

a) Konzeptionelle Gestaltungsmacht im Abgleich mit Kommunikationsdiensten .................

b) Konkrete Steuerungsfähigkeiten ............... 176

II. Rechtliche Zuordnung . ....................... 178

1. Rechtliche Unterscheidbarkeit von Suchmaschinen und Kommunikationsdiensten im Lichte des Privilegs für HostProvider 
a) Anknüpfungen am Gesetzestext $\ldots \ldots \ldots \ldots \ldots \ldots 178$

b) Unklare Rechtsprechung ................ 180

2. Primäre Zuordnung zu den Access- oder Caching-Diensten? 181

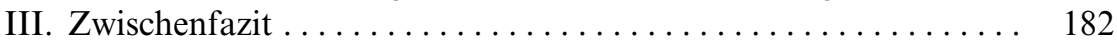

D. Fazit .................................... 183

Kapitel 3: Perspektiven des Haftungsrechts der Intermediäre: Die Störerhaftung und ihre Weiterentwicklung ...

$\S 9$ Normative Annäherung an das Problem der

Inanspruchnahme der Intermediäre als Gatekeeper . .

A. Die Inpflichtnahme der Intermediäre als Gatekeeper ......... 187

I. Gatekeeper .............................. 187

II. Das Spezifikum der rechtlichen Inpflichtnahme der Intermediäre als Gatekeeper ............................. 188

B. Analytischer Rahmen zur Bewertung von Gatekeeping-Regimen nach Kraakman ..................................... 189

I. Darstellung ................................ 190

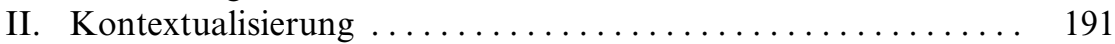

C. Fazit ......................................... 194

$\S 10$ Die Störerhaftung als kontextangemessenes Instrument des bürgerlichen Rechts zur Realisierung der Verantwortung der Intermediäre? . . . . . . . . . . . . . . . . . . . . . . 195

A. Grundlagen der Störerhaftung $\ldots \ldots \ldots \ldots \ldots \ldots \ldots \ldots \ldots . \ldots \ldots$

I. Zweck .................................... 196

II. Anwendungsbereich . . . . . . . . . . . . . . . . . . . . . . . 198

1. Allgemeines .............................. 198

2. Keine Haftung des Intermediärs als Täter . . . . . . . . . 198

3. Keine Subsidiarität gegenüber Ansprüchen gegen den Primärverursacher . . . . . . . . . . . . . . . . . . . . . 200

III. Voraussetzungen der Störerhaftung unter besonderer

Berücksichtigung der Intermediäre . . . . . . . . . . . . . 202

1. Relevante Störungshandlungen $\ldots \ldots \ldots \ldots \ldots \ldots \ldots . \ldots 202$

2. Prüfpflichten im Rahmen der Störerhaftung ........... 203

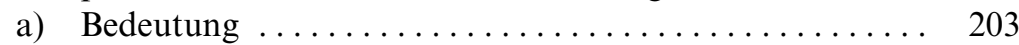

b) Übergeordnete Kriterien ...................... 204

aa) Grad der Betroffenheit der Rechtsgüter .......... 204

bb) Wirtschaftliche Zumutbarkeit bzw. Gefährdung des Geschäftsmodells 
cc) Gefahrgeneigtheit des Dienstes . . . . . . . . . . 205

dd) Sozialadäquanz $\ldots \ldots \ldots \ldots \ldots \ldots \ldots \ldots \ldots$

ee) Finanzielle Vorteile? ................ 206

c) Prüfprogramm für Kommunikationsdienste

bei Persönlichkeitsrechtsverletzungen ............. 207

aa) Initiierung des Prüfprogramms durch Notifizierung des Diensteanbieters .................... 208

(1) Szenario I: Keine „unschwere Erkennbarkeit““ der Rechtsverletzung . ................ 208

(2) Szenario II: „Unschwer erkennbare“ Rechtsverletzung auf Grundlage des behaupteten Sachverhalts .............. 210

bb) Pflicht zur Sachverhaltsermittlung ........... 211

cc) Mögliche Konsequenzen .................. 211

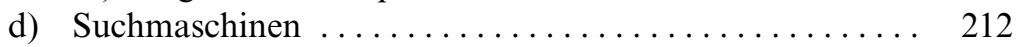

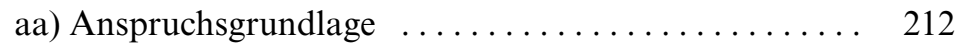

bb) Grundsätzliche Wertungen .............. 213

cc) Spezifische Prüfpflichten

für Suchmaschinenbetreiber ............... 214

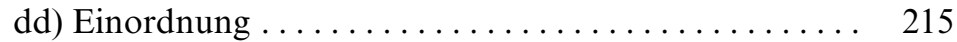

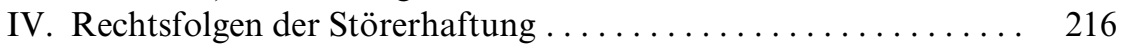

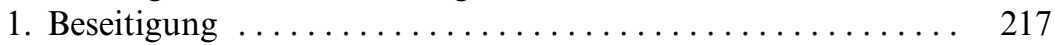

a) Allgemeine Bedeutung .................. 217

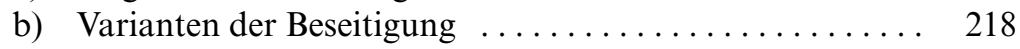

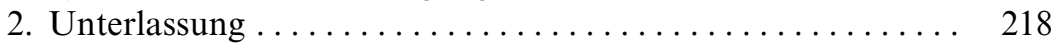

3. Erstbegehungs- und Wiederholungsgefahr; Verhältnis von Kenntnisnahme und Prüfpflicht ................... 219

4. Begrenzung der Störerhaftung durch das Verbot allgemeiner Überwachungspflichten ................. 220

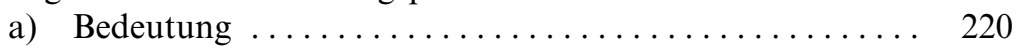

b) Allgemeine vs. „spezifische“ Überwachungspflichten .... 220

aa) Die „Kerntheorie“ ..................... 221

bb) Filtersysteme ........................ 224

5. Kosten der Inanspruchnahme des Störers . . . . . . . . . 225

V. Sperrung durch die Haftungsprivilegierungen? ........... 226

1. Unionsrechtliche Ausgangslage ................ 226

2. Übertragung auf den mitgliedstaatlichen Kontext ........ 226

B. Bewertung der Störerhaftung im Lichte der Verantwortung der Intermediäre . . . . . . . . . . . . . . . . . . . . . . . . . . . . . 229

I. Dogmatische Einwände gegen die Störerhaftung .......... 229

II. Problematische Übertragung richterlicher Aufgaben? ........ 231 


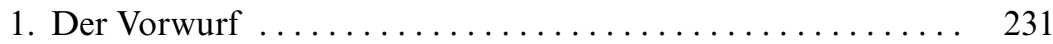

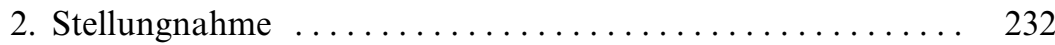

III. Die grundsätzlichen Vorzüge der Störerhaftung .......... 234

1. Flexible und kontextangemessene Ausformung der Verantwortung der Intermediäre $\ldots \ldots \ldots \ldots \ldots \ldots \ldots . \ldots 235$

2. Der Gedanke der Prozeduralisierung .............. 235

3. Grenzen des Prozeduralisierungsgedankens und ihre

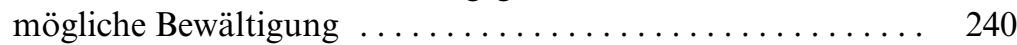

IV. Offene Flanken der Störerhaftung $\ldots \ldots \ldots \ldots \ldots \ldots \ldots .241$

1. Die Unangemessenheit der Anforderungen an eine die Prüfpflicht auslösenden Notifizierung ............... 242

a) Unangemessene Begründungslasten ............. 242

b) Anreiz zum Blindflug . . . . . ................ 244

c) Implikationen rechtlicher Bewertungen . . . . . . . 245

2. Ineffizienz wegen zu strenger Anforderungen an die Erstbegehungs- bzw. Wiederholungsgefahr? ........... 246

a) Der Ansatzpunkt der Kritik . ............... 246

b) Differenzierung zwischen Urheberrecht und Persönlichkeitsrecht . . . . . . . . . . . . . . . . 247

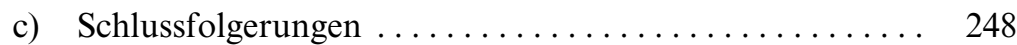

3. Ausschluss einer Schadensersatzhaftung; Verhältnis zum Verkehrspflichtenkonzept ................... 249

4. Zweifelhafte grundrechtliche Verortung $\ldots \ldots \ldots \ldots \ldots .252$

C. Fazit ................................... 254

$\S 11$ Folgerungen für das Recht der Haftung der Intermediäre .............................. 257

A. Die Bedeutung der Haftungsprivilegierungen für die Haftung der Intermediäre ................................ 257

B. Die Zwecke des Rechts der Haftungsprivilegierungen .......... 259

I. Blick zurück in die USA . . . . . . . . . . . . . . . . . . . . . . . 259

II. Modifizierung der Zwecke des Rechts der Haftungsprivilegierung durch Einführung einer „Good Samaritan“-Regelung ........ 260

C. Elemente einer Reform des Haftungsrechts der Intermediäre . . . . 262

I. Neukonzeption der Einteilung in spezifische Dienste ........ 263

II. Aufgabe der Unterscheidung zwischen aktiver und passiver Rolle .............................. 265

III. Elemente verbesserter Anreizstrukturen . . . . . . . . . . . . 267

1. Die Kenntnisnahme als Anknüpfungspunkt intermediärer Verantwortung im Haftungsrecht . . . . . . . . . . . . . . . 
a) Vermittlung eines Wissensstandes unterhalb positiver Kenntnis . . . . . . . . . . . . . . . . . . . . 268

b) Bezugspunkt der Kenntnis . . . . . . . . . . . . . . . . 269

2. Fortentwicklung der Substanziierungslasten im Rahmen der Störerhaftung . . . . . . . . . . . . . . . . . . . . . . . . . 270

3. Umgang der Diensteanbieter mit Notifizierungen ....... 271

D. Die Zukunft des Verbots allgemeiner Überwachungspflichten . . . 273

I. Auswirkungen eines verstärkten Anreizes zur privaten Kontrolle im Sinne einer „Good Samaritan"-Regel . . . . . . . . . . . . 274

II. Ausgestaltung der Reichweite des Verbots ............... 275

III. Zulässigkeit manueller Kontrollen .................. 276

F. Fazit .................................. 278

$\S 12$ Neuere sektorspezifische Zuschreibungen von

Verantwortlichkeit als Modifikation der Störerhaftung ..... 281

A. Die Modifizierung der Störerhaftung zur Durchsetzung des

Persönlichkeitsrechts durch das NetzDG ............... 281

I. Hintergrund ............................ 281

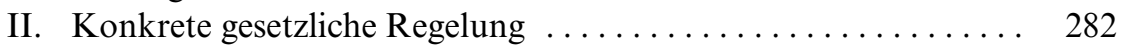

1. Allgemeines ............................ 282

2. Organisationspflichten ..................... 283

3. Bußgelder ............................. 283

III. Bewertung vor dem Hintergrund der Verantwortung

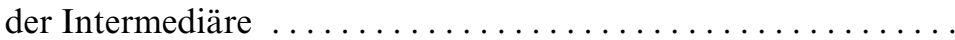

1. Positivierung der Verantwortung der Intermediäre durch Anknüpfung an die Störerhaftung . ............... 284

2. Keine „Privatisierung“ der Rechtsdurchsetzung ......... 286

3. Verkürzung der Rechte Einzelner durch Ausgestaltung des Beschwerdeverfahrens . . . . . . . . . . . . . . . . . . . 286

4. Verantwortungsbezogene Aspekte der Verhältnismäßigkeit 288

a) Gemeinwohlsicherung als legitimes Ziel .......... 288

b) Effektivität der Heranziehung der Intermediäre und Alternativen ......................... 288

c) Anreizstrukturen und Overblocking ............... 289

IV. Fazit zum NetzDG . . . . . . . . . . . . . . . . . . . . 292

B. Die Modifizierung der Wertungen der ECRL und der Störerhaftung zur Durchsetzung des Urheberrechts durch die DSM-RL . . . . . 294

I. Hintergrund ................................ 294

II. Konkrete gesetzliche Regelung . .................. 295

1. Etablierung der Primärhaftung über das Instrument der öffentlichen Wiedergabe 
2. Einschränkungen der Haftung . . . . . . . . . . . . . . 296

a) Obliegenheiten des Diensteanbieters .............. 296

aa) Anstrengungen zur Einholung einer Erlaubnis ..... 296

bb) Branchenübliche Anstrengungen zur

Nichtverfügbarkeit von Inhalten, Filterpflichten . . . 297

cc) Notice-and-Stay-Down . . . . . . . . . . . . . . . . . 299

b) Abgestufte Ausgestaltung

der Handlungsobliegenheiten $\ldots \ldots \ldots \ldots \ldots \ldots \ldots . \quad 300$

3. Nutzerrechte .......................... 301

III. Bewertung vor dem Hintergrund der Verantwortung

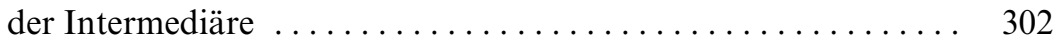

1. Differenzierte Positivierung der Verantwortung der Intermediäre . . . . . . . . . . . . . . . . . . . 302

2. Explizite Regelung der Nutzerrechte und Verhältnis zum Rechtsschutzinstrumentarium der Mitgliedstaaten ....... 303

3. Filterpflichten und ihre Konsequenzen ............. 304

a) Vorbemerkung: Die Konkretisierung der Anforderungen an Diensteanbieter in Abhängigkeit der technischen Entwicklung ................... 305

b) Verstoß gegen das Verbot allgemeiner Überwachungspflichten ............. 306

c) Versto $ß$ gegen grundrechtliche Garantien . ........ 307

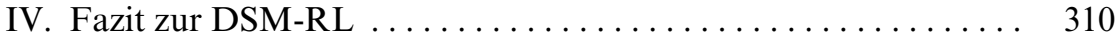

C. Fazit ................................. 310

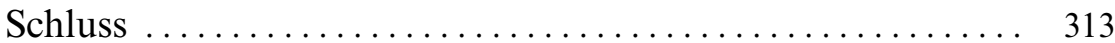

$\S 13$ Abschließende Betrachtung ................. 315

$\S 14$ Zusammenfassung $\ldots \ldots \ldots \ldots \ldots \ldots \ldots \ldots \ldots \ldots \ldots \ldots \ldots \ldots \ldots \ldots$

Literaturverzeichnis .............................. 325

Register ..................................... 349 



\section{Abkürzungsverzeichnis}

Hinweise: Bei unspezifischen Personenbezeichnungen wird auf die gleichzeitige Verwendung der femininen und maskulinen Form verzichtet. Beide Formen umfassen als generische Begriffe alle Geschlechtsidentitäten.

Alle Quellen des Internets wurden zuletzt am 14. Februar 2021 abgerufen.

$\begin{array}{ll}\text { a.F. } & \text { alte Fassung } \\ \text { Abs. } & \text { Absatz, Absätze } \\ \text { AcP } & \text { Archiv für die civilistische Praxis } \\ \text { AEUV } & \text { Vertrag über die Arbeitsweise der Europäischen Union } \\ \text { AfP } & \text { Zeitschrift für das gesamte Medienrecht } \\ \text { AG } & \text { Amtsgericht } \\ \text { AGB } & \text { Allgemeine Geschäftsbedingungen } \\ \text { Alt. } & \text { Alternative } \\ \text { AöR } & \text { Archiv des öffentlichen Rechts } \\ \text { ARSP } & \text { Archiv für Rechts- und Sozialphilosophie } \\ \text { Art. } & \text { Artikel } \\ \text { AtomG } & \text { Atomgesetz } \\ \text { Aufl. } & \text { Auflage } \\ \text { AVMD-RL } & \text { Richtlinie (EU) 2018/1808 des Europäischen Parlaments und des } \\ & \text { Rates vom 14. November 2018 zur Änderung der Richtlinie } \\ & \text { 2010/13/EU zur Koordinierung bestimmter Rechts- und Verwal- } \\ & \text { tungsvorschriften der Mitgliedstaaten über die Bereitstellung au- } \\ & \text { diovisueller Mediendienste (Richtlinie über audiovisuelle Medien- } \\ \text { BeckRS } & \text { dienste) im Hinblick auf sich verändernde Marktgegebenheiten } \\ \text { Beschl. } & \text { beck-online.RECHTSPRECHUNG } \\ \text { BGB } & \text { Beschluss } \\ \text { BGBl. } & \text { Bürgerliches Gesetzbuch } \\ \text { BGH } & \text { Bundesgesetzblatt } \\ \text { BT-Drucks. } & \text { Bundesgerichtshof } \\ \text { BVerfG } & \text { Bundestagsdrucksache } \\ \text { BVerfGE } & \text { Bundesverfassungsgericht } \\ \text { bzw. } & \text { Entscheidungen des Bundesverfassungsgerichts } \\ \text { C.D. Cal. } & \text { beziehungsweise } \\ \text { Cal. } & \text { United States District Court for the Central District of California } \\ \text { Cal.App. 4th } & \text { Supreme Court of California } \\ \text { Cal. Ct. App } & \text { California Appellate Reports, Fourth Series } \\ \text { CDA } & \text { California Court of Appeal } \\ \text { Cir. } & \text { Communications Decency Act (Title V, Telecommunications Act of } \\ \text { CR } & \text { 1996, 104th Congress, Public Law No. 104-104) } \\ & \text { United States Court of Appeals } \\ \text { Computer und Recht } \\ \end{array}$


D.D.C.

D. Mass.

D. Md.

D.N.J.

ders.

dies.

DMCA

DS-GVO

DS-RL

DSM-RL

E.D. Mo.

E.D. Pa.

E.D. Tex. ECRL

EGMR

EL

EMRK

Enforcement-RL

et al.

etc.

EU

EuGH

EurUP

EUV

EuZW

f., ff.

F.2d

F.3d

F.Supp.

F.Supp. 2d

F.Supp. 3d

FAZ

Florida Sup. Ct.

Fn.

GA
United States District Court for the District of Columbia

United States District Court for the District of Massachusetts

United States District Court for the District of Maryland

United States District Court for the District of New Jersey

derselbe

dieselbe(n)

Digital Millennium Copyright Act 1998 (105th Congress, Public Law No. 105-304)

Verordnung (EU) 2016/679 des Europäischen Parlaments und des

Rates vom 27. April 2016 zum Schutz natürlicher Personen bei der Verarbeitung personenbezogener Daten, zum freien Datenverkehr und zur Aufhebung der Richtlinie 95/46/EG

Richtlinie 95/46/EG des Europäischen Parlaments und des Rates vom 24. Oktober 1995 zum Schutz natürlicher Personen bei der Verarbeitung personenbezogener Daten und zum freien Datenverkehr Richtlinie (EU) 2019/790 des Europäischen Parlaments und des Rates vom 17. April 2019 über das Urheberrecht und die verwandten Schutzrechte im digitalen Binnenmarkt und zur Änderung der Richtlinien 96/9/EG und 2001/29/EG

United States District Court for the Eastern District of Missouri

United States District Court for the Eastern District of Pennsylvania

United States District Court for the Eastern District of Texas

Richtlinie 2000/31/EG des Europäischen Parlaments und des Rates vom 8. Juni 2000 über bestimmte rechtliche Aspekte der Dienste der Informationsgesellschaft, insbesondere des elektronischen Geschäftsverkehrs, im Binnenmarkt

Europäischer Gerichtshof für Menschenrechte

Ergänzungslieferung

Konvention zum Schutz der Menschenrechte und Grundfreiheiten

Richtlinie 2004/48/EG des Europäischen Parlaments und des Rates vom 29. April 2004 zur Durchsetzung der Rechte des geistigen

Eigentums

et alii

et cetera

Europäische Union

Gerichtshof der Europäischen Union

Zeitschrift für Europäisches Umwelt- und Planungsrecht

Vertrag über die Europäische Union

Europäische Zeitschrift für Wirtschaftsrecht

folgende

Federal Reporter, Second Series

Federal Reporter, Third Series

Federal Supplement

Federal Supplement, Second Series

Federal Supplement, Third Series

Frankfurter Allgemeine Zeitung

Supreme Court of Florida

Fußnote(n)

Generalanwalt, Generalanwältin 
GG

ggf.

GRCh

GRUR

GRUR Int

GWB

Hervorh.

HPflG

Hrsg.

Hs.

InfoSoc-RL

insb.

itrb

IuKDG

iVm

jurisPR-StrafR

jurisPR-WettbR

JurPC

$\mathrm{JuS}$

$\mathrm{JZ}$

$\mathrm{K} \& \mathrm{R}$

KG

KritV

LG

lit.

Ls.

LuftVG

M.D. Fla.

MMR

MMR-Beilage

MüKo BGB

MüKo StGB

MüKo ZPO

mwN

N.C. Ct. App.

N.D. Cal.

n.F.

N.Y. Sup. Ct.

NetzDG

NJOZ

NJW

NJW-RR

$\mathrm{Nr}$.

NStZ
Grundgesetz für die Bundesrepublik Deutschland

gegebenenfalls

Charta der Grundrechte der Europäischen Union

Gewerblicher Rechtsschutz und Urheberrecht

GRUR International Journal of European and International IP

Law

Gesetz gegen Wettbewerbsbeschränkungen

Hervorhebung

Haftpflichtgesetz

Herausgeber

Halbsatz

Richtlinie 2001/29/EG des Europäischen Parlaments und des Rates vom 22. Mai 2001 zur Harmonisierung bestimmter Aspekte des Urheberrechts und der verwandten Schutzrechte in der Informationsgesellschaft

insbesondere

IT-Rechtsberater

Gesetz zur Regelung der Rahmenbedingungen für Informationsund Kommunikationsdienste

in Verbindung mit

juris PraxisReport Strafrecht

juris PraxisReport Wettbewerbsrecht

Internet-Zeitschrift für Rechtsinformatik und Informationsrecht

Juristische Schulung

JuristenZeitung

Kommunikation \& Recht

Kammergericht

Kritische Vierteljahresschrift für Gesetzgebung und

Rechtsprechung

Landgericht

littera

Leitsatz

Luftverkehrsgesetz

United States District Court for the Middle District of Florida

Zeitschrift für IT-Recht und Recht der Digitalisierung

MultiMedia und Recht - Beilage

Münchener Kommentar zum Bürgerlichen Gesetzbuch

Münchener Kommentar zum Strafgesetzbuch

Münchener Kommentar zur Zivilprozessordnung

mit weiteren Nachweisen

North Carolina Court of Appeals

United States District Court for the Northern District of California

neue Fassung

New York Supreme Court

Netzwerkdurchsetzungsgesetz

Neue Juristische Online-Zeitschrift

Neue Juristische Wochenschrift

NJW Rechtsprechungs-Report Zivilrecht

Nummer

Neue Zeitschrift für Strafrecht 


\begin{tabular}{|c|c|}
\hline NVwZ & Neue Zeitschrift für Verwaltungsrecht \\
\hline NZKart & Neue Zeitschrift für Kartellrecht \\
\hline OLG & Oberlandesgericht \\
\hline OWiG & Ordnungswidrigkeitengesetz \\
\hline RG & Reichsgericht \\
\hline RGZ & Entscheidungen des Reichsgerichts in Zivilsachen \\
\hline RhPfVerfGH & Verfassungsgerichtshof Rheinland-Pfalz \\
\hline RL & Richtlinie \\
\hline $\mathrm{Rn}$. & Randnummer(n) \\
\hline RStV & Rundfunkstaatsvertrag \\
\hline RW & Rechtswissenschaft \\
\hline S. & Satz, Sätze; Seite(n) \\
\hline S.Ct. & Supreme Court Reporter \\
\hline S.D. N.Y. & United States District Court for the Southern District of New York \\
\hline S.E. $2 \mathrm{~d}$ & South Eastern Reporter, Second Series \\
\hline Sec. & Section \\
\hline So. $2 \mathrm{~d}$ & Southern Reporter, Second Series \\
\hline sog. & sogenannte $(\mathrm{n} / \mathrm{r})$ \\
\hline StGB & Strafgesetzbuch \\
\hline StPO & Strafprozessordnung \\
\hline StVG & Straßenverkehrsgesetz \\
\hline TDG & Teledienstegesetz \\
\hline TMG & Telemediengesetz \\
\hline u.a. & unter anderem \\
\hline U.S. & United States Reports \\
\hline UAbs. & Unterabsatz \\
\hline UrhG & Urheberrechtsgesetz \\
\hline Urt. & Urteil \\
\hline $\mathrm{v}$. & von, vom \\
\hline Var. & Variante \\
\hline Verf. & Verfasser \\
\hline Vgl. & vergleiche \\
\hline vs. & versus \\
\hline WL & Westlaw \\
\hline WRP & Wettbewerb in Recht und Praxis \\
\hline z.B. & zum Beispiel \\
\hline ZaöRV & Zeitschrift für ausländisches öffentliches Recht und Völkerrecht \\
\hline ZD & Zeitschrift für Datenschutz \\
\hline ZEuP & Zeitschrift für Europäisches Privatrecht \\
\hline ZfPW & Zeitschrift für die gesamte Privatrechtswissenschaft \\
\hline ZHR & Zeitschrift für das gesamte Handelsrecht und Wirtschaftsrecht \\
\hline $\mathrm{ZPO}$ & Zivilprozessordnung \\
\hline ZRP & Zeitschrift für Rechtspolitik \\
\hline ZUM & Zeitschrift für Urheber- und Medienrecht \\
\hline ZUM-RD & Zeitschrift für Urheber- und Medienrecht - Rechtsprechungsdienst \\
\hline
\end{tabular}




\section{Einführung}





\section{Einleitung}

\section{A. Haftung für Inhalte Dritter als Kernfrage des Verhältnisses von Internet und Regulierung}

Wer sich mit dem Verhältnis von Recht und Internet befasst, kommt an John Perry Barlow kaum vorbei. 1996 formulierte der US-amerikanische Poet, Essayist und Bürgerrechtler seine monumentale „Unabhängigkeitserklärung des Cyberspace“, in der er sich an die Regierungen der alten Welt, „giants of flesh and steel", wandte und diese aufforderte, die Welt des Cyberspace nicht zu betreten. „You are not welcome among us. You have no sovereignty where we gather. "' Seine Haltung steht sinnbildlich für die euphorische Vorstellung der damaligen Zeit, mit dem und im Internet entstehe eine neue Gesellschaftsordnung jenseits territorialstaatlicher Ordnungen. „Cyberspace does not lie within your borders. “2 Diese neue Ordnung sei mit dem veralteten Souveränitätskonzept staatlicher Ordnungen unvereinbar. Deren auf Recht und Zwang beruhendes Herrschaftssystem könne im Cyberspace keine Anwendung finden. „Your legal concepts of property, expression, identity, movement, and context do not apply to us. They are all based on matter, and there is no matter here. ${ }^{\text {"3 }} \mathrm{Im}$ Cyberspace werde eine neue Gemeinschaft entstehen. „We will create a civilization of the Mind in Cyberspace. May it be more humane and fair than the world your governments have made before. ${ }^{" 4}$

Barlows Erklärung hallt bis heute nach. Sie bietet noch immer einen Reibungspunkt für all jene, die sich mit dem Internet und seiner normativen Struktur befassen. Die Erklärung buchstabierte eine zu der Zeit weit verbreitete Haltung aus, die den Cyberspace als etwas völlig Neues und Einzigartiges betrachtete, für das alte Denkweisen, Kategorien und vor allen Dingen das Recht nicht mehr passten. Dieser „Internet Exceptionalism“ drang

\footnotetext{
${ }^{1}$ Barlow, Electronic Frontier Foundation (08.02.1996), www.eff.org/de/cyberspace-ind ependence.

${ }^{2}$ Barlow, Electronic Frontier Foundation (08.02.1996), www.eff.org/de/cyberspace-ind ependence.

${ }^{3}$ Barlow, Electronic Frontier Foundation (08.02.1996), www.eff.org/de/cyberspace-ind ependence.

${ }^{4}$ Barlow, Electronic Frontier Foundation (08.02.1996), www.eff.org/de/cyberspace-ind ependence.
} 
bis in juristische Diskurse vor und prägte diese. ${ }^{5}$ Vielfach war die Forderung anzutreffen, das Recht müsse diese Außergewöhnlichkeit des Cyberspace anerkennen und diesen schützen. Nicht selten war und ist die Rede von einer „Konstitutionalisierung ohne Staat“. 6

Weniger geläufig als Barlows Erklärung selbst ist der Grund bzw. Anlass, der Barlow dazu brachte, diesen Text überhaupt zu schreiben. Anfang des Jahres 1995 hatten US-Politiker begonnen, sich der in ihren Augen unzulässigen Verbreitung pornografischer bzw. „obszöner“ Inhalte im Internet zu widmen und hierauf eine Antwort zu formulieren. Nach einem kontroversen Gesetzgebungsprozess kam es zur Verabschiedung des „Telecommunications Act“, der einen als „Communications Decency Act“ übertitelten Abschnitt beinhaltete. ${ }^{7}$ Dieser verbot unter Strafe u.a. die Verbreitung von ,indecent material" über das Internet. ${ }^{8}$ Wenig überraschend wurde dieser Passus in den USA als erheblicher Eingriff in die traditionell kaum beschränkbare Redefreiheit nach dem ersten Zusatzartikel der US-Verfassung angesehen. Dass das Gesetz dennoch verabschiedet und von US-Präsident Clinton am 8. Februar 1996 unterzeichnet wurde, versetzte Barlow so sehr in Rage, dass dieser eine E-Mail an einige hunderte Bekannte verschickte. Diese E-Mail wurde zu seiner erwähnten Erklärung. Der CDA kann somit als Auslöser für eine Protestbewegung angesehen werden, deren Forderungen Barlow in seiner Erklärung auf einen programmatisch-ideologischen Ansatz verdichtete. ${ }^{9}$

Die Regelungen wurden wenig überraschend sofort gerichtlich angegriffen und vom US-amerikanischen Supreme Court gut ein Jahr später tatsächlich für verfassungswidrig erklärt und aufgehoben. ${ }^{10}$ Der CDA verschwand

${ }^{5}$ Prominent geworden ist insbesondere der Beitrag von Johnson und Post, die die Entgrenzung des Internets und die damit einhergehende Schwächung territorialer Regulierung tradierter Prägung diskutierten: „The rise of an electronic medium that disregards geographical boundaries throws the law into disarray by creating entirely new phenomena that need to become the subject of clear legal rules but that cannot be governed, satisfactorily, by any current territorially based sovereign“, Johnson/Post, Stanford Law Review 48 (1996), 1367 (1375). Vgl. auch American Civil Liberties Union v. Reno, 929 F.Supp. 824, 844 (E.D. Pa. 1996): „The Internet is [...] a unique and wholly new medium of worldwide human communication."

${ }^{6}$ Teubner, ZaöRV 2003, 1 (5).

${ }^{7}$ 104th Congress, Public Law No. 104-104, Title V. Vgl. hierzu auch Kosseff, The Twenty-Six Words that Created the Internet, S. $58 \mathrm{ff}$.

${ }^{8}$ 104th Congress, Public Law No. 104-104, Title V Sec. 502: „Whoever [...] in interstate or foreign communications [...] by means of a telecommunications device knowingly [...] makes, creates, or solicits, and [...] initiates the transmission of any [...] communication which is $[\ldots]$ indecent $[\ldots]$ shall be fined $[. .$.$] . “$

${ }^{9}$ Vgl. Higgins, Electronic Frontier Foundation (23.02.2016), www.eff.org/de/deeplinks/ 2016/02/webs-first-blackout-protest-cda-20-years-later; Kosseff, The Twenty-Six Words that Created the Internet, S. $77 \mathrm{f}$.

${ }^{10}$ Vgl. Reno v. ACLU, 521 U.S. 844, 874 ff. (1997). 
damit jedoch keineswegs vollständig von der Bildfläche der Gesetzgebung. Nicht Gegenstand der Kritik und dieser Aufhebungsentscheidung war nämlich eine andere Regelung des CDA. Dessen $\S 230,{ }^{11}$ der vom Gesetzgeber den Untertitel „Online Family Empowerment" erhielt, ${ }^{12}$ enthält Aussagen darüber, welche Maßnahmen von bestimmten Diensteanbietern zu treffen sind, um die Verbreitung von „offensive material“" zu verhindern und inwiefern sie hierfür in Haftung genommen werden können. Diese Regelung war auf relativ unscheinbare, kaum kontroverse Weise als Anpassungsvorschlag in das Verfahren zur Verabschiedung des „Telecommunications Act“ eingebracht worden und wurde von der Öffentlichkeit zunächst kaum beachtet. ${ }^{13}$ Etwa zur selben Zeit begannen in Europa parallel gelagerte Diskussionen. ${ }^{14}$ In Deutschland nahm die Popularität des Internets in Wirtschaft und Gesellschaft spürbar zu; gleichzeitig nahm man allerdings auch schon früh eine „unübersehbare Flut von Rechtsproblemen“ wahr. ${ }^{15}$ Die Politik erkannte einen Handlungsbedarf, der auch die Frage umfasste, in welcher Art und Weise Diensteanbieter für Inhalte in Verantwortung genommen werden können. Nach einem ebenfalls nicht komplikationsfreien Gesetzgebungsprozess kam es zur Verabschiedung des IuKDG, das mit dem TDG die Haftung von bestimmten Diensteanbietern adressierte. ${ }^{16}$

Die Frage nach der Verantwortlichkeit für Verhalten Dritter im Internet ist damit in etwa so alt wie das Internet selbst, ${ }^{17}$ jedenfalls sofern man auf den Zeitraum abstellt, als es zur Technologie der Massen avancierte. Fast schon historischen Wert hat in diesem Zusammenhang das strafgerichtliche Verfahren vor dem AG München, bei dem der Geschäftsführer des in Deutschland ansässigen Providers CompuServe erstinstanzlich wegen Verbreitung pornographischer Schriften schuldig gesprochen wurde, weil entsprechende Inhalte, die Dritte im Netzwerk der CompuServe in den USA hochgeladen hatten, in Deutschland abrufbar waren. ${ }^{18}$ Die damals kein ganzes Jahr alte Regelung des $\S 5$ Abs. 2 TDG a.F., wonach Diensteanbieter für fremde Inhalte nicht verantwortlich sind, wenn sie von diesen keine Kenntnis haben oder ihnen die Verhinderung der Nutzbarkeit des betreffenden Inhalts nicht

\footnotetext{
${ }^{11} 47$ U.S.C. $\$ 230$.

${ }^{12}$ 104th Congress, Public Law No. 104-104, Title V Sec. 509.

${ }^{13} \mathrm{Vgl}$. Kosseff, The Twenty-Six Words that Created the Internet, S. $67 \mathrm{f}$.

${ }^{14}$ Vergleichbare Regelungen existieren in verschiedener Form in sehr vielen Rechtssystemen, vgl. Secretary-General of the OECD, The Economic and Social Role of Internet Intermediaries, S. 73.

${ }^{15}$ Spindler, ZUM 1996, 533 (533).

${ }^{16}$ Näher dazu noch unten $\S 4$ B. I.

${ }^{17}$ Vgl. Sobola, in: Auer-Reinsdorff/Conrad, Handbuch IT- und Datenschutzrecht, §42

${ }^{18}$ AG München, Urt. v. 28.05.1998 - 8340 Ds 465 Js 173158/95, NJW 1998, 2836 ff. CompuServe.
} Rn. 6. 
möglich oder zumutbar ist, ließ das Gericht nicht zur Anwendung kommen. ${ }^{19}$ Das Urteil hatte zwar keinen Bestand, ${ }^{20}$ führte jedoch nach Bekanntwerden nicht nur zu Protesten vor Goethe-Instituten in den USA und Vorwürfen gegenüber CompuServe eines „Kniefalls“ vor der deutschen Justiz, ${ }^{21}$ sondern auch zur Verlagerung von Servern aus Deutschland ins Ausland..$^{22}$ Dennoch zeigte der Fall sehr früh nach Erlass des TDG a.F. auf, dass die Frage nach einer Privilegierung von Diensteanbietern gegenüber der Inanspruchnahme durch Betroffene einer Rechtsverletzung und deren genauen Voraussetzungen eine zentrale Rolle bei der Ausgestaltung der rechtlichen Erfassung des Internets und seiner gesellschaftlichen Relevanz einnehmen würden.

Ein Vierteljahrhundert später ist in diesem Regelungsgebiet keineswegs Klarheit geschweige denn Ruhe eingekehrt. Einerseits haben sich im Markt der Diensteanbieter einige wenige Akteure als besonders einflussreich etablieren können, denen kraft ihrer Funktion und Reichweite nicht selten eine „strukturierende“ Funktion bei der öffentlichen Kommunikation zugeschrieben wird. ${ }^{23}$ Dies betrifft etwa Unternehmen wie Google oder Facebook. Andererseits wird aus rechtspolitischer Perspektive ein nicht unerheblicher Teil der Verantwortung für das zunehmend als problematisch empfundene Klima des Diskurses im Internet den Diensteanbietern ebenso zugewiesen wie die scheinbar kaum beherrschbare Verbreitung urheberrechtswidriger Inhalte über Dienste wie YouTube. Nicht selten ist von der Gefährdung der Demokratie durch diese Unternehmen die Rede..$^{24}$ Damit ist schon auBerrechtlich ein gewisses Spannungsfeld grob skizziert. In diesem bewegt sich auch die Verhandlung der juristischen Frage, ob und inwieweit eine Verantwortung dieser Unternehmen für von Dritten erzeugte Inhalte besteht bzw. begründbar ist. Versteht man die Berücksichtigung des außerrechtlichen Kontexts als eine Aufgabe der Rechtswissenschaft, ${ }^{25}$ liegt es nahe, den rechtlichen Regeln, die die Haftung und damit auch die Verantwortung der Diensteanbieter entscheidend prägen, gerade mit Blick auf ihre sozialen Auswirkungen nachzugehen.

${ }^{19}$ Vgl. AG München, Urt. v. 28.05.1998 - 8340 Ds 465 Js 173158/95, NJW 1998, 2836 (2839f.) - CompuServe.

${ }^{20}$ Vgl. LG München I, Urt. v. 17.11.1999 - 20 Ns 465 Js 173158/95, NJW 2000, 1051 f. CompuServe.

${ }^{21}$ Sieber, JZ 1996, 429 (429).

${ }^{22}$ Vgl. Sieber, Verantwortlichkeit im Internet, S. 3.

${ }^{23}$ Schulz, Rechtsstandort Hamburg (16.06.2016), www.rechtsstandort-hamburg.de/fil eadmin/rechtsstandort-hamburg/docs/2017-06-16-rede-schulz-wolfgang.pdf. In den Augen von Pasquale, in: Saetnan/Schneider/Green, The Politics of Big Data, S. 110 (122), übernehmen ,vast conglomerates like Facebook and Google effectively [...] the role of global communication regulators".

${ }^{24}$ Vgl. Zuboff, The New York Times, 26.01.2020.

${ }^{25}$ Vgl. nur Hoffmann-Riem, Zeitschrift für Rechtssoziologie 2018, 20 (22 f.); Grimm, in: Hassemer/Hoffmann-Riem/Limbach, Grundrechte und soziale Wirklichkeit, S. 39 (42 ff.). 


\section{B. Gegenstand}

Gegenstand dieser Untersuchung sind primär diejenigen Rechtsregeln, die wesentliche Aussagen über die Verantwortung der Diensteanbieter für Inhalte Dritter beinhalten. Das betrifft zunächst die mögliche Haftung für die Verbreitung solcher Inhalte. Mit Haftung ist hier im Wesentlichen die Unterlassungs-, aber auch die Schadensersatzhaftung gemeint. Hier stehen die Privilegierungen einer solchen Haftung im Fokus. Diese sind im Wesentlichen in den Art. $12 \mathrm{ff}$. ECRL und den $\$ \S 7 \mathrm{ff}$. TMG niedergelegt, wobei die Privilegierung für „Host-Provider“ gemäß Art. 14 ECRL und $§ 10$ TMG eine besonders gewichtige Rolle einnimmt. Neben diesen Bestimmungen wird auBerdem die Entwicklung zentraler vergleichbarer Vorschriften in den USA beleuchtet, die teilweise bereits einleitend erwähnt wurden. Der Gesamtbestand dieser Regeln wird im Folgenden auch als das Recht der Haftungsprivilegierungen bezeichnet.

Neben den Haftungsprivilegierungen ist aber auch der Haftungsgrund in den Blick zu nehmen. Dieser ist in der EU primär im Recht der Mitgliedstaaten geregelt. In Deutschland geht es hier insbesondere um die allgemeinen Grundsätze der Störerhaftung, wie sie vom BGH in jüngerer Zeit mit Blick auf die Diensteanbieter bedeutend weiterentwickelt wurden. Generell stehen Haftungsgrund und Haftungsprivilegierung in diesem Kontext nicht unabhängig nebeneinander, sondern nehmen auch jenseits ihrer Kausalbeziehung - ohne Haftung keine Haftungsprivilegierung - in vielfältiger Weise aufeinander Einfluss und Bezug. Aufgrund der Vielfalt der möglichen Formen der Verletzung verschiedener Rechtsgüter, die durch die Geschäftsmodelle der Diensteanbieter ermöglicht oder erleichtert werden, verspricht der Abgleich zweier Teilbereiche der Rechtsordnung, die strukturell vor vergleichbaren Herausforderungen stehen, aber dennoch unterschiedlichen Eigenlogiken unterworfen sind, ein Kontrast- und damit Erkenntnispotenzial. Neben dem Persönlichkeitsrecht dient als zweites Anschauungsbeispiel daher der urheberrechtliche Kontext der Inpflichtnahme von Diensteanbietern.

\section{Ziel}

Ausgehend vom Verständnis der Rechtswissenschaft als Versuch, nicht nur das geltende Recht zu beschreiben, sondern aufzuzeigen, wie das Recht sein könnte, ${ }^{26}$ ergeben sich danach die folgenden konkreten Forschungsfragen, deren Beantwortung diese Untersuchung gewidmet ist: Spiegelt das geltende

${ }^{26}$ „Was Rechtens sei (quid sit iuris), d.i. was die Gesetze an einem gewissen Ort und zu einer gewissen Zeit sagen oder gesagt haben, kann er wohl noch angeben; aber, ob das, was 
Recht die besondere Rolle, die die Diensteanbieter heutzutage einnehmen, noch angemessen wider? Welche Spielräume lassen die geltenden Regeln, diese besondere Rolle besser zu reflektieren? Ist es damit Anspruch und Haltung gleichermaßen, diese besondere Rolle angemessen zu würdigen, liegt es nahe, ein besonderes Augenmerk auf die Position der Dienstenutzerinnen zu richten. Diese verfügen oftmals nicht über die Mittel, ihre Interessen gegenüber global agierenden Unternehmen effektiv wahrzunehmen. ${ }^{27}$ Ziel dieser Untersuchung ist es also, einen kritisch-konstruktiven Beitrag zur Fortentwicklung des Rechts zu leisten und hierbei besonders die Position der schwächeren Seite eines ungleichgewichtigen Verhältnisses im Blick zu behalten.

sie wollten, auch recht sei, und das allgemeine Kriterium, woran man überhaupt Recht sowohl als Unrecht (iustum et iniustum) erkennen könne, bleibt ihm wohl verborgen, wenn er nicht eine Zeitlang jene empirischen Prinzipien verläßt, die Quellen jener Urteile in der bloßen Vernunft sucht (wiewohl ihm dazu jene Gesetze vortrefflich zum Leitfaden dienen können), um zu einer möglichen positiven Gesetzgebung die Grundlage zu errichten. Eine bloß empirische Rechtslehre ist [...] ein Kopf, der schön sein mag, nur schade! daß er kein Gehirn hat", Kant, Metaphysische Anfangsgründe der Rechtslehre, S. 37 f. (Hervorh. im Original). $\mathrm{Zu}$ den epistemologischen Konsequenzen eines solchen Verständnisses vgl. Metzger, Extra legem, intra ius, S. $48 \mathrm{f}$.

${ }^{27}$ Vgl. Di Fabio, Grundrechtsgeltung in digitalen Systemen, S. 79. Dieser Befund ist umso bedeutsamer, je eher es um Fragen mit Relevanz für die Persönlichkeitsentfaltung geht, vgl. Kube, in: Isensee/Kirchhof, Handbuch des Staatsrechts, §91 Rn. 7. 


\section{Gang der Untersuchung}

Das erste Kapitel stellt die historische Entwicklung des Rechts der Haftungsprivilegierungen dar. Dabei wird der Weg von den US-amerikanischen Regelungen zu denjenigen in der ECRL und im TMG beleuchtet. Ziel dieses Kapitels ist es, einen umfassenden Überblick über die einschlägigen rechtlichen Grundlagen zu liefern, die eine Aussage über die privilegierte Haftung der Intermediäre für von Dritten begangene Rechtsverletzungen treffen, sowie insoweit eine erste kritische Einordnung vorzunehmen.

Das zweite Kapitel verfolgt das Ziel, die problematischen und grundlegenden Aspekte der überkommenen Rechtslage zu kartieren. Erforderlich hierfür ist zunächst eine phänomenologische Zuordnung der Diensteanbieter. Diese erfolgt in drei Schritten: zum einen ist eine funktionale Einordnung vorzunehmen. Zum anderen liefert eine Analyse der begrifflichen Zugriffe auf die im Zentrum dieser Untersuchung stehenden Akteure wertvolle Einsichten. Hier zeigt sich, dass insbesondere bei technisch konstituierten Entitäten die Wahl der Begrifflichkeiten eine entscheidende Rolle dabei spielen kann, wie wir diese Akteure wahrnehmen. Daraus folgen nicht selten Rückschlüsse normativer Natur. Ziel des Kapitels ist es schließlich, solche normativen Rückschlüsse im geltenden Recht aufzuzeigen und kritisch zu hinterfragen.

Im Anschluss an diese allgemeineren und grundlegenden Perspektiven geht das dritte Kapitel auf die Perspektiven einer weiteren künftigen Ausgestaltung ein, die ein kritischer Blick auf Haftungsgrund und Haftungsprivilegierung aufzeigen kann. Hier erweist sich insbesondere das Institut der Störerhaftung als grundsätzlich geeignetes Instrument, um das Spannungsfeld zwischen der Durchsetzung von Schutzrechten und betroffenen gegenläufigen Interessen aufzulösen. Allerdings ist auch die Störerhaftung im Detail nicht gänzlich frei von kritischen Aspekten, die es herauszustellen gilt. Eine Synthese des so entwickelten Blicks auf die Haftungsprivilegierungen und die Störerhaftung ermöglicht es, konkrete Folgerungen sowohl auf nationaler wie auf europäischer Ebene zu benennen und insoweit das Reformpotenzial dieser Regelungen aufzuzeigen. In diesem Zusammenhang ist auch auf neuere gesetzliche Entwicklungen in diesem Kontext einzugehen, die sowohl die Durchsetzung von Persönlichkeitsrechten als auch des Urheberrechts betreffen. Ersteres betrifft das deutsche NetzDG, letzteres die jüngste Reform des unionalen Urheberrechts. 
Abschließende Betrachtungen und eine Zusammenfassung der gefundenen Ergebnisse beenden die Abhandlung. 


\section{Kapitel 1 \\ Entwicklung des Rechts der Haftungsprivilegierungen}

Grundlage jeder Auseinandersetzung mit der Sachgerechtigkeit rechtlicher Regeln ist eine Analyse ihres normativen Bestands. Zweck dieses Kapitels ist es also, die rechtliche Ausgestaltung der Haftungsprivilegierungen in ihrer Entwicklung hin zum heutigen status quo darzustellen. Die Haftungsprivilegierung für die Diensteanbieter als Grundmodus - mit der Bejahung einer Haftung in Ausnahmefällen - ist historisch gesehen kein Zufall, sondern beruht auf Prämissen und Einsichten, die im Wesentlichen aus der Frühphase der Nutzung des Internets durch die breite Gesellschaft stammen. Die Idee, für bestimmte Fälle Transporteure von Dritten erzeugter Informationen bzw. Inhalte von einer Haftung hierfür auszunehmen, wird historisch oftmals den USA zugeordnet. Dort brachte der Gesetzgeber mit §230 CDA erstmals den Gedanken der Haftungsfreistellung zum Ausdruck. Die europäische und deutsche Rechtslage - also die ihrerseits von der ECRL determinierten $\S \S 7 \mathrm{ff}$. TMG - sind geschichtlich ohne diese Norm sowie das in den USA für das Urheberrecht erstmals entwickelte Notice-and-TakedownVerfahren (\$512 DMCA) nicht erklärbar. Dies ist Anlass genug, diese beiden US-amerikanischen Regelungskomplexe an den Beginn dieses Kapitels zu stellen.

Dabei wird nicht übersehen, dass die Entwicklung der US-amerikanischen Regeln zur Haftung und ihrer europäischen Pendants sich nicht zwingend nur nicht linear darstellen lassen, sondern dass sie sich vielfach zeitlich überlagert haben. Das ändert allerdings nichts an dem Umstand, dass viele der hier relevanten Dienste in den USA beheimatet sind und die Entwicklung in den USA schon deshalb stets einen - obschon nicht immer quantifizierbaren - Einfluss auf die europäische und deutsche Perspektive ausübte und die Herangehensweise an diese Dienste zumindest bislang prägte. Gerade der Umstand, dass im Kontext dieser Untersuchung relevante Argumentationsmuster sowohl im US-amerikanischen als auch im europäischen Kontext anzutreffen sind, ist hierfür ein Beleg. Ziel ist es also, die Entwicklung in den USA zu skizzieren ( $(3)$, bevor der Blick dann auf die ECRL und das TMG gelenkt wird (§4). Eine kritische Durchsicht der dargestellten Regelungskomplexe mit Blick auf gemeinsame Motive und Unterschiede schließt das Kapitel ab (§5). 



\section{USA}

Gegenstand dieses Abschnitts ist es, die Entwicklung der Verantwortungszuweisung an die Diensteanbieter nachzuzeichnen. Ausgangspunkt hierbei sind zentrale Regelungen in den USA. Der bereits erwähnte $\$ 230$ CDA gilt als Ursprungsform einer Haftungsprivilegierung für Diensteanbieter des Internets (A.). Auch das bereits angesprochene Kontrastpotenzial des Urheberrechts lässt sich in den USA an einer konkreten Norm festmachen (B.). Das in $\$ 512$ DMCA niedergelegte Notice-and-Takedown-Verfahren setzt einen Kontrapunkt zur rigiden Immunisierung in $\$ 230 \mathrm{CDA}$ und etabliert so einen Korridor an möglichen Verantwortungszuweisungen, in den sich historisch auch die Regelungen auf Unionsebene sowie in Deutschland einordnen lassen.

\section{A. $\S 230$ Communications Decency Act}

Die zentrale Aussage der Norm lautet:

„(c) Protection for ,Good Samaritan“ blocking and screening of offensive material

(1) Treatment of publisher or speaker

No provider or user of an interactive computer service shall be treated as the publisher or speaker of any information provided by another information content provider.

(2) Civil liability

No provider or user of an interactive computer service shall be held liable on account of -

(A) any action voluntarily taken in good faith to restrict access to or availability of material that the provider or user considers to be obscene, lewd, lascivious, filthy, excessively violent, harassing, or otherwise objectionable, whether or not such material is constitutionally protected; or

(B) any action taken to enable or make available to information content providers or others the technical means to restrict access to material described in paragraph (1).

$[\ldots]$

(e) Effect on other laws

(1) No effect on criminal law

Nothing in this section shall be construed to impair the enforcement of section 223 or 231 of this title, chapter 71 (relating to obscenity) or 110 (relating to sexual exploitation of children) of title 18, or any other Federal criminal statute." 
Im Folgenden soll der Versuch unternommen werden, die Entstehung und die unterschiedlichen Auslegungsfähigkeiten dieser weitreichenden Privilegierung, die auch schon als „Core pillar of internet freedom" des Internets und als „Law that gave us the modern internet" ${ }^{\text {"2 }}$ bezeichnet wurde, herauszuarbeiten (I.), um anschließend ihre Handhabung kritisch darzustellen (II.), die Regelung in ihren verfassungsrechtlichen Kontext einzuordnen (III.) sowie neuere Entwicklungen aufzuzeigen (IV.).

\section{Entstehungsgeschichte}

Praktisch mit Beginn der Nutzung des Internets als Medium zur Information und zur Verbreitung von Meinungen durch die breite Masse der Bevölkerung behielten die entsprechenden Diensteanbieter, insbesondere die Betreiber von Foren, sich in ihren AGB vor, bestimmte Inhalte zu entfernen bzw. nicht $\mathrm{zu}$ veröffentlichen, die gegen ihre Regelwerke verstoßen. ${ }^{3} \mathrm{Zu}$ Beginn der 1990er Jahre stellte sich für die Gerichte in den USA die Frage, inwiefern die entsprechenden Diensteanbieter für verleumderische (,defamation“) oder sonstige grob anstößige Inhalte (,obscenity“) auf ihren Plattformen zur Verantwortung gezogen werden können.

Die Gerichte behalfen sich hierbei mit einer Einordnung der Diensteanbieter in die etablierte, im analogen Kontext entwickelte und maßgeblich in das Common Law verweisende ${ }^{4}$ Dogmatik des ersten Zusatzartikels der USBundesverfassung, nach der für die haftungsrechtliche Bewertung zwischen den Kategorien ,publisher“, „distributor“ und „common carrier“ zu unterscheiden sei. ${ }^{5}$ Hiernach haften ,publisher“, z.B. klassische Printmedien oder Buchverlage, für Inhalte Dritter vollumfänglich, also auch ohne Kenntnis des Inhalts, während ein „distributor“ (z.B. Büchereien oder Verlage) nur bei positiver Kenntnis oder fahrlässiger Unkenntnis der beanstandeten Inhalte haftet $^{6}$ und ein „common carrier“ (in erster Linie Telekommunikationsun-

${ }^{1}$ Jeong, The Verge (01.08.2017), www.theverge.com/2017/8/1/16072680/cda-230-stop-e nabling-sex-traffickers-act-liability-shield-senate-backpage.

${ }^{2}$ Khanna, The Atlantic (12.09.2013), www.theatlantic.com/business/archive/2013/09/t he-law-that-gave-us-the-modern-internet-and-the-campaign-to-kill-it/279588.

${ }^{3}$ Vgl. für die USA Ehrlich, Berkeley Technology Law Journal 17 (2002), 401 (402); zu Einzelheiten im deutschen Recht vgl. Bräutigam/Sonnleithner, in: Hornung/Müller-Terpitz, Rechtshandbuch Social Media, S. 35 (54, 60 f.).

${ }^{4}$ Vgl. Davis, New Mexico Law Review 32 (2002), 75 (78 ff.).

${ }^{5}$ Ehrlich, Berkeley Technology Law Journal 17 (2002), 401 (403); vgl. auch Barnes v. Yahoo!, Inc., 570 F.3d 1096, 1104 (9th Cir. 2009).

${ }^{6}$ Vgl. Sylvain, Connecticut Law Review 50 (2018), 203 (233 mit Fn. 146). So hatte der Supreme Court bereits 1959 die Pönalisierung eines Buchhändlers wegen des Verkaufs eines Buches beanstandet, das die Gerichte als „obszön“ angesehen hatten, ohne dass dem Buchhändler selbst Kenntnis dieses Buches unterstellt werden konnte. Gerade das Abse- 
ternehmen) als technische Infrastrukturanbieter kaum einem Haftungsrisiko unterliegt. ${ }^{7}$ Es überrascht nicht, dass insbesondere die Haftung als „distributor" den Ausgangspunkt der möglichen Inpflichtnahme von Intermediären darstellte. Denn bereits in der Entscheidung Smith v. California des US Supreme Court von 1959, in der es um die Haftung eines Buchverkäufers für den Besitz von Büchern mit „obszönen“ Inhalten ging, ${ }^{8}$ sind die Konfliktlinien zu erkennen, die auch die heutige Diskussion um den richtigen regulativen Umgang mit den Diensteanbietern prägen. Einerseits gilt insoweit die prinzipielle Wertung, dass ein rechtlicher Vorwurf für einen Verbreiter dritterzeugter Inhalte wegen dieser nur dann haltbar sei, wenn eine Kenntnis dieser Inhalte vorliege. Andererseits gilt die pragmatische Einsicht, dass dieses Prinzip zugleich Anreiz sein kann, die Augen vor der Gewahrwerdung von Inhalten zur Vermeidung von rechtlichen Risiken zu verschließen. ${ }^{9}$

Während vereinzelte Gerichtsentscheidungen dazu tendierten, Diensteanbieter, die keine gesonderte Prüfung von Inhalten vornehmen, als „distributor" anzusehen und damit einer materiell beschränkten Haftung zu unterwerfen, ${ }^{10}$ löste Stratton Oakmont v. Prodigy Services Co. eine kleine Revolution aus, die den Erlass des $§ 230$ CDA zur Folge hatte. ${ }^{11}$ Die Wertpapierbank Stratton Oakmont hatte den Dienstanbieter Prodigy wegen verleumderischer Aussagen in Anspruch genommen, die in dessen Forum „Money Talk" geäußert worden waren. Der New York Supreme Court hielt Prodigy für einen „publisher“, weil Prodigy öffentlich behauptete, die Inhalte in sei-

hen von der Kenntnis des Inhalts des Buches als rechtlichen Anknüpfungspunkt hielt das Gericht mit der Rede- und Pressefreiheit für unvereinbar, vgl. Smith v. California, 361 U.S. 147, 152 f. (1959). Das Gericht legte hier indes nicht abstrakt fest, welche Maßstäbe an die Kenntnis positiv anzulegen seien, um eine solche Haftung zu begründen, vgl. Smith v. California, 361 U.S. 147, 154 (1959). In einem der Entscheidungsmehrheit beipflichtenden Votum hielt der Richter Frankfurter jedoch fest, dass die Mehrheitsentscheidung keineswegs dahin zu deuten sei, dass ,a bookseller who insulates himself against knowledge [...] is thereby free to maintain an emporium for smut", Smith v. California, 361 U.S. 147, 161 (1959). Die „Smith Rule“ gilt nach wie vor, und zwar auch für Diensteanbieter des Internets, Webseitenbetreiber und andere Personen bzw. Organisationen, die dritterzeugte Inhalte verbreiten, vgl. Kosseff, The Twenty-Six Words that Created the Internet, S. 27.

${ }^{7}$ Ehrlich, Berkeley Technology Law Journal 17 (2002), 401 (403), mwN.

${ }^{8} \mathrm{Vgl}$. oben $\S 3$ Fn. 6 .

${ }^{9}$ Vgl. Kosseff, The Twenty-Six Words that Created the Internet, S. 28.

${ }^{10}$ Vgl. für den hiesigen Kontext wohl erstmals Cubby v. CompuServe, 776 F.Supp. 135, 140 f. (S.D. N.Y. 1991); Zittrain, Harvard Journal of Law \& Technology 19 (2006), 253 (258), weist allerdings darauf hin, dass das Gericht hier in der faktischen Annahme irrte, eine Kontrolle der hochgeladenen Inhalte vorab sei nicht möglich; vgl. ferner Davis, New Mexico Law Review 32 (2002), 75 (83f.).

${ }^{11}$ Kosseff, The Twenty-Six Words that Created the Internet, S. 3: „In the two decades since Section 230's passage, those twenty-six words have fundamentally changed American life." 
nem Angebot zu kontrollieren und hierzu eine Filtersoftware einsetzte. ${ }^{12}$ Der Einsatz dieser Software und ihre Entscheidung sei gleichbedeutend mit einer inhaltlichen Stellungnahme zu den entsprechenden Inhalten. Eine Entscheidung über den Verbleib eines Inhalts in dem Forum sei somit als ein Äquivalent zu einer redaktionellen Entscheidung anzusehen. ${ }^{13}$ Dass diese Bemühungen, die Foren von rechtswidrigen bzw. verleumderischen Inhalten freizuhalten, hier nicht erfolgreich waren, gereichte Prodigy somit gerade zum Nachteil. Weil damit aber ein finanziell nicht unerheblicher Anreiz bestand, Bemühungen solcher Art einzustellen oder herunterzufahren, um in den Genuss der beschränkten Haftung zu kommen, schritt der US-amerikanische Gesetzgeber ein und verabschiedete u.a. §230 CDA. ${ }^{14}$

Mit dieser Regelung, konkret mit dem oben zitierten Unterabschnitt $\S 230(c)(1)$, wird vorgesehen, dass „,provider“ oder Nutzer eines „,interactive computer service“ nicht als „publisher“ oder „speaker“ im Hinblick auf Inhalte Dritter anzusehen sind. Damit sollte eine vollumfängliche Haftung im oben erläuterten Sinne ausgeschlossen werden. Mit §230(c)(2) wurde ferner die Haftung für jegliche Inhalte ausgeschlossen, soweit freiwillig ,in good faith" Maßnahmen zum Blockieren oder Filtern beanstandungswürdiger Inhalte getroffen wurden. ${ }^{15}$ Die Haftungsprivilegierung sollte damit die Entwicklung und den Einsatz effektiver Filtertechnologien ermöglichen und hierzu einen positiven Anreiz setzen. ${ }^{16}$ Die Diensteanbieter sollten gerade

${ }^{12}$ Vgl. Stratton Oakmont v. Prodigy Services Co., 1995 WL 323710 1, 4 (N.Y. Sup. Ct. 1995).

${ }^{13}$ Vgl. Stratton Oakmont v. Prodigy Services Co., 1995 WL 323710 1, 4 (N.Y. Sup. Ct. 1995).

${ }^{14}$ Vgl. zur Gesetzgebungsgeschichte der Regelung im Nachgang der Entscheidung Stratton Oakmont v. Prodigy ausführlich Kosseff, The Twenty-Six Words that Created the Internet, S. $59 \mathrm{ff}$.

${ }^{15}$ Die Norm stellt dabei entscheidend auf die Perspektive des Diensteanbieters selbst ab (,[...] that the provider [...] considers [...]"). Dies kann als eine zentrale Weichenstellung im US-amerikanischen Recht bezeichnet werden, weil den Diensten damit ein erheblicher Spielraum eingeräumt wird, eigene private Standards für zulässige Kommunikation zu entwickeln. Dies lässt sich zwar durch den Zusammenhang zu dem vom Gesetzgeber beabsichtigten Einsatz von Filtertechnologien erklären, die selbst auch von dem jeweiligen Diensteanbieter entwickelt oder zumindest hinsichtlich der inhaltlichen Ausrichtung der Technologie in irgendeiner Form ausgewählt werden. Über die Legitimation solcher Diensteanbieter, von gesetzlichen Maßstäben völlig unabhängige Präferenzen zu entwickeln oder festzulegen, ist damit jedoch noch kein Urteil getroffen.

${ }^{16}$ Vgl. Ehrlich, Berkeley Technology Law Journal 17 (2002), 401 (405 f.); Citron/Wittes, Fordham Law Review 86 (2017), 401 (404); Kuczerawy, Intermediary Liability and Freedom of Expression in the EU, S. 68. Vgl. außerdem $\$ 230$ (b)(4) CDA: „It is the policy of the United States to remove disincentives for the development and utilization of blocking and filtering technologies [...]"; dies soll allerdings nur im Hinblick auf die elterliche Kontrolle über den Zugang ihrer Kinder zu unangemessenen Inhalten gelten. 
dazu motiviert werden, Technologien einzusetzen, um „offensive material“ zu blockieren bzw. zu filtern. ${ }^{17}$ Sie sollten zum "guten Samariter“ zum Schutze von Persönlichkeitsrechten werden, wie die Überschrift des entsprechenden Unterabschnitts des $\S 230 \mathrm{CDA}$ plakativ verdeutlicht. ${ }^{18}$ Es wäre daher unzutreffend, dem US-Gesetzgeber die Absicht zu unterstellen, mit der Norm eine pauschale Haftungsfreistellung von Diensten, die rechtswidrige Inhalte Dritter tolerieren oder gar fördern, um ihrer selbst willen bezweckt zu haben. ${ }^{19}$ Bei der Abwägung der Redefreiheit und gegenläufigen Rechtspositionen bzw. sonstigen, die Redefreiheit beschränkenden Handlungsverboten lässt sich damit keine abstrakte normative Aussage über das Rangverhältnis treffen. Gerade dies ist jedoch in der Handhabung der Norm durch die Gerichte oftmals eingetreten. Denn wie im Folgenden aufgezeigt werden soll, verstanden die Gerichte den Zweck der Regelung überwiegend darin, einen „hands off"-Ansatz zu bevorzugen bzw. nur hierin einen Grund für deren Immunisierung zu sehen.

\section{Auslegung von $\$ 230$ CDA durch die Gerichte}

Ziel dieses Abschnitts ist es, die wesentlichen Aspekte und Entwicklungsschritte der Anwendung von $\$ 230$ CDA durch die Gerichte in den USA herauszuarbeiten. In der Gesamtschau wird deutlich, dass die Entscheidungspraxis nahezu durchweg providerfreundlich ausfiel und die Gerichte sich von der ursprünglichen Intention des Gesetzgebers distanzierten.

\section{Keine Differenzierung zwischen „Publisher" und „Distributor" führt zur} Immunisierung auch bei Kenntnis

Die erste maßgebliche und bis heute die Rechtsprechung prägende Entscheidung ist Zeran v. $A O L,{ }^{20}$ die als Ausgangspunkt der extensiven Auslegung des $\$ 230$ CDA angesehen werden kann. ${ }^{21}$ Der Kläger des Verfahrens sah sich einem „Shitstorm“22 ausgesetzt, nachdem in einem Forum von AOL der Verkauf von Bekleidung angepriesen wurde, die einen terroristischen Anschlag in den USA verherrlichte und die seine Telefonnummer als Kontaktmöglichkeit angab. ${ }^{23}$ Trotz einer sich hieran anschließenden längeren

${ }^{17}$ Vgl. Citron/Wittes, Fordham Law Review 86 (2017), 401 (404).

${ }^{18}$ Vgl. zur Forderung der Übernahme dieses Prinzips in der EU noch unten $\S 11$ B. II.

${ }^{19}$ Vgl. Citron/Wittes, Fordham Law Review 86 (2017), 401 (406).

${ }^{20}$ Zeran v. AOL, Inc., 129 F.3d 327 (4th Cir. 1997).

${ }^{21}$ Vgl. Lukmire, NYU Annual Survey of American Law 66 (2010), 371 (384): ,by far the most influential case on section 230"; vgl. auch Tremble, Fordham Law Review 86 (2017), 825 (843 ff.).

${ }^{22}$ www.duden.de/rechtschreibung/Shitstorm.

${ }^{23}$ Vgl. Zeran v. AOL, Inc., 129 F.3d 327, 329 (4th Cir. 1997). 
Korrespondenz zwischen dem Kläger und AOL vermochte das Unternehmen nicht, die immer wieder aktualisierten verleumderischen Inhalte zu beseitigen bzw. deren weitere Veröffentlichung zu unterbinden. Der Kläger nahm deshalb AOL wegen „negligence“, also der Verletzung einer (deliktischen) Sorgfaltspflicht in Anspruch. ${ }^{24}$ Das Gericht führte aus, dass $\$ 230$ CDA es verbiete, AOL als „publisher“ eines Inhalts Dritter anzusehen..$^{25}$ Dies gelte nicht nur im Anwendungsbereich eines Anspruchs wegen „defamation“, sondern auch im Allgemeinen, also auch bezogen auf andere Klagegründe. ${ }^{26}$ Die vom Kläger vorgetragene Rechtsauffassung, dass §230 CDA sich nur auf die „publisher liability“ beziehe, die Haftung als „distributor" davon jedoch unberührt bleibe, lehnte das Gericht in folgenreicher Weise ab. ${ }^{27}$ Die Haftung als Verbreiter sei lediglich eine Unterkategorie der Haftung als „publisher“. ${ }^{28}$ Der „distributor" sei - jedenfalls im materiellen Anwendungsbereich des Äußerungsrechts (,defamation law“) - wie derjenige zu behandeln, der einen Inhalt veröffentliche, weil und soweit er seine Infrastruktur (,facilites") zur Verbreitung von Inhalten zur Verfügung stelle...$^{29}$ Dahinter stehe die Erwägung, dass andernfalls das Risiko rechtlicher Konflikte in einen Abschreckungseffekt (,,chilling effect“) münden würde; Diensteanbieter hätten keinen Anreiz mehr, Informationen überhaupt zu veröffentlichen. ${ }^{30}$ Das Gericht kam so zum Ergebnis, der vom Kläger geltend gemachte

${ }^{24}$ Die Wahl dieses Klagegrundes und nicht etwa „defamation“ sollte gerade das Risiko vermeiden, dass das Begehren als unter §230(c) CDA unzulässige Behandlung von AOL als „speaker“ bewertet werden könnte, vgl. Kosseff, The Twenty-Six Words that Created the Internet, S. 85.

${ }^{25}$ Vgl. Zeran v. AOL, Inc., 129 F.3d 327, 332 ff. (4th Cir. 1997).

${ }^{26},,[\ldots] \S 230$ creates a federal immunity to any cause of action that would make service providers liable for information originating with a third-party user", Zeran v. AOL, Inc., 129 F.3d 327, 330 (4th Cir. 1997). Dass das Gericht damit ein recht kühne These aufstellt, die eine möglichst breite Wirkung erzeugen soll, zeigt Lukmire, NYU Annual Survey of American Law 66 (2010), 371 (395 ff.), auf und weist zudem nach, dass das Gericht so weit gar nicht hätte gehen müssen, um im konkreten Fall AOL dennoch am Privileg teilhaben zu lassen.

${ }^{27}$ Die vom Gericht gegebene Begründung lässt die Haftungsprivilegierung insgesamt in nur zwei Bereichen als unanwendbar erscheinen, nämlich in den Bereichen des geistigen Eigentums und des Bundesstrafrechts. Insgesamt ist Zeran als Ausgangspunkt der expansiven Handhabung des Haftungsprivilegs anzusehen, vgl. Tremble, Fordham Law Review 86 (2017), 825 (843 ff.).

${ }^{28}$ Damit setzt sich das Gericht in seiner Argumentationsstruktur in Widerspruch, da es einerseits gerade keine spezifische Konstellation der „,defamation“ behandeln will, andererseits aber in dieser dogmatischen Kategorie verbleibt, vgl. Lukmire, NYU Annual Survey of American Law 66 (2010), 371 (396).

${ }^{29}$ Vgl. Zeran v. AOL, Inc., 129 F.3d 327, 332 (4th Cir. 1997); vgl. auch Barrett v. Rosenthal, 51 Cal.Rptr.3d 55, 65 f. (Cal. 2006).

${ }^{30}$ Vgl. Zeran v. AOL, Inc., 129 F.3d 327, 331 (4th Cir. 1997). 
Anspruch behandele AOL als „publisher“ und sei daher von §230(c) CDA gesperrt. Damit spielte es für das Gericht keine Rolle, ob der Diensteanbieter Kenntnis von einem rechtsverletzenden Inhalt hat oder nicht. Auch im ersteren Falle ist die Haftung nach $\S 230$ (c) CDA ausgeschlossen. Anstrengungen zur eigenständigen Kontrolle des eigenen Einflussbereichs, wie es die Bezeichnung als „Good Samaritan“-Privileg nahelegen, braucht der Diensteanbieter danach überhaupt nicht mehr zu leisten. ${ }^{31}$

In Doe v. $A O L^{32}$ nahm die Mutter eines elf Jahre alten Jungen AOL in Anspruch, nachdem sie das Unternehmen darauf hingewiesen hatte, dass ein Nutzer Bild- und Videoaufnahmen u.a. ihres Sohnes, die die beiden und andere Jungen bei sexuellen Handlungen zeigten, aufgenommen und bei AOL vermarktet hatte. AOL unterband diese Aktivitäten trotz der Hinweise der Klägerin nicht. Ihre Klage gegen AOL hatte bis hin zum Florida Supreme Court keinen Erfolg, weil die Gerichte entsprechende Ansprüche als von $\S 230$ (c) CDA gesperrt ansahen.$^{33}$ Der Florida Supreme Court verwies in seiner Begründung auf Zeran und machte sich das Argument zu eigen, eine Differenzierung zwischen der Haftung als „publisher“ und als „distributor“ sei mit Blick auf $\S 230$ CDA nicht geboten. ${ }^{34}$ Neben der Tragik des Falles, die die Gerichte insoweit nicht dazu bringen konnte, eine Ausnahme zur Privilegierung zu konstruieren, ist an der Entscheidung insbesondere die abweichende Meinung durch Richter Lewis bemerkenswert. Zum einen verweist er auf die bereits angesprochene Ratio des Gesetzgebers, eine Privilegierung nur für solche Dienste vorzusehen, die ein "good-faith monitoring program“ einsetzen, um die Verbreitung von ,illicit and improper materials“" zu verhindern. ${ }^{35}$ Zum anderen kritisiert er die ausbleibende Differenzierung zwischen der Haftung als „publisher“ oder „distributor“, wie sie hier skizziert wurde, als prinzipiellen Widerspruch zur Intention des Gesetzgebers: ${ }^{36}$

„In Zeran and the majority view here, however, this statement that an ISP shall not be treated as a ,publisher or speaker" of third-party information has been interpreted to mean not only that an ISP can never be subject to liability [...] as a ,publisher ${ }^{6}$ of third-party information [...], but also that an ISP can never be subject to liability based upon its own patently irresponsible role as a distributor who has allegedly been given actual notice of materials published on its service [...] This flies in the face of the very purpose of the Communications Decency Act. [...] What conceivable good could a statute purporting to promote ISP self-policing efforts do if, by virtue of the courts' interpretation of that statute, an ISP which is specifically made aware of child pornography being distributed [...]

${ }^{31}$ Vgl. Sylvain, Connecticut Law Review 50 (2018), 203 (213); Citron/Wittes, Fordham Law Review 86 (2017), 401 (409).

${ }^{32}$ Doe v. AOL, Inc., 783 So.2d 1010 (Florida Sup. Ct. 2001).

${ }^{33}$ Vgl. Doe v. AOL, Inc., 783 So.2d 1010, 1013 ff. (Florida Sup. Ct. 2001).

${ }^{34}$ Vgl. Doe v. AOL, Inc., 783 So.2d 1010, 1017 f. (Florida Sup. Ct. 2001).

${ }^{35}$ Doe v. AOL, Inc., 783 So.2d 1010, 1019 (Florida Sup. Ct. 2001).

${ }^{36}$ Vgl. Doe v. AOL, Inc., 783 So.2d 1010, 1023 ff. (Florida Sup. Ct. 2001). 
through [...] its service, may, with impunity, do absolutely nothing, and reap the economic benefits flowing from the activity? ${ }^{637}$

Als Konsequenz dieser beiden Entscheidungen immunisierte §230(c) CDA die Diensteanbieter nicht nur für die Haftung als „publisher“, also ohne Kenntnis eines Inhalts, sondern auch für die Fälle, in denen Kenntnis des von $\S 230$ CDA grundsätzlich erfassten Inhalts unzweifelhaft besteht. ${ }^{38}$

\section{Das ,Information Content Provider"-Kriterium und die Anforderungen an eine haftungsbegründende „,material contribution“}

$\S 230$ (c)(1) CDA befreit von der Haftung nur insoweit, als es um Inhalte von „another information content provider" geht. ${ }^{39}$ Hat derjenige, der sich auf die Privilegierung beruft, den Inhalt selbst ganz oder teilweise erstellt, ist somit fraglich, ob das Privileg des $\S 230$ CDA Anwendung finden kann. Die Frage stellt sich umso dringlicher, als Intermediäre im Lauf der Zeit ihre Geschäftsmodelle und Angebote immer wieder fortentwickelt und verändert haben. Gerade die Präsentation ihres Angebots unter Einbeziehung von „Usergenerated content", aber auch die Art und Weise der Erhebung von Informationen bei ihren Nutzern lässt somit die Frage aufkommen, ob die strenge binäre Einordnung einer Information bzw. eines Inhalts als ausschließlich nutzergeneriert oder eben durch den Intermediär selbst erstellt bzw. veranlasst sich im Lichte des $\$ 230 \mathrm{CDA}$ aufrechterhalten lässt. Jedenfalls lag und liegt es für von bestimmten Inhalten Betroffene im Sinne einer Beeinträchtigung von geschützten Rechten nahe, bei der Inanspruchnahme von Diensteanbietern diese als Ersteller ihres Angebots darzustellen, um damit das Kriterium der Personenverschiedenheit zwischen Diensteanbieter und Ersteller des Inhalts auszuhebeln und die Privilegierung entfallen zu lassen.

Zwar hatte Fair Housing Council v. Roommates.com als eine der wenigen prominenten Entscheidungen in den USA, die die Anwendung von $\S 230$ CDA ablehnten,$^{40}$ statuiert, dass das Privileg nur insoweit gelte, als ein Diensteanbieter rein passiv Inhalte anbiete, die vollständig von einem Dritten erstellt wurden. ${ }^{41}$ Daraus folge, dass eine Suchbörse für Mitbewohner sich

${ }^{37}$ Doe v. AOL, Inc., 783 So.2d 1010, 1024 f. (Florida Sup. Ct. 2001).

${ }^{38}$ Das bezeichnet Sylvain, Connecticut Law Review 50 (2018), 203 (233), als positivrechtliche Reform der bereits im tradierten common law angelegten Differenzierung zwischen ,publishers“ und „distributors“.

${ }^{39} \S 230$ (f)(3) CDA definiert den „Information content provider“ als „,any person or entity that is responsible, in whole or in part, for the creation or development of information provided through the Internet or any other interactive computer service."

${ }^{40}$ Vgl. noch FTC v. Accusearch, 570 F.3d 1187, 1197 ff. (10th Cir. 2009); dort auch ausführlich zur Frage der Verantwortlichkeit für einen Inhalt im Sinne der Definition eines „Information Content Provider" in §230(f)(3) CDA.

${ }^{41}$ Vgl. Fair Housing Council v. Roommates.com, 521 F.3d 1157, 1162 f. (9th Cir. 2008). 
dann nicht auf $\S 230$ CDA berufen könne, wenn diese selbst Informationen von ihren Nutzern erhebt ${ }^{42}$ und diese Erhebung zur Bedingung der Nutzung ihrer Dienste macht ${ }^{43}$ und damit eine ,material contribution“ $\mathrm{zu}$ dem rechtswidrigen Inhalt leiste. ${ }^{44}$ Das Gericht selbst suchte in seiner Entscheidungsbegründung jedoch, diese Aussage auf die Fälle zu beschränken, in denen die Diensteanbieter rechtswidrige Inhalte provozierten (,encourage illegal content"). ${ }^{45}$

Diese Entscheidung, selbst nicht frei von argumentativen Inkonsistenzen, ${ }^{46}$ blieb jedoch ein Einzelfall. ${ }^{47}$ Die Voraussetzung einer ,material contribution" wurde bemüht, um Internetseiten aus dem Privileg des $§ 230$ CDA herausfallen zu lassen, sobald diese die Erstellung von Inhalten durch Dritte fördern oder gar nur erlauben. In Hill v. Stubb wies der North Carolina Court of Appeals dieses Ansinnen jedoch in einem Fall ab und forderte für das Bejahen einer „material contribution“ im eben erläuterten Sinne, dass die betroffene Internetseite den Inhalt eines Dritten effektiv kontrolliert oder dass sie anderweitig Handlungen vornimmt, die praktisch gesprochen die Herstellung eines illegalen Inhalts sicherstellen (,ensure“) ${ }^{48}$ In Jones v. The

${ }^{42}$ Bedeutsam ist insoweit auch die Feststellung, dass es in Bezug auf denselben Inhalt mehrere „provider" geben könne, nämlich die Nutzer einerseits und Roommates.com andererseits, vgl. Fair Housing Council v. Roommates.com, 521 F.3d 1157, 1165 (9th Cir. 2008).

${ }^{43}$ Vgl. Fair Housing Council v. Roommates.com, 521 F.3d 1157, 1165 ff. (9th Cir. 2008). Das Abfragen von Informationen stellt demnach eine inhaltsbezogene Tätigkeit dar, die sich in der Darstellung des konkreten, vom Nutzer angegeben Inhalt niederschlägt. Der abweichenden Meinung, die eine viel engere Interpretation des „development“ vorzieht, entgegnet die entscheidungstragende Mehrheit geradezu plakativ: „The projectionist in the theater may push the last button before a film is displayed on the screen, but surely this doesn't make him the sole producer of the movie“, Fair Housing Council v. Roommates.com, 521 F.3d 1157, 1166 f. (2008).

${ }^{44}$ Vgl. Fair Housing Council v. Roommates.com, 521 F.3d 1157, 1167 f. (9th Cir. 2008). In Carafano v. Metrosplash.com, Inc., 339 F.3d 1119, 1124 (2003), hatte das Gericht noch apodiktisch festgestellt, dass kein Nutzerprofil eines sozialen Mediums einen Inhalt im Sinne von $§ 230$ CDA aufweisen könne, bis ein Nutzer diesen erstelle. Der Diensteanbieter könne daher nie selbst der Information Content Provider im Sinne der Vorschrift sein. Dieser Haltung stellt sich die jüngere Entscheidung von 2008 nunmehr entgegen.

${ }^{45}$ Fair Housing Council v. Roommates.com, 521 F.3d 1157, 1175 (9th Cir. 2008).

${ }^{46}$ Vgl. dazu Tremble, Fordham Law Review 86 (2017), 825 (849, 854 ff.).

${ }^{47}$ Vgl. auch Tremble, Fordham Law Review 86 (2017), 825 (850 ff.).

${ }^{48}$ Vgl. Hill v. StubHub, 727 S.E.2d 550, 561 (N.C. Ct. App. 2012). Unklar bleibt hier, was mit „effectiv(ely) control“ überhaupt gemeint sein soll. Denkbar wären faktische Kontrollmöglichkeiten vor der Veröffentlichung eines Inhalts, also die Veröffentlichung eines Inhalts durch den Diensteanbieter vorbehaltlich einer (redaktionellen?) Prüfung. Faktisch wird so für diesen eine Rolle gefordert, die derjenigen des Dritten/Nutzers praktisch gleichkommt, um das Privileg des $\$ 230$ CDA fallenzulassen. Eine solche Gleichstellung ist aber im Wortlaut der Norm in keiner Weise angelegt, jedenfalls nicht in dem Sinne, dass diese 
Dirty nahm ein Anbieter am Haftungsprivileg teil, der sich zum Ziel gesetzt hatte, „dirt“, also verleumderisches oder anrüchiges Material jedweder Art zu veröffentlichen. Dafür wollte er Nutzer einladen, ebensolche Inhalte einzusenden, woraufhin der Betreiber ausgewählte Einsendungen versehen mit eigenen Kommentaren veröffentlichte. ${ }^{49}$ Diese Auswahl an eingesendeten Inhalten Dritter könne, so der Court of Appeals for the Sixth Circuit, nicht als „material contribution“ angesehen werden, weil genau dies der traditionellen Tätigkeit eines „publishers“ entspreche. ${ }^{50}$ Obwohl alle äußeren Anzeichen des Designs der Website darauf angelegt waren, persönlichkeitsrechtsverletzende Inhalte zum Zwecke des Abrufs zu speichern, fordere das Formular zum Hochladen von solchen Inhalten die Nutzer lediglich dazu auf, Neuigkeiten mitzuteilen (,tell us what's happening“) und könne daher nicht als eine ,material contribution“ angesehen werden..$^{51}$

Zwar setzten die verschiedenen obergerichtlichen Entscheidungen unterschiedliche Akzente, während im Schrifttum oftmals jene gerichtlichen Ansätze zur Verantwortungszuweisung an die Diensteanbieter besonders kritisch bewertet wurden. ${ }^{52}$ Dennoch lässt sich als Resümee festhalten, dass die Abgrenzung zwischen nutzergenerierten Inhalten und solchen Inhalten, die auf Veranlassung des Providers selbst erstellt wurden, äußerst streng und eher kleinteilig durch die Gerichte vorgenommen wurde. ${ }^{53}$

\section{Negierung des ,Treatment"-Kriteriums}

$\S 230$ CDA verbietet es, Diensteanbieter als „publisher or speaker“ eines Inhalts „zu behandeln“. Rein grammatikalisch betrachtet kann eine Inanspruchnahme eines Diensteanbieters damit dann erfolgversprechend sein, wenn die Begründung des Anspruchs gerade nicht dazu führt, dass der

Gleichstellung für eine Haftung des Providers erforderlich wäre. §230(f)(3) CDA spricht insofern eine klare Sprache: eine teilweise Verantwortlichkeit genügt, um als „Information content provider“ zu gelten. Wieso dann erst eine sinngemäß als vollständig zu verstehende Kontrolle die Privilegierung beseitigt, erschließt sich nicht ohne Weiteres.

${ }^{49}$ Vgl. Jones v. Dirty World Entertainment Recordings, 755 F.3d 398, 402 f. (6th Cir. 2014).

${ }^{50}$ Vgl. Jones v. Dirty World Entertainment Recordings, 755 F.3d 398, 416 (6th Cir. 2014).

${ }^{51}$ Vgl. Jones v. Dirty World Entertainment Recordings, 755 F.3d 398, 416 (6th Cir. 2014).

${ }^{52}$ Kosseff, The Twenty-Six Words that Created the Internet, S. 166, sieht etwa in Fair Housing Council v. Roommates.com, 521 F.3d 1157 (9th Cir. 2008) ein Anzeichen für die „Erosion“ des in $§ 230$ CDA niedergelegten Prinzips. Die Entscheidung markiere einen Wendepunkt, da die Gerichte seitdem restriktiver bei der Gewährung des Haftungsprivilegs entschieden, vgl. Kosseff, The Twenty-Six Words that Created the Internet, S. 180; vgl. auch Goldman, Tulane Journal of Technology and Intellectual Property 20 (2017), 1 (6): „perhaps the most significant remaining incursion into Zeran's defense-favorable immunity. “

${ }^{53}$ Vgl. auch Citron/Wittes, Fordham Law Review 86 (2017), 401 (408). 
Diensteanbieter als „publisher or speaker“ „behandelt“ wird. Eine Reihe von Klägern versuchten genau dies zu begründen, indem sie sich auf die Verletzung behaupteter Schutzpflichten durch die Diensteanbieter beriefen, die - so die Argumentation - normativ eine andere Ebene betreffen. Die Gerichte lehnten solche Ansprüche gegen die Diensteanbieter jedoch gerade mit der Begründung ab, die Begehren würden diese als „,publisher“ im Sinne von $\$ 230$ (c)(1) CDA behandeln, weil eben nur diese Inhalte entfernten. ${ }^{54}$

Soweit Gerichte vereinzelt doch annahmen, eine Klage gegen einen Diensteanbieter „,behandele“ diesen nicht als „publisher“, lagen diesen Fällen jedoch durchweg Fallkonstellationen zugrunde, die kaum als verallgemeinerungsfähig bezeichnet werden können und denen damit kein übergreifender, interpretationsleitender Charakter zugeschrieben werden kann. So lehnte etwa der Court of Appeals for the Ninth Circuit in Doe v. Internet Brands $^{55}$ zwar die Privilegierung aus $\S 230 \mathrm{CDA}$ ab. Hier wich das faktische Geschehen allerdings deutlich von den „klassischen“ Fällen einer möglichen Haftung von Diensteanbietern für Inhalte Dritter ab. Denn hier hatte nicht ein Dritter (als Primärverursacher) einen Inhalt selbst erstellt. Vielmehr hatte die Klägerin eine Vermittlungsplattform für Models in Anspruch genommen, nachdem Dritte über diesen Dienst ihre Kontaktdaten herausgefunden und infolgedessen Straftaten gegen ihre Person begangen hatten, obwohl der Diensteanbieter von der Gefährlichkeit der Dritten und früherer ähnlicher Vorfälle wusste. Das Gericht stützte sich hier maßgeblich auf die Erwägung, dass die von dem Dienst unterlassene Warnung nur durch diesen selbst hätte erfolgen können, sodass insoweit kein drittgenerierter Inhalt vorgelegen hätte. Dies habe zur Folge, dass auch die Ratio von $\$ 230$ CDA nicht greife. ${ }^{56}$ Obgleich damit das Gericht eine äußer(st)e Grenze für das Haftungsprivileg anerkannte, nämlich die Begehung einer eigenen Pflichtverletzung durch den Diensteanbieter, kann die Entscheidung daher nicht als prinzipieller Umschwung bei der Auslegung von §230 CDA im Rahmen der geläufigen äuBerungsrechtlichen Ansprüche angesehen werden.

In Hassell v. Bird ließ eine Rechtsanwältin eine Verfügung gegenüber einer ehemaligen Klientin, verleumderische Bewertungen bei dem Bewertungsdienst Yelp zu unterlassen, gegenüber Yelp als nicht am Verfahren beteiligte

${ }^{54}$ Vgl. Barnes v. Yahoo!, Inc., 570 F.3d 1096, 1103 (9th Cir. 2009); vgl. auch Jane Doe No. 1 v. Backpage.com, 817 F.3d 12, 20 (1st Cir. 2016). Abgesehen von der Tautologie dieses Arguments - wer welche Pflichten zum Entfernen von Inhalten hat, ist ja gerade Gegenstand der juristischen Auseinandersetzung - weisen die entsprechenden Diensteanbieter jedoch zunehmend Elemente der Moderation bzw. der (reaktiven) Überwachung ihrer Sphären jedenfalls anhand ihrer eigenen „Gemeinschaftsstandards“ auf, die sie bisweilen in die Nähe eines klassischen „publisher“ rücken lassen.

${ }^{55}$ Doe No. 14 v. Internet Brands, Inc., 824 F.3d 846 (9th Cir. 2016).

${ }^{56}$ Vgl. Doe No. 14 v. Internet Brands, Inc., 824 F.3d 846, 851 ff. (9th Cir. 2016). 
„nonparty“ vollstrecken. Der Court of Appeals of California verneinte die Rechtsfrage, ob diese vollstreckungsrechtliche Inanspruchnahme zu einer Behandlung von Yelp als „publisher" und damit zur Anwendbarkeit von $\$ 230$ CDA führe. ${ }^{57}$ Die Besonderheit dieses Falles liegt allerdings darin begründet, dass hier die Klägerin bereits gegen den Primärverursacher vorgegangen war und einen Titel erlangt hatte, der in den meisten Fällen jedoch kaum Aussicht auf Erfolg verspricht. Zudem stellte sich der California Supreme Court dieser Sichtweise dezidiert entgegen und ist der Auffassung, dass auch die Vollstreckung einer gegenüber dem Primärverursacher erlangten Verfügung gegen den Diensteanbieter als Dritten darauf hinauslaufe, diesen als „publisher" zu behandeln. ${ }^{58}$ Deshalb hob es die Berufungsentscheidung aufgrund des $\S 230 \mathrm{CDA}$ auf. ${ }^{59}$

In dem vor dem District Court for the Northern District of California verhandelten Fall Airbnb v. San Francisco kann man noch am ehesten einen verallgemeinerungsfähigen Sachverhalt entdecken. Hier hatten die städtischen Behörden von San Francisco Airbnb, ein Vermittler von Privatwohnungen als Übernachtungsgelegenheit, verpflichtet, nur solche Wohnungsangebote in seinem Angebot zu listen, die bei der Stadt registriert sind und für den Fall der Missachtung dieser Anordnung ein Bußgeld vorgesehen. Airbnb klagte gegen diese Maßnahmen und berief sich auf $\$ 230 \mathrm{CDA}$, weil - so die Auffassung des Unternehmens - es für Inhalte Dritter, nämlich der Wohnungsinserenten, zur Verantwortung gezogen und insoweit als deren „publisher“ angesehen würde, da das Unternehmen nunmehr verpflichtet sei, alle Wohnungsangebote vorab zu kontrollieren. Das Gericht sah dies anders und betonte, die städtischen Maßnahmen beträfen das Verhalten der Klägerin selbst, nämlich wie diese ihr Geschäftsmodell organisiere. Die städtische Maßnahme beträfe daher nicht die Ausübung der freien Rede Dritter. ${ }^{60}$ Die Entscheidung erging jedoch im Eilrechtsschutz. Zu einer abschließenden Entscheidung kam es nicht mehr, da die Parteien schließlich noch einen Vergleich schlossen. ${ }^{61}$

\footnotetext{
${ }^{57}$ Vgl. Hassell v. Bird, Cal.App. 4th 1336, 1363 ff. (Cal. Ct. App. 2016).

${ }^{58}$ Vgl. Hassell v. Bird, 5 Cal.5th 522, $541 \mathrm{f}$. (Cal. 2018).

${ }^{59}$ Vgl. aber das beachtliche Sondervotum in Hassell v. Bird, 5 Cal.5th 522, $567 \mathrm{ff}$. (Cal. 2018).

${ }^{60}$ Vgl. Airbnb v. City and County of San Francisco, 217 F.Supp. 3d 1066, 1072 ff. (N.D. Cal. 2016).

${ }^{61}$ Vgl. money.cnn.com/2017/05/01/technology/airbnb-san-francisco-settlement-agree ment/index.html.
} 


\section{Engführung des Ausnahmenkatalogs}

Schließlich wird die Tendenz der Rechtsprechung, §230 CDA grundsätzlich zugunsten von „unfettered free speech“ und damit stets zugunsten der Diensteanbieter auszulegen, ${ }^{62}$ auch bei den im Gesetz vorgesehenen Ausnahmen bzw. Konkurrenzregeln nach §230(e) CDA offenkundig. Insbesondere lehnen es die Gerichte ab, zivilrechtliche Ansprüche gegen Provider zu bejahen, die sich auf die Begehung von Straftaten beziehen und damit eigentlich von der Ausnahme nach §230(e)(1) CDA hätten profitieren können. Dies begründete etwa der District Court for the Eastern District of Texas in Doev. Bates damit, dass so jede vorsätzliche Handlung eines Diensteanbieters und die damit begründete strafrechtliche Relevanz des Falles die von $\$ 230$ CDA bezweckte Immunisierung unterliefe. ${ }^{63}$ Die Ausnahmeregel adressiere, so ein anderes Gericht, außerdem nur die staatliche Verfolgung bzw. Ahndung von Straftaten, nicht jedoch privatrechtliche Begehren. ${ }^{64}$ Den Klägern verbleibe schließlich die Geltendmachung von Ansprüchen gegenüber den Schädigern. ${ }^{65}$

\section{Verfassungsrechtlicher Kontext des $\$ 230$ CDA}

Für das Verständnis der weitgehenden Haftungsfreistellung der Diensteanbieter ist eine verfassungsrechtliche Kontextualisierung, insbesondere mit Blick auf die Redefreiheit nach dem ersten Zusatzartikel der US-Bundesverfassung, hilfreich. ${ }^{66}$ Hier ist zunächst von Bedeutung, dass $§ 230 \mathrm{CDA}$ die legislative Antwort auf eine Gerichtsentscheidung war, die einen Diensteanbieter haftungsrechtlich dafür sanktionierte, dass er eine redaktionelle Kontrolle über Inhalte Dritter ausübte. ${ }^{67}$ Gerade diese Sanktionswirkung wollte $\S 230$ CDA beseitigen, indem die Diensteanbieter für jedwede positive Ausübung dieser redaktionellen Kontrolle eben keine Sanktion mehr befürchten sollten. ${ }^{68}$ Die Ratio dieser Vorgehensweise basiert maßgeblich auf

${ }^{62}$ Kritisch insoweit Lukmire, NYU Annual Survey of American Law 66 (2010), 371 (389); vgl. zum verfassungsrechtlichen Kontext des §230 CDA noch sogleich §3 A. III.

${ }^{63}$ Vgl. Doe v. Bates, 2006 WL 3813758 1, 4 f. (E.D. Tex.).

${ }^{64}$ Vgl. Obado v. Magedson, 2014 WL 3778261 1, 8 (D.N.J. 2014).

${ }^{65}$ Vgl. M.A. v. Village Voice Media Holdings, 809 F.Supp. 2d 1041-10591055 (E.D. Mo. 2011).

${ }^{66} \mathrm{Im}$ Ansatz ähnlich Schmoll, Die deliktische Haftung der Internet-Service-Provider, S. 200 .

${ }^{67}$ Vgl. Stratton Oakmont v. Prodigy Services Co., 1995 WL 3237101 (N.Y. Sup. Ct. 1995); dazu schon oben $\S 3$ A. I.

${ }^{68}$ Vgl. Wyden, der als einer der „Väter“ von $\S 230 \mathrm{CDA}$ gilt, zitiert bei Zara, Wired (01.03.2017), www.wired.com/2017/01/the-most-important-law-in-tech-has-a-problem; vgl. ferner Franks, Huffington Post (18.12.2013), www.huffingtonpost.com/mary-anne-fra 
der verfassungsrechtlichen Redefreiheit nach dem Ersten Zusatzartikel der US-Verfassung. Deren Schutzbereich wird zum einen sehr weit ausgelegt, sodass eine kaum überschaubare Vielfalt menschlichen Verhaltens als Meinungsäußerung gewertet werden kann. ${ }^{69} \mathrm{Zum}$ anderen kennt der Erste $\mathrm{Zu}$ satzartikel keinen expliziten Schrankenvorbehalt, sondern nur ein kleines und in seinen Voraussetzungen zumeist sehr streng gehandhabtes Reservoir an verfassungsrechtlich akzeptablen Motiven zur Beschränkung der Redefreiheit. ${ }^{70}$ Gerade weil der Schutzbereich der Redefreiheit danach so weit ausgelegt wird, kann eine Kontrolle bzw. Beeinflussung redaktioneller Art potenziell immer einen Eingriff darstellen. ${ }^{71}$

Durchaus kritisch fällt insoweit auch die Einleitung der ersten Entscheidung des Court of Appeals for the Ninth Circuit zu §230 CDA aus, der kraft seiner örtlichen Zuständigkeit u.a. für Kalifornien und den Bundesstaat Washington ein enorm bedeutungsvolles Gericht ist. Das Gericht betonte hier, es gebe keinen dem Cyberspace inhärenten, zwingenden Grund, die Redefreiheit im digitalen Bereich anders anzuwenden als im analogen. Es sei die politische Präferenz des Gesetzgebers gewesen, mittels der Haftungsimmunisierung die Ausübung der Redefreiheit im Internet gleichsam keinerlei Beschränkungen zu unterwerfen. ${ }^{72}$ Gerade diese teleologische Verknüpfung

nks/section-230-the-lawless-internet_b_4455090.html: selektive Wahrnehmung der legislativen Absichten hinter $\$ 230 \mathrm{CDA}$.

${ }^{69}$ Vgl. kritisch hierzu Schauer, William \& Mary Law Review 56 (2015), $1613 \mathrm{ff}$.

${ }^{70}$ Ihren Ursprung hat diese Praxis neben dem bereits im Wortlaut angelegten rigorosen Ansatz in dem Prinzip des „marketplace of ideas“, das erstmals der Richter Holmes in seiner abweichenden Meinung in Abrams v. United States zum Ausdruck brachte: „the best test of truth is the power of the thought to get itself accepted in the competition of the market", Abrams v. United States, 250 U.S. 616, 630 (1919). Der einflussreiche Richter Brandeis hielt wenige Jahre später fest: „freedom to think as you will and to speak as you think are means indispensable to the discovery and spread of political truth, [...] [with free speech and assembly], discussion affords ordinarily adequate protection against the dissemination of noxious doctrine“, Whitney v. California, 274 U.S. 357, 375 (1927). Dementsprechend stellte der Supreme Court sehr hohe Hürden für die Annahme einer nicht mehr zulässigen Meinungsäußerung auf, die nämlich ,inciting or producing imminent lawless action" müsse und „likely to incite or produce such action" sei, vgl. Brandenburg v. Ohio, 395 U.S. 444, 447 (1969). Für eine Einordnung mit Blick auf digitale Kommunikation vgl. Lombardi, American Affairs 3, 1 (2019), $198 \mathrm{ff}$.

${ }^{71}$ Vgl. Citron/Wittes, Fordham Law Review 86 (2017), 401 (412), die allerdings später zu Recht darauf hinweisen, dass eine Reform von $\$ 230$ CDA nicht zwingend als Beschränkung der freien Rede bewertet werden müsse (420). In diesem Sinne auch Balkin, Pepperdine Law Review 36 (2009), 427 (434): ,[\$230 CDA] is not required by First Amendment doctrine" sowie Tushnet, George Washington Law Review 76 (2008), 986 (1004); anders insoweit aber o.A., Harvard Law Review 131 (2018), 2027 ff., wo eine (US-) verfassungsrechtliche Aufladung von $§ 230$ CDA befürwortet wird.

${ }^{72}$ Vgl. Batzel v. Smith, 333 F.3d 1018, 1027 (9th Cir. 2003). 
des juristischen Instruments der Haftungsprivilegierung und eines ,,robusten“ Verständnisses der Redefreiheit machte es jedoch von Anfang an schwer, die Breitenwirkung des Gesetzes restriktiv zu handhaben, ohne darin zugleich eine Beschränkung des verfassungsrechtlichen Prinzips zu erblicken. ${ }^{73}$ Die Verknüpfung der grundrechtlichen Redefreiheit mit dem Haftungsprivileg zeigt sich in den USA nicht zuletzt in einer gesetzlichen Maßnahme des USBundesgesetzgebers, der im sog. „SPEECH Act“ im Jahr 2010 die Gerichte verpflichtete, jenen ausländischen Gerichtsurteilen wegen Verleumdung die Anerkennung zu versagen, die entweder den US-amerikanischen Schutzstandard der Redefreiheit verletzten oder in den USA als Verstoß gegen $§ 230$ CDA aufgefasst würden. ${ }^{74}$ Gleichwohl ist in den USA verschiedentlich auch auf den breiten Ermessensspielraum des Gesetzgebers zur Regulierung der Verantwortlichkeit der Diensteanbieter hingewiesen worden. ${ }^{75}$ Insbesondere gebe es kein verfassungsrechtlich geschütztes Recht, als Diensteanbieter ein Geschäftsmodell ohne jegliches Haftungsrisiko zu betreiben. ${ }^{76}$ Auch in den USA entspricht es zwar nicht der allgemeinen Meinung, dass eine robuste Haftungsimmunisierung wie $§ 230$ CDA der Redefreiheit bzw. all ihren Trägern uneingeschränkt zugutekommen müsse. ${ }^{77}$ Nach Ansicht mancher Stimmen bevorzugt eine solche Regel vielmehr gerade diejenigen, die sich sowieso schon in einer gesellschaftlich mächtigeren Position befinden und verhindert jeden Versuch, die von uneingeschränkter Redefreiheit Benachteiligten besser zu schützen. ${ }^{78}$ Diese konträren Auffassungen über den Zusammenhang von Haftungsprivileg und Redefreiheit spiegeln anschaulich wieder, wie Aufforderungen zu einer gesetzgeberischen Reaktion und Legitimationspotenziale im Rahmen der haftungsrechtlichen Regulierung der Intermediäre oft auf den grundrechtlichen Kontext Bezug nehmen und damit potenziell gegenüber Angriffen auf jene Privilegierungen abschotten.

\section{Neuere Entwicklungen}

Die vorangegangene Darstellung der Entwicklung von $§ 230$ CDA und seiner Handhabung durch die Gerichte ist - bei aller territorial und kontext-spezifisch bedingter Tendenz zur Bereitschaft, gesellschaftliche Konflikte durch

${ }^{73}$ Vgl. Kosseff, The Twenty-Six Words that Created the Internet, S. 111.

${ }^{74}$ 111th Congress, Public Law No. 111-223, Sec. $3 \S 4102$.

${ }^{75}$ Vgl. Tushnet, George Washington Law Review 76 (2008), 986 (1004 f.).

${ }^{76}$ Vgl. Tushnet, George Washington Law Review 76 (2008), 986 (1008 mit Fn. 95).

${ }^{77}$ Kosseff, The Twenty-Six Words that Created the Internet, S. 225, meint gar, ohne $\S 230$ CDA würden Intermediäre viel eher Inhalte aufgrund von Beschwerden entfernen; die Norm verhelfe damit der Redefreiheit zur größeren Wirksamkeit; differenziert-fragend dagegen Balkin, Columbia Law Review 118 (2018), 2011 (2046).

${ }^{78}$ Vgl. Citron/Wittes, Fordham Law Review 86 (2017), 401 (420). 
den Markt lösen zu lassen und insbesondere staatliche Übergriffe in die Wirtschaft als illegitim abzulehnen ${ }^{79}$ - auch in den USA nicht ohne Kritik geblieben. Die Entscheidung Jane Doe No. 1 v. Backpage ${ }^{80}$ - bei der der Forenbetreiber von Backpage.com trotz Kenntnis von der Bewerbung sexueller Ausbeutung von Kindern wegen $\S 230$ CDA nicht haftete ${ }^{81}$ - hatte die Aufmerksamkeit der Öffentlichkeit auf bisweilen als untragbar empfundene Ergebnisse der Anwendung von $\S 230 \mathrm{CDA}^{82}$ gelenkt, was auch im politischen Prozess nicht unbemerkt blieb. Auch diverse Skandale und eine allgemeine Missstimmung bezüglich der Tätigkeit großer Diensteanbieter wie Facebook oder Twitter im Nachgang zu den Präsidentschaftswahlen in den USA im Jahr $2016^{83}$ heizten die öffentliche Stimmung zusätzlich auf und erzeugten einen politischen Handlungsdruck, „etwas“ gegen die Macht dieser Firmen „zu unternehmen“.

Vor diesem Hintergrund verabschiedete der US-Bundesgesetzgeber ein Gesetz, das es u.a. unter Strafe stellt, einen Dienst mit der Absicht der Erleichterung oder Ermöglichung der Prostitution einer anderen Person zu betreiben,${ }^{84}$ das weitere Strafvorschriften entsprechend verschärft, ${ }^{85}$ den Opfern solcher Prostitution in bestimmten Fällen einen Anspruch auf Schadensersatz gegenüber den Diensteanbietern gewährt, ${ }^{86}$ sowie eine Klarstellung in $\S 230$ CDA einfügt, dass das Haftungsprivileg solchen Ansprüchen sowie den entsprechenden Strafvorschriften nicht entgegensteht. ${ }^{87}$ Nahezu zeitgleich mit Erlass dieser unter dem Akronym SESTA/FOSTA bekannten Reform ging indes das US-Justizministerium bereits aufgrund existierender Vorschriften strafrechtlich gegen die Betreiber von Backpage.com vor. Insoweit zeigt sich, dass die politische Großwetterlage, die für erheblichen Druck auf die zuständigen Institutionen sorgte, bereits unabhängig von der Reform Wirkungen zeitigte. ${ }^{88}$ $44 \mathrm{ff}$.

${ }^{79}$ Vgl. zur „Californian Ideology“ Barbrook/Cameron, Science as Culture 6 (1996),

${ }^{80}$ Jane Doe No. 1 v. Backpage.com, 817 F.3d 12 (1st Cir. 2016).

${ }^{81} \mathrm{Vgl}$. dazu Kosseff, The Twenty-Six Words that Created the Internet, S. $253 \mathrm{ff}$.

${ }^{82}$ Vgl. Chamberlain, Fordham Law Review 87 (2019), 2171 (2186).

${ }^{83}$ Vgl. www.spiegel.de/politik/ausland/russland-und-die-us-wahl-2016-fuer-donald-tru mp-gegen-alle-anderen-a-1244043.html.

${ }^{84} 115$ th Congress, Public Law No. 115-164, Sec. 3.

${ }^{85} 115$ th Congress, Public Law No. 115-164, Sec. 5. Dies betrifft insbesondere die Reform der Definition einer Teilnahmehandlung, die nunmehr „knowingly assisting, supporting, or facilitating" einer entsprechenden Haupttat bedeutet.

${ }^{86} 115$ th Congress, Public Law No. 115-164, Sec. 3, § 2421A.(c). Diesen Anspruch darf alternativ auch der jeweils zuständige Justizminister des Bundesstaates geltend machen, vgl. Sec. 6 .

${ }^{87} 115$ th Congress, Public Law No. 115-164, Sec. 4.

${ }^{88}$ Dies betraf allerdings nicht die Etablierung eines zivilrechtlichen Anspruchs gegen 
Die verabschiedete, hier in aller Kürze dargestellte Reform traf kaum überraschend auf erhebliche Kritik. So widersprach insbesondere der CoSponsor der ursprünglich verabschiedeten Fassung von $\$ 230$ CDA, Senator Wyden, der Reform, da diese die Verfolgung Krimineller erschwere, die groBen Technologieunternehmen auf Kosten ihrer kleineren Konkurrenten bevorteile und Innovation verhindere. ${ }^{89}$ Interessenverbände, Bürgerrechtsorganisationen ${ }^{90}$ und selbst das US-Justizministerium ${ }^{91}$ äußerten sich kritisch und warfen dem Gesetzgeber vor, die Zensur im Internet zu fördern und damit die Redefreiheit zu beschneiden. ${ }^{92}$ In juristischer Perspektive wurde die unbestimmte und mehrdeutige Formulierung der Reform kritisiert, ${ }^{93}$ und zwar selbst von jenen Stimmen, die sich ansonsten gegen die weitreichende Immunisierung der Diensteanbieter aussprechen. ${ }^{94}$ Andererseits, und dies mag ebenfalls ein Symptom des sich offenbar drehenden politischen Windes sein, befürwortete die Internet Asssociation, hinter der sich namhafte Technologieunternehmen befinden, die Reform. ${ }^{95}$

Bei der Bewertung lässt sich in der Tat das Dilemma nicht leugnen, in dem sich betroffene Unternehmen befinden können. Denn entweder sie verschlieBen sich jedweder Beschäftigung mit nutzergenerierten Inhalten und der Be-

die Forenbetreiber, die aufgrund der Ermächtigung der Justizminister zu dessen Beitreibung zu einer praktisch garantierten Kompensation der Opfer führt, vgl. Goldman, First Amendment Law Review 17 (2019), 279 (286f.).

${ }^{89}$ Wyden, Pressemitteilung vom 08.11.2017, www.wyden.senate.gov/news/press-release s/wyden-issues-warning-about-sesta.

${ }^{90}$ Vgl. American Civil Liberties Union (07.11.2017), www.aclu.org/letter/aclu-letter-o pposing-sesta.

${ }^{91}$ Vgl. U.S. Department of Justice, Views on H.R. 1865, S. 2; in einem gerichtlichen Verfahren, in dem die Klägerin die einstweilige Unterbindung des Vollzugs von SESTA/FOSTA begehrte, verteidigte der US-Justizminister indes die Reform, vgl. Woodhull Freedom Foundation v. U.S., 334 F.Supp. 3d 185, 194 (D.D.C. 2018).

${ }^{92}$ Vgl. noch Center for Democracy \& Technology (04.08.2017), cdt.org/insights/jointletter-on-free-speech-concerns-with-s-1693-sesta; Jeong, The Verge (01.08.2017), www.the verge.com/2017/8/1/16072680/cda-230-stop-enabling-sex-traffickers-act-liability-shield-s enate-backpage.

${ }^{93}$ Vgl. Chamberlain, Fordham Law Review 87 (2019), 2171 (2195 ff.); Kosseff, The Twenty-Six Words that Created the Internet, S. 272.

${ }^{94}$ Citron/Jurecic, Lawfare (28.03.2018), www.lawfareblog.com/fosta-new-anti-sex-tra fficking-legislation-may-not-end-internet-its-not-good-law-either, betonen denn auch in ihrer Kritik den Umstand, dass die Reform handwerklich nicht sonderlich gelungen sei und die ihrer Auffassung nach bestehenden Einwände gegen die weitreichende Immunisierung der Intermediäre nicht vollumfänglich bzw. in systematischer Weise adressiert würden.

${ }^{95}$ Vgl. Internet Association, Pressemitteilung vom 03.11.2017, internetassociation. org/statement-in-support-of-the-bipartisan-compromise-to-stop-enabling-sex-trafficking -act-sesta. 
wertung dieser, oder aber die aktive Moderation führt zur Bejahung des Wissenselements in Betreff der kriminalisierten Inhalte, wodurch sich Unternehmen bzw. ihr Führungspersonal beim fehlerhaften Übersehen eines solchen Inhalts oder seiner falschen Bewertung einem erheblichen Strafbarkeitsrisiko aussetzen. ${ }^{96}$ Damit wird letztlich die ursprüngliche Intention hinter der Haftungsprivilegierung des $\S 230 \mathrm{CDA}$ in ihr Gegenteil verkehrt. Denn diese wollte ja gerade das Risiko der Kenntnis eines rechtswidrigen Inhalts und den hieraus entstehenden Anreiz, durch das eigene Verhalten gar nicht erst diese Kenntnis zu erlangen, abmildern und den Diensteanbietern vielmehr als Belohnung für eine verantwortungsvolle Regulierung ihres Einflussbereichs das Risiko der Haftung wegen der hiermit in aller Regel verbundenen Kenntnisnahme abnehmen. Es ist daher zu bezweifeln, dass dies der richtige Weg zur weiteren Ausgestaltung des Haftungsprivilegs ist. Allerdings lässt allein die Verabschiedung der Reform, gleich wie man inhaltlich zu ihr steht, als positives Anzeichen für den Umstand sehen, dass §230 CDA keineswegs als der „heilige Gral“ des Internetrechts anzusehen ist, wie es in der Debatte bislang mitunter den Anschein hat. ${ }^{97}$

\section{Fazit}

Abschließend lässt sich für $\S 230 \mathrm{CDA}$ insgesamt eine ausgesprochen rigide Immunisierung der Diensteanbieter festhalten. Die legislativ beabsichtigte Anreizfunktion dahingehend, tatsächlich Maßnahmen zur eigenständigen Kontrolle ihrer Dienste vorzunehmen, hat sich überwiegend nicht realisiert. Wie auch schon eingangs erwähnt, war es die unzweifelhafte und in der Überschrift des Gesetzes sogar wiedergegebene Intention von § 230 CDA, die Privilegierung nur solchen Unternehmen zukommen zu lassen, die von sich aus ihre Inhalte auf „offensive material“ hin durchsuchen und entsprechende Inhalte sperren..$^{90}$ Dennoch und womöglich gerade weil diese Intention nicht expliziten Eingang in den Gesetzeswortlaut selbst gefunden hat, hat sich die Anreizwirkung in der gerichtlichen Praxis bislang kaum durchsetzen können. ${ }^{99}$ Ein Diensteanbieter haftet nur, wenn er die Erstellung entsprechender Inhalte selbst anregt, während es auf den Versuch, Inhalte entsprechend dem soeben dargestellten Zweck eigenständig und freiwillig zu überwachen, kaum

\footnotetext{
${ }^{96}$ Vgl. Goldman, First Amendment Law Review 17 (2019), 279 (288).

${ }^{97}$ Ähnlich auch Citron/Jurecic, Lawfare (28.03.2018), www.lawfareblog.com/fosta-newanti-sex-trafficking-legislation-may-not-end-internet-its-not-good-law-either: Vorschläge zu einer grundsätzlichen Reform von $\S 230 \mathrm{CDA}$, are no longer laughably out of the question."

${ }^{98}$ Vgl. Doe No. 14 v. Internet Brands, Inc., 824 F.3d 846, 851 f. (9th Cir. 2016).

${ }^{99}$ Vgl. auch Citron/Wittes, Fordham Law Review 86 (2017), 401 (408); Sylvain, Connecticut Law Review 50 (2018), 203 (255).
} 
mehr ankommt. ${ }^{100}$ So immunisiert $\$ 230 \mathrm{CDA}$ einen gegenüber Rechtsverletzungen komplett passiven Diensteanbieter genauso wie jenen, der im Sinne des Ideals eines ,guten Samariters" versucht, seine Sphäre auf solche Inhalte hin zu kontrollieren. Nach Sylvain besteht damit eine „Rationalitätsfalle“, die einen ökonomischen Anreiz zu moralisch fragwürdigem Verhalten setzt, ${ }^{101}$ weil in einer solchen Situation (jedenfalls kein ökonomisch sinnvoller) kein Grund existiert, tatsächlich den Weg des „guten Samariters“ zu gehen. Die gerade nicht anvisierte „blanket liability protection“" ${ }^{“ 102}$ setzt im Gegenteil den Anreiz, Inhalte nicht zu löschen, weil dies für die jeweils betroffenen Geschäftsmodelle insofern schädlich sein könnte, als weniger abrufbare Inhalte mit einer geringeren Attraktivität im Markt einhergehen. Versteht man die betreffenden Judikate insbesondere der höheren Gerichte als einzelne, fallbezogene Hinweise an entsprechende Unternehmen, wie diese erfolgreiche Geschäftsmodelle modellieren können, ohne Haftungsrisiken befürchten zu müssen, kann der Einfluss der hier dargestellten Entwicklung insbesondere der Rechtsprechung durchaus eine entscheidende Rolle zur Entwicklung der im Internet agierenden Branchen zugeschrieben werden. ${ }^{103}$

Dennoch hat sich im Diskurs eine Dominanz der Befürworter einer möglichst umfassenden Immunisierung der betroffenen Diensteanbieter etabliert. Kritiker, die keineswegs für eine Abschaffung des §230 CDA, sondern für eine behutsame Reform plädieren, bleiben bislang in der Minderheit. ${ }^{104}$ Dahinter mag eine zur Zeit des Erlasses der Norm vorherrschende, bis heute noch nicht verklungene Präferenz zu einem „Internet exceptionalism“ auszumachen sein, ${ }^{105}$ wie er eingangs skizziert wurde. Dahinter steht die Grundhaltung, das Internet als Kulturtechnik ${ }^{106}$ sei so neuartig und revolutionär, dass tradierte Überzeugungen, Institutionen und Regulierungsansätze schon

${ }^{100}$ Vgl. Sylvain, Connecticut Law Review 50 (2018), 203 (243).

${ }^{101}$ Vgl. Sylvain, Connecticut Law Review 50 (2018), 203 (257 f.): ,,moral hazard“; dort auch zu einer möglichen Neuinterpretation der Norm, die freilich bislang von den Gerichten nicht in Betracht gezogen wird.

${ }^{102}$ Beale, Duke Law \& Technology Review 16 (2018), 333 (344); Citron/Wittes, Fordham Law Review 86 (2017), 401 (409): „Granting immunity to platforms designed in part or in whole for illegal activity would seem absurd to the CDA's drafters. "Offen bleibt, wie sich die bei Kosseff, The Twenty-Six Words that Created the Internet, S. 95, zitierte Aussage des Senators Wyden hierzu verhält, eine solche umfassende Haftungsprivilegierung sei gerade sein Ziel gewesen.

${ }^{103}$ Vgl. Sylvain, Connecticut Law Review 50 (2018), 203 (245f.).

${ }^{104}$ Vgl. die obigen Hinweise sowie Leary, Harvard Journal of Law \& Public Policy 41 (2018), $553 \mathrm{ff}$.

${ }^{105}$ Kosseff, The Twenty-Six Words that Created the Internet, S. 78: „Internet exceptionalism is at the heart of Section 230.“

${ }^{106}$ Vgl. Coy, Informatik Spektrum 31 (2008), $30 \mathrm{ff}$. 
deshalb nicht einfach zu übertragen seien. Nicht zuletzt wird mit der Entwicklung des Internets als Technik für die Massen implizit oder gar explizit auch ein Akt der intellektuellen und gesellschaftlichen Befreiung gesehen, die es ohne dieses Privileg nie gegeben hätte. ${ }^{107}$ In diesem Sinne wird Diensteanbietern ein gleichsam aufklärerisches Potenzial beigemessen. ${ }^{108}$ Oft fällt dabei aber unter den Tisch, dass sie immer noch gewinnorientierte Unternehmen sind. Die „robuste Natur“ digitaler Kommunikation ist daher nach den Befürwortern einer weitgehenden Immunisierung in Kauf zu nehmen, ${ }^{109}$ während der Schutz geistigen Eigentums demgegenüber eine deutliche Aufwertung erfährt, wie der folgende Abschnitt aufzeigen wird.

\section{B. $\S 512$ Digital Millennium Copyright Act}

Denn während §230 CDA die Privilegierung wie aufgezeigt recht kompromisslos verfolgt, erfolgt für Urheberrechtsverstöße durch den US-Gesetzgeber eine Regelung, die betroffenen Schutzrechten eine ungleich stärkere Rolle einräumt. Der Umgang mit potenziell urheberrechtsverletzenden Inhalten wurde mit dem Digital Millennium Copyright Act 1998 neu geregelt. Dessen „Online Copyright Infringement Liability Limitation Act“ führte das sog. Notice-and-Take-Down-Verfahren ein, das in $\S 512$ geregelt ist. ${ }^{110}$ Diese Norm sieht in den Absätzen (a) bis (d) Haftungsprivilegierungen vor, die für verschiedene Diensteanbieter bzw. verschiedene Situationen unterschiedliche Handlungsobliegenheiten vorsehen. Die Vorschrift differenziert insoweit zwischen „Transitory Digital Network Communications“ (\$ 512(a)), „System Caching" (§512(b)), „Information Residing on Systems or Networks At Direction of Users" (§512(c)) und „Information Location Tools“ (§ 512(d)). Für die Zwecke dieser Untersuchung ist die Variante „Information Residing on Systems or Networks At Direction of Users", die den Haftungsprivilegierungen für Host-Provider unter Art. 14 ECRL sehr ähnelt, besonders relevant. Diese lautet :

„(c) Information Residing on Systems or Networks At Direction of Users. -

(1) In general. - A service provider shall not be liable for monetary relief, or, except as provided in subsection (j), for injunctive or other equitable relief, for infringement of

\footnotetext{
${ }^{107}$ Vgl. Kosseff, The Twenty-Six Words that Created the Internet, S. 274 f.: „Prodigy allowed me to travel the world and live in it."

${ }^{108}$ Wiederum Kosseff, The Twenty-Six Words that Created the Internet, S. 276: ,[...] to turn the Internet into a closed, one-way street [...] is not a world to which I want to return. I had seen the world before and after the creation of the modern Internet, and I prefer the latter version."

${ }^{109}$ Vgl. Zeran v. AOL, Inc., 129 F.3d 327, 330 (4th Cir. 1997).

${ }^{110} 17$ U.S.C. $\$ 512$.
} 
copyright by reason of the storage at the direction of a user of material that resides on a system or network controlled or operated by or for the service provider, if the service provider -

(A)

(i) does not have actual knowledge that the material or an activity using the material on the system or network is infringing;

(ii) in the absence of such actual knowledge, is not aware of facts or circumstances from which infringing activity is apparent; or

(iii) upon obtaining such knowledge or awareness, acts expeditiously to remove, or disable access to, the material;

(B) does not receive a financial benefit directly attributable to the infringing activity, in a case in which the service provider has the right and ability to control such activity; and

(C) upon notification of claimed infringement as described in paragraph (3), responds expeditiously to remove, or disable access to, the material that is claimed to be infringing or to be the subject of infringing activity."

Die weiteren Abschnitte von $\S 512$ DMCA sind systematisch nicht ganz einfach zu durchschauen. §512(e) DMCA sieht eine Sonderregel für „,nonprofit educational institutions" vor; §512(f) sieht eine Sonderhaftung für Personen vor, die bewusst falsche Angaben im Kontext einer Nutzerbeschwerde tätigen; § 512(g) DMCA regelt bestimmte Konsequenzen einer Entfernung bzw. Löschung eines Inhalts, insb. das hier besonders relevante sog. „,counternotice"-Verfahren; §512(h) DMCA legt die Voraussetzungen einer gerichtlichen Vorladung (,subpoena“) zur Identifikation der rechtsverletzenden Person fest; für die Zwecke dieser Untersuchung von besonderer Relevanz ist §512(i) DMCA, der die allgemeinen Bedingungen für die Haftungsprivilegierung aller Diensteanbieter festschreibt („Conditions for Eligibility“); §512(k) DMCA nimmt außerdem eine Definition des hier verwendeten Begriffs „Service Provider“ vor, und zwar einerseits für jene Dienste im Sinne von §512(a) DMCA, die man technisch gesprochen Access-Provider nennen kann $(\S 512(\mathrm{k})(1)(\mathrm{A}))$ und andererseits alle übrigen Dienste $(\S 512(\mathrm{k})(1)(\mathrm{B}))$; schließlich hält § 512(m) DMCA zum Schutze der Privatsphäre fest, dass kein Abschnitt der Norm von einer Überwachung des Dienstes durch seinen Anbieter bzw. einer präventiven Suche nach inkriminierenden Tatsachen abhängig gemacht werden darf.

Im Folgenden sollen Entstehungsgeschichte und die konkrete Anwendung von § 512 DMCA erläutert (I.-II.) sowie mit Bezug auf den Gegenstand dieser Untersuchung kritisch erörtert werden (III.).

\section{Entstehungsgeschichte}

Inhaltlicher Anlass der gesetzlichen Reform war die Unsicherheit über die Standards der Haftung von Diensteanbietern infolge divergierender gerichtlicher Entscheidungen. Teilweise wurde eine direkte (Primär-)Haftung vorgesehen, die nicht einmal Absicht oder Kenntnis über die rechtsverletzende 
Handlung erforderte. ${ }^{111}$ Teilweise wurden Ansprüche nur gegenüber Teilnehmern der Verletzungshandlung mit Kenntnis bezüglich dieser in Betracht gezogen. ${ }^{112}$ Wiederum andere Gerichte forderten nach der sog. ,vicarious liability doctrine" ${ }^{113}$ (Haftung für fremdes Verschulden) zwar keine Kenntnis, setzten aber ein „right and ability to control the infringing actions" sowie ein finanzielles Interesse aus der Verletzungshandlung voraus. ${ }^{114}$ Dieser „threestandard approach" wurde in wissenschaftlichen Stellungnahmen teils ebenso kritisch bewertet wie von den betroffenen Industriezweigen, während die Urheberrechtslobby einer gesetzlichen Reform kritisch gegenüberstand. ${ }^{115}$

Die Clinton-Regierung hatte 1993 die Gründung einer Information Infrastructure Task Force veranlasst, die mit der Artikulation und Implementierung einer Vision für die Zukunft der Informationsinfrastruktur beauftragt wurde. ${ }^{116}$ Deren Working Group on Intellectual Property Rights kam hier zu dem Schluss, dass die bestehende Rechtslage angemessen und der Weg über eine Haftung der Diensteanbieter nach Maßgabe der Rechtsprechung besser geeignet sei, Urheberrechtsverletzungen zu verhindern. ${ }^{1{ }^{17}}$ Einen konkreten Handlungsauftrag an den US-Gesetzgeber formulierte sodann der Urheberrechtsvertrag der WIP, der Weltorganisation für geistiges Eigentum, von 1996. ${ }^{118}$ Dieser sah in seinen Erwägungsgründen vor, ein Gleichgewicht zwischen den Interessen der Urheber und der Allgemeinheit herzustellen, ohne allerdings explizit auf die Haftung von Diensteanbietern einzugehen. ${ }^{119}$ Gemeinsam mit dem WIPO Performances and Phonograms Treaty aus demselben $\mathrm{Jahr}^{120}$ etablierte er eine völkerrechtliche Regelungsstruktur für den

${ }^{111}$ Vgl. etwa Playboy Enterprises v. Frena, 839 F.Supp. 1552, 1559 (M.D. Fla. 1993).

${ }^{112}$ Vgl. Religious Technology Center v. Netcom, 907 F.Supp. 1361, 1369 (N.D. Cal. 1995).

${ }^{113}$ Vgl. dazu Ott, GRUR Int 2007, 14 (20).

${ }^{114}$ Shapiro, Bernstein \& Co. v. H.L. Green, 316 F.2d 304, 307 (2nd Cir. 1963); vgl. ergänzend Holznagel, Notice and Take-Down-Verfahren als Teil der Providerhaftung, S. $19 \mathrm{f}$.

${ }^{115}$ Vgl. Halpern, Seton Hall Legislative Journal 23 (1999), 359 (377 ff.); vgl. auch Ravn, Ohio State Law Journal 60 (1999), 755 (783 ff.).

${ }^{116}$ Vgl. Information Infrastructure Task Force, Intellectual Property and the National Information Infrastructure, S. 1.

${ }^{117}$ Information Infrastructure Task Force, Intellectual Property and the National Information Infrastructure, S. $117 \mathrm{ff}$., insbesondere S. 122: ,[...] it is - at best - premature to reduce the liability of any type of service provider"; , , [t]his would encourage intentional and willful ignorance".

${ }^{118}$ Vgl. www.wipo.int/treaties/en/ip/wct/index.html.

${ }^{119}$ Ein entsprechender Passus war zwar vorgesehen, wurde jedoch vor Verabschiedung gestrichen, vgl. Halpern, Seton Hall Legislative Journal 23 (1999), 359 (384 mit Fn. 151).

${ }^{120}$ www.wipo.int/treaties/en/ip/wppt/. 
urheberrechtlichen Umgang mit digitalen Medien und forderte eine nationalstaatliche Implementierung auch von den USA ein.

Der US-Gesetzgeber hielt sich hierbei jedoch nicht an die Empfehlung der Working Group, den Diensteanbietern eine noch stärkere bzw. aktivere Rolle beim Umgang mit urheberrechtsverletzenden Inhalten zuzuweisen, sondern verabschiedete den DMCA mit der erwähnten Regelung in $\S 512$ DMCA. Die gesetzgeberische Begründung für § 512 DMCA äußerte insoweit die Position, einerseits „strong incentives for service providers and copyright owners to cooperate to detect and deal with copyright infringements that take place in the digital networked environment" vorzusehen, andererseits Rechtssicherheit für Diensteanbieter zu erhöhen. ${ }^{121} \S 512$ DMCA sei daher nicht als Haftungsfreistellung zu verstehen, sondern als Anreiz für die Diensteanbieter, ihre Angebote auf rechtsverletzende Inhalte hin zu überprüfen. ${ }^{122}$ Dennoch waren zum Zeitpunkt des Erlasses des DMCA die Potenziale und Herausforderungen kaum abzusehen, die die Digitalisierung im Allgemeinen sowie das Internet im Besonderen mit sich gebracht haben. ${ }^{123}$ Insoweit spricht viel für die Vermutung, dass die Genese nicht nur von $\S 230$ CDA, sondern auch von $\S 512$ DMCA den Fokus vor allen Dingen auf die Erhaltung und Förderung von Innovationsfreundlichkeit legte. ${ }^{124}$

\section{Handhabung des Notice-and-Takedown-Verfahrens nach $\$ 512$ DMCA}

Im Folgenden soll nun die Regelung des §512 DMCA überblicksartig dargestellt werden. Diese orientiert sich insbesondere an den Handlungsanforderungen, die an die Diensteanbieter gestellt werden, um in den Genuss der Haftungsprivilegierung zu kommen.

\section{Allgemeines}

Der Regelung des $\S 512$ DMCA wird, ähnlich wie $\S 10$ TMG und entsprechende Vorgängervorschriften in Deutschland, ${ }^{125}$ eine Filterfunktion zugeschrieben, die die haftungsrechtlichen Grundierungen für Ansprüche wegen

${ }^{121}$ H.R. Rep. No. 1096 (1998), S. 72.

${ }^{122}$ H.R. Rep. No. 1096 (1998), S. 73.

${ }^{123}$ Das leitet Holznagel, Notice and Take-Down-Verfahren als Teil der Providerhaftung, S. 21, daraus ab, dass sog. P2P-Dienste sowie Suchmaschinen in ihrer heutigen Funktion kaum absehbar gewesen seien und vielmehr eine thematische Anknüpfung an das Problem von Hyperlinks erfolgt sei.

${ }^{124}$ Kritisch zur historischen Perspektive Hassanabadi, Berkeley Technology Law Journal 26 (2011), 405 (415f.).

${ }^{125}$ Vgl. zu § 5 TDG a.F. unten $§ 4$ B. Nach Holznagel, Notice and Take-Down-Verfahren als Teil der Providerhaftung, S. 24, spielt zumindest in den USA die dogmatische Einord- 
Urheberrechtsverstößen prinzipiell unangetastet lässt. ${ }^{126}$ Der hier besonders relevante Abschnitt §512(c) DMCA erfasst grundsätzlich sowohl monetäre Schadensersatzansprüche wie auch Unterlassungsbegehren ${ }^{127}$ und stellt Diensteanbieter von grundsätzlich frei in Bezug auf Urheberrechtsverstöße, die durch ,storage at the direction of a user of material that resides on a system or network controlled or operated by or for the service provider" entstehen, ${ }^{128}$ sofern die genannten Voraussetzungen eingehalten werden.

\section{Speicherung ,, at the direction of a user"}

Voraussetzung ist demnach zunächst, dass eine Speicherung eines Inhalts „,at the direction of a user" vorliegt. Eine Vorabkontrolle bzw. deren Vorbehalt durch den Diensteanbieter steht der Bejahung dieser Voraussetzung nicht entgegen, da §512 DMCA gerade diese Möglichkeit als positive Handlungsobliegenheit nach Erhalt einer entsprechenden notice aufstellt. ${ }^{129}$ Vielmehr ist eine Privilegierung erst dann nicht mehr möglich, wenn der Inhalt „resides on the system or network operated by or for the service provider through its own acts or decisions ".${ }^{130}$ Auch die automatisierte Erleichterung der Auffindbarkeit von Inhalten durch den Diensteanbieter auf seiner Webseite steht der Annahme nicht entgegen, dass diese ,at the direction of a user" gespeichert sind. ${ }^{131}$ Zur Begründung verweist der District Court for the Northern District of California auf die Definitionen von Diensteanbietern in $\S 512(\mathrm{k})$ DMCA. Dort schließt nämlich nur die engere Definition eines Anbieters in $\S 512(\mathrm{k})(1)(A)$, die sich auf Access-Provider im Sinne von $\S 512(\mathrm{a})$ bezieht, eine „modification to the content of the material as sent or received" aus der Definition aus. Für alle anderen Fälle, für die $§ 512(k)(1)(B)$ gilt, ist eine solche Modifikation nicht vorgesehen. ${ }^{132}$

nung als „Vorfilter“ oder Modifikation einer bestehenden Haftung keine Rolle, entscheidend sei die Einsicht, dass - auch nach dem expliziten Willen des US-Gesetzgebers - der DMCA keine Auswirkung auf die Interpretation und Anwendung der Haftungstheorien haben solle. In diesem Sinne auch Neumann, Die Haftung der Intermediäre im Internationalen Immaterialgüterrecht, S. 315.

${ }^{126}$ Vgl. H. Rep. No. 1051 (1998), Part 2, S. 50.

${ }^{127}$ Für letztere gilt aber die Ausnahmeregel des §512(j) DMCA.

${ }^{128}$ Vgl. aber Holznagel, Notice and Take-Down-Verfahren als Teil der Providerhaftung, S. 25, der auf die Privilegierung auch für Tätigkeiten in Zusammenhang mit dem Speichern hinweist.

${ }^{129}$ Vgl. CoStar Group v. Loopnet, 164 F.Supp. 2d 688, 702 (D. Md. 2001).

${ }^{130}$ CoStar Group v. Loopnet, 164 F.Supp. 2d 688, 701 (D. Md. 2001).

${ }^{131}$ Vgl. IO Group v. Veoh Networks, 586 F.Supp. 2d 1132, 1147 f. (N.D. Cal. 2008).

${ }^{132}$ Vgl. IO Group v. Veoh Networks, 586 F.Supp. 2d 1132, 1147 (N.D. Cal. 2008). 


\section{Die sog. ,threshold requirements“}

Um in den Genuss einer der sog. „,safe harbors“ der Vorschrift zu kommen, müssen sodann die sog. ,threshold requirements" ${ }^{\text {"133 }}$ eingehalten werden. Von grundsätzlicher Bedeutung sind dabei die Anforderungen des §512(i)(1) DMCA.

\section{a) „Repeat Infringer Policy“ und "Standard Technical Measures“: §512(i) DMCA}

§512(i) DMCA setzt spezielle Voraussetzungen der Haftungsprivilegierung fest, die insbesondere auf den Einsatz von Technologie (,Accommodation of technology") abzielen, um mit urheberrechtsverletzenden Inhalten bzw. Nutzern umzugehen. So müssen gemäß §512(i)(1) die Richtlinien der entsprechenden Diensteanbieter wiederholte Verstöße mit einem Ausschluss von ihrem Angebot sanktionieren (,repeat infringer policy“). ${ }^{134} \mathrm{Nach}$ §512(i)(1)(B) DMCA müssen die Anbieter selbst „standard technical measures" nach Maßgabe des §512(i)(2) DMCA einhalten und durchsetzen. ${ }^{135}$

Die „reasonable implementation“ einer solchen unternehmensseitigen Richtlinie ist dann anzunehmen, wenn diese ein wirksames Notifizierungssystem und ein Verfahren zum Umgang mit solchen (dem DMCA entsprechenden) Notifizierungen vorhält sowie der Diensteanbieter nicht aktiv Urheberrechtsinhaber daran hindert, für Notifizierungen erforderliche Informationen zu erlangen (,actively prevent copyright owners from collecting information needed to issue such notifications"). ${ }^{136}$ Es muss also ein ,funktionsfähiges Hinweissystem“ vorliegen. ${ }^{137}$ Der Court of Appeals for the Ninth Circuit betont jedoch, dass dieses Notifizierungssystem nicht nur den betroffenen Rechteinhabern selbst offenstehen darf, sondern daneben auch ein allgemeines System einzurichten ist, das diese zur Meldung von (mutmaßlich) rechtsverletzenden Inhalten nutzen können. ${ }^{138}$ Insgesamt verbleibt aber dem Diensteanbieter ein recht großzügiger Spielraum zur konkreten Anwendung der „repeat infringer policy“, wie §512(i)(1)(A) DMCA auch verdeutlicht (,in appropriate circumstances“). Die Einbeziehung von Tech-

${ }^{133}$ Vgl. IO Group v. Veoh Networks, 586 F.Supp. 2d 1132, 1142 (N.D. Cal. 2008).

${ }^{134}$ Vgl. Holznagel, GRUR Int 2007, 971 (974).

${ }^{135} \S 512(\mathrm{i})(1)(\mathrm{b})$ DMCA.

${ }^{136}$ Perfect 10 v. CCBill, 488 F.3d 1102, 1109 (9th Cir. 2007).

${ }^{137}$ Holznagel, Notice and Take-Down-Verfahren als Teil der Providerhaftung, S. 29.

${ }^{138}$ Vgl. Perfect 10 v. CCBill, 488 F.3d 1102, 1113 (9th Cir. 2007). Ob man dieser Passage mit Holznagel, Notice and Take-Down-Verfahren als Teil der Providerhaftung, S. 29, die Aussage zuschreiben kann, das Gericht habe dort ein allen Nutzern offenstehendes System eingefordert, ist allerdings zweifelhaft, da in der Passage selbst hinsichtlich der „,non-party notices“ nur von „other copyright holders“ die Rede ist. 
nologie zur Durchsetzung des haftungsrechtlichen Regelungsregimes zeigt sich sodann insbesondere in der Pflicht der Diensteanbieter, „standard technical measures“ einzuführen, die gemäß §512(i)(2) DMCA auf einem Industriekonsens infolge eines transparenten Prozesses entwickelt sowie diskriminierungsfrei zugänglich sein müssen und die zudem keine unverhältnismäßigen Kosten für die Unternehmen darstellen. ${ }^{139}$

Die unternehmensseitig zu erlassenden Richtlinien sind in ihrer Zielsetzung mit dem bereits skizzierten Anliegen des § 230(b)(3), (4) und (c)(2) CDA durchaus vergleichbar, da sie einen Anreiz zur Kontrolle der Angebote setzen und dies mit dem ,sicheren Hafen“ der Haftungsfreistellung belohnen. Anders als dort werden im Rahmen von §512 DMCA jedoch konkrete unternehmensseitige Umsetzungsmaßnahmen gefordert, deren rechtliche Anerkennung eine entscheidende Rolle einnimmt. Ein weiterer konzeptioneller Unterschied liegt in der prinzipiellen Ausrichtung des § 512(i) DMCA auf die Entfernung des Nutzers bzw. seines Zugangs und nicht des Inhalts. ${ }^{140}$ Obwohl §512(c) DMCA selbst die Entfernung des Inhalts im Rahmen des Noticeand-Takedown-Verfahren erfordert, lässt sich aus der grundlegenden Bedeutung des §512(i) DMCA und seiner Forderung eines zielgerichteten Technologieeinsatzes, ohne den der ,sichere Hafen“ gar nicht erst erreicht werden kann, ableiten, dass bei Urheberrechtsverstößen mit der Sperrung des Nutzers gerade im Vergleich zu §230 DMCA eine deutlich härtere Sanktion vorgesehen ist. Nur auf den ersten Blick ist dies mit dem breiten Schutz der freien Rede nach dem ersten Zusatzartikel der US-Verfassung erklärbar, denn bislang hat die Rechtsprechung der US-Gerichte eine Erstreckung des Schutzbereichs des ersten Zusatzartikels auf urheberrechtliche Nutzungen nur deshalb nicht bejaht oder vertieft thematisiert, weil die sog. , fair use“-Doktrin ${ }^{141}$ grundsätzlich bereits in der Lage sei, Fälle der legitimen Ausübung der freien Rede aufzufangen. ${ }^{142}$ Daraus lässt sich also gerade nicht der Schluss ziehen, $\S 230$ CDA sei hinsichtlich des angeordneten Sanktionsregimes (der betroffenen Diensteanbieter) laxer, weil es in Bezug zur freien Rede um „mehr“ gehe.

${ }^{139}$ Vgl. dazu auch Holznagel, Notice and Take-Down-Verfahren als Teil der Providerhaftung, S. 31. Dies wird später auch bei der Reform des europäischen Urheberrechts eine bedeutsame Rolle spielen, vgl. unten $\S 12 \mathrm{~B}$.

${ }^{140}$ Vgl. Perfect 10 v. CCBill, 340 F.Supp. 2d 1077, 1088 (C.D. Cal. 2004).

${ }^{141} 17$ U.S.C. § 107; vgl. dazu umfassend Förster, Fair Use, passim.

${ }^{142}$ A\&M Records v. Napster, 239 F.3d 1004, 1028 (9th Cir. 2001); Religious Technology Center v. Netcom, 923 F.Supp. 1231, 1258 (N.D. Cal. 1995); kritisch hierzu Wang, University of Pennsylvania Journal of Constitutional Law 13 (2011), 1471 (1484 ff.), mwN. 


\section{b) Keine tatsächliche Kenntnis oder Missachtung von „Red Flags“"}

Als weiterer Bestandteil der „threshold requirements“ darf der Diensteanbieter keine positive Kenntnis ${ }^{143}$ von der Rechtswidrigkeit haben oder sich der faktischen Umstände, aus denen die rechtsverletzende Tätigkeit offensichtlich hervorgeht, nicht bewusst sein (,,apparent knowledge“). ${ }^{144}$ Diese negative Tatbestandsvoraussetzung für die Haftungsfreistellung bezieht sich hier auf ein „specific knowledge“, ${ }^{145}$ das also den konkreten Fall meint und nicht die generalisierte Kenntnis, dass es etwa im Angebot eines Diensteanbieters wie YouTube generell zu Urheberrechtsverletzungen kommt. ${ }^{146}$ „Apparent knowledge" ist mit einem einfachen Fahrlässigkeitsmaßstab nicht vergleichbar, vielmehr geht es um sog. „,red flags of obvious infringement", ${ }^{147}$ vor denen der Diensteanbieter die Augen gleichsam verschlossen haben muss, um die Privilegierung gemäß §512(c)(1)(A)(ii) DMCA zu verlieren. Dieser „,red flag“-Test besteht aus zwei Elementen: zunächst muss festgestellt werden, dass die subjektive „,awareness“ von den Umständen der Rechtsverletzung gegeben ist. Sodann muss das Gericht zu der Überzeugung gelangen, dass die Rechtsverletzung objektiv „apparent“ für einen vernünftigen Beobachter unter den gleichen Bedingungen wie für den betroffenen Diensteanbieter gewesen wäre. ${ }^{148}$

Grundsätzlich wird der „red flag"-Test eher streng gehandhabt. Die Hürde für die Bejahung von „,apparent knowledge“ wird hoch angesetzt, da ansonsten die Grenze zur (Primär-)Haftung wegen eines „contributory infringement", also Haftung als Teilnehmer der Haupttat, nicht mehr erkennbar wäre. ${ }^{149}$ Dahinter steckt also das Ziel, die Primär- und die Sekundärhaftung systematisch voneinander zu trennen. Wenn der US-Gesetzgeber meint, dass „,apparent knowledge“ etwa dann gegeben sei, wenn das Material auf "clearly [...] a pirate site" gespeichert sei, ${ }^{150}$ die Rechtsprechung aber postuliert, sogar der Betrieb der Internetseiten „,illegal.net“ und „stolencelebritypics.com" stelle keine „red flag" dar ${ }^{151}$ ist allerdings nicht anzunehmen,

${ }^{143} \S 512(\mathrm{c})(1)(\mathrm{A})(\mathrm{i})$ DMCA.

${ }^{144} \S 512$ (c)(1)(A)(ii) DMCA: ,[...] is not aware of facts or circumstances from which infringing activity is apparent".

${ }^{145}$ Ausführlich Viacom v. YouTube, 676 F.3d 19, 30 ff. (2nd Cir. 2012).

${ }^{146} \mathrm{Vgl}$. Holznagel, Notice and Take-Down-Verfahren als Teil der Providerhaftung, S. 31.

${ }^{147}$ S. Rep. No. 1090 (1998), S. 48.

${ }^{148}$ Vgl. S. Rep. No. 1090 (1998), S. 44; H.R. Rep. No. 1051 (1998), S. 53; Hassanabadi, Berkeley Technology Law Journal 26 (2011), 405 (414).

${ }^{149}$ So Holznagel, GRUR Int 2007, 971 (974).

${ }^{150}$ S. Rep. No. 1090 (1998), S. 48.

${ }^{151}$ Perfect 10 v. CCBill, 488 F.3d 1102, 1114 (9th Cir. 2007); kritisch hierzu Chang, Cardozo Arts \& Entertainment Law Journal 28 (2010), 195 (203 f.); vertiefend zur Recht- 
dass die Privilegierung von Diensteanbietern allzu oft an diesem Merkmal scheitern wird. Welche Rolle die Rechtspraxis von den Diensteanbietern erwartet, formuliert der District Court for the Central District of California ausdrücklich in UMG Recordings v. Veoh Networks. Hier hält das Gericht fest, dass eine „notice“, die eine Untersuchung der Fakten und Umstände erforderlich mache, um einen Inhalt als rechtsverletzend zu identifizieren, einer Bejahung einer ,,red flag“ bereits prinzipiell entgegenstehe. ${ }^{152}$ Vielmehr bedürfe es „willful ignorance of readily apparent infringement“, um eine „,red flag" bejahen zu können. ${ }^{153}$ In Viacom v. YouTube formuliert es der Court of Appeals for the Second Circuit noch deutlicher: eine „red flag“ setze einen „deliberate effort to avoid guilty knowledge“ voraus. ${ }^{154}$ Begründet wird diese strenge, providerfreundliche Handhabung mit dem Hinweis auf das Verbot allgemeiner Monitoringpflichten im Sinne von $\S 512(m)$ DMCA. ${ }^{155}$

\section{c) Kein ,right and ability to control"}

Schließlich setzt §512(c)(1)(B) bzw. (d)(2) DMCA voraus, dass der Diensteanbieter ,does not receive a financial benefit directly attributable to the infringing activity, in a case in which the service provider has the right and ability to control such activity". Auf den ersten Blick erscheint dieses negative Merkmal identisch zur Anspruchsvoraussetzung der deliktischen Haftung für fremdes Verschulden unter sog. „vicarious liability“. ${ }^{156}$ Trotz der Berufung auf die gesetzgeberische Konzeption, durch die Privilegierung keine Modifikation der Haftungsgrundlagen vornehmen zu wollen (zu denen die Figur der „vicarious liability“ gehört), erreicht die Praxis mitunter keine klare Trennung zwischen dieser Figur und dem negativen Merkmal ,no right and ability to control" ${ }^{157}$ Indes ergibt sich schon aus der notwendigen tatsächlichen Fähigkeit zum „takedown“ einerseits und dem Erfordernis einer

sprechung der US-Gerichte Hassanabadi, Berkeley Technology Law Journal 26 (2011), 405 (416 ff.).

${ }^{152}$ UMG Recordings v. Veoh Networks, 665 F.Supp. 2d 1099, 1108 (C.D. Cal. 2009); zustimmend Viacom v. YouTube, 676 F.3d 19, 32 (2nd Cir. 2012).

${ }^{153}$ UMG Recordings v. Veoh Networks, 665 F.Supp. 2d 1099, 1108 (C.D. Cal. 2009).

${ }^{154}$ Viacom v. YouTube, 676 F.3d 19, 35 (2nd Cir. 2012).

${ }^{155}$ Vgl. Holznagel, Notice and Take-Down-Verfahren als Teil der Providerhaftung, S. 33 .

${ }^{156}$ Vgl. bereits Shapiro, Bernstein \& Co. v. H.L. Green, 316 F.2d 304, 307 (2nd Cir. 1963): „When the right and ability to supervise coalesce with an obvious and direct financial interest in the exploitation of copyrighted materials [...] the purposes of copyright law may be best effectuated by the imposition of liability upon the beneficiary of that exploitation." Vertiefend hierzu Polygram International Publishing v. Nevada/TIG, 855 F.Supp 1314, 1324 ff. (D. Mass. 1994).

${ }^{157}$ Explizite Nivellierung beider Figuren bei Perfect 10 v. CCBill, 488 F.3d 1102, 1117 (9th Cir. 2007). 
„repeat infringer policy“ andererseits ein technisches und ein rechtliches Element der Kontrolle, sodass es hier insoweit um mehr gehen muss als um die rein technische Fähigkeit zur Reaktion. ${ }^{158}$ Vielmehr muss auch eine rechtliche Möglichkeit des Zugriffs auf einzelne rechtsverletzende Inhalte gegeben sein. ${ }^{159}$ Eine Abstraktion der erforderlichen Maßstäbe erscheint jedoch denkbar schwierig, da dies stets vom konkreten Angebot eines Diensteanbieters abhängt. ${ }^{160}$

Der Erhalt finanzieller Vorteile im Falle eines solchen „,right and ability to control" setzt schließlich die Anwendbarkeit der Privilegierung außer Kraft. Damit ist offenbar nur der finanzielle Vorteil gemeint, der sich unmittelbar aus einer rechtsverletzenden Handlung ergibt. ${ }^{161}$ Das können Gebühren sein, die die Dienste von den Nutzern selbst einfordern, betrifft aber insbesondere auch die Finanzierung von entsprechenden Diensten durch Werbung.

\section{Anforderungen und Konsequenzen einer ,"notice“}

Hält die „notice“ sodann die formalen Anforderungen ein, ${ }^{162}$ ist der Diensteanbieter angehalten, unverzüglich den gerügten Inhalt zu entfernen oder den Zugang zu ihm zu sperren. ${ }^{163}$ Entscheidende Konsequenz einer solchen formal korrekten Notifizierung ist die fehlende Pflicht des Diensteanbieters, in eine selbstständige Prüfung des juristischen Vorwurfs der Rechtsverletzung einzutreten. ${ }^{164}$ Vielmehr ist er aus dem juristischen Konflikt im Grundsatz entlassen. ${ }^{165}$

In dem Gewand einer Immunisierung des Diensteanbieters gegenüber sämtlichen Ansprüchen infolge eines redlich (,good faith“) vorgenommenen „takedowns"166 - insoweit ist ebenfalls von einer „Good Samaritan“-RegeS. 35 .

${ }^{158}$ Vgl. auch Holznagel, Notice and Take-Down-Verfahren als Teil der Providerhaftung,

${ }^{159}$ So auch Hendrickson v. eBay, 165 F.Supp. 2d 1082, 1093 f. (C.D. Cal. 2001).

${ }^{160} \mathrm{Vgl}$. in diesem Sinne IO Group v. Veoh Networks, 586 F.Supp. 2d 1132, 1151 (N.D. Cal. 2008).

${ }^{161} \mathrm{Vgl}$. Holznagel, Notice and Take-Down-Verfahren als Teil der Providerhaftung, S. 37.

${ }^{162}$ Für das Hosting vgl. insbesondere $\S 512$ (c)(3)(i)-(vi) DMCA. Zu beachten ist dabei, dass die in diesem Katalog aufgeführten Anforderungen nur im Wesentlichen (,substantially“) zu erfüllen sind. §512(c)(2)(B) DMCA hält ein Fehlerfolgenregime parat, dass für eine unvollständige „notice“ dennoch Verhaltenspflichten für den Diensteanbieter vorsieht (je nachdem, welche formalen Anforderungen die „notice“ nicht einhält).

${ }^{163} \S 512(\mathrm{c})(1)(\mathrm{C})$ DMCA.

${ }^{164}$ Vgl. Zittrain, Harvard Journal of Law \& Technology 19 (2006), 253 (266); Holznagel, GRUR Int 2007, 971 (972, 977 f.).

${ }^{165}$ Insoweit unterscheidet sich die Rechtslage in den USA fundamental von derjenigen in Europa, vgl. dazu unten $\S 10$ A. III.

${ }^{166}$ Ausweislich $\S 512(\mathrm{~g})(1)$ DMCA gilt dies unabhängig davon, ob der Inhalt sich schlussendlich als rechtsverletzend erweist oder nicht. 
lung die Rede ${ }^{167}$ - sieht §512(g) DMCA sodann die Möglichkeit vor, dass der Nutzer, dessen Inhalt gesperrt wurde, mittels einer sog. , counter-notice“168 den Inhalt doch wieder verfügbar machen kann. Die Immunisierung des Diensteanbieters greift nämlich nur dann ein, wenn er den betroffenen Nutzer benachrichtigt und dessen ggf. eingereichte „counter-notice“ dem Rechteinhaber und ursprünglichen „Melder“ mit dem Hinweis zukommen lässt, den Inhalt nach zehn Tagen wieder verfügbar zu machen und dies dann tatsächlich tut, sofern nicht der Rechteinhaber ihn benachrichtigt, dass er gerichtlich gegen den Nutzer, um dessen Inhalt es geht, vorgehen werde. ${ }^{169}$

\section{Kritik}

§512 DMCA ist - ähnlich wie § 230 CDA - Gegenstand vieler Debatten, die sich hierbei insbesondere um die wohlfahrtsökonomisch sinnvolle und gerechte Zuordnung von konfligierenden Interessen drehen. Diese sind hier kurz darzustellen.

\section{Allgemeines}

Durch das Notice-and-Takedown-Verfahren sucht der Gesetzgeber, den Diensteanbieter im Eskalationsfall von dem juristischen Konflikt nahezu vollständig abzuschirmen und so aus der Verantwortung zu entlassen. Hierbei kommt allerdings wiederum das wirtschaftliche und strategische Übergewicht der Diensteanbieter zum Tragen. Dieses versetzt die Anbieter einerseits in die Lage, die technischen Konfigurationen zur Umsetzung des Notice-and-Takedown-Verfahrens so zu gestalten, dass Nutzer kaum Hoffnung auf die Erfolgsaussichten einer ,counter-notice“ haben dürften. ${ }^{170}$ Andererseits kann es sich aber auch von Rechteinhabern mit besonderen vertraglichen Vereinbarungen zunutze gemacht werden, was zulasten der Dienstenutzer gehen kann. ${ }^{171}$ In der Praxis scheint das Verfahren außerdem selten

${ }^{167}$ Vgl. H. Rep. No. 1051 (1998), Part 1, S. 26.

${ }^{168}$ Diese muss den Anforderungen des §512(g)(3) DMCA genügen.

${ }^{169} \S 512(\mathrm{~g})(2)$ DMCA.

${ }^{170}$ So berichten Perel/Elkin-Koren, Stanford Technology Law Review 19 (2016), 473 (506), dass etwa bei YouTube die Funktion zur „counter-notice“ erst im System sichtbar wird, wenn man sich zum Bestreiten des Vorwurfs der Rechtsverletzung entschieden hat.

${ }^{171}$ Paradigmatisch der bei PerellElkin-Koren, Stanford Technology Law Review 19 (2016), 473 (506f.) geschilderte Fall: hier hatte ein Nutzer eine „,counter-notice“ gestellt und sich dabei auf die Urheberrechtsschranke „fair use“ (vgl. dazu oben§3 Fn. 141) gestützt. YouTube stellte den beanstandeten Inhalt dennoch nicht, wie von $\S 512(\mathrm{~g})(2)$ DMCA vorgesehen, wieder her und berief sich hierbei auf eine mit einem bestimmten Rechteinhaber vertraglich vorgesehene Pflicht, beanstandete Inhalte trotz einer solchen „,counter-notification“ unverfügbar zu halten. 
praktische Anwendung zu finden. ${ }^{172}$ Das ruft Zweifel an einem solchen Modell hervor, bei dem die Diensteanbieter nur wenig Anreiz haben, einer Notifizierung tatsächlich in der Sache nachzugehen. ${ }^{173}$ Andererseits kann man in der starken prozeduralen Komponente des Notice-and-Takedown-Verfahrens auch einen prinzipiell lobenswerten Ansatz sehen, soweit zum einen das Eigenverantwortlichkeitsprinzip betont wird, das Rechteinhaberinnen grundsätzlich zur Wahrnehmung ihrer Rechte mobilisieren will, und soweit zum anderen eben auch eigenständige Sorgfaltspflichten der Diensteanbieter statuiert werden, um ihre Rolle bei der Begehung von Urheberrechtsverletzungen zu unterstreichen.

Die Differenzierung zwischen dem prozedural ausgestalteten Haftungsregime im DMCA und der rigiden Immunisierung der Diensteanbieter nach CDA ist jedenfalls frappierend. ${ }^{174}$ Diese Divergenz deutet darauf hin, dass die Repräsentanz kommerzieller Interessen aufseiten der Urheber deutlich ausgeprägter zur Geltung kommt als diejenige der von „Hassrede“ bzw. Verleumdung Betroffenen, ${ }^{175}$ obwohl gerade den Interessensvertretern der Urheberrechtsinhaber ein nicht unerheblicher Einfluss auf das Gesetzgebungsverfahren zugeschrieben wurde. ${ }^{176}$ So erweist sich $\S 512$ DMCA zwar durchaus als Kompromiss zweier (halbwegs äquivalent repräsentierter) Interessengruppen. Holznagel sieht in der Regelung dennoch einen „Punktsieg“ für die Seite der Diensteanbieter, weil aktive Überwachungspflichten verhindert wurden und stattdessen eine einzelfallbezogene Steuerungspflicht etabliert wurde, deren Initiierung den betroffenen Rechteinhabern obliegt. ${ }^{177}$

Genau dies scheint in Stellungnahmen des deutschsprachigen Schrifttums teilweise jedoch gerade als Erfolg gewertet zu werden. Gelobt wird die durch $\S 512$ DMCA vorgenommene Akzentuierung der Konfliktlage als (vorgeblich) rein privatrechtlich, insoweit es nicht den Gerichten obliege, die Rechtmäßigkeit eines gerügten Inhalts zu prüfen, sondern vielmehr die Risiken unter den betroffenen Privatrechtssubjekten verteilt würden. ${ }^{178}$ Dies hat jedoch zugleich zur Folge, dass die Diensteanbieter selbst praktisch sehr selten eine Haftung riskieren. Denn die Anreizstrukturen überantworten es regel-

${ }^{172}$ Vgl. Urban/Quilter, Santa Clara Computer \& High Technology Law Journal 22 (2006), 621 (679).

${ }^{173}$ Vgl. Tushnet, George Washington Law Review 76 (2008), 986 (1003); Maier, Remixe auf Hosting-Plattformen, S. $187 \mathrm{f}$.

${ }^{174}$ Vgl. auch Tushnet, George Washington Law Review 76 (2008), 986 (1004).

${ }^{175}$ Vgl. Holznagel, Notice and Take-Down-Verfahren als Teil der Providerhaftung, S. 21.

${ }^{176}$ Vgl. Amirmahani, Berkeley Technology Law Journal 30 (2015), 865 (872).

${ }^{177}$ Vgl. Holznagel, Notice and Take-Down-Verfahren als Teil der Providerhaftung, S. $22 \mathrm{f}$.

${ }^{178}$ Vgl. Schapiro, ZUM 2014, 201 (209); Specht, ZUM 2017, 114 (121). 
mäßig den Betroffenen, sich für oder gegen eine gerichtliche Auseinandersetzung zu entscheiden, während die Diensteanbieter selbst eine überlegene, weil ein Zuwarten auf die Entscheidung anderer Akteure erlaubende Position innehaben. ${ }^{179}$ Umgekehrt bedeutet dies zugleich, dass Rechteinhaberinnen zur quasipolizeilichen Kontrolle von entsprechenden Diensten gezwungen werden, ${ }^{180}$ und zugleich die Nutzer gleichsam (im untechnischen Sinne) im Auftrag ${ }^{181}$ bzw. in Stellvertretung der Diensteanbieter delegierte Aufgaben wahrnehmen.

Ferner erweist sich das gegenwärtige Regelungsregime unter § 512 DMCA als Anreiz zur (mehr oder weniger) ungeprüften Akzeptanz von entsprechenden „notices“ und dementsprechenden Löschung von Inhalten. ${ }^{182}$ Den Diensteanbietern wird einerseits unterstellt, ihnen fehlten oftmals hinreichende Ressourcen, um die genauen Umstände einzelner Beschwerden zu prüfen und eine rechtliche Prüfung vorzunehmen, zu der sie, wie bereits dargestellt, unter $\S 512$ DMCA allerdings auch nicht verpflichtet sind. ${ }^{183} \mathrm{Ob}$ diese Annahme auf tatsächlicher Ebene trägt, sei hier dahingestellt. Jedenfalls sollte eine sinnvolle Regulierung darauf bedacht sein, keine einseitigen Anreizstrukturen zu verursachen, um Diensteanbietern gar nicht erst die Gelegenheit zu eröffnen, sich auf einseitige Anreizsysteme zurückzuziehen und die gesetzlich überantwortete Verantwortung zu ignorieren. Hierbei steht nicht nur der Gesetzgeber in der Pflicht; die Rechtsprechung vermag hier ggf. sogar flexibler auf Fehlanreize im Einzelfall zu reagieren. Andererseits macht man es den Diensteanbietern auch sehr einfach, sich in ,gutem Glauben“ an die „notice“ des Rechteinhabers ${ }^{184}$ aus komplexen Prüf- und Abwägungserfordernissen herauszuhalten, ohne zu bedenken, dass die „Gutgläubigkeit“" der Rechteinhaber selbst stets mit einer objektiven, neutralen Perspektive auf die Beurteilung eines konkreten Inhalts einherginge. ${ }^{185}$

${ }^{179}$ Vgl. Ott, GRUR Int 2008, 563 (569); sehr kritisch Halpern, Seton Hall Legislative Journal 23 (1999), 359 (406): ,the [DMCA] [...] hinders the progress of arts because its provisions do not provide any incentives for service providers to prevent infringement."

${ }^{180}$ So explizit Perfect 10 v. CCBill, 488 F.3d 1102, 1113 (9th Cir. 2007); zustimmend Viacom v. YouTube, 718 F.Supp. 2d 514, 524 (S.D. N.Y. 2010). Dass die Rechteinhaber nicht schärfer gegen § 512 DMCA vorgegangen sind, erklärt Holznagel, Notice and TakeDown-Verfahren als Teil der Providerhaftung, S. 65, mit Kooperationsvereinbarungen zwischen größeren Rechteinhabern und den Diensteanbietern. Dieses Argument enthebt jedoch nicht von einer Analyse der Gesetzesfolgen bzw. der Empirie unter dem geltenden Recht.

${ }^{181}$ Vgl. Gillespie, Georgetown Law Technology Review 2 (2018), 198 (201).

${ }^{182}$ Vgl. Chen et al., Updating 17 U.S.C. §512's Notice and Takedown Procedure for Innovators, Creators, and Consumers, S. 8.

${ }^{183}$ Vgl. Holznagel, GRUR Int 2014, 105 (112f.): Weiterleitung der „notice“ entlässt den Diensteanbieter aus dem Streit.

${ }^{184} \mathrm{Vgl} . \S 512(\mathrm{~g})(1)$ DMCA.

${ }^{185}$ Vgl. PerellElkin-Koren, Stanford Technology Law Review 19 (2016), 473 (501); in- 
Auch die rechtspraktische Perspektive offenbart die Mängel des $§ 512$ DMCA. Auf die zunehmende Verlagerung der Durchsetzungsmechanismen weg von menschlich gesteuerten und verhandelten Konflikten hin zum algorithmisch gesteuerten und abgewickelten Verfahren, das sich im Rahmen des Notice-and-Takedown-Verfahrens insbesondere in der massenhaften Erstellung entsprechender „notices“ sowie des automatisch gesteuerten Umgangs mit diesen zeigt, hat der DMCA keine adäquate Antwort parat. Insbesondere im Hinblick auf die Erklärbarkeit bzw. der Transparenz, Nachprüfbarkeit und Korrigierbarkeit entsprechender Mechanismen ist dies problematisch. ${ }^{186}$ Zwar erscheint das „,counter-notice“-Verfahren nach $§ 512(\mathrm{~g})$ DMCA als durchaus geeignet, eine „notice“ einer gewissen Prüfung zu unterziehen und so zumindest in Bezug auf die hier beteiligten Personen eine Reaktionsmöglichkeit herzustellen. ${ }^{187}$ Hinsichtlich der Nachprüfbarkeit der Entscheidungen des Diensteanbieters erscheint es aber heikel, bis zur Einreichung einer „,counter-notice“ den gerügten Inhalt unverfügbar zu halten, weil sich in einem schnelllebigen medialen und kommunikativen Umfeld, in dem augenscheinlich Aufmerksamkeitsspannen immer weiter absinken, ${ }^{188}$ eine Sperrung von mindestens zehn Tagen $(\$ 512(\mathrm{~g})(2)(\mathrm{B})$ DMCA) faktisch als Behinderung von meinungsrelevanten Inhalten und damit vom Meinungsdiskurs selbst angesehen werden kann. ${ }^{189}$ Fehlerhaft wäre indes der Schluss, dass ein solches Verfahren, das die vorübergehende Sperrung eines Inhalts vorsieht, in jedem Falle im Hinblick auf die Äußerungsfreiheit ab-

soweit erscheint die Annahme von Holznagel, Notice and Take-Down-Verfahren als Teil der Providerhaftung, S. 72, nehme man das Kriterium der Gutgläubigkeit ernst, sei für eine hinreichend tiefe Prüfung durch die Diensteanbieter gesorgt, ihrerseits etwas gutgläubig.

${ }^{186}$ PerellElkin-Koren, Stanford Technology Law Review 19 (2016), 473 (494 ff.), legen diese drei „proxies“ für ihr Verständnis von „Accountability“ zugrunde.

${ }^{187}$ Perel/Elkin-Koren, Stanford Technology Law Review 19 (2016), 473 (499), mwN, kritisieren allerdings die fehlende Kontaktmöglichkeit für die Suchmaschinenbetreiber (§ 512(d) DMCA). Dabei ist die generelle Anwendbarkeit des §512(g) auf § 512(d) DMCA jedoch alles andere als eindeutig, denn $\S 512(\mathrm{~g})(2)$ DMCA spricht recht klar nur von „material residing at the direction of a subscriber", also dem Fall des Hostings von Inhalten nach $\S 512$ (c) DMCA; vgl. dazu auch Holznagel, Notice and Take-Down-Verfahren als Teil der Providerhaftung, S. 56 mit Fn. 301.

${ }^{188} \mathrm{Vgl}$. Schweiger, Der (des)informierte Bürger im Netz, S. 15; relativierend aus kommunikationswissenschaftlicher Perspektive Geiß, Die Aufmerksamkeitsspanne der Öffentlichkeit, S. 309, der zu dem Schluss kommt, dass die intensive Nutzung des Internets und ein entsprechend unkonzentrierter Mediennutzungsstil die Aufmerksamkeitsspanne nicht zwingend negativ beeinträchtige.

${ }^{189}$ Vgl. Chen et al., Updating 17 U.S.C. §512's Notice and Takedown Procedure for Innovators, Creators, and Consumers, S. 7; vgl. auch Urban/Quilter, Santa Clara Computer \& High Technology Law Journal 22 (2006), 621 (636f.), die später von einer „,extrajudicial temporary restraining order, based solely on the copyright holder's allegation of copyright infringement" sprechen (639). 
zulehnen sei. Vielmehr kann das wünschenswerte Ziel der Nachprüfbarkeit einer „notice“ auch durch unmittelbare Einbindung aller Beteiligten und einer zeitnahen (vorläufigen) Entscheidung durch den Diensteanbieter ebenso effektiv sein.

\section{Merkmale des , Safe Harbor" im Einzelnen}

Im Folgenden soll nun auf einige spezifische Merkmale des §512 DMCA kritisch eingegangen werden. Die konkrete Handhabung der einzelnen Tatbestandsmerkmale des $\S 512$ DMCA ist insoweit zu kritisieren, als diese eine Haftung von Diensteanbietern faktisch erschwert bzw. unmöglich macht.

\section{a) Keine ,Red Flags" in Sicht?}

So entlässt insbesondere die hohe Hürde des soeben schon angesprochenen „red flag“-Test im Sinne von §512(c)(1)(A)(i) DMCA die Diensteanbieter nahezu aus jeglicher Verantwortung, sich mit der rechtlichen Qualität der in ihrem Angebot verfügbar gemachten Inhalte zu befassen. Wenn dies damit begründet wird, die Unternehmen träfe nicht die Pflicht, die tatsächliche Rechtswidrigkeit eines Inhalts zu prüfen, ${ }^{190}$ ist die Vereinbarkeit dieser Feststellung mit dem Wortlaut von §512(c)(1)(A)(ii) DMCA zumindest zweifelhaft, weil es dort eben um den Anschein einer rechtsverletzenden Aktivität geht $\left(,,[\ldots]\right.$ infringing activity is apparent“). ${ }^{191}$ Auch systematisch ist diese Feststellung fraglich, denn der Court of Appeals for the Ninth Circuit setzt damit für §512(c)(1)(A)(ii) DMCA einen Maßstab an, der effektiv auf das Bejahen eines „actual knowledge“ hinausläuft. ${ }^{192}$ Jedenfalls bedeutet diese Haltung im Ergebnis, dass eine Verantwortlichkeit des Diensteanbieters in diesem Kontext nur unter einem erheblichen Begründungsaufwand erreicht werden kann. Bedenklich mutet insoweit die Argumentation an, die „notice“ an den Auktionsdienst eBay mit einem Hinweis auf illegale Kopien eines Films sei unzureichend, um „,actual knowledge“ oder ,awareness of apparent infringing activity " zu begründen. ${ }^{193}$ Überdies erzeugt diese Herangehensweise kaum einen Anreiz für Diensteanbieter, mit Rechteinhaberinnen zu kooperieren und geeignete Strategien zu entwickeln, um kooperativ und effektiv die Rechtsdurchsetzung zu gewährleisten. ${ }^{194}$

\footnotetext{
${ }^{190}$ Vgl. Perfect 10 v. CCBill, 488 F.3d 1102, 1114 (9th Cir. 2007).

${ }^{191}$ Chang, Cardozo Arts \& Entertainment Law Journal 28 (2010), 195 (204): , , [...] under the court's interpretation [...], the red flag test is effectively dead."

${ }^{192}$ So auch Chang, Cardozo Arts \& Entertainment Law Journal 28 (2010), 195 (204).

${ }^{193}$ So Hendrickson v. eBay, 165 F.Supp. 2d 1082, 1091 (C.D. Cal. 2001), obwohl der Kläger eBay unterrichtet hatte, welche eBay-Nutzer die Rechtsverletzung begingen.

${ }^{194}$ Vgl. auch Chang, Cardozo Arts \& Entertainment Law Journal 28 (2010), 195 (208).
} 
Von den Verteidigern einer möglichst niedrigschwelligen Immunisierung durch $\S 512$ DMCA wird im Hinblick auf den „red flag"-Test indes oftmals unter Verweis auf $\S 512(\mathrm{~m})(1)$ DMCA mit dem Verbot einer allgemeinen Monitoringpflicht argumentiert, das durch eine zu laxe Handhabung des Tests unterlaufen werde. ${ }^{195}$ Das erweckt jedoch Zweifel. Denn zunächst bedürfte es mit dieser strengen Handhabung der Regelung in $\S 512(\mathrm{~m})$ DMCA eigentlich nicht, weil bereits mit dem so interpretierten „red flag"-Test gerade kein Anreiz gesetzt würde, ein solches aktives Monitoring durchzuführen. AuBerdem kann mit einer vom Einzelfall abhängigen, ggf. sogar nur durch einen Hinweis ${ }^{196}$ ausgelösten Verpflichtung zum Auffinden weiterer geschützter Werke von einem allgemeinen Monitoring noch nicht die Rede sein. Schließlich ist ohnehin bedeutsam, dass es insoweit auf die Nichtverfügbarkeit von „standard technical measures" ankommt. Sehen solche technischen Maßnahmen die Möglichkeit eines Monitorings von Inhalten vor, würde ein wie auch immer zu definierendes Verbot eines allgemeinen Monitorings ohnehin nicht greifen. ${ }^{197}$

\section{b) Die Macht der Diensteanbieter vs. ,right and ability to control"}

Hinsichtlich des „threshold requirement“, weder rechtlich noch faktisch eine Kontrollmöglichkeit über rechtsverletzende Tätigkeiten innezuhaben, besteht in der Praxis noch ein erhebliches Unsicherheitspotenzial, ${ }^{198}$ da eine gesicherte Linie in der Rechtsprechung bislang nicht auszumachen ist. Dies erleichtert es den Diensteanbietern ebenfalls, sich aus Konflikten herauszuhalten. Zugleich erscheint die Bedeutung der Diensteanbieter, deren Geschäftsmodell die kuratierte, strukturierte Darstellung von Inhalten ist und die deshalb gerade darauf aus sind, ihre Nutzer zu binden, ${ }^{199}$ insoweit nicht angemessen abgebildet, weil der Zuordnung von Risiken bzw. Gefahrenquellen und deren Beherrschbarkeit sowie der Korrespondenz von Vorteilen und Nachteilen als Grundsatz „,der allgemeinen deliktischen Sorgfaltspflicht ${ }^{“ 200}$ nicht hinreichend Rechnung getragen wird. Unter diesem Ge-

${ }^{195}$ Vgl. Viacom v. YouTube, 718 F.Supp. 2d 514, 528 f. (S.D. N.Y. 2010); Lee, Columbia Journal of Law \& the Arts 32 (2008), 233 (253); Holznagel, Notice and Take-Down-Verfahren als Teil der Providerhaftung, S. 33.

${ }^{196}$ Dabei ist offen, ob nur ein nach $\S 512$ DMCA tauglicher Hinweis eine solche Pflicht auslösen sollte. Die besseren Argumente sprechen dafür, hier nicht allein den Maßstab für eine „notice“ nach DMCA heranzuziehen, weil diese und das Begründen einer „red flag“ gerade nicht deckungsgleich sind und auch nicht sein sollten, wie selbst Viacomv. YouTube, 718 F.Supp. 2d 514, 525 (S.D. N.Y. 2010), konzediert.

${ }^{197}$ Vgl. auch Fitzner, GRUR Int 2012, 109 (110).

${ }^{198}$ Kurzer Überblick bei Maier, Remixe auf Hosting-Plattformen, S. $155 \mathrm{f}$.

${ }^{199} \mathrm{Vgl}$. dazu noch unten $\S 8 \mathrm{~A}$.

${ }^{200}$ So treffend Wagner, in: MüKo BGB, §823 Rn. 455. 
sichtspunkt wäre es demgegenüber angemessener, die technischen Fähigkeiten der Diensteanbieter, die in Form großer Konzerne ja gerade deren Vorteil ausmachen und deretwegen sie ja gerade einen so großen Zulauf haben, ${ }^{201}$ als solche anzuerkennen und die gesetzlichen Anreize dementsprechend so zu verteilen, dass die technischen Möglichkeiten entsprechend der Interessenlage abgerufen werden können. ${ }^{202}$ Dies würde es hier erfordern, das „threshold requirement", nach dem die Diensteanbieter sich dazu veranlasst sehen, sich von ihren technischen Kontrollmöglichkeiten um ihrer Immunisierung willen gerade fernzuhalten, abzuschaffen oder zumindest dahingehend zu modifizieren, dass der verhältnismäßige Einsatz von technischen Kontrollmöglichkeiten einer (wie auch immer gestalteten) Haftungsprivilegierung nicht entgegensteht. Auch in diesem Punkt zeigt sich damit eine weitere Parallele zu § 230 DMCA, bei dem die sogar im Wortlaut vorgesehene Aufforderung an die betroffenen Diensteanbieter, ihre Steuerungsmöglichkeiten auch zu nutzen, praktisch und veranlasst durch die dargestellte Rechtsprechung leerläuft.

\section{Fazit}

Zusammenfassend zeigt sich, dass $§ 512$ DMCA einerseits zwar durchaus einen positiven Anteil an der Herausbildung des digitalen Ökosystems hatte. Zugleich sind aber punktuell auch Zweifel angebracht, ob die Regelung die juristischen Herausforderungen, die sich teilweise bereits wenige Monate nach Erlass des DMCA ergeben haben, wirksam auffängt. ${ }^{203}$ Andererseits ist allerdings ebenso festzustellen, dass die sehr weitgehende Prozeduralisierung der Verantwortung von Diensteanbietern hier ein hohes $\mathrm{Ma}$ an Berechenbarkeit sowohl für die Anbieter selbst als auch der Rechteinhaber im Umgang mit vermuteten Rechtsverletzungen erzeugt. Insbesondere die Intention, möglichst alle betroffenen Parteien in das Verfahren einzubeziehen ist begrüßenswert. ${ }^{204}$ Damit kann abschließend festgehalten werden, dass die Regelungen zum Notice-and-Takedown-Verfahren - gerade im Vergleich zu $\S 230$ CDA - eine im Grundansatz sinnvolle Normierung von Handlungsanforderungen der Diensteanbieter darstellen. Die Anreizstrukturen der Regelung in §512 DMCA erweist sich allerdings teilweise als nicht minder kri-

${ }^{201}$ Zutreffend weist Gillespie, Georgetown Law Technology Review 2 (2018), 198 (202), darauf hin, dass ohne jedwede technische Steuerung, insbesondere ohne „Content Moderation“ ein entsprechender Dienst leicht gestaltet werden könnte, zugleich aber am Markt kaum Chancen auf Erfolg hätte.

${ }^{202}$ Zur Berücksichtigung technischer Fähigkeiten bei der Konkretisierung rechtlicher Erwartungen vgl. noch unten $\S 12$ B. III. 3. a).

${ }^{203}$ Vgl. Hassanabadi, Berkeley Technology Law Journal 26 (2011), 405 (415 f.).

${ }^{204}$ Vgl. auch Beckmann, Verantwortlichkeit von Online-Diensteanbietern in Europa und den Vereinigten Staaten von Amerika, S. $284 \mathrm{f}$. 
tisch. Insbesondere ist hier die die Motivation bzw. die Konsequenz der Normierung des Notice-and-Takedown-Verfahrens zu nennen, Diensteanbieter von einer Befassung mit den Konflikten ihrer Nutzer abzuhalten. Die Benennung der hieraus folgenden Konsequenzen und deren eingehende Erörterung bleiben dem Kontext des europäischen Regelungskomplexes vorbehalten, der an entscheidenden Stellen bewusst Anleihen am soeben dargestellten Notice-and-Takedown-Verfahren nach §512 DMCA genommen hat und so insbesondere bei den Anreizstrukturen Pfadabhängigkeiten geschaffen hat, die auch heute noch den rechtlichen Zugriff auf die Diensteanbieter in der EU prägen. 



\section{$\S 4$ \\ Europäische Union \& Deutschland}

Der soeben erläuterten Entwicklung der Rechtslage in den USA und der kritischen Beobachtung der Handhabung zweier zentraler Regelungen für den Umgang der Diensteanbieter mit Inhalten ihrer Nutzerinnen soll nun die Rechtslage in der Europäischen Union und in Deutschland gegenübergestellt werden. Ziel ist es, die Pfadabhängigkeiten aufzuzeigen und so einen reflektierten Blick auf die Entstehungsbedingungen der europäischen Regelungen zu ermöglichen. Die nachstehenden Ausführungen folgen dabei der zeitlichen Abfolge des Erlasses verschiedener Regelungen auf nationaler und europäischer Ebene. Vor 1997 hatte die Frage nach der Haftung von Intermediären noch keine spezifische gesetzliche Regelung erfahren (A.). Im Jahr 1997 erließ der deutsche Gesetzgeber das TDG,${ }^{1}$ dessen $\S 5$ die Verantwortlichkeit der hiervon erfassten Diensteanbieter regelte (B.), bevor diese Bestimmungen infolge der danach verabschiedeten ECRL (C.) angepasst bzw. umgesetzt wurden (D.).

\section{A. Rechtslage in Deutschland vor Erlass des TDG}

Vor Erlass des $\mathrm{TDG}^{2}$ waren Rechtsprechung und Rechtswissenschaft gezwungen, die aufkommende Nutzung des Internet und die sich hieraus ergebenden Fragestellungen aufgrund überkommener Figuren und Institute zu lösen. Mangels gefestigter Rechtsprechung wurden hinsichtlich der Haftung für Inhalte Dritter viele, teils konträre Auffassungen vertreten, ${ }^{3}$ die vor allem um die richtige Bestimmung des Sorgfaltsmaßstabs kreisten. Soweit ersichtlich ergingen vor Erlass des TDG keine gerichtlichen Entscheidungen, die

${ }^{1}$ Art. 1 Informations- und Kommunikationsdienste-Gesetz, BGB1. I 1997, S. 1870. Das IuKDG wurde auch als sog. Multimediagesetz des Bundes bezeichnet, vgl. Freytag, Haftung im Netz, S. 1; zum IuKDG insgesamt Engel-Flechsig/Maennel/Tettenborn, NJW 1997, $2981 \mathrm{ff}$.

${ }^{2}$ Gemeint ist hier die Phase zwischen der Liberalisierung des Telekommunikationsmarktes und Erlass des TDG. Für einen Überblick zur Rechtslage bis zur Liberalisierung siehe nur Bleisteiner, Rechtliche Verantwortlichkeit im Internet, S. $138 \mathrm{ff}$.

${ }^{3}$ Allgemein zur Unsicherheit in der rechtlichen Bewertung Freytag, ZUM 1999, 185 (185f.). 
sich unmittelbar mit der Haftung von Diensteanbietern im Sinne dieser Untersuchung befassten. Dafür waren aber viele Konfliktlagen bekannt, die ähnliche Rechtsfragen aufwarfen, nämlich für Printmedien, ${ }^{4}$ Rundfunk und Fernsehen, ${ }^{5}$ die sog. Mailbox-Systeme zur Kommunikation und Datenaustausch $^{6}$ sowie insbesondere Post- und Telekommunikationsdienste. ${ }^{7}$ Eine theoretisch auf die Thematik dieser Untersuchung übertragbare Entscheidung traf der BGH bereits 1976, als er festhielt, dass Fernsehanstalten und ihre für eine Sendung verantwortliche Mitarbeiter nicht für jedwede Äußerung Dritter passivlegitimiert und damit verantwortlich seien. ${ }^{8}$, ,[Es] widerspräche [...] dem Wesen des Mediums und seiner Funktion, es neben oder gar an Stelle des eigentlichen Urhebers der Äußerung in Anspruch nehmen zu können. " Indes sprach das Gericht auch hier schon davon, dass es bei der Frage der Haftung darauf ankomme, „den Besonderheiten Rechnung zu tragen, die sich aus seiner [des Mediums] Rolle [...] ergeben “. ${ }^{10}$ Später entschied derselbe Senat, dass der Verleger einer Zeitschrift als Störer für Unterlassungsansprüche passivlegitimiert sei, da er an der Verbreitung der $\mathrm{Pu}$ blikation bestimmter Äußerungen mitwirke, auch wenn er sie sich nicht zu

${ }^{4}$ Vgl. zur Abgrenzung des Maßes der Sorgfaltspflicht des Verlegers zwischen redaktionellem und Werbeteil BGH, Urt. v. 20.06.1972 - VI ZR 26/71, NJW 1972, 1658 (1659); ähnlich (Beschränkung der Sorgfaltspflicht auf grobe Verstöße bei Anzeigen, auch im Lichte der Grenze der Pressefreiheit in Art. 5 Abs. 2 GG) Urt. v. 30.06.1972 - I ZR 1/71, GRUR 1973, 203 (204) - Badische Rundschau; Urt. v. 26.04.1990 - I ZR 127/88, GRUR 1990, 1012 (1014) - Pressehaftung I; Urt. v. 19.03.1992 - I ZR 166/90, NJW 1992, 3093 (3094) - Ausländischer Inserent. Zur Unterlassungshaftung des Setzers einer Druckschrift KG, Urt. v. 26.09.1986 - 9 U 1849/86, AfP 1987, 427 f. Die Haftung auf Unterlassen des Versandhändlers als Störer sah der BGH nicht unter der presserechtlichen Kategorie der Zumutbarkeit begrenzt, vgl. BGH, Urt. v. 03.02.1976 - VI ZR 23/72, NJW 1976, 799 (800) - Alleinimporteur; dazu auch schon Urt. v. 15.01.1957 - I ZR 56/55, GRUR 1957, 352 ff. Taeschner (Pertusin II).

${ }^{5}$ Aus zivilrechtlicher Perspektive BGH, Urt. v. 06.04.1976 - VI ZR 246/74, NJW 1976, 1198 ff. - Panorama. Aus strafrechtlicher Perspektive EGMR, Urt. v. 23.09.1994 36/119/431/510, NStZ 1995, 237 ff., zur redaktionellen Entscheidung, eine Zusammenfassung eines Interviews mit rassistischen Äußerungen der Gesprächspartner zu senden. Generell lässt sich festhalten, dass bei Fragen der Haftung von Journalisten bzw. Verlegern die Annahme einer solchen Haftung zu Recht sehr hohen Hürden gegenübersteht.

${ }^{6}$ LG Stuttgart, Urt. v. 17.11.1987 - 17 O 478/87, JurPC 1992, 1714 (1716), lehnte eine Haftung eines Betreibers eines Mailbox-Systems ab und verwies für die Bejahung eines Unterlassungsanspruchs auf die Verantwortlichkeit des Verlegers einer Zeitung für den Anzeigenteil. Zum Mailbox-System vgl. ferner Edwards, The Atlantic (04.11.2016), www.t heatlantic.com/technology/archive/2016/11/the-lost-civilization-of-dial-up-bulletin-boa rd-systems/506465.

${ }^{7}$ Vgl. dazu Spindler, ZUM 1996, 533 (541 f.).

${ }^{8}$ Vgl. BGH, Urt. v. 06.04.1976 - VI ZR 246/74, NJW 1976, 1198 (1199) - Panorama.

${ }^{9}$ BGH, Urt. v. 06.04.1976 - VI ZR 246/74, NJW 1976, 1198 (1199) - Panorama.

${ }^{10}$ BGH, Urt. v. 06.04.1976 - VI ZR 246/74, NJW 1976, 1198 (1199) - Panorama. 
eigen mache. ${ }^{11}$ In diesen Entscheidungen lassen sich bereits erste Ansätze zur Bestimmung der Verantwortlichkeit für fremde Inhalte erkennen, die zum Teil noch deutlich später rezipiert wurden.

Für die Diskussion im Schrifttum sei exemplarisch auf zwei Stimmen verwiesen, die sich bei der haftungsrechtlichen Einordnung der Diensteanbieter zur damaligen Zeit gegenüberstanden. Sieber sah - allerdings auf die strafrechtliche Perspektive bezogen ${ }^{12}$ - nur in Fällen einer „extremen Gefahrsteigerung" eine gesteigerte Sorgfaltspflicht begründet. Im Übrigen entspreche das Risiko der Versendung strafbarer Inhalte über einen Internetdienst den „allgemeinen Missbrauchsmöglichkeiten“ und sei daher insoweit nicht relevant. ${ }^{13}$ Spindler verwies hingegen auf die Grundlagen der Verkehrspflichtendogmatik, nämlich die Eröffnung einer Gefahrenquelle. ${ }^{14}$ Deliktsrechtlich müsse zwar nicht für alle denkbaren Risiken vorgesorgt werden; der Schuldner der Sorgfaltspflicht könne sich aber nicht auf die ausschließlich bestimmungsgemäße Nutzung verlassen. ${ }^{15}$ Der Verweis auf die reine Vermittlungsfunktion der Diensteanbieter sei nicht geeignet, diese deliktsrechtliche Einschätzung zu entkräften, da deren Tätigkeit die Risiken und eingetretenen Schäden durch ortsunabhängige Verbreitung potenziere. ${ }^{16}$ Entscheide sich der Anbieter gegen die Moderation seines Dienstes, müsse „er sich so behandeln lassen, als hätte er ohne jegliche Sicherungsvorkehrungen eine Gefahrenquelle eröffnet", woraus die Haftung für Beiträge Dritter folge. ${ }^{17}$ Während auf die Frage der Störerhaftung andernorts vertieft einzugehen sein wird, lässt sich hier für die Rechtslage vor Erlass des TDG festhalten, dass sich sowohl in der Rechtsprechung als auch im Schrifttum bereits frühzeitig

${ }^{11}$ Vgl. BGH, Urt. v. 27.05.1986 - VI ZR 169/85, NJW 1986, 2503 (2504) - Ostkontakte; vgl. dazu auch Spindler, ZUM 1996, 533 (538). Zum Zueigenmachen fremder Inhalte vgl. noch unten $\S 4$ D. I. 2.

${ }^{12}$ Diese beschränkte Perspektive steht durchaus stellvertretend für die Debatten dieser Zeit, vgl. Freytag, Haftung im Netz, S. 12. Ob im Sinne der Einheit der Rechtsordnung die Wertungen zwischen straf- und zivilrechtlicher Ebene zwingend gleichlaufen müssen, wie es Sieber, JZ 1996, 429 (437), vertritt, ist allerdings zweifelhaft. Dies zeigt sich gerade in Fällen, in denen an der Verbreitung rechtswidriger Inhalte ein offenkundig schützenswertes Interesse besteht, beispielsweise um die Öffentlichkeit im Rahmen von Berichterstattung über Missstände zu informieren, wie es etwa bei EGMR, Urt. v. 23.09.1994 36/119/431/510, NStZ 1995, 237 (238), offenkundig wird.

${ }^{13}$ Sieber, JZ 1996, 494 (502).

${ }^{14}$ Spindler, ZUM 1996, 533 (536); ihm stimmt Waldenberger, ZUM 1997, 176 (183 ff.), $\mathrm{zu}$.

${ }^{15}$ Spindler, ZUM 1996, 533 (536).

${ }^{16}$ Spindler, ZUM 1996, 533 (536).

${ }^{17}$ Spindler, ZUM 1996, 533 (537). Damit greift er letztlich genau den Differenzierungsansatz auf, dem Stratton Oakmont v. Prodigy Services Co., 1995 WL 3237101 (N.Y. Sup. Ct. 1995), sowie die ursprünglich intendierte Absicht hinter $\$ 230$ CDA zugrunde lagen. 
die Problem- bzw. Konfliktlagen manifestierten, die auf das Recht als Folge der zunehmenden Verbreitung des Internets in alle gesellschaftlichen Bereiche zukommen würden und die teilweise auch heute noch nicht abschließend geklärt sind.

\section{B. TDG}

Im europäischen Kontext stellt die Regelung des §5 TDG die erste prominente Thematisierung der Haftungsfrage durch einen Gesetzgeber dar. Deren Entstehungsbedingungen sollen im Folgenden kurz skizziert werden, um damit die Grundlage für die Erörterung der noch heute geltenden Art. 14, 15 ECRL und $\S \S 7 \mathrm{ff}$. TMG zu legen, die sich in vielen Einzelheiten an $\S 5$ TDG orientieren.

\section{Hintergrund}

Bereits 1995 wurde ein Handlungsbedarf erkannt, um die Rahmenbedingungen für die immer populärer werdenden Internetdienste herzustellen. ${ }^{18}$ Ebenso wie in den USA lag eine der entscheidenden Erwägungen in dem Schutz der hier involvierten Unternehmen und ihrer Investitions- und Standortbedingungen ${ }^{19}$ wozu sich insbesondere die Haftungsfrage verhalten musste,$^{20}$ weil die rechtliche Unsicherheit als Belastung bzw. Risiko für die Entwicklung des Internets wahrgenommen wurde. ${ }^{21}$ Nicht sonderlich überraschend lagen schon früh die ersten Konfliktpotenziale im bundesdeutschen Föderalismussystem begründet: der Bund hielt sich für weitgehend zuständig, während die Länder dies selbstredend anders sahen. ${ }^{22}$ Ein mitunter chaotischer Prozess - teilweise arbeiteten Bund und Länder an konkurrierenden Regelungswerken ${ }^{23}$ - mündete schließlich in eine gemeinsame Erklärung von Bund und Ländern, die primär die Innovationsoffenheit in den Blick nahm

\footnotetext{
${ }^{18}$ Vgl. Bleisteiner, Rechtliche Verantwortlichkeit im Internet, S. 74; Freytag, Haftung im Netz, S. 17; Matthies, Providerhaftung für Online-Inhalte, S. 35.

${ }^{19}$ So u.a. die Auffassung des 1995 von Bundeskanzler Kohl eingerichteten Rates für Forschung, Technologie und Innovation beim Bundeskanzler, vgl. BT-Drucks. 13/4000, S. 42: „Um zu einer freien Entfaltung der Marktkräfte bei den neuen Multimedia-Diensten zu gelangen [...], sollte in national einheitliches Medienordnungsrecht geschaffen werden. Dabei sollten Regelungen auf ein notwendiges Maß beschränkt werden.“

${ }^{20}$ Freytag, Haftung im Netz, S. 17.

${ }^{21}$ Vgl. Eifert, in: Eifert/Gostomzyk, Netzwerkrecht, S. 9 (10).

${ }^{22}$ Weiterführend dazu Freytag, Haftung im Netz, S. 18; Bleisteiner, Rechtliche Verantwortlichkeit im Internet, S. 74 ff., mwN.

${ }^{23}$ Vgl. Bleisteiner, Rechtliche Verantwortlichkeit im Internet, S. 74.
} 
und die Bestrebung zum Ausdruck brachte, zwar an einer parallelen Regelung in Bund und Ländern festzuhalten, die Vorgaben aber weitgehend identisch auszugestalten. ${ }^{24}$ Dieser Erklärung folgend kam es zur Verabschiedung u.a. des $\$ 5$ TDG, der am 1. August 1997 in Kraft trat und damit als erste Regelung im europäischen Rechtsraum die Frage der Haftung der Diensteanbieter unmittelbar adressierte. ${ }^{25}$

\section{Konzeption von $\$ 5 T D G$}

$\S 5$ TDG hatte folgenden Wortlaut:

(1) Diensteanbieter sind für eigene Inhalte, die sie zur Nutzung bereithalten, nach den allgemeinen Gesetzen verantwortlich.

(2) Diensteanbieter sind für fremde Inhalte, die sie zur Nutzung bereithalten, nur dann verantwortlich, wenn sie von diesen Inhalten Kenntnis haben und es ihnen technisch möglich und zumutbar ist, deren Nutzung zu verhindern.

(3) Diensteanbieter sind für fremde Inhalte, zu denen sie lediglich den Zugang zur Nutzung vermitteln, nicht verantwortlich. Eine automatische und kurzzeitige Vorhaltung fremder Inhalte auf Grund Nutzerabfrage gilt als Zugangsvermittlung.

(4) Verpflichtungen zur Sperrung der Nutzung rechtswidriger Inhalte nach den allgemeinen Gesetzen bleiben unberührt, wenn der Diensteanbieter unter Wahrung des Fernmeldegeheimnisses gemäß $\S 85$ des Telekommunikationsgesetzes von diesen Inhalten Kenntnis erlangt und eine Sperrung technisch möglich und zumutbar ist.

Die im Vergleich zu Access- bzw. Cache-Providern gesteigerte qualitative Nähe der Diensteanbieter zu der Information des Dritten kommt hier insbesondere in den Absätzen 2 und 3 zum Ausdruck. ${ }^{26}$ Der Gesetzgeber sah in dieser Nähe denn auch eine „Garantenstellung [des Diensteanbieters] für die Verhinderung der Übermittlung [rechtswidriger Inhalte] an Dritte“, wenn diese „bewußt zum Abruf bereit [gehalten werden] ${ }^{\circ .}{ }^{27}$ Damit sollte für $\S 5$ Abs. 2 TDG auf subjektiver Seite ein Gleichlauf zum Vorsatzkriterium des allgemeinen Straf- und Ordnungswidrigkeitenrechts, aber auch zu den zivilrechtlichen Grundsätzen hergestellt werden. ${ }^{28}$

${ }^{24}$ Vgl. Matthies, Providerhaftung für Online-Inhalte, S. 36 f.; die Erklärung ist abgedruckt bei Engel-Flechsig, ZUM 1997, $231 \mathrm{ff}$.

${ }^{25}$ Vgl. Frey, Die Haftung von Host-Providern für Immaterialgüterrechtsverletzungen, S. 115, der § 5 TDG sowie §512 DMCA insoweit eine „Vorreiterrolle“ zuschreibt.

${ }^{26}$ Vgl. Hollenders, Mittelbare Verantwortlichkeit von Intermediären im Netz, S. 206.

${ }^{27}$ BT-Drucks. 13/7385, S. 20.

${ }^{28}$ BT-Drucks. 13/7385, S. 20. 


\section{Handhabung}

\section{1. „Filterfunktion“, Darlegungs- und Beweislast}

Nach der Konzeption des Regierungsentwurfs sollten, sofern die Voraussetzungen der Norm vorliegen, die Rechtsfolgen sich nach den allgemeinen Regeln der Rechtsordnung richten. ${ }^{29}$ Die insoweit als „Filter" bezeichnete Funktion des $\S 5$ TDG war freilich nicht unumstritten. ${ }^{30}$ Der BGH sah die Tatbestandsmerkmale des $\S 5$ Abs. 2 TDG als den anspruchsbegründenden Merkmalen im Sinne von $\S 823$ BGB zugehörig an, für die der Gläubiger die Darlegungs- und Beweislast trage, und schloss sich damit der Auffassung an, die § 5 TDG eine „Filterfunktion“ zuschrieb. ${ }^{31}$ Dafür stritt auch der Wortlaut der Norm (,... nur dann verantwortlich ..."), der ein Regel-AusnahmeVerhältnis nahelegte. ${ }^{32}$ Damit stellte er sich u.a. Spindler entgegen, der argumentiert hatte, als Haftungsprivilegierung stelle § 5 TDG eine Ausnahme zum allgemeinen Haftungsregime und damit eine für den Diensteanbieter günstige Tatsache dar. ${ }^{33}$

\section{Bereithalten eines fremden Inhalts}

Gegenüber der ECRL noch als eigenständiges Tatbestandsmerkmal ausgestaltet, setzte $\$ 5$ Abs. 2 TDG voraus, dass Inhalte „,bereitgehalten“ werden. ${ }^{34}$ Diese Voraussetzung verhielt sich in ihrem textlichen Gehalt jedoch schon zu den damaligen Formen und Bedingungen vielfältiger Geschäftsmodelle im Internet sehr unscharf. ${ }^{35}$

Die entscheidende Weichenstellung für die Anwendung der Privilegierung nach $§ 5$ TDG war sodann die Frage, ob es sich um einen eigenen (§5 Abs. 1 TDG) oder fremden Inhalt ( 55 Abs. 2-4 TDG) handelte, für die der Diensteanbieter in Anspruch genommen wurde. Die Annahme eines fremden In-

${ }^{29}$ BT-Drucks. 13/7385, S. 20.

${ }^{30}$ Eine solche Funktion nehmen Engel-Flechsig/Maennel/Tettenborn, NJW 1997, 2981 (2984), an; vgl. zur Gegenauffassung Freytag, ZUM 1999, 185 (189).

${ }^{31}$ BGH, Urt. v. 23.09.2003 - VI ZR 335/02, NJW 2003, 3764 (3764 f.).

${ }^{32} \mathrm{Vgl}$. Hoffmann/Volkmann, in: Spindler/Schuster, Recht der elektronischen Medien, TMG $§ 10$ Rn. 54.

${ }^{33}$ Spindler, NJW 1997, 3193 (3198), gesteht allerdings zugleich zu, dass der Beweis des Nichtvorliegens einer Tatsache, hier der fehlenden Kenntnis, kaum zu erbringen sei, sodass deshalb Darlegungs- und Beweislasten entsprechend abgestuft in Anschlag zu bringen seien.

${ }^{34}$ Dazu ausführlich Freytag, Haftung im Netz, S. $162 \mathrm{ff}$.

${ }^{35} \mathrm{Vgl}$. Freytag, Haftung im Netz, S. 163 f. Es lässt sich eine gewisse Kontinuität insoweit nicht leugnen, als auch unter dem status quo eine Unschärfe der juristischen Inbezugnahme der Geschäftsmodelle festzustellen ist. Darauf wird noch einzugehen sein, vgl. ausführlich unten $\S 8$. 
halts setzte nach Auffassung vieler Stimmen eine Distanzierung von dem transportierten Inhalt voraus, ohne die nicht auszuschließen sei, dass der Diensteanbieter selbst für diesen einstehen will. ${ }^{36}$ Anknüpfend hieran war ebenso entscheidend für eine Inanspruchnahme des Diensteanbieters, ob der Diensteanbieter des Privilegs verlustig ging, weil er sich einen Inhalt zu Eigen gemacht hat ${ }^{37}$ und dieser daher als eigener zu behandeln war. ${ }^{38}$ Diese Abgrenzung ist auch noch heute noch von Bedeutung. ${ }^{39}$

\section{Kenntnis}

„Kenntnis“ im Sinne von $\S 5$ Abs. 2 TDG setzte die positive Kenntnis des konkreten, in Rede stehenden Inhalts voraus. Ob eine Haftung auch für einen bedingten Vorsatz in Betracht kam, war nicht abschließend geklärt. ${ }^{40}$ Ein Kennenmüssen als hinreichende Bedingung wurde aber jedenfalls überwiegend abgelehnt ${ }^{41}$ obgleich vereinzelt auch auf die mögliche Treuwidrigkeit einer Berufung auf mangelnde Kenntnis hingewiesen wurde. ${ }^{42}$ Der Maßstab der Kenntnis bezog sich außerdem nur auf den Inhalt selbst, nicht auf seine rechtliche Bewertung als rechtswidrig. ${ }^{43}$

\section{Technische Möglichkeit und Zumutbarkeit der Verhinderung der Nutzung von Inhalten}

Schließlich setzte die Haftung ausweislich $\S 5$ Abs. 2 TDG weiterhin voraus, dass die Verhinderung der Nutzung des Inhalts technisch möglich und zumutbar ist. ${ }^{44}$ Dabei sollte es nicht nur um die technische, sondern auch um die

\footnotetext{
${ }^{36}$ Vgl. Spindler, NJW 1997, 3193 (3196). Dies beruhte auf der im Presserecht tradierten Figur der sog. Verbreiterhaftung, bei der die Verbreitung einer Äußerung ohne eigene Distanzierung von ihrem Inhalt in eine eigene Äußerung des Verbreitenden umschlagen soll, was selbst dann gilt, wenn die verbreitete Tatsache als von einem Dritten stammend gekennzeichnet wird, vgl. BGH, Urt. v. 29.10.1968 - VI ZR 180/66, GRUR 1969, 147 (150) - Korruptionsvorwurf; Urt. v. 20.06.1969 - VI ZR 234/67, NJW 1970, 187 (189) - Hormoncreme; Urt. v. 26.11.1996 - VI ZR 323/95, NJW 1997, 1148 (1149) - Stern-TV; vgl. dazu auch Burkhardt/Peifer, in: Wenzel, Das Recht der Wort- und Bildberichterstattung, Kap. 10 Rn. 208 ff.

${ }^{37}$ Vgl. schon BT-Drucks. 13/7385, S. 19.

${ }^{38}$ Vgl. ausführlich zur Rechtslage unter $§ 5$ TDG Klein, Haftung von Social-SharingPlattformen, S. $76 \mathrm{ff}$.

${ }^{39}$ Dazu sogleich $\S 4$ D. I. 2.

${ }^{40}$ Dafür wohl BT-Drucks. 13/7385, S. 20, wo von vorsätzlichem Handeln die Rede ist; dagegen Spindler, NJW 1997, 3193 (3196).

${ }^{41}$ Vgl. BGH, Urt. v. 23.09.2003 - VI ZR 335/02, NJW 2003, 3764 (3764); Engel-Flechsig/Maennel/Tettenborn, NJW 1997, 2981 (2985); Spindler, NJW 1997, 3193 (3196).

${ }^{42}$ So Spindler, NJW 1997, 3193 (3197).

${ }^{43}$ Vgl. Freytag, Haftung im Netz, S. $180 \mathrm{ff}$.

${ }^{44}$ Dies hatte das AG München, Urt. v. 28.05.1998 - 8340 Ds 465 Js 173158/95, NJW
} 
wirtschaftliche Zumutbarkeit der Verhinderung gehen. ${ }^{45}$ Auf dieses Merkmal verzichtete der Gesetzgeber zwar mit der ersten Reform des TDG ${ }^{46}$ mit der die ECRL in nationales Recht umgesetzt wurde. Er ging jedoch davon aus, dass die technische Möglichkeit und Zumutbarkeit der Entfernung bzw. Sperrung als „allgemeiner Grundsatz“ weiterhin Geltung beanspruche. ${ }^{47}$

\section{ECRL}

Der Erlass der ECRL stellte für das Haftungsrecht der Diensteanbieter in der EU einen Systemwechsel dar, ${ }^{48}$ der auch als Zäsur für die mitgliedstaatlichen Vorschriften zur Haftung für fremde Inhalte bezeichnet worden ist. ${ }^{49}$ Die ECRL ist bis heute Kernstück der Regelungen zur Haftung der Diensteanbieter und steht als solches im Zentrum dieser Untersuchung. Im Folgenden sollen Hintergrund (I.) und Konzeption (II.) erläutert werden, bevor der Blick auf die einzelnen Regelungen zur Haftung wandert. Hier spielen insbesondere die Privilegierung für Host-Provider (III.) und das allgemeine Verbot von Überwachungspflichten (IV.) eine entscheidende Rolle.

\section{Hintergrund}

Bereits im Frühjahr 1997, also noch vor Erlass des TDG, hatte die Europäische Kommission in ihrer „Europäischen Initiative für den elektronischen Geschäftsverkehr" die Priorität auf die Entwicklung eines sicheren rechtlichen Rahmens gelegt, um ,den elektronischen Geschäftsverkehr in Europa kräftig an[zu]kurbeln. “50 Hierzu wurden die „Schaffung eines günstigen ord-

1998, 2836 (2839) - CompuServe, in der bereits erwähnten strafrechtlichen Verurteilung des Geschäftsführers von CompuServe Deutschland wegen Verbreitung pornographischer Schriften mit der Begründung bejaht, einem Repräsentanten einer deutschen Rechtsperson sei die Zumutbarkeit auch hinsichtlich des Datenspeichers des US-amerikanischen Mutterunternehmens vorzuwerfen. Dies sah die Berufungsinstanz - LG München I, Urt. v. 17.11.1999 - 20 Ns 465 Js 173158/95, NJW 2000, 1051 (1051f.) - CompuServe - anders. Vgl. zu den Reaktionen auf das amtsgerichtliche Urteil oben $\S 1$ Fn. 21, 22 und den zugehörigen Text. Die Darstellung bei Spindler, MMR 2018, 48 (48), wonach dieses Verfahren den Anlass gab, über die Einführung von Haftungsprivilegierungen nachzudenken, ist zweifelhaft, da jedenfalls zum Zeitpunkt der Entscheidung das TDG schon galt und dieses in der Entscheidung selbst umfänglich rezipiert wurde.

${ }^{45}$ Vgl. Spindler, NJW 1997, 3193 (3197).

${ }^{46}$ Vgl. §11 TDG in der Fassung gemäß Art. 1 des Elektronischer GeschäftsverkehrGesetz v. 14.12.2001, BGB1. I 2001, S. 3721.

${ }^{47}$ Vgl. BT-Drucks. 14/6098, S. 25.

${ }^{48}$ Vgl. Hollenders, Mittelbare Verantwortlichkeit von Intermediären im Netz, S. 207.

${ }^{49}$ Vgl. Klein, Haftung von Social-Sharing-Plattformen, S. 75.

${ }^{50} \mathrm{KOM}(97) 157$ endg., S. 3. 
nungspolitischen Rahmens ${ }^{\text {“51 }}$ und die „Förderung eines günstigen unternehmerischen Umfelds ${ }^{652}$ als notwendig erachtet. Der Erlass der ECRL wurde hier zwar noch nicht ausdrücklich in Aussicht gestellt. ${ }^{53}$ Der Vorschlag der Kommission für die ECRL vom 23. Dezember $1998^{54}$ griff diese Prioritäten jedoch prominent auf und setzte diese eben u.a. mit den Art. 12 ff. um, ${ }^{55}$ die einen Schwerpunkt des Entwurfs darstellten. ${ }^{56}$ Was die Haftung von HostProvidern betrifft, orientiert die ECRL sich u.a. an $\$ 5$ TDG,${ }^{57}$ dessen abgestuftes Haftungsmodell sie teilweise übernahm, sowie an $\S 512$ DMCA. ${ }^{58}$ Insbesondere die Regelungen in $\S 512$ (c)(A) DMCA und Art. 14 ECRL sind sich - trotz des wesentlichen Unterschieds im Anwendungsbereich der Normkomplexe ${ }^{59}-$ sehr ähnlich, soweit es um die Anforderungen an die Haftungsfreistellung geht. ${ }^{60}$

Die Ähnlichkeiten zwischen $\$ 230$ CDA und der ECRL sind hingegen nicht sonderlich ausgeprägt. So ist schon der intendierte Anwendungsbereich grundverschieden: während die ECRL (wie auch $\S 5$ TDG) als sektorübergreifende Regelung potenziell jedes Rechtsgebiet erfasst, sollte $\$ 230$ CDA von vornherein insbesondere nicht die Verantwortlichkeit für Urheberrechtsverletzungen regeln. ${ }^{61}$

${ }^{51} \mathrm{KOM}(97) 157$ endg., S. 4, $21 \mathrm{ff}$.

${ }^{52} \mathrm{KOM}(97) 157$ endg., S. 5, $30 \mathrm{ff}$.

${ }^{53}$ Hoeren, MMR 1999, 192 (193), betont denn auch, dass die Richtlinie keine nennenswerte Vorgeschichte aufzuweisen habe.

${ }^{54} \mathrm{KOM}(1998) 586$ endg.

${ }^{55}$ Vgl. zum Zusammenhang auch Maennel, MMR 1999, 187 (187).

${ }^{56}$ Vgl. Maennel, MMR 1999, 187 (191).

${ }^{57} \mathrm{Vgl}$. Kuczerawy, Intermediary Liability and Freedom of Expression in the EU, S. 73; Beckmann, Verantwortlichkeit von Online-Diensteanbietern in Europa und den Vereinigten Staaten von Amerika, S. 270; Hoeren, MMR 1999, 192 (193).

${ }^{58}$ Vgl. Spindler, in: Spindler/Schmitz, TMG, § 10 Rn. 1; BT-Drucks. 14/6098, S. 22. Kritisch Bröhl, MMR 2001, 67 (71), der die fehlende Übernahme des „schlanke[n] Regelungsansatz[es] des geltenden $\S 5$ TDG“ beklagt.

${ }^{59}$ Vgl. Holznagel, Notice and Take-Down-Verfahren als Teil der Providerhaftung, S. 85 mit Fn. 9.

${ }^{60} \mathrm{Vgl}$. auch Beckmann, Verantwortlichkeit von Online-Diensteanbietern in Europa und den Vereinigten Staaten von Amerika, S. 285.

${ }^{61}$ Allerdings meint Beckmann, Verantwortlichkeit von Online-Diensteanbietern in Europa und den Vereinigten Staaten von Amerika, S. 240, unzutreffend, §230 CDA regele die Haftung nur für Ehrverletzungen. Das mag zwar im Ansatz der wesentliche Anwendungsfall im Sinne des Gesetzgebers gewesen sein. Im Gesetz findet diese Begrenzung jedenfalls keinen Niederschlag. 


\section{Konzeption}

\section{Allgemeines, Systematik}

Wie auch schon $\S 5$ TDG nimmt die ECRL der Sache nach keine positive Normierung von Haftungsvoraussetzungen vor, sondern schränkt diese im Sinne der bereits erwähnten „Filterlösung " negativ ein. ${ }^{62}$ Sie unterteilt in dem mit „Verantwortlichkeit der Vermittler“ übertitelten Abschnitt die Regelungen zur Verantwortlichkeit nach den einzelnen funktionsspezifischen Tätigkeiten - und trifft die Differenzierung damit gerade nicht nach den Akteuren $^{63}$ - sowie der allgemeinen Regel in Art. 15. Art. 12 betrifft die „reine Durchleitung", Art. 13 adressiert „Caching"-Dienste und Art. 14 betrifft die hier vor allem interessierenden „Hosting“-Anbieter. Art. 15 statuiert, dass keinen der vorgenannten Dienste „allgemeine Verpflichtung[en]“ zur Überwachung der von ihnen übermittelten oder gespeicherten Informationen oder zur aktiven Nachforschung nach Umständen, die auf eine rechtswidrige Tätigkeit hinweisen" auferlegt werden dürfen.

\section{Persönlicher Anwendungsbereich der Art. $12 \mathrm{ff}$.}

\section{a) Grundlagen}

Der Abschnitt 4 der ECRL betrifft in persönlicher Hinsicht „Dienste der Informationsgesellschaft“. Art. 2 lit. a verweist auf Art. 1 Nr.2 RL 98/34/EG, die allerdings durch die RL (EU) 2015/1535 aufgehoben und neu gefasst wurde, und die grundlegende Regelungen für „Dienste der Informationsgesellschaft" vorsieht. ${ }^{64}$ Art. 1 Abs. 1 lit. b RL (EU) 2015/1535 als insoweit maßgebliche Vorschrift definiert nunmehr als Dienstleistung der Informationsgesellschaft ,jede in der Regel gegen Entgelt elektronisch im Fernabsatz und auf individuellen Abruf eines Empfängers erbrachte Dienstleistung". Diese Kriterien unterliegen angesichts der technischen Entwicklung der letzten Jahre in vielerlei Hinsicht Zweifeln, ob sie tatsächlich noch sachangemessen sind, die hier allerdings nicht ausgebreitet werden können. ${ }^{65}$ Ausweislich Erwägungsgrund 18 S. 1 ECRL soll der Begriff des Dienstes der Informationsgesellschaft weit ausgelegt werden. Erwägungsgrund 18 S. 5 nimmt dabei schon die einzelnen Tätigkeiten vorweg, die im Fokus des Abschnitts 4 liegen.

${ }^{62}$ Vgl. Maennel, MMR 1999, 187 (191).

${ }^{63}$ Vgl. Marly, in: Grabitz/Hilf/Nettesheim, Recht der EU, 40. EL 2009, ECRL Vor. Abschnitt 4 Rn. 5.

${ }^{64}$ Veraltet insofern Chmelik, Social Network Sites - Soziale Netzwerke, S. 76; vgl. auch Riordan, The Liability of Internet Intermediaries, S. $387 \mathrm{ff}$.

${ }^{65}$ Vgl. hierzu nur Lodder, in: Lodder/Murray, EU Regulation of E-Commerce, S. 15 (23 ff.). 
Aufgrund der Verweisung werden nur ,in der Regel gegen Entgelt“" erbrachte Dienstleistungen erfasst. Damit ist die ECRL hinsichtlich der Dienste der Informationsgesellschaft im Grundsatz nur auf wirtschaftliche Tätigkeiten anwendbar. ${ }^{66}$ Dass es der ECRL allerdings nicht allein um die Entgeltlichkeit im engeren Sinne einer Gegenleistung geht, belegt Erwägungsgrund 18 S. 4, demzufolge der Annahme der Wirtschaftlichkeit nicht per se entgegensteht, wenn die in Anspruch genommenen Dienste nicht von demjenigen vergütet werden, der sie empfängt. ${ }^{67}$ Insoweit liegt die Definition auf einer Linie mit der Auslegung der Dienstleistungsfreiheit gemäß Art. 57 AEUV, bei der diese Feststellung ebenso Gültigkeit beansprucht. ${ }^{68}$ Damit sind seit jeher auch ,kostenlose“ Dienste wie soziale Medien oder Suchmaschinen erfasst. ${ }^{69}$ Das ist nunmehr auch sekundärrechtlich insoweit reflektiert, als mit Art. 3 Abs. 1 UAbs. 2 Digitale-Inhalte-RL (EU) 2019/770 erstmals eine explizite Anerkennung der Hingabe personenbezogener Daten als Gegenleistung erfolgt ist. ${ }^{70} \mathrm{Nach}$ alldem steht es jedenfalls nicht grundsätzlich in Zweifel, dass Diensteanbieter im Sinne dieser Untersuchung „Dienste der Informationsgesellschaft" betreiben. ${ }^{71}$

\section{b) Suchmaschinen als Sonderfall}

Hinsichtlich der Anwendbarkeit der Art. 14, 15 ECRL auf Suchmaschinen bedarf dies insoweit der Spezifizierung, als deren Einbeziehung in die ECRL in Art. 21 Abs. 2 ECRL unter einen Prüfvorbehalt gestellt wird und damit augenscheinlich de lege lata nicht erfasst sein soll. ${ }^{72}$ Allerdings ging der EuGH in seiner Entscheidung Google France und Google von einer Anwendbarkeit des Art. 14 ECRL zumindest in diesem Sachverhalt aus. Hier ging es

${ }^{66}$ Anders Marly, in: Grabitz/Hilf/Nettesheim, Recht der EU, 40. EL 2009, ECRL Vor. Abschnitt 4 Rn. 6.

${ }^{67}$ Es ist anzunehmen, dass es dem Unionsgesetzgeber hier primär um werbefinanzierte Geschäftsmodelle ging, vgl. Marly, in: Grabitz/Hilf/Nettesheim, Recht der EU, 40. EL 2009, ECRL Art. 2 Rn. 7.

${ }^{68}$ Vgl. EuGH, Urt. v. 15.09.2016 - C-484/14, NJW 2016, 3503 (Rn. 37 ff.) - McFadden; Urt. v. 11.09.2014 - C-291/13, MMR 2016, 63 (Rn. 28 f.) - Papasavvas.

${ }^{69}$ Vgl. Riordan, The Liability of Internet Intermediaries, S. 389.

${ }^{70}$ Vgl. hierzu Mischau, ZEuP 2020, 335 (339ff.). Zur Diskussion um die rechtliche Einordnung und den Umgang mit Verträgen über digitale Güter vgl. Grünberger, AcP 218 (2018), 213 ff.; speziell zu Daten als (Gegen-)Leistung vgl. Metzger, AcP 216 (2016), $817 \mathrm{ff}$.

${ }^{71}$ Für Facebook explizit nunmehr EuGH, Urt. v. 03.10.2019 - C-18/18, NJW 2019, 3287 (Rn. 22) - Glawischnig-Piesczek; vgl. auch schon Urt. v. 16.02.2012 - C-360/10, MMR 2012, 334 (Rn. 27) - SABAM/Netlog; für Suchmaschinen wie Google ist dies nicht ganz eindeutig, richtigerweise aber ebenfalls anzunehmen, vgl. ferner Martini, in: BeckOK Informations- und Medienrecht, TMG § 2 Rn. 8.

${ }^{72}$ Darauf zieht sich auch Milker, Die Umsetzung des »Rechts auf Vergessenwerden« im deutschen Recht, S. 39, zurück. 
um das von Google betriebene System AdWords (heute Google Ads), das es Wirtschaftsteilnehmerinnen gegen Entgelt ermöglicht, anhand bestimmter Stichwörter bei bestimmten Suchanfragen Werbeanzeigen bei Google zu platzieren, die den Nutzerinnen der Suchmaschine bei einem Suchvorgang angezeigt werden. Hier bejahte der EuGH eine Speicherung der Werbeanzeigen als Informationen der Werbekunden, die insoweit Nutzer des Diensteanbieters seien, sodass es sich bei Google insoweit um einen Dienst der Informationsgesellschaft handele, der prinzipiell unter Art. 14 ECRL falle. ${ }^{73}$ $\mathrm{Ob}$ die Voraussetzungen der Privilegierung im Einzelfall vorlägen - hier komme es insbesondere auf dessen ,passive Rolle ${ }^{\text {"77 }}$ bzw. seine „Neutralität ${ }^{\text {"75 }}$ an - sei durch die mitgliedstaatlichen Gerichte zu klären.

Während für Google Ads oder Vorschaubilder eine Zuordnung unter Art. 14 ECRL noch eher einleuchten mag, ist diese hinsichtlich der reinen Darstellung von Suchergebnissen - die Diskussion ist überwiegend unter dem Stichwort Hyperlinks geläufig - ungleich komplexer. Denn erstens hat der EuGH seine Auffassung nur hinsichtlich des entscheidungserheblichen Sachverhalts zum Ausdruck gebracht und gerade keine pauschale Positionierung bezüglich aller bei Suchmaschinen möglichen Konstellationen vorgenommen ${ }^{76}$ Zweitens spricht Art. 21 Abs. 2 ECRL recht explizit von der vorbehaltenen Prüfung der Erstreckung der ECRL auf Suchmaschinen, woraus im Umkehrschluss abgeleitet werden kann, dass ohne diese Erstreckung eine Anwendbarkeit gerade nicht gegeben ist. Drittens scheint Art. 14 ECRL selbst nicht auf die Verbreitung von Hyperlinks bzw. generell die Tätigkeit von Suchmaschinen zugeschnitten zu sein. ${ }^{77}$

Die Entwicklung der als Vollharmonisierung konzipierten Regelungen zur Vermittlerhaftung ${ }^{78}$ spricht indes eine andere Sprache. So haben nach dem ersten Bericht der Europäischen Kommission zur Anwendung der ECRL vier Mitgliedstaaten bei der Umsetzung der ECRL Suchmaschinen explizit in die Regelungen zur Verantwortlichkeit aufgenommen, wobei Spanien und Portugal sich hierbei an der Regelung des Art. 14 ECRL orientieren. ${ }^{79}$ Hier-

\footnotetext{
${ }^{73}$ Vgl. EuGH, Urt. v. 23.03.2010 - C-236/08, NJW 2010, 2029 (Rn. 110 f.) - Google France und Google.

${ }^{74}$ Vgl. EuGH, Urt. v. 23.03.2010 - C-236/08, NJW 2010, 2029 (Rn. 113, 119) - Google France und Google.

${ }^{75}$ Vgl. EuGH, Urt. v. 23.03.2010 - C-236/08, NJW 2010, 2029 (Rn. 114) - Google France und Google.

${ }^{76}$ Das verkennt Kovacs, Die Haftung der Host-Provider für persönlichkeitsrechtsverletzende Internetäußerungen, S. 325.

${ }^{77}$ Vgl. Spindler, in: Spindler/Schmitz, TMG, § 7 Rn. 84.

${ }^{78}$ Vgl. Sieber/Liesching, MMR-Beilage 8/2007, 1 (8); mit gewichtigen Argumenten anderer Auffassung aber Stalla-Bourdillon, in: Taddeo/Floridi, The Responsibilities of Online Service Providers, S. 275 (282 f.).

${ }^{79} \mathrm{Vgl}$. KOM(2003) 702 endg., S. 15; die spanische Version ist abgedruckt bei Kovacs,
} 
gegen erhob die Kommission explizit keine Bedenken ${ }^{80}$ Im Lichte der soeben angesprochenen EuGH-Entscheidung, die jedenfalls in der Tendenz Suchmaschinen ebenfalls der ECRL und ggf. deren Art. 14 zuordnet, ist daraus mithin zu schlussfolgern, dass eine Anwendung der Haftungsprivilegierung auf Suchmaschinen mit der ECRL vereinbar ist. ${ }^{81}$ Damit ist zumindest eine Weichenstellung für die parallele Rechtsfrage unter den $\S \S 7$ ff. TMG getroffen. ${ }^{82}$

\section{Die Rolle des Diensteanbieters im System der Art. $12 \mathrm{ff}$.}

Gesonderte Beachtung verdient die Rolle des Diensteanbieters, die die ECRL diesem als normative Erwartung zuschreibt. Wie sich im Verlauf dieser Untersuchung immer wieder zeigen wird, ist es gerade die Frage nach dieser Rolle, die einzelne juristische Fragestellungen im System der ECRL, aber auch die Verantwortlichkeit im übergeordneten Sinne erheblich beeinflusst. Ausgangspunkt für die hierbei entscheidende Rechtsprechungslinie des EuGH ist Erwägungsgrund 42 S. 1-2 ECRL.$^{83}$ Hiernach erfassen die Art. 12 ff. nur Fälle,

„in denen die Tätigkeit des Anbieters von Diensten der Informationsgesellschaft auf den technischen Vorgang beschränkt ist, ein Kommunikationsnetz zu betreiben und den $\mathrm{Zu}$ gang zu diesem zu vermitteln, über das von Dritten zur Verfügung gestellte Informationen übermittelt oder zum alleinigen Zweck vorübergehend gespeichert werden, die Übermittlung effizienter zu gestalten. Diese Tätigkeit ist rein technischer, automatischer und passiver Art, was bedeutet, daß der Anbieter eines Dienstes der Informationsgesellschaft weder Kenntnis noch Kontrolle über die weitergeleitete oder gespeicherte Information besitzt."

Die Haftung der Host-Provider für persönlichkeitsrechtsverletzende Internetäußerungen, S. 328.

${ }^{80} \mathrm{Vgl} . \mathrm{KOM}(2003) 702$ endg., S. 15.

${ }^{81}$ Vgl. auch Elixmann, Datenschutz und Suchmaschinen, S. 137 f.; Kovacs, Die Haftung der Host-Provider für persönlichkeitsrechtsverletzende Internetäußerungen, S. 325 ff.; Sieber/Höfinger, in: Hoeren/Sieber/Holznagel, Handbuch Multimedia-Recht, Teil 18.1 Rn. 100; vorsichtiger aber Paal, ZEuP 2016, 591 (620), der die Bedeutung des Einzelfalls betont.

${ }^{82}$ Weiterführend dazu unten $\S 8 \mathrm{C}$.

${ }^{83}$ Die Erwägungsgründe finden sich in der Präambel und nicht im verfügenden Teil des Rechtsaktes, sodass ihnen grundsätzlich keine Rechtsverbindlichkeit zukommt, vgl. EuGH, Urt. v. 19.11.1998 - C-162/97, BeckRS 2004, 74578 (Rn. 54) - Nilsson u.a.; Urt. v. 24.11.2005 - C-136/04, BeckRS 2005, 70929 (Rn. 32) - Deutsches Milch-Kontor. Daraus folgt, dass Abweichungen vom Wortlaut des Rechtsaktes durch die Erwägungsgründe nicht gerechtfertigt sind. Dennoch sind sie wichtige und wertvolle Anhaltspunkte zur Interpretation, die als politisch konsentierte Begründung eines Rechtsaktes auch demokratische Legitimation aufweisen können. Dies berücksichtigt auch der EuGH, wie sich nicht zuletzt an dem hier referenzierten Beispiel zeigt. 
Zwar klingt in Erwägungsgrund $42 \mathrm{~S} .1$ die ausschließliche Bezugnahme auf Fälle der „reinen Durchleitung" im Sinne von Art. 12 und das „Caching“ nach Art. 13 ECRL an. ${ }^{84}$ Indes ändert dies nichts daran, dass der entsprechende Erwägungsgrund kraft seines Wortlauts („Die in dieser Richtlinie hinsichtlich der Verantwortlichkeit festgelegten Ausnahmen [...]") die Erfassung aller Diensteanbieter jedenfalls zulässt. ${ }^{85}$ Der EuGH betont denn auch stets die technische, automatische und passive Natur der Tätigkeit des Diensteanbieters als wesentliche Voraussetzung der Privilegierung nach den Art. 12 ff. ECRL. Für die Thematik dieser Untersuchung ist dies insbesondere bei Art. 14 von Bedeutung. Aus dieser Erwägung heraus endet für das Gericht die Immunisierung stets dort, woder Anbieter eine „aktive Rolle“ wahrnimmt, die ihm - in Anknüpfung an den bereits erwähnten Erwägungsgrund 42 S. 2 - ,Kenntnis oder Kontrolle“ über die rechtsverletzende Information verschaffen kann. ${ }^{86}$ Diese Formulierung überrascht insofern, als dass der Erwägungsgrund von technischen, automatischen oder passiven Funktionen als denkbaren Zuschreibungen ausgeht, nicht allerdings von ,aktiven“ oder ,neutralen“. ${ }^{87}$

Ein Fall der ,aktiven Rolle“ soll etwa dann vorliegen, wenn der Anbieter eine „Hilfestellung“ leistet, die etwa - im Fall von eBay - in der Präsentation der rechtsverletzenden Information oder ihrer Bewerbung zu sehen sein kann. ${ }^{88}$ Im Falle des von Google betriebenen Systems Google Ads (damals

${ }^{84}$ So auch GA Jääskinen, Schlussantrag v. 09.12.2010 - C-324/09, juris, Rn. $141 \mathrm{f}$. L'Oréal SA; Van Eecke, Common Market Law Review 48 (2011), 1455 (1482); wohl auch Kuczerawy, Intermediary Liability and Freedom of Expression in the EU, S. 59, 97; wenig überzeugend meint aber Schapiro, Unterlassungsansprüche gegen die Betreiber von Internet-Auktionshäusern und Internet-Meinungsforen, S. 340 f., die Feststellung eines Zusammenhangs zwischen Art. 14 und Erwägungsgrund 42 verbiete sich aus „dogmatischen Gesichtspunkten“, sei aber aus teleologischen Gründen dennoch Auslegungsdirektive. Holznagel, Notice and Take-Down-Verfahren als Teil der Providerhaftung, S. 121, untermauert diese Sichtweise mit einem systematischen Argument: aus Art. 14 Abs. 2 lasse sich ableiten, dass Hosting-Dienste erst in diesem Fall nicht mehr privilegiert seien, sodass es nicht einsichtig sei, Erwägungsgrund 42 S. 2 uneingeschränkt auf alle Fälle der Art. 12 ff. ECRL anzuwenden. Zwingend ist dies freilich nicht, denn Art. 14 Abs. 2 lässt sich auch als spezielle Ausnahme vom Regelfall der Privilegierung (unter den in Abs. 1 genannten Voraussetzungen) interpretieren, die nichts über die generelle normative Erwartung der ECRL an die Rolle der Diensteanbieter aussagt, um die es hier geht. Holznagel scheint dies selbst auch insoweit einzugestehen, als er Art. 14 Abs. 2 als ,keineswegs praxisrelevante[n]“ Fall ansieht.

${ }^{85}$ Im Ergebnis auch Spindler, NJW 2002, 921 (922).

${ }^{86}$ EuGH, Urt. v. 23.03.2010 - C-236/08, NJW 2010, 2029 (Rn. 114, 120) - Google France und Google; Urt. v. 12.11.2011 - C-324/09, MMR 2011, 596 (Rn. 113) - L'Oréal SA; Urt. v. 11.09.2014 - C-291/13, MMR 2016, 63 (Rn. 41, 44) - Papasavvas.

${ }^{87}$ Vgl. Holznagel, Notice and Take-Down-Verfahren als Teil der Providerhaftung, S. 120 .

${ }^{88}$ EuGH, Urt. v. 12.11.2011 - C-324/09, MMR 2011, 596 (Rn. 116) - L’Oréal SA. 
AdWords) hatte Google bei der Auswahl der Stichwörter zugelassen, dass bei Suchen nach geschützten Marken Werbeanzeigen auch auf Nachahmungen der betreffenden Marken verwiesen. Hier entschied der EuGH zwar nicht abschließend über die Anwendbarkeit von Art. 14 auf entsprechende Ansprüche ${ }^{89}$ Generell hält der Gerichtshof es jedoch für entscheidend, die „Rolle [von] Google bei der Abfassung der den Werbelink begleitenden Werbebotschaft oder bei der Festlegung oder der Auswahl der Schlüsselwörter" in den Blick zu nehmen..$^{90}$

In grundsätzlicher Diktion stellt er zudem fest, dass jedenfalls die Entgeltlichkeit eines Geschäftsmodells, das mit Gewinnerzielungsabsicht etabliert wurde, das aber zugleich für Rechtsverletzungen ausgenutzt werden kann, nicht zur Verneinung des Privilegs führen könne. ${ }^{91}$ Die Grenze insoweit dürfte in der Tat erst dann überschritten sein, wenn der Diensteanbieter selbst die Rechtsverletzung anregt, wie dies im entschiedenen Fall etwa zu bejahen wäre, soweit Google die rechtsverletzenden Stichwörter selbst vorschlägt. ${ }^{92}$ Für hier besonders relevante Fallgestaltungen hat der EuGH hingegen Art. 14 für offenkundig einschlägig gehalten, ohne dies weiter zu begründen. ${ }^{93}$

\section{Privilegierung des Hostings (Art. 14)}

Zentrale und hier wichtigste Vorschrift der ECRL für die Haftung und Verantwortung der Diensteanbieter ist Art. 14. Zunächst sollen kurz die Tatbestandsmerkmale und Rechtsfolgen von Art. 14 erläutert werden (1.). Anschließend sind die von Art. 14 ECRL vorgesehenen Rechtsfolgen und die hiervon erfassten Regelungsbereiche zu erörtern (2.).

${ }^{89}$ EuGH, Urt. v. 23.03.2010 - C-236/08, NJW 2010, 2029 (Rn. 119) - Google France und Google.

${ }^{90}$ EuGH, Urt. v. 23.03.2010 - C-236/08, NJW 2010, 2029 (Rn. 118) - Google France und Google.

${ }^{91}$ EuGH, Urt. v. 23.03.2010 - C-236/08, NJW 2010, 2029 (Rn. 116) - Google France und Google.

${ }^{92} \mathrm{Vgl}$. Jaeschke, EuZW 2010, 426 (427).

${ }^{93}$ Vgl. EuGH, Urt. v. 16.02.2012 - C-360/10, MMR 2012, 334 (Rn.27) - SABAM/Netlog. 


\section{Voraussetzungen}

\section{a) Speicherung von Informationen}

Anders als bei Art. 12 f. geht es bei der Speicherung im Sinne von Art. 14 ECRL um eine solche, die nicht lediglich Nebenfolge der Gewährung von Diensten zum Zwecke der Durchleitung bzw. zum „Caching“ ist. ${ }^{94}$ Hier ist die Speicherung, also die Einschreibung von Informationen in den Serverspeicher, ${ }^{95}$ vielmehr das finale Ziel, zu dessen Erreichung der Diensteanbieter tätig ist. Beim Merkmal der Speicherung kommt es nicht auf Eigentumsoder Besitzverhältnisse hinsichtlich dieses Speichers, sondern auf die Fähigkeit zum Zugriff auf den konkreten Inhalt an.$^{96}$ Auch das Medium bzw. die Form der Speicherung ist nicht entscheidend. ${ }^{97}$

\section{b) Durch einen Nutzer eingegebene Informationen}

Sodann muss die gespeicherte Information „von einem Nutzer eingegeben“ sein. Wer in diesem Sinne „Nutzer“ ist, bestimmt Art. 2 lit. d. Danach kann dies auch eine juristische Person sein. Maßgeblich ist die Inanspruchnahme des Dienstes, „um Informationen zu erlangen oder zugänglich zu machen“. Hier stellt sich die Frage, wie im Einzelnen die Grenzziehung zwischen von „durch einen Nutzer eingegebenen“ und sonstigen Informationen zu erfolgen hat. Handelt es sich im Einzelfall nicht um eine vom Nutzer eingegebene Information, stehen dem Diensteanbieter die Haftungsprivilegierungen der ECRL, insbesondere Art. 14 nicht zur Verfügung. Damit rückt insbesondere das Verhältnis der Diensteanbieter und ihrer Gestaltungs- und Einwirkungsmöglichkeiten zu ihren Nutzerinnen in den Vordergrund.

Der einfachste Fall ist die physische Erstellung des Inhalts, also durch die Eingabe von informationstechnisch verarbeitungsfähigen Informationen durch den Nutzer. In diesem Sinne geht es bei Art. 14 um einen technischen Vorgang ${ }^{98}$ der unmittelbar zur Verfügbarkeit dieser Information für den Diensteanbieter führt. Stellt der Diensteanbieter einen eigenen Inhalt her, handelt es sich jedenfalls im Kontext des Art. 14 ECRL schon begrifflich nicht um eine „,von einem Nutzer eingegebene Information“. Abstrakt gesprochen kommt es allein auf die technische Kontrolle des Informationsflusses an. ${ }^{99}$ Die Abgrenzung im Fall der Kuratierung von Inhalten, die ein

${ }^{94} \mathrm{Vgl}$. Kuczerawy, Intermediary Liability and Freedom of Expression in the EU, S. 61.

${ }^{95}$ Vgl. EuGH, Urt. v. 12.11.2011 - C-324/09, MMR 2011, 596 (Rn. 110) - L'Oréal SA.

${ }^{96}$ So wohl die überwiegende Auffassung im Schrifttum. Überzeugend Pankoke, Von der Presse- zur Providerhaftung, S. 51 f.; vgl. auch Hoffmann/Volkmann, in: Spindler/ Schuster, Recht der elektronischen Medien, TMG § 10 Rn. 1, 14; Paal, in: BeckOK Informations- und Medienrecht, TMG $\S 10$ Rn. 11, mwN.

${ }^{97} \mathrm{Vgl}$. Spindler, in: Spindler/Schmitz, TMG, §10 Rn. 11.

${ }^{98}$ Vgl. Spindler, NJW 2002, 921 (923).

${ }^{99}$ Vgl. Chmelik, Social Network Sites - Soziale Netzwerke, S. 93. 
zentrales Element der Tätigkeit der Diensteanbieter darstellt, ${ }^{100}$ ist alles andere als trivial. Gerade die Geschäftsmodelle, die entscheidend auf nutzergenerierten Inhalten basieren und hieraus ihren wirtschaftlichen Nutzen ziehen, ${ }^{101}$ lassen eine valide abstrakte Grenzziehung kaum zu. Dies wird erschwert durch die soeben angesprochene Fokussierung von Art. 14 auf rein technische Vorgänge der Eingabe, sodass insoweit bereits der Spielraum fehlt, um auf normative und damit mehr Flexibilität ermöglichende Kriterien wie insbesondere die Zurechenbarkeit abzustellen. ${ }^{102}$ Konkretisierende Differenzierungskriterien könnten sich ferner etwa an der Frage orientieren, ob ein Dritter die Generierung einer Information veranlasst hat oder nicht. ${ }^{103,104}$

\section{c) Speicherung im Auftrag des Nutzers}

Eine weitere entscheidende Abgrenzung liegt sodann in der Frage, ob der Diensteanbieter die Information „im Auftrag [des] Nutzers“ speichert oder ob der Anbieter selbst die tatsächliche oder rechtliche Herrschaft über die Information ausübt. ${ }^{105}$ Damit erweist sich dieses Tatbestandsmerkmal als wesentlich offener für normative Zuordnungen, als es bei der Frage nach der Eingabe der Information durch einen Nutzer der Fall ist. Die rechtliche Herrschaft über die Information ist nicht schon dann gegeben, wenn der Dienste-

${ }^{100}$ Vgl. unten $\S 6$ B.

${ }^{101}$ Hier sind vor allen Dingen die bekannten kommunikationsbezogenen Dienste wie YouTube und Facebook zu nennen.

${ }^{102}$ Vgl. Klein, Haftung von Social-Sharing-Plattformen, S. 49.

${ }^{103}$ Der in BT-Drucks. 16/11173, S. 3 abgedruckte Entwurf für ein Gesetz zur Änderung des TMG sah in Art. 1 Nr. 5 vor, $§ 10$ TMG dahingehend zu ändern, dass die Speicherung „auf Veranlassung eines Nutzers“ erfasst werden sollte. Dass diese Bestrebung sich nicht durchsetzen konnte, steht allerdings der Interpretation von § 10 TMG in seiner aktuellen Fassung nicht entgegen, da eine solche dessen Wortlaut nicht sprengen würde.

${ }^{104}$ Vgl. Chmelik, Social Network Sites - Soziale Netzwerke, S. 94 ff. Um eine nicht vom Nutzer eingegebene, sondern diensteeigene Information handelt es sich danach etwa bei der Rekombination verschiedener Daten, aus der sich neue Informationen ergeben, ohne dass ein Dienstenutzer an der Erstellung der einzelnen Daten aktiv mitgewirkt hätte. Als Beispiel für diese Kategorie ist der Fall denkbar, dass ein Diensteanbieter aufgrund eigener Erkenntnisse bestimmter Informationen, die sich aus dem Verhalten des Nutzers ergeben, diesem zuordnet. So ist etwa denkbar und bereits Realität, dass eine Gesichtserkennungssoftware von Facebook hochgeladene Bilder auf ihr bekannte Gesichter absucht und diese sodann in dem Bild markiert; vgl. dazu www.verbraucherzentrale.de/wissen/digitale-welt/ datenschutz/gesichtserkennung-bei-facebook-das-sollten-nutzer-wissen-23818. Hier ist in der Regel eine eigene Information des Diensteanbieters anzunehmen, für die er als „,content provider" haftet. Denn andernfalls müsste eine ggf. kaum bewusste Entscheidung von Dritten als Nutzer des Dienstes zur aktiven „Produktion“ einer Information unterstellt werden, was unter datenschutzrechtlichen Kautelen bereits Zweifel hervorriefe.

${ }^{105}$ Vgl. Freytag, GRUR-Prax 2010, 355 (357). 
anbieter sich - etwa durch seine AGB - das Recht einräumt, einzelne vom Nutzer stammende Informationen zu sperren oder zu löschen, da dies die von Art. 14 beabsichtigte Pflicht zu eben dieser Löschung bzw. Sperrung zur Erlangung der Privilegierung ihrem Sinn nach unterliefe. ${ }^{106}$ Nach Ansicht mancher Stimmen soll eine rechtliche Herrschaft des Diensteanbieters begründet sein, wenn ein Fall des Zueigenmachens einer Information im Sinne der BGH-Rechtsprechung vorliegt. ${ }^{107}$ Die Begründung dieser Auffassung verlangt jedoch einige systematische Verrenkungen, ${ }^{108}$ die indes nicht erforderlich sind, weil - jedenfalls nach Auffassung des EuGH zur Rolle der Diensteanbieter betreffend der Art. $12 \mathrm{ff}$. im soeben dargelegten Sinne - im Falle einer zu Eigen gemachten Information der Diensteanbieter schon keine „neutrale Rolle“ wahrnehmen dürfte.

Auf das Bestehen eines gültigen Rechtsverhältnisses zwischen Nutzerin und Diensteanbieter kommt es nicht an. Notwendig, aber auch hinreichend ist das Vorliegen der technischen Beziehung. ${ }^{109}$ Auch hier wird die starke Betonung der technischen Qualität offenkundig, auf die die Haftungsprivilegierungen insgesamt abstellen.

\section{d) Keine Kenntnis von der rechtswidrigen Information} (Art. 14 Abs. 1 lit. a Var. 1)

Sodann kommt es für die Anwendbarkeit der Privilegierung im Einzelfall darauf an, ob der Diensteanbieter Kenntnis von der rechtsverletzenden Information hatte oder nicht. ${ }^{110}$ Der Tatbestand des Art. 14 differenziert zwi-

${ }^{106}$ Zutreffend Freytag, GRUR-Prax 2010, 355 (357).

${ }^{107}$ Vgl. Matthies, Providerhaftung für Online-Inhalte, S. 141. Vgl. zur Figur des Zueigenmachens sogleich unter $\S 4$ D. I. 2.

${ }^{108}$ So sieht sich Matthies, Providerhaftung für Online-Inhalte, S. 144, zu einer entsprechenden Auslegung von Art. 14 nicht imstande, weil das Erfordernis eines Nutzerauftrags nicht in den Art. $12 \mathrm{f}$. enthalten, ein entsprechendes Auslegungserfordernis aber auch dort erforderlich sei, weshalb eine entsprechende Handhabung des „Eingebens" angezeigt sei. Dem folgt Klein, Haftung von Social-Sharing-Plattformen, S. $50 \mathrm{f}$.

${ }^{109}$ Vgl. EuGH, Urt. v. 15.09.2016 - C-484/14, NJW 2016, 3503 (Rn. 50 ff.) - McFadden.

${ }^{110}$ Der Maßstab der Kenntnis war im Gesetzgebungsvorhaben nicht unumstritten. So setzte sich das Parlament insbesondere dafür ein, im Rahmen von Abs. 1 lit. a auch die grob fahrlässige Unkenntnis aufzunehmen, um im Falle deren Bejahung die Anwendung des Privilegs zu verhindern, vgl. Marly, in: Grabitz/Hilf/Nettesheim, Recht der EU, 40. EL 2009, ECRL Art. 14 Rn. 2. Das lehnte die Kommission, der sich der Rat anschloss, jedoch $\mathrm{ab}$ und begründete dies damit, dass die Regelungen zur Verantwortlichkeit einen, ,äusserst wichtigen und heiklen Bereich“ darstellten, ,bei dem im Zuge der Erarbeitung des ursprünglichen Vorschlags besondere Anstrengungen unternommen wurden, in enger Abstimmung mit den Beteiligten zu einer vernünftigen Kompromißlösung zu gelangen, die allen Interessen, die im Spiele sind, gerecht wird“, KOM/99/0427 endg., unter 2.3.3; vgl. Marly, in: Grabitz/Hilf/Nettesheim, Recht der EU, 40. EL 2009, ECRL Art. 14 Rn. 5 f., Art. 12 Rn. 1. 
schen dem Grundfall, bei der es um die „tatsächliche Kenntnis von der rechtswidrigen Tätigkeit oder Information " geht ${ }^{111}$ und verschärft für Schadensersatzansprüche den Haftungsmaßstab, indem er zusätzlich (,und“) verlangt, dass der Diensteanbieter sich der „Tatsachen oder Umstände bewußt [war], aus denen die rechtswidrige Tätigkeit oder Information offensichtlich wird". ${ }^{112}$

Von grundsätzlicher Bedeutung sind insoweit zwei Fragen: der Bezugspunkt der Kenntnis und ihr konkreter Maßstab.

Hinsichtlich des Bezugspunkts sind im Prinzip zwei Möglichkeiten denkbar, nämlich die Tatsachen, aus denen sich die Rechtsverletzung ergibt und die Feststellung der Rechtswidrigkeit im rechtlichen Sinne. Der Wortlaut von Art. 14 Abs. 1 lit. a ECRL ist insoweit nicht eindeutig. Der EuGH scheint hier nicht ganz widerspruchsfrei zu argumentieren. Einerseits führt er aus, Art. 14 erfasse im Sinne der „praktischen Wirksamkeit“ alle Konstellationen, „in denen sich der betreffende Anbieter in der einen oder anderen Weise solcher Tatsachen oder Umstände bewusst war." ${ }^{\text {"113 }}$ Dies spricht für die Tatsachengrundlage als hinreichenden Bezugspunkt. Andererseits müsse aber das mit dem Fall befasste Gericht des Mitgliedstaates feststellen, „ob sich der Betreiber etwaiger Tatsachen oder Umstände bewusst war, auf deren Grundlage ein sorgfältiger Wirtschaftsteilnehmer die Rechtswidrigkeit hätte feststellen müssen. " ${ }^{114}$ Dies scheint eher für eine Sichtweise zu sprechen, die auch die konkrete Feststellung der Rechtswidrigkeit unter Art. 14 ECRL erforderlich macht. Dieser Sichtweise neigen auch viele Stimmen im Schrifttum zu. ${ }^{115}$ Das bedeutet allerdings auch, dass es zunächst in den Verantwortungsbereich des Nutzers fällt, den Diensteanbieter auf die Rechtswidrigkeit eines Inhalts hinzuweisen, sofern nicht der Anbieter von sich aus tätig geworden ist und hierbei die rechtswidrige Information erkannt hat. ${ }^{116}$

Hinsichtlich des Maßstabs der Kenntnis verweist der EuGH im Ausgangspunkt die Beurteilungshoheit an die mit dem jeweiligen Konflikt befassten Gerichte, ${ }^{117}$ gibt jedoch einige Auslegungsdirektiven vor. Auch diese erscheinen jedoch in ihrer Intention nicht gänzlich widerspruchsfrei. Denn allgemein hat es den Anschein, dass der EuGH eine großzügige Prüfung der Kenntnis vorsehen will, wenn er die „praktische Wirksamkeit“ von Art. 14

${ }^{111}$ Art. 14 Abs. 1 lit. a Var. 1 ECRL.

${ }^{112}$ Art. 14 Abs. 1 lit. b ECRL.

${ }^{113}$ EuGH, Urt. v. 12.11.2011 - C-324/09, MMR 2011, 596 (Rn. 121) - L'Oréal SA.

${ }^{114}$ EuGH, Urt. v. 12.11.2011 - C-324/09, MMR 2011, 596 (Rn. 122) - L'Oréal SA.

${ }^{115}$ Vgl. Spindler, in: Spindler/Schmitz, TMG, §10 Rn. 28 f.; Hoffmann/Volkmann, in: Spindler/Schuster, Recht der elektronischen Medien, TMG $§ 10$ Rn. 23; Paal, in: BeckOK Informations- und Medienrecht, TMG $\S 10$ Rn. $29 \mathrm{f}$.

${ }^{116} \mathrm{Vgl}$. kritisch hierzu noch unter $\S 10$ B. IV. 1.

${ }^{117}$ Vgl. EuGH, Urt. v. 12.11.2011 - C-324/09, MMR 2011, 596 (Rn. 120) - L’Oréal SA. 
betont und deshalb alle Fälle zu erfassen sind, in denen sich der Diensteanbieter ,in der einen oder anderen Weise“ jener Umstände bewusst sei. ${ }^{118}$ So sei die Kenntniserlangung sowohl ,auf Grund einer aus eigenem Antrieb vorgenommenen Prüfung“ als auch durch eine entsprechende „Anzeige“ (eines Dritten) möglich. ${ }^{119}$ Diese Aussage ist in ihrer Tendenz eher anbieterfeindlich, da sie den Maßstab der Kenntniserlangung eher großzügig zu sehen scheint und daher jedwede Art der Kenntniserlangung zulässt. Die erste Alternative überlässt es dem Diensteanbieter dabei, eigenmächtig Umstände zutage zu tragen, die die Rechtswidrigkeit eines Inhalts offenbaren. Denn eine rechtliche Pflicht hierzu kommt schon wegen Art. 15 ECRL nicht in Betracht. Ohnehin wäre die Kenntniserlangung infolge einer solchen eigeninitiativen Prüfung für den Nutzer schwer nachweisbar. ${ }^{120}$ In aller Regel wird eine entsprechende Kenntnis damit erst durch eine Notifizierung des Diensteanbieters zu begründen und nachzuweisen sein. Dabei ist es von eminenter Bedeutung, welche Anforderungen an diese zu stellen sind, da sie letztlich für die Haftungsfrage entscheidend ist. Der EuGH scheint dies zu antizipieren und gibt - nun aber in der Bestrebung, die Diensteanbieter vor einem allzu schnellen Verlust der Privilegierung bewahren zu wollen - vor, dass nicht hinreichend genaue bzw. substanziierte Notifizierungen nicht schon zur Annahme der Kenntnis führen dürften. ${ }^{121}$ Dies ist offenkundig anbieterfreundlich gemeint. Andererseits seien aber auch solche Anzeigen als Anhaltspunkt anzusehen, den die Gerichte der Mitgliedstaaten zu berücksichtigen hätten. ${ }^{122}$ Die Anknüpfung an eine hinreichend genaue und substanziierte Notifizierung ist nach dem EuGH also hinreichend, aber nicht zwingend notwendig. ${ }^{123}$

Zusammenfassend lässt sich also festhalten, dass Intention und gewünschte Verhaltensanreize in der Perspektive des EuGH zwischen „,praktischer Wirksamkeit" - in der Regel eine Chiffre für eine robuste bzw. großzügige Interpretation - und Anbieterschutz oszillieren. Dies betrifft im Grundsatz sowohl die Frage des Bezugspunkts der Kenntnis und - in ausgeprägterem Maße - den Aspekt des Maßstabs der Kenntnis selbst. Die insoweit nur groben Vorgaben des EuGH haben den BGH im Fall YouTube dazu veranlasst, dem EuGH die Frage zur Vorabentscheidung vorzulegen, ob die Kenntnis sich auf ,konkrete rechtswidrige Tätigkeiten oder Informationen beziehen muss" oder ob der Kreis der die Kenntnis auslösenden Tatsachen oder Umstände insoweit auch weiter gezogen werden könne. ${ }^{124}$ Der BGH

${ }^{118}$ EuGH, Urt. v. 12.11.2011 - C-324/09, MMR 2011, 596 (Rn. 121) - L'Oréal SA.

${ }^{119}$ EuGH, Urt. v. 12.11.2011 - C-324/09, MMR 2011, 596 (Rn. 122) - L'Oréal SA.

${ }^{120}$ Vgl. Frey, ZUM 2019, 40 (44).

${ }^{121}$ Vgl. EuGH, Urt. v. 12.11.2011 - C-324/09, MMR 2011, 596 (Rn. 122) - L'Oréal SA.

${ }^{122}$ Vgl. EuGH, Urt. v. 12.11.2011 - C-324/09, MMR 2011, 596 (Rn. 122) - L'Oréal SA.

${ }^{123}$ Anders wohl Frey, Die Haftung von Host-Providern für Immaterialgüterrechtsverletzungen, S. 282.

${ }^{124}$ BGH, Beschl. v. 13.09.2018 - I ZR 140/15, GRUR 2018, 1132 (Rn. 44) - YouTube; 
selbst tendiert zur ersteren Auslegung und begründet dies damit, dass die sich aus Art. 14 Abs. 1 lit. b ergebenden Pflichten nur im Hinblick auf konkrete Informationen erfüllt werden könnten. ${ }^{125}$ Mithin sei die Kenntniserlangung nur im Hinblick auf eine konkrete Rechtsverletzung möglich, die für den Diensteanbieter identifizierbar sein müsse. ${ }^{126}$ Damit werden jedoch potenziell diejenigen, die entsprechende Anzeigen bzw. Beschwerden den Diensteanbietern zur Kenntnis bringen (wollen), nicht unerheblichen Darlegungs- und Begründungspflichten tatsächlicher und rechtlicher Natur unterworfen. ${ }^{127}$

Nicht weiter vertieft werden braucht die Frage, auf wessen Kenntnis es im Rahmen von Art. 14 genau ankommt - ob also auf die menschliche Kenntnisnahme abgestellt werden muss oder auch die automatisierte Kenntnis allein genügt. Materiell ist die Auffassung der Rechtsprechung und Teilen des Schrifttums, dass es generell, also rechtsgebietsübergreifend, ${ }^{128}$ nur auf die menschliche Kenntnisnahme ankomme, ${ }^{129}$ wenig überzeugend, weil sie die technischen Fähigkeiten vieler Diensteanbieter und deren Einsatz in der Praxis vieler Geschäftsmodelle gänzlich ausblendet. Letztlich ist die Frage aber zumeist nur für die exakte Bestimmung des Zeitpunkts der Kenntniserlangung von Bedeutung.

um die im Grunde gleiche Rechtsfrage geht es auch im Parallelverfahren Beschl. v. 20.09.2018 - I ZR 53/17, ZUM 2018, 870 (Rn. 35 f.) - uploaded.

${ }^{125}$ Vgl. BGH, Beschl. v. 13.09.2018 - I ZR 140/15, GRUR 2018, 1132 (Rn. 45) - YouTube. Erwähnenswert ist in dem Zusammenhang, dass der BGH hier offensichtlich versucht, den EuGH zu einer Verknüpfung der Anforderungen an die Kenntniserlangung im Rahmen von Art. 14 mit der BGH-Rechtsprechung zur Störerhaftung zu animieren, wie die Referenzierung auf das Urt. v. 17.08.2011 - I ZR 57/09, MMR 2012, 178 (Rn. 20, 28) Stiftparfüm (die Referenzierung der Rn. 21 ist offenkundig ein Versehen) deutlich macht. Der Generalanwalt scheint dieser Auffassung zuzuneigen, ohne aber auf die Störerhaftung in diesem Kontext einzugehen, vgl. GA Øe, Schlussantrag v. 16.07.2019 - C-682/18, juris, Rn. 172 ff. - YouTube.

${ }^{126}$ Vgl. BGH, Beschl. v. 13.09.2018 - I ZR 140/15, GRUR 2018, 1132 (Rn. 45) - YouTube. Im Schrifttum scheint diese Sicht tendenziell auch von der Mehrheit vertreten zu werden, vgl. Frey, ZUM 2019, 40 (44); Spindler, in: Spindler/Schmitz, TMG, § 10 Rn. 24, zur Lage in Deutschland unter Berufung auf den EuGH.

${ }^{127}$ Siehe dazu noch unten $\S 10$ B. IV. 1 .

${ }^{128}$ Für die strafrechtliche Sichtweise kommt es selbstredend auf die Verantwortung und damit die Kenntnis des Handelnden an.

${ }^{129}$ Vgl. Holznagel, Notice and Take-Down-Verfahren als Teil der Providerhaftung, S. 123 f.; Spindler, GRUR 2011, 101 (103), der nicht vollkommen überzeugend auf BGH, Urt. v. 19.04.2007 - I ZR 35/04, NJW 2007, 2636 (Rn. 31) - Internet-Versteigerung II, verweist, wo es aber um die Frage des Vorsatzes im Rahmen der Beteiligtenhaftung ging. 
e) Kein Bewusstsein über Tatsachen und Umstände, aus denen die Rechtsverletzung offensichtlich wird (Art. 14 Abs. 1 lit. a Var. 2)

Während der EuGH für die erste Variante von Art. 14 Abs. 1 lit. a ECRL bislang nur grobe Auslegungsdirektiven vorgegeben hat, liegt für die Var. $2^{130}$ der Bestimmung immerhin eine Leitlinie zur Bildung eines normativen Erwartungshorizonts vor. Insoweit hielt er nämlich in der Entscheidung L'Oréal fest, dass sich der Diensteanbieter bereits dann nicht auf Art. 14 Abs. 1 lit. a Var. 2 ECRL berufen könne, ,wenn er sich etwaiger Tatsachen oder Umstände bewusst war, auf deren Grundlage ein sorgfältiger Wirtschaftsteilnehmer die in Rede stehende Rechtswidrigkeit hätte feststellen [...] müssen. ${ }^{\text {"131 }}$ Damit wird die subjektive Komponente letztlich mit einem eher objektivierten, jedenfalls aber normativen Kriterium operationalisiert. Dies ist einerseits hilfreich, um auf verschiedene Fallgestaltungen flexibel reagieren zu können, muss sich andererseits aber auch den Vorwurf der mangelnden Bestimmtheit gefallen lassen. Dies gilt aber letztlich nicht in ausgeprägterem Maße als für die meisten Versuche der Rechtsordnung, für die Anordnung rechtlicher Folgen oder der Etablierung von Handlungspflichten auf subjektive bzw. interne Kriterien in der Sphäre der rechtlich Betroffenen abzustellen. ${ }^{132}$

Das obige Zitat des EuGH, wonach es auf den „sorgfältigen Wirtschaftsteilnehmer" ankomme, betrifft nur die Privilegierung gegenüber Schadensersatzansprüchen in Art. 14 Abs. 1 lit. a Var. 2 ECRL. Zwar könnte man meinen, dass der Gerichtshof alle Arten der Inanspruchnahme im Blick hat, wenn er von „den in Art. 14 Abs. 1 lit. a [ECRL] niedergelegten Regeln“ spricht, ${ }^{133}$ bevor er den „,sorgfältigen Wirtschaftsteilnehmer" dann ein zweites Mal erwähnt. ${ }^{134}$ Daraus lässt sich allerdings nicht schlussfolgern, dass der Maßstab des ,sorgfältigen Wirtschaftsteilnehmers“ auf alle Modalitäten der Kenntnis im Sinne von Art. 14 Abs. 1 lit. a ECRL anwendbar ist. ${ }^{135}$ Ein solches Verständnis kann der Gerichtshof hier schlechterdings nicht im Sinn gehabt haben, ${ }^{136}$ gerade weil seine Formulierung erkennbar nur auf Var. 2

${ }^{130}$ Siehe aber oben $\S 4$ Fn. 114.

${ }^{131}$ EuGH, Urt. v. 12.11.2011 - C-324/09, MMR 2011, 596 (Rn. 120) - L’Oréal SA.

${ }^{132}$ Das Paradebeispiel ist hier die Bestimmung und Feststellung des Vorsatzes im Strafrecht, wo ebenfalls oft auf äußere Umstände oder Normativierungen abgestellt wird, um den subjektiven Tatbestand zu prüfen, vgl. nur Hörnle, JZ 2019, 440 (442 f.).

${ }^{133}$ EuGH, Urt. v. 12.11.2011 - C-324/09, MMR 2011, 596 (Rn. 121) - L'Oréal SA.

${ }^{134}$ Vgl. EuGH, Urt. v. 12.11.2011 - C-324/09, MMR 2011, 596 (Rn. 122) - L'Oréal SA.

${ }^{135}$ So offenbar Frey, Die Haftung von Host-Providern für Immaterialgüterrechtsverletzungen, S. 148; wohl auch Maier, Remixe auf Hosting-Plattformen, S. 160.

${ }^{136}$ Vgl. explizit Spindler, ZUM 2017, 473 (482): ,Für zivilrechtliche Schadensersatzansprüche genügt die Kenntnis evidenter Umstände, nicht dagegen für jede andere Verantwortlichkeit. Eine solche Unterscheidung würde keinen Sinn machen, wenn eine allge- 
gemünzt ist. ${ }^{137}$ Sofern es um Unterlassungsansprüche geht, ist stets der Maßstab der positiven Kenntnis maßgeblich. ${ }^{138}$

\section{f) Unverzügliche Entfernung oder Sperrung nach Kenntnis- bzw.} Bewusstseinserlangung (Art. 14 Abs. 1 lit. b)

Hat der Diensteanbieter Kenntnis oder Bewusstsein über rechtsverletzende Inhalte im erläuterten Sinne, verlangt Art. 14 Abs. 1 lit. b ECRL die unverzügliche Entfernung oder Sperrung dieses Inhalts, um dennoch in den Genuss der Privilegierung zu kommen. Insoweit stellt Art. 14 ECRL also ein Verfahren auf, das Elemente des Notice-and-Takedown-Verfahrens aufgreift und knüpft damit in deutlich erkennbarer Weise an die bereits erörterte Regelung des $\S 512(\mathrm{c})(\mathrm{C})$ DMCA an. ${ }^{139}$

\section{Rechtsfolgen, erfasste Regelungsbereiche}

Liegen die Voraussetzungen von Art. 14 ECRL in der jeweils maßgeblichen Konstellation vor, haftet der Diensteanbieter nicht bzw. ist - in der Diktion der ECRL - für die Rechtsverletzung nicht verantwortlich. Die begriffliche Differenzierung zwischen Haftung und Verantwortlichkeit scheint primär der deutschen Fassung der ECRL zu entspringen, da beispielsweise die englische Fassung von der „liability“ spricht, von der die nach den Art. 12-14 erfassten Dienste befreit sein sollen. Bereits deshalb wäre es nicht überzeugend, den Begriff mit einem eigenständigen normativen Gehalt zu versehen, um beispielsweise den Verschuldensmaßstab zum Differenzierungskriterium zwischen von Art. 14 ECRL erfassten und nicht erfassten Ansprüchen zu unterscheiden. ${ }^{140}$

Grundsätzlich sollen alle Teilrechtsgebiete erfasst sein, auf die die ECRL Anwendung findet. Von den gemäß Art. 1 Abs. 5 ECRL ausgeschlossenen Bereichen ist für die Thematik dieser Untersuchung insbesondere die DSGVO als Nachfolgeregelung der DS-RL (Art. 1 Abs. 5 lit. b ECRL) von gesteigerter Bedeutung. ${ }^{141}$ Prinzipiell scheinen sich Art. 1 Abs. 5 lit. b ECRL

meine Benachrichtigung über rechtswidrige Inhalte bereits für die Kenntnis nach Art. 14 Abs. 1 Alt. 1 E-Commerce-RL genügen würde"; vgl. auch Chmelik, Social Network Sites Soziale Netzwerke, S. 99 f.; letztlich auch Nolte/Wimmers, GRUR 2014, 16 (24); Leistner, ZUM 2012, 722 (726).

${ }^{137}$ Vgl. EuGH, Urt. v. 12.11.2011 - C-324/09, MMR 2011, 596 (Rn. 120) - L’Oréal SA: „In dieser letztgenannten Hinsicht [...]“.

${ }^{138} \mathrm{Vgl}$. dazu noch unten $\S 11$ C. III. 1.

${ }^{139}$ Vgl. Hollenders, Mittelbare Verantwortlichkeit von Intermediären im Netz, S. 256; Spindler, in: Spindler/Schmitz, TMG, §10 Rn. 49.

${ }^{140}$ Insoweit überzeugend (unter Abstellen auf Regelungen allein im BGB) Hollenders, Mittelbare Verantwortlichkeit von Intermediären im Netz, S. $46 \mathrm{f}$.

${ }^{141}$ Vgl. zum Verhältnis von ECRL und DS-RL auch Stalla-Bourdillon, in: Taddeo/Floridi, The Responsibilities of Online Service Providers, S. 275 (284 ff.). 
und Art. 2 Abs. 4 DS-GVO in ihrem normativen Gehalt zu widersprechen und damit eine Anwendungslücke für Fälle mit datenschutzrechtlichem Bezug zu lassen. Nach Art. 2 Abs. 4 DS-GVO bleiben die Art. 12 ff. ECRL von der DS-GVO jedoch lediglich „unberührt“, woraus gerade keine Unvereinbarkeit beider Regelungsregime folgt. Außerdem ist hier auch der Grundsatz lex posterior derogat legi priori zu beachten. ${ }^{142}$ Demnach kann nicht angenommen werden, dass der Unionsgesetzgeber mit Erlass des Art. 2 Abs. 4 DS-GVO eine solche Anwendungslücke schaffen wollte, zumal diese Normierung keine Entsprechung in der alten DS-RL findet und demnach eine echte Neuregelung mit deklaratorischem Charakter darstellt. Das bedeutet, dass im Falle von Tätigkeiten, die von den Art. 12 ff. ECRL erfasst sind, beide Regelungskomplexe nebeneinander anwendbar sind. ${ }^{143}$ Die gegenläufige Auffassung ${ }^{144}$ findet keinerlei Grundlage im Gesetz und kann auch keine systematisch überzeugenden Gründe geltend machen, die ein Spezialitätsverhältnis zwischen der DS-GVO und der ECRL insoweit rechtfertigen würden. Aus der Entscheidung Promusicae, in der der EuGH noch festhielt, dass eine Verpflichtung eines Access-Providers zur Herausgabe personenbezogener Daten von Personen, die an einer illegalen Tauschbörse für Musik teilgenommen hatten, den von Art. 1 Abs. 5 lit. b ECRL statuierten Vorrang des Datenschutzrechts verletze, ${ }^{145}$ folgt nichts anderes. Denn das Gericht ließ die Möglichkeit der grundrechtskonformen Ausgestaltung entsprechender Auskunftspflichten, die sich mittelbar auch auf die Art. 12 ff. ECRL beziehen können, explizit offen. ${ }^{146}$

Die hier vertretene parallele Anwendbarkeit darf allerdings nicht dazu führen, dass solche Regelungen, die eine bestimmte Konstellation in spezieller Art und Weise regeln, durch den anderen Rechtsakt unterlaufen würden. Das bedeutet insbesondere, dass Regelungen, die spezifische Pflichten und Folgen als Konsequenz von Datenverarbeitungsvorgängen - zu denken ist hier insbesondere an die Art. 24, 82 ff. DS-GVO - nicht durch die Privilegierungen der ECRL leerlaufen können. Umgekehrt gilt dies ebenso, sodass die in Art. 82 Abs. 3 DS-GVO vorgesehene Exkulpationsmöglichkeit des Datenverarbeiters nicht zur Anwendung kommt, soweit es um die Verantwortung für fremde Inhalte geht. Im Ergebnis ist damit festzuhalten, dass

\footnotetext{
${ }^{142}$ Vgl. zu diesem Ruffert, in: Calliess/Ruffert, EUV/AEUV, AEUV Art. 288 Rn. 13.

${ }^{143}$ So auch Zerdick, in: Ehmann/Selmayr, DS-GVO, Art. 2 Rn. 17; Bäcker, in: BeckOK Datenschutzrecht, DS-GVO Art. 2 Rn. 33; Wagner, ZD 2018, 307 (310).

${ }^{144}$ Vgl. Kühling/Raab, in: Kühling/Buchner, DS-GVO, Art. 2 Rn. 32; Ennöckl, in: Sydow, DS-GVO, Art. 2 Rn. 17.

${ }^{145}$ Vgl. EuGH, Urt. v. 29.01.2008 - C-275/06, NJW 2008, 743 (Rn. 57) - Promusicae.

${ }^{146}$ Vgl. EuGH, Urt. v. 29.01.2008 - C-275/06, NJW 2008, 743 (Rn. 67 f.) - Promusicae. Kritisch unter dem Blickwinkel der Harmonisierung entsprechender Auskunftsansprüche Spindler, GRUR 2008, 574 (577).
} 
ein Anspruch gegen einen Diensteanbieter nicht am Vorrang der DS-GVO scheitert.

\section{$I V$. Verbot der allgemeinen Überwachungspflicht (Art. 15)}

Eine weitere zentrale Vorschrift zur Verantwortlichkeit der Diensteanbieter im Sinne der ECRL ist deren Art. 15. Nach Art. 15 Abs. 1 darf den Diensteanbietern ,keine allgemeine Verpflichtung“ auferlegt werden, ,die von ihnen übermittelten oder gespeicherten Informationen $\mathrm{zu}$ überwachen oder aktiv nach Umständen zu forschen, die auf eine rechtswidrige Tätigkeit hinweisen."

\section{Bedeutung}

Das so formulierte Verbot allgemeiner Überwachungspflichten soll verhindern, dass Diensteanbieter zu einer Polizei des digitalen Raums werden, die diesen anlasslos überwachen bzw. - dies ist insoweit entscheidend - hierzu rechtlich gezwungen werden. ${ }^{147}$ Denn eine haftungsauslösende Kenntnis infolge eigeninitiativ ausgeführter Recherchen hat der EuGH bereits in Bezug zu Art. 14 ECRL für möglich erachtet, ${ }^{148}$ sodass diese Einsicht auch im Kontext von Art. 15 Geltung beansprucht.

Art. 15 Abs. 1 ECRL selbst spricht nicht von der Verantwortlichkeit, ist also keine Haftungsregel im engeren Sinne, sondern markiert nur eine Grenze der Möglichkeit, Diensteanbieter für einen Inhalt in Verantwortung zu nehmen. ${ }^{149}$ Denn eine Verantwortlichkeit darf eben nicht zu einer von der Norm erfassten Pflicht führen. ${ }^{150}$ Dabei wird der Gehalt der Regelung teilweise nur als deklaratorisch bezeichnet, da die jeweils dritten Absätze der Art. 12-14 ohnehin deren Grundaussage entsprechend modifizierten. ${ }^{151}$ Das ist jedoch nur insoweit richtig, als diese Regelungen im Einzelfall bestimmte Möglichkeiten mitgliedstaatlicher Institutionen zur Anordnung konkreter Pflichten vorsehen, die sich mit der Grundaussage von Art. 15 ECRL decken. Diese Norm muss aber jedenfalls nicht allein aus systematischen Gesichtspunkten auf diese Fälle beschränkt bleiben. Dass sie womöglich auch wei-

${ }^{147}$ Vgl. Lodder, in: Lodder/Murray, EU Regulation of E-Commerce, S. 15 (53).

${ }^{148}$ Vgl. oben § 4 Fn. 119.

${ }^{149}$ Zwar wäre auch eine gesetzliche Pflicht zu solchen Überwachungsmaßnahmen jenseits der Inanspruchnahme durch einen Nutzer an Art. 15 zu messen. Für den Kontext dieser Untersuchung geht es aber hauptsächlich um die beschriebene Grenze der möglichen Haftung eines Diensteanbieters gegenüber einem Dritten.

${ }^{150}$ Vgl. Marly, in: Grabitz/Hilf/Nettesheim, Recht der EU, 40. EL 2009, ECRL Art. 15 Rn. 3.

${ }^{151}$ Vgl. Marly, in: Grabitz/Hilf/Nettesheim, Recht der EU, 40. EL 2009, ECRL Art. 15 Rn. 5. 
tergehend zu verstehen ist, legt überdies Erwägungsgrund 48 ECRL nahe, demzufolge die ECRL - insoweit geht es der Sache nach maßgeblich um Art. 15 - der mitgliedstaatlichen Auferlegung von Sorgfaltspflichten an Host-Provider nicht entgegensteht, ,,um bestimmte Arten rechtswidriger Tätigkeiten aufzudecken und zu verhindern". Art. 15 ECRL und die genannten Verantwortlichkeitsregelungen sind demnach nicht ohne Weiteres deckungsgleich. ${ }^{152}$

Art. 15 Abs. 1 ECRL ist ferner dahingehend zu verstehen, dass das statuierte Verbot sich nicht nur gegen eine Haftung für unterlassene Kontrollen in der Vergangenheit richtet, sondern auch ein solches Verbot für in die $\mathrm{Zu}$ kunft gerichtete Begehren gegen Diensteanbieter aufstellt. ${ }^{153}$

\section{Reichweite}

Um den genauen Gehalt des Verbots allgemeiner Überwachungspflichten erfassen zu können, stellt sich die Frage, wann ein Fall einer solchen von Art. 15 ECRL verbotenen Überwachungspflicht vorliegt. Konkret ist fraglich, wann eine Überwachungspflicht im Sinne der Norm eine „allgemeine“ ist. Denn im Umkehrschluss zum Wortlaut der Norm sind „nichtallgemeine“ Überwachungspflichten jedenfalls mit Blick auf Art. 15 ECRL zulässig.

\section{a) Allgemeine vs. spezifische Überwachungspflichten}

Dementsprechend legt auch Erwägungsgrund 47 S. 1 ECRL nahe, dass das in Art. 15 Abs. 1 statuierte Verbot mit Betonung auf der Allgemeinheit solcher Überwachungspflichten zu lesen ist. Nach Erwägungsgrund 47 S. 2 soll dies nämlich nicht „Überwachungspflichten in spezifischen Fällen“ betreffen, „die von einzelstaatlichen Behörden nach innerstaatlichem Recht getroffen werden" können. Mithin ist für die Reichweite der normativen Anordnung des Art. 15 Abs. 1 ECRL entscheidend, was unter einer unzulässigen ,allgemeinen“, „proaktiven"154 bzw. „generellen" ${ }^{\text {"155 }}$ und einer im Gegensatz dazu zulässigen „spezifischen“ Überwachung zu verstehen ist, die zu zulässigen „Verhinderungspflichten" ${ }^{156}$ führen kann. Die Rechtsprechung des EuGH verhält sich zu der genauen Abgrenzung bislang nicht abschließend, sondern nur punktuell und ohne eine bislang erkennbare Bemühung, diese auch begrifflich festzuziehen. ${ }^{157}$ Teilweise ergibt sich sogar eine in sich widersprüchliche Linie in der Rechtsprechung.

${ }^{152}$ So auch Lodder, in: Lodder/Murray, EU Regulation of E-Commerce, S. 15 (53).

${ }^{153}$ Vgl. Van Eecke, Common Market Law Review 48 (2011), 1455 (1464).

${ }^{154}$ Vgl. nur Hoffmann/Volkmann, in: Spindler/Schuster, Recht der elektronischen Medien, TMG $§ 7$ Rn. 35.

${ }^{155}$ EuGH, Urt. v. 16.02.2012 - C-360/10, MMR 2012, 334 (Rn. 33) - SABAM/Netlog.

${ }^{156} \mathrm{Vgl}$. Holznagel, ZUM 2018, 350 (351).

${ }^{157}$ Kritisch insoweit auch Metzger, GRUR 2012, 384 (385). 


\section{aa) Unzulässigkeit von Filtersystemen}

Zwei parallel gelagerte Fälle aus Belgien betrafen die die belgische Verwertungsgesellschaft SABAM. Im ersten Rechtsstreit hatte diese das Unternehmen Scarlet Extended, einen Access-Provider, mit dem Ziel in Anspruch genommen, den rechtswidrigen Austausch von geschützten Musiktiteln zu unterbinden und hierbei einen elektronischen Filter zu nutzen. ${ }^{158} \mathrm{Im}$ zweiten Rechtsstreit war Gegner das Unternehmen Netlog, das ebenfalls von SABAM darauf in Anspruch genommen wurde, den Austausch geschützter Werke zu unterbinden und hierbei einen Filter einzusetzen. ${ }^{159}$ In beiden Fällen lief das klägerische Begehren in den Ausgangsentscheidungen darauf hinaus, die Klagegegner zum Einsatz von Filtersystemen zu verpflichten, um entsprechende rechtswidrige Handlungen zu unterbinden. Die jeweils mit dem Fall befassten Gerichte legten die Verfahren dem EuGH zur Vorabentscheidung vor, um die Grenze einer möglichen Verpflichtung mit Blick auf das Verbot allgemeiner Überwachungspflichten auszuloten.

Konkret war in beiden Fällen fraglich, ob es mit Art. 15 Abs. 1 ECRL vereinbar sei, die betroffenen Diensteanbieter zu verpflichten, ein Filtersystem, „das unterschiedslos auf alle seine Kunden anwendbar ist, präventiv, auf ausschließlich seine eigenen Kosten und zeitlich unbegrenzt einzurichten. " ${ }^{160}$ Der Gerichtshof hält insoweit einen Fall unzulässiger allgemeiner Überwachungspflichten im Sinne von Art. 15 Abs. 1 ECRL für gegeben, wenn ein Diensteanbieter dazu verpflichtet würde, „sämtliche Daten jedes Einzelnen seiner Kunden aktiv zu überwachen, um jeder künftigen Verletzung von Rechten des geistigen Eigentums vorzubeugen. "“161 Die Einführung eines solchen Filtersystems bedeute nach Auffassung des Gerichtshofs, dass sämtliche Kommunikationsinhalte der Nutzer eines Dienstes identifiziert würden, um sodann jene zu ermitteln, die unter ein Schutzrecht fielen bzw. anderweitig rechtswidrig seien und diese Inhalte dann entsprechend zu sperren. ${ }^{162}$ Weil damit sämtliche Kommunikation aller Kunden überwacht werden müssten, verbiete es Art. 15 ECRL, einem Host-Provider die Pflicht zur Verwendung eines Filtersystems aufzuerlegen. ${ }^{163}$ Ähnlich hatte sich der

\footnotetext{
${ }^{158}$ Vgl. EuGH, Urt. v. 24.11.2011 - C-70/10, MMR 2012, 174 (174 f.) - Scarlet Extended/SABAM.

${ }^{159}$ Vgl. EuGH, Urt. v. 16.02.2012 - C-360/10, MMR 2012, 334 (335) - SABAM/Netlog.

${ }^{160}$ EuGH, Urt. v. 24.11.2011 - C-70/10, MMR 2012, 174 (Rn. 29) - Scarlet Extended/SABAM; Urt. v. 16.02.2012 - C-360/10, MMR 2012, 334 (Rn. 26) - SABAM/Netlog.

${ }^{161}$ EuGH, Urt. v. 24.11.2011 - C-70/10, MMR 2012, 174 (Rn. 36) - Scarlet Extended/SABAM; Urt. v. 16.02.2012 - C-360/10, MMR 2012, 334 (Rn. 34) - SABAM/Netlog.

${ }^{162}$ Vgl. EuGH, Urt. v. 24.11.2011 - C-70/10, MMR 2012, 174 (Rn. 38) - Scarlet Extended/SABAM; Urt. v. 16.02.2012 - C-360/10, MMR 2012, 334 (Rn. 36) - SABAM/Netlog.

${ }^{163}$ Vgl. EuGH, Urt. v. 24.11.2011 - C-70/10, MMR 2012, 174 (Rn. 39 f.) - Scarlet Extended/SABAM; Urt. v. 16.02.2012 - C-360/10, MMR 2012, 334 (Rn. 36 ff.) - SABAM/Netlog.
} 
EuGH schon zuvor in der Entscheidung L'Oréal geäußert, wo es hieß, ein Diensteanbieter dürfe nicht dazu verpflichtet werden, ,aktiv alle Angaben eines jeden seiner Kunden zu überwachen, um jeder künftigen Verletzung von Rechten des geistigen Eigentums über die Seite dieses Anbieters vorzubeugen. " ${ }^{164}$

\section{bb) Automatisierte Techniken und Mittel zur Nachforschung}

Eine mit der soeben erläuterten Position nicht gänzlich in Einklang zu bringende Auffassung äußerte der EuGH jüngst in der vielbeachteten Entscheidung Glawischnig-Piesczek. Hier ging es um einen Unterlassungsanspruch, den eine österreichische Politikerin gegenüber Facebook geltend gemacht hatte, nachdem dort über sie ein Beitrag zu lesen war, der nach der Feststellung des vorlegenden Gerichts diffamierend und damit rechtswidrig war. Die wesentlichen Rechtsfragen lauteten hier, ob Facebook auch für ,wortund/oder sinngleiche" Wiederholungen entsprechender Äußerungen in Anspruch genommen werden kann, ohne dass dem Unternehmen diese (erneut) zur Kenntnis gebracht wurden sowie ob die Entfernung dieser auch weltweit $\mathrm{zu}$ erfolgen habe. ${ }^{165}$

Soweit es die Verhinderungspflicht betreffend wortgleicher Inhalte betrifft, geht der EuGH davon aus, dass ein „spezifischer Fall“ im Sinne des Erwägungsgrund 47 S. 1 dann vorliege, wenn es um eine „konkrete Information" gehe, „die vom betreffenden Hosting-Anbieter [...] gespeichert wurde und deren Inhalt von einem zuständigen Gericht [...] analysiert und beurteilt wurde, das diese Information nach Abschluss seiner Würdigung für rechtswidrig erklärt hat. “166 Da die Gefahr einer späteren erneuten Verbreitung des Inhalts bestehe, sei die Pflicht zur Sperrung auch bei wortgleichen Inhalten gegeben, ,ganz gleich, wer den Auftrag zur Speicherung dieser Informationen gegeben hat. " ${ }^{\text {167 }}$

Was die Frage nach der Verhinderung ,sinngleicher" Inhalte anbetrifft, versteht der EuGH hierunter solche Informationen, ,die eine Aussage vermitteln, deren Inhalt im Wesentlichen unverändert bleibt und daher sehr wenig von dem Inhalt abweicht, der zur Feststellung der Rechtswidrigkeit geführt hat. ${ }^{\text {"168 }}$ Weil die Rechtswidrigkeit sich insoweit nicht aus einzelnen

\footnotetext{
${ }^{164}$ EuGH, Urt. v. 12.11.2011 - C-324/09, MMR 2011, 596 (Rn. 139) - L'Oréal SA (Hervorh. v. Verf).

${ }^{165}$ Vgl. EuGH, Urt. v. 03.10.2019 - C-18/18, NJW 2019, 3287 (3287f.) - GlawischnigPiesczek; die Frage nach der weltweiten Entfernung ist hier nicht von Bedeutung.

${ }^{166}$ EuGH, Urt. v. 03.10.2019 - C-18/18, NJW 2019, 3287 (Rn. 35) - Glawischnig-Piesczek.

${ }^{167}$ EuGH, Urt. v. 03.10.2019 - C-18/18, NJW 2019, 3287 (Rn. 37) - Glawischnig-Piesczek.

${ }^{168}$ EuGH, Urt. v. 03.10.2019 - C-18/18, NJW 2019, 3287 (Rn. 39) - Glawischnig-Piesczek.
} 
Begriffen, sondern aus der mit diesen vermittelten Aussage ergebe, müsse die gerichtliche Verfügung zur Verhinderung weiterer Schäden beim Betroffenen sich auf nicht wortgleiche Inhalte beschränken. Sie könne vielmehr auch solche erfassen, „Zwar leicht unterschiedlich formuliert“ sind, „aber im Wesentlichen die gleiche Aussage" vermittelten. ${ }^{169}$ Das sei schon deshalb geboten, damit nicht die Verfolgung von Persönlichkeitsrechtsverletzungen durch jeweils minimale Änderungen der rechtsverletzenden Inhalte erschwert werden könnte, da ansonsten die Betroffenen jeweils immer wieder neue Verfahren anstrengen müssten. ${ }^{170}$

Schließlich, und in diesem Kontext entscheidend, dürfe aber die Pflicht zur Verhinderung sinngleicher Inhalte nicht das Gleichgewicht der betroffenen Interessen zu sehr beeinträchtigen, weshalb die Instanz, die die Verhinderungspflicht erlasse, „spezifische Einzelheiten [...] gebührend“ identifizieren müsse. ${ }^{171}$ Diese Identifikation von Einzelheiten, die einen Inhalt zu einem sinngleichen mache, müsse insbesondere so hinreichend genau sein, dass sie den Diensteanbieter nicht dazu zwängen, „eine autonome Beurteilung dieses Inhalts vorzunehmen. "172 Dadurch werde ein hinreichender Schutz von Persönlichkeitsrechten erzielt, während die Diensteanbieter nicht über Gebühr belastet würden, „da die Überwachung und das Nachforschen [...] auf die Informationen beschränkt sind, die die in der Verfügung genau bezeichneten Einzelheiten enthalten, und da ihr diffamierender Inhalt sinngleicher Art den Hosting-Anbieter nicht verpflichtet, eine autonome Beurteilung vorzunehmen, so dass er auf automatisierte Techniken und Mittel zur Nachforschung zurückgreifen kann.“173 Mit der Unzulässigkeit der „autonomen Beurteilung" geht praktisch das Verbot einher, von den Diensteanbietern manuelle Kontrollen der Ergebnisse der automatisierten Filterung zu verlangen. ${ }^{174}$

\section{cc) Verhältnismäßigkeit von Verhinderungspflichten}

Neben den speziell Art. 15 ECRL zugeordneten Rechtsgrundsätzen im soeben erläuterten Sinne thematisiert der EuGH teilweise auch grundrechtliche Aspekte von Verhinderungspflichten. So bleibt er in den SABAM-Entschei-

${ }^{169}$ EuGH, Urt. v. 03.10.2019 - C-18/18, NJW 2019, 3287 (Rn. 40 f.) - GlawischnigPiesczek.

${ }^{170}$ Vgl. EuGH, Urt. v. 03.10.2019 - C-18/18, NJW 2019, 3287 (Rn. 41) - GlawischnigPiesczek.

${ }^{171}$ EuGH, Urt. v. 03.10.2019 - C-18/18, NJW 2019, 3287 (Rn. 43 ff.) - GlawischnigPiesczek.

${ }^{172}$ EuGH, Urt. v. 03.10.2019 - C-18/18, NJW 2019, 3287 (Rn. 45) - Glawischnig-Piesczek.

${ }^{173}$ EuGH, Urt. v. 03.10.2019 - C-18/18, NJW 2019, 3287 (Rn. 46) - Glawischnig-Piesczek (Hervorh. v. Verf.).

${ }^{174}$ Vgl. Holznagel, ZUM 2019, 910 (911). 
dungen nicht bei der Feststellung der Unvereinbarkeit von Verhinderungspflichten mit der ECRL stehen, sondern nimmt ausführlich zu den betroffenen grundrechtlichen Garantien aus der GRCh Stellung. ${ }^{175}$ Hierzu gehörten einerseits die potenziell bedrohten Schutzrechte, insbesondere das Recht am geistigen Eigentum (Art. 17 Abs. 2 GRCh) und die unternehmerische Freiheit der Diensteanbieter (Art. 16 GRCh) ${ }^{176}$ Andererseits seien aber auch die Implikationen von Prüf- und Verhinderungspflichten auf die Freiheitssphären der anderen Nutzerinnen eines Dienstes zu berücksichtigen, die durch eine übermäßige Pflicht zur Überwachung und ggf. Sperrung von Inhalten vor allen Dingen in ihrem Recht auf Schutz ihrer personenbezogenen Daten (Art. 8 GRCh) und ihrer Informationsfreiheit (Art. 11 GRCh) beeinträchtigt würden. ${ }^{177}$ In beiden Fällen sieht der Gerichtshof mit der insoweit identischen Argumentation einen angemessenen Ausgleich dieser Positionen für nicht gegeben an, weshalb die in Rede stehenden Verhinderungspflichten mit der GRCh unvereinbar seien bzw. die einschlägigen Sekundärrechtsakte, ,ausgelegt im Hinblick auf die sich aus dem Schutz der anwendbaren Grundrechte ergebenden Anforderungen", solchen Maßnahmen entgegenstünden. ${ }^{178}$ In der Entscheidung Glawischnig-Piesczek fehlen dagegen entsprechende Ausführungen oder Verweise. Hier begnügt sich der EuGH mit kurzen Hinweisen allein auf die Rechtsposition der Diensteanbieter. ${ }^{179}$

\section{b) Zwischenfazit}

In den $S A B A M$-Entscheidungen positioniert sich der EuGH damit recht deutlich gegen Verpflichtungen, mittels Filtertechnologien den gesamten Bestand an Kommunikation innerhalb des eigenen Angebots zu erfassen. Die Risiken für die Freiheitssphären der hiervon betroffenen Nutzer liegen dabei auf der Hand. Insbesondere die Erfassung einer Vielzahl personenbezogener Daten, die bei einer solch umfassenden Überwachung anfallen würden, sowie die potenzielle Einbuße im Bestand der für die Informationsfreiheit relevan-

${ }^{175}$ Zum Verhältnis des Sekundärrechts im Vergleich zur zunehmenden Bedeutung der GRCh in diesem Kontext vgl. noch unten $\S 11 \mathrm{~A}$.

${ }^{176}$ Vgl. EuGH, Urt. v. 24.11.2011 - C-70/10, MMR 2012, 174 (Rn. 42 ff.) - Scarlet Extended/SABAM; Urt. v. 16.02.2012 - C-360/10, MMR 2012, 334 (Rn. 40 ff.) - SABAM/Netlog.

${ }^{177}$ Vgl. EuGH, Urt. v. 24.11.2011 - C-70/10, MMR 2012, 174 (Rn. 50 ff.) - Scarlet Extended/SABAM; Urt. v. 16.02.2012 - C-360/10, MMR 2012, 334 (Rn. 48 ff.) - SABAM/Netlog.

${ }^{178}$ EuGH, Urt. v. 24.11.2011 - C-70/10, MMR 2012, 174 (Rn. 54) - Scarlet Extended/SABAM; vgl. Urt. v. 16.02.2012 - C-360/10, MMR 2012, 334 (Rn. 52) - SABAM/Netlog.

${ }^{179}$ Vgl. EuGH, Urt. v. 03.10.2019 - C-18/18, NJW 2019, 3287 (Rn. 44, 46) - Glawischnig-Piesczek. 
ten Inhalte verdeutlichen das Bedrohungspotenzial, das von umfassenden Verhinderungspflichten ausgeht. In der Entscheidung Glawischnig-Piesczek fehlen solche Ausführungen hingegen. Insoweit ist zwischen dem SABAMKomplex und der Entscheidung Glawischnig-Piesczek eine nicht unerhebliche argumentative Unwucht auszumachen. Das bestätigt auch ein Blick auf die Art und Weise, wie die drei Entscheidungen den grundrechtlichen Kontext des Streitstoffes reflektieren. Aus der Perspektive der Diensteanbieter erweisen sich die ihnen auferlegten Pflichten, gleich wie man zu der skizzierten Abgrenzung von allgemeinen und spezifischen Überwachungspflichten im Einzelfall steht, als unmittelbare Auflage für ihr unternehmerisches Geschäftsmodell. Es ist deshalb einerseits im Grundansatz einsichtig, dass der EuGH in den $S A B A M$-Entscheidungen zur Vereinbarkeit von Verhinderungspflichten mit grundrechtlichen Garantien insbesondere aus der GRCh Stellung nimmt. ${ }^{180}$ Andererseits sind diese Ausführungen prozessual lediglich als obiter dicta einzuordnen, da der Gerichtshof in beiden Fällen die betreffenden Filterpflichten schon für mit Art. 15 Abs. 1 ECRL unvereinbar erklärt hatte. ${ }^{181}$ Umso mehr fällt dann aber auf, dass in der Entscheidung Glawischnig-Piesczek im Grunde kaum noch eine grundrechtliche Verortung des Einsatzes ,automatisierter Techniken“ erfolgt, jedenfalls und insbesondere nicht hinsichtlich der Auswirkungen auf andere Dienstenutzer, sondern nur ansatzweise hinsichtlich der Auswirkungen solcher Pflichten auf den betroffenen Diensteanbieter selbst. ${ }^{182}$ Wenn eine dieser Entscheidungen eine solche Einordnung jedoch notwendig gemacht hätte, dann diese. ${ }^{183}$ Hier zeigt sich eine grundrechtliche Leerstelle in der Rechtsprechung des EuGH.

Die erläuterten Entscheidungen offenbaren damit ein nicht ganz widerspruchsfreies Bild, was die Vereinbarkeit des Einsatzes technischer Mittel zur Verhinderung weiterer Rechtsverletzungen mit Art. 15 Abs. 1 ECRL betrifft. Denn während die beiden $S A B A M$-Entscheidungen dem Einsatz von Filtersystemen zur automatisierten Überprüfung von Inhalten eine recht deutliche Absage erteilen, scheint die Tendenz in Glawischnig-Piesczek gerade in die entgegengesetzte Richtung zu gehen, soweit an entscheidender Stelle in den Urteilsgründen ,automatisierte Techniken“184 als entscheidendes Mittel angesehen werden, um Pflichten der Diensteanbieter verhältnismäßig auszu-

\footnotetext{
${ }^{180}$ Vgl. Kastl, GRUR 2016, 671 (676f.); Metzger, GRUR 2012, 384 (385).

${ }^{181}$ Vgl. das eindeutige und unabhängig von der grundrechtlichen Ebene gefundene Ergebnis bei EuGH, Urt. v. 24.11.2011 - C-70/10, MMR 2012, 174 (Rn. 40) - Scarlet Extended/SABAM.

${ }^{182}$ Vgl. EuGH, Urt. v. 03.10.2019 - C-18/18, NJW 2019, 3287 (Rn. 44, 46) - Glawischnig-Piesczek.

${ }^{183}$ Im Ansatz ebenso Spindler, NJW 2019, 3274 (3275 f.); Paal, JZ 2020, 92 (94).

${ }^{184}$ EuGH, Urt. v. 03.10.2019 - C-18/18, NJW 2019, 3287 (Rn. 46) - Glawischnig-Piesczek.
} 
gestalten, insbesondere diesen keine überspannten Prüfpflichten aufzuerlegen. Was die Formulierung von konkreten normativen Erwartungen an den Umgang mit den Folgen aus dem Einsatz dieser Techniken angeht, fällt das Urteil indes unterkomplex aus. Für den Gerichtshof ist hier nur die Zielfunktion entscheidend: die automatisierten Techniken müssen identische und sinngleiche, das heißt „im Wesentlichen die gleiche Aussage“ vermittelnde Inhalte erkennen. Die Pflicht zur Erfassung identischer Inhalte dürfte noch eher handhabbar sein, während die Sinngleichheit ungleich schwieriger zu ermitteln ist. ${ }^{185}$

Freilich bestehen zwischen dem $S A B A M$-Komplex und der Entscheidung Glawischnig-Piesczek auch Unterschiede, die im jeweiligen Sachverhalt und den jeweils gerichtlich beantragten Maßnahmen ihren Ursprung haben. Bei den $S A B A M$-Entscheidungen ging es um eine allgemeine, sehr breite und grundsätzlich alle Nutzer eines Dienstes erfassende Filterpflicht ${ }^{186}$ während bei Glawischnig-Piesczek die Pflicht nur bereits gerichtlich bezeichnete Inhalte betreffen soll. Dass im letzteren Fall auch die Erfassung „sinngleicher“ Inhalte Teil der Verhinderungspflichten eines Diensteanbieters sein sollen, lässt den Unterschied zu den $S A B A M$-Entscheidungen allerdings nicht kategorial, sondern vielmehr nur graduell erscheinen. Jedenfalls macht sich der EuGH in Glawischnig-Piesczek augenscheinlich nicht die Mühe, die aufgestellten Grundsätze von seiner bisherigen Rechtsprechung zu Art. 15 ECRL dergestalt abzugrenzen, dass diese Entscheidung lediglich als Einzelfall wahrgenommen werden könnte.

\section{Fazit}

Zusammenfassend bleibt man also bei der Bewertung der Rechtsprechung des EuGH unter dem Blickwinkel des Verbots allgemeiner Überwachungspflichten mit einem gemischten Eindruck zurück. Die früheren Entscheidungen zum SABAM-Komplex legen eine eher anbieterfreundliche Lesart des Art. 15 Abs. 1 ECRL nahe, die den Gerichten der Mitgliedstaaten überdies den recht deutlichen Auftrag übermitteln, die externen Effekte der Inpflichtnahme der Diensteanbieter insbesondere bei deren Nutzern im Blick zu behalten. Die Wende hin zu der eher betroffenenfreundlichen Perspektive, die solche externen Effekte zumindest nicht mehr explizit anspricht, scheint mit zwei Motiven erklärbar, die nicht unabhängig voneinander gedacht werden können. Das eine Motiv betrifft den Umstand, dass es in der Entscheidung Glawischnig-Piesczek um Persönlichkeitsrechtsverletzungen und nicht den Schutz des geistigen Eigentums ging. Offenbar scheint hier der EuGH einen qualitativen Unterschied zu sehen, obgleich zwischen diesen beiden Rechts-

\footnotetext{
${ }^{185}$ Kritisch insoweit auch Paal, JZ 2020, 92 (93).

${ }^{186}$ Das betont auch Holznagel, ZUM 2018, 350 (355).
} 
positionen kein explizites Vorrangverhältnis existiert. Es spricht zwar für eine stärkere Inpflichtnahme zum Schutz des Persönlichkeitsrechts, dass deren Träger sich in der Regel nicht auf einen starken institutionalisierten Schutz verlassen können sowie die Täter in der Regel schwerer greifbar sind und damit geringere Chancen bei der Rechtsverfolgung bestehen ${ }^{187}$ Unter dem bestehenden Rechtsrahmen lässt sich eine solche Motivation allerdings kaum ohne eine Abstrahierung entsprechender Maßstäbe anhand der sektorübergreifenden Regelungen der ECRL ausbuchstabieren, was Auswirkungen eben auch auf die Position der geistigen Eigentumsrechte und anderer Rechtsgüter zeitigt. ${ }^{188}$ Das zweite Motiv ist rechtspolitischer Natur. So ist in den Diskussionen der vergangenen Jahre um die Debattenkultur im Netz und die richtigen rechtlichen Rahmenbedingungen eine unschwer erkennbare Entwicklung im europäischen Raum hin zu einer stärkeren Inpflichtnahme von Diensteanbietern festzustellen, die auch vor dem EuGH nicht Halt zu machen scheint, der insbesondere seit der Google Spain-Entscheidung ${ }^{189}$ bisweilen eine kritischere Haltung an den Tag legt. Ausgangspunkt und Fokus dieser Diskussionen waren und sind oft, wenn auch nicht ausschließlich die Rechtspositionen von Individuen gegenüber diesen Unternehmen. Ob die Entwicklung insgesamt zu einer stärkeren Differenzierung nach betroffenen Schutzgütern neigen wird, bleibt nach dem bisher bekannten Entscheidungsmaterial allerdings abzuwarten.

\section{Die Inanspruchnahme von Diensteanbietern im deutschen Recht}

Der Fokus galt bislang dem Unionsrecht. Beim Richtlinienrecht kommt es allerdings auch maßgeblich auf das mitgliedstaatliche Umsetzungsrecht an. Dies gilt nicht zuletzt für den deutschen Kontext, der sich durch eine Rechtsprechungstradition auszeichnet, die historisch der ECRL um Längen vorausgeht, was wenig überraschend teilweise zu Friktionen führt.

Im bürgerlichen Recht ist im Grundsatz anerkannt, dass die zivilrechtliche Verantwortung (die dann in Unterlassungs- und Schadensersatzansprüche mündet) zunächst einmal bei demjenigen liegt, der die Rechtsverletzung unmittelbar begangen hat. ${ }^{190}$ Das Vorgehen gegen den Primärverursacher selbst ist allerdings mühsam und bietet oftmals keine hohen Realisierungschancen. ${ }^{191}$ Damit ist die Frage aufgeworfen, inwieweit Diensteanbieter unter dem

\footnotetext{
${ }^{187}$ So auch Holznagel, ZUM 2018, 350 (353).

${ }^{188}$ So auch die Vermutung von Holznagel, ZUM 2018, 350 (350 f.).

${ }^{189}$ Vgl. EuGH, Urt. v. 13.05.2014 - C-131/12, NJW 2014, 2257 ff. - Google Spain SL.

${ }^{190} \mathrm{Vgl}$. Hofmann, JuS 2017, 713 (714).

${ }^{191}$ Vgl. Wagner, in: MüKo BGB, § 823 Rn. 836.
} 
überkommenen bürgerlichen Recht für von ihnen nicht begangene, aber von ihnen weiterverbreitete Rechtsverletzungen in Anspruch genommen werden können. Dies soll hier zunächst überblicksartig dargestellt werden (I.-III.), bevor die Privilegierung der Host-Provider nach $\S 10$ TMG als Umsetzung des Art. 14 ECRL in den Blick genommen wird (IV.).

\section{Haftung für eigene Inhalte}

In den Kategorien des deutschen Rechts ist zunächst grundsätzlich denkbar, einen Diensteanbieter wegen von ihm selbst stammender, also „eigener“ Inhalte in Anspruch zu nehmen (1.). Dies ist im Wesentlichen im bürgerlichen Recht des BGB geregelt. ${ }^{192}$ Für die Thematik der vorliegenden Untersuchung ist materiell-rechtlich zuvörderst § 1004 BGB als Grundnorm der Täterhaftung zu nennen. Das Gesetz bezeichnet hier den Schuldner der negatorischen ${ }^{193}$ und quasi-negatorischen ${ }^{194}$ Ansprüche als "Störer" ${ }^{\text {“195 }}$, obwohl es bei $\S 1004$ BGB in seiner unmittelbaren Anwendung noch nicht um die Störerhaftung geht.

Neben den tatsächlich eigenen Inhalten hat der BGH jedoch eine weitere Kategorie zur normativen Zuordnung von Inhalten geschaffen, die zwar streng genommen keine eigenen Inhalte des Äußernden sind, die die Rechtsprechung aber dennoch als eigene behandelt. Gerade jene Kategorie, die unter dem Stichwort „Zueigenmachen“ fremder Informationen geläufig ist, offenbart die teils schwierige Gemengelage aus Unionsrecht und nationalem (Richter-)Recht, das den Gegenstand dieser Untersuchung auszeichnet (2.).

${ }^{192}$ In der Sache sind auch andere Normen relevant, aus denen sich entsprechende Ansprüche ergeben können, namentlich sind dies insbesondere die Unterlassungs- und Schadensersatzansprüche wegen Urheberrechtsverletzungen nach $\S 97$ UrhG.

${ }^{193}$ Der Begriff verweist auf die actio negatoria im römischen Recht, die dem Eigentümer einen Abwehranspruch gegen die „Anmaßung eines dinglichen Rechts“ an die Hand gab, Raff, in: MüKo BGB, § 1004 Rn. 10. Heutzutage eignet sich der Begriff im eigentlichen Sinne nicht mehr, da die auf $\S 1004$ BGB (in direkter oder analoger Anwendung) gestützten Ansprüche nunmehr generell die Abwehr von eingetretenen oder drohenden Störungen meint, vgl. ders., in: MüKo BGB, § 1004 Rn. 14.

${ }^{194}$ Damit sind solche Ansprüche gemeint, die die deliktischen Ansprüche ( $\$ 823$ Abs. 2, 824, 826 BGB) ergänzen, vgl. Schapiro, Unterlassungsansprüche gegen die Betreiber von Internet-Auktionshäusern und Internet-Meinungsforen, S. 22.

${ }^{195}$ Diesen Umstand nutzen die beiden zuständigen Senate des BGH auch dazu, sich begrifflich voneinander abzugrenzen, ohne dabei auf rhetorische Spitzen zu verzichten, vgl. BGH, Urt. v. 04.04.2017 - VI ZR 123/16, NJW 2017, 2029 (Rn. 18) - klinikbewertungen.de: „Unmittelbarer Störer - in der Diktion des I. Zivilsenats „Täter“ [...].“ Zur Bedeutung dieser begrifflichen Divergenz siehe noch $\S 10$ A. II. 2. 


\section{Tatsächlich eigene Inhalte}

\section{a) Grundlagen}

Soweit es um tatsächlich eigene Inhalte des Diensteanbieters geht, kommt bei diesen grundsätzlich nur die Haftung als sog. „content provider“ in Betracht, wie $\S 7$ Abs. 1 TMG deklaratorisch feststellt. ${ }^{196} \mathrm{Da} \S 10 \mathrm{TMG}$, der als Gegenstück zu $\$ 7$ Abs. 1 „fremde Informationen“ in den Blick nimmt, die Vorschrift des Art. 14 ECRL umsetzt, ist insbesondere bei der Eingabe von Informationen durch einen Nutzer nicht von eigenen Informationen des Diensteanbieters auszugehen ${ }^{197}$ da ansonsten die intendierte Privilegierung ausgehöhlt würde. Umgekehrt ist aber auch nur in diesem Fall die Möglichkeit der Privilegierung gerechtfertigt. Damit können Informationen jenseits ihrer Eingabe durch einen Nutzer theoretisch auch als eigene Informationen des Diensteanbieters bewertet werden, für die eine eigenständige Täterhaftung in Betracht kommt.

Generell ist bei der Prüfung eines Inhalts darauf, ob er als „eigener" zu bewerten ist, nicht ausschließlich auf die ursprüngliche Schaffung von Daten bzw. Informationen abzustellen, sondern auch darauf, ob andere Umstände ersichtlich sind, die eine Einordnung als eigene Information notwendig machen. Die technische Kontrolle über den Vorgang des Verfügbarmachens einer von einem Dritten geschaffenen Information kann insoweit zur Annahme eines eigenen Inhalts führen. ${ }^{198}$ Kein Fall einer Haftung für eigene Inhalte liegt vor, wenn der Diensteanbieter es mit seinem Geschäftsmodell darauf anlegt, die Rechte Dritter zu verletzen. ${ }^{199}$ Auch hier kommt eine Zurechnung für die entsprechend begangenen Rechtsverletzungen Dritter in der Regel allenfalls als Teilnehmer, jedenfalls aber über die Figur der Störerhaftung in Betracht. ${ }^{200}$

Bei Verstößen gegen handlungsbezogene Rechtspflichten wie etwa im Urheber- und Markenrecht ist die Rechtswidrigkeit mitunter einfacher festzustellen als bei erfolgsbezogenen Rechtsverletzungen, weil hinsichtlich des Eintritts einer bestimmten rechtlichen Tatsache wie etwa einer Persönlichkeitsrechtsverletzung das Handlungsunrecht weniger bedeutsam ist als der Eintritt eines bestimmten Erfolgs. ${ }^{201}$ Die Handlungsbezogenheit vieler Tat-

\footnotetext{
${ }^{196}$ Vgl. auch Spindler, in: Spindler/Schmitz, TMG, §7 Rn. 6.

${ }^{197}$ Vgl. BGH, Urt. v. 04.07.2013 - I ZR 39/12, NJW 2014, 552 (Rn. 19) - Terminhinweis mit Kartenausschnitt; Paal, in: BeckOK Informations- und Medienrecht, TMG § 7 Rn. 28; Altenhain, in: MüKo StGB, Vor. §7 Rn. 16.

${ }^{198}$ Vgl. BGH, Urt. v. 04.07.2013 - I ZR 39/12, NJW 2014, 552 (Rn. 22) - Terminhinweis mit Kartenausschnitt.

${ }^{199}$ Bestenfalls missverständlich Wagner, in: MüKo BGB, $\S 823$ Rn. 840.

${ }^{200}$ Vgl. BGH, Urt. v. 15.01.2009 - I ZR 57/07, MMR 2009, 625 (Rn. 18, 21) - Cybersky; vgl. dazu noch unten $\S 10$ A. III. 2. b) cc).

${ }^{201}$ Vgl. Holznagel, Notice and Take-Down-Verfahren als Teil der Providerhaftung,
} 
bestände des Urheberrechts erklärt auch, weshalb sich in jüngerer Zeit Entwicklungen abzeichnen, die solche Diensteanbieter, die das Hochladen und Abspielen von urheberrechtlich geschützten Werken ermöglichen, zunehmend als Akteure einer eigenständigen Wiedergabe ansehen. Namentlich betrifft dies Portale wie YouTube, dessen Geschäftsmodell mit Blick auf seine Haftung für Urheberrechtsverletzungen Anlass und Gegenstand eines Vorlagebeschlusses des BGH sind, bei dem es genau um die Frage geht, ob YouTube eine öffentliche Wiedergabe im Sinne des Art. 3 InfoSoc-RL vornimmt. ${ }^{202}$ Das Gericht ist insoweit allerdings eher skeptisch, da das Hochladen des Videomaterials automatisch und ohne vorherige Kontrolle durch das Unternehmen erfolge ${ }^{203}$ und dieses deshalb vor Hinweis durch den Rechteinhaber keine Kenntnis von den Urheberrechtsverletzungen habe. ${ }^{204}$ Auf der Linie der Zuordnung einer Wiedergabehandlung durch die Diensteanbieter selbst liegt auch der Ansatz des reformierten Urheberrechts der EU. So verpflichtet Art. 17 Abs. 1 UAbs. 1 DSM-RL die Mitgliedstaaten, „Diensteanbieter für das Teilen von Online-Inhalten" als Akteure der öffentlichen Wiedergabe anzusehen. Diese Einordnung dient als Hebel für die weiteren in diesem Artikel vorgesehenen Pflichten. ${ }^{205}$ Gerade hier zeigt sich also, dass die eigenständige Handlung eines Diensteanbieters eine Art Einfallstor darstellen kann, neue Pflichten und Sorgfaltsmaßstäbe zu etablieren bzw. zu legitimieren.

S. 95 f. Dies ist auch der Grund für die Differenzierung der herrschenden Auffassung zur Frage, ob das Handlungs- oder das Erfolgsunrecht beim deliktischen Anspruch auf Schadensersatz maßgeblich ist. Für unmittelbare Rechtsgutsverletzungen gilt letzteres mit der Folge der indizierten Rechtswidrigkeit, für mittelbare gilt ersteres mit der zusätzlichen eigenständigen Voraussetzung einer Sorgfaltspflichtverletzung, vgl. dazu nur Wagner, in: MüKo BGB, $\S 823$ Rn. 7, mwN.

${ }^{202}$ Vgl. BGH, Beschl. v. 13.09.2018 - I ZR 140/15, GRUR 2018, 1132 (Rn. 22 ff.) YouTube. $\mathrm{Ob}$ die These der Exklusivität zwischen einer Wiedergabehandlung und der Anwendbarkeit des Art. 14 ECRL (vgl. Rn. 19 sowie Frey, ZUM 2019, 40 (42)) ohne weiteres überzeugt, ist jedoch zweifelhaft. Dagegen spricht jedenfalls die neue Regelung des Art. 17 Abs. 3 DSM-RL, die einen Gleichlauf von Wiedergabehandlung und Tätigkeit als Diensteanbieter vorsieht.

${ }^{203}$ Vgl. BGH, Beschl. v. 13.09.2018 - I ZR 140/15, GRUR 2018, 1132 (Rn. 31) - YouTube.

${ }^{204}$ Vgl. BGH, Beschl. v. 13.09.2018 - I ZR 140/15, GRUR 2018, 1132 (Rn. 34) - YouTube. Ebenso skeptisch zeigt sich GA Øe, Schlussantrag v. 16.07.2019 - C-682/18, juris, Rn. 66 ff. - YouTube.

${ }^{205}$ Vgl. zu Art. 17 DSM-RL noch unten $§ 12$ B. 


\section{b) "Autocomplete“}

Einen Sonderfall bei der Kategorie der Haftung für eigene Inhalte stellt die sog. „Autocomplete"-Funktion von Suchmaschinen dar. Hierbei handelt es sich um automatisch erzeugte Vorschläge zur Vervollständigung von Eingaben in das Textfeld einer Suchmaschine. Dabei richten sich die Vorschläge zur Vervollständigung, die auch aus Wortkombinationen bestehen können, nach der Eingabe der ersten Buchstaben oder Wörter durch den Nutzer. Als Beispiel, das bereits Gegenstand einer prominenten Entscheidung des BGH war, ist etwa denkbar, dass die Eingabe des Namens die Vervollständigungsfunktion Vorschläge unterbreitet, die in ihrem Sinnzusammenhang ggf. persönlichkeitsrechtsverletzend sein können. So hatte der BGH zu entscheiden, ob der Vorschlag zur Vervollständigung einer Sucheingabe durch die Wörter „Betrug“ oder „Scientology“ nach Eingabe des Namens einer bestimmten Person eine Unterlassungs- und Geldentschädigungshaftung von Google auslöst. Dabei war insbesondere fraglich, ob die Vorschläge der Suchmaschine als deren eigene Inhalte anzusehen sind. Der BGH ist der Auffassung, dass Nutzerinnen einer Suchmaschine in der Regel inhaltlich sinnvolle Ergänzungsvorschläge erwarteten. Dies sei auch für die Attraktivität einer bestimmten Suchmaschine von entscheidender Bedeutung, weshalb den vorgeschlagenen Begriffen die Aussage zu entnehmen sei, zwischen ihnen und den bereits vom Nutzer eingegebenen Buchstaben oder Wörtern bestehe ein sachlicher Zusammenhang. ${ }^{206}$ Diese Verknüpfungen würden nur vom Suchmaschinenbetreiber selbst vorgenommen, also insbesondere nicht von Dritten erstellt. ${ }^{207}$ Damit stellt sich der BGH auch der Vorinstanz, dem OLG Köln, entgegen, das dem Vervollständigungsvorschlag keinen eigenständigen Aussagegehalt von Google zugeordnet hatte. ${ }^{208}$

Dies überzeugt, denn tatsächlich wäre kein Dritter in der Lage, auch nur mittelbar (etwa durch gezielte Beeinflussung des entsprechenden Programms durch wiederholte Suchanfragen) eine solche statistische Relevanz von Begriffspaaren zu erzeugen, um als faktischer Urheber eines Vorschlags zur Vervollständigung angesehen zu werden. ${ }^{209}$ Außerdem ließe ein solcher Verweis auf die Verursachung durch Dritte die Fähigkeit von Google außer Betracht, bestimmte Vorschläge zu unterbinden. ${ }^{210}$ Die Annahme eines eigenen Inhalts lässt sich auch nicht mit der Plattitüde ablehnen, bei Google

\footnotetext{
${ }^{206}$ Vgl. BGH, Urt. v. 14.05.2013 - VI ZR 269/12, NJW 2013, 2348 (Rn. 16) - Autocomplete.

${ }^{207}$ Vgl. BGH, Urt. v. 14.05.2013 - VI ZR 269/12, NJW 2013, 2348 (Rn. 17) - Autocomplete.

${ }^{208}$ Vgl. OLG Köln, Urt. v. 10.05.2012 - 15 U 199/11, MMR 2012, 840 (842f.) - Autocomplete.

${ }^{209}$ Vgl. auch Ladeur, JZ 2013, 792 (793).

${ }^{210} \mathrm{Vgl}$. Kastl, Automatisierung im Internet, S. 214.
} 
säßen keine „verwegene [...] Programmierer“, die mittels der Vorschläge dem Nutzer subjektive Wertungen zu übermitteln suchten. ${ }^{211}$ Denn gerade auf solche Intentionen vom Personal juristischer Personen kommt es bei den $\S \S 7 \mathrm{ff}$. TMG nicht an, soweit diese auf die Unternehmen selbst abstellen. Dies gilt erst recht nicht bei der ECRL, die grundsätzlich auf kommerzielle Tätigkeiten bezogen ist.

\section{c) Fazit}

Zusammenfassend lässt sich festhalten, dass die technische Entwicklung sich von den Internetforen der 1990er Jahre, bei denen noch recht statisch davon ausgegangen werden konnte, dass deren Betreiber typischerweise keine eigenen Inhalte erstellen, deutlich entfernt hat. Die unterschiedlichen Funktionen von Diensteanbietern, deren Breite im Prinzip nur durch die technischen Bedingungen der Nutzung des Internets beschränkt ist, eröffnen immer wieder die Möglichkeit, auch das Vorliegen eines eigenen Inhalts im Rechtssinne in Erwägung zu ziehen. Dies ändert aber nichts daran, dass die Zurechnung von Handlungen Dritter jenseits der Fälle eigener Informationen auch in Zukunft von entscheidender Bedeutung bleiben wird, wenn es um die haftungsrechtliche Erfassung von Diensteanbietern geht, die ihren Nutzerinnen das Hochladen und Verbreiten von Inhalten ermöglichen.

\section{Die Kategorie des Zueigenmachens fremder Inhalte als Bruchstelle im Verhältnis zum Unionsrecht}

\section{a) Grundlagen}

Ein überaus relevantes Instrument für eine solche Zurechnung ist die Figur des Zueigenmachens fremder Informationen. ${ }^{212}$ Sie fußt mittelbar auf der gesetzlichen Differenzierung zwischen eigenen ( $\$ 7$ Abs. 1 TMG) und fremden (§§ 8 Abs. 1, 9 S. 1, 10 TMG) Informationen. ${ }^{213}$ Wesenskern des Zueigenmachens ist die Feststellung, ob ,der in Anspruch Genommene nach außen erkennbar die inhaltliche Verantwortung für die von ihm als Äußerungen anderer Personen veröffentlichten Inhalte übernimmt".${ }^{214}$ Die Prüfung eines solchen Zueigenmachens setzt einen normativen Zurechnungsakt voraus,

\footnotetext{
${ }^{211}$ So Härting, Internetrecht, Rn. 2511.

${ }^{212}$ Soweit ersichtlich in der Rechtsprechung erstmals erwähnt von OLG Köln, Urt. v. 28.05.2002 - 15 U 221/01, MMR 2002, 548 (548).

${ }^{213}$ Diese Unterscheidung war schon $\S 5$ TDG immanent und vom Willen des damaligen Gesetzgebers getragen, vgl. BT-Drucks. 13/7385, S. 19. Seitdem hat der Gesetzgeber die Existenz dieser Figur bei den Reformen des Telemedienrechts bestätigt, vgl. BTDrucks. 14/6098, S. 23 (zu den $\S \S 8$ ff. TDG a.F.); BT-Drucks. 16/3078, S. 15 (unveränderte Übernahme der Regeln zur Verantwortlichkeit).

${ }^{214}$ BGH, Urt. v. 02.07.2019 - VI ZR 494/17, ZUM 2019, 942 (Rn. 62).
} 
der einen großen Spielraum belässt, Kriterien für dessen Feststellung zu entwickeln. ${ }^{215}$ Demgemäß hat auch der BGH in der Entscheidung, in der er die Existenz dieser Rechtsfigur bestätigte, festgestellt, es komme bei der Abgrenzung auf ,eine objektive Sicht auf der Grundlage einer Gesamtbetrachtung aller relevanten Umstände" an. ${ }^{216}$

Mag man hierin einen prinzipiell sehr dehnbaren Ansatz zur Prüfung des Zueigenmachens sehen, mahnt das Gericht mittlerweile aber Zurückhaltung bei der Annahme eines Zueigenmachens an. ${ }^{217}$ Für ein Zueigenmachen spreche aber insbesondere, ,wenn der Portalbetreiber eine inhaltlich-redaktionelle Überprüfung der auf seinem Portal eingestellten Nutzerbewertungen auf Vollständigkeit und Richtigkeit vornimmt “ ${ }^{218}{ }^{218}$ Weitere Kriterien können „die Art der Datenübernahme, ihr Zweck und die konkrete Präsentation der Inhalte" sein. ${ }^{219}$ Für ein Zueigenmachen spreche es auch, wenn Inhalte Dritter ,,inhaltlich-redaktionell auf Vollständigkeit und Richtigkeit kontrolliert oder [ausgewählt] “" werden. ${ }^{220}$ Dem Zueigenmachen einer Information stehe aber nicht schon grundsätzlich entgegen, dass Nutzer des Dienstes den Betreiber in der Regel nicht als klassischen „,content provider" wahrnehmen. ${ }^{221}$ Denn auch dann könne die Integration fremder Inhalte in das eigene Angebot einen zurechenbaren Anschein der Identifikation mit diesen erwecken. ${ }^{222}$ Auch die „Kenntlichmachung eines fremden Inhalts als solchen“ schließe dies nicht unbedingt aus. ${ }^{223}$

${ }^{215}$ Vgl. schon Pelz, ZUM 1998, 530 (532).

${ }^{216}$ BGH, Urt. v. 12.11.2009 - I ZR 166/07, MMR 2010, 556 (Rn. 23) - marions-kochbuch.de; zuletzt Urt. v. 02.07.2019 - VI ZR 494/17, ZUM 2019, 942 (Rn. 62); vgl. aber schon das Urt. v. 06.04.1976 - VI ZR 246/74, NJW 1976, 1198 (1199) - Panorama.

${ }^{217}$ Vgl. BGH, Urt. v. 24.07.2018 - VI ZR 330/17, ZUM-RD 2019, 203 (Rn. 33); Urt. v. 27.02.2018 - VI ZR 489/16, NJW 2018, 2324 (Rn.28) - Internetforum; Urt. v. 04.04.2017 - VI ZR 123/16, NJW 2017, 2029 (Rn. 18) - klinikbewertungen.de, mwN.

${ }^{218}$ BGH, Urt. v. 04.04.2017 - VI ZR 123/16, NJW 2017, 2029 (Rn. 18) - klinikbewertungen.de; eine Prüfung durch den Diensteanbieter sah schon das Urt. v. 11.03.2004 - I ZR 304/01, NJW 2004, 3102 (3103) - Internet-Versteigerung I, offenbar als hinreichend an.

${ }^{219}$ KG, Beschl. v. 10.07.2009 - 9 W 119/08, MMR 2010, 203 (204); sehr ähnlich bereits OLG Köln, Urt. v. 28.05.2002 - 15 U 221/01, MMR 2002, 548 (548).

${ }^{220}$ BGH, Urt. v. 19.03.2015 - I ZR 94/13, MMR 2015, 726 (Rn. 25) - Hotelbewertungsportal.

${ }^{221}$ BGH, Urt. v. 12.11.2009 - I ZR 166/07, MMR 2010, 556 (Rn. 27) - marions-kochbuch.de.

${ }^{222}$ Vgl. BGH, Urt. v. 12.11.2009 - I ZR 166/07, MMR 2010, 556 (Rn. 27) - marionskochbuch.de.

${ }^{223}$ BGH, Urt. v. 12.11.2009 - I ZR 166/07, MMR 2010, 556 (Rn. 27) - marions-kochbuch.de. Freilich stellte das Gericht nicht auf die eigene Differenzierung ab, als es dem EuGH ein Verfahren zur Vorabentscheidung vorlegte, in denen es um die Einordnung von YouTube unter Art. 14 ECRL ging; hier stellt das Gericht die insoweit entscheidenden Beweggründe für die Vorlage auf die Differenzierung des EuGH in ,aktiv“ oder „passiv“ 
Als Möglichkeit zur aktiven Steuerung der Beurteilung eines Inhalts nach diesen Kriterien lassen sich teilweise Anleihen bei presserechtlichen Argumentationsfiguren erkennen, wobei die Lage in den Einzelheiten durchaus unklar ist. So soll etwa nach einem älteren Urteil des OLG Köln die eigene Distanzierung - eine tradierte Kategorie des Presserechts zur Abgrenzung eigener Behauptungen von der Verbreitung Informationen Dritter ${ }^{24}-$ gegenüber wiedergegebenen Inhalten als weiteres Kriterium gelten. ${ }^{225}$ Ähnliches vertrat der I. Zivilsenat des BGH bereits, indem er in einem Fall das Zueigenmachen bejahte, weil ,,aus der Sicht eines objektiven Nutzers eine ernsthafte und genügende Distanzierung des Diensteanbieters" nicht erfolgt sei. ${ }^{226}$ Allein das Ausbleiben einer solchen Distanzierung sieht der VI. Zivilsenat des BGH aber offenbar nicht (mehr) als hinreichende Bedingung für das Bejahen des Zueigenmachens an. ${ }^{227}$ Dahinter verbirgt sich wiederum eine presserechtliche Rechtsprechungslinie, die - geprägt durch den EGMR ${ }^{228}-$ gerade diese Distanzierungspflicht zur Vermeidung eines Zueigenmachens und damit der Haftung ablehnt. ${ }^{229}$ In diese Richtung deutet auch das BVerfG wenn es ausführt, „,dass Zeitungen sich üblicherweise nicht alle veröffentlichten Inhalte zu Eigen machen, auch wenn sie sich nicht jeweils ausdrücklich von ihnen distanzieren. “230 Danach ist es nicht ausgeschlossen, die Kriterien zur Distanzierung im Presserecht zumindest im Ansatz auf die Prüfung des Zueigenmachens zu übertragen. ${ }^{231}$ Die Möglichkeit der aktiven Steuerung der Wiedergabe von Inhalten in diesem Kontext geht indes nicht so weit, dass der Wille des Diensteanbieters allein maßgeblich wäre. Dieser muss in ir-

dar, vgl. Beschl. v. 13.09.2018 - I ZR 140/15, GRUR 2018, 1132 (Rn. 41 ff.) - YouTube; ähnlich auch die Vorlagefragen in Beschl. v. 20.09.2018 - I ZR 53/17, ZUM 2018, 870 (Rn. 31 ff.) - uploaded.

${ }^{224} \mathrm{Vgl}$. oben $\S 4$ Fn. 36.

${ }^{225}$ Vgl. OLG Köln, Urt. v. 28.05.2002 - 15 U 221/01, MMR 2002, 548 (548).

${ }^{226}$ BGH, Urt. v. 12.11.2009 - I ZR 166/07, MMR 2010, 556 (Rn. 27) - marions-kochbuch.de.

${ }^{227}$ Vgl. BGH, Urt. v. 04.04.2017 - VI ZR 123/16, NJW 2017, 2029 (Rn. 20) - klinikbewertungen.de.

${ }^{228}$ Vgl. EGMR, Urt. v. 14.12.2006 - 76918/01, Rn. 33 - Verlagsgruppe News GmbH v. Österreich.

${ }^{229}$ Vgl. BGH, Urt. v. 17.12.2013 - VI ZR 211/12, NJW 2014, 2029 (Rn. 19) - Sächsische Korruptionsaffäre; vgl. auch Burkhardt, in: Wenzel, Das Recht der Wort- und Bildberichterstattung, Kap. 4 Rn. 103, $110 \mathrm{ff}$.

${ }^{230}$ BVerfG, Beschl. v. 24.05.2005 - 1 BvR 1072/01, BVerfGE 113, 63 (83) - Junge Freiheit.

${ }^{231}$ Vgl. Hoffmann/Volkmann, in: Spindler/Schuster, Recht der elektronischen Medien, TMG $\S 7$ Rn. 16; ablehnend Paal, in: BeckOK Informations- und Medienrecht, TMG $\S 7$ Rn. 31a. 
gendeiner Weise nach außen erkennbar werden, um ein Zueigenmachen der Information abzulehnen. ${ }^{232}$

Insgesamt ist zur Figur des Zueigenmachens festzustellen, dass die Rechtsprechung bislang kaum auf einen gesicherten Bestand einzelner Tatbestandsvoraussetzungen verweisen kann. ${ }^{233}$ Das ermöglicht ihr zwar einerseits eine flexible Handhabung. Andererseits ist damit ein zentraler Ansatzpunkt der Kritik benannt, nämlich die fehlende abstrakte Berechenbarkeit der relevanten Kriterien, die die eigentlich klare Differenzierung zwischen eigenen und fremden Inhalten verwässert. Daneben ist allerdings ein weiterer, de lege lata deutlich gewichtigerer Kritikpunkt die zweifelhafte Vereinbarkeit der Figur des Zueigenmachens mit den Vorgaben des Unionsrechts.

\section{b) Verhältnis zum Unionsrecht}

Ansatzpunkt dieser unionsrechtlichen Skepsis ist die Tatsache, dass die ECRL selbst nicht zwischen eigenen und fremden Informationen unterscheidet und demnach erst recht nicht die Figur des Zueigenmachens anerkennt. Nach Art. 14 ECRL kommt es, wie bereits erwähnt, allein darauf an, ob die Information von einem Nutzer eines Diensteanbieters eingegeben wurde. Ist dies der Fall, kommt eine Privilegierung grundsätzlich in Betracht. Andernfalls steht jedenfalls Art. 14 ECRL einer Inanspruchnahme grundsätzlich nicht entgegen. Dementsprechend sieht ein Großteil der Stimmen im Schrifttum die Figur des Zueigenmachens als mit Art. 14 ECRL unvereinbar an. ${ }^{234}$ Stattdessen solle es allein auf die „Herrschaftsmacht des Diensteanbieters" ankommen. ${ }^{235}$ Danach ist es mehr als zweifelhaft, ob die Unterscheidung zwischen eigenen und fremden Inhalte unionsrechtlich Bestand haben kann. ${ }^{236}$

Mittlerweile betitelt der BGH jedoch die Frage, ob ein von einem Dritten erstellter Inhalt dennoch im Sinne von $\S 7$ Abs. 1 TMG eine eigene Information darstellen kann, zwar nach wie vor als Zueigenmachen solcher Informationen. Bei der Subsumtion bezieht sich das Gericht dann aber auf die Abgrenzung zwischen einer aktiven oder passiven Rolle, wie sie die bereits dargestellte Rechtsprechung des EuGH prägt. ${ }^{237}$ So hat der BGH in einem

${ }^{232}$ Vgl. zutreffend Härting, Internetrecht, Rn. 2516; Kovacs, Die Haftung der HostProvider für persönlichkeitsrechtsverletzende Internetäußerungen, S. $140 \mathrm{f}$.

${ }^{233}$ Kritisch auch Schapiro, Unterlassungsansprüche gegen die Betreiber von InternetAuktionshäusern und Internet-Meinungsforen, S. 353.

${ }^{234}$ Vgl. nur Fischer, Die Einbindung von Providern in die Durchsetzung von Urheberrechten, S. 179 ff.; Spindler, in: Spindler/Schmitz, TMG, §7 Rn. 8; Sieber/Höfinger, in: Hoeren/Sieber/Holznagel, Handbuch Multimedia-Recht, Teil 18.1 Rn. 39; Paal, in: BeckOK Informations- und Medienrecht, TMG § 7 Rn. 34.

${ }^{235}$ Paal, in: BeckOK Informations- und Medienrecht, TMG $§ 7$ Rn. 33.

${ }^{236}$ Vgl. Spindler, in: Spindler/Schmitz, TMG, §10 Rn. 16.

${ }^{237}$ Vgl. oben $\S 4$ C. II. 3. 
Fall ein Zueigenmachen aufgrund seiner bisherigen Rechtsprechung angenommen und als Konsequenz festgestellt, der Diensteanbieter habe „damit die Rolle eines neutralen Vermittlers verlassen und eine aktive Rolle übernommen“. ${ }^{238}$ Das Verhältnis der Abgrenzungen „aktiv/passiv“ und zu Eigen oder nicht zu Eigen gemachter Informationen ist hiernach nicht ganz klar. ${ }^{239}$ Viel spricht jedoch dafür, dass der BGH seine eigene Linie fortzusetzen sucht und hierbei lediglich rhetorisch die Kategorien des EuGH aufnimmt, um seine Entscheidungen unionsrechtlich abzusichern. Damit scheint sich die Rechtsprechung des BGH zwar nicht gänzlich vom Konzept des Zueigenmachens zu verabschieden. Dennoch werden die Bemühungen des BGH offenkundig, seine Rechtsprechungslinie mit den Vorgaben des EuGH zu integrieren ${ }^{240}$ die, wie bereits erläutert, selbst auf einer eigenwilligen Interpretation der ECRL, insbesondere ihrer Erwägungsgründe beruhen. ${ }^{241}$

Solange dieser kombinierende Ansatz unter der dogmatischen Kategorie des Zueigenmachens erfolgt, lässt sich jedoch die These, der BGH schwenke nur rhetorisch auf die Linie des EuGH ein, nicht stichhaltig widerlegen. Denn immerhin geht es hier um zwei sich grundsätzlich unvereinbar gegenüberstehenden Positionen: das technische Gepräge der ECRL und der demgegenüber eher normativ geprägte Zugang des $\mathrm{BGH} .{ }^{242}$ Es bleibt daher die Einsicht, dass ein Konflikt zwischen der deutschen Kategorisierung zwischen eigenen und fremden Inhalten und dem Konzept der ECRL besteht. ${ }^{243} \mathrm{Da}$ es letzterer darum geht, die Haftung für alle von Nutzern bei einem Diensteanbieter eingegebene Informationen zu regeln, unterfallen ihr nach dieser Sichtweise auch jene, die nach deutschem Telemedienrecht als zu Eigen ge-

${ }^{238}$ BGH, Urt. v. 04.04.2017 - VI ZR 123/16, NJW 2017, 2029 (Rn. 20) - klinikbewertungen.de.

${ }^{239}$ Vgl. Spindler, MMR 2018, 48 (49): „eine Art doppelte Prüfung“; wohl konzilianter Specht, ZUM 2017, 114 (117), der zufolge beide Konzepten „ganz ähnlich“ seien; das Nebeneinander beider Konzepte betont auch Paal, in: BeckOK Informations- und Medienrecht, TMG $\$ 7$ Rn. 34a; dagegen meint Hacker, MMR 2018, 779 (782), es handele sich „teilweise um identische Kriterien“.

${ }^{240}$ Von manchen Stimmen im Schrifttum war bereits zuvor ein ähnlicher Ansatz vorgeschlagen worden, vgl. Leistner, ZUM 2012, 722 (738); ihm folgt Chmelik, Social Network Sites - Soziale Netzwerke, S. 138 f.; wohl auch Klein, Haftung von Social-Sharing-Plattformen, S. $73 \mathrm{f}$.

${ }^{241}$ Vgl. oben $\S 4$ C. II. 3.

${ }^{242}$ Vgl. auch Spindler, in: Spindler/Schmitz, TMG, TMG $§ 7$ Rn. 8.

${ }^{243}$ Vgl. aber Marly, in: Grabitz/Hilf/Nettesheim, Recht der EU, 40. EL 2009, ECRL Vor. Abschnitt 4 Rn. 2, der auf die implizite Grundannahme der Differenzierung zwischen eigenen und fremden Inhalten auch in der ECRL hinweist und diese Abgrenzung daher als zentralen Gegenstand der Art. 12 ff. bezeichnet; dagegen meint Frey, Die Haftung von Host-Providern für Immaterialgüterrechtsverletzungen, S. 122, „fremde Informationen“ seien nicht zwingend immer solche, die von einem Nutzer „eingegeben“ sind. 
machte Informationen gelten. Das hieße, dass der Diensteanbieter für eine zu Eigen gemachte Information richtlinienkonform dennoch zu privilegieren wäre. ${ }^{244}$ Inwiefern also die Integration zwischen der Figur des Zueigenmachens und dem Konzept des EuGH auf einem sicheren Fundament steht, ist nach den bisherigen Erkenntnissen offen und trägt zum Eindruck eines nicht in sich schlüssigen und unionsrechtskonformen Haftungssystems bei.

\section{Haftung für fremde Inhalte: Das Privileg des $\S 10$ TMG}

Schließlich ist auf die in $\S 10$ TMG geregelte Haftungsprivilegierung einzugehen. Diese setzt Art. 14 ECRL um und markiert damit die Grundlage der begrenzten Möglichkeit der Inanspruchnahme von Diensteanbietern für von ihnen transportierte Inhalte Dritter.

\section{Allgemeines}

Der Regelung in $\S 10$ TMG wird, wie auch schon $\S 5$ TDG, ${ }^{245}$ eine „Filterfunktion" zugeordnet, die die geltend gemachten Ansprüche gegenüber Diensteanbietern beschränkt. ${ }^{246}$ Insbesondere begründet sie keine strafrechtliche Garantenstellung. ${ }^{247}$ Die Norm soll - wie der Gegenstand der $\$ \S 7 \mathrm{ff}$. TMG insgesamt - sowohl entgeltliche, als auch unentgeltliche Tätigkeiten erfassen und geht damit über die Art. $12 \mathrm{ff}$. ECRL hinaus. ${ }^{248}$

\section{Voraussetzungen}

\section{a) Abspeichern einer fremden Information für einen Nutzer}

In Umsetzung von Art. 14 ECRL erfordert § 10 TMG zunächst das Abspeichern von Inhalten für einen Nutzer, ohne dass es auf die Eigentumsverhältnisse am Server ankommt. Maßgeblich ist auch hier die technische Herrschaft, die dem Diensteanbieter einen Zugriff ermöglicht. ${ }^{249}$ Was die Fremdheit der Information anbetrifft, wurde bereits darauf hingewiesen, dass in richtlinienkonformer Auslegung primär nur auf die Eingabe der Information durch einen Nutzer ankommen kann. ${ }^{250}$ Insoweit deutet noch eher die Aus-

\footnotetext{
${ }^{244} \mathrm{Vgl}$. Paal, in: BeckOK Informations- und Medienrecht, TMG $\S 10$ Rn. 34, mwN.

${ }^{245}$ Vgl. oben $\$ 4$ Fn. 30, 31.

${ }^{246}$ Vgl. Hoeren, Internetrecht, S. 510.

${ }^{247}$ Vgl. Spindler, in: Spindler/Schmitz, TMG, Vor. $\S 7$ ff. Rn. 33.

${ }^{248}$ Zum persönlichen Anwendungsbereich der ECRL siehe bereits oben $\S 4$ C. II. 2. Diese überschießende Umsetzung begegnet keinen Bedenken, vgl. Paal, in: BeckOK Informations- und Medienrecht, TMG § 7 Rn. 19.

${ }^{249} \mathrm{Vgl}$. Rhode, Publizistische Sorgfalt und redaktionelle Rechtspflichten, S. 180; Spindler, in: Spindler/Schmitz, TMG, § 10 Rn. 12, mwN.

${ }^{250} \mathrm{Vgl}$. oben $§ 4$ Fn. 197 und den zugehörigen Text.
} 
sage von $\S 10$ S. 2 TMG auf das richtige unionsrechtliche Verständnis hin. Es kommt demnach maßgeblich darauf an, ob der Diensteanbieter Einfluss auf den Inhalt ausübt bzw. ausüben konnte, den der Nutzer bei ihm gespeichert hat. ${ }^{251}$

\section{b) Keine Kenntnis}

\section{aa) Positive Kenntnis eines konkreten Inhalts}

Wie auch unter Art. 14 ECRL ist im Rahmen von $\S 10$ TMG entscheidend, ob der Diensteanbieter Kenntnis von der ,rechtswidrigen Handlung oder Information" hat. Ohne Kenntnis haftet der Diensteanbieter nämlich nicht. Im Falle von Schadensersatzansprüchen ist zusätzlich erforderlich, dass dem Diensteanbieter auch „,keine Tatsachen oder Umstände bekannt sind, aus denen die rechtswidrige Handlung oder die Information offensichtlich wird". Daraus lässt sich bereits schlussfolgern, dass die Norm grundsätzlich positive Kenntnis voraussetzt. Nach überwiegender Auffassung soll auch das bewusste Sich-Verschließen hiervon nicht erfasst sein, da es auf die tatsächliche Kenntnis ankomme. ${ }^{252}$ Hieraus wird ferner abgeleitet, dass die Kenntnis sich auf einen hinreichend konkretisierten Inhalt beziehen muss. Insoweit sollen nach Spindler die zur Störerhaftung entwickelten Kriterien anwendbar sein, die also auf die „unschwere“ Erkennbarkeit abstellen. ${ }^{253}$

\section{bb) Kenntnis der Rechtswidrigkeit}

$\mathrm{Ob} \S 10$ TMG die Kenntnis der Rechtswidrigkeit eines Inhalts erfordert, ist nicht ganz eindeutig. Der Wortlaut selbst legt eine Differenzierung zwischen Handlungen und Informationen nahe, da nur in § $10 \mathrm{~S} .1 \mathrm{Nr} .1$ Var. 1 TMG von der „Kenntnis von der rechtswidrigen Handlung“ die Rede ist, während dies bei rechtswidrigen Informationen ( $(10 \mathrm{~S} .1 \mathrm{Nr} .1$ Var. 2 TMG) selbst theoretisch nicht der Fall zu sein bräuchte. ${ }^{254} \mathrm{Ob}$ eine solche trennscharfe Differenzierung zwischen Handlungen und Informationen durchweg durchgehalten werden kann, braucht hier allerdings nicht vertieft werden. Denn wie schon soeben ausgeführt, ${ }^{255}$ schließt die Kenntnis bei Art. 14 ECRL die

${ }^{251}$ Vgl. Spindler, in: Spindler/Schmitz, TMG, § 10 Rn. 16.

${ }^{252}$ Vgl. Paal, in: BeckOK Informations- und Medienrecht, TMG $§ 10$ Rn. 24; Spindler, in: Spindler/Schmitz, TMG, § 10 Rn. 23; BGH, Urt. v. 23.09.2003 - VI ZR 335/02, NJW 2003, 3764 (3764); anders aber Hoffmann/Volkmann, in: Spindler/Schuster, Recht der elektronischen Medien, TMG $\S 10$ Rn. 36.

${ }^{253}$ Vgl. Spindler, in: Spindler/Schmitz, TMG, §10 Rn. 24. Auf die Probleme, die mit dieser Kategorie verbunden sind, wird noch später eingegangen, vgl. unten $\S 10$ B. IV. 1.

${ }^{254}$ So BT-Drucks. 14/6098, S. 25; vgl. auch Paal, in: BeckOK Informations- und Medienrecht, TMG $\S 10$ Rn. 28; Chmelik, Social Network Sites - Soziale Netzwerke, S. 129.

${ }^{255} \mathrm{Vgl}$. oben $\S 4$ C. III. 1. d). 
Kenntnis der Rechtswidrigkeit mit ein. ${ }^{256}$ Aus unionsrechtlicher Perspektive ist also die subjektive Erfassung der Rechtswidrigkeit ebenfalls erforderlich, um das Privileg entfallen zu lassen. Lässt sich die Kenntnis der Rechtswidrigkeit nicht bejahen, ist der Diensteanbieter dagegen grundsätzlich unter $\S 10$ TMG privilegiert. ${ }^{257}$

\section{cc) Kenntniserlangung durch automatisierte Systeme?}

Im Kontext dieser Untersuchung von besonderem Interesse ist die Frage, auf wen es für die Kenntniserlangung ankommt. Konkret ist fraglich, ob eine Kenntniserlangung auch durch softwarebasierte Systeme erfolgen kann oder ob stets eine menschliche Gewahrwerdung erforderlich (und nachzuweisen) ist, um eine Haftung zu begründen. Ersteres wird von der wohl überwiegenden Auffassung im Schrifttum abgelehnt, ${ }^{258}$ da eine rechtliche Subsumtion erforderlich sei, die softwarebasierte Systeme noch nicht zu leisten imstande seien. Die Ratio der Privilegierung die Diensteanbieter liege insoweit gerade in der Freistellung der Diensteanbieter von quantitativ und technisch nicht leistbaren Einschätzungen. Gerade in Zusammenhang mit der Voraussetzung, dass die Kenntnis sich auch auf die Rechtswidrigkeit beziehen muss, würde andernfalls wohl das in Art. 15 ECRL formulierte Verbot allgemeiner Überwachungspflichten verletzt. Insoweit entsteht allerdings ein nicht unerheblicher Widerspruch zur jüngst geäußerten Auffassung des EuGH in der Entscheidung Glawischnig-Piesczek, der bei der Bestimmung der Reichweite von unter Art. 15 ECRL noch zulässigen Verhinderungspflichten davon spricht, dass diese nur mit Hilfe automatisierter Techniken durchgeführt werden dürfen bzw. der Diensteanbieter nicht zur manuellen Nachkontrolle gezwungen werden kann. ${ }^{259}$ Dem könnte man zwar entgegenhalten, dass es nur um die Identifikation weiterer Inhalte geht, bei denen der ursprüngliche Inhalt durch eine menschliche Prüfung als rechtswidrig beurteilt wurde. Dies würde allerdings nichts daran ändern, dass eine automatisierte Erkennung auch von sinngleichen Inhalten zu irgendeinem Zeitpunkt stattfindet. Es liegt deshalb eher fern, die Kenntnisnahme durch automatisierte Systeme pau-

${ }^{256}$ Vgl. Spindler, in: Spindler/Schmitz, TMG, § 10 Rn. 29; Paal, in: BeckOK Informations- und Medienrecht, TMG $\S 10$ Rn. 30; Chmelik, Social Network Sites - Soziale Netzwerke, S. 129.

${ }^{257} \mathrm{Vgl}$. zu den problematischen Konsequenzen dieses Umstandes unten $§ 10$ B. IV. 1, $\S 11$ C. III. 1.

${ }^{258}$ Vgl. Hoffmann/Volkmann, in: Spindler/Schuster, Recht der elektronischen Medien, TMG §10 Rn. 30; Spindler, in: Spindler/Schmitz, TMG, §10 Rn. 43; Paal, in: BeckOK Informations- und Medienrecht, TMG $\S 10$ Rn. 26; Altenhain, in: MüKo StGB, TMG $\S 10$ Rn. 22; Chmelik, Social Network Sites - Soziale Netzwerke, S. $132 \mathrm{f}$.

${ }^{259}$ Vgl. EuGH, Urt. v. 03.10.2019 - C-18/18, NJW 2019, 3287 (Rn. 46) - GlawischnigPiesczek; ausführlich dazu oben $\S 4$ C. IV. 2 a) bb). 
schal abzulehnen. Vielmehr bietet sich eine differenzierte Sichtweise an, die auf den Kontext der potenziellen Rechtsverletzung sowie die Fähigkeiten solcher Systeme ebenso Rücksicht nimmt wie auf die Komplexität der rechtlichen Bewertung im Einzelfall.

\section{c) Unverzügliche Entfernung oder Sperrung nach Kenntniserlangung}

Hat der Diensteanbieter Kenntnis erlangt, eröffnet § 10 S. 1 Nr. 2 TMG ihm entsprechend Art. 14 ECRL eine weitere Möglichkeit, sich von der Haftung zu befreien, indem er „unverzüglich“ tätig wird, um die Information zu entfernen oder den Zugang zu ihr zu sperren. Insoweit ist zunächst bemerkenswert, dass das Gesetz selbst den Erfolg der Entfernung bzw. Sperrung nicht voraussetzt, sondern nur ein redliches Bemühen. ${ }^{260}$ Zumindest soweit der Diensteanbieter mit dem betroffenen Nutzer in einem Vertragsverhältnis steht, dürften der Entfernung bzw. Sperrung rechtswidriger Inhalte vertragliche Pflichten aber gerade nicht entgegenstehen. ${ }^{261}$ Ohne eine bestehende Vertragsbeziehung dürften die Pflichten des Diensteanbieters, etwa einer Suchmaschine, tatsächlich eher auch auf zumutbare Bemühungen beschränkt sein.

\section{d) Keine ,aktive Rolle“}

In Anwendung der bereits erläuterten Rechtsprechung des EuGH übernimmt auch der BGH die bereits angesprochene schwierige Verknüpfung zwischen dem Konzept der ,aktiven Rolle“ und der hieraus ggf. resultierenden Möglichkeit, von der Rechtsverletzung Kenntnis zu nehmen oder sich über diese Kontrolle zu verschaffen. ${ }^{262}$ Insoweit hält er nunmehr fest, dass im Falle einer solchen aktiven Rolle der Diensteanbieter sich „nicht auf das Haftungsprivileg der Art. 14 Abs. 1, Art. 15 Abs. 1 der [ECRL], §7 Abs. 2 TMG berufen“" könne. ${ }^{263}$

${ }^{260} \mathrm{Vgl}$. Hoffmann/Volkmann, in: Spindler/Schuster, Recht der elektronischen Medien, TMG $\S 10$ Rn. 44.

${ }^{261}$ Vgl. auch Paal, in: BeckOK Informations- und Medienrecht, TMG $\S 10$ Rn. 44.

${ }^{262}$ Vgl. BGH, Urt. v. 05.02.2015 - I ZR 240/12, MMR 2015, 674 (Rn. 53) - Kinderhochstühle im Internet III; Urt. v. 16.05.2013 - I ZR 216/11, MMR 2014, 55 (Rn. 37) - Kinderhochstühle im Internet II; Urt. v. 17.08.2011 - I ZR 57/09, MMR 2012, 178 (Rn. 23) Stiftparfüm.

${ }^{263}$ BGH, Urt. v. 17.08.2011 - I ZR 57/09, MMR 2012, 178 (Rn. 23) - Stiftparfüm; vgl. auch die vorgenannten Urteile in $\S 4$ Fn. 262. Wieso bei dieser Aussage kein Verweis auf $\S 10 \mathrm{TMG}$ erfolgt, bleibt allerdings unklar. 
e) Grenze der Haftung: allgemeine Überwachungspflichten ( $\$ 7$ Abs. 2 TMG)

Das soeben erläuterte Verbot, den Diensteanbietern ,allgemeine Überwachungspflichten" aufzuerlegen, setzt §7 Abs. 2 TMG im nationalen Recht um. Auch hinsichtlich §10 TMG wird dieses Verbot gemeinhin als Grenze jedweder Verhaltenspflicht angesehen, die zu einer Kenntnisnahme rechtswidriger Inhalte führen könnte. Nicht zuletzt deshalb wird von manchen Stimmen im Schrifttum auch bei der genauen Bestimmung des Maßstabs der Kenntnis, wie soeben diskutiert, eine providerfreundliche Lesart herangezogen. ${ }^{264}$ Dies verdeutlicht ebenfalls den Zusammenhang zwischen dem Privileg des $§ 10 \mathrm{TMG}$ und dem Verbot allgemeiner Überwachungspflichten. ${ }^{265}$

\section{Darlegungs- und Beweislast}

Hinsichtlich der Darlegungs- und Beweislast sollen die vom BGH getroffenen Aussagen zu $§ 5$ TDG a.F. ${ }^{266}$ weiterhin Geltung beanspruchen. Hiernach trifft die Darlegungs- und Beweislast jedenfalls hinsichtlich der Kenntnis den Nutzer, der einen Diensteanbieter in Anspruch nimmt. ${ }^{267}$ Der Wortlaut des $\S 10$ S. 1 TMG spricht allerdings gerade vom Gegenteil (,,[...] nicht verantwortlich, sofern [...]"). ${ }^{268}$ Dies trägt zum allgemeinen Eindruck bei, demzufolge die Bürde der hinreichend substanziierten Notifizierung von Diensteanbietern nicht nur materiell, sondern auch beweisrechtlich durch den Notifizierenden zu tragen ist. ${ }^{269}$

\section{E. Kritische Annäherung in institutioneller Perspektive}

Jenseits der konkreten materiell-rechtlichen Analyse und Kritik, die den Kapiteln 2 und 3 vorbehalten ist, lassen sich mit Blick auf die Regelungen zur Haftungsprivilegierung der Diensteanbieter problematische Aspekte erkennen, die die Herausforderungen gesetzgeberischer Natur in diesem Regelungsbereich hervorheben. Eine solche institutionelle Perspektive verweist insbesondere auf die Problematik eines koordinierten europäischen Vorgehens. Dieser Perspektive widmet sich der folgende Abschnitt.

${ }^{264} \mathrm{Vgl}$. Hoffmann/Volkmann, in: Spindler/Schuster, Recht der elektronischen Medien, TMG § 10 Rn. 23; Paal, in: BeckOK Informations- und Medienrecht, TMG §10 Rn. 29.

${ }^{265}$ Kritisch dazu noch unten $\S 10$ A. IV. 4. und $\S 11$ D.

${ }^{266} \mathrm{Vgl}$. oben $\S 4$ Fn. 31.

${ }^{267}$ So Hoffmann/Volkmann, in: Spindler/Schuster, Recht der elektronischen Medien, TMG $\S 10$ Rn. 54.

${ }^{268}$ Vgl. Spindler, in: Spindler/Schmitz, TMG, $§ 10$ Rn. 74. So auch die Begründung des Regierungsentwurfs zum EGG, vgl. BT-Drucks. 14/6098, S. 23.

${ }^{269} \mathrm{Vgl}$. dazu noch unten $\S 10 \mathrm{~B}$. IV. 1. 


\section{Probleme eines materiell horizontalen Ansatzes}

Zunächst ist es bemerkenswert, dass die EU mit der ECRL einen horizontalen, also im Prinzip jede Rechtsmaterie umfassenden Ansatz verfolgt, um im Grundsatz jede Rechtsverletzung dem Regime der Art. 12 ff. zu unterwerfen. Theoretisch kann ein solcher horizontaler Ansatz dabei hilfreich sein, eines hohes Maß an Rechtssicherheit in der Praxis der betroffenen Akteure zu vermitteln. ${ }^{270}$ Denn dieser Ansatz erlaubt es den Unternehmen, ihr Handeln umfassend nach den gesetzlichen Anforderungen auszurichten und so die Transaktionskosten für die Ausrichtung an verschiedenen Standards zu vermeiden.

Materiell ist es jedoch nicht nur von Vorteil, vielgestaltige Herausforderungen, die womöglich vielfältige Lebensbereiche betreffen, mit einer Norm zu regeln. Das hat sich nicht zuletzt in den USA gezeigt, wo §230 CDA für viele verschiedene Geschäftsmodelle des Internets zur Anwendung kommt. Dies führt zu einer normativen Überlastung, die dann mitunter auch ungerechte Ergebnisse produziert hat, wie sie oben skizziert wurden. Nicht zuletzt zeigt sich auch bei der Anwendung des $\$ 10 \mathrm{TMG}$, dass es sehr schwierig sein kann, die Sachgerechtigkeit einer hochabstrakten Regelung gerade angesichts einer rasanten Entwicklung der von ihr betroffenen Lebenswelt aufrechtzuerhalten. ${ }^{271}$ Beredtes Zeugnis hierfür liefert schon ein Blick in die Kommentarliteratur, bei der die Etablierung einer Reihe von stark ausdifferenzierten Oberkategorien für die Anwendung der Privilegierung in die Entwicklung von Bereichsdogmatiken zu münden scheint. ${ }^{272}$ Das kann man jedenfalls für kritikwürdig erachten, ${ }^{273}$ weil das Postulat einer als materiell einheitlich konzipierten Regelung so kaum durchgehalten wird. Dies zeigt sich nicht zuletzt an den Schwierigkeiten der Rechtsprechung, ausgehend von einer solchen einheitlichen Regelung eine Vielzahl verschiedener Konstellationen sinnvoll zu erfassen. Dass es überhaupt zu einer solchen Vielgestaltigkeit der Anwendungskonstellationen einer einzelnen Norm gekommen ist, liegt freilich vor allen Dingen an der Vielfalt der mittlerweile entstandenen Geschäftsmodelle der digitalen Welt, die mitunter ganz verschiedener Lösungsansätze bedürfen. So mögen etwa Sorgfaltsanforderungen bei der Prüfung von nutzergenerierten Inhalten in Abhängigkeit des jeweils betrof-

${ }^{270}$ So auch Beckmann, Verantwortlichkeit von Online-Diensteanbietern in Europa und den Vereinigten Staaten von Amerika, S. 241.

${ }^{271}$ Vgl. Beckmann, Verantwortlichkeit von Online-Diensteanbietern in Europa und den Vereinigten Staaten von Amerika, S. 242.

${ }^{272}$ Vgl. insoweit die Vielzahl an „Einzelfällen“ und die Vielzahl von Anwendungsfällen der Störerhaftung in der Kommentierung von Spindler, in: Spindler/Schmitz, TMG, § 10 Rn. 77 ff., $97 \mathrm{ff}$.

${ }^{273}$ Vgl. auch Spindler, MMR 2018, 48 (51). 
fenen Diensteanbieters und des spezifischen Geschäftsmodells anzusetzen sein. Das lässt sich zwar gewiss durch den Gebrauch von Generalklauseln und „weichen“ Kriterien wie etwa der Zumutbarkeit auffangen, stößt jedoch bisweilen auch an Grenzen, die insbesondere durch das Gebot allgemeingültiger Maßstäbe in der Rechtsprechung determiniert sind. Von einer ,vorbildlichen Klarheit" der in der ECRL niedergelegten Tatbestände kann also vor dem Hintergrund jedenfalls angesichts der dargelegten faktischen Entwicklungen und Herausforderungen nicht die Rede sein. ${ }^{274}$

\section{Herausforderungen der unionsrechtlichen Harmonisierung des Haftungsregimes}

Ferner erweist sich der Ansatz einer Vollharmonisierung im Unionsrecht zwar im Grundsatz als uneingeschränkt begrüßenswert. Dieses Ziel bringt jedoch ebenfalls Herausforderungen mit sich.

\section{Im Gesetz angelegte Probleme}

So ist hinsichtlich der Kompetenzgrundlage und der hiermit verbundenen Zielvorgaben Skepsis angebracht, ob die ausschließlich wirtschaftlich geprägte Legitimation der ECRL ${ }^{275}$ zukunftsfähig ist. Zwar ist zweifellos ein grenzüberschreitend einheitlicher rechtlicher Rahmen nicht nur erforderlich, um für die Diensteanbieter eine hinreichend verlässliche Rechtsgrundlage für ihre Tätigkeit aufzufinden, die entsprechende Investitionen erlaubt. ${ }^{276}$ Eine solche verlässliche Grundlage kann angesichts der Transnationalität der Internetkommunikation nur durch die Union gegeben werden. ${ }^{277} \mathrm{Im}$ globalen Ringen um die beste regulative Vorgehensweise hat ein unionaler Ansatz zweifellos auch einen eigenen Wert und eine hiermit verbundene Bedeutung, die eine nationale Regelung in der Regel kaum erreichen könnte. Zugleich ist aber auch festzustellen, dass die ECRL systematisch kaum imstande ist, komplizierte Fragen insbesondere im Bereich der Meinungsfreiheit einer angemessenen Antwort zuzuführen. Denn insbesondere die Meinungsfreiheit

\footnotetext{
${ }^{274}$ So noch Pankoke, Von der Presse- zur Providerhaftung, S. 123.

${ }^{275}$ Aufgeführt sind diese zu Beginn des Richtlinientextes. Danach stützte man sich auf die Art. 47 Abs. 2, 55 und 95 EGV und damit ausschließlich auf Kompetenzen im Bereich der Freizügigkeit und des Binnenmarktes.

${ }^{276}$ Vgl. Erwägungsgrund 40 S. 1 ECRL: „Bestehende und sich entwickelnde Unterschiede in den Rechtsvorschriften und der Rechtsprechung der Mitgliedstaaten hinsichtlich der Verantwortlichkeit von Diensteanbietern, die als Vermittler handeln, behindern das reibungslose Funktionieren des Binnenmarktes, indem sie insbesondere die Entwicklung grenzüberschreitender Dienste erschweren und Wettbewerbsverzerrungen verursachen."
}

${ }^{277} \mathrm{Vgl}$. Wielsch, RW 10 (2019), 84 (89). 
repräsentiert sinnbildlich - trotz aller Harmonisierungstendenzen auch im Bereich des Grundrechtsschutzes - die verschiedenen rechtskulturellen und verfassungsdogmatischen Unterschiede der Mitgliedstaaten. Dies hat der Unionsgesetzgeber beim Erlass der ECRL auch ausweislich des Erwägungsgrundes 9 S. 3 selbst gesehen. ${ }^{278}$ Es ist zwar nicht davon auszugehen, dass die Grundannahme hinter dieser Aussage - nämlich dass entsprechende Konflikte besser bei den Mitgliedstaaten aufgehoben sind - sich in absehbarer Zeit politisch oder rechtlich ${ }^{279}$ grundlegend wandeln wird. Der Unionsgesetzgeber wird vielmehr weiterhin auf einem schmalen Grat wandern müssen, bei dem auf der einen Seite das legitime Interesse an einem einheitlichen Rechtsrahmen für die Tätigkeit der Diensteanbieter zu beachten ist und auf der anderen Seite die unterschiedlichen, kontextsensiblen Priorisierungen der Mitgliedstaaten hinsichtlich ihrer grund- bzw. menschenrechtlichen Garantien ebenso anerkennenswert sind. In Zeiten zunehmend komplexer werdender Mehrheitsfindung auf europäischer Ebene ist dies politisch gewiss kein leichtes Unterfangen.

\section{Die Rolle der Rechtsprechung}

Die oben bereits angesprochene Problematik der Kompetenzabgrenzung wird verschärft durch die institutionelle Dynamik zwischen den verschiedenen Organen der Europäischen Union. So ist das unionsrechtliche Haftungsregime nicht bloß durch eine ausdifferenzierte Rechtsprechung des EuGH geprägt, der in jüngerer Zeit verstärkt seine Rolle als „Grundrechtsgericht“ $\mathrm{zu}$ entdecken und auszubilden scheint. ${ }^{280}$ Auch die Rolle der mitgliedstaatlichen Institutionen im Verhältnis zum EuGH erweist sich im Einzelfall als kompliziert. Denn einerseits ist eine binnenmarktübergreifende harmonisierte Rechtslage und Rechtsanwendung wünschenswert. ${ }^{281}$ Andererseits verweist der EuGH dem Subsidiaritätsgedanken folgend viele Subsumtionsfragen an die mitgliedstaatlichen Gerichte. ${ }^{282}$ Für die Etablierung eines unionsweit einheitlichen Maßstabs ist dies nicht unproblematisch. ${ }^{283}$ Realistisch

${ }^{278}$ Vgl. auch Marly, in: Grabitz/Hilf/Nettesheim, Recht der EU, 40. EL 2009, ECRL Vor. Abschnitt 4 Rn. 73.

${ }^{279}$ Gemäß Art. 4 Abs. 2 EUV achtet die Union die „,nationale Identität“ der Mitgliedstaaten, „die in ihren grundlegenden politischen und verfassungsmäßigen Strukturen [...] zum Ausdruck kommt."

${ }^{280}$ Nicht ohne Zufall wird als Hauptschauplatz dieser Entwicklung vor allen Dingen der Digitalbereich gesehen, wo die EU verstärkt aktiv wird, vgl. etwa Kühling, NVwZ 2014, 681 (684 f.). Kritisch dazu allerdings Franzius, ZaöRV 75 (2015), 383 (398).

${ }^{281} \mathrm{Vgl}$. Erwägungsgrund 7, 50, Art. 1 ECRL.

${ }^{282}$ Vgl. etwa EuGH, Urt. v. 12.11.2011 - C-324/09, MMR 2011, 596 (Rn. 120) - L'Oréal SA, für die Anwendung des Kenntnismaßstabs.

${ }^{283}$ Vgl. dazu auch Stalla-Bourdillon, in: Taddeo/Floridi, The Responsibilities of Online 
betrachtet scheint hier der Trend jedoch weiter in Richtung einer starken Rolle des EuGH zu weisen. Dass dies jedoch nicht zu einer Entwertung der Verfassungsrechtsordnungen der Mitgliedstaaten führen muss, hat jüngst das BVerfG nachdrücklich unter Beweis gestellt. Seine Entscheidung, die Grundrechtsgarantien der GRCh eigenständig zur Entfaltung zu bringen, ${ }^{284}$ kann insoweit durchaus als Signal interpretiert werden, dass eine zunehmende Harmonisierung und Vereinheitlichung des Grundrechtsschutzes auf der Ebene des Unionsrechts nicht zu Lasten der Gerichte der Mitgliedstaaten gehen muss. Das sollte auch als Anreiz zur Fortentwicklung des hier im Fokus stehenden Regelungsbereichs aufgefasst werden.

\section{Rechtspolitische Aktivitäten des Gesetzgebers}

Schließlich lässt sich außerdem ein immer aktiverer Gesetzgeber beobachten. Insoweit geht die Tendenz zwar, wie bereits erwähnt, in Richtung verstärkter Harmonisierung. ${ }^{285}$ Zugleich ist allerdings auch festzustellen, dass der Unionsgesetzgeber in jüngerer Zeit eher rechtsgebietsspezifisch aktiv geworden ist, etwa in Form von Art. 17 der DSM-RL, ${ }^{286}$ der Fortentwicklung der AVMD-RL ${ }^{287}$ sowie den Maßnahmen zur Bekämpfung terroristischer Inhalte ${ }^{288}$ und zur Bekämpfung des sexuellen Missbrauchs und der Ausbeutung von Kindern. ${ }^{289}$ In all diesen Fällen war bzw. ist die Rolle der Diensteanbieter

Service Providers, S. 275 (279 ff.); ferner Kuczerawy, Intermediary Liability and Freedom of Expression in the EU, S. 99.

${ }^{284}$ Vgl. BVerfG, Beschl. v. 06.11.2019 - 1 BvR 276/17, NVwZ 2020, 63 (Rn. 50 ff.) Recht auf Vergessen II.

${ }^{285}$ Riordan, The Liability of Internet Intermediaries, S. 15.

${ }^{286} \mathrm{Vgl}$. dazu noch unten $\S 12 \mathrm{~B}$.

${ }^{287}$ Die Richtlinie (EU) 2018/1808 vom 14.11.2018 hat die AVMD-RL weitreichenden Änderungen insbesondere im Hinblick auf die nunmehrige Erfassung von nutzergenerierten Videos unterzogen und einen hier maßgeblichen Art. 28b eingefügt, der „unbeschadet der Artikel 12 bis 15 [ECRL]" die Mitgliedstaaten verpflichtet, Maßnahmen zu treffen, um Minderjährige sowie die Allgemeinheit vor bestimmten Inhalten zu schützen. Gemäß Art. 28b Abs. 3 UAbs. 2 S. 3 sollen diese Maßnahmen aber mit Art. 15 ECRL ,in Einklang stehen". Kritisch dazu Kuczerawy, Intermediary Liability and Freedom of Expression in the EU, S. 94.

${ }^{288}$ Vgl. dazu den Vorschlag der Kommission für eine Verordnung des Europäischen Parlaments und des Rates zur Verhinderung der Verbreitung terroristischer Online-Inhalte v. 12.09.2018, $\operatorname{COM(2018)~} 640$ final. Insbesondere dessen Art. 6 sieht insoweit ,proaktive Maßnahmen“ vor, die allerdings nach Auffassung der Kommission keine Abweichung vom Grundsatz des Art. 15 ECRL darstellen sollen, wie sich aus Erwägungsgrund 19 des Vorschlags ergibt. Zum Verhältnis des Vorschlags und Art. 14 ECRL siehe Erwägungsgrund 5 des Vorschlags.

${ }^{289}$ Vgl. dazu Richtlinie 2011/93/EU zur Bekämpfung des sexuellen Missbrauchs und der sexuellen Ausbeutung von Kindern sowie der Kinderpornografie, deren Art. 25 die Mitgliedstaaten verpflichtet, die unverzügliche Entfernung von entsprechenden Internetseiten sicherzustellen. 
generell sowie insbesondere das für sie relevante Regelungsregime in der ECRL von erheblicher Bedeutung. Dies zeigt, dass der vom Anspruch her als harmonisierend beabsichtigte Rechtsrahmen der ECRL zur Begründung eines „klaren Regelwerks ${ }^{\text {“290 }}{ }^{29}$ ittlerweile dieser Aufgabe nicht mehr durchweg gerecht wird. Das zentrale Ziel der Richtlinie, nämlich eine grenzüberschreitende rechtssichere Grundlage für Dienstanbieter zu schaffen, ist angesichts der Unterschiede in der gerichtlichen Anwendung einzelner Privilegierungen auf verschiedene Dienste bislang nicht wirklich erreicht. ${ }^{291} \mathrm{Ob}$ weitere Kodifikationen diesem Eindruck werden entgegenwirken können, bleibt mit einer gewissen Skepsis abzuwarten.

\section{Die Herausforderung föderal verteilter Gesetzgebungskompetenzen}

In einem föderal ausgestalteten System wie dem deutschen, das sei hier nur kurz angedeutet, ist zudem fast zwangsläufig ein überkomplexes Geflecht von Zuständigkeiten zu erwarten. ${ }^{292}$ Beim Versuch, die vielgestaltigen regulativen Herausforderungen des Internets insbesondere hinsichtlich der Netzkommunikation kompetenzrechtlich hinreichend aufzufangen, stellt dieser Umstand eine zusätzliche Herausforderung dar. ${ }^{293}$ In Zeiten sich rasant weiterentwickelnder Geschäftszweige und -modelle fördert ein horizontaler Ansatz in einem föderalen System nicht unbedingt die Reaktionsschnelligkeit des Gesetzgebers. ${ }^{294}$ Das muss nicht immer von Nachteil sein, denn die Notwendigkeit der Einbeziehung mehrerer kooperationswilliger Akteure kann unzweifelhaft zu wohlüberlegten und sinnvollen Fortentwicklungen führen. In diesem Kontext überwiegt allerdings der Eindruck, dass die föderale Kompetenzverteilung eher Teil des Problems als der Lösung ist. ${ }^{295}$ Symptomatisch hierfür ist nicht zuletzt die schwierige Abgrenzung bzw. Überlappung von Rundfunk- und Telemedienrecht. Die ursprünglich noch weniger problematische Trennung von Inhalt und Technik, ${ }^{296}$ die das Kompetenzge-

\footnotetext{
${ }^{290} \mathrm{Vgl}$. Erwägungsgrund 50 ECRL.

${ }^{291}$ Vgl. auch Nolte/Wimmers, GRUR 2014, 16 (16).

${ }^{292}$ Vgl. Beckmann, Verantwortlichkeit von Online-Diensteanbietern in Europa und den Vereinigten Staaten von Amerika, S. 241.

${ }^{293}$ Das hat auch, jedoch nicht in erster Linie mit der offenen Frage nach einer neuen grundrechtlichen Kategorie für die neuen Medien zu tun; vgl. zu den grundrechtsdogmatischen Fragen insoweit nur Franzius, JZ 2016, $650 \mathrm{ff}$.

${ }^{294}$ Vgl. etwa die Aufzählung der weitreichenden Abgrenzungsprobleme im Dunstkreis des Rundfunkrechts bei Vesting, in: Binder/Vesting, Rundfunkrecht, RStV § 1 Rn. 13 ff.

${ }^{295}$ Vgl. auch Vesting, in: Binder/Vesting, Rundfunkrecht, RStV § 1 Rn. 41 ff.; ebenso kritisch und mit besonderem Augenmerk auf die medienpolitischen Konsequenzen Eifert, in: Eifert/Gostomzyk, Medienföderalismus, S. $7 \mathrm{ff}$.

${ }^{296}$ Positiviert ist diese Trennung etwa in $§ 1$ Abs. 4 TMG sichtbar.
} 
füge in den ersten Jahrzehnten des GG entscheidend prägte, ${ }^{297}$ gerät mittlerweile immer stärker unter Druck. ${ }^{298}$ Die Debatte um die kompetenzrechtliche Einordnung des NetzDG liefert hierfür ein beredtes Zeugnis ab. ${ }^{299}$ Die 2009 eingeführte Regelung des Art.91c GG, die die Kooperation von Bund und Ländern „,bei der Planung, der Errichtung und dem Betrieb [von] informationstechnischen Systemen " ${ }^{\text {"300 }}$ und damit eine eigentlich unzulässige Mischverwaltung erlaubt, ${ }^{301}$ ist insoweit ebenso ein positives Indiz für die Notwendigkeit, das tradierte Kompetenzgefüge zur Wahrung der Handlungsfähigkeit im digitalen Bereich zu überwinden. Unzweifelhaft dürfte jedenfalls die Einsicht sein, dass das System der Gesetzgebungskompetenzen unter dem GG für den Kontext dieser Untersuchung jedenfalls mittelbar eine erhebliche Bedeutung entfaltet.

${ }^{297}$ Vgl. insbesondere BVerfG, Urt. v. 28.02.1961 - 2 BvG 1/60, BVerfGE 12, 205 (225 ff., 236 ff.) - 1. Rundfunkentscheidung; dazu auch Bleisteiner, Rechtliche Verantwortlichkeit im Internet, S. $78 \mathrm{f}$.

${ }^{298}$ Kritisch insoweit auch Vesting, in: Binder/Vesting, Rundfunkrecht, RStV §1 Rn. $21 \mathrm{f}$.

\footnotetext{
${ }^{299}$ Vgl. dazu Schiff, MMR 2018, 366 (366 f.).

${ }^{300}$ Art. 91c Abs. 1 GG.

${ }^{301}$ Vgl. Heun/Thiele, in: Dreier, GG, Art. 91c Rn. 6, dort auch zur Gegenauffassung.
} 



\section{Gemeinsamkeiten und Unterschiede in übergeordneter Perspektive}

Ein ausführlicher Vergleich der US-amerikanischen und unionsrechtlichen Haftungsprivilegierungen kann hier nicht geleistet werden. Es geht vielmehr darum, allgemeine Entwicklungslinien der US-amerikanischen und europäischen bzw. nationalen Rechtslage aufzuzeigen. Dies kann einen Beitrag zur besseren kritischen Reflexion des status quo leisten und damit Reformbestrebungen den Weg weisen.

\section{A. Die Grundsatzentscheidungen für eine Haftungsprivilegierung}

Im Ansatz treffen die beiden hier dargestellten Rechtsordnungen ähnliche Grundentscheidungen. Im Gesetzestext selbst ähneln sich §230 CDA und die europäischen Regeln insoweit, als sie für eine Haftung die Kenntnis des jeweils betroffenen Inhalts voraussetzen. Die Haftungsfreistellung wurde in den USA allerdings durch die Rechtsprechung bedeutend erweitert, wie die oben besprochene Entscheidung Zeran aufzeigt, ${ }^{1}$ die in ihrer Konsequenz letztlich auf die Irrelevanz der Kenntnis hinausläuft und die Diensteanbieter so weitgehend von der Haftung ausnimmt. Die noch größere Parallele ist zwischen $\$ 512$ DMCA und Art. 14 ECRL auszumachen. Obgleich in der EU allenfalls implizit ein Notice-and-Takedown-Verfahren geregelt ist, ${ }^{2}$ orientiert sich diese Bestimmung ebenso wie ihr Vorgänger $-\S 5$ TDG a.F. - an der US-amerikanischen Normierung zum Schutz des Urheberrechts, insbesondere indem sie mit der Notifizierung Rechteinhabern ein Instrument an die Hand gibt, um einen Handlungsdruck bei den Diensteanbietern zum Schutz ihrer Rechtspositionen zu erzeugen.

Systematisch handelt es sich bei den Grundentscheidungen für eine Haftungsprivilegierung um recht einzigartige Regelungsansätze insoweit, als

\footnotetext{
${ }^{1}$ Vgl. oben $\S 3$ A. II. 1.

${ }^{2}$ Art. 14 ECRL selbst setzt nicht die Notifizierung voraus, sondern stellt nur auf die Kenntnisnahme ab, die auch Ergebnis einer Mitteilung an den Diensteanbieter sein kann, vgl. Holznagel, GRUR Int 2007, 971 (972); ders., GRUR Int 2014, 105 (109).
} 
man jenseits des Internets kaum auf gesetzlich normierte Verantwortlichkeitsbegrenzungen stößt. ${ }^{3}$ Historisch gesehen ist die Verschiebung der Perspektive auf die Haftungsfrage im Vergleich zum Umgang mit anderen, sich in der Entwicklung befindlichen Technologien wie etwa der Einsatz von Kraftfahrtzeugen als gewöhnliches Fortbewegungsmittel durchaus bemerkenswert. ${ }^{4}$ Zwar lässt sich dieser Umstand einerseits mit der beim Betrieb von Kraftfahrzeugen deutlich größeren Gefahr für Leib und Leben erklären. ${ }^{5}$ Andererseits geht es aber hier wie dort um den Umgang mit den kausal durch menschlichen Einsatz von Technologien eintretenden Risiken, ${ }^{6}$ der im Fall der Kraftfahrzeuge freilich unmittelbarer wirkt als bei der Nutzung von Angeboten der Diensteanbieter. In der historischen Perspektive fällt es jedenfalls auf, dass angesichts der sonstigen Erfahrungen bzw. Beobachtungen in der rechtlichen Begleitung technischer Entwicklung grundlegend kritische Stimmen gegenüber einer Haftungsprivilegierung für Diensteanbieter kaum Gehör fanden bzw. schnell verstummten.

$\mathrm{Zu}$ bedenken ist hier schließlich noch, dass es in der EU womöglich nicht einmal einer Haftungsfreistellung bedurft hätte, weil die betreffenden Diensteanbieter auch ohne eine solche nicht ohne Weiteres für jede von ihnen vermittelte Rechtsverletzung haftbar gemacht werden können. So hat der EuGH im Ansatz ähnliche Hürden für die Haftung des Betreibers eines echten, physischen Marktplatzes für Markenverletzungen aufgestellt. Unter explizitem Verweis auf die bereits erwähnte Entscheidung L'Oréal sei auch diesem Marktplatzbetreiber eine „generelle und ständige Überwachung“ grundsätzlich nicht zumutbar. ${ }^{8}$ Zulässig seien dagegen Verpflichtungen zu „Maßnahmen [...], die dazu beitragen zu vermeiden, dass erneute derartige Verletzungen durch denselben Händler auftreten". ${ }^{9}$ Ob daraus abgeleitet werden kann, dass konkrete Maßnahmen zur Unterbindung von Rechtsverletzungen erst bei - einer Art. 14 ECRL vergleichbaren - Kenntnis der entsprechenden Handlungen verlangt werden dürfen ${ }^{10}$ geht aus der EntscheiS. 113 .

${ }^{3}$ Vgl. Frey, Die Haftung von Host-Providern für Immaterialgüterrechtsverletzungen,

${ }^{4}$ Vgl. Freytag, Haftung im Netz, S. 17, der auf die $§ 7$ StVG, $§ 1$ HPflG, $\$ 33$ LuftVG, $\S 25$ AtomG, verweist, bei denen der Gesetzgeber jeweils mit einer Gefährdungshaftung reagierte, während die Reaktion hier in einer Haftungsbegrenzung liegt. Kritisch insoweit auch Lehmann, ZUM 1999, 180 (183); Matthies, Providerhaftung für Online-Inhalte, S. 101.

${ }^{5}$ Zur historischen Perspektive auf $§ 7$ StVG vgl. nur Schirmer, RW 9 (2018), 453 (457 ff.).

${ }^{6}$ Für die Halterhaftung dezidiert Schirmer, RW 9 (2018), 453 (463).

${ }^{7}$ So auch Matthies, Providerhaftung für Online-Inhalte, S. 101; vgl. aber Bleisteiner, Rechtliche Verantwortlichkeit im Internet, S. $211 \mathrm{f}$.

${ }^{8}$ EuGH, Urt. v. 07.07.2016 - C-494/15, MMR 2016, 676 (Rn. 34) - Tommy Hilfiger.

${ }^{9}$ EuGH, Urt. v. 07.07.2016 - C-494/15, MMR 2016, 676 (Rn. 34) - Tommy Hilfiger.

${ }^{10}$ So Hofmann, GRUR 2016, 1064 (1065). 
dung des EuGH selbst nicht hervor. ${ }^{11}$ Insgesamt zeigt sich hier in praktischer Perspektive, dass das Verhältnismäßigkeitsprinzip auch jenseits der normierten Haftungsprivilegien Einfluss auf das Haftungsrecht nimmt. In den USA war hingegen das Haftungsrisiko ohne eine Immunisierung durchaus real, wie ja nicht zuletzt die angesprochene Entscheidung Stratton Oakmont bewiesen hatte, ${ }^{12}$ sodass ein materiell überzeugendes Bedürfnis für eine Privilegierung dort unzweifelhaft bestand.

\section{B. Innovationssicherung als Leitidee}

Allen hier verglichenen Regelungsbereichen war und ist immer noch als Leitmotiv die Absicherung technischer Innovation immanent. Mit der Auslegung der Haftungsprivilegierungen befasste Gerichte fragen immer wieder, inwiefern die Inanspruchnahme eines Diensteanbieters diesen wirtschaftlich gefährden könnte. ${ }^{13}$ Die von den Befürwortern einer weitreichenden Immunisierung stets vorgetragene innovationsfördernde Wirkung von Regelungen wie derjenigen des $\$ 230 \mathrm{CDA}$ verliert mit der Zeit jedoch zunehmend ihre argumentative Überzeugungskraft. Denn während in den 1990er-Jahren ein allzu rigides Zurechnungsregime womöglich in der Lage gewesen wäre, Innovation und Diskurs abzuwürgen, lässt sich der Wert einer solchen „Subventionierung für die Internet-Branche ${ }^{\text {"14 }}$ nach heutigen Maßstäben zumindest bezweifeln. Mit der zunehmenden Ingebrauchnahme des Internets durch große Teile der Gesellschaft konnte sich nämlich einerseits dessen Risikopotenzial für die öffentliche Kommunikation in erheblicher Weise manifestieren, ${ }^{15}$ andererseits generiert die Branche mittlerweile einen Umsatz in Höhen, die nicht die Annahme rechtfertigen, dass Klagen von Individuen ein finanziell bedeutendes Risiko darstellen (werden). ${ }^{16}$

${ }^{11} \mathrm{Zu}$ weit ginge es allerdings, daraus abzuleiten, dass es bei einer Reform des Haftungsrechts nicht zu einer Verschärfung des Haftungsmaßstabs kommen dürfe, wie Hofmann, JZ 2018, 746 (749), aus der vorgenannten EuGH-Entscheidung schlussfolgert.

${ }^{12} \mathrm{Vgl}$. oben $\S 3$ A. I.

${ }^{13}$ Vgl. BGH, Urt. v. 26.11.2015 - I ZR 174/14, NJW 2016, 794 (Rn. 27) - Access-Provider.

${ }^{14}$ So Matthies, Providerhaftung für Online-Inhalte, S. 102.

${ }^{15}$ Vgl. dazu nur Citron, Hate Crimes in Cyberspace, S. 35 ff. und passim; Fawzi, CyberMobbing, S. 45 ff. und passim; Lembke, Kollektive Rechtsmobilisierung gegen Digitale Gewalt, S. 3 f.

${ }^{16}$ Vgl. Citron/Wittes, Fordham Law Review 86 (2017), 401 (411 ff.); vgl. ferner Fair Housing Council v. Roommates.com, 521 F.3d 1157, 1164 (9th Cir. 2008) bei Fn. 15: „The Internet is no longer a fragile new means of communication that could easily be smothered in the cradle by overzealous enforcement of laws and regulations applicable to brick-andmortar businesses. [...] [Its] vast reach into the lives of millions is exactly why we must be 
Für die mittlerweile zu einer lebhaften und umsatzstarken entwickelten Technologiebranche ist eine haftungsrechtliche Absicherung jedenfalls in einem weitreichenden Ausmaß nicht mehr erforderlich. ${ }^{17}$ Soweit eine andere Sichtweise geltend gemacht wird, weil das Augenmerk etwa Start-ups gilt, für die ein Haftungsrisiko teilweise noch als Marktzugangsschranke angesehen wird, lässt sich dem zweierlei entgegnen. Zum einen besteht jenseits der im GG bzw. in der GRCh grundrechtlich verbürgten Unternehmensfreiheiten kein rechtlich geschütztes Interesse an einer unternehmerischen Tätigkeit ohne jedes Haftungsrisiko. ${ }^{18}$ Es gehört letztlich in jedem vom Einsatz von Technologie geprägten Lebensbereich zum regulären Zyklus des Fortschritts, dass dieser mit steigenden Sorgfaltspflichten einhergeht, die kleinere Marktteilnehmer mitunter aus dem Markt drängt. ${ }^{19}$ Zum anderen kann der Schutz kleinerer Unternehmen insoweit jedenfalls auch als Differenzierungsmerkmal für unterschiedlich ausgestaltete Sorgfaltspflichten dienen, wie es etwa bei der noch zu erörternden Regelung des Art. 17 DSM-RL der Fall ist. ${ }^{20}$ Ein pauschaler Verweis auf den Schutz technischer Innovation zur Rechtfertigung von Haftungsprivilegierungen als solche kann demgegenüber nicht überzeugen.

careful not to exceed the scope of the immunity provided by Congress and thus give online businesses an unfair advantage over their real-world counterparts, which must comply with laws of general applicability."

${ }^{17}$ Vgl. Lembke, Kollektive Rechtsmobilisierung gegen Digitale Gewalt, S. 19; Eifert, in: Eifert/Gostomzyk, Netzwerkrecht, S. 9 (13); ders., in: Eifert/Hoffmann-Riem, Innovation, Recht und öffentliche Kommunikation, S. 255 (256 ff.); zweifelhaft dagegen Hofmann, JZ 2018, 746 (753): intuitive Richtigkeit der „Verfügbarkeit des Internets durch weitreichende Haftungsfreistellungen“.

${ }^{18}$ Überzeugend Holznagel, ZUM 2018, 350 (353): „Es besteht [...] kein schützenswertes Interesse an einem besonders kostengünstigen Betrieb entsprechender Plattformen.“

${ }^{19}$ Vgl. Spindler, in: Eifert/Hoffmann-Riem, Innovation, Recht und öffentliche Kommunikation, S. 67 (91). In manchen Bereichen ist es sogar gerade erwünscht, dass nur bestimmte wenige Akteure in der Lage sind, trotz bestehender Haftungsrisiken einen Marktzutritt zu wagen, etwa wenn es um den Betrieb von Atomkraftwerken geht. Die Risiken von Diensteanbietern in diesem Kontext sind damit natürlich keineswegs vergleichbar. Der Vergleich dient hier lediglich zur Verdeutlichung, dass der Verweis auf den Schutz von und die Innovationsfähigkeit der Start-ups keineswegs ein überall und in jedem Kontext durchschlagendes Argument gegen Sorgfaltsstandards darstellen kann.

${ }^{20} \mathrm{Vgl}$. dazu unten $\S 12 \mathrm{~B}$. 


\section{Vergleich der Anreizwirkungen}

Während also die Sicherung der Innovationskraft der aufkommenden Internetbranchen eine wesentliche Gemeinsamkeit zwischen den Ansätzen der USA und der EU darstellt, erweisen sich die hierbei verfolgten Konzepte bzw. die erwünschten Anreizeffekte zwischen den USA und Europa hingegen durchaus als unterschiedlich. So war es die klare Intention des Gesetzgebers in den USA, auch im Rahmen des §230 CDA den Unternehmen hinreichend Spielraum zu belassen, um eigenständig und freiwillig Überwachungsinstrumente zu entwickeln. Insoweit war es insbesondere die Absicht, durch die weitreichende Privilegierung die Diensteanbieter zur Wahrnehmung ihrer Rolle als „gute Samariter“ zu animieren, ${ }^{21}$ denen der Staat nicht über die Schulter zu schauen, also nicht zu bestimmen habe, in welcher Art und Weise sie ihren Dienst „moderieren“ “. ${ }^{22}$ Das kann nicht in gleicher Weise für die EU behauptet werden. Hier legt zum einen die herausgehobene Stellung der Kenntnisnahme als entscheidende Voraussetzung einer Inpflichtnahme eine Passivität der Diensteanbieter nahe. ${ }^{23}$ Zum anderen und unmittelbar damit in Zusammenhang beherrscht das Ideal der Tätigkeit „,rein technischer, automatischer und passiver Art ${ }^{\text {‘22 }}$ die Rechtsprechung des EuGH und - wenngleich in mittelbarer Weise - auch diejenige des BGH. Diese normative Erwartung setzt einen Anreiz gerade in die entgegengesetzte Richtung. Gerade die Untätigkeit bietet sicheren Schutz vor Haftung. Sofern allerdings eine Kenntnis anzunehmen ist, beziehen die Haftungsregeln durchaus Stellung zum sachlichen Maßstab des Umgangs mit Inhalten: sind diese rechtswidrig, müssen sie entfernt werden, um eine Haftung zu vermeiden.

$\mathrm{Zu}$ diesen beiden Polen liegt dagegen das Notice-and-Takedown-Verfahren, wie es in $\S 512$ DMCA geregelt ist, teilweise quer. Einerseits ist hier die Rolle der Diensteanbieter durchaus aktiver als bei $\$ 230$ CDA. Denn die positive Kenntnis kann zu einer Haftung führen, wie §512(1)(A)(i) DMCA ausdrücklich anordnet. Andererseits besteht allerdings auch hier ein Anreiz dahingehend, sich mit den Notifizierungen im Rahmen des Notice-andTakedown-Verfahrens nicht näher auseinanderzusetzen. Denn das Gesetz will die Diensteanbieter ausdrücklich von der materiellen Prüfung der Nutzeranliegen ausnehmen. Die Diensteanbieter können sich also auf eine „me-

\footnotetext{
${ }^{21}$ Freilich wurde bereits auch aufgezeigt, dass die Anreizwirkung des $§ 230$ CDA in der Rechtspraxis derzeit kaum zur Entfaltung kommt, da die Diensteanbieter unabhängig entsprechender freiwilliger Bemühungen in den Genuss des Privilegs kommen, vgl. oben $\S 3$ A. II. 1.

${ }^{22}$ Vgl. Grimmelmann, Yale Journal of Law \& Technology 17 (2015), 42 (104).

${ }^{23}$ Vgl. Spindler, in: Eifert/Hoffmann-Riem, Innovation, Recht und öffentliche Kommunikation, S. 67 (79f., 91 f.).

${ }^{24}$ Erwägungsgrund 42 ECRL.
} 
chanische" Position zurückziehen, indem sie nach einer formal korrekten Notifizierung einen Inhalt entfernen und diesen aufgrund der „,counternotice" ggf. wieder verfügbar machen. Die hier etablierte Anreizstruktur lässt sich demnach nicht vollständig dem Paradigma der Neutralität zuordnen, wie es in der ECRL der Fall ist. Dennoch verlangt auch die ECRL keine tiefere materielle Befassung des Diensteanbieters mit dem durch ihn erst ermöglichten juristischen Konflikt. Demnach stellen die Regelungen in der ECRL teilweise eine Emanzipation von ihrem US-amerikanischen Vorbild dar.

\section{Fazit}

Die Analyse bestehender Rechtsinstitute, die Diensteanbieter - jedenfalls in ihrer Handhabung in der Rechtspraxis - weitestgehend von jedweder Haftung für Inhalte Dritter befreit, hat aufgezeigt, dass der konzeptionelle Ursprung des Haftungsprivilegs in den US-amerikanischen Regelungen des $\S 230$ CDA und des $\S 512$ DMCA zu erblicken ist. Erstere hatte jedoch nicht von vornherein den Zweck, Diensteanbieter generell von einer Haftung zu befreien, sondern diese vielmehr dazu zu veranlassen, ihre Verantwortung für den Schutz von Persönlichkeitsrechten und anderen Schutzrechten mit Bedeutung für die Rede- bzw. Meinungsfreiheit wahrzunehmen. Die insoweit zum Ausdruck kommende, marktbezogene Erwartung, die Entwicklung verschiedener Umgangsweisen werde sich auch im Wettbewerb der Diensteanbieter niederschlagen, sodass gleichsam die „beste“ Unternehmenspraxis beim Umgang mit rechtswidrigen Inhalten sich durchsetzen werde, scheint sich allerdings bislang jedenfalls nicht zu bestätigen. Vielmehr haben klassische „Lock-in“-Effekte ${ }^{25}$ dazu geführt, dass sich ein Großteil der Internetnutzerinnen auf wenige große Dienste beschränkt. ${ }^{26}$

${ }^{25} \mathrm{Vgl}$. hierzu und zu anderen wettbewerbshindernden Effekten der Plattformökonomie Schneider, in: Saetnan/Schneider/Green, The Politics of Big Data, S. 129 (130 ff.). Vgl. zu deren wettbewerbsrechtlicher Bewältigung auch §18 Abs. 2a, Abs. 3a GWB; hierzu nur Podszun/Schwalbe, NZKart 2017, $98 \mathrm{ff}$. Auch das unionsrechtliche Datenschutzrecht greift mit Art. 20 DS-GVO diese Problematik mittlerweile auf und stellt damit sektorspezifisches Wettbewerbsrecht dar; unzutreffend insoweit Jülicher/Röttgen/Schönfeld, ZD 2016, 358 (360 f.), die das Recht auf Datenportabilität auch dem informationellen Selbstbestimmungsrecht zuordnen wollen, ohne hierbei die Sprengung dessen konzeptioneller Grenzen als Kontrollmittel gegen spezifische Datenverarbeitungen hinreichend zu begründen.

${ }^{26}$ Die Ökonomie hat diese Netzwerkeffekte, die sich insbesondere auch auf Kommunikationsdienste anwenden lassen, schon vor längerer Zeit in den Blick genommen, vgl. etwa Katz/Shapiro, Journal of Economic Perspectives 8 (1994), 93 (95 ff., 100 f.); vgl. ergänzend Kellner, Die Regulierung der Meinungsmacht von Internetintermediären, S. 57, mwN. 
Aus einer übergeordneten Perspektive lassen sich in der jüngeren Vergangenheit unterschiedliche Trends ausmachen. ${ }^{27}$ Einerseits sind in Europa Bestrebungen zur Privatisierung bzw. Deregulierung im Bereich der Kommunikation sichtbar geworden, wie sie etwa im Bereich des Telekommunikationsrechts zum Ausdruck kommen. Oft stand bzw. steht die (ggf. regulierte) Selbstregulierung ${ }^{28}$ der entsprechenden Akteure im Vordergrund. ${ }^{29}$ Entsprechende Forderungen nach einer solchen Selbstregulierung stehen sinnbildlich für die übergeordnete Position, die eine möglichst beeinträchtigungsfreie Entscheidungsfähigkeit der Diensteanbieter fordert. Zugleich lassen sich aber andererseits auch Gegenbewegungen ausmachen, die insbesondere die Abwehr von Gefahren im Bereich der Kommunikationsregulierung und der Einhegung des öffentlichen Diskurses im Netz zum Zielwert haben. Insoweit werden entsprechend Schutzvorkehrungen als rechtliche Handlungskategorie in den Mittelpunkt gestellt. ${ }^{30}$ Diese Entwicklung spiegeln auch die hier im Fokus stehenden Regelungskomplexe in den USA und in Europa wider, die zwischen diesen beiden Polen oszillieren.

In welche Richtung die Entwicklung ihren Lauf nehmen wird, lässt sich noch nicht abschließend beurteilen. Allerdings ist es durchaus bemerkenswert, dass gerade in jüngerer Zeit in den ansonsten in der Tendenz eher regulierungskritisch eingestellten USA $^{31}$ Stimmen an Auftrieb zu gewinnen scheinen, die sich für eine stärkere gesetzliche Verantwortungszuweisung an die Diensteanbieter einsetzen. Das Beispiel der DS-GVO ${ }^{32}$ mag hier ggf. als Blaupause dienen, inwiefern rechtspolitische Errungenschaften der EU auch in den USA zu einem teilweisen Umdenken führen können. Ob diese Entwicklung eines Tages auch die hier im Fokus stehende Regelung des $§ 230$ CDA erfassen wird, die jüngst Gegenstand hitziger, gleichwohl nicht immer bestens informierter Debatten im politischen Kontext war, ${ }^{33}$ ist zum gegenwärtigen Zeitpunkt noch nicht abzusehen. Insoweit sollte man allerdings den verfassungsrechtlichen Kontext nicht außer Acht lassen. Dieser gewährt in der EU und in Deutschland dem Gesetzgeber und der Rechtsprechung auch

${ }^{27}$ Vgl. allgemein zu den Herausforderungen der Medienregulierung Eifert/HoffmannRiem, in: Schulte/Schröder, Handbuch des Technikrechts, S. 667 (679 ff.).

${ }^{28}$ Vgl. zur Selbstregulierung im digitalen Kontext Hoffmann-Riem, in: Fehling/ Schliesky, Neue Macht- und Verantwortungsstrukturen in der digitalen Welt, S. 27 (36 ff.).

${ }^{29} \mathrm{Vgl}$. Hoffmann-Riem, in: Eifert/Hoffmann-Riem, Innovation, Recht und öffentliche Kommunikation, S. 9 (20).

${ }^{30} \mathrm{Vgl}$. wiederum Hoffmann-Riem, in: Eifert/Hoffmann-Riem, Innovation, Recht und öffentliche Kommunikation, S. 9 (20 ff.).

${ }^{31}$ Vgl. nur den Nachweis oben in $\$ 3$ Fn. 79.

${ }^{32}$ Vgl. unten den $\S 13$ Fn. 10 zugehörigen Text.

${ }^{33} \mathrm{Vgl}$. zu den jüngsten politischen Diskussionen etwa Laslo, Wired (13.08.2019), www. wired.com/story/fight-over-section-230-internet-as-we-know-it. 
im Umfeld der Kommunikationsfreiheiten einen deutlich größeren Spielraum als in den USA. Daran wird sich gewiss so schnell auch nichts ändern. ${ }^{34}$

${ }^{34}$ Erst kürzlich beschied der Supreme Court in einem Rechtsstreit einen Antrag auf writ of certiorari abschlägig, der $\$ 230$ CDA ggf. hätte zu Fall bringen können, vgl. Herrick v. Grindr, 140 S.Ct. 221 (2019). Zum Hintergrund des Verfahrens vgl. Goldberg, Lawfare (14.08.2019), www.lawfareblog.com/herrick-v-grindr-why-section-230-communicationsdecency-act-must-be-fixed. 


\section{Phänomenologische Annäherungen an die Intermediäre und die Problematik ihrer Konzeption im geltenden Recht}

Eine reflektierte Analyse auf bestimmte Akteure bezogener rechtlicher Regeln setzt zunächst einen phänomenologisch klaren Blick auf diese Akteure voraus. Denn ohne eine Klärung funktionaler und begrifflicher Zusammenhänge ist insbesondere in einem stark technisch geprägten Lebensbereich eine juristische reflektierte Analyse nur schwerlich realisierbar. Zugleich liegen dem Regelungsgegenstand dieser Untersuchung auch gewisse grundlegende Wertungen zugrunde, die mit einem Blick auf das einschlägige juristisches Fallmaterial allein noch nicht vollständig erfasst werden können. In diesem Sinne liegen diesem Kapitel mehrere Anliegen zugrunde. Zunächst ist ein Blick auf die Funktionsweise und die technische Verortung der Diensteanbieter im Zusammenhang netzbasierter Kommunikation zu werfen ( $§ 6)$. Sodann ist zu erörtern, welchen epistemischen Wert die verschiedenen Ansätze zur begrifflichen Beschreibung des Agierens der hier im Fokus stehenden Akteure haben und welche Auswirkungen solche begrifflichen Zuschreibungen auf das normative Verständnis zeitigen (§7) können. Der Grundansatz dieser Fragen ist damit ein deskriptiver, da von dem faktischen Beobachtungsgegenstand ausgegangen wird. Zugleich legen aber sowohl die begrifflichen wie auch die funktionalen Zusammenhänge Perspektiven auf den Beobachtungsgegenstand frei, die nicht mehr rein deskriptiver Natur sind. Bereits der Beschreibung von technischen Funktionszusammenhängen ist stets eine normative Komponente immanent, weil und soweit diese Grundlage normativer Ableitungen sind. Dies zeigt sich beim Gegenstand dieser Untersuchung in besonderer Weise. Auch mit der Wahl der begrifflichen $\mathrm{Zu}$ schreibung ist eine Haltung oder eine Herangehensweise verbunden, die nicht selten normative Konsequenzen zeitigt.

Die normative Natur des zunächst nur technisch beschriebenen Modells der Diensteanbieter wird sodann bei im dritten Abschnitt dieses Kapitels deutlich. Denn es sind gerade jene Ungenauigkeiten bzw. teilweise ein veraltetes technisches Vorstellungsbild, die die europäische und nationale Perspektive auf das Recht ihrer Haftung immer noch prägen und somit in struktureller Hinsicht ein defizitäres Haftungsregime erkennbar werden lassen. Diese Defizite liegen systematisch klar trennbar den rechtlichen Einzelheiten 
des juristischen Fallmaterials voraus und verdienen es daher, gesondert herausgestellt zu werden ( $(8)$, bevor im darauffolgenden Kapitel das Augenmerk der dogmatischen Analyse zuzuwenden ist. 


\section{Funktionale Zuordnung der Intermediäre}

Eine phänomenologische Annäherung an die Diensteanbieter setzt eine Auseinandersetzung mit den funktionalen und technischen Zusammenhängen ihrer Aktivität voraus. Dies ist Ziel und Gegenstand dieses Abschnitts. Zunächst ist eine technische Verortung im Modell netzbasierter Kommunikation vorzunehmen (A.), wobei der Begriff der Kommunikation insofern weit zu verstehen ist, als er sich im informationstechnischen Sinne auf jeden „Austausch von Informationen nach bestimmten Regeln" bezieht. ${ }^{1}$ Das Internet ist in diesem Sinne ebenfalls nur eine Sammlung von Einzelnetzen, „, die weltweit über viele Kommunikationsverbindungen miteinander verbunden sind." ${ }^{2}$ Sodann erfolgt eine Darstellung der wesentlichen Funktionen der Diensteanbieter, soweit sie für den Zweck dieser Untersuchung von Bedeutung sind (B.).

\section{A. Schichtenbasierte Modelle des Internets}

Zunächst ist eine Zuordnung der Diensteanbieter im informationstechnischen Design von Kommunikationsnetzen vorzunehmen. Diese erfolgt hier primär anhand des „Open Systems Interconnection“-Modells (OSI), das ein „Referenzmodell für herstellerunabhängige Kommunikationssysteme bzw. eine Design-Grundlage für Kommunikationsprotokolle und Computernetze" darstellt. ${ }^{3}$ Das Modell ist von der International Organization for Standardization, besser bekannt als ISO, und der International Telecommunication Union als Standard deklariert und veröffentlicht. ${ }^{4}$ Es beinhaltet insgesamt sieben Schichten. Jede dieser Schichten ist für die Kommunikation zwischen zwei Systemen von Bedeutung, denn zwischen diesen Systemen

\footnotetext{
${ }^{1}$ Mandl, Internet Internals, S. 1.

${ }^{2}$ Mandl, Internet Internals, S. 31.

${ }^{3}$ Schmidt/Pruß, in: Auer-Reinsdorff/Conrad, Handbuch IT- und Datenschutzrecht, $\S 3$ Rn. 62. Die Verwendung dieses Modells erfolgt hier und auch andernorts nur zur phänomenologischen Beschreibung, in der technischen Realität beruhen nur wenige Protokolle auf diesem Modell, vgl. Mandl, Internet Internals, S. 3.

${ }^{4}$ ISO: ISO/IEC 749:1994, vgl. handle.itu.int/11.1002/1000/2820; ITU: ITU-T Recommendation X.200, 07/94, vgl. www.itu.int/ITU-T/recommendations/rec.aspx?rec=2820.
} 
muss eine Information alle Schichten durchlaufen. ${ }^{5}$ Diese sollen hier grob anhand der sie prägenden Akteure dargestellt werden, um ein hinreichendes Differenzierungspotenzial zu ermöglichen, wenn es um die Bewertung verschiedener Konstellationen des Rechts der Diensteanbieter, insbesondere im Falle der von Dritten begangenen Rechtsverletzung geht. ${ }^{6}$

\section{Darstellung}

Die Schichten 1-3 des OSI-Modells beinhalten die physikalische bzw. Bitübertragungs- ${ }^{7}$, Sicherungs- und die Netzwerkschicht. In der physikalischen Schicht geht es um die grundlegenden Funktionen, die für die internetbasierte Kommunikation essenziell sind. Sie sind in der Regel durch ein physisches Medium miteinander verbunden. ${ }^{8}$ Die Sicherungsschicht erzeugt logische Verbindungen der Datenpakete und damit für eine funktionierende Verbindung zwischen Endgerät und Übertragungsmedium. ${ }^{9}$ In der Netzwerkschicht wird das sog. Routing, also die Wahl der Datenwege durch das Netz organisiert. ${ }^{10}$ Diese Schichten dienen damit insgesamt nur der Signalübertragung bzw. dem Telekommunikationsvorgang an sich. ${ }^{11}$ Damit haben diese Bereiche in aller Regel nichts mit der Übertragung von kognitiv fassbaren Inhalten zu tun, wie sie etwa in $\S 10 \mathrm{TMG}$ gemeint sind. Ihnen kann deshalb auch kaum eine inhaltliche Kontrolle über durch sie übertragene Inhalte zugeschrieben werden. In der Transportschicht (Schicht 4) wird die Zustellung einzelner Datenpakete wahrgenommen bzw. einzelnen Anwendungen zugeordnet, ${ }^{12}$ sodass eine eindeutige Zuordnung zu den signalübertragenden Schichten nicht mehr möglich ist. ${ }^{13}$ In den anwendungsbezogenen Schichten 5-7 des OSI-Modells sind die Kommunikationssteuerungs-, Dar-

\footnotetext{
${ }^{5}$ Vgl. Schmidt/Pruß, in: Auer-Reinsdorff/Conrad, Handbuch IT- und Datenschutzrecht, $\S 3$ Rn. 63.

${ }^{6}$ Vgl. auch Riordan, The Liability of Internet Intermediaries, S. $36 \mathrm{f}$.

${ }^{7}$ Schütz, in: Geppert/Schütz, TKG, §6 Rn. 36.

${ }^{8}$ Vgl. International Telecommunication Union, Open Systems Interconnection Basic Reference Model, S. 49.

${ }^{9}$ Vgl. Schmidt/Pruß, in: Auer-Reinsdorff/Conrad, Handbuch IT- und Datenschutzrecht, $\$ 3$ Rn. 64.

${ }^{10}$ Vgl. Schütz, in: Geppert/Schütz, TKG, §6 Rn. 36.

${ }^{11}$ Vgl. Schütz, in: Geppert/Schütz, TKG, §6 Rn. 37.

${ }^{12}$ Vgl. Schmidt/Pruß, in: Auer-Reinsdorff/Conrad, Handbuch IT- und Datenschutzrecht, §3 Rn. 64.

${ }^{13}$ Damit ergeben sich hier bereits erste Konfliktpotenziale im Hinblick auf die im Fokus dieser Untersuchung stehenden Art. $12 \mathrm{ff}$. ECRL, $\S \S 7 \mathrm{ff}$. TMG. In aller Regel wird für Fälle der Transportschicht Art. 12 ECRL bzw. § 8 TMG einschlägig sein. Vgl. zu diesem Themenkomplex statt vieler Müller, MMR 2019, $426 \mathrm{ff}$.
} 
stellungs- und die Anwendungsschichten enthalten. ${ }^{14}$ Insbesondere die Anwendungsschicht selbst ist diejenige, mit der alle Nutzer des Internets interagieren. ${ }^{15}$ Hier sind die sog. Anwendungsprotokolle wie FTP, HTTP oder SMPT, die die Oberfläche für die Eingabe, den Transport und Abruf nutzergenerierter Inhalte darstellen. ${ }^{16}$ Auf der Anwendungsschicht spielen sich auch die rechtlich relevanten Konflikte um den Zugriff der Diensteanbieter auf die Art und Weise der Datenübertragung und damit auch auf ihre inhaltliche Kontrolle ab. ${ }^{17}$

Eine ähnliche Einteilung in Schichten nimmt das sog. TCP/IP-Modell vor. Hier sind es vier Schichten: Netzwerkzugangsschicht, Vermittlungsschicht, Transportschicht und wiederum die Anwendungsschicht. ${ }^{18}$ Für den Zweck dieser Untersuchung unterscheiden sich diese Modelle allerdings nicht wesentlich, denn auch hier sind die Intermediäre der obersten Schicht, nämlich der Anwendungsschicht zuzuordnen.

\section{Bedeutung für die Thematik dieser Untersuchung}

Der Wert der mit dem Schichtenmodell verbundenen Perspektive auf die Infrastruktur des Internets ergibt sich aus der hier hervorgehenden, klaren phänomenologischen Zuordnung verschiedener Funktionen und Dienste entweder auf die technische oder die inhaltliche bzw. anwendungsbezogene Ebene. Während die technischen Schichten dieses Modells grundsätzlich wenig oder kaum mit den transportierten Inhalten zu tun haben sollen und deshalb insbesondere auch zur sog. „Netzneutralität“ verpflichtet sein können, ${ }^{19}$ spielt die äußere Anwendungs- bzw. Inhaltsschicht insoweit eine deutlich größere Rolle. ${ }^{20}$ Die Zuordnung der inhaltlichen Perspektive bzw. kognitiven Dimension der transportieren Informationen findet hier statt. Etwas Ähnliches postuliert das sog. „Ende-zu-Ende“-Prinzip: „,it counsels that the ,intelligence' in a network should be located at the top of a layered system - at its, ends', where users put information and applications onto the

\footnotetext{
${ }^{14}$ Vgl. Schmidt/Pruß, in: Auer-Reinsdorff/Conrad, Handbuch IT- und Datenschutzrecht, $\S 3$ Rn. 64.

${ }^{15}$ Vgl. Schmidt/Pruß, in: Auer-Reinsdorff/Conrad, Handbuch IT- und Datenschutzrecht, §3 Rn. 64.

${ }^{16} \mathrm{Vgl}$. Mandl, Internet Internals, S. 2; Riordan, The Liability of Internet Intermediaries, S. 40.

${ }^{17}$ Hier sind nun insbesondere die im Fokus dieser Untersuchung stehenden Art. 15 ECRL, §§ 7, 10 TMG maßgeblich.

${ }^{18} \mathrm{Vgl}$. Mandl, Internet Internals, S. 2 f.

${ }^{19}$ Vgl. Art. 3 Abs. 3 VO (EU) 2015/2120; vgl. ferner Lemley/Lessig, UCLA Law Review 48 (2001), 925 (931).

${ }^{20}$ Vgl. Bridy, Boston University Journal of Science \& Technology Law 24 (2018), 193 (205).
} 
network“ “. ${ }^{21}$ Die Enden dienen hier als Anknüpfung für die funktionale Spezialisierung mit Blick auf nutzerrelevante Anwendungen, damit die dazwischen liegenden, technischen Schichten gerade anwendungs- und inhaltsunspezifisch ihre Leistungen erbringen. ${ }^{22}$ Die so im Grundsatz erfolgte Abkopplung von Signalübertragung und Inhalt ist keine technische Petitesse, sondern hat ein enormes gesellschaftliches Potenzial, weil sie die Weiterentwicklung anwendungsorientierter Lösungen nicht in die Hände einer kleinen Gruppe von Experten legt, sondern einem viel größeren Kreis zur Verfügung steht. ${ }^{23}$

Diese zunächst rein phänomenologischen Zuschreibungen können in normativer Perspektive erklären, weshalb inhaltsbezogene Regulierungszugriffe wie etwa $\$ 230$ CDA, $\$ 512$ DMCA oder Art. 14 ECRL an den anwendungsbezogenen Schichten ansetzen, also an den „Enden“ netzbasierter Kommunikation. Denn gerade die Enden vermitteln untechnisch gesprochen nicht nur Datenpakete, sondern Wörter, Bilder, allgemein gesprochen kognitiv unmittelbar erfassbare Informationen -, ,[t]he application layer is the Internet's human-experiential layer. " ${ }^{24}$ Aus den vielfältigen Schlussfolgerungen dieser Feststellungen seien hier drei explizit ausgewiesen. ${ }^{25}$ Erstens ist in den hier erwähnten Schichtenmodellen bzw. dem „Ende-zu-Ende“-Prinzip die Einsicht immanent, dass an den unmittelbar anwendungsbezogenen Funktionen eine ,aktive“, inhaltliche Ungleichbehandlung von Informationen dem Wesenskern der Struktur netzbasierter Kommunikation entspricht. ${ }^{26}$ Zweitens folgt aus den hier skizzierten Beobachtungen, dass einer „mustcarry"-Regel im Sinne eines Diskriminierungsverbotes in diesen Bereichen, die einer Übertragung des Prinzips der Netzneutralität auf die Inhaltsebene gleichkäme, im Grundsatz mit Skepsis zu begegnen ist. ${ }^{27}$ Dies gilt jedenfalls, soweit es um nutzergenerierte Inhalte geht. ${ }^{28}$ Das bedeutet allerdings nicht,

${ }^{21}$ Lemley/Lessig, UCLA Law Review 48 (2001), 925 (930).

${ }^{22}$ Vgl. Solum/Chung, Notre Dame Law Review 79 (2004), 815 (847); Bridy, Boston University Journal of Science \& Technology Law 24 (2018), 193 (199f.).

${ }^{23}$ Vgl. Lemley/Lessig, UCLA Law Review 48 (2001), 925 (931 f.); Bridy, Boston University Journal of Science \& Technology Law 24 (2018), 193 (203).

${ }^{24}$ Bridy, Boston University Journal of Science \& Technology Law 24 (2018), 193 (205).

${ }^{25}$ Aus der Governance-Perspektive ist das „Ende-zu-Ende“-Prinzip allerdings nicht völlig ideologiefrei, sondern steht vielmehr in der Tradition des „Internet Exceptionalism“, wie Pohle/Thiel, in: Borucki/Schünemann, Internet und Staat, S. 57 (60), hervorheben.

${ }^{26}$ Vgl. Bridy, Boston University Journal of Science \& Technology Law 24 (2018), 193 $(205,219)$. Darauf wird mit Blick auf die rechtlichen Regelungen der Haftungsprivilegierungen noch zurückzukommen sein, vgl. unten $\S 8 \mathrm{~B}$.

${ }^{27}$ Vgl. Schulz, AfP 2017, 373 (379); Paal, Intermediäre, S. 32; Schulz/Dankert, Die Macht der Informationsintermediäre, S. 72 f.; Bridy, Boston University Journal of Science \& Technology Law 24 (2018), 193 (219).

${ }^{28}$ Damit ist insbesondere keine Stellungnahme bezüglich einer ,must-carry“-Regel für 
dass nicht auch Dienste in der Anwendungsschicht mitunter Diskriminierungsverboten unterliegen können, etwa wenn es um den Versand von E-Mails geht. ${ }^{29}$ Drittens steigt der Rechtfertigungsdruck, umso eher ein inhaltsbezogenes Haftungsrisiko für Diensteanbieter die Akteure in den „mittleren“"Schichten bzw. zwischen den „Enden“ trifft. Im Ansatz reflektieren die Art. 12 ff. ECRL diese Aussage, da sie höhere Anforderungen an ein solches Haftungsrisiko stellen, als es bei Art. 14 ECRL der Fall ist. Folglich ist also die Anknüpfung regulativer Zugriffe auf Diensteanbieter an Inhalten prinzipiell zutreffend, da einzig diese unmittelbar zwischen den Nutzerinnen (am „Ende“) und den Inhalten stehen.

Zugleich sollte man aber einer zu strengen Einteilung von Diensten in die verschiedenen Schichten ebenfalls mit Skepsis begegnen, da die generelle Entwicklung des Internets, wie bereits angedeutet wurde,${ }^{30} \mathrm{zu}$ einer Verwässerung der ursprünglich klaren Trennung von Technik und Inhalt führt. AuBerdem sind die Grundannahmen der technischen Beschreibung von solchen Diensten und insbesondere ihrer Kategorisierung nicht immer trennscharf voneinander abgrenzbar. Nicht zuletzt bedient die Verortung von Unternehmen wie Facebook und Google am „Ende“ eines netzbasierten Kommunikationsvorgangs ein unzutreffendes Narrativ, das diesen Unternehmen mit Blick auf den Kommunikationsvorgang als solchen eine unbedeutende, randständige Rolle zuschreibt. ${ }^{31}$ Das hier vorgestellte Modell bietet also einen guten abstrakten Blick auf die technische Komponente der Diensteanbieter. Eine schematisierende, unreflektierte Übertragung gilt es insoweit aber zu vermeiden.

\section{B. Funktionen der Intermediäre}

Im Folgenden werden Dienste in den Blick genommen, soweit sie für diese Untersuchung von Bedeutung sind. Als Oberbegriff für sämtliche Diensteanbieter, die die Kommunikation ihrer Nutzer untereinander und zu einer (wie auch immer gestalteten) allgemeinen Öffentlichkeit organisieren, lässt hier der Begriff „Kommunikationsdienst“ als Unterkategorie der Diensteanbieter verwenden. In ihm materialisiert sich der primäre Zweck, nämlich die Kommunikation als mehrseitiger Austausch von Informationen, Inhal-

\footnotetext{
journalistische Angebote verbunden, wie sie etwa in $\S 81$ des Medienstaatsvertrags enthalten ist, den die Regierungschefinnen der Länder am 5. Dezember 2019 beschlossen haben, vgl. www.rlp.de/fileadmin/rlp-stk/pdf-Dateien/Medienpolitik/ModStV_MStV_u nd_JMStV_2019-12-05_MPK.pdf.

${ }^{29}$ Vgl. Balkin, Columbia Law Review 118 (2018), 2011 (2037 mit Fn. 119).

${ }^{30} \mathrm{Vgl}$. oben den Nachweis in $\S 4$ Fn. 298 und den zugehörigen Text.

${ }^{31}$ Vgl. Balkin, Columbia Law Review 118 (2018), 2011 (2037 mit Fn. 119).
} 
ten etc. Zudem verweist das kommunikationsbezogene Element auf die besondere Bedeutung dieser Dienste für die Verantwortung mit Blick auf nutzergenerierte Inhalte und Kommunikation. Der Begriff dient im Verlauf dieser Untersuchung insbesondere zur Abgrenzung von Suchmaschinen, bei denen sich zwar mitunter ähnlich Probleme mit Blick auf diese Thematik ergeben, die aber dennoch fundamentale Unterschiede aufweisen, die an verschiedenen Punkten dieser Untersuchung eine Rolle spielen.

\section{Kommunikationsdienste}

Kommunikationsdienste lassen sich in allgemeine und in bereichsspezifische Dienste unterteilen. ${ }^{32}$ Erstere erfassen insbesondere die bekannten Akteure, also Facebook, Twitter etc., sowie Dienste wie YouTube, Instagram oder Flickr, die in allgemeiner Weise einem bestimmten Zweck dienen, nämlich dem Teilen von Videos, Bildern oder anderen Dokumenten jedweder Natur. ${ }^{33}$ Bereichsspezifische Dienste sind insbesondere zur Aufnahme beruflicher (z.B. LinkedIn, Xing), zwischenmenschlicher (sog. Dating-Portale) oder interessengeleiteter Kontakte sowie zur Verwirklichung spezifischer, punktueller Ziele wie etwa der Vermittlung von Übernachtungsgelegenheiten (z.B. Airbnb) geläufig. Aufgrund des Fokus dieser Untersuchung auf nutzergenerierten Inhalten sind insbesondere die allgemeinen, weder thematisch noch personell zugeschnittenen Dienste von besonderem Interesse. Vor allem aufgrund ihrer Eigenschaft als allgemeiner Sammelplatz für den kommunikativen und informativen Austausch sind diese Dienste zudem besonders mitgliederstark. Sie sind damit in einer anderen Situation als kleine, spezifischere Dienste. ${ }^{34}$

Die Funktionen von Kommunikationsdiensten sind äußerst vielfältig. Allgemein ermöglichen sie in erster Linie soziale Interaktion, die sich nicht auf individuelle Kommunikation beschränkt, sondern diese „mit größeren Sozialgebilden (Gruppen) verknüpft". ${ }^{35}$ Die kommunikative Funktion kommt hier nicht allein im individuellen oder gruppenbezogenen Austausch von Informationen und Meinungen zur Geltung, sondern bezieht das Arrangement des kommunikativen Handelns um Informationen herum mit ein. Damit ist insbesondere ein Wandel traditionell auf Einseitigkeit ausgerichteter Angebots- und Nutzungsformen von Informationen verbunden: „Austausch

\footnotetext{
${ }^{32}$ Vgl. zum Folgenden auch Pille, Meinungsmacht sozialer Netzwerke, S. 90 ff.

${ }^{33}$ Mit Blick auf das Haftungsproblem ist eine selbstredend mögliche, weitergehende Differenzierung hier jedoch nicht geboten.

${ }^{34}$ Mit Blick auf die Meinungsbildungsrelevanz vgl. auch Pille, Meinungsmacht sozialer Netzwerke, S. 92.

${ }^{35}$ Hohlfeld/Godulla, in: Hornung/Müller-Terpitz, Rechtshandbuch Social Media, S. 11 (12).
} 
statt bloßer Zurschaustellung, Kommentieren statt Konsumieren, Identitätsmanagement statt Selbstmarketing. In den weltumspannenden Zyklus des Austauschs werden per Link Hinweise auf Fundstücke aus dem Internet genauso eingespeist wie selbstproduzierte digitale Inhalte, die in allen multimedialen Aggregatzuständen verfügbar gemacht werden. “36

Konkret weisen viele Dienste im Grunde ähnliche Funktionen auf: das Erstellen, Veröffentlichen, Kommentieren von oder die anderweitige Reaktion auf Inhalte, das Weiterleiten dieser sowie die Möglichkeit, Personen oder Institutionen zu ,abonnieren“, also regelmäßige Aktualisierungen von deren Präsenzen und neue Inhalte zu erhalten sowie sich mit anderen Personen zu vernetzen. ${ }^{37}$ Zentrales Element der inhaltsbezogenen Funktionen ist bei vielen Kommunikationsdiensten ein „Newsfeed“, „Timeline“, „Stream“ oder anders genannter virtueller Ort innerhalb eines Dienstes, der zum Austausch der Inhalte fungiert und die Nutzer ständig mit „Updates“ versorgt. Hier werden je nach persönlicher und allgemeiner Konfiguration des Dienstes laufend neue Inhalte organisiert dargestellt. Dargestellt werden stets nutzergenerierte Inhalte, mitunter aber auch diensteseitig „empfohlene“ Inhalte. Die Auswahl der angezeigten Beiträge erfolgt nicht zufällig, sondern in der Regel als Ergebnis kuratierter Informationsflüsse. ${ }^{38}$ Diese Funktion bildet gleichsam den Nukleus der hier im Fokus stehenden Funktion der Vermittlung nutzergenerierter Inhalte. Denn die Interaktion mit nutzergenerierten Inhalten hängt entscheidend von der Sichtbarkeit im „Newsfeed“ ab. Insoweit ist insbesondere von „Facebook als Ort der zufälligen Nachrichtennutzung" die Rede. ${ }^{39}$ Wenn also rechtsverletzende Inhalte bei Kommunikationsdiensten eingestellt werden, erlangen sie ihre Sichtbarkeit nicht nur durch den gezielten, also „suchenden“ Abruf, der dann allerdings die Kenntnis der Existenz und Auffindbarkeit voraussetzt, sondern auch durch die „zufällige“ bzw. aufgrund der Empfehlung im „Newsfeed“ oder in anderen Empfehlungsmechanismen erfolgenden Darstellung. Die Funktionalität der Kommunikationsdienste bedingt also die spezifische, wirkungsbezogene Qualität begangener Rechtsverletzungen, die das Recht der Haftungsprivilegierungen zu so einem wichtigen Instrument zur rechtlichen Aufarbeitung der hiermit entstehenden Konflikte macht.

\footnotetext{
${ }^{36}$ Hohlfeld/Godulla, in: Hornung/Müller-Terpitz, Rechtshandbuch Social Media, S. 11 (13).

${ }^{37}$ Vgl. Schmidt/Taddicken, in: Schmidt/Taddicken, Handbuch Soziale Medien, S. 23 (24ff.).

${ }^{38}$ Vgl. Thorson/Wells, Communication Theory 26 (2016), 309 (312 f.), die die ,,algorithmische Kuratierung" konzeptionell als Produkt bzw. Verbindung journalistischer, strategischer, personaler und sozialer Kuratierung verstehen (314ff.).

${ }^{39} \mathrm{Vgl}$. Steiner et al., in: Weber/Mangold/Hofer, Meinungsbildung in der Netzöffentlichkeit, S. 17 (20 f.), deren Studienergebnisse diese Zuschreibung freilich teilweise relativieren (32 ff.).
} 
Aus der Digitalität der über Kommunikationsdienste erfolgten Interaktionen folgen weitere Eigenschaften, die man mit Schmidt als Teil ihrer „kommunikativen Architektur" bezeichnen kann und die sie von anderen Medien kategorial unterscheiden: Persistenz, also die dauerhafte Speicherung von Inhalten; Kopierbarkeit, also die Möglichkeit ihrer Duplikation und erneuter Verfügbarmachung; Skalierbarkeit, also die potenziell erhebliche Streubreite hinsichtlich der Reichweite bestimmter Inhalte; sowie Durchsuchbarkeit, also ihre Eigenschaft, potenziell unbeschränkt auffindbar zu sein. ${ }^{40}$ Diese Eigenschaften deuten unmittelbar die praktische Bedeutsamkeit sowohl der hinter den Kommunikationsdiensten stehenden Akteure als auch der rechtlichen Regeln im Fokus dieser Untersuchung an. Die Bedeutung der Akteure „hinter“ den Kommunikationsdiensten leuchtet unmittelbar ein. Denn die kommunikative Architektur bedarf selbstredend eines „Architekten", der deren Eigenschaften und Bedeutung im Einzelfall steuern kann. Der Einfluss des Architekten hierbei erschöpft die Bedeutung der aufgezählten „architekturrelevanten“ Eigenschaften jedoch nicht abschließend, denn jeder Inhalt kann - theoretisch - jenseits des verwendeten Dienstes „weiterleben“, sei es als echte Kopie oder als „Screenshot“.

In rechtlicher Hinsicht beeinflussen die aufgezählten Faktoren die Relevanz von rechtsverletzenden Inhalten in erheblicher Weise, weil ihre Wirkmacht sich einer gewöhnlichen rechtlichen Subsumtion entzieht bzw. diese die Folgen oftmals nicht hinreichend adressiert. Denn der Beeinträchtigungsgrad einer Persönlichkeitsrechtsverletzung steigt durch ihre Persistenz, Kopierbarkeit, potenzielle Reichweite sowie an der Durchsuchbarkeit und Auffindbarkeit mitunter erheblich an. Gleiches gilt für die Verletzung von Urheberrechten oder sonstigen Schutzrechten. Nicht nur im „Hinblick auf Privatsphäre erzeugen soziale Medien also ein Dilemma. “41 Denn den unbestrittenen Vorteilen der Nutzung von Kommunikationsdiensten steht eine qualitativ neue Dimension der rechtlichen Risiken gegenüber.

\section{Suchmaschinen}

Nun gilt es, auf die Funktionsweise von Suchmaschinen näher einzugehen. Einschränkend muss allerdings schon vorweg darauf hingewiesen werden, dass aufgrund begrenzten Wissens hierüber notwendigerweise nur eine oberflächliche Beschreibung möglich ist. Welche Kriterien genau Dienste wie Google zur Darstellung von Suchergebnissen verwenden sowie insgesamt die Funktionsweise des Rankings ist nach wie vor kaum hinreichend beschrieben, geschweige denn von Google transparent gemacht. ${ }^{42}$

\footnotetext{
${ }^{40}$ Schmidt, Social Media, S. $36 \mathrm{ff}$.

${ }^{41}$ Vgl. Schmidt, Social Media, S. 39.

${ }^{42}$ Vgl. hierzu Jürgens/Stark/Magin, in: Stark/Dörr/Aufenanger, Die Googleisierung der
} 
Die Tätigkeit von Suchmaschinendiensten lässt sich grob in drei Phasen bzw. Komponenten unterteilen ${ }^{43}$ : das Sammeln von Daten, genannt „Crawling“; die Aufbereitung und Speicherung dieser Daten, genannt „Indexing“; sowie die Suche in den Daten und die Präsentation eines Suchergebnisses, genannt „Retrieval“. Zur Dokumentenbeschaffung durchsucht der Dienst mithilfe des „Crawlers“, mitunter auch als „Spider" oder „Robot" bezeichnet, zunächst systematisch und mit andauernder periodischer Wiederholung Webseiten und extrahiert die Inhalte aller - technisch auffindbaren - Seiten. ${ }^{44}$ Bestandteil des Crawlings ist in unterschiedlicher Ausgestaltung auch die Speicherung der besuchten Webseiten bzw. bestimmter komprimierter Daten dieser Webseiten in einem Datenbestand, bis der Crawler diese aktualisiert. ${ }^{45}$ Dieser Speicher ist öfters auch als „Cache“ bekannt. Das „caching“ erlaubt eine deutlich schnellere Verarbeitung von Suchanfragen, da so eine Verarbeitung des Originaldokuments nicht mehr notwendig ist. ${ }^{46}$ Im Anschluss werden die so gewonnen Daten durch den Dienst in einen Index übertragen, wo Analysen durchgeführt werden, um die aufgefundenen Seiten zu strukturieren. ${ }^{47}$ Der Suchmaschinenindex ist auf den Servern des Suchmaschinendienstes gespeichert und enthält Informationen über jedes indizierte Dokument, wobei diese Informationen noch nicht strukturiert, geschweige untereinander verknüpft sind. Bis hierhin erfolgen die Tätigkeiten der Suchmaschine nutzerunabhängig, also von sich aus. ${ }^{48}$

Dies ändert sich erst mit einer erfolgten Suchanfrage. Auf eine Suchanfrage werden entsprechend der Programmierung für relevant erachtete Webseiten identifiziert, in eine bestimmte Reihenfolge gebracht und dem Suchenden präsentiert (,retrieval“). Die Bestimmung der Reihenfolge wird auch als Ranking bezeichnet, das als „,charaktergebendes Merkmal“449 den „Kern der

Informationssuche, S. 98 (113 ff.); Pasquale, The Black Box Society, S. 64 f.; zu Forderungen nach mehr Transparenz vgl. etwa Kaiser/Reiling, in: Unger/Ungern-Sternberg, Demokratie und künstliche Intelligenz, S. 85 (103 f.).

${ }^{43}$ Vgl. Schmidt/Pruß, in: Auer-Reinsdorff/Conrad, Handbuch IT- und Datenschutzrecht, $\S 3$ Rn. 401.

${ }^{44}$ Vgl. Lewandowski, Suchmaschinen verstehen, S. 37. Dass alle Webseiten des Internets durchsucht werden ist dabei nicht schon zwingend, vielmehr muss der „Crawler" grundsätzlich mit „Uniform Resource Locators“ (besser bekannt als URL) „gefüttert“ werden, vgl. Langville/Meyer, Google's PageRank and Beyond, S. 15 f. Jede Verlinkung bzw. jeder Verweis einer erfassten Webseite auf andere Seiten, Anwendungen, Bilder u.Ä. wird zudem weiterverfolgt und ebenfalls erfasst, sodass die ursprünglich „gefütterten“ URL nie den gesamten Bestand der erfassten Informationen darstellen, vgl. Schmidt/Pruß, in: AuerReinsdorff/Conrad, Handbuch IT- und Datenschutzrecht, §3 Rn. $406 \mathrm{f}$.

${ }^{45} \mathrm{Vgl}$. Brunn, Cache me if you can, S. $28 \mathrm{f}$.

${ }^{46} \mathrm{Vgl}$. Brunn, Cache me if you can, S. 29.

${ }^{47}$ Vgl. Langville/Meyer, Google's PageRank and Beyond, S. 19 f.

${ }^{48}$ Vgl. Langville/Meyer, Google's PageRank and Beyond, S. 21.

${ }^{49}$ Siegmund, Suchmaschinenalgorithmen im Kartellrecht der EU und der USA, S. 56. 
Suchmaschine [bildet], der sie zu etwas Besonderem macht" ", ${ }^{50}$ da dieser Schritt nicht nur vom Nutzer abhängt, sondern die Qualität dieses Schrittes über die Qualität der Suchmaschine als solche entscheidet. ${ }^{51}$ Denn mit dem Ranking versucht jeder Suchmaschinendienst die größtmögliche Relevanz der Suchergebnisse für den Nutzer zu erzeugen. ${ }^{52}$ Die sortierte Suchergebnisliste wird dem Nutzer angezeigt, wobei in der Regel bereits Inhalte der jeweils verlinkten Webseite schon in der Suchmaske selbst angezeigt werden (sog. „Snippets"). Auch die URL selbst bzw. Teile davon werden angezeigt. Ob weitere Informationen dargestellt werden, hängt von der Natur des Treffers ab. Sucht man etwa „Haus“ bei Google, werden dem Ergebnis für den Wikipedia-Artikel „Haus“ bereits die einzelnen Abschnitte des Eintrags bei Wikipedia angezeigt. Bei kommerziell relevanten Ergebnissen werden auBerdem mitunter auch Links zu konkreten gewerblichen Inhalten jeweils einzelnen Ergebnissen zugeordnet dargestellt.

\section{Vergleichende Betrachtungen}

Im Folgenden seien kurz die Unterschiede (1.) und Gemeinsamkeiten (2.) von Kommunikationsdiensten und Suchmaschinen reflektiert, soweit dies für diese Untersuchung von Bedeutung ist.

\section{Unterschiede zwischen Kommunikationsdiensten und Suchmaschinen}

Nach den bisherigen Beschreibungen von Kommunikationsdiensten und Suchmaschinen ist deren Unterschiedlichkeit in vielerlei Hinsicht offenkundig. Sie werden im Grundsatz für vollkommen unterschiedliche Zwecke genutzt und vermitteln ganz eigene Chancen und Risiken. Eine indizielle Bestätigung dieser Unterschiedlichkeit liegt nicht zuletzt in der Grundverschiedenheit der gegenwärtigen Marktsituation. So ist im Markt der Suchmaschinen Google der mit weitem Abstand marktmächtigste Akteur. Der Marktanteil dieses Dienstes liegt seit Jahren stabil bei ca. 90 Prozent. ${ }^{53}$ Das mag auch daran liegen, dass die einmal getroffene Entscheidung für die Verwendung von Google von vielen Nutzern nicht mehr revidiert wird. ${ }^{54}$ Die hiermit beschriebene Dominanz begründet nicht nur eine marktbeherrschende Stellung im Bereich der Suchmaschinen, ${ }^{55}$ sondern reicht bis in den

\footnotetext{
${ }^{50}$ Lewandowski, Suchmaschinen verstehen, S. 90.

${ }^{51}$ Vgl. auch Schmidt/Pruß, in: Auer-Reinsdorff/Conrad, Handbuch IT- und Datenschutzrecht, §3 Rn. $423 \mathrm{f}$.

${ }^{52} \mathrm{Vgl}$. zu den Implikation noch unten $\S 8$ C. I 1. b).

${ }^{53}$ Vgl. www.statista.com/statistics/216573/worldwide-market-share-of-search-engines; Lewandowski, Suchmaschinen verstehen, S. 6.

${ }^{54} \mathrm{Vgl}$. Unkel, Informationsselektion mit Suchmaschinen, S. 67, mwN.

${ }^{55}$ Vgl. nur Siegmund, Suchmaschinenalgorithmen im Kartellrecht der EU und der USA, S. $247 \mathrm{ff}$.
} 
Raum sprachlicher und kultureller Praxis hinein ${ }^{56}$ wenn etwa der Vorgang des Suchens nach einer Information mittlerweile sogar im Duden als ,googeln" aufgenommen ist. ${ }^{57}$

Der Markt von Kommunikationsdiensten zeichnet sich demgegenüber durch einen etwas höheren Differenzierungsgrad und ein höheres Entwicklungstempo aus. Für vergleichbare Zwecke oder Bereiche finden sich durchaus mehrere Dienste. Doppelmitgliedschaften sind bei diesen nicht selten, was im Vergleich zu Suchmaschinen bzw. Google schon per se für einen weiter entwickelten Wettbewerb spricht. Auch die wirtschaftliche Entwicklung von Märkten in diesem Bereich zeichnet sich durch eine höhere Dynamik aus. ${ }^{58}$ Dienste schießen schnell empor, gewinnen an wirtschaftlicher Bedeutung, verschwinden mitunter aber ebenso schnell wieder bzw. finden nur noch in einem Nischensegment Platz. ${ }^{59}$ Insoweit ist auch nicht davon auszugehen, dass diese Dynamik in einem konsolidierten Umfeld aufgehen wird bzw. ein solcher Übergang schon stattfindet. Denn geringe Markteintrittskosten, Netzwerkeffekte auch zugunsten von jungen Unternehmen, eine generell hohe Verfügbarkeit von Nutzerdaten sowie die permanente Weiterentwicklung digitaler Technologien als insoweit relevante Faktoren werden wohl bis auf Weiteres von Bedeutung bleiben. ${ }^{60}$

\section{Wesentliche Gemeinsamkeiten}

Gleichwohl weisen Kommunikationsdienste und Suchmaschinen wesentliche Gemeinsamkeiten auf. Zwei seien im Folgenden kurz erläutert und mit Blick auf den Kontext dieser Untersuchung reflektiert.

${ }^{56}$ Stark, in: Stark/Dörr/Aufenanger, Die Googleisierung der Informationssuche, S. 1 (1): „Googeln“ als ,globale Kulturtechnik“; zum Internet als Kulturtechnik vgl. schon den Nachweis oben in $\S 3$ Fn. 106.

${ }^{57}$ www.duden.de/rechtschreibung/googeln.

${ }^{58}$ Vgl. zur Dynamik der Internetbranche auch Volmar, Digitale Marktmacht, S. $312 \mathrm{ff}$.

${ }^{59}$ Insoweit sei etwa an die Dienste MySpace oder StudiVZ erinnert. In den USA stürzte die Zahl von Besuchern auf myspace.com von 32,35 Millionen im Oktober 2014 auf 13,32 Millionen im Oktober 2016 ab, vgl. de.statista.com/statistik/daten/studie/39035/umfrage /unique-visitors-auf-myspacecom. Eine Abfrage bei trends.google.de nach dem weltweiten Interesse nach dem Suchergebnis „myspace“ seit 2004 ergibt ebenfalls eine Gauß'sche Glockenkurve, bei der ab 2012/2013 kaum noch ein nennenswertes Interesse zu verzeichnen ist. Die Zahl der Besuche bei den sog. „VZ“-Diensten lag im Juni 2007 bei 88 Millionen, erreichte ihren Höchststand im März 2010 (451 Millionen) und lag schon im Mai 2012 bei nur noch 45 Millionen, vgl. de.statista.com/statistik/daten/studie/ 197007/umfrage/anzahlder-nutzer-der-vz-netzwerke; auch hier ergibt eine Abfrage bei trends.google.de nach dem Interesse in Deutschland nach dem Suchbegriff „studivz“ einen ähnlichen Befund.

${ }^{60}$ Vgl. Volmar, Digitale Marktmacht, S. $315 \mathrm{ff}$. 


\section{a) Dominanz weniger Akteure}

Obschon man im Bereich von Kommunikationsdiensten nicht in gleicher Weise von einer Dominanz eines einzelnen Akteurs sprechen kann, sind auch hier durchaus dominante Akteure vorhanden. Insbesondere Facebook ist ein nennenswertes Beispiel. Für das Unternehmen ergeben die weltweiten Marktanteile für das Jahr 2019 monatliche Werte zwischen rund 64 und 74 Prozent. ${ }^{61}$ Im Bereich von Videodiensten existieren zwar viele Dienste mit ähnlichem Angebot, gleichwohl hat YouTube hier ebenfalls eine dominante Stellung inne. Insgesamt weisen die Märkte, in denen die hier im Fokus stehenden Akteure tätig sind, deutliche Tendenzen zu oligopolistischen bzw. monopolistischen Strukturen auf. ${ }^{62}$ Aus rechtlicher Perspektive ist der $\mathrm{Zu}$ griff auf das Konzept der Marktmacht in jüngerer Zeit verstärkt in den Fokus gerückt. ${ }^{63}$ In der juristischen Praxis nehmen die Versuche ebenfalls zu, auf die Marktmacht einzelner Akteuren eine Antwort zu finden. ${ }^{64}$ In Deutschland tat sich hier jüngst das Bundeskartellamt hervor, das Facebook die Zusammenführung von Daten seiner Nutzer untersagte. ${ }^{65}$

In dem hier nur angerissenen Befund einer Dominanz weniger, global tätiger Akteure und Unternehmen werden Zusammenhänge zum Gegenstand dieser Untersuchung in vielfältiger Art erkennbar. So adressieren einzelne, neuere gesetzliche Regelungen nur bestimmte, kraft spezifischer, „harter" Kriterien als besonders einflussreich markierte Diensteanbieter ${ }^{66}$ wenngleich dies nicht zwingend mit einer Marktmacht im juristischen Sinne einhergeht. Bei der Frage der Drittwirkung der Grundrechte deutet die jüngere verfassungsgerichtliche Rechtsprechung ebenfalls an, jedenfalls in bestimmten Fragen dieses grundrechtsdogmatische Instrument nur gegenüber besonders mächtigen bzw. (gesamt-)gesellschaftlich relevanten Akteuren zu entfalten. ${ }^{67}$ Auch im rechtspolitischen Kontext ist nicht selten eine Zuspitzung

${ }^{61}$ Bezugspunkt der Statistik ist insoweit die Kategorie „Social-Media-Seiten“, vgl. de.statista.com/statistik/daten/studie/241601/umfrage/marktanteile-fuehrender-socialmedia-seiten-weltweit.

${ }^{62}$ Vgl. Schneider, in: Saetnan/Schneider/Green, The Politics of Big Data, S. 129 (130 ff.); Staab, Digitaler Kapitalismus, S. 103 ff. und passim; Hoffmann-Riem, in: Augstein, Reclaim Autonomy, S. 121 (128ff.).

${ }^{63} \mathrm{Vgl}$. jüngst nur Grothe, Datenmacht in der kartellrechtlichen Missbrauchskontrolle, passim; Volmar, Digitale Marktmacht, passim.

${ }^{64}$ Instruktive Übersicht bei Hoffer/Lehr, NZKart 2019, 10 ff. Aus populärwissenschaftlicher Perspektive vgl. Galloway, The Four, passim.

${ }^{65}$ Vgl. Bundeskartellamt, Pressemitteilung vom 07.02.2019, www.bundeskartellamt.de/ SharedDocs/Meldung/DE/Pressemitteilungen/2019/07_02_2019_Facebook.html, kritische Einordnung der Entscheidung bei Körber, NZKart 2019, $187 \mathrm{ff}$.

${ }^{66} \mathrm{Vgl}$. Art. 2 Nr. 6, 17 Abs. 6 DSM-RL (vgl. dazu noch unten $\S 12$ B.); 1 Abs. 2 NetzDG (vgl. dazu noch unten $\S 12$ A.).

${ }^{67}$ Vgl. insbesondere BVerfG, Beschl. v. 11.04.2018 - 1 BvR 3080/09, NVwZ 2018, 813 
mancher Stellungnahmen auf einzelne Unternehmen wie Facebook oder Google festzustellen. Das übersetzt sich nicht zwingend in die Entwicklung und Anwendung allgemeiner Rechtsregeln. Die Singularität und die besondere Sichtbarkeit dieser Unternehmen lässt einen Zusammenhang aber nicht unplausibel erscheinen. All diese Entwicklungen hätten zumindest in dieser Form nicht ohne eine Konzentration entsprechender Märkte auf bestimmte einzelne Akteure stattgefunden.

\section{b) Kuratierung von Inhalten}

Die hier wesentlich bedeutsamere Gemeinsamkeit von Kommunikationsdiensten und Suchmaschinen liegt allerdings in einem anderen Aspekt begründet. In beiden Bereichen liegt das Proprium in der Verfügbarmachung von Informationen, die ohne diese Angebote kaum möglich wäre. In beiden Bereichen genügt die bloße Referenzierung, also der Verweis auf diese im Sinne der Übergabe einer mit Dokumenten gefüllten Kiste keineswegs. Vielmehr müssen diese Informationen aufbereitet, kuratiert werden, um für die Nutzer sinnvoll kognitiv verarbeitet werden zu können. ${ }^{68}$ Die etymologischen Ursprünge des Wortes - „curare“, also „Sorge tragen“ oder „sich kümmern“ - weisen bereits auf die Funktion und die Relevanz der Kuratierung hin. ${ }^{69}$ Denn mit zunehmender Komplexität vieler Gesellschaftsbereiche gestaltet sich deren Navigation als immer herausfordernder. Der heutzutage wohl noch am ehesten geläufige Verwendungskontext des Wortes verdeutlicht dies anschaulich. So geht das im Laufe der letzten Jahrhunderte sich stetig wandelnde Verständnis von Kunst mit einer erheblichen Zunahme an Artefakten oder Verhaltensweisen einher, die sich hierunter fassen lassen. Mit dem wachsenden Korpus an als Kunst anerkannter Gegenstände und Ideen schrumpft aber beständig die Möglichkeiten, diese in Museen oder anderen Kontexten in ihrer Gesamtheit zu präsentieren. Das führt zur Notwendigkeit, unter dem Bestand von Kunst eine Auswahl zu treffen. Diese Funktion nahm und nimmt der Kurator ein, der Sammlungen, Ausstellungen etc. organisiert und hierbei die Entscheidung trifft, was zu sehen sein soll. ${ }^{70}$

Der Wert der Kuratierung ${ }^{71}$ liegt danach nicht allein darin, welches Artefakt es in die Auswahl schafft, sondern eben auch darin, welches nicht auf-

(Rn. 41) - Stadionverbot; sehr kritisch dazu aber Hellgardt, JZ 2018, 901 ff.; Michl, JZ 2018, $910 \mathrm{ff}$.

${ }^{68}$ Vgl. Bhaskar, Curation, S. 1 ff., 136 f., 223 ff. und passim; Rosenbaum, Curation Nation, S. 1 ff., 128 f. und passim; Balkin, Columbia Law Review 118 (2018), 2011 (2038, 2040 ff.); Groys, Google, Words beyond Grammar, S. $11 \mathrm{f}$.

${ }^{69}$ Vgl. Bhaskar, Curation, S. 67.

${ }^{70}$ Vgl. Bhaskar, Curation, S. $67 \mathrm{ff}$.

${ }^{71}$ Vgl. aber die pejorative Zuschreibung bei Wagner/Eidenmüller, ZfPW 2019, 220 (234): Verbraucherpräferenzen als „Ergebnis einer kuratierten, drastischer formuliert: manipulierten Informationssphäre“. 
genommen wird. ${ }^{72}$ Die erforderliche Auswahl, Organisation oder Anordnung bestimmter Artefakte oder Inhalte ist damit zwingend mit einem Akt des Wertens verbunden. Will der Kurator erfolgreich sein, muss also auch seine Tätigkeit bestimmten Ansprüchen genügen. Hinter jeder Form und jedem Kontext des Kuratierens steht also das Ziel, einen Mehrwert jenseits dessen zu produzieren, was gesammelt bzw. organisiert wird. ${ }^{73}$ Im Idealfall geht dies mit einer Zeitersparnis beim Konsum einher, denn aus der Vielfalt der Artefakte wird eine überschaubare Menge. In diesem Kontext erhebt nur noch Expertenwissen den Anspruch, in einem bestimmten Feld „alles“ zu kennen. Insoweit gleichen sich Kuratoren von Kunst und die Diensteanbieter des Internets. Bei Letzteren übertrifft der „overload“ an Informationen denjenigen an Kunst sogar um ein Vielfaches. Dies gilt gleichermaßen für Kommunikationsdienste und Suchmaschinen..$^{74}$ Insoweit ist also die Bedeutung der Akteure hinter diesen Diensteanbietern als gleichsam informationsordnende Instanz festzustellen.

Die jeweiligen Folgen der primären Funktion des Kuratierens von Inhalten gewinnen in verschiedenen Kontexten dieser Untersuchung an Bedeutung. Ihre Erörterung bleibt damit dem jeweils einschlägigen Kontext vorbehalten. Hier genügt der Hinweis, dass das Phänomen der Kuratierung mit dem Kontext der Haftungsprivilegierungen in einem unmittelbaren Zusammenhang insoweit steht, als der Handlungskomplex der Kuratierung der Diensteanbieter conditio sine qua non für die Verbreitung von Inhalten ist. Ihre positive Entscheidung, wann, wo und wie Inhalte von Nutzern dargestellt werden sollen oder können, kann nicht hinweg gedacht werden, ohne dass der Erfolg einer Rechtsverletzung in ihrer speziellen Form innerhalb eines Dienstes entfiele.

\section{Zusammenfassung}

Abschließend lässt sich festhalten, dass die hier in den Blick genommenen Akteure sich zwar funktional und in wesentlichen Eigenschaften unterscheiden, dennoch aber mit Bezug auf die informationsvermittelnde Funktion auch wesentliche Merkmale miteinander gemein haben. Diese Einsicht spielt für eine kritische Analyse des Rechts der Haftungsprivilegierungen eine we-

\footnotetext{
${ }^{72}$ Vgl. Bhaskar, Curation, S. $82 \mathrm{ff}$.

${ }^{73}$ Rosenbaum, Curation Nation, S. 3: ,[C]uration is about adding value from humans who add their qualitative judgement to whatever is being gathered and organized." Vgl. auch Bhaskar, Curation, S. 85.

${ }^{74}$ Bei jedem Besuch von Facebook beträgt die Zahl potenziell relevanter Inhalte für diese Person nach Angabe eines Entwicklers 1500, die erst durch die Kuratierung durch Facebook auf eine deutlich kleinere Zahl reduziert wird, die von Nutzern realistischerweise an einem Tag konsumiert werden können, vgl. Bhaskar, Curation, S. 238.
} 
sentliche Rolle, insoweit ihre gemeinsame Verarbeitung in einem einheitlichen Regelungsregime naheliegenderweise Friktionen erzeugt. ${ }^{75}$

\footnotetext{
${ }^{75}$ Vgl. unten $\S 8$.
} 



\section{Begriffliche Zuordnung als Element des rechtlichen Zugriffs auf Intermediäre}

Eine Untersuchung über die Rolle bestimmter Akteure erfordert zunächst Klarheit über das Bezugsobjekt und einen analytischen Rahmen, der neben dieser faktischen Bestimmung auch das Feld absteckt, das es dann normativ $\mathrm{zu}$ erkunden gilt. Insoweit sind bereits die verschiedenen Begrifflichkeiten von Interesse, die im Zusammenhang mit den hier thematisierten Unternehmen und deren Funktionen verwendet werden. Ohne Anspruch auf Vollständigkeit sollen die wichtigsten Begriffe im Folgenden schlagwortartig erläutert werden. Für jeden der hier angesprochenen Begriffe ist sodann darauf einzugehen, inwieweit er bereits in rechtlichen Regelungen (gesetzlicher oder judikativer Natur) rezipiert ist, wobei dies selbstredend nur dann relevant ist, wenn und soweit diese Regelungen thematischen Bezug zum Gegenstand dieser Untersuchung haben. ${ }^{1}$ Schließlich sind die hier vorgestellten Begriffe in ihrer Überzeugungskraft einander gegenüberzustellen.

Dabei ist allerdings noch zu berücksichtigen, dass die hier dargestellten Begrifflichkeiten teilweise synonym, teilweise als Ober- und Unterkategorien verwendet werden. Zweck dieses Abschnitts ist es deshalb nicht, diese Verwendungen grundsätzlich in Frage zu stellen, sondern jeden Begriff für sich zum Gegenstand einer semantischen Reflexion zu machen, um zu einem reflektierteren begrifflichen Verständnis zu gelangen, das den Weg und das Ziel dieser Untersuchung zu erhellen vermag.

Dass Begriffe in rechtswissenschaftlichen Diskursen von enormer Bedeutung sind, ist insoweit keine sonderlich neue Erkenntnis. Gerade in netzpolitischen Debatten ist die Wahl der Begriffe von überragender Bedeutung. Denn mit Blick auf viele technische Entwicklungen, bei denen eine erhebliche soziale Relevanz und ein mitunter eher begrenztes technisches Verständnis sich gegenüberstehen, können begriffliche Zugriffe für den allgemeinen wie auch für den Fachdiskurs entscheidende Bedeutung erlangen und oftmals auch normatives Potenzial entfalten. ${ }^{2}$ Dass der semantische Gehalt von Be-

${ }^{1}$ Insbesondere der Plattformbegriff findet sich in sehr vielen rechtlichen Regelungen wieder, die mit dem Thema dieser Untersuchung nichts zu tun haben.

${ }^{2}$ Das gilt unabhängig davon, ob in juristischen Debatten mit Metaphern (vgl. Münkler, Der Staat 55 (2016), 181 (205 ff.)), Leitbildern (vgl. dazu Braun, Leitbilder im Recht, 
griffen dabei subjektiv unklar bzw. nicht abschließend definierbar bleiben kann, steht ihrer Verwendung nicht entgegen. ${ }^{3}$ Dennoch sollte man beim Gebrauch besonders suggestiver Begriffe Vorsicht walten lassen. Ihr rein deskriptiver Charakter enthebt ihren Gebrauch jedenfalls nicht per se jedweder Rechtfertigungsbedürftigkeit. ${ }^{4}$

\section{A. Plattformen}

Für die hier relevanten Dienste und deren Betreiber ist die Metapher der Plattform weit verbreitet. Diese „Leitmetapher“ ist in nahezu jedem Kontext der Befassung mit Themen der digitalen Welt anzutreffen. ${ }^{5}$ Zugleich erscheint die semantische Durchdringung des Begriffs bisweilen eher unterkomplex. ${ }^{6}$ Dies rechtfertigt es, diesen an den Anfang der hier vorzunehmenden begrifflichen Annäherungen an den Beobachtungsgegenstand dieser Untersuchung zu stellen.

\section{Begriffsverständnisse}

\section{Allgemeine Verständnis}

Nicht unbedeutend ist bereits der Umstand, dass der Duden für den Begriff „Plattform" sechs unterschiedliche Bedeutungsebenen ausmacht. ${ }^{7}$ Neben „(mit einem Geländer gesicherte) ebene Fläche auf hohen Gebäuden, Türmen o. Ä.“ oder einer „Fläche am vorderen oder hinteren Ende älterer Straßen- oder Eisenbahnwagen zum Ein- und Aussteigen" wird - für den Kontext dieser Untersuchung wohl am relevantesten - eine Plattform definiert als „Ort oder Personenkreis, der dem Austausch und der Verbreitung von Ideen, Anschauungen oder Produkten dient" ${ }^{8}{ }^{8}$ Als Beispiele möglicher Begriffsverwendungen nennt der Duden verschiedene Verwendungsweisen: „die Universität dürfe keine Plattform für politische Propaganda bieten, sagte der Rektor", „die Messe ist auch eine Plattform für technische Innovationen“ und ,im Internet eine Plattform für Existenzgründer einrichten“.

passim; Schoch, NVwZ 2008, 241 (241)) oder Schlüsselbegriffen (vgl. hierzu auch Braun, Leitbilder im Recht, S. 30 ff., mwN) gearbeitet wird.

${ }^{3}$ Zur Verweisungsstruktur von Begriffen und Bedeutungsintentionen vgl. Poscher, JZ 2013, 1 (10), mwN.

${ }^{4}$ Vgl. nur Buchheim, Die Verwaltung 49 (2016), 55 (70 ff.).

${ }^{5}$ Seiler, in: Baechler et al., Medienlinguistik 3.0, S. 63 (63).

${ }^{6}$ Vgl. Seiler, in: Baechler et al., Medienlinguistik 3.0, S. 63 (64 ff.).

${ }^{7}$ www.duden.de/rechtschreibung/Plattform.

${ }^{8}$ Eine politische Konnotation des Begriffs erfolgt aus der Übernahme der amerikanisch-englischen Verwendung erstmals Mitte des 19. Jahrhunderts, vgl. den Abschnitt „Etymologie" unter www.dwds.de/wb/Plattform. 
Gillespie weist darauf hin, dass viele mögliche Bedeutungsvariationen, selbst die metaphorischen, im Ausgangspunkt auf eine erhöhte, plane Oberfläche verweisen, die das Stattfinden einer zeitlich nachfolgenden Handlung erleichtern sollen. Dem Begriff wohne damit ein vorausschauendes oder vorwegnehmendes (,anticipatory“), nicht jedoch kausales Element inne, das seine Neutralität hinsichtlich der vorzunehmenden Handlung unterstreiche. Zugleich sei ihm eine progressive, weil offene, egalitäre sowie zugängliche und damit ermächtigende Funktionalität immanent, ${ }^{9}$, ,promising to support those who stand upon it" ${ }^{10}$ Damit spielen neben den physischen Eigenschaften Zuschreibungen ideologischer Natur eine mindestens gleichwertige Rolle: „In any of ,platform“s [sic] senses, being raised, level and accessible are ideological features as much as physical ones. "11

Diese Einsichten gewinnen vor allem im Kontext von „User-generated content" an Überzeugungskraft, indem sie das innovationsfördernde, aufklärerische und damit von den Fesseln des „,analogen Leviathans“ befreiende Potenzial der Plattformen des Internets unterstreichen. Gerade hier wird die Interdependenz von der allgemeinen Forderung nach einem freien Internet und den spezifischen Zuschreibungen durch Neutralität fördernde bzw. (tatsächlich oder vorgeblich) fordernde Regulierungszugriffe wie $\$ 230 \mathrm{CDA}$, $\S 512$ DMCA und der ECRL offenkundig: Wer für ein möglichst wenig reguliertes Internet ist, muss dessen Dienste als Plattform in dem beschriebenen Sinne begreifen, denn nur dieses Paradigma schirmt sie als neutral ab und gewährleistet ihre ermächtigende Funktion. Wer die Neutralitätsthese dagegen anzweifelt, stellt damit zugleich den Freiheitsgedanken in Frage. In keiner dieser beiden argumentativen Figuren werden die Betreiber der Plattformen noch als eigenständige Akteure wahrgenommen - sie sind gleichsam die Plattform und haben damit keinen Subjektcharakter mehr.

Oftmals schreiben sich die betreffenden Unternehmen jedoch selbst noch eine andere, leicht veränderte Rolle zu, nämlich diejenige als Garant der Kommunikationsfreiheit ihrer Nutzer, die sie mit ihrem Angebot wesentlich förderten, wenn nicht gar ermöglichten. ${ }^{12}$ Bezogen auf die Plattformmeta-

${ }^{9}$ Vgl. Gillespie, New Media \& Society 12 (2010), 347 (350 f.). Die Debatte um Offenheit, insbesondere im Zusammenhang mit „Free“ oder „Open Source Software“ zeichnet Morozov scharfzüngig nach; insbesondere habe diese Bewegung kaum die Freiheit aller, erst recht nicht der Nutzer im Blick gehabt, sondern nur die Freiheit der Entwickler, vgl. Morozov, The Baffler 22 (2013), 125 (125); zum Begriff „open“ vgl. ders., The Baffler 22 (2013), 125 (127).

${ }^{10}$ Gillespie, New Media \& Society 12 (2010), 347 (350).

${ }^{11}$ Gillespie, New Media \& Society 12 (2010), 347 (351).

${ }^{12}$ Vgl. zur Einordnung von Suchmaschinen unter den ersten Zusatzartikel der USVerfassung etwa Volokh/Falk, Journal of Law, Economics \& Policy 8 (2012), 883 (886 ff.); anders aber sowohl in Bezug auf die einfache Durchleitung von fremden Inhalten als auch auf die editierte Transmission der von Dritten erzeugten Inhalte Benjamin, Duke Law 
pher stellen sich die Betreiber danach als aktive Garanten der ,erhöhten Fläche“ dar, die mit ihrer Tätigkeit diese erst „erbaut" hätten. Diese Rollenzuschreibung hat oftmals strategische Gründe, die insbesondere auf ihre Position im politischen Diskurs (vor allem in den USA) ausgerichtet sind, wo das Argument jeweils abhängig von dem konkreten Sachbezug ebenfalls gegen Regulierung abschirmen bzw. diese abschwächen soll.

Dabei lässt sich eine gewisse Pfadabhängigkeit von dem US-amerikanisch radizierten Ideal der öffentlichen Kommunikation als „marketplace of ideas" ${ }^{\text {"13 }}$, also dem öffentlichen Marktplatz für den Austausch von Informationen und Meinungen, zur Entwicklung und Verwendung der Plattformmetapher kaum leugnen. Denn die Idealisierung eines solchen MarktplatzParadigmas setzt logisch einen physischen oder ideellen Ort voraus, der als solcher Marktplatz fungieren kann. Je größer dieser Ort ist, desto eher hat auch die „Suche nach der Wahrheit“, die die Proponenten des MarktplatzIdeals zum Ziel ausrufen, Aussicht auf Erfolg. ${ }^{14}$ Haftungsprivilegierungen wie $\$ 230 \mathrm{CDA}$, aber auch entsprechende Rechtsinstitute im hiesigen Kontext, ${ }^{15}$ helfen also den betroffenen Unternehmen, sich als virtueller Ort jener Tätigkeit darzustellen, die den Kern des Ideals eines solchen Marktplatzes der Ideen ausmacht. ${ }^{16}$ Idealisierend können sie von sich behaupten, ihre Plattform sei als ein solcher Marktplatz anzusehen. ${ }^{17}$

Journal 60 (2011), 1673 (1682 ff.); vgl. dazu auch Whitney, Knight First Amendment Institute (27.02.2018), knightcolumbia.org/content/search-engines-social-media-and-editorial-analogy.

${ }^{13}$ Vgl. dazu schon die Nachweise oben bei § 3 Fn. 70.

${ }^{14}$ Vgl. Medeiros, Social Media + Society 3 (2017), 1 (2).

${ }^{15}$ So verweist etwa BVerfG, Beschl. v. 25.06.2009 - 1 BvR 134/03, NJW-RR 2010, 470 (Rn. 66) - Pressespiegel, auf die „Rechtsfigur der Eröffnung eines Marktes der Meinungen“ im Kontext der Begründung von Haftungsprivilegierungen (Hervorh. v. Verf.).

${ }^{16}$ So auch Medeiros, Social Media + Society 3 (2017), 1 (2); dass man das Marktplatzideal indes auch anders instrumentalisieren kann, zeigt sich bei OLG München, Beschl. v. 24.08.2018 - 18 W 1294/18, NJW 2018, 3115 (Rn. 26) - Hassrede, wo das Gericht diese Funktion Facebook zuschreibt und deshalb zum Ergebnis kommt, das Unternehmen sei von der mittelbaren Drittwirkung der Meinungsfreiheit betroffen und könne deshalb keine unter Art. 5 GG zulässige Meinungsäußerung entfernen.

${ }^{17}$ Augenscheinlich sieht der Supreme Court dies ähnlich: „While in the past there may have been difficulty in identifying the most important places (in a spatial sense) for the exchange of views, today the answer is clear. It is cyberspace - the ,vast democratic forums of the Internet" in general [...], and social media in particular", Packingham v. North Carolina, 137 S.Ct. 1730, 1735 (2017). „These websites can provide perhaps the most powerful mechanisms available to a private citizen to make his or her voice heard. They allow a person with an Internet connection to ,become a town crier with a voice that resonates farther than it could from any soapbox '[...]", Packingham v. North Carolina, 137 S.Ct. 1730, 1737 (2017). 
Die Plattformmetapher erweist sich damit zumindest in den Augen mancher Beobachter als gerade flexibel genug, um im Sinne der betroffenen Unternehmen „the best of both worlds“ gewährleisten zu können. ${ }^{18}$ Sie erlaubt es, einen Mittelweg zwischen der Betonung ihrer Rolle als aktiver und passiver Mittler ${ }^{19} \mathrm{zu}$ beschreiten, um sich jeweils kontextadäquat zu positionieren. ${ }^{20}$ Diese Fähigkeit, unter einem Begriff verschiedene Rollen eines Akteurs $\mathrm{zu}$ besetzen, ist dabei keineswegs kontingent, sondern begründet sich wiederum in der beobachtbaren Tendenz, eine enorme Vielfalt sozialer Praxis unter einzelne Begriffe zu subsumieren und damit für ihre Verwender nicht mehr sichtbar zu machen. ${ }^{21}$ Das macht die Verwendung der PlattformMetapher einerseits so attraktiv, andererseits aber so schwer zu entziffern.

\section{Rechtliche Rezeption des Plattformbegriffs}

Der Begriff der Plattform hat im Recht an verschiedenen Orten eine Positivierung erhalten. Von Interesse sind diese im Folgenden, soweit sie ansatzweise einen Bezug zum Kontext dieser Untersuchung aufweisen. ${ }^{22}$ Die Rechtsordnung kennt den Plattformbegriff insbesondere im Rundfunkrecht, wo die $\S \S 52$ ff. RStV Plattformen im Hinblick auf Zugangs- und Vielfaltssicherung einer Regulierung unterwerfen. Damit soll vor allen Dingen der Konvergenz der Übertragungswege Rechnung getragen werden, die es Netzbetreibern ermöglicht, die vormals prägende Trennung zwischen Inhalt und Übertragungsweg $^{23} \mathrm{zu}$ überwinden und damit eine vertikale Integration in Medienmärkten zu etablieren. ${ }^{24} \mathrm{Im}$ rundfunkrechtlichen Sinne sind Plattformen lediglich in Bezug auf ihre Betreiber definiert: Plattformanbieter ist nämlich, ,,wer auf digitalen Übertragungskapazitäten oder digitalen Datenströmen Rundfunk und vergleichbare Telemedien (Telemedien, die an die Allgemeinheit gerichtet sind) auch von Dritten mit dem Ziel zusammenfasst, diese Angebote als Gesamtangebot zugänglich zu machen oder wer über die Auswahl für die Zusammenfassung entscheidet ". ${ }^{25}$ Die hierin zum Ausdruck kommende Konnotation des Plattformbegriffs lässt sich zwar nicht vollumfänglich, aber doch wesentlich dem im Duden beschriebenen Verständnis als „Ort zur Verbreitung von Ideen und Produkten“ zuordnen. Zugleich spricht sie, insbesondere wenn sie Infrastrukturunternehmen als „Flaschenhälse“

\footnotetext{
${ }^{18}$ So Volokh, zitiert bei Cohen, New York Times, 20.05.2012.

${ }^{19} \mathrm{Zu}$ den Implikationen dieser Konnotationen vgl. unten $\S 8$ B.

${ }^{20}$ Vgl. Gillespie, New Media \& Society 12 (2010), 347 (356 f.).

${ }^{21}$ Vgl. Seiler, in: Baechler et al., Medienlinguistik 3.0, S. 63 (68 mit Fn. 2).

${ }^{22}$ Ausgenommen ist damit insbesondere der kartellrechtliche Kontext; vgl. dazu nur Kumkar, Online-Märkte und Wettbewerbsrecht, passim.

${ }^{23}$ Dazu schon oben $\$ 4$ Fn. 297.

${ }^{24}$ Vgl. Wagner, in: Binder/Vesting, Rundfunkrecht, RStV $\S 52$ Rn. 4.

${ }^{25} \S 2$ Abs. 2 Nr. 13 Hs. 1 RStV.
} 
problematisiert, ${ }^{26}$ auch in der Sache Parallelen zu den hier relevanten Konstellationen und den betroffenen Unternehmen an, die ebenfalls den Zugang zum Endkunden steuern und damit entscheiden können, welche Inhalte diese wie wahrnehmen können. ${ }^{27}$

Die Verwendung des Plattformbegriffs in der Rechtsprechung lässt sich durchaus als etabliert bezeichnen. Bezog der BGH in Zivilrechtsstreitigkeiten anfangs den Begriff tatsächlich auf einen physischen Gegenstand, ${ }^{28}$ verwendete das Gericht bereits in den 1960er-Jahren in Strafsachen als Metapher. ${ }^{29}$ In zivilrechtlichen Streitigkeiten gewann die Metapher erst zu Beginn des 21. Jahrhunderts an Bedeutung, als die ersten Versteigerungsportale im Internet ihren Betrieb aufnahmen.$^{30}$ Eine begriffliche Reflexion fand jedoch, soweit ersichtlich, in der Rechtsprechung kaum statt. Semantisch interessant erscheint hier noch eine Entscheidung des BVerfG zur Zulässigkeit von Werbemaßnahmen von Kliniken, bei denen das Gericht von deren Darstellung auf eigenen Internetpräsenzen als „passive Darstellungsplattform“ spricht. ${ }^{31}$

Zwar sollte man den Einfluss solcher Begriffsverwendungen in einzelnen Judikaten nicht überbewerten. Es liegt allerdings mehr als nahe, dass insbesondere zu einem solch frühen Zeitpunkt der Entwicklung des Lebensbereichs Internet die Bezeichnung einer Internetpräsenz als ,passive Plattform“ semantisch überaus prägende Kraft mit Auswirkungen auch auf juristische Verständnisse und Konzepte entwickelte. Die normative Erwartung des EuGH im Kontext der Haftungsprivilegierungen für Host-Provider, die passiv bzw. neutral zu sein haben, ${ }^{32}$ liefert hierfür ein beredtes Zeugnis ab. Ein weiteres anschauliches Beispiel findet sich im jüngst erfolgten Vorlagebeschluss des BGH, mit dem das Gericht Fragen zur Anwendung der InfoSocRL und der ECRL auf Urheberrechtsverstöße über das Angebot von YouTube dem EuGH zur Vorabentscheidung gem. Art. 267 AEUV vorlegte. ${ }^{33} \mathrm{Im}$ Hinblick auf die Anwendbarkeit der Haftungsprivilegierung des Art. 14 ECRL auf eine „Internetplattform“ sei im Grundsatz maßgeblich, ob der

${ }^{26}$ Vgl. Wagner, in: Binder/Vesting, Rundfunkrecht, RStV $\$ 52$ Rn. 16.

${ }^{27}$ Gleichwohl sind die $\$ \S 52 \mathrm{ff}$. RStV selbst materiell kaum auf soziale Medien zugeschnitten, vgl. Schiff, MMR 2018, 366 (367). Zur Einordnung unter die Gatekeeper-Diskussion vgl. noch unten $\S 9$ A.

${ }^{28}$ Vgl. BGH, Beschl. v. 04.03.1957 - GSZ 1/56, juris, Rn. 2: Der Kläger des Verfahrens verletzte sich beim Besteigen der vorderen "Plattform des Motorwagens“.

${ }^{29}$ Vgl. BGH, Urt. v. 04.06.1964 - 3 StR 5/64, juris, Rn. 17: die KPD versuche, sich ,in der Bundesrepublik eine Plattform zu schaffen“.

${ }^{30}$ Vgl. BGH, Urt. v. 07.11.2001 - VIII ZR 13/01, juris, Rn. 40: „Auktionsplattform“.

${ }^{31}$ BVerfG, Beschl. v. 17.07.2003 - 1 BvR 2115/05, juris, Rn. 20 - Klinikwerbung im Internet; vgl. im Anschluss hieran auch BGH, Urt. v. 09.10.2003 - I ZR 167/01, juris, Rn. 36 - Arztwerbung im Internet.

${ }^{32} \mathrm{Vgl}$. oben $\S 4$ C. II. 3.

${ }^{33}$ Vgl. BGH, Beschl. v. 13.09.2018 - I ZR 140/15, GRUR 2018, 1132 ff. - YouTube. 
betreffende Anbieter sich darauf beschränke, seine Leistung „mittels rein technischer und automatischer Verarbeitung der von seinen Kunden eingegebenen Daten neutral zu erbringen". ${ }^{34}$ Sei dies nicht der Fall, spiele er also eine ,aktive Rolle, [...] die ihm eine Kenntnis dieser Daten oder eine Kontrolle über sie [verschaffe]", komme eine Privilegierung nicht mehr in Betracht. ${ }^{35}$ Auf die Bewertung dieser Abgrenzungen wird noch zurückzukommen sein. ${ }^{36}$ Hier ist jedenfalls deutlich sichtbar, wie die Abgrenzung von Aktivität und Passivität semantisch stark mit dem Plattformbegriff verknüpft ist und dieser dadurch normatives Potenzial entfaltet.

Auch im NetzDG finden Plattformen prominent Erwähnung. So ist insbesondere bei der Bestimmung des Gegenstandes und Anknüpfungspunktes des Gesetzes in $\S 1$ Abs. 1 S. 1-3 NetzDG nur von Plattformen die Rede. Gleichwohl hat sich insoweit, nicht zuletzt aufgrund des Titels des Gesetzes und der in $\S 1$ Abs. 1 S. 1 NetzDG vorgenommenen Legaldefinition, eher die Diktion der sozialen Netzwerke etabliert. Auf diese ist sogleich einzugehen.

\section{II. Überzeugungskraft}

Trotz der soeben dargelegten unterschiedlichen Bedeutungsebenen, die dem Plattformbegriff zugewiesen werden können, prägt die zu Beginn erläuterte Konnotation des Begriffs, die gerade implizit und damit oftmals ohne eine bewusste Reflexion Wirkungen entfaltet, nach hier vertretener Auffassung das Verständnis der Plattform-Metapher. ${ }^{37}$

Es liegt zwar im Wesen von Metaphern begründet, dass diese in Funktion oder Beschaffenheit nicht mit dem beschriebenen Begriff identisch sein können. ${ }^{38}$ Das Plattformparadigma vermag dennoch für die Zwecke dieser Untersuchung auch deshalb nicht zu überzeugen, weil es - semantisch äußerst suggestiv - davon ausgeht, ,auf“ der Plattform bestünde wie auch auf einer physischen Plattform, einem Gleis, eine halbwegs gleichwertige (flache) Ausgangslage für jeden, der sie betritt, ein „level playing field“. Gerade weil aber

${ }^{34}$ BGH, Beschl. v. 13.09.2018 - I ZR 140/15, GRUR 2018, 1132 (Rn. 42) - YouTube; im Ansatz kritisch hierzu Riordan, The Liability of Internet Intermediaries, S. 32 (,fails to supply an all-encompassing definition").

${ }^{35}$ BGH, Beschl. v. 13.09.2018 - I ZR 140/15, GRUR 2018, 1132 (Rn. 42) - YouTube.

${ }^{36} \mathrm{Vgl}$. unten $\S 8 \mathrm{~B}$.

${ }^{37}$ Kritische Einwände, die wie etwa Andreessen, blog.pmarca.com (16.09.2007), pmarchive.com/three_kinds_of_platforms_you_meet_on_the_internet.html, eine Reduktion des Begriffs bzw. die Rückkehr zu dessen informationstechnischer Grundlage forderten, können mittlerweile als gescheitert angesehen werden.

${ }^{38}$ Ansonsten handelte es sich nämlich schlichtweg um eine Tautologie, wie Gillespie, Alexander von Humboldt Institut für Internet und Gesellschaft (24.08.2017), www.hiig.de/ die-plattform-metapher-revisited, zutreffend bemerkt. 
die Zusammenstellung bzw. Kuratierung und Sortierung von Inhalten durch die betreffenden Unternehmen sehr stark von den divergierenden Interessen einzelner Nutzer, Gruppen, Staaten oder Staatengemeinschaften abhängt, liegt schon die Annahme eines solchen „level playing field" in Bezug auf das Informations- und Kommunikationsverhalten aller Nutzer einer Plattform eher fern. Wenn für verschiedene Akteure Informationen und Inhalte unterschiedlich verfügbar sind, ${ }^{39}$ kann es zumindest zwischen diesen Akteuren keine gemeinsame Tatsachengrundlage im Sinne der identischen Verfügbarkeit von Inhalten geben. Bestenfalls bestehen sich überlappende Informationssphären, schlechtestenfalls kommt es zur Bildung der vielfach erwähnten Filterblasen. ${ }^{40}$ In keinem Fall aber kann in einem solchen Umfeld von ebenen Plattformen in einem metaphysischen Sinne gesprochen werden, auf denen allen Nutzern eine identische Informationsumgebung zur Verfügung steht. ${ }^{41}$ Auch die Rolle der die „Plattform“ betreibenden Unternehmen erweckt, wie bereits oben angeklungen ist, Skepsis. Denn es ist schlichtweg nicht realistisch, bei solchen privat beherrschten Umgebungen, in denen die Unternehmen rechtliche, ökonomische, technische und nicht zuletzt kulturelle Bedingungen beeinflussen, ${ }^{42}$ von einer im praktischen Sinne ähnlich be-

${ }^{39} \mathrm{Vgl}$. etwa Gillespie, Custodians of the Internet, S. $187 \mathrm{ff}$.

${ }^{40}$ Pariser, The Filter Bubble, passim; vgl. auch Sunstein, Yale Law Journal 110 (2000), 71 ( $78 \mathrm{ff}$.). Die notwendige Konsequenz hieraus, das erforderliche Aufbrechen solcher Polarisierungsprozesse durch die „Arbeit des Negativen“, findet dialektische Anklänge bereits bei Hegel, Phänomenologie des Geistes, S. 27: „Aber nicht das Leben, das sich vor dem Tode scheut und von der Verwüstung rein bewahrt, sondern das ihn erträgt und in ihm sich erhält, ist das Leben des Geistes, Er gewinnt seine Wahrheit nur, indem er in der absoluten Zerrissenheit sich selbst findet. Diese Macht ist er nicht als das Positive, welches von dem Negativen wegsieht, wie wenn wir von etwas sagen, dies ist nichts oder falsch, und nun, damit fertig, davon weg zu irgend etwas anderem übergehen; sondern er ist diese Macht nur, indem er dem Negativen ins Angesicht schaut, bei ihm verweilt, Dieses Verweilen ist die Zauberkraft, die es in das Sein umkehrt. "(Hervor. v. Verf.). Dass die Einbindung von konträren oder abweichenden Perspektiven in ein Gruppengefüge der Polarisierung entgegenwirken kann, zeigt Postema, The Harvard Review of Philosophy 17 (2010), 36 (41 ff.), auf, der darauf hinweist, dass deliberativer Konsens schon auf prinzipieller Ebene nur ein regulatives Ideal darstelle, das einen am Ende immer noch bestehenden Dissens auch konzeptionell zu integrieren vermöge. Der Konsens stehe dagegen eher im Verdacht, ein Symptom zu großer sozialer Homogenität zu sein und damit das Produkt deliberativer Dysfunktion darzustellen.

${ }^{41}$ Gerade hierin unterscheiden sich die Intermediäre als Informationsmedien fundamental von klassischen Medien. Letztere erlauben die Verhandlung von Informationen und Meinungen auf gemeinsamer Grundlage, während ersteren der ,common corpus of public discourse" abgeht, wie Gillespie, Custodians of the Internet, S. 195, zutreffend feststellt (ohne aber vom Plattformparadigma an dieser Stelle abzurücken).

${ }^{42}$ Vgl. Burgess/Green/Rebane, in: Friese et al., Handbuch Soziale Praktiken und Digitale Alltagswelten, S. 1 (2): YouTube als „Mäzen“ (Patron) kollektiver Kreativität. 
einflussungsfreien Umgebung zu sprechen, wie es beim Erbauer und Betreiber eines physisch hervorgehobenen, allen in gleicher Weise zur Verfügung stehenden Ortes zum Austausch der Fall ist. Schließlich hilft die Metapher dabei, die Verantwortung der Betreiberunternehmen zu verwässern bzw. sie vor dieser Frage abzuschirmen. Denn so wie der Betreiber einer physischen Plattform nicht für das Verhalten der auf ihr laufenden Personen verantwortlich ist, kann dies nach dieser Lesart auch bei den digitalen Plattformen der Fall sein. ${ }^{43}$ Semantisch kann eine Plattform durchaus Infrastruktur und zugleich Institution sein, ${ }^{44}$ logisch nicht.

Dennoch ist die Verwendung des Begriffs in Theorie ${ }^{45}$ und Praxis ${ }^{46}$ durchaus verbreitet bzw. etabliert. Dies mag durchaus mit einer längeren Verwendungstradition und der Eingängigkeit sowohl im Alltag als auch in spezifischen Gruppen der Gesellschaften, aber eben auch mit einem assoziationsreichen und gerade deswegen diffusen Begriffsverständnis im oben erläuterten Sinne zusammenhängen. Ist damit bereits nicht zu erwarten, dass die Verwendung der Metapher sich eines Tages überwinden lassen wird, so sollten diese Gedanken jedenfalls zu einem reflektierteren Umgang mit ihr anregen.

\section{B. Soziale Netzwerke}

Ebenso in allgemeinen wie auch in fachspezifischen Diskursen ist außerdem der Begriff des ,sozialen Netzwerks“ sehr häufig anzutreffen. Auch insoweit lohnt sich eine tiefergehende Reflexion.

${ }^{43}$ Gillespie, Alexander von Humboldt Institut für Internet und Gesellschaft (24.08.2017), www.hiig.de/die-plattform-metapher-revisited.

${ }^{44}$ Auf die Spitze getrieben bei Klonick, Harvard Law Review 131 (2018), 1598 (1603 f.): „These platforms are both the architecture for publishing new speech and the architects of the institutional design that governs it" (Hervorh. v. Verf.).

${ }^{45}$ Aus medienlinguistischer Perspektive vgl. Seiler, in: Baechler et al., Medienlinguistik 3.0, S. 63 (71 ff.).

${ }^{46}$ Zumindest auf der Ebene der Organe der EU taucht der Begriff sowohl in offiziellen Dokumenten (Europäische Kommission, Umgang mit illegalen Online-Inhalten, $\operatorname{COM}(2017) 555$ final) als auch in Organigrammen, insbesondere bei der Bezeichnung des für die ECRL zuständigen Referats F.2 „Elektronischer Handel \& Plattformen“ der Generaldirektion CONNECT, vgl. ec.europa.eu/info/sites/info/files/organisation_charts/or ganisation-chart-dg-connect_de.pdf. 


\section{Begriffsverständnisse}

\section{Allgemeines Verständnis}

Netzwerke verweisen typischerweise auf eine dezentral-heterarchische Kultur der menschlichen Kommunikation, in der die Rollen von Sender und Empfänger variabel sind und die sich durch eine gewisse Dynamik auszeichnet. ${ }^{47}$ In diesem Sinne steht der Begriff des Netzwerks auch für ein allgemeines, viele Fachdiskurse durchziehendes und diese prägendes Kulturphänomen, das eng mit der Etablierung der Computer und insbesondere des Internets als Technologie der Massen zusammenhängt. ${ }^{48}$ „Neben die „Gesellschaft der Individuen“ und die „Gesellschaft der Organisationen“ tritt nach Vesting die „Gesellschaft der Netzwerke“. ${ }^{49}$ Der insoweit eher positiven Konnotation werden aber ebenso problematische Implikationen der Vernetzung gegenübergestellt. So taugt etwa nach Gärditz die Vernetzung eher als „rechtswissenschaftliche Problembeschreibung “, ${ }^{50}$ denn „Netzwerke entindividualisieren Zurechnung und lassen Verantwortlichkeit diffundieren". ${ }^{51}$

Soziale Netzwerke - als Begriff wohl erstmals 1969 notiert $^{52}$ - bezeichnen zunächst generell „Erscheinungsform[en] sozialer Interaktionsstrukturen “..$^{53}$ Nach verbreitetem, jedenfalls aber nicht exklusivem Gebrauch sind mit sozialen Netzwerken hier im Ausgangspunkt internetbasierte Kommunikationsmedien gemeint, die zum Austausch von Informationen zwischen ihren „Mitgliedern“" ${ }^{44}$ genutzt werden. ${ }^{55}$ Sie zeichnen sich dadurch aus, „dass sie es ermöglichen, Informationen aller Art mithilfe der digital vernetzten Medien anderen zugänglich zu machen " ${ }^{\text {"56 }}$ und zur Pflege bestehender und Knüpfung neuer sozialer Beziehungen genutzt werden können. ${ }^{57}$ Der Begriff entbehrt allerdings nicht einer gewissen Redundanz insoweit, als Medien per se „sozial" sind, weil sie Bestandteil von sozialem Handeln sind..$^{58}$

${ }^{47}$ Vgl. Vesting, Die Medien des Rechts: Computernetzwerke, S. 56.

${ }^{48}$ Vgl. Vesting, Die Medien des Rechts: Computernetzwerke, S. $151 \mathrm{ff}$.

${ }^{49}$ Vesting, Die Medien des Rechts: Computernetzwerke, S. 153.

${ }^{50}$ Gärditz, Der Staat 54 (2015), 113 (114).

${ }^{51}$ Gärditz, Der Staat 54 (2015), 113 (114).

${ }^{52}$ Vgl. Kleiner, Die urheberrechtliche Wirksamkeit von Nutzungsbedingungen sozialer Netzwerke, S. 19.

${ }^{53}$ Kähler, Zeitschrift für Soziologie 1975, 283 (283).

${ }^{54} \mathrm{Ob}$ man sich tatsächlich als Mitglieder registrieren muss, ist freilich von Fall zu Fall unterschiedlich.

${ }^{55} \mathrm{Vgl}$. Gieseler, Öffentliche Kommunikation auf sozialen Netzwerkplattformen, S. 14 f.; Ziegler, Urheberrechtsverletzungen durch Social Sharing, S. 10.

${ }^{56}$ Taddicken/Schmidt, in: Schmidt/Taddicken, Handbuch Soziale Medien, S. 3 (4).

${ }^{57}$ Vgl. Taddicken/Schmidt, in: Schmidt/Taddicken, Handbuch Soziale Medien, S. 3 (5).

${ }^{58}$ Vgl. Taddicken/Schmidt, in: Schmidt/Taddicken, Handbuch Soziale Medien, S. 3 (4). 
Die der breiten Allgemeinheit geläufigen Netzwerke sind dabei thematisch offen. ${ }^{59}$ Allerdings herrscht auch insoweit keine einheitliche funktionale Beschreibung zur Abgrenzung verschiedener Dienste. Denkbar ist eine allgemeine Oberkategorie, die sog. Messenger-Dienste (z.B. WhatsApp oder Snapchat), Blogging-Dienste (z.B. Twitter), Bewertungsportale und ,soziale Netzwerke im engeren Sinne“" (insbesondere Facebook) vereint. ${ }^{60}$ Zwecks schärferer Konturierung lassen sich aber die genannten Kategorien auch begrifflich trennen, sodass nur Dienste wie Facebook subsumiert werden. ${ }^{61}$

\section{Rechtliche Rezeption}

Mit $\S 1$ NetzDG hat der Terminus „soziales Netzwerk“ eine prominente Legaldefinition erfahren. Hiernach sind soziale Netzwerke „Telemediendiensteanbieter, die mit Gewinnerzielungsabsicht Plattformen im Internet betreiben, die dazu bestimmt sind, dass Nutzer beliebige Inhalte mit anderen Nutzern teilen oder der Öffentlichkeit zugänglich machen" ". ${ }^{62}$ Ausgenommen sind Plattformen mit selbst verantworteten journalistisch-redaktionellen gestalteten Inhalten. Gesetzgeberische Intention der Bestimmung des Anwendungsbereichs auf diese Weise ist es, ,in erster Linie Dienste im Sinne von $\S 10$ $\mathrm{TMG}^{\text {“ }} \mathrm{zu}$ erfassen. ${ }^{63}$ Die Begründung des Regierungsentwurfs geht insoweit davon aus, dass jedenfalls im Zeitpunkt der Verabschiedung drei Netzwerkbetreiber vom NetzDG erfasst werden, ohne diese zu benennen, und ,bei weiteren sieben Netzwerken [...] eine Einbeziehung denkbar" ist. ${ }^{64}$ Bei den genannten drei Netzwerkanbietern dürfte es sich um Facebook, Twitter und YouTube handeln.

${ }^{59}$ Vgl. Müller-Terpitz, in: Eifert/Gostomzyk, Netzwerkrecht, S. 45 (45).

${ }^{60}$ Vgl. Müller-Terpitz, in: Eifert/Gostomzyk, Netzwerkrecht, S. 45 (45).

${ }^{61}$ In diese Richtung wohl Europäische Kommission, Umgang mit illegalen OnlineInhalten, COM(2017) 555 final, S. 2. Chmelik, Social Network Sites - Soziale Netzwerke, S. 39 f., 210 f., lehnt etwa die Subsumtion von Twitter unter den Begriff ab, weil dort die Pflege sozialer Kontakte nicht im Vordergrund stünde, eine gegenseitige Bestätigung der Beziehung („Freundschaft“) nicht erforderlich sei, der Dienst also primär zur Verbreitung und Gewinnung von Informationen diene. Das trifft zwar wohl überwiegend zu, allerdings konzediert er selbst, dass auch Dienste wie Twitter „gemeinschaftliche Strukturen“ aufwiesen (S. 211). Jedenfalls semantisch ist die Differenzierung daher alles andere als zwingend.

${ }^{62}$ Die Einschränkung auf Unternehmen mit Gewinnerzielungsabsicht erfolgt hier aus kompetenzrechtlichen Gründen, vgl. dazu Schiff, MMR 2018, 366 (366 f.) Damit ist indes nichts über das richtige kommunikationswissenschaftliche Verständnis gesagt, vgl. MüllerTerpitz, in: Eifert/Gostomzyk, Netzwerkrecht, S. 45 (46).

${ }^{63}$ BT-Drucks. 18/12356, S. 18.

${ }^{64}$ BT-Drucks. 18/12356, S. 16. Zur Kritik insoweit vgl. Liesching, in: Spindler/Schmitz, TMG, NetzDG $\S 1$ Rn. 36 ff. 
Im Unionsrecht sind soziale Netzwerke mittlerweile ebenfalls rezipiert. So verweist die RL (EU) 2018/1808, die die sog. AVMD-RL anpasst, in den Erwägungsgründen 4 und 5 auf soziale Netzwerke, ohne diese aber näher zu erläutern. Im Wettbewerbsrecht hat der Netzwerkbegriff ebenfalls kürzlich eine prominente Erwähnung erfahren. So sind in der bereits erwähnten Regelung in $\S 18$ Abs. IIIa GWB ${ }^{65}$ Netzwerke ebenfalls angesprochen und als „mehrseitige Märkte“ definiert. Diese Regelung wird weit ausgelegt und soll die hier gemeinten sozialen Netzwerke daher ebenfalls erfassen. ${ }^{66}$

\section{II. Überzeugungskraft}

In seiner grundlegenden Konnotation als Geflecht menschlicher Beziehungen stellt der Begriff ,soziales Netzwerk" kein Spezifikum des Internets dar. ${ }^{67}$ Schon dies stellt seine Eignung als tauglicher Anknüpfungspunkt in Frage. ${ }^{68}$ Zudem blendet auch er den Netzwerkbetreiber als zentralen Akteur mit erheblichen Entscheidungs- und Einwirkungsbefugnissen aus ${ }^{69}$ wenngleich nicht in gleichem Ausmaß, wie es beim Plattform-Begriff der Fall ist. Es ist bei der Verwendung des Begriffs jedenfalls unklar bzw. nicht durchweg konsentiert, dass mit „sozialen Netzwerken“ die Betreiber selbst und gerade nicht die Gesamtheit der auf ihnen registrierten und miteinander verbundenen Mitgliedern gemeint ist. Auch dieser Begriff eignet sich daher nicht zur präzisen Beschreibung derjenigen Akteure, die den Gegenstand dieser Untersuchung darstellen. ${ }^{70}$

\footnotetext{
${ }^{65}$ Vgl. dazu schon oben $§ 5$ Fn. 25.

${ }^{66}$ Müller-Terpitz, in: Eifert/Gostomzyk, Netzwerkrecht, S. 45 (46 f.).

${ }^{67}$ Übersicht bei Kähler, Zeitschrift für Soziologie 1975, 283 (285 ff.).

${ }^{68}$ Vgl. Chmelik, Social Network Sites - Soziale Netzwerke, S. 37; Vesting, Die Medien des Rechts: Computernetzwerke, S. 57.

${ }^{69}$ Plastisches Beispiel bei Ladeur, in: Bommes/Tacke, Netzwerke in der funktional differenzierten Gesellschaft, S. 143 (160), der für Bewertungsdienste von einer „selbstorganisierten Entstehung von Regeln" spricht, ohne dabei die Architektur der entsprechenden Berechnungsregeln durch die Betreiber in den Blick zu nehmen.

${ }^{70}$ Chmelik, Social Network Sites - Soziale Netzwerke, S. 38 f., meint dem Problem durch Verwendung des Begriffs „,social network service“ aus dem Weg gehen zu können, weil hier die Funktion als Dienstleistung sichtbar werde; ähnlich wohl Pille, Meinungsmacht sozialer Netzwerke, S. 88 ff., oder auch Beher/Hilgert/Mämecke, in: Bommes/Tacke, Netzwerke in der funktional differenzierten Gesellschaft, S. 289 (289 ff.): „social network platforms"/SNP. Das ist aus der akteursbezogenen Perspektive nachvollziehbar, geht aber nicht hinreichend auf die Tätigkeit der Unternehmen ein.
} 


\section{Diensteanbieter}

Sucht man also nach einem Begriff, der semantisch deutlich macht, welches Phänomen er adressieren will, liegt der Begriff Diensteanbieter tatsächlich um ein Vielfaches näher.

\section{Begriffsverständnisse}

\section{Allgemeines Verständnis}

Der Begriff Diensteanbieter bezeichnet laut Duden eine „Person oder Einrichtung, Firma, die gegen Gebühr bestimmte Dienste, eine Dienstleistung anbietet ". ${ }^{\text {.1 }}$ Damit ist zum einen deutlich, wer gemeint ist, nämlich eine mit einer bestimmten Absicht handelnde Person. Zum anderen steht hier eindeutig die wirtschaftliche Komponente im Vordergrund. Ein Diensteanbieter ist also ein primär mit wirtschaftlichen Motiven tätiger Akteur. Auf einen bestimmten Bereich lässt sich der Begriff in seinem allgemeinen Verständnis dagegen nicht beschränken, sodass der Zusammenhang zum digitalen Raum sich nicht ohne Weiteres jedem erschließt.

\section{Rechtliche Rezeption}

Der Begriff des Diensteanbieters ist rechtlich breit rezipiert. Er ist insbesondere Anknüpfungspunkt für die Vorschriften zur Haftung in der ECRL und in den $\S \S 7$ ff. TMG. Im TMG, das insoweit auf die entsprechende Bestimmung des TDG zurückgeht, ${ }^{72}$ ist der Diensteanbieter legaldefiniert als ,jede natürliche oder juristische Person, die eigene oder fremde Telemedien zur Nutzung bereithält oder den Zugang zur Nutzung vermittelt" ${ }^{.73}$ Erst in der rechtlichen Rezeption wird der Begriff auf internetbasierte Anwendungen beschränkt und damit gegenüber ,analogen“ Dienstleistungen abgegrenzt, selbst wenn diese in Form von Datenträgern erbracht werden. ${ }^{74}$

Der unionsrechtlich determinierte Begriff des „Dienstes der Informationsgesellschaft" verweist sekundärrechtlich auf die Bestimmung des Art. 1 Abs. 1 lit. b RL (EU) 2015/1535 als Nachfolgervorschrift, die wiederum von Art. 2 lit. a ECRL in Bezug genommen wird. Primärrechtlich ist durchaus eine Nähe zur Grundfreiheit des Dienstleistungsverkehrs gegeben, ${ }^{75}$ ohne

${ }^{71}$ www.duden.de/rechtschreibung/Diensteanbieter.

${ }^{72}$ Vgl. Spindler, in: Spindler/Schmitz, TMG, §2 Rn. 1.

${ }^{73} \S 2$ S. 1 Nr. 1 Hs. 1 TMG.

${ }^{74} \mathrm{Vgl}$. Kovacs, Die Haftung der Host-Provider für persönlichkeitsrechtsverletzende Internetäußerungen, S. 60.

${ }^{75}$ Vgl. Marly, in: Grabitz/Hilf/Nettesheim, Recht der EU, 40. EL 2009, ECRL Art. 2 Rn. 15. 
aber die Abgrenzungen der Dienstleistungsfreiheit zu übernehmen. ${ }^{76}$ Prominent wird der Begriff nunmehr auch in der DSM-RL aufgegriffen. ${ }^{77}$ Diese gestaltet den Begriff für den dort geregelten Bereich des Urheberrechts fort, indem die Kategorie „Diensteanbieter für das Teilen von Online-Inhalten“ neu geschaffen wird. Diese ist gemäß Art. 2 Nr. 6 UAbs. 1 DSM-RL definiert als

„Anbieter eines Dienstes der Informationsgesellschaft, bei dem der Hauptzweck bzw. einer der Hauptzwecke darin besteht, eine große Menge an von seinen Nutzern hochgeladenen, urheberrechtlich geschützten Werken oder sonstigen Schutzgegenständen zu speichern und der Öffentlichkeit Zugang hierzu zu verschaffen, wobei dieser Anbieter diese Inhalte organisiert und zum Zwecke der Gewinnerzielung bewirbt."

Ausweislich Erwägungsgrund 62 S. 2 DSM-RL sollen nur solche Dienste erfasst sein, „die auf dem Markt für Online-Inhalte eine wichtige Rolle spielen, indem sie mit anderen Online-Inhaltsdiensten, wie Audio- und VideoStreamingdiensten, um dieselben Zielgruppen konkurrieren ". Dies betrifft in diesem Kontext insbesondere, aber nicht ausschließlich den Dienst YouTube. ${ }^{78}$ Für die nicht erfassten Diensteanbieter gelten weiterhin die allgemeinen Regeln der (Sekundär-)Haftung, in Deutschland also etwa der Störerhaftung, und zwar unabhängig davon, ob dies im Einzelfall vorteilhaft oder nachteilig für den betreffenden Diensteanbieter ist. ${ }^{79}$

\section{II. Überzeugungskraft}

Für den Begriff der Diensteanbieter streitet primär, dass er im Gegensatz zu den vorher diskutierten Begrifflichkeiten bei weitem nicht so vielen verschiedenen Bedeutungsebenen zugänglich ist. Vielmehr ist seine Bedeutung eindeutig funktional konnotiert und damit in personaler Hinsicht leicht handhabbar. Dafür ist er allerdings nicht trennscharf der digitalen oder analogen Sphäre zuordenbar. Die Stärke des Begriffs ist damit zugleich seine Schwäche. Mag das „Anbieten eines Dienstes“ zwar nicht semantisch so aufgeladen sein wie die oben erläuterten Begriffe, insbesondere wie derjenige der Plattform, so ist der Begriff umgekehrt so unspezifisch, dass eine funktionelle Zuschreibung über die reine Aktivität des Anbietens kaum möglich ist. Insofern weist der Begriff „Diensteanbieter“ die Eigenschaft auf, die die Plattformmetapher lediglich, wenngleich äußerst suggestiv, insinuiert: Der Anbieter tut nicht viel mehr, als einen Dienst anzubieten. Darüber hinaus weist

${ }^{76}$ Spindler, in: Spindler/Schmitz, TMG, §2 Rn. 2; Husovec, Injunctions against Intermediaries in the European Union, S. 88.

${ }^{77}$ Ausführlicher dazu unten $\S 12$ B.

${ }^{78}$ Vgl. Hofmann, ZUM 2019, 617 (618).

${ }^{79}$ Vgl. Dreier, GRUR 2019, 771 (775). 
der Begriff keinerlei Spezifika auf, die seine Verwendung im Hinblick auf diese Untersuchung als besonders dringlich erscheinen lassen würden.

Wenn der Begriff bislang und im Folgenden dennoch Verwendung findet, liegt dies zum einen an pragmatischen Erwägungen stilistischer oder syntaktischer Natur. Zum anderen wäre insbesondere in Anbetracht der Verwendung des Begriffs im geltenden Recht, insbesondere im TMG, eine vollständige Ausblendung des Begriffs untunlich, wenn der genaue Wortlaut oder der Zweck einzelner Bestimmungen oder Gerichtsentscheidungen herausgearbeitet werden soll.

\section{Vorzugswürdiger Begriff: (Informations-)Intermediäre}

Im Sinne des genannten Ziels, ein reflektiertes begriffliches Vorverständnis an den Anfang der kritischen Reflexion zu stellen, ist der Begriff (Informations-)Intermediär am geeignetsten, wie im Folgenden kurz aufzuzeigen ist.

\section{Begriffsverständnisse}

\section{Allgemeines Verständnis}

Der Begriff „Intermediär“ eine artifizielle Schöpfung. Der Duden kennt ihn lediglich als Adjektiv und schreibt ihm die Bedeutung als ,in der Mitte liegend“, „dazwischen befindlich“ und „ein Zwischenglied bildend“ zu. ${ }^{80}$ Das Substantiv kann folglich „Mittler" bzw. „Vermittler“ oder Zwischen- bzw. Bindeglied bedeuten. Mancherorts wird diese Vermittlerfunktion noch qualifiziert, etwa dahingehend, dass der Intermediär eine Voraussetzung „dafür schafft, dass der unmittelbar Handelnde seine Tätigkeit ausüben kann. “81 Informationsintermediären kann folglich funktional die Vermittlung von Informationen zugeschrieben werden. ${ }^{82}$ Der Begriff, wie er in diesem Kontext immer häufiger verwendet wird, beschränkt sich jedoch nicht auf eine einfache Vermittlungsfunktion oder gar nur auf „(inhalts-)neutrale“ Tätigkeiten.$^{83}$ Denn neben der reinen Vermittlungsfunktion erschließen, sortieren und verbreiten Intermediäre diese Inhalte. ${ }^{84}$ Demgemäß versteht die OECD unter „Internet Intermediaries“" solche Dienste, die ihre Nutzer im Internet miteinander verbinden oder Transaktionen zwischen ihnen erleichtern. „They give

\footnotetext{
${ }^{80} \mathrm{Vgl}$. www.duden.de/rechtschreibung/intermediaer.

${ }^{81}$ Ohly, ZUM 2015, 308 (308).

${ }^{82}$ Vgl. Schulz/Dankert, Die Macht der Informationsintermediäre, S. 15.

${ }^{83}$ So aber etwa noch der Dreizehnte Zwischenbericht der Enquete-Kommission „Internet und digitale Gesellschaft" v. 19.03.2013, BT-Drucks. 17/12542, S. 16.

${ }^{84}$ Vgl. Lischka, itrb 2018, 235 (236); ausführlich dazu noch unten $\S 8$ A. II. 2.
} 
access to, host, transmit and index content, products and services originated by third parties on the Internet or provide Internet-based services to third parties. " 85

Oft sind qualifizierende Begrifflichkeiten anzutreffen, insbesondere die Informationsintermediäre ${ }^{86}$ aber auch Medienintermediäre ${ }^{87}$ oder Internetintermediäre. ${ }^{88,89}$ Entscheidend scheint jedenfalls zu sein, dass sie Kommunikation im Internet ermöglichen. ${ }^{90}$ Gerade bei der Verwendung dieses Begriffs wird aber mitunter zusätzlich (begriffliche) Verwirrung gestiftet, wenn Plattformen als Teilbereich von Intermediären bezeichnet werden..$^{91}$ Dies mag allerdings auch dafür sprechen, dass die Verwendung des Begriffs der Plattform mittlerweile derart konsolidiert ist, dass ein solcher referenzieller Begriffsgebrauch nicht mehr als ungewöhnlich bezeichnet werden kann.

Welche Bereiche von Diensten der Begriff umfassen soll, wird nicht einheitlich bewertet. Während manche auch Internetanschlussanbieter hierunter fassen wollen, ${ }^{92}$ ziehen andere den Begriff enger. ${ }^{93}$ Die bereits angesprochene Definition der OECD erfasst etwa auch die Access-Provider. ${ }^{94}$ Gegen ein so weites Verständnis spricht allerdings, dass Access-Provider nach herkömmlichen Verständnis nur den Zugang zum Internet als solchen vermitteln, nicht jedoch den unmittelbaren Zugang zu Inhalten..$^{95}$ Die Prägung des

${ }^{85}$ Secretary-General of the OECD, The Economic and Social Role of Internet Intermediaries, S. 9; ders., The Role of Internet Intermediaries in Advancing Public Policy Objectives, S. 20.

${ }^{86} \mathrm{Vgl}$. Schulz/Dankert, Die Macht der Informationsintermediäre, S. $15 \mathrm{ff}$.

${ }^{87}$ Vgl. Paal, Intermediäre, S. 18 ff.

${ }^{88}$ Vgl. Wagner, in: MüKo BGB, § 823 Rn. 838; Lang, AöR 143 (2018), 220 (221); Kellner, Die Regulierung der Meinungsmacht von Internetintermediären, S. 21 ff. und passim.

${ }^{89}$ Die im Bereich des Kapitalmarktrechts ebenfalls geläufige Verwendung des Begriffs der Informationsintermediäre spielt hier keine Rolle.

${ }^{90} \mathrm{Vgl}$. Kuczerawy, Intermediary Liability and Freedom of Expression in the EU, S. 2.

${ }^{91}$ Vgl. Ohly, ZUM 2015, 308 (309); Stark/Magin/Jürgens, Ganz meine Meinung?, S. 20.

${ }^{92}$ Vgl. Ohly, ZUM 2015, 308 (308); Wielsch, ZGE 2018, 1 (2); wohl auch Hofmann, ZUM 2019, 617 (618).

${ }^{93}$ Von der Reichweite der Haftungsprivilegien her denkt etwa Hollenders, Mittelbare Verantwortlichkeit von Intermediären im Netz, S. 34 f.; dagegen scheint Hoeren, in: Westphalen/Thüsing, Vertragsrecht und AGB-Klauselwerke, E-Commerce-Verträge Rn. 119, unter Intermediären offenbar nur Suchmaschinen und Auktionshäuser zu verstehen.

${ }^{94} \mathrm{Vgl}$. Secretary-General of the OECD, The Economic and Social Role of Internet Intermediaries, S. 9.

${ }^{95}$ Davon unberührt bleibt die Frage der Anwendbarkeit der $\S \S 7 \mathrm{ff}$., insbesondere des $\S 8$ TMG auf Access-Provider. Systematisch unstimmig, aber in Anwendung der ECRL zutreffend hat der Gesetzgeber auch die Access-Provider dem $§ 8$ TMG unterstellt, obwohl die Vermittlung des technischen Zugangs zum Internet gegen Entgelt ein „Telekommunikationsdienst" gemäß $\S 3 \mathrm{Nr}$. 24 TKG darstellt und damit entsprechend der Negativbestimmung des Anwendungsbereichs des TMG (§1 Abs. 1 TMG) von dessen Regelungen 
Gegenstands dieser Untersuchung durch die ECRL, insbesondere deren Art. 14, legt es nahe, hier ein engeres Begriffsverständnis der Intermediäre zugrunde zu legen. Danach umfassen Informationsintermediäre einerseits soziale Netzwerke im engeren Sinne (insbesondere Facebook), Suchmaschinen (insbesondere Google) und Nachrichtenaggregatoren (z.B. reddit), ${ }^{96}$ andererseits aber insbesondere auch Dienste für nutzergenerierte Inhalte (z.B. YouTube und Twitter). ${ }^{97}$

\section{Rechtliche Rezeption}

Die InfoSoc-RL bezieht sich in den Erwägungsgründen 33, 59 der englischen Fassung auf „intermediaries“, die in der deutschen Version als Vermittler bezeichnet werden. Insbesondere ihr Erwägungsgrund 59 S. 1-2 verdient hier eine besondere Erwähnung:

„Insbesondere in der digitalen Technik können die Dienste von Vermittlern immer stärker von Dritten für Rechtsverstöße genutzt werden. Oftmals sind diese Vermittler selbst am besten in der Lage, diesen Verstößen ein Ende zu setzen. [...]“

Konsequent sieht denn auch Art. 8 Abs. 3 InfoSoc-RL vor, dass gegen „intermediaries“ der Weg zur Erlangung einer gerichtlichen Anordnung zur Verfügung stehen muss, wenn „deren Dienste von einem Dritten zur Verletzung eines Urheberrechts oder verwandter Schutzrechte genutzt werden. ${ }^{\text {“98 }}$ Ähnliches ist auch in Art. 11 Enforcement-RL formuliert. In der bemerkenswert präzisen Formulierung wird deutlich, dass der unionsrechtliche Gesetzgeber bereits zu einem frühen Zeitpunkt um das Machtpotenzial der Intermediäre wusste und bereit war, hieraus konkrete Pflichten abzuleiten. Eine ähnliche Wertung ist auch der ECRL in ihrem Erwägungsgrund 40 S. 6 immanent, wo ebenfalls in Zusammenhang mit Vermittlern bzw. ,intermediaries" davon die Rede ist, deren technische Fähigkeiten auch für die Begründung von Rechtspflichten zu verwerten. Auch die Erwägungsgründe 14, 40 sprechen in der englischen Fassung von der "liability of intermediaries“.

ausgenommen ist; zur Auflösung dieser rechtssystematischen Unstimmigkeiten vgl. Frey, MMR 2014, 650 (651 ff.).

${ }^{96}$ Vgl. Stark/Magin/Jürgens, Ganz meine Meinung?, S. 20.

${ }^{97}$ Die von Schulz/Dankert, Die Macht der Informationsintermediäre, S. 23 f., vorgenommene, nicht weiter begründete Unterteilung in „Plattformen für nutzergenerierte Inhalte" und „Micro-Blog-Plattformen“ wird hier nicht übernommen; nach dem hiesigen Verständnis umfassen erstere auch letztere, obschon sich rechtliche Konfliktpotenziale in der Empirie durchaus unterscheiden dürften, da etwa bei YouTube persönlichkeitsrechtsverletzende Äußerungen durchaus möglich sind, hier aber Urheberrechtsverstöße in einem deutlich höherem Umfang möglich sind als etwa bei Twitter.

${ }^{98} \mathrm{Vgl}$. hierzu Husovec, Injunctions against Intermediaries in the European Union, passim. 
Im deutschen Recht ist mittlerweile ebenfalls eine Positivierung des Begriffs Intermediär abzusehen. So definiert der am 5. Dezember 2019 von den Regierungschefinnen der Länder verabschiedete Medienstaatsvertrag als „Medienintermediär jedes Telemedium, das auch journalistisch-redaktionelle Angebote Dritter aggregiert, selektiert und allgemein zugänglich präsentiert, ohne diese zu einem Gesamtangebot zusammenzufassen " ${ }^{99}$ Die Verwendung des Wortes ,auch" verdeutlicht, dass hiernach insbesondere auch Dienste im Sinne des $\S 10$ TMG bzw. Art. 14 ECRL erfasst werden können. ${ }^{100}$ Offen bleibt dagegen bislang, ob Suchmaschinendienste hierunter fallen sollen, insbesondere, weil sie im Vergleich zu einer früheren Entwurfsversion nicht mehr explizit als Beispiel bei der Legaldefinition genannt werden.

Insgesamt ist festzuhalten, dass der Begriff Intermediär vor allen Dingen in jüngerer Zeit eine starke rechtliche Aufwertung erfährt, was als Beleg dafür gelten kann, dass die Rolle bzw. Funktion der hiervon erfassten Akteure verstärkt in den Blick rückt.

\section{II. Überzeugungskraft}

Auf Grundlage dieser Verständniszuschreibungen ist zunächst zu beachten, dass der Begriff „Vermittler" nicht perfekt geeignet ist, um die Funktionsweisen von sozialen Netzwerken, Suchmaschinen, aber auch anderen Angeboten hinreichend abzubilden. Denn Vermittler kann im Ausgangspunkt jede natürliche oder juristische Person sein, die eine Information von einer Entität zu einer anderen transportiert. Wie aber auch schon angeklungen ist, hat es mit der Benennung der Funktion als zwischen zwei Entitäten bestimmte Informationen vermittelnde Instanz hier indes nicht sein Bewenden. ${ }^{101}$ Das berücksichtigen neuere Zuschreibungen wie diejenige im Entwurf des neuen Medienstaatsvertrags durchaus. ${ }^{102}$ Dieser hebt die Multifunktionalität dieser Dienste hervor, die gerade über eine reine Vermittlungsleistung hinausgehen. Unter Berücksichtigung dieser Einsicht ist der Begriff „Intermediär" für diese Untersuchung geeignet, denn er ist in wertender Perspektive hinreichend neutral und verkürzt nicht schon semantisch den $\mathrm{Zu}-$

\footnotetext{
${ }^{99}$ Vgl. §2 Abs. 2 Nr. 16 des von Regierungschefinnen der Länder beschlossenen Medienstaatsvertrags, vgl. www.rlp.de/fileadmin/rlp-stk/pdf-Dateien/Medienpolitik/ModStV MStV_und_JMStV_2019-12-05_MPK.pdf.

${ }^{100}$ Das scheint insbesondere für Facebook denkbar bzw. wahrscheinlich. Bei einem Dienst wie Twitter wäre dies allerdings nicht mehr ganz so eindeutig, weil hier, soweit ersichtlich, eine Aggregation und Selektion journalistisch-redaktioneller Angebote in der Regel nicht erfolgt.

${ }^{101}$ Vgl. aber Schulz/Dankert, Die Macht der Informationsintermediäre, S. 15: „Informationsintermediäre bieten also in der Regel keinen eigenen Inhalt an [...].“

${ }^{102} \mathrm{Vgl}$. oben $\S 7$ Fn. 99.
} 
griff auf die verschiedenen Rollen, die die hier relevanten Unternehmen im Rahmen ihrer Tätigkeit wahrnehmen. ${ }^{103}$ Zudem rückt er die betroffenen Akteure in den Fokus und liefert so auf der semantischen Ebene ein präziseres Beschreibungsangebot, welches dann auch für die Bewertung normativer Fragen hilfreich sein kann.

Wie bereits erwähnt, kann der Begriff Intermediär indes nicht ausschließlich zur Verwendung kommen. Es mögen hin und wieder pragmatische Erwägungen auch Anlass geben, beispielsweise von Diensteanbietern zu sprechen, um die Phänomene im Fokus dieser Untersuchung bzw. deren rechtliche Verarbeitung sinnvoll zu erfassen. Eine solche rechtliche Verarbeitung kann insbesondere in der ECRL und dem TMG gegeben sein, die hinsichtlich der hier interessierenden Haftungsprivilegierungen primär von Diensteanbietern sprechen. Folglich ist im Rahmen dieser Untersuchung von Intermediären und Diensteanbietern die Rede. Diese Begriffe werden hier synonym verwendet.

\section{E. Fazit}

Begriffe, insbesondere Metaphern, prägen Debatten. Das gilt nicht nur für den juristischen Diskurs, sondern auch für viele weitere kommunikative Settings. Das verbindende Element ist in dem Umstand begründet, dass das Recht als sozio-normative Institution umso erfolgreicher ist, je eher es sich nicht systemisch entkoppelt, sondern stets auf die soziale Realität fokussiert bleibt. ${ }^{104}$ In diesem Sinne ist es von erheblicher Bedeutung, wie man begrifflich mit privaten Akteuren umgeht, deren Geschäftsmodell darauf ausgelegt ist, Dritten die Ausübung ihrer Freiheitsrechte zu ermöglichen, die diesen Vorgang bzw. dessen Kontext zugleich aber auch kommerziell verwerten. Einzelne Unternehmen waren und sind bislang insoweit erfolgreich, bei der Frage möglicher regulatorischer Zugriffe nicht nur im rechtspolitischen Bereich ihre Interessen geschickt $\mathrm{zu}$ verfolgen, sondern ihre Funktionalität auch diskursiv zu prägen. ${ }^{105}$ Gerade der Gegenstand dieser Untersuchung ist anfällig für diskursive Kurzschlüsse, weil mit der Wahl der Terminologie zumeist auch eine partikulare ideologische Fahne gehisst wird. ${ }^{106}$ Dies ist insbesondere dann riskant, wenn dies von den Verwendern des Begriffs unbemerkt bleibt. Bei Äußerungen von betroffenen Unternehmen und ihren Interessenvertretern lassen sich aufgrund der zumeist relativ klar strukturier-

${ }^{103}$ Vgl. auch Riordan, The Liability of Internet Intermediaries, S. 28.

${ }^{104}$ Treffend Oermann, Gewährleistung der Möglichkeit internetbasierter Kommunikation, S. 38.

${ }^{105}$ Vgl. Gillespie, New Media \& Society 12 (2010), 347 (348).

${ }^{106}$ Vgl. Riordan, The Liability of Internet Intermediaries, S. 29. 
ten Interessenlage diese Motive auch in einer entsprechenden Begriffsverwendung entdecken und die dahinterliegende Strategie dadurch aufbrechen. Bei unbeteiligten und hinsichtlich der Wirkung der Begriffe zumeist unreflektiert agierenden Akteuren ist dies zumeist schwieriger. Insbesondere die Rechtsprechung nimmt insoweit eine wichtige Rolle ein, weil sich hier entsprechende Begrifflichkeiten über den Weg des juristischen Vorverständnisses $^{107}$ hin zu konkreten Sollensanforderungen materialisieren können. Aus den vorangegangenen Ausführungen wird deutlich, dass viele Judikate entsprechende Muster bedienen.

Für den Kontext dieser Untersuchung ist die Einsicht entscheidend, dass terminologische Klarheit nie schon mit der Wahl von Begriffen selbst zu erreichen ist, sondern dass eine tiefergehende Reflexion herausfordernd, aber lohnenswert sein kann, um Vorverständnisse, Tendenzen und biases zu erkennen und zu vermeiden. In diesem Sinne stellt die hiesige Thematik denn auch einen Paradefall eines ,semantischen Kampfes“ dar. ${ }^{108}$ Die Verwendung von Begriffen wie „Plattform“, „, soziales Netzwerk“ oder „Intermediär“lässt sich mit Felder als „Bezeichnungs- bzw. Benennungskonkurrenz" verstehen, bei der - noch im Vorfeld der echten juristischen Zuordnung - verschiedene Ausdrücke als ,interessenspezifische Versprachlichungstechniken“ ${ }^{109}$ miteinander konkurrieren. ${ }^{110}$ Diese Auseinandersetzungen sind alles andere als folgenlos, sondern nehmen auf „Wirklichkeitswahrnehmung und -konstitution" ${ }^{\prime 111}$ entscheidenden Einfluss und strukturieren das Feld der juristischen Arbeit entscheidend vor. ${ }^{112}$ Im hiesigen Kontext ist diese Einsicht umso entscheidender, gerade weil die begriffliche Annäherung so schwer fällt. ${ }^{113}$

${ }^{107}$ Vgl. insoweit nur Esser, Vorverständnis und Methodenwahl in der Rechtsfindung, S. 99 ff.; Hassemer, ARSP 72 (1986), 195 (210 f.).

${ }^{108}$ Vgl. zu den Verwendungskontexten dieses Begriffs die Nachweise bei Felder, Der Staat 2010, 543 (549).

${ }^{109}$ Felder, Der Staat 2010, 543 (547).

${ }^{110} \mathrm{Vgl}$. Felder, Der Staat 2010, 543 (551 ff.).

${ }^{111}$ Felder, Der Staat 2010, 543 (570).

${ }^{112}$ Vgl. Felder, Der Staat 2010, 543 (570): „Der jeweilige Fachwissenschaftler neigt zu einer Betrachtung der Streitigkeiten von einem ontischen Blickwinkel aus [...], der fachkommunikativ interessierte Diskurslinguist fokussiert die sprachlichen Mittel, welche die jeweiligen Protagonisten strategisch einsetzen. Es handelt sich dabei um zwei komplementäre Ebenen der Erkenntnis. Die eine Ebene ist ohne die andere nicht zu denken [...].“

${ }^{113} \mathrm{Vgl}$. Schulz/Dankert, Die Macht der Informationsintermediäre, S. 63. 


\section{Strukturelle Defizite bei der Zuweisung von Verantwortung an die Intermediäre für Rechtsverletzungen}

Ein zentrales Paradigma, das oftmals als Argument gegen eine stärkere regulative Ausgestaltung der Rolle der Intermediäre in Stellung gebracht wird, bezieht sich auf ihre vorgebliche Rolle lediglich als Ermöglichungsbedingung von Kommunikation, die sie demnach lediglich als rein technischer neutraler Transporteur fremder Informationen darstellt. ${ }^{1}$ Dies muss allerdings insoweit bezweifelt werden, als die Intermediäre, sei es aus rechtlichen oder aus praktischen Erwägungen heraus, eine solche Machtstellung in Betreff der über sie ausgetauschten Inhalte entwickeln, die ihr Verständnis als rein technisch oder neutral nicht mehr als angemessen erscheinen lässt. Deshalb erweisen sich die im Fokus dieser Untersuchung stehenden rechtlichen Regeln, die die Intermediäre von der Verantwortlichkeit für von ihnen transportierten Inhalte ausnehmen, als strukturell unangemessen. Dies aufzuzeigen ist Ziel dieses Abschnitts.

Konkret erweist sich das Konzept „Host-Providing“ als zentraler Anknüpfungspunkt der Haftungsfreistellungen in Art.14 ECRL bzw. §10 TMG mit der tatsächlich wahrgenommenen Funktion der Intermediäre als unvereinbar und deshalb strukturell defizitär (A.) Das in diesem Zusammenhang stets anzutreffende normative Leitbild der „neutralen“ bzw. „passiven“ Diensteanbieter verweist insoweit ebenfalls auf eine strukturell relevante Prämisse, die es zu hinterfragen gilt (B.). Einen Sonderfall stellen in diesem Zusammenhang schließlich Suchmaschinendienste dar, die zumindest teilweise ebenfalls in das Regelungsgefüge der hier im Fokus stehenden Haftungsprivilegierungen eingeordnet werden (C.).

${ }^{1}$ Vgl. etwa Pasquale, Theoretical Inquiries in Law 17 (2016), 487 (494ff.). 


\section{A. Host-Providing als funktional ungenügende Kategorie zur Beschreibung intermediärer Dienste}

Die hier im Fokus stehenden Regelungen unterstellen die Intermediäre einem beschränkten Haftungsmodell und setzen hierfür - in unterschiedlicher Erscheinungsform und Ausprägung - tatbestandlich an der ,Speicherung von Informationen" auf Weisung durch bzw. aufgrund von eingegebenen Informationen der Nutzer an. Das normative Leitbild, in welches sich die Tätigkeit der Diensteanbieter einfügt, suggeriert damit nicht mehr als einen rein technischen Vorgang der Speicherung von Informationen, für den sich Internetnutzer einzelner Diensteanbieterinnen bedienen. Im Folgenden soll aufgezeigt werden, weshalb dies in konzeptioneller Hinsicht problematisch ist.

Dazu ist zunächst herauszuarbeiten, inwiefern die hier dargestellten Rechtsordnungen die Abhängigkeit der Haftungsprivilegierung von einem Speicherauftrag des Nutzers eines Dienstes und damit ein Kontrollverhältnis zwischen Diensteanbieter und -nutzer in den Vordergrund stellen, sowie welche Konsequenzen hiermit verbunden sind (I.). Sodann gilt es, die Verfügungsmacht der Intermediäre über die von ihnen gespeicherten Informationen zu skizzieren (II.). Eine Beschränkung des normativen Leitbilds der Diensteanbieter als Host-Provider bildet diese Verfügungsmacht jedoch nicht hinreichend ab (III.).

\section{De lege lata vermitteltes normatives Leitbild intermediärer Dienste}

\section{USA}

In den USA wurde für die Privilegierungsnorm des § 512(c)(1) DMCA bereits herausgearbeitet, dass die insoweit maßgebliche Voraussetzung in der Speicherung von Inhalten auf Weisung des Nutzers liegt. ${ }^{2}$ Eine solche Speicherung ,at the direction of a user" soll nicht schon zu verneinen sein, wenn der Diensteanbieter diese Inhalte vorab kontrolliert oder anderweitig modifiziert. ${ }^{3}$ Danach stehen das Kuratieren bzw. automatische Ordnen von Inhalten im Sinne des Geschäftsmodells vieler Diensteanbieter der Anwendung

\footnotetext{
${ }^{2}$ Vgl. oben $\S 3$ B. II. 2.

${ }^{3}$ Vgl. IO Group v. Veoh Networks, 586 F.Supp. 2d 1132, 1146 ff. (N.D. Cal. 2008), wo das Gericht auf $\S 512(k)(1)(A)$ DMCA verweist. Diese Norm gilt für „Service Provider“ im Sinne von §512(a) DMCA, der der Sache nach Access-Provider nach hiesigem Verständnis meint. Nur insoweit gilt die Einschränkung, dass eine „modification to the content or the material as sent or received" der Anwendbarkeit entgegensteht, während diese Einschränkung für Host-Provider im Sinne des hiesigen Verständnisses nach §512(k)(1)(B) DMCA nicht gilt.
} 
nicht entgegen. Entscheidend ist die Entscheidung der Nutzerin, ob der Inhalt überhaupt veröffentlicht werden soll. ${ }^{4}$ Daraus lässt sich ableiten, dass, solange eine ebensolche Speicherung auf Veranlassung bzw. Weisung einer Nutzerin vorliegt, die Wirkmächtigkeit der Diensteanbieter im Rahmen des von ihnen angebotenen Dienstes letztlich nicht mehr der entscheidende Faktor ist, was die Anwendbarkeit der Privilegierungsnorm betrifft.

\section{EU und Deutschland}

Ein ähnlicher Befund ist auch für das Unionsrecht festzustellen. Die bereits angesprochene Aussage des Erwägungsgrundes 42 S. 1 ECRL verdeutlicht, dass es allein auf den „technischen Vorgang“ ankommt, bei dem über ein Kommunikationsnetz „von Dritten zur Verfügung gestellte Informationen übermittelt [...] werden“. Dies betont auch der EuGH, der das Privileg des Art. 14 ECRL nur dann als gegeben ansieht, „wenn das Verhalten [...] auf das eines, Vermittlers' in dem vom Gesetzgeber im Rahmen des Abschnitts 4 der [ECRL] gewollten Sinn beschränkt bleibt. " Insoweit erfolgt ebenso eine unmittelbare Verknüpfung zu Erwägungsgrund $42 .{ }^{6} \mathrm{Nach}$ dem EuGH genügt unter Art. 14 ECRL, dass ein Dienst eine entsprechende Speicherung von Informationen vornimmt. ${ }^{7}$ Auch hier kommt es also ebenfalls auf einen technischen Vorgang der Speicherung von Informationen an. Eine weitere funktionale Differenzierung legt Art. 14 ECRL selbst nicht nahe und wird auch vom $\mathrm{EuGH}$ weder unmittelbar oder interpretationsleitend vorgenommen. Auf der Ebene des TMG setzt sich diese Linie wenig überraschend fort. Auch hier kommt es maßgeblich darauf an, ob eine technische Speicherung von Informationen überhaupt gegeben ist. ${ }^{8}$ Eine solche Herangehensweise erlaubt es, eine erhebliche Bandbreite an verschiedenen Phänomenen ein und

\footnotetext{
${ }^{4}$ Vgl. Holznagel, Notice and Take-Down-Verfahren als Teil der Providerhaftung, S. 25.

${ }^{5}$ EuGH, Urt. v. 23.03.2010 - C-236/08, NJW 2010, 2029 (Rn. 112) - Google France und Google.

${ }^{6}$ Vgl. EuGH, Urt. v. 23.03.2010 - C-236/08, NJW 2010, 2029 (Rn. 113) - Google France und Google.

${ }^{7}$ Vgl. EuGH, Urt. v. 12.11.2011 - C-324/09, MMR 2011, 596 (Rn. 110) - L’Oréal SA. Zwar schränkt der EuGH sodann die Anwendbarkeit des Art. 14 ECRL insoweit ein, als der Diensteanbieter nur eine bestimmte „Rolle“ einnehmen darf, um am Privileg teilhaben zu können. Das betrifft aber zum einen nicht mehr die hier interessierende technische Ebene. Zum anderen sind diese Einschränkungen aus anderen Gründen ebenso zweifelhaft, wie sogleich aufgezeigt werden soll $(\S 8$ B.).

${ }^{8}$ Das wird, soweit ersichtlich, im Schrifttum kaum in Frage gestellt, vgl. nur Hollenders, Mittelbare Verantwortlichkeit von Intermediären im Netz, S. 111; Matthies, Providerhaftung für Online-Inhalte, S. 30; Schapiro, Unterlassungsansprüche gegen die Betreiber von Internet-Auktionshäusern und Internet-Meinungsforen, S. 17.
} 
derselben Norm zu unterstellen. Denn als Speicherung von Informationen lassen sich schließlich beliebig viele Geschäftsmodelle verstehen. ${ }^{9}$

\section{Verfügungsmacht der Intermediäre über gehostete Inhalte}

In allen soeben dargestellten Fällen suggerieren die relevanten Rechtsordnungen damit ein typisierendes Ideal des Speicherns von Inhalten auf Weisung und für Dritte, das die Funktionalität des nach der Speicherung ablaufenden Prozesses und dessen Auswirkungen auf andere, rechtlich geschützte Güter überwiegend ausblendet. Dabei zeigt sich die Verfügungsmacht der Intermediäre auf mehreren Ebenen in deutlicher Weise.

\section{Zugangskontrolle}

Die erste Ebene, die eine Verfügungsmacht von Kommunikationsdiensten beschreibt, ist die Ebene des Zugangs zu ihren Diensten. So lassen Dienste wie Facebook und Twitter zwar potenziell alle Nutzer des Internets zu ihren Diensten zu. Sie behalten sich aber qua ihrer AGB explizit vor, ${ }^{10}$ einzelne Nutzer von ihren Diensten fernzuhalten bzw. auszuschließen und machen dies von mehr oder weniger detaillierten Bedingungen abhängig. Sie vermögen kraft dieser Rechte in vielfältiger Weise über den Zugang zu ihrem Angebot und den damit verbundenen „kommunikativen Funktionen“ zu verfügen. ${ }^{11}$ Nicht selten steht die Transparenz konkreter Entscheidungen über den (verweigerten) Zugang bzw. über die Entfernung in Zweifel, ${ }^{12}$ was zum Machtungleichgewicht zwischen Diensteanbieter und -nutzer beitragen kann. Auch in Zusammenhang mit dem Informationsverhalten der Nutzer dieser Dienste - laut jüngsten Erhebungen nutzen 34 Prozent aller Erwachsenen in Deutschland soziale Medien zur Informationsgewinnung ${ }^{13}$ - ist die Frage des Zugangs bedeutsam, vermögen die Diensteanbieter doch aufgrund solcher vorbehaltener Zugangskontrollen bzw. durch die Fähigkeit zum Ausschluss einzelner Nutzer von ihrem Angebot darüber zu entscheiden, wer zur Informationsgewinnung und zum kommunikativen Handeln ihre Dienste nutzen kann.

\footnotetext{
${ }^{9}$ Vgl. Schapiro, Unterlassungsansprüche gegen die Betreiber von Internet-Auktionshäusern und Internet-Meinungsforen, S. $336 \mathrm{ff}$.

${ }^{10}$ Vgl. Ziff. 3.1. der AGB von Facebook, de-de.facebook.com/terms; vgl. Ziff. 1., 4. der AGB von Twitter, twitter.com/de/tos.

${ }^{11}$ Vgl. Wielsch, in: Eifert/Gostomzyk, Netzwerkrecht, S. 61 (62).

${ }^{12}$ Vgl. Hindelang, Freiheit und Kommunikation, S. 302.

${ }^{13}$ Vgl. Newman, Reuters Institute Digital News Report 2019, S. 87; vgl. ferner Kaiser/ Reiling, in: Unger/Ungern-Sternberg, Demokratie und künstliche Intelligenz, S. 85 (92 f.); Zimmer/Kunow, in: die medienanstalten, Vielfaltsbericht der Medienanstalten, S. 46 (46).
} 
Die Frage des Zugangs ist in ihrer Bedeutung keineswegs zu vernachlässigen. Mit Blick auf diesen Aspekt formulieren Befürworter wie auch Skeptiker verschiedener Geschäftsmodelle und -praktiken der Intermediäre das ermächtigende als auch das negative Potenzial von entsprechenden Diensten, insbesondere soweit sie von einer großen Zahl genutzt werden. Gemeinsamer Nenner dieser Positionen ist das theoretisch hohe demokratische Potenzial dieser Dienste, insoweit sie tradierte und - so viele Zuschreibungen - verkrustete Strukturen öffentlicher Kommunikation zulasten einzelner, mächtiger Gatekeeper und zugunsten der Vielen aufbrechen. ${ }^{14}$ Die Proponenten sehen hierin ausschließlich oder überwiegend positive Auswirkungen ${ }^{15}$ während die Skeptiker auf die zunehmende Fragmentierung der Öffentlichkeit hinweisen. ${ }^{16}$ Konkret ist insbesondere die Gewährleistung eines fairen $\mathrm{Zu}$ gangs aller zu entsprechenden „öffentlichen Orten“ sowie dessen Beeinträchtigung problematisch. ${ }^{17}$ Dieser Aspekt erinnert nicht zufällig an einen jüngeren Beschluss des BVerfG, in dem es genau um die Frage des Zugangs zu solchen privat beherrschten Orten ging. Hier führt das Gericht aus, dass „für spezifische Konstellationen" eine gleichheitsrechtliche Bindung Privater anzunehmen sein könne. Dies sei bei einem auf das Hausrecht gestützten Ausschluss von Veranstaltungen dann der Fall, wenn diese ,aufgrund eigener Entscheidung der Veranstalter einem großen Publikum ohne Ansehen der Person geöffnet werden“ und „für die Betroffenen in erheblichem Umfang über die Teilnahme am gesellschaftlichen Leben [entscheiden]. " ${ }^{18}$ Die Durchführung solcher Veranstaltungen führe „von Verfassungs wegen“ zu einer „besondere[n] rechtliche[n] Verantwortung“ ${ }^{19}{ }^{19}$ Die so formulierte demokratische Aufladung privat organisierter Veranstaltungen verdeutlicht die besondere Bedeutung, die deren Veranstalter für den Zugang hierfür einnehmen. Der Schritt vom Besuch eines Fußballspiels, um den es bei der Entscheidung des BVerfG ging, zum Zugang zu digital konstituierten Kommunikationsräumen ist in dieser Perspektive kein besonders großer mehr. ${ }^{20}$ Ent-

\footnotetext{
${ }^{14}$ Vgl. Klonick, Harvard Law Review 131 (2018), 1598 (1665f.).

${ }^{15}$ Vgl. etwa Benkler, The Wealth of Networks, S. $176 \mathrm{ff}$., 212 ff.; Kersten, Schwarmdemokratie, S. 128 f.; Ingold, Der Staat 56 (2017), 491 (511).

${ }^{16}$ Vgl. Habermas, Ach, Europa, S. 162; Vesting, in: Unger/Ungern-Sternberg, Demokratie und künstliche Intelligenz, S. 33 (44ff.); Neuberger, in: Neuberger/Nuernbergk/ Rischke, Journalismus im Internet, S. 19 (29); Just/Latzer, Media, Culture \& Society 39 (2017), 238 (248).

${ }^{17} \mathrm{Im}$ Ansatz auch Hindelang, Freiheit und Kommunikation, S. 304.

${ }^{18}$ BVerfG, Beschl. v. 11.04.2018 - 1 BvR 3080/09, NVwZ 2018, 813 (Rn. 41) - Stadionverbot.

${ }^{19}$ BVerfG, Beschl. v. 11.04.2018 - 1 BvR 3080/09, NVwZ 2018, 813 (Rn. 41) - Stadionverbot.

${ }^{20}$ Vgl. nur Schweitzer, ZEuP 2019, 1 (5); Wagner, GRUR 2020, 329 (335); zur Kritik der Entscheidung des BVerfG vgl. oben $\S 6$ Fn. 67.
} 
scheidend hieran ist an dieser Stelle allerdings nicht die grundrechtliche Perspektive, sondern die Einsicht, dass die Zugangskontrolle im Allgemeinen ein Aspekt rechtlicher Zuschreibungen sein kann und sollte. Dies gilt auch für die Privilegierungen der Intermediäre, die aus der haftungsrechtlichen Perspektive deren Tätigkeit nicht weniger genau adressieren sollte als der grundrechtliche Zugriff.

Bereits angesichts der mehr oder weniger stark ausgeprägten, hier skizzierten Fähigkeit der Diensteanbieter, den Zugang zu ihren Diensten zu kontrollieren, sind Zweifel daran angebracht, inwiefern ihre Tätigkeit mit einer Speicherung von Informationen hinreichend beschrieben ist. Dabei spielt es keine Rolle, ob diese Speicherung wie im Falle des § 10 TMG „für“ einen Nutzer erfolgt, oder ob dieser lediglich solche Informationen eingibt, wie es Art. 14 ECRL verlangt.

\section{Gestaltungsmacht bei der Kommunikationsordnung}

Bei der Errichtung und Durchsetzung einer kommunikativen Ordnung in ihrer Einflusssphäre kommt den Intermediären ebenfalls ein erhebliches Gewicht zu, das mit einer Beschränkung auf den technischen Speichervorgang nicht hinreichend beschrieben ist. Dieses äußert sich in mehreren Aspekten, die zum Zwecke der systematisierenden Erfassung hier getrennt dargestellt werden, ohne dass in der Sache eine solche Trennung stets gelingen kann.

\section{a) Design des kommunikativen Umfelds}

Ein wesentlicher Aspekt dieser Macht der Intermediäre zur Gestaltung des kommunikativen Umfelds ihrer Dienste ergibt sich aus den Parametern, die sie beim Design der Benutzeroberfläche setzen, und die sich für die Nutzer als festgelegte, ihren Handlungsrahmen positiv wie negativ begrenzende Faktoren darstellen. ${ }^{21}$ Das betrifft sehr vielfältige Parameter, die sich stark von Dienst zu Dienst unterscheiden. So sind etwa die Entscheidungen über die Zulässigkeit der anonymen Autorschaft oder eine Begrenzung auf 280 Zeichen für einen „Tweet" bei Twitter ebenso als solche Parameter zu verstehen wie die Entscheidung, mit wem eine bestimmte Äußerung in einem bestimmten Kommunikationsdienst geteilt werden oder sichtbar sein soll. ${ }^{22}$ Auch die Entscheidung, in welcher Form Nutzerinnen eines Dienstes mit dessen Anbieter in Kontakt treten können, zählt hierzu. Solche Präfigurationen fallen selten vom Himmel, sondern bringen stets bestimmte Prioritäten der Intermediäre zum Ausdruck - ob bewusst oder unbewusst, explizit oder implizit ${ }^{23}$

\footnotetext{
${ }^{21}$ Vgl. Lessig, Code and other Laws of Cyberspace, S. 89.

${ }^{22}$ Vgl. Sylvain, Connecticut Law Review 50 (2018), 203 (224 f.).

${ }^{23}$ Vgl. Sylvain, Connecticut Law Review 50 (2018), 203 (225 f.).
} 
sodass die Bezeichnung als „normative Technologie“ grundsätzlich als angemessen erscheint. ${ }^{24}$ Das ist per se nicht verwerflich ${ }^{25}$ sondern letztlich eine Selbstverständlichkeit, wenn man die verhaltenssteuernde bzw. verhaltensbegrenzende Funktion von Software anerkennt, wie sie u.a. Lessig bereits beschrieben hat. ${ }^{26}$ Diese Gestaltungsmacht setzt sich in Bezug auf konkrete Inhalte fort, die den Gegenstand eines einzelnen Diensteanbieters ausmachen bzw. für diesen als entscheidender Bestandteil des Angebots angesehen werden.

\section{b) Filterung, Sortierung und Personalisierung von Inhalten}

Drei weitere Faktoren, die die Gestaltungsmacht von Host-Providern zur Geltung bringen und im Kontext der Privilegierung dieser Dienste Bedeutung entfalten, sind die Filterung, Sortierung und Personalisierung von Inhalten. ${ }^{27}$ Alle drei Faktoren beziehen sich auf die Fähigkeit und das evidente ökonomische Interesse der Intermediäre an der Organisation dieser ,gehosteten" Inhalte.

Mit Filterung ist hier die technische Macht gemeint ${ }^{28}$ Inhalte überhaupt zum Bestandteil auffindbarer Informationen zu machen. ${ }^{29}$ Kommunikationsdienste üben hiermit - neben dem Zutritt zum Dienst überhaupt - eine zweite Ebene der Zugangskontrolle aus, die hier die technische Fertigkeit meint, das Hochladen bestimmter Inhalte schon per se zu unterbinden. ${ }^{30}$ Mit Sortierung ist hier vor allen Dingen die Sammlung, strukturierende Gewichtung und Aggregation von Inhalten gemeint. ${ }^{31}$ Sie betrifft die algorithmisch determinierte Rangfolge der Darstellung von Inhalten und entscheidet damit wesentlich über deren Wahrnehmbarkeitschancen. Bestandteil der Sortierprozesse kann dabei auch die häufig ausgemachte Abhängigkeit der Darstellung bestimmter Inhalte von der Person des Dienstenutzers und dessen

${ }^{24}$ Vgl. Dankert, KritV 2015, 49 (52 ff.).

${ }^{25}$ Vgl. aber Hoffmann-Riem, in: Unger/Ungern-Sternberg, Demokratie und künstliche Intelligenz, S. 129 (156f.), der von einer Beschränkung der Verhaltensautonomie spricht.

${ }^{26} \mathrm{Vgl}$. oben $\S 8$ Fn. 21.

${ }^{27}$ Nach Stark/Magin/Jürgens, Ganz meine Meinung?, S. 21.

${ }^{28}$ Zum Filtern von Inhalten als Machtproblem vgl. auch Hoffmann-Riem, AöR 137 (2012), 509 (533 ff.).

${ }^{29}$ Vgl. Stark/Magin/Jürgens, Ganz meine Meinung?, S. 21; zur Funktionsweise und einem ersten rechtlichen Zugriff vgl. Ingold, in: Unger/Ungern-Sternberg, Demokratie und künstliche Intelligenz, S. 184 (192 ff.).

${ }^{30} \mathrm{Im}$ Kontext der Haftungsprivilegien ist dieser Aspekt im Sinne des hier verfolgten Ziels freilich noch eher unkritisch, denn wenn Facebook das Hochladen eines bestimmten Inhalts schon per se unterbindet, „,speichert“ es schlichtweg keinen Inhalt. Die Motivlage oder das Abhängigkeitsverhältnis des Nutzers gegenüber dem Unternehmen spielen insoweit schlicht keine Rolle.

${ }^{31}$ Vgl. Stark/Magin/Jürgens, Ganz meine Meinung?, S. 15; Lischka, itrb 2018, 235 (236). 
persönliche, in seiner Macht stehende Konfiguration des Dienstes, aber auch die umfassende Verarbeitung der bei dessen Nutzung eines Dienstes anfallenden Daten sein. ${ }^{32}$ Diese Phänomene können insgesamt unter dem Stichwort der Personalisierung zusammengefasst werden. ${ }^{33}$

Die Sortierungs- und Personalisierungsprozesse ermöglichen es Diensten, das „Erlebnis“ für Nutzer möglichst attraktiv zu gestalten und damit zugleich deren möglichst hohe Verweildauer zu erzielen. Diese ist für den wirtschaftlichen Erfolg maßgeblich, der primär durch eine hohe Attraktivität für den Anzeigenmarkt bedingt ist. ${ }^{34}$ Letztlich kommt also der Bestimmung und Erzielung personaler Relevanz durch algorithmisch gesteuerte Systeme entscheidende Bedeutung zu. ${ }^{35}$ Denn je höher die Relevanz der dargestellten Inhalte, desto attraktiver ist der Dienst für die Nutzerinnen und der Dienst für seine Anzeigenkunden. Eine kontinuierliche Optimierung der Sortiermechanismen erlaubt es, das Design eines Dienstes stetig im Sinne des erhöhten „user engagement“ anzupassen. ${ }^{36}$ Dies ist entscheidend, denn jeder transportierte Inhalt eines Dritten, der zu mehr ",user engagement “ - also zur aktiven Verwendung der Angebote durch Nutzer - führt und so die Nutzer zur weiteren Nutzung motiviert, hat aus Sicht der Diensteanbieter einen monetären Wert, weil und soweit diese vorrangig mit Anzeigen Geld verdienen. ${ }^{37}$ Die hierin zum Ausdruck kommende Anreizwirkung kann folglich nicht unterschätzt werden. Dies klingt auch beim BGH an, der bei der Frage des Zueigenmachens berücksichtigt, dass in der Gesamtbetrachtung auch eine wirtschaftliche Zuordnung eines fremden Inhalts an den Diensteanbieter selbst eine Rolle spielen kann..$^{38}$

All diese Phänomene, deren kritische Erschließung und Diskussion in Fachdiskursen wie auch in der Allgemeinheit einerseits in jüngerer Zeit bedeutenden Fortschritt erzielt hat, andererseits aber nach wie vor durch die

${ }^{32}$ Vgl. Helbig, in: Mohabbat Kar/Thapa/Parycek, (Un)berechenbar?, S. 339 (344 ff.).

${ }^{33}$ Vgl. Stark/Magin/Jürgens, Ganz meine Meinung?, S. 21; Ungern-Sternberg, in: Unger/Ungern-Sternberg, Demokratie und künstliche Intelligenz, S. 3 (10).

${ }^{34}$ Vgl. LischkalStöcker, Digitale Öffentlichkeit, S. 64; Ungern-Sternberg, in: Unger/Ungern-Sternberg, Demokratie und künstliche Intelligenz, S. 3 (9); Drexl, ZUM 2017, 529 (532f.).

${ }^{35} \mathrm{Vgl}$. Ingold, in: Unger/Ungern-Sternberg, Demokratie und künstliche Intelligenz, S. 184 (190f.).

${ }^{36}$ Vgl. Stöcker/Lischka, in: Mohabbat Kar/Thapa/Parycek, (Un)berechenbar?, S. 364 (372f.).

${ }^{37}$ Vgl. Tremble, Fordham Law Review 86 (2017), 825 (837ff.); Drexl, ZUM 2017, 529 (533); siehe auch Bergen, Bloomberg (02.04.2019), www.bloomberg.com/news/features/20 19-04-02/youtube-executives-ignored-warnings-letting-toxic-videos-run-rampant.

${ }^{38}$ Vgl. BGH, Urt. v. 12.11.2009 - I ZR 166/07, MMR 2010, 556 (Rn. 27) - marionskochbuch.de; kritisch insoweit aber Schapiro, Unterlassungsansprüche gegen die Betreiber von Internet-Auktionshäusern und Internet-Meinungsforen, S. $361 \mathrm{f}$. 
Problematik mangelnder Transparenz der betreffenden Unternehmen geprägt ist, lassen sich mit dem technischen Speicherungsprozess, den die Haftungsprivilegierungen in den Fokus rücken, kaum hinreichend darstellen. Die Fähigkeit der Intermediäre, die Rezeptionschancen von kommunikativen Akten wesentlich zu beeinflussen, ${ }^{39}$ blenden diese Regeln komplett aus.

\section{c) Reaktive Zugriffe auf einzelne Inhalte}

Schließlich behalten sich die Diensteanbieter in ihren AGB umfassende Möglichkeiten vor, bestimmte Inhalte zu entfernen, die mit den AGB bzw. anderweitigen „Gemeinschaftsstandards“ unvereinbar sind. So heißt es bei Facebook, dass das Unternehmen solche Inhalte „entfernen oder blockieren“ kann, die gegen die Nutzungsbedingungen bzw. „Gemeinschaftsstandards“ verstoßen. ${ }^{40}$ Ähnliche Regelungen finden sich in unterschiedlicher Ausgestaltung bei anderen Diensteanbietern. ${ }^{41}$ Unter dem Geflecht dieser Regelungen und der hierauf fußenden Praxis der Diensteanbieter, das vielfach auch als "Content Moderation“ beschrieben wird, ${ }^{42}$ haben die Unternehmen sowie die ihrer Aufsicht unterstehenden Dienstleister weitreichende Befugnisse, welche Inhalte in welchem Kontext entfernt oder nicht entfernt werden.

Das ist zunächst weder überraschend noch per se kritikwürdig, denn der Betrieb eines solchen Dienstes wäre ohne eine entsprechende Praxis kaum zu leisten. ${ }^{43}$ Die Art und Weise, wie Diensteanbieter dabei vorgehen, kann theoretisch sogar als wettbewerbsrelevantes Unterscheidungskriterium dienen ${ }^{44}$ und damit Wohlfahrtseffekte hervorrufen. Dies ändert aber nichts daran, dass die private Ordnung der Kommunikation jedenfalls bei solchen Diensten, die von einem erheblichen Teil der Bevölkerung zum kommunikativen Austausch und zum Informationsgewinn genutzt werden, die Regelsetzer in

${ }^{39}$ Vgl. Schulz, AfP 2017, 373 (376).

${ }^{40} \mathrm{Vgl}$. Ziff. 3.2 der AGB, de-de.facebook.com/terms.

${ }^{41}$ Vgl. für Twitter Ziff. 4 der AGB, twitter.com/de/tos, sowie die vielfältigen „Regeln und Richtlinien“, die jeweils ebenfalls die Entfernung von Inhalten im Einzelfall vorsehen, help.twitter.com/de/rules-and-policies; ebenso findet sich bei YouTube ein komplexes Arrangement von Regeln, vgl. insbesondere den Abschnitt „Entfernen von Inhalten durch YouTube" unter www.youtube.com/static?gl=DE\&template=terms\&hl=de sowie die allgemeinen Richtlinien unter support.google.com/youtube/topic/9223153.

${ }^{42} \mathrm{Vgl}$. dazu umfassend Gillespie, Custodians of the Internet, passim; Roberts, Behind the Screen, passim.

${ }^{43}$ Vgl. nur Gillespie, Custodians of the Internet, S. $5 \mathrm{ff}$.; Yoo, George Washington Law Review 78 (2010), 697 (703 ff.).

${ }^{44}$ Vgl. Gillespie, Georgetown Law Technology Review 2 (2018), 198 (202); ferner Schweitzer, ZEuP 2019, 1 (7f.), die bei einem bestehenden Wettbewerb aus der Wahlfreiheit der Nutzer eine „Richtigkeitsgewähr“ der privaten Regelregime ableitet, die selbst bei einer marktbeherrschenden Position eines Diensteanbieters (wenngleich nur bedingt) gelten soll (9). 
eine sehr mächtige Position im Hinblick auf Grundrechtspostulate kommunikativer Freiheit rücken lässt. ${ }^{45}$ Diese Position lässt sich normativ auch nicht mehr allein durch die $\S \S 305$ ff. BGB bewältigen. Denn zum einen lassen sich mittels der AGB-Kontrolle jene privat gesetzten Regeln und Entscheidungen nicht mehr adäquat erfassen, die in ihre Infrastruktur eingeschrieben sind, da es ja dort gerade nicht mehr „Vertragsbedingungen“ im Sinne von $\S 305$ Abs. $1 \mathrm{~S} .1$ BGB geht. ${ }^{46}$ Zum anderen existiert ein breites Arrangement von ebenfalls „verhaltensregulierenden Bestimmungen“ jenseits der eigentlichen Nutzungsbedingungen, dessen wirksame Einbeziehung unter $\S 305$ Abs. 2 BGB und damit auch dessen rechtliche Verbindlichkeit mitunter zumindest fraglich erscheint ${ }^{47}$ Dies verdeutlicht, dass zur rechtlichen Verarbeitung dieser Aspekte neben der AGB-Kontrolle der Blick auf weitere Teile der Rechtsordnung erforderlich ist. Dies gilt nicht zuletzt für das Recht der Haftungsprivilegierungen.

\section{d) Zwischenfazit}

Die hier dargestellte Gestaltungsmacht der Intermediäre in Bezug auf die Ordnung der über sie vermittelten Kommunikation steht in allen hier betonten Aspekten selbstverständlich nicht frei im Raum, sondern ist logisch und normativ stets von den Inhalten abhängig, die - soweit es den Gegenstand dieser Untersuchung betrifft - nicht von ihnen selbst, sondern stets von den Nutzern ihrer Angebote stammen. Das Anliegen der vorangegangenen $\mathrm{Zu}-$ ordnung ist es demnach nicht, diesen Zusammenhang aufzulösen und einer alleinigen Verantwortung der Intermediäre das Wort zu reden, sondern auf die besondere Funktion der Diensteanbieter selbst hinzuweisen. Der Umstand, dass die von Nutzerinnen erzeugten Inhalte bezüglich ihrer Reichweite mit der Gestaltungsmacht der Intermediäre unauflösbar verbunden sind, führt daher zur Feststellung, dass diese eine bedeutende Funktion in Bezug auf die Ordnung der Kommunikation in ihren Angeboten innehaben.

Diese Feststellung einer so weitreichenden Verfügungsmacht der Kommunikationsdienste, die sich insbesondere in technischer Gestalt offenbart und damit zugleich opak bleibt, zeitigt für das Konzept demokratischer Deliberation und damit für den öffentlichen Meinungsbildungsprozess erheb-

\footnotetext{
${ }^{45}$ Vgl. etwa Lang, AöR 143 (2018), 220 (243), mwN; auch die Verfassungsrechtsprechung deutet in den letzten Jahren an, aus dieser Erkenntnis grundrechtsdogmatische Folgerungen mit Blick auf die Grundrechtsbindung Privater vorzunehmen, vgl. prominent BVerfG, Beschl. v. 11.04.2018 - 1 BvR 3080/09, NVwZ 2018, 813 (Rn. 41) - Stadionverbot; sowie Urt. v. 22.02.2011 - 1 BvR 699/06, BVerfGE 128, 226 (249) - Fraport.

${ }^{46}$ Vgl. Schweitzer, ZEuP 2019, 1 (4).

${ }^{47}$ Wielsch, in: Eifert/Gostomzyk, Netzwerkrecht, S. 61 (64 ff.); zur Diskrepanz zwischen Gemeinschaftsstandards und internen Richtlinien und Praxen der Intermediäre vgl. auch Klonick, Harvard Law Review 131 (2018), 1598 (1638ff.).
} 
liche Folgen ${ }^{48}$ Die Aufgabe, diese aufzuarbeiten, ist demgegenüber keinem (Teil-)Rechtsgebiet allein überantwortet, sondern bedarf in grundlegender Hinsicht vielmehr einer umfassenden Reflexion der vorangehenden Überlegungen. Dies ist insbesondere in dem Kontext dieser Untersuchung und mit Blick auf das normative Ideal geboten, das Regelungen wie Art. 14 ECRL oder § 10 TMG immanent ist. Denn die Verfügungsmacht in dem hier skizzierten Sinne bleibt in diesem Ideal unterbelichtet, wie im Folgenden näher zu begründen ist.

\section{Speicherung von Informationen als unangemessenes Merkmal für die Tätigkeit der Intermediäre}

Im Lichte des vorangegangenen Abschnitts, dessen Fokus der tatsächlichen Seite galt, ist nunmehr aufzuzeigen, weshalb die rechtliche Konzeption der Speicherung von Informationen für Dritte kritikwürdig ist. ${ }^{49}$ Gerade die Verfügungsmacht der Intermediäre in Bezug auf die von ihnen ",gehosteten“ Inhalte stellt sich als besonders prägendes Merkmal dar, das eine entsprechende Einordnung der entsprechenden Unternehmen als „Host“ in Zweifel zieht. $^{50}$

\section{Fokus auf der technischen Lokalisierung ,,gespeicherter “Informationen}

Diese Macht zeigt sich insbesondere in der Fähigkeit, die Benutzererfahrung bei einem bestimmten Dienst vollumfänglich zu bestimmen, was sich nicht zuletzt in dem Oberflächen- und Funktionsdesign widerspiegelt, wie es vorhin beschrieben wurde. ${ }^{51}$ Das Abstellen auf den Vorgang des Speicherns von Informationen beschreibt die soeben skizzierte Entscheidungsarchitektur, bei der es maßgeblich auf die Diensteanbieter selbst ankommt, nicht hinreichend. Denn die Speicherung im Sinne der Art. 14 ECRL, §10 TMG trägt eine Konnotation als einfacher technischer Vorgang in sich, bei dem es nur

${ }^{48}$ Vgl. weiterführend dazu etwa Helbig, in: Mohabbat Kar/Thapa/Parycek, (Un)berechenbar?, S. 339 (349 ff.); Kaiser/Reiling, in: Unger/Ungern-Sternberg, Demokratie und künstliche Intelligenz, S. 85 (93 ff.); Lischka/Stöcker, Digitale Öffentlichkeit, S. 28 ff.; Sunstein, \#republic, S. $85 \mathrm{ff}$.

${ }^{49}$ Historisch gesehen ist es kein Zufall, dass bereits in einem frühen Stadium der tatsächlichen Entwicklung und der juristischen Debatte schon Gegenstand von Diskussionen war, ob bei der Frage der Verantwortlichkeit der Unternehmen auf das Element der Nutzbarmachung von Speicher- bzw. Serverkapazität, also eine positive Handlung, oder aber auf die unzureichende oder unterbliebene Kontrolle der Inhalte, also auf ein Unterlassen abzustellen sei, vgl. Freytag, Haftung im Netz, S. 12.

${ }^{50} \mathrm{Vgl}$. ansatzweise auch Mengden, Zugangsfreiheit und Aufmerksamkeitsregulierung, S. 377.

${ }^{51}$ Vgl. auch Sylvain, Connecticut Law Review 50 (2018), 203 (218). 
auf dessen Initiierung durch den Nutzer ankommt. Was im Anschluss passiert, blendet diese gesetzliche Konzeption aus oder versucht dies allenfalls mit dem Paradigma der Passivität zu erfassen. ${ }^{52}$

Zwar mag es zutreffen, dass technisch betrachtet ein Teil der von den Intermediären angebotenen Leistungen darin besteht bzw. generell wesentlich davon abhängt, Serverkapazitäten zur Verfügung zu stellen. Es trifft aber selten zu, dass eine Information für einen Nutzer abgespeichert wird und diese Information dann technisch und inhaltlich unbeeinträchtigt auf den Servern eines Dienstes mit anderen Nutzern ausgetauscht wird. ${ }^{53}$ Das juristische Kurzschließen der inhaltlichen Zuordnung im Sinne der personellen Zurechnung von Inhalten mit der technischen Frage, wer für wen Speicherkapazitäten zur Verfügung stellt, kann nicht überzeugen, weil es zwei normativ unterschiedlich zu bewertende Ebenen miteinander vermengt und dadurch den Blick auf die hierin liegenden Sachfragen trübt.

Anstatt eine materielle Zuordnung nach Risikosphären vorzunehmen, lenkt die Rechtslage den Blick auf die - ebenfalls nicht einfach zu beantwortende - Frage nach der technischen Lokalisierung einer Information. ${ }^{54}$ Das Abstellen beim Tatbestandsmerkmal „durch einen Nutzer eingegeben“ auf den technischen Vorgang ${ }^{55}$ unterstreicht den Befund, dass eine gesetzliche Fokussierung auf die technische Komponente wenig normativen Spielraum lässt, der aber notwendig wäre, um die Bandbreite der Einflussmöglichkeiten verschiedener intermediärer Dienste hinreichend abzubilden. Insbesondere die Dienste mit erheblicher Reichweite und damit einem bedeutsamen Einfluss auf die Ausübung grundrechtlich verbürgter Kommunikationsfreiheiten, der bis zur faktischen Fähigkeit reicht, einzelne Kommunikate zu entfernen, sind mit der Anknüpfung des Rechts der Haftungsprivilegierungen an den technischen Vorgang der Speicherung nicht hinreichend beschrieben.

${ }^{52}$ Dazu sogleich $\S 8$ B.

${ }^{53}$ Vgl. Sylvain, Connecticut Law Review 50 (2018), 203 (271).

${ }^{54} \mathrm{Im}$ Ansatz scheint dies Bleisteiner, Rechtliche Verantwortlichkeit im Internet, S. 56, ähnlich zu sehen, wenn er von „Mischformen“ der Dienste spricht, die eine funktionale Differenzierung anhand der tradierten Kategorien teilweise unmöglich mache und daher für eine schwerpunktmäßige Zuordnung plädiert. Durchaus bezeichnend ist die hier zutage tretende Meinungsverschiedenheit hinsichtlich der Frage, ob eine „Färbung“ (im Sinne politischer Couleur) eines Host-Providers dergestalt, dass nur bestimmten Inhalten Kapazitäten zur Verfügung gestellt werden, diesen zu einem Content-Provider macht, sodass jedenfalls eine Immunisierung als Host-Provider abzulehnen ist. Während Sieber, JZ 1996, 429 (434), das Host-Providing als ,bloß formale technische Unterstützung“ ansieht, befürwortet Bleisteiner, Rechtliche Verantwortlichkeit im Internet, S. 56, in diesem Fall die Möglichkeit, dem Dienst das Privileg abzusprechen.

${ }^{55}$ Vgl. oben $\$ 4$ Fn. 98, 102 und den zugehörigen Text; siehe auch Pankoke, Von der Presse- zur Providerhaftung, S. 175, der (allerdings affirmativ) vom „technischen Gepräge“ des Art. 14 ECRL spricht. 
Diese Diensteanbieter kommen damit unangemessen leicht in den Genuss solcher Privilegierungen.

Positiv formuliert sollte eine haftungsrechtliche Privilegierung, die zwar mit Blick auf das Prinzip der Eigenverantwortung grundsätzlich weiterhin möglich sein, aber zugleich den Beitrag der Intermediäre nicht aus den Augen verlieren sollte, nach hier vertretener Auffassung tatbestandlich an eine Handlung oder einen Zustand anknüpfen, der die privilegierte Tätigkeit in differenzierungsfähiger Weise markieren kann. Denn nur durch eine solche gesetzlich markierte Differenzierung ließe sich das Abweichen von einem rechtlichen Normalzustand - nämlich die rechtliche Verantwortung für kausal bedingte Rechtsgutsverletzungen - rationalisieren. Bei der „Speicherung von Informationen“, die wie gesehen in beliebig vielen Kontexten angenommen werden kann, stellt sich ein solcher Effekt hingegen nicht ein. Daran ändert auch der Umstand nichts, dass die Privilegierung tatbestandlich noch an weitere Voraussetzungen geknüpft ist. Denn eigentlich bräuchte es keinerlei positivierter Tatbestandsvoraussetzungen jenseits etwa der Kenntnisnahme von einem rechtswidrigen Inhalt. Mit einem derartigen Mangel an Differenzierung ließe sich aber erst recht nicht begründen, weshalb bestimmte Tätigkeiten im Gegensatz zu anderen überhaupt privilegiert sein sollen.

\section{Normativ unterstellte Unabhängigkeit der Dienstenutzer}

Das gesetzlich vermittelte Ideal der Speicherung von Informationen trübt außerdem insoweit den Blick auf die tatsächliche Rolle der Intermediäre, als bei klassischen „User-generated content“-Diensten die Darstellung solcher Inhalte im Rahmen von Sortierungs- und Empfehlungsprozessen, in denen sich das bereits angesprochene Phänomen der Kuratierung manifestiert, ${ }^{56}$ immer maßgeblich von den Diensteanbietern selbst abhängt. Die Nutzer entscheiden sich zwar selbstständig und freiwillig, die mit der Nutzung der Dienste einhergehenden Kuratierungsfunktionen zu gebrauchen. Dies betrifft allerdings nur die Verschaffung des ersten Zugangs zu einem entsprechenden Angebot, die außerdem ihrerseits vielfältigen Bedingungen und Motivationen unterliegen kann. Die nachfolgende Nutzung ist zwar vielfachen Variablen unterworfen, die ebenfalls im Einflussbereich der Nutzer stehen und die ebenfalls auf die Selektion und Art und Weise der Darstellung bestimmter Inhalte Einfluss nehmen. Dieses Einflusspotenzial steht jedoch bereits in einem Verhältnis der Abhängigkeit zu den bereits angesprochenen Elementen der Verfügungsmacht der Intermediäre, deren kuratierende Funktion jedenfalls bei einem großen Aufkommen von Nutzerinhalten als bedeutsamer zu bewerten ist als eine einzelne Entscheidung der Nutzer. Die

\footnotetext{
${ }^{56} \mathrm{Vgl}$. oben $\S 6$ B. III. 2. b).
} 
Nutzerinnen entscheiden gerade nicht durchweg selbstständig, welche Inhalte sie sehen bzw. wer ihre Inhalte sehen soll, ${ }^{57}$ sondern bekommen in erster Linie die Inhalte zuerst angezeigt, die die Sortierungsprozesse als prioritär einstufen. Das ist mit dem idealtypischen Vorstellungsbild, das die rechtlichen Regelungen derzeit suggerieren, bei dem die Diensteanbieter lediglich eine reaktive Wirkmacht entfalten und bis dahin gleichsam technische Erfüllungsgehilfen ihrer Nutzer darstellen, jedoch nicht in Einklang zu bringen. Denn das geltende Recht sieht in der Tendenz eher den Dienstenutzer als eigenverantwortlich handelnden und entscheidenden Akteur an. Ob der normative Blickwinkel nun in der Speicherung „für einen Nutzer“ (§10 TMG) oder der Speicherung „,von durch einen Nutzer eingegebenen Informationen“ (Art. 14 ECRL) liegt, ist hierbei irrelevant. Beide Perspektiven greifen die skizzierte Bedeutung der Diensteanbieter nicht hinreichend auf.

Letztlich erscheint es außerdem kaum vorstellbar, dass ein Geschäftsmodell exakt den Tatbestand des Privilegs für Host-Provider erfüllt, ohne aber auch Eigenschaften aufzuweisen, die über das bloße Speichern von durch einen Nutzer eingegebenen Information hinausgehen und damit eine Verfügungsmacht untermauern, wie sie hier skizziert wurde ${ }^{58}$ Das Kriterium der Speicherung von Informationen, wie es in Art. 14 ECRL und \$10 TMG niedergelegt ist, ist damit nicht geeignet, die funktionale Bandbreite der Intermediäre abzubilden. ${ }^{59}$

\section{B. Passivität/Neutralität als unangemessenes Leitbild der haftungsrechtlichen Zuordnung der Intermediäre}

Der rechtliche Vorteil der Haftungsprivilegierung soll nach dem EuGH nur solchen Diensteanbietern gewährt werden, die sich neutral, also rein technisch, automatisch und passiv verhalten. ${ }^{60}$ Der Diensteanbieter muss unter diesen Bedingungen eine gewisse Distanz zu seinen Nutzern halten, um die Anwendung des Haftungsprivilegs zu rechtfertigen, ${ }^{61}$ da er sich ansonsten „,in

${ }^{57}$ Dies gilt auch in Anbetracht von mitunter bestehenden Möglichkeiten, den Adressatenkreis von „Posts“ etwa bei Facebook mittlerweile recht spezifisch eingrenzen zu können.

${ }^{58}$ Vgl. Van Eecke, Common Market Law Review 48 (2011), 1455 (1473); vgl. auch GA Jääskinen, Schlussantrag v. 09.12.2010 - C-324/09, juris, Rn. 149 - L'Oréal SA, der insoweit eine überaus kritische Haltung zum bestehenden Rechtsrahmen erkennen lässt.

${ }^{59} \mathrm{Vgl}$. zu den Konsequenzen dieser Feststellung noch unten $§ 11 \mathrm{C}$.

${ }^{60}$ Vgl. EuGH, Urt. v. 23.03.2010 - C-236/08, NJW 2010, 2029 (Rn. 114) - Google France und Google; Urt. v. 12.11.2011 - C-324/09, MMR 2011, 596 (Rn. 113) - L'Oréal SA.

${ }^{61}$ Vgl. Van Eecke, Common Market Law Review 48 (2011), 1455 (1482), der zwar auch 
das Lager eines Content-Providers" begibt. ${ }^{62}$ Diese Rechtsprechung findet beim BGH wie auch beim EGMR ${ }^{63}$ deutlichen Widerhall und gehört damit mittlerweile zum dogmatischen Bestand des Rechts der Haftungsprivilegierungen. Wie im Folgenden aufzuzeigen gilt, bestehen gegenüber einem solchen Neutralitätsprinzip erhebliche Bedenken. Dieses ist mit Blick auf HostProvider durch eine rechtssystematisch zweifelhafte Lesart der ECRL durch den EuGH erst so bedeutsam geworden (I.). Die Abgrenzung zwischen einer „aktiven Rolle" und Neutralität bzw. einer passiven Rolle gelingt in der Rechtspraxis kaum (II.) und erscheint zudem auch prinzipiell nur schwer umsetzbar (III.).

\section{Rechtssystematische Zweifel am Begründungsansatz des EuGH}

Zweifel stellen sich schon bei der Begründung des EuGH ein, weshalb die Privilegierungsnorm des Art. 14 ECRL voraussetzen soll, dass der Diensteanbieter sich neutral zu verhalten habe. In der Entscheidung Google France und Google verweist der Gerichtshof ohne weitere Begründung auf Erwägungsgrund $42 \mathrm{~S} .2^{64}$ und gibt damit zu verstehen, dass er diesen auf sämtliche Privilegierungsnormen anwenden will.

Dieser rechtssystematische Begründungsansatz erweist sich bei näherer Betrachtung als verfehlt. Denn wie bereits angesprochen, ist der Wortlaut von Erwägungsgrund 42 S. 1 ECRL mehrdeutig, da er alle Ausnahmen der Verantwortlichkeit in der ECRL zu adressieren scheint. ${ }^{65}$ Tatsächlich spricht die Entstehungsgeschichte des Erwägungsgrundes aber eher dafür, dass dieser tatsächlich nur auf die Art. 12, 13 ECRL Anwendung finden sollte. ${ }^{66}$

davon ausgeht, dass die von Art. 14 ECRL erfassten Diensteanbieter „will almost necessarily have some degree of involvement with their users" (1483). Hierunter scheint er aber nur einen eher beschränkten technischen Rahmen zu meinen („tools to allow users to upload, categorize and display the information").

${ }^{62}$ Frey, ZUM 2019, 40 (43); vgl. auch Klein, Haftung von Social-Sharing-Plattformen, S. 37.

${ }^{63}$ Vgl. EGMR, Urt. v. 16.06.2015 - 64569/09, NJW 2015, 2863 (Rn. 128, 146) - Delfi.

${ }^{64}$ Vgl. EuGH, Urt. v. 23.03.2010 - C-236/08, NJW 2010, 2029 (Rn. 113) - Google France und Google.

${ }^{65}$ Vgl. oben Text zu $§ 4$ Fn. 85. Diese Auffassung hat auch augenscheinlich die Bundesregierung bei der Begründung des Entwurfs des Elektronischer Geschäftsverkehr-Gesetz angeleitet, vgl. BT-Drucks. 14/6098, S. 22 f.: „Die Ausnahmen von der Verantwortlichkeit nach allgemeinen Vorschriften decken die Fälle ab, in denen die Tätigkeit des Anbieters von Diensten der Informationsgesellschaft auf den technischen Vorgang beschränkt ist, [...] von Dritten zur Verfügung gestellte Informationen zu speichern."

${ }^{66}$ Vgl. Protokoll des Rates der Europäischen Union v. 12.01.2000, 13858/99, Ziff. 9.1.f).; Altenhain, in: MüKo StGB, TMG Vor. § 7 Rn. 18; Schapiro, Unterlassungsansprüche gegen die Betreiber von Internet-Auktionshäusern und Internet-Meinungsforen, S. 340 . 
Zudem scheint mit Erwägungsgrund $46 \mathrm{~S}$. 1 ein eigenständiger, nur auf HostProvider anwendbarer Erwägungsgrund zu existieren, ${ }^{67}$ sodass viel für einen rechtssystematischen Fehlschluss aufseiten des EuGH spricht. Diese Auffassung nahm in prominenter Weise auch Generalanwalt Jääskinen in seinem Schlussantrag im L'Oréal-Verfahren ein. Statt auf den Erwägungsgrund 46 abzustellen, schlug er vor, innerhalb eines Geschäftsmodells funktional danach zu differenzieren, ob im konkreten Einzelfall eine Inspektion oder Kontrolle durch den Diensteanbieter vorgenommen werde. Entsprechend sei die Anwendbarkeit des Art. 14 ECRL zu bejahen oder zu verneinen. ${ }^{68}$ Wie bereits erläutert, hat der EuGH diesen Vorschlag allerdings nicht aufgegriffen, sondern die Haftungsprivilegierung weiterhin von der Neutralität des Diensteanbieters abhängig gemacht. ${ }^{69}$

Das rechtssystematische Argument allein ist hier allerdings noch nicht hinreichend, um die Kritik an dem Neutralitätsdogma zu begründen. Schließlich wird in der Literatur von manchen auch aus dem Telos des Art. 14 ECRL selbst eine Neutralitätspflicht abgeleitet. ${ }^{70}$ Das Neutralitätskriterium überzeugt aber auch aus grundsätzlicheren Erwägungen nicht.

\section{Normative Brüche bei der Anwendung des Kriteriums der Neutralität}

\section{Inkohärenz der Rechtsprechung}

\section{a) Normative Maßstäbe innerhalb der ECRL}

Denn der EuGH bejaht das Vorliegen der von ihm geschaffene Figur der „aktiven Rolle“, wenn und soweit der Anbieter die rechtsverletzenden Tatsachen „kennt oder kontrolliert ${ }^{\text {"71 }}$ oder sich die „Kenntnis [...] oder eine Kontrolle verschaffen [kann] “. ${ }^{72}$ Diese unterschiedlichen Maßstäbe sind in

${ }^{67}$ Vgl. GA Jääskinen, Schlussantrag v. 09.12.2010 - C-324/09, juris, Rn. 142 L'Oréal SA.

${ }^{68}$ Vgl. GA Jääskinen, Schlussantrag v. 09.12.2010 - C-324/09, juris, Rn. $143 \mathrm{f}$. L'Oréal SA; vgl. zu innerhalb der EU verschiedenen Ansätzen auch Van Eecke, Common Market Law Review 48 (2011), 1455 (1469 ff.).

${ }^{69}$ Vgl. EuGH, Urt. v. 12.11.2011 - C-324/09, MMR 2011, 596 (Rn. 113) - L'Oréal SA.

${ }^{70}$ So etwa Schapiro, Unterlassungsansprüche gegen die Betreiber von Internet-Auktionshäusern und Internet-Meinungsforen, S. 341; Klein, Haftung von Social-Sharing-Plattformen, S. 56 ff., plädiert für eine teleologische Reduktion des Anwendungsbereichs von Art. 14 ECRL, ohne sich mit der Problematik der Begründung durch den EuGH auseinanderzusetzen.

${ }^{71}$ EuGH, Urt. v. 23.03.2010 - C-236/08, NJW 2010, 2029 (Rn. 117) - Google France und Google.

${ }^{72}$ EuGH, Urt. v. 12.11.2011 - C-324/09, MMR 2011, 596 (Rn. 113) - L'Oréal SA. Sofern man von einem Gegensatz zwischen einem ,automatischen“ Verhalten und der 
sich selbst inkohärent. ${ }^{73}$ Denn sie offenbaren eine grundsätzliche Verquickung zweier völlig unterschiedlicher Perspektiven auf die Rolle der Intermediäre im Lichte des von ihnen erwarteten Verhaltens, das eine Privilegierung erlaubt. Denn während das Kriterium der „aktiven Rolle“ semantisch objektiver Natur ist - Aktivität als Verhalten, Zustand oder Wirkung ist eine objektiv feststellbare Tatsache ${ }^{74}$ - und sich damit äußerlich manifestiert, trifft dies auf Kriterien wie Kenntnis, (Verschaffung von) Kontrolle oder gar dem Kennenmüssen nicht zu. Diese sind erheblich von subjektiven Einschätzungen oder normativen Zuschreibungen geprägt.

Nicht zuletzt ist die Vermengung der neutralen Rolle mit dem Kriterium der Möglichkeit der Kenntnisnahme deshalb kritisch, weil die allermeisten Diensteanbieter sich theoretisch immer Kenntnis von Inhalten verschaffen können, wenn und weil sie ihre technische Infrastruktur und damit ihr gesamtes Geschäftsmodell darauf ausrichten, diese Möglichkeit gerade nicht aus der Hand zu geben. ${ }^{75}$ Im eigentlichen Sinne müssten diese Diensteanbieter also immer eine ,aktive Rolle“ einnehmen, ${ }^{76}$ der Zweck der vom EuGH insoweit vorgenommenen Einschränkung der Haftung wäre somit normativ unterlaufen. Anschaulich wird diese Einsicht beim Vorlagebeschluss des BGH in Sachen YouTube. Hier fragt das Gericht, ob der Dienst eine ,aktive Rolle" einnehme, was die Anwendbarkeit von Art. 14 ECRL ausschließen würde. Sei dies zu verneinen, stelle sich sodann die Frage, wie das Maß der Kenntnis zu bestimmen sei. ${ }^{77}$ Unter dem Verständnis des EuGH im erläuterten Sinne setzt die ,aktive Rolle“, die zur Verneinung der Haftungsprivilegierung führt, aber die Möglichkeit der Kenntnisnahme voraus. Deren Verneinung und die anschließende Frage nach der Kenntnisnahme, die auch deren Möglichkeit im konkreten Fall einschließt, ${ }^{78}$ erscheint daher normativ widersprüchlich.

Kontrolle von Inhalten ausgeht (so wohl Kastl, Automatisierung im Internet, S. 160), überzeugt dies nicht, weil man in einem „von selbst erfolgenden“ bzw. „durch Selbststeuerung erfolgten Verhalten“ (www.duden.de/rechtschreibung/automatisch) und der Kontrolle nicht begriffliche Gegensätze erblicken kann.

${ }^{73} \mathrm{Vgl}$. auch Kuczerawy, Intermediary Liability and Freedom of Expression in the EU, S. 98; Stalla-Bourdillon, in: Taddeo/Floridi, The Responsibilities of Online Service Providers, S. 275 (280 f.); anders Kastl, Automatisierung im Internet, S. 160, die sogar von einer komplementären Funktion spricht, die die Unsicherheit bei der Subsumtion beseitigen könne.

${ }^{74}$ Vgl. die Bedeutungen bei www.duden.de/rechtschreibung/Aktivitaet.

${ }^{75}$ Vgl. Ott, K\&R 2012, 387 (391).

${ }^{76}$ Vgl. auch Stalla-Bourdillon, in: Taddeo/Floridi, The Responsibilities of Online Service Providers, S. 275 (287).

${ }^{77}$ Vgl. BGH, Beschl. v. 13.09.2018 - I ZR 140/15, GRUR 2018, 1132 (Rn. 42 ff.) YouTube.

${ }^{78}$ Richtigerweise bezieht sich das Kriterium der Möglichkeit der Kenntnisnahme auch auf den konkret in Rede stehenden Inhalt, wie die Formulierung bei EuGH, Urt. 


\section{b) Widersprüchliche Wertungen zwischen ECRL und Datenschutzrecht}

Ein weiterer Beleg der Inkohärenz, mit der der EuGH Diensteanbietern begegnet, ergibt sich aus einem Vergleich seiner Haltung unter der ECRL einerseits und dem europäischen Datenschutzrecht andererseits. ${ }^{79}$ So traf der Gerichtshof mit Blick auf Art. 14 ECRL die Feststellung, dass Google mit seinem System Google Ads „Informationen des Nutzers dieses Dienstes, nämlich des Werbenden, in einem Kommunikationsnetz übermittelt, das den Internetnutzern zugänglich ist, und bestimmte Daten, wie z.B. die vom Werbenden ausgewählten Schlüsselwörter, den Werbelink und die diesen begleitende Werbebotschaft sowie die Adresse der Website des Werbenden, speichert, d.h., sie in den Speicher seines Servers schreibt. ${ }^{\text {"80 }}$ Dies genügte dem Gerichtshof allerdings noch nicht, Google das Haftungsprivileg des Art. 14 ECRL abzusprechen. ${ }^{81}$ Entscheidend sei vielmehr, ,, welche Rolle Google bei der Abfassung der den Werbelink begleitenden Werbebotschaft oder bei der Festlegung oder der Auswahl der Schlüsselwörter gespielt hat. "682 In der datenschutzrechtlich konnotierten Entscheidung Google Spain, bei der es um die Verpflichtung des Suchmaschinenbetreibers zur Auslistung bestimmter Suchergebnisse ging, hielt der EuGH hingegen in aller Klarheit fest, dass es der Suchmaschinenbetreiber Google sei, der ,[ü]ber die Zwecke und Mittel der genannten Tätigkeit und somit der in deren Rahmen vom Suchmaschinenbetreiber selbst ausgeführten Verarbeitung personenbezogener Daten entscheidet [...], so dass er als für diese Verarbeitung „Verantwortlicher“ iSv [Art. 2 lit. d DS-RL] anzusehen ist. ${ }^{\text {"83 }}$ Die zwei hier in Bezug gesetzten Positionierungen des EuGH beruhen zwar im praktischen Sinne auf unterschiedlichen Funktionen. Rechtssystematisch lassen sich das unionsrechtliche Datenschutzrecht und die ECRL auch nicht gegeneinander ausspielen. ${ }^{84}$ Auf der Metaebene ist dennoch die Vermutung nicht gänzlich in Abrede zu stellen, dass hier mit mindestens zweierlei Maß gemessen wird, da einerseits eine umfassende Zwecksetzungsfähigkeit unterstellt wird (Google Spain), andererseits aber dem gleichen Diensteanbieter eine völlig untergeordnete Funk-

v. 12.11.2011 - C-324/09, MMR 2011, 596 (Rn. 113) - L’Oréal SA, verdeutlicht („dieser Daten").

${ }^{79}$ Vgl. zum Folgenden Stalla-Bourdillon, in: Taddeo/Floridi, The Responsibilities of Online Service Providers, S. 275 (287 f.).

${ }^{80}$ EuGH, Urt. v. 23.03.2010 - C-236/08, NJW 2010, 2029 (Rn. 111) - Google France und Google.

${ }^{81}$ Vgl. EuGH, Urt. v. 23.03.2010 - C-236/08, NJW 2010, 2029 (Rn. 112) - Google France und Google.

${ }^{82}$ EuGH, Urt. v. 23.03.2010 - C-236/08, NJW 2010, 2029 (Rn. 118) - Google France und Google.

${ }^{83}$ EuGH, Urt. v. 13.05.2014 - C-131/12, NJW 2014, 2257 (Rn. 33) - Google Spain SL.

${ }^{84}$ Zum Verhältnis der beiden Regelungskomplexe vgl. oben §4 C. III. 2. 
tion unterstellt wird. Dafür ist nicht zuletzt die fehlgeleitete Position des EuGH zum Neutralitätsprinzip unter der ECRL verantwortlich. Ohne diese wäre es dem Gerichtshof deutlich leichter gefallen, einen konsistenten Blick auf die Praxis der Intermediäre zu entwickeln.

\section{Inkohärenz mit Blick auf wirtschaftliche Ausrichtung der Diensteanbieter}

Das Neutralitätskriterium verweist außerdem auf den problematischen Umgang der ECRL und der Gerichte mit der Besonderheit der finanziellen Interessen, die hinter dem Ringen um die Anwendbarkeit der Haftungsprivilegierungen stehen. Die ECRL gilt zwar gemäß ihres Art. 2 für entgeltliche Aktivitäten, sodass unter dem geltenden Regelungsregime allein das finanzielle Interesse nicht zugleich zu einer „,nicht-neutralen“ Rolle im Sinne des $\mathrm{EuGH}^{85}$ und damit zur Unanwendbarkeit der Art. $12 \mathrm{ff}$. führen kann.$^{86}$ Dies ist aber insofern kritisch, als die kommerzielle Ausrichtung - sofern sie zum wirtschaftlichen Erfolg und damit zum Wachstum führt - die Diensteanbieter aus marktökonomischen Gründen dazu drängt, die materielle Distanz zu ihren Nutzern bzw. deren Inhalten zu relativieren. Denn wollen diese Unternehmen weiterhin dieser Marktlogik folgen und wirtschaftlich wachsen, sind sie darauf angewiesen, solche Inhalte zu bevorzugen, die das ,user engagement", also die Aktivität der Nutzer steigen lässt. ${ }^{87}$ Wirtschaftliche Ausrichtung und inhaltliche Neutralität vertragen sich damit nicht. Auch insoweit ist also Neutralität als leitendes Ideal der Organisation von Geschäftsmodellen der Diensteanbieter kaum plausibel.

\section{Neutralität als prinzipiell unplausibler Maßstab}

Neben den konkreten, positiv-rechtlich konnotierten Schwierigkeiten bei der Umsetzung des Neutralitätskriteriums sind schließlich auch grundlegendere Einwände gegen das Neutralitätskriterium angezeigt. Neutralität als Motiv für die Beschreibung der Tätigkeit der Intermediäre ist im Kontext ihrer regulativen Erfassung ebenso ungeeignet (1.) wie als Narrativ für Technologien generell (2.).

\section{Neutrale Intermediäre?}

Die Entscheidung, Inhalte zu veröffentlichen, kann schon insoweit nicht als neutral bezeichnet werden, als sie stets aus einem bestimmten Grund getrof-

${ }^{85}$ Vgl. EuGH, Urt. v. 12.11.2011 - C-324/09, MMR 2011, 596 (Rn. 115) - L’Oréal SA.

${ }^{86}$ Dem EuGH stimmt Ott, K\&R 2012, 387 (389), insoweit zu.

${ }^{87}$ Vgl. Stöcker/Lischka, in: Mohabbat Kar/Thapa/Parycek, (Un)berechenbar?, S. 364 (372f.), sowie oben $\S 8$ Fn. 37. 
fen wird. Auch unbedenkliche Motive wie die Ermöglichung oder Erleichterung der Wahrnehmung kommunikativer Freiheitsrechte Dritter, die Ermöglichung der Anprangerung politischer oder gesellschaftlicher Missstände oder der Austausch von Katzenvideos sind nicht inhaltlich neutral, sondern wertegeleitet bzw. jedenfalls -geprägt. Die Entscheidung für oder gegen die erleichterte Verbreitung bestimmter Inhalte kann nur aufgrund bestimmter Prioritäten getroffen werden, die einer Bezeichnung als neutral entgegenstehen. Insoweit lässt sich die Praxis der „Content Moderation“, wie sie als Geflecht von AGB und Unternehmenspraxis einzelner Diensteanbieter beschrieben wird, nicht als neutral bzw. passiv bezeichnen. ${ }^{88}$

Auch die konkrete Art und Weise der Verbreitung dritterzeugter Inhalte kann nie neutral sein. Allein ihre Sortierung ist notwendigerweise mit Wertungen verbunden. Weder die Sortierung der „Timeline“ bzw. des „Newsfeed“ von Facebook oder ähnlicher Kanäle von anderen Kommunikationsdiensten, noch die Reihenfolge von Suchergebnissen bei Suchmaschinen sind jemals neutral. ${ }^{89}$ Denn entweder treffen die Diensteanbieter Entscheidungen über die Sortierung, die sie in die technische Infrastruktur einspeisen, oder sie stellen die Inhalte in umgekehrter zeitlicher Reihenfolge dar. Beide Fälle lassen sich nicht als neutrales Verhalten des Diensteanbieters bezeichnen.

Im ersten Fall hängt die Entscheidung, in welcher Reihenfolge eine solche Darstellung erfolgt, von sehr vielfältigen Faktoren $a b,{ }^{90}$ über die gemeinhin eher nur spekuliert werden kann, da sie jedenfalls nicht technisch nachweisbar bzw. hinreichend beschrieben sind. ${ }^{91}$ Auch ohne vollständige Transparenz in dieser Hinsicht lässt sich allerdings kaum bestreiten, dass ökonomische Erwägungen die Darstellung von Inhalten erheblich beeinflussen..$^{92}$ Mithin kann die sortierte Darstellung von Inhalten Dritter wie auch eigener Inhalte der Diensteanbieter nicht als neutral bezeichnet werden. ${ }^{93}$

${ }^{88}$ Vgl. auch Sylvain, Connecticut Law Review 50 (2018), 203 (223f.). Zumindest der Terminologie nach anders, aber auch deshalb nicht minder kritikwürdig Kellner, Die Regulierung der Meinungsmacht von Internetintermediären, S. 79, $83 \mathrm{ff}$.

${ }^{89}$ Vgl. Unkel, Informationsselektion mit Suchmaschinen, S. 84; Kaiser/Reiling, in: Unger/Ungern-Sternberg, Demokratie und künstliche Intelligenz, S. 85 (103); mit Blick auf die Anreizstruktur Eifert, in: Eifert/Gostomzyk, Netzwerkrecht, S. 9 (34); vgl. ferner Klonick, Harvard Law Review 131 (2018), 1598 (1621 f.).

${ }^{90}$ Für einen Einblick zum Newsfeed von Facebook vgl. Oremus, Slate (03.01.2016), www.slate.com/articles/technology/cover_story/2016/01/how_facebook_s_news_feed_alg orithm_works.html.

${ }^{91}$ Vgl. Pasquale, The Black Box Society, S. 71.

${ }^{92}$ Für einen Überblick über Facebook in diesem Zusammenhang vgl. Galloway, The Four, S. $90 \mathrm{ff}$.

${ }^{93} \mathrm{Ob}$ diese Feststellung zwingend mit einer Bejahung der Trägerschaft der Meinungsfreiheit aus Art. 5 Abs. 1 S. 1 GG einhergeht, ist damit allerdings noch nicht gesagt. Dass dieser Einordnung eher mit Skepsis zu begegnen ist, wird noch andernorts dargelegt (vgl. 
Auch im zweiten Fall, also bei der „objektiven“ Darstellung von Inhalten rein nach dem Zeitpunkt ihrer Erstellung liegt unzweifelhaft eine wertegebundene Entscheidung vor, weil sie zeitliche Aktualität anderen denkbaren Kriterien vorzieht. Dies begünstigt - jedenfalls bei Kommunikationsdiensten - diejenigen Nutzer, die immer wieder neue Inhalte hochladen und benachteiligt jene, die dies nicht tun. Dies dürfte zwar faktisch nicht allzu häufig das präferierte Modell sein, zeigt aber strukturell die permanente Verwiesenheit der konkreten Darstellung dritterzeugter Inhalte auf menschliche Entscheidungen auf.

In den USA scheint in den parallel gelagerten Debatten die Einsicht in die prinzipielle Schwierigkeit der Beschreibung von Intermediären und ihren Geschäftsmodellen als „neutral“ noch eher konsentiert zu sein, als es im europäischen Kontext der Fall ist. So wird gegenüber in jüngerer Zeit vermehrt vorgetragenen politischen Forderungen, die Anwendung von $§ 230$ CDA stärker auf ,neutrale“ Dienste zu beschränken, ${ }^{94}$ immer wieder darauf hingewiesen, dass Intermediäre wie Facebook eben gerade nicht „neutral“ agieren, ${ }^{95}$ ein Neutralitätsprinzip der genannten Regelung von jeher fremd sei. ${ }^{96}$ Vielmehr erwarten die dortigen Regelungen, wie bereits aufgezeigt wurde, ${ }^{97}$ von den Diensteanbietern eine eigenständige, in diesem Sinne freiwillige Überwachung und Moderation ihrer Dienste. Neutralität könnte einer solchen Erwartung nicht diametraler gegenüberstehen.

\section{Neutrale Technologie?}

Auch Technologie im Allgemeinen, insbesondere wie sie im Kontext der Digitalisierung von Relevanz ist, entzieht sich einer Bewertung als „neutral“. Es trifft zwar zu, dass ihre Nutzung stets von den sie benutzenden Menschen abhängt. ${ }^{98}$ Sie kann daher nicht von vornherein mit einem menschlichen Bias

unten $\S 10$ B. IV. 4). Ferner ist es prinzipiell unschädlich, wenn viele Dienste ihren Nutzern anbieten, von einer automatischen Sortierung der „Timeline“ zu einer chronologischen zu wechseln, wie es etwa bei Facebook oder Twitter möglich ist. Denn jedenfalls Facebook lässt eine dauerhafte Änderung dieser Einstellung wohl nicht zu, vgl. Luckerson, Time (09.07.2015), time.com/3951337/facebook-chronological-order.

${ }^{94}$ Vgl. dazu Ingram, Columbia Journalism Review (08.08.2019), www.cjr.org/the_med ia_today/platform-bias.php.

${ }^{95}$ Vgl. ausführlich Gillespie, Custodians of the Internet, S. 24 ff.; ferner Klonick, Harvard Law Review 131 (2018), 1598 (1661).

${ }^{96}$ Vgl. prägnant Kosseff, The Regulatory Review (15.07.2019), www.theregreview.org/ 2019/07/15/kosseff-correcting-persistent-myth-about-law-that-created-the-internet; vgl. ferner oben $\S 6$ Fn. 26 und den zugehörigen Text zur Einsicht, dass dies letztlich auch dem Prinzip der technischen Infrastruktur des Internets als Kommunikationsmedium entspricht.

${ }^{97} \mathrm{Vgl}$. oben $\S 3$ A. I.

${ }^{98}$ Vgl. mit Blick auf „künstliche Intelligenz“ Wischmeyer, AöR 143 (2018), 1 (14f.); 
gleichgesetzt werden kann. ${ }^{99}$ Das ändert aber nichts daran, dass Technologie, insbesondere ihre Infrastruktur, nicht neutral sein kann, weil sie, wenn sie von Menschen benutzt werden soll, dieser Nutzung schon von vornherein Grenzen setzt. ${ }^{100}$ Das hat namentlich Lessig in seiner breit rezipierten Studie „Code and other Laws of Cyberspace" eindrücklich beschrieben. ${ }^{101}$ Die Architektur des privat beherrschten Cyberspace entfalte eine rechtliche bzw. normative Funktion, weil sie determiniere, was Menschen dort tun und nicht tun könnten. ${ }^{102}$ Damit sei diese Architektur, der „Code“, Macht - und seine Beschaffenheit demnach politischer Gestaltung zugänglich. ${ }^{103}$ Eine Beschreibung von Technologie als neutrales Instrument geht mithin fehl, ${ }^{104}$ weil sie die Fähigkeit kollektiver Selbstbestimmung ebenso beschränkt wie sie die Rolle des Menschen als gestaltenden Akteur der Technologie übergeht. Technolo-

ferner Herberger, NJW 2018, 2825 (2827), dort auch zur etymologisch begründeten Skepsis gegenüber diesem Schlagwort.

${ }^{99}$ Vgl. Laidlaw, Regulating Speech in Cyberspace, S. 35.

${ }^{100}$ Vgl. insoweit die einflussreiche Arbeit von Winner, Daedalus 109 (1980), 121 ff., der vor den Konsequenzen einer rein sozialkonstruktivistischen Perspektive auf Technologie warnt und an deren politische Qualität erinnert. Eine kritische Bestandsaufnahme seiner Thesen findet sich bei Katzenbach, Die Regeln digitaler Kommunikation, S. $153 \mathrm{ff}$.

${ }^{101}$ Vgl. aber auch die wichtigen Vorarbeiten von Reidenberg, Texas Law Review 76 (1998), $553 \mathrm{ff}$.

${ }^{102}$ Lessig, Code and other Laws of Cyberspace, S. 59; für eine kritische Reflexion der Arbeiten von Reidenberg und Lessig mit Blick auf den Konflikt deterministischer und sozialkonstruktivistischer Perspektiven auf Technologie vgl. Katzenbach, Die Regeln digitaler Kommunikation, S. $201 \mathrm{ff}$.

${ }^{103}$ Lessig, Code and other Laws of Cyberspace, S. 59: „,Power', in this account, is just another word for constraints that humans can do something about. [...] But the architecture of cyberspace is power in this sense; how it is could be different. Politics is how we decide [...]" (Hervorh. im Original); vgl. auch schon oben $\S 8$ Fn. 100. Sehr kritisch hierzu allerdings Hildebrandt, Smart Technologies and the End(s) of Law, S. 164 f., die u.a. Lessig vorwirft, unter dem Deckmantel seines Ansatzes nichts weniger als eine verdeckte (und damit illegitime) Verhaltenssteuerung zu propagieren, die Menschen als „rational agents“ und Recht als rein utilitaristisches Mittel begreife, um letztlich nur auf Grundlage von Effizienz und Wirksamkeit politische Ziele erreichen zu wollen. Diese Kritik überzeugt schon deshalb nicht, weil sie den Ansatz von Lessig fälschlicherweise in den Zusammenhang eines „regulatory paradigm“ im Sinne einer Konzeption des Rechts als neutrales und wertfreies Steuerungsmittel stellt, das nunmehr auch Recht und Rechtstheorie heimgesucht (,infested“) habe (dies., Smart Technologies and the End(s) of Law, S. 164). Sie ignoriert damit aber Lessigs zentrales Anliegen, die Möglichkeit des politischen Zugriffs auf das Cyberspace nicht nur über das Recht selbst, sondern eben auch über technische Strukturen zu erläutern. Es ist gerade sein zentrales Anliegen, die Rolle des Rechts hinter diesen Strukturen zu betonen. Der Vorwurf, Lessig entwickle gegen die Manipulation von Individuen kein kritisches Potenzial (S. 185), geht denn auch fehl, weil gerade das seine Sorge ist (vgl. Lessig, Code and other Laws of Cyberspace, S. $95 \mathrm{ff}$.).

${ }^{104} \mathrm{Vgl}$. insoweit auch Lessig, Code, S. $292 \mathrm{f}$. 
gie ist aber in den Worten von Hoffmann-Riem „ein Akt sozialer Gestaltung, in dem Ziele und Wertungen verarbeitet werden".${ }^{105}$ Ein rechtlich vermitteltes Idealbild von „,neutralen“ Intermediären ignoriert diese Einsicht und kann damit kein geeigneter normativer Maßstab sein, um Handlungspotenzial im Allgemeinen und die Haftung von Diensteanbietern im Besonderen sinnvoll abzustecken.

\section{Unklare Zuordnung von Suchmaschinendiensten im System der Haftungsprivilegien}

Zuletzt erweist sich das Recht der Haftungsprivilegierungen für Intermediäre auch mit Blick auf Suchmaschinendienste als strukturell defizitär. Auch diesen kommt eine erhebliche Gestaltungsmacht bei der Vermittlung von nicht selbst produzierten Inhalten zu (I.). Vor diesem Hintergrund lassen sich diese Dienste jenseits der „Autocomplete“-Konstellation derzeit nicht widerspruchsfrei in das System der Art. 12 ff. ECRL, $\S \S 7 \mathrm{ff}$. TMG einpassen (II.).

\section{Gestaltungsmacht bei der Sortierung und Verfügbarmachung eines bedeutsamen Wissensbestandes}

\section{Die Rolle von Suchmaschinendiensten bei der Verfügbarkeit von Wissen}

a) Abhängigkeit des Wissensbestandes von dessen Auffindbarkeit

Über das Internet lassen sich fast unbeschränkt Wissensbestände auffinden. Die bloße Existenz dieses Wissens genügt zu dessen Abruf allerdings bei weitem nicht. Sofern die Kenntnis über die Lokalisierung einer genauen Ressource nicht bekannt ist, ist man auf Suchmaschinen als Wegweiser zwingend angewiesen. ${ }^{106}$ Die konkrete Aufbereitung von Suchergebnissen, insbesondere ihre Reihenfolge, kann in ihrer Bedeutung für Informationsgewinnung und damit mittelbar für die Meinungsbildung nicht unterschätzt werden. ${ }^{107}$

${ }^{105}$ Hoffmann-Riem, AöR 142 (2017), 1 (29).

${ }^{106}$ Vgl. Unkel, Informationsselektion mit Suchmaschinen, S. 62 ff., mwN; Lewandowski, Suchmaschinen verstehen, S. 2 ff.; Schulz/Held/Laudien, Suchmaschinen als Gatekeeper in der öffentlichen Kommunikation, S. 20 f.; Brunn, Cache me if you can, S. $190 \mathrm{f}$; Dörr/Natt, ZUM 2014, 829 (829); vgl. aus der Rechtsprechung BGH, Urt. v. 17.07.2003 - I ZR 259/00, NJW 2003, 3406 (3410) - Paperboy; Urt. v. 01.04.2004 - I ZR 317/01, NJW 2004, 2158 (2160) - Schöner Wetten.

${ }^{107} \mathrm{Vgl}$. Hartl, Suchmaschinen, Algorithmen und Meinungsmacht, S. 43 ff.; Kellner, Die Regulierung der Meinungsmacht von Internetintermediären, S. $69 \mathrm{ff}$.; jüngst auch BVerfG, Beschl. v. 06.11.2019 - 1 BvR 276/17, NVwZ 2020, 63 (Rn. 105) - Recht auf Vergessen II. 
Ohne eine einfache Auffindbarkeit bei Google - der mit Abstand bedeutendste Suchmaschinendienst weltweit ${ }^{108}$ - kann es sich als mitunter fast unmöglich erweisen, Informationen aufzufinden ${ }^{109}$ Suchmaschinendienste stellen damit einen besonders prägnanten und empirisch überaus bedeutsamen Aspekt der allgemeinen Entwicklung des Umgangs mit Wissen unter den Bedingungen des Internets dar. Angesichts der erheblichen Akkumulation von Informationen in Form von Daten steht insoweit nicht mehr die Verfügbarkeit und schnelle Erreichbarkeit von Wissen selbst im Vordergrund, wie es früher der Fall gewesen sein mag. Vielmehr ist das Kuratieren der verfügbaren Informationen mittlerweile eines der zentralen Stichwörter in der digitalen Wissensgesellschaft. ${ }^{10}$

\section{b) Organisation der Suchmaschinendienste}

Der Betrieb einer Suchmaschine erfordert hierbei vielfach Entscheidungen darüber, wie Informationen organisiert und priorisiert werden ${ }^{111}$ welche Informationen den Nutzern zur Verfügung gestellt werden sollen ${ }^{112}$ und welche Informationen hierbei für die Nutzer von besonderer Relevanz sind und deshalb eine prominente Platzierung einnehmen sollen. ${ }^{113}$ Eine „richtige“ Sortierung ist dabei weder möglich noch der behauptete Anspruch. ${ }^{114}$ Vielmehr geht es, ähnlich wie schon zuvor im Rahmen des Host-Providings ausgeführt, um die Ermittlung von Relevanz als dem grundlegenden Ziel der Suchmaschinendienste. ${ }^{115}$ In noch viel deutlicherem Ausmaß kommt es hier darauf an, bereits mit den ersten gelisteten Suchergebnissen für die Nutzer relevante Informationen bereitzustellen, weil das Durchmustern sämtlicher Suchergebnisse nicht den Regelfall darstellt. ${ }^{116}$

${ }^{108}$ Vgl. nur Unkel, Informationsselektion mit Suchmaschinen, S. 66 f., mwN.

${ }^{109}$ Vgl. Paal/Hennemann, JZ 2017, 641 (643). Die Besorgnis um und die Ausgestaltung der Sicherung der Meinungsvielfalt unter diesen Bedingungen ist wenig überraschend Gegenstand vieler Stellungnahmen im Schrifttum; zum bestehenden Rechtsrahmen vgl. statt vieler Dörr/Schuster, in: Stark/Dörr/Aufenanger, Die Googleisierung der Informationssuche, S. 262 (268ff.); für Reformoptionen vgl. statt vieler Paal, Intermediäre, S. 25 ff. Zum neuen Medienstaatsvertrag, wie er von den Regierungschefinnen der Länder beschlossen wurde (vgl. oben §6 Fn. 28) vgl. Hartung, FAZ, 09.08.2019.

${ }^{110}$ Vgl. schon oben $\S 6$ Fn. 38; vgl. zur rechtlichen Dimension des Wissens nur Hoffmann-Riem, in: Röhl, Wissen, S. 160 (160 ff.)

${ }^{111}$ Vgl. Volokh/Falk, Journal of Law, Economics \& Policy 8 (2012), 883 (888).

${ }^{112}$ Vgl. Volokh/Falk, Journal of Law, Economics \& Policy 8 (2012), 883 (890).

${ }^{113}$ Vgl. Lischka/Stöcker, Digitale Öffentlichkeit, S. 20 ff.; Volokh/Falk, Journal of Law, Economics \& Policy 8 (2012), 883 (899). Zum Kriterium der Relevanz vgl. auch schon oben $\S 8$ Fn. 35 und den zugehörigen Text.

${ }^{114}$ Vgl. Lewandowski, Suchmaschinen verstehen, S. 89.

${ }^{115}$ Vgl. Lewandowski, Suchmaschinen verstehen, S. 89.

${ }^{116}$ Vgl. Unkel, Informationsselektion mit Suchmaschinen, S. $74 \mathrm{ff}$. 
Mit der so skizzierten Bedeutung des Relevanzkriteriums als „zentrale Zielgröße" der Qualität einer Suchmaschine ${ }^{117}$ geht zudem eine Tendenz zur Personalisierung einher. ${ }^{118}$ Dem liegt die bereits beschriebene Logik der ökonomischen Anreize zugrunde. Weil Dienste wie Google insbesondere mit Anzeigen Geld verdienen, haben sie ein eminentes Interesse daran, ihre Suchergebnisse so zu strukturieren, dass entsprechende Potenziale möglichst weitgehend ausgeschöpft werden. Deshalb kommt es stets darauf an, für die Person des Suchenden diejenigen Ergebnisse (und sonstigen Inhalte) prioritär anzuzeigen, bei denen ein monetärer Gewinn am wahrscheinlichsten erscheint. Eine solche Personalisierung ist nicht stets, sondern allenfalls in Extremfällen gleichbedeutend mit der bereits angesprochenen Problematik der Filterblasen. ${ }^{119}$ Gleichwohl ist die Personalisierung auch diesseits solcher $\mathrm{Zu}$ schreibungen ein relevanter Faktor, wenn es um die Funktion von Suchmaschinendiensten bei der Verfügbarmachung von Wissen im digitalen Kontext geht.

\section{Einwirkungspotenziale der Suchmaschinenbetreiber}

Im Folgenden sei kurz skizziert, inwieweit Suchmaschinenbetreiber im Hinblick auf die Verfügbarmachung von Informationen eine Gestaltungsmacht ausüben. Dies lässt sich strukturell zum einen im Abgleich mit Kommunikationsdiensten ergründen (a)). Zum anderen sind auch konkrete Zugriffsmöglichkeiten bekannt (b)).

\section{a) Konzeptionelle Gestaltungsmacht im Abgleich mit Kommunikationsdiensten}

Konzeptionell bestehen zwischen Suchmaschinen und Kommunikationsdiensten hinsichtlich ihres Einflusses auf die Verfügbarkeit und Sichtbarkeit von Inhalten, die auch im Rahmen der Inanspruchnahme von Bedeutung sind, grundlegende Unterschiede. Denn während die Entfernung eines Inhalts bei Facebook dessen Existenz gleichsam aufhebt, ${ }^{120}$ soweit dieser nicht noch andernorts verfügbar ist, lässt eine Entfernung eines Suchergebnisses

${ }^{117}$ Unkel, Informationsselektion mit Suchmaschinen, S. 80.

${ }^{118} \mathrm{Vgl}$. Lewandowski, Suchmaschinen verstehen, S. 90; Bozdag, Ethics and Information Technology 15 (2013), 209 (213 ff.); vgl. ferner Ungern-Sternberg, in: Unger/Ungern-Sternberg, Demokratie und künstliche Intelligenz, S. 3 (10f.); zu Relativierungen insoweit aber Kellner, Die Regulierung der Meinungsmacht von Internetintermediären, S. 70 f.; Unkel, Informationsselektion mit Suchmaschinen, S. $83 \mathrm{f}$., jeweils mwN.

${ }^{119}$ Vgl. zu Filterblasen die Nachweise oben in $\$ 7$ Fn. 40; ferner Lewandowski, in: Taddeo/Floridi, The Responsibilities of Online Service Providers, S. 61 (73).

${ }^{120}$ Dies betrifft freilich nicht die Möglichkeit, Inhalte über „Screenshots“ oder andere Mittel trotz Entfernung weiterhin abrufen oder verbreiten zu können. 
den referenzierten Inhalt selbst unberührt. ${ }^{121}$ Einerseits ist also die qualitative Beziehung eines Suchmaschinenbetreibers zur transportierten Information schwächer, weil sie nicht direkt auf diese einwirken kann. Andererseits ist aber der Einfluss auf die Sichtbarkeit von Inhalten, wie bereits aus den vorangegangen Ausführungen deutlich geworden ist, keinesfalls geringer. Denn die eben schon beschriebene essenzielle Bedeutung von Suchmaschinendiensten für die Auffindbarkeit von Informationen vermittelt deren Betreibern eine überragende Bedeutung im Hinblick auf die Wirkmacht der von Dritten erzeugten Inhalte. Insoweit lässt sich von einem funktionalen Äquivalent zu Kommunikationsdiensten hinsichtlich der Einwirkungsfähigkeit auf solche Inhalte sprechen. Ebenso wie Facebook aufgrund bestimmter Kriterien bestimmte Inhalte im „Newsfeed“ weiter oben platziert und damit die Wahrnehmungswahrscheinlichkeit bedeutend erhöht, vollbringt Google bei der Sortierung von Suchergebnissen ähnliches. Lediglich der Kontext und die Modalität unterscheiden sich. Bei Facebook erscheint der „Newsfeed“ ohne Weiteres nach Aufsuchen des Dienstes, während bei einer Suche bei Google eine weitere positive Handlung der Nutzerin erforderlich ist und diese zugleich den Rahmen der dargestellten Inhalte entscheidend determiniert. Aber auch bei Diensten wie Facebook oder Twitter hat eine ähnliche Determinierung stattgefunden, nämlich durch das vorherige Kommunikationsverhalten in dem Dienst. Insbesondere durch gezielte Kontaktaufnahmen („,Freundschaftsanfragen") mit anderen Personen (z.B. Facebook) oder das „Folgen“ von Personen und Institutionen (z.B. Twitter, YouTube) bringen Nutzer so ebenfalls inhaltliche Präferenzen bisweilen sehr deutlich zum Ausdruck. ${ }^{122}$

\section{b) Konkrete Steuerungsfähigkeiten}

Neben der allgemeinen Steuerung der Verfügbarmachung des vorhandenen Wissensbestandes zeigt sich die Wirkmächtigkeit von Suchmaschinen auch in ihrer Fähigkeit, konkret und einzelfallspezifisch in den Ablauf der Suchanfrage einzugreifen. Das betrifft zum einen die bereits thematisierte „Autocomplete"-Funktion von Google, von der schon länger bekannt ist, dass sie die einzelfallbezogene Unterdrückung oder Anpassung von bestimmten Vorschlägen zur Vervollständigung von Eingaben zulässt, sodass Rechtsordnungen wie die deutsche dies zur Durchsetzung von Persönlichkeitsrechten auch einfordern. ${ }^{123}$

${ }^{121}$ Vgl. Kellner, Die Regulierung der Meinungsmacht von Internetintermediären, S. 82 f.; differenzierend, aber letztlich ebenso Mengden, Zugangsfreiheit und Aufmerksamkeitsregulierung, S. $377 \mathrm{f}$.

${ }^{122}$ Vgl. Kaiser/Reiling, in: Unger/Ungern-Sternberg, Demokratie und künstliche Intelligenz, S. 85 (87).

${ }^{123} \mathrm{Vgl}$. dazu schon oben $\S 4$ D. I. 1. b). 
Suchmaschinendienste entfernen aber auch bestimmte Suchergebnisse infolge rechtlicher Verpflichtungen hierzu. Das betrifft im europäischen Kontext insbesondere die vielfach diskutierten Fälle eines „Rechts auf Vergessenwerden", ${ }^{124}$ aber auch Fälle des Jugendschutzes. Trotz gegenläufiger Terminologie handelt es sich dabei technisch nicht um eine Entfernung bzw. Löschung aus dem Index der Suchmaschine, sondern um die Nichtaufnahme einzelner Suchergebnisse in die Treffermenge. ${ }^{25}$

Dass Google aber auch darüber hinaus in der Lage ist, die technische Arbeitsweise seines Dienstes bei der Zusammenstellung von Suchergebnissen in systematischer Weise zu beeinflussen und dies mutmaßlich auch in seine Praxis inkorporiert hat, obwohl es jahrelang behauptet hatte, genau dies nicht zu tun, behaupten journalistische Recherchen der jüngeren Vergangenheit. ${ }^{126}$ Zum Teil wenig überraschend legen die - im Einzelfall von einer Sprecherin des Unternehmens bestrittenen - Ergebnisse dieser Recherchen folgende Tatsachen nahe: Im Bereich des E-Commerce werden bei Suchergebnissen generell größere Unternehmen (z.B. eBay oder Amazon) gegenüber kleineren bevorzugt; manche Unternehmen werden aktiv durch Mitarbeiter von Google beraten, wie sie eine bessere Platzierung bei Suchergebnissen aktiv erreichen können; der Mechanismus des „Crawling“ aktualisiert manche Webseiten oder Bereiche öfters als andere und trägt so zu deren verbesserter Sichtbarkeit bei; es werden sog. „Blacklists“ zur Unterdrückung bestimmter Suchergebnisse geführt; im Rahmen der bekannten Praxis, den Suchalgorithmus von externen „Evaluatoren“ prüfen zu lassen und diese Änderungen vorschlagen zu lassen, ${ }^{127}$ erfolgen teilweise auch konkrete Anweisungen von Google an diese Evaluatoren, damit diese bestimmte Änderungen vorschlagen ${ }^{128}$ Diese Erkenntnisse - sofern tatsächlich zutreffend verdeutlichen das Ausmaß der Steuerungsfähigkeiten von Google im Hinblick nicht nur auf die konkrete Sichtbarkeit einzelner Inhalte, sondern auch auf die systemische Funktionsweise seines Suchmaschinendienstes.

${ }^{124}$ Vgl. EuGH, Urt. v. 13.05.2014 - C-131/12, NJW 2014, 2257 ff. - Google Spain SL; BVerfG, Beschl. v. 06.11.2019 - 1 BvR 276/17, NVwZ 2020, 63 ff. - Recht auf Vergessen II; vgl. auch Kellner, Die Regulierung der Meinungsmacht von Internetintermediären, S. 73 f.

${ }^{125} \mathrm{Vgl}$. Lewandowski, Suchmaschinen verstehen, S. 44.

${ }^{126}$ Vgl. Grind et al., The Wall Street Journal, 15.11.2019; vgl. auch schon die von Kellner, Die Regulierung der Meinungsmacht von Internetintermediären, S. 72 f., aufgeführten, indes nicht validierten Vorwürfe gegenüber Google.

${ }^{127} \mathrm{Vgl}$. www.google.com/intl/de/search/howsearchworks/algorithms.

${ }^{128}$ Vgl. Grind et al., The Wall Street Journal, 15.11.2019. 


\section{Rechtliche Zuordnung}

Vor dem Hintergrund der bisherigen Überlegungen ist es fraglich, ob die Suchmaschinendienste für die in Hyperlinks verlinkten Webseiten bzw. den Inhalt des Suchindex ${ }^{129}$ unter die Haftungsprivilegierung des $\S 10$ TMG subsumiert werden können. Es wurde bereits ausgeführt, dass die unionsrechtliche Perspektive eine Zuordnung unter die Art. 12 ff. ECRL zumindest erlaubt, bei der Frage der Haftungsprivilegierung in einem konkreten Einzelfall allerdings nicht eindeutig ist. ${ }^{130}$

Ziel der nachfolgenden Überlegungen ist keine endgültige Festlegung im Sinne einer abschließenden Bejahung oder Verneinung der Anwendbarkeit einzelner Normen wie etwa dem genannten § 10 TMG. Vielmehr soll aufgezeigt werden, dass eine Zuordnung von Suchmaschinendiensten zu dieser Norm möglich ist, weil sich insbesondere die Rechtsprechung bislang sehr ambivalent äußert (1.) und auch ein Blick auf die anderen Privilegierungsnormen des TMG insoweit nicht schlauer macht (2.). So verstärkt sich der Eindruck eines Regelungsregimes, das seinen Gegenstand in funktionaler Hinsicht nicht wirklich effektiv und sachgerecht zu adressieren vermag.

\section{Rechtliche Unterscheidbarkeit von Suchmaschinen und} Kommunikationsdiensten im Lichte des Privilegs für Host-Provider

\section{a) Anknüpfungen am Gesetzestext}

Prima facie lässt sich feststellen, dass die Darstellung von Suchergebnissen im Vergleich zum „klassischen“ Fall dritterzeugter Inhalte im untechnischen Sinne nicht gleichermaßen als Darstellung gespeicherter Inhalte im Sinne von Art. 14 ECRL bzw. § 10 TMG angesehen werden kann. Auch ein verständiger Laie dürfte zwischen der Benutzung einer Suchmaschine wie Google und anderen Diensten mit Blick auf Verwendungszweck und Funktionalität erhebliche Unterschiede erkennen. Dementsprechend votieren nicht wenige Stimmen im Schrifttum gegen die Zuordnung unter $\$ 10$ TMG. ${ }^{131}$ Oft geschieht dies unter Verweis auf eine Stellungnahme der Bundesregierung im Kontext der Verabschiedung der Vorgängervorschrift § 11 TDG a.F., in der die Bundesregierung explizit dessen Erstreckung auf Suchmaschinen abgelehnt hatte. ${ }^{132}$ Es ist allerdings auch schon darauf hingewiesen worden, dass

${ }^{129}$ Zur Bedeutung der Unterscheidung insoweit Sieber/Liesching, MMR-Beilage 8/2007, 1 (10f.).

${ }^{130} \mathrm{Vgl}$. oben $\S 4$ C. II. 2. b).

${ }^{131}$ Vgl. Sieber/Liesching, MMR-Beilage 8/2007, 1 (14); Hoffmann/Volkmann, in: Spindler/Schuster, Recht der elektronischen Medien, TMG Vor. $\$ \$ 7 f$. Rn. 39; Altenhain, in: MüKo StGB, TMG Vor. $\$ 7$ Rn. 53 ff.; Spindler, in: Spindler/Schmitz, TMG, Vor. §§ 7 ff. Rn. 51, 84, mwN.

${ }^{132}$ Vgl. BT-Drucks. 14/6098, S. 37. 
diese Lesart dieser Äußerung nicht die einzige denkbare ist. Vielmehr ist auch die gegenteilige Interpretation keineswegs unplausibel. ${ }^{133}$ Denn in dieser ÄuBerung heißt es nach der Ablehnung der expliziten Aufnahme der Suchmaschinen, dass ,[i]m Hinblick auf die Komplexität der damit zusammenhängenden Fragen [...] zunächst die weitere Entwicklung in Wissenschaft und Rechtsprechung zu verfolgen [ist]. Ohne spezielle Beschränkungen der ziviloder strafrechtlichen Verantwortlichkeit bleibt es für Hyperlinks bei der Haftung nach allgemeinen Vorschriften." ${ }^{134}$ Das steht demnach der Annahme einer planwidrigen Regelungslücke als Grundvoraussetzung einer analogen Anwendung nicht entgegen. ${ }^{135}$

Auch die für eine Analogie erforderliche vergleichbare Interessenlage lässt sich nicht von der Hand weisen. Diese liegt in der soeben beschriebenen Fähigkeit von Suchmaschinenbetreibern begründet, auf die Sichtbarkeit von Informationen dergestalt einzuwirken, dass ein funktionales Äquivalent zu $\S 10$ TMG jedenfalls nicht von vornherein auszuschließen ist.

Denn Suchmaschinendienste sind sowohl gemäß $§ 1$ Abs. 1 S. 1 TMG, ${ }^{136}$ als auch im Sinne von $\S 2$ Abs. 1 S. $3 \mathrm{RStV}^{137}$ als Telemedien zu bewerten. Ebenso sind sie Diensteanbieter im Sinne von $\S 2$ S. 1 Nr. 1 TMG. ${ }^{138}$ Ihre Verortung unter den $\S \S 7 \mathrm{ff}$. TMG ist damit grundsätzlich angezeigt. Dort erscheinen sie nach hier vertretener Auffassung am ehesten der Haftungsprivilegierung des $§ 10$ TMG zuordenbar. ${ }^{139}$ Denn die technischen Vorgänge bei der Nutzung eines Suchmaschinendienstes lassen sich - wenngleich nicht in ebenso evidenter Weise wie es bei Kommunikationsdiensten der Fall ist ebenfalls als Speicherung von Inhalten im Sinne von $\S 10$ TMG bewerten. So ist insbesondere das „Caching“ von Webseiten ein Vorgang der Speicherung. Dies hat als zugestandene Tatsache Eingang in mehrere gerichtliche Entscheidungen betreffend der Haftung von Suchmaschinen gefunden. ${ }^{140}$ Auf die Frage der Fremdheit dieser Informationen kommt es jedenfalls nach der

${ }^{133}$ Vgl. Sieber/Höfinger, in: Hoeren/Sieber/Holznagel, Handbuch Multimedia-Recht, Teil $18.1 \mathrm{Rn} .98 \mathrm{f}$.

${ }^{134}$ BT-Drucks. 14/6098, S. 37.

${ }^{135} \mathrm{Vgl}$. Sieber/Höfinger, in: Hoeren/Sieber/Holznagel, Handbuch Multimedia-Recht, Teil 18.1 Rn. 101.

${ }^{136}$ Vgl. Martini, in: BeckOK Informations- und Medienrecht, TMG $§ 1$ Rn. 7; Sieber/ Liesching, MMR-Beilage 8/2007, 1 (4); mit Blick auf den Anwendungsbereich des TMG ebenso OLG Hamburg, Urt. v. 26.05.2011 - 3 U 67/11, MMR 2011, 685 (686) - Snippets.

${ }^{137}$ So auch die Begründung zum 9. Rundfunkänderungsstaatsvertrag, abgedruckt bei Schulz, in: Binder/Vesting, Rundfunkrecht, RStV §2 Rn. 71.

${ }^{138}$ Vgl. Martini, in: BeckOK Informations- und Medienrecht, TMG $\S 2$ Rn. 8.

${ }^{139}$ So letztlich auch Ott, WRP 2006, 691 (699), der eine analoge Anwendung des $§ 11$ TDG a.F. diskutiert und bejaht.

${ }^{140}$ Vgl. BGH, Urt. v. 29.04.2010 - I ZR 69/08, NJW 2010, 2731 (2732) - Vorschaubilder I; OLG Hamburg, Urt. v. 26.05.2011 - 3 U 67/11, ZUM-RD 2011, 670 (671). 
vorrangigen unionsrechtlichen Sichtweise nicht an. ${ }^{141}$ Vielmehr ist die Herkunft der Information entscheidend, also ob sie vom Diensteanbieter selbst eingegeben wurde oder nicht. ${ }^{12}$ Das lässt sich jedenfalls bei Abstellen auf die (Suchmaschinen-)Nutzerperspektive nicht völlig klar beantworten, weil unter diesem Blickwinkel auch anzunehmen sein könnte, dass eine Suchmaschine immer nur den Zugang zu Informationen bietet, die im eigentlichen Sinne nicht von ihr selbst stammen. ${ }^{143}$ Die Suchmaschine stellt die Informationen im Rahmen eines Suchvorgangs systematisch geordnet zur Verfügung. Der Suchmaschinendienst ist also insoweit nicht lediglich technischer Transmissionsriemen, sondern eigenständig handelnder und ggf. so auch wahrgenommener Akteur. ${ }^{144}$ Damit sind zwar noch nicht alle sich potenziell stellenden Fragen bei der Zuordnung von Suchmaschinen abgehandelt. Die voranstehenden Überlegungen zeigen jedoch zumindest auf, dass das System der Art. $12 \mathrm{ff}$. ECRL und folgerichtig auch der $\$ \S 7 \mathrm{ff}$. TMG teilweise überfordert scheint, soweit es um die Zuordnung von Suchmaschinen geht. Dies setzt sich in der Rechtsprechung fort.

\section{b) Unklare Rechtsprechung}

Wie bereits aus den obigen Ausführungen zur Anwendbarkeit der ECRL auf Suchmaschinen erkennbar wurde, ${ }^{145}$ ist nach dem Stand der Rechtsprechung eine Zuordnung der Suchmaschinen für die Vermittlung von Hyperlinks bzw. den Inhalten des Suchindex zu dem Regelungsregime der Art. 14 ECRL, § 10 TMG gegenwärtig nicht ausgeschlossen. Denn es wurde, erstens, bereits darauf hingewiesen, dass Suchmaschinenbetreiber nach der Auffassung des EuGH dennoch grundsätzlich als Diensteanbieter unter Art. 14 ECRL fallen können. ${ }^{146}$ Gleichwohl betraf diese Frage in der betreffenden Entscheidung des EuGH explizit nur den Fall der Inanspruchnahme im Kontext von Google Ads, also gerade nicht die Darstellung von Suchergebnissen. Zweitens führte der BGH in der Entscheidung Vorschaubilder I in einem obiter dictum unter Verweis auf die soeben angesprochene Entscheidung des EuGH in Sachen Google France und Google ohne tiefergehendere Erörterung aus, dass die Darstellung von Vorschaubildern einer Suchmaschine auch unter

${ }^{141}$ Dazu schon oben $\S 4$ D. I. 2. b). Dies scheint LG Frankfurt/M., Urt. v. 26.10.20172-03 O 190/16, MMR 2018, 251 (Rn. 36), zu verkennen.

${ }^{142}$ Vgl. Altenhain, in: MüKo StGB, TMG Vor. §7 Rn. 16.

${ }^{143}$ So Sieber/Höfinger, in: Hoeren/Sieber/Holznagel, Handbuch Multimedia-Recht, Teil 18.1 Rn. 120; ablehnend Altenhain, in: MüKo StGB, TMG Vor. § 7 Rn. 37 mit Fn. 136.

${ }^{144}$ Dagegen meint Spindler, in: Spindler/Schmitz, TMG, Vor $\$ § 7 \mathrm{ff}$. Rn. 84, Suchmaschinen würden von $§ 10$ TMG nicht erfasst, weil diese nicht das Merkmal „Informationsweiterleitung" beinhalteten, das aber für Suchmaschinen charakteristisch sei.

${ }^{145} \mathrm{Vgl}$. oben $\S 4$ C. II. 2. b).

${ }^{146} \mathrm{Vgl}$. oben $\S 4 \mathrm{Fn} .73$. 
Art. 14 ECRL falle. ${ }^{147}$ In der Entscheidung Internetforum greift das Gericht diese Haltung auf, indem es seiner Begründung, warum Google als Suchmaschinenbetreiber nur geringere Prüfpflichten bei der Inanspruchnahme wegen einer Persönlichkeitsrechtsverletzung aufzuerlegen seien, gleichsam als Obersatz bzw. Einleitung der entscheidenden Passage einen Verweis auf die Entscheidung Vorschaubilder I voranstellt und seine Haltung damit bekräftigt. ${ }^{148}$ Drittens, und hier womöglich noch aussagekräftiger, ist der Verweis des BGH im Kontext der Konkretisierung der Prüfpflichten eines Suchmaschinenbetreibers auf eine Entscheidung des LG Hamburg. ${ }^{149}$ In dieser hatte das Gericht bei der Prüfung der Störerhaftung eines Suchmaschinenbetreibers wegen einer Persönlichkeitsrechtsverletzung explizit den $\S 10$ TMG als mögliche Privilegierungsnorm angesprochen und nur wegen der Rechtsprechung des BGH zu dessen Unanwendbarkeit auf Unterlassungsansprüche abgelehnt. ${ }^{150}$ Diese Verweisung auf andere Entscheidungen, in denen offenkundig von der grundsätzlichen Zuordnung von Suchmaschinendiensten unter § 10 TMG ausgegangen wird, scheint also für den BGH nicht beanstandungswürdig zu sein. Das alles entspricht zwar kaum einer eindeutigen und klaren Positionierung zu dieser Rechtsfrage. Dennoch spricht viel dafür, dass der BGH bei der Inanspruchnahme eines Suchmaschinenbetreibers wegen der Anzeige eines bestimmten Suchergebnisses von der Anwendbarkeit des $§ 10 \mathrm{TMG}$ ausgeht. ${ }^{151}$

\section{Primäre Zuordnung zu den Access- oder Caching-Diensten?}

Hierfür spricht auch ein rechtssystematischer Blick auf die $\S \S 8,9$ TMG als denkbare Alternativen zur Anwendbarkeit des §10 TMG. Bei beiden Normen stellen sich allerdings ebenso Bedenken gegenüber ihrer Anwendung ein.

${ }^{147}$ Vgl. BGH, Urt. v. 29.04.2010 - I ZR 69/08, NJW 2010, 2731 (Rn. 39) - Vorschaubilder I; kritisch dagegen Spindler, in: Spindler/Schmitz, TMG, Vor. $\$ \S 7$ ff. Rn. 84, der allerdings die vom EuGH recht klar vorgenommene Trennung zwischen der personellen Anwendbarkeit und dem Vorliegen der Voraussetzungen des Privilegierungstatbestands im Einzelnen zu übergehen scheint.

${ }^{148}$ Vgl. BGH, Urt. v. 27.02.2018 - VI ZR 489/16, NJW 2018, 2324 (Rn. 33) - Internetforum.

${ }^{149}$ Vgl. BGH, Urt. v. 27.02.2018 - VI ZR 489/16, NJW 2018, 2324 (Rn. 36) - Internetforum.

${ }^{150}$ Vgl. LG Hamburg, Urt. v. 07.11.2014 - 324 O 660/12, NJW 2015, 796 (799); die Aufhebungsentscheidung des OLG Hamburg, Urt. v. 10.07.2018 - 7 U 125/14, NJOZ 2019, 730 ff., betraf nicht diesen Aspekt.

${ }^{151}$ Vgl. auch OLG Köln, Urt. v. 13.10.2016 - 15 U 173/15, NJOZ 2016, 1814 (Rn. 56) Internetforum; Urt. v. 13.10.2016 - 15 U 189/15, BeckRS 2016, 18916 (Rn.93), wobei beide Entscheidungen auch eine Zuordnung unter $§ 9$ TMG nicht ausschließen. 
Gegen die oftmals als einschlägig bewertete Privilegierungsnorm des $\S 8$ $\mathrm{TMG}^{152}$ spricht der Umstand, dass ein Suchmaschinendienst die von ihm empfohlenen Informationen „,auswählt“, was gemäß Art.12 Abs. 1 lit.c ECRL bzw. \$ 8 Abs. 1 S. 1 Nr. 3 TMG aber zum Ausschluss der Privilegierung führt. ${ }^{153}$ Jedenfalls lässt sich angesichts des beschriebenen Einflusses von Google auf die Darstellung von Suchergebnissen nicht von einer völlig unbeeinflussten Informationsübermittlung sprechen. ${ }^{154}$ Auch hinsichtlich der Anwendbarkeit des $\S 9$ TMG bestehen Zweifel. ${ }^{155}$ Denn bei der Verwendung von Suchmaschinen geht es jedenfalls nicht immer nur um die Steigerung der Effizienz der Übermittlung von Informationen, sondern im Kern um deren Verfügbarmachung.

\section{Zwischenfazit}

Wie bereits betont, geht es hier nicht darum, eine Zuordnung von Suchmaschinendiensten unter Art. 14 ECRL, §10 TMG (oder auch die anderen Haftungsprivilegierungen) abschließend zu bejahen oder zu verneinen. Die voranstehenden Überlegungen verdeutlichen aber, dass Suchmaschinendienste trotz ihrer Funktion als Transporteur von dritterzeugten Inhalten sich derzeit nur schwer unter den geltenden Rechtsrahmen fassen lassen, obwohl sie mitunter sehr ähnliche Einwirkungsfähigkeiten hinsichtlich dieser Inhalte aufweisen. Die Kontrastierung mit dem aktuell bestehenden Rechtsrahmen zeigt, dass dieser die Funktionen von Suchmaschinendiensten nicht nur nicht vollständig reflektiert, sondern im Dickicht unklarer Aussagen der Rechtsprechung für mehr Verwirrung als für Klarheit sorgt. Die von der Rechtsordnung angenommene, insbesondere in der bereits angesprochenen Entscheidung Google France und Google zum Ausdruck kommende Erwartung, auch ein Suchmaschinenbetreiber wie Google möge sich auf eine technische Vermittlerrolle beschränken, um in den Genuss der Privilegierung in Art. 14 ECRL zu kommen, ist in diesem Kontext ebenfalls in besonderem Ausmaß kritikwürdig. Diese Haltung sollte von einem stärker auf Wesen, Funktionalität und Interessenlage beim Betrieb von Suchmaschinen geprägten Verständnis ersetzt werden.

${ }^{152}$ Vgl. ausführlich Sieber/Liesching, MMR-Beilage 8/2007, 1 (11 ff.).

${ }^{153}$ So Milker, Die Umsetzung des »Rechts auf Vergessenwerden« im deutschen Recht, S. 39; LG Frankfurt/M., Urt. v. 26.10.2017-2-03 O 190/16, MMR 2018, 251 (Rn. 37) (zur Berufungsentscheidung vgl. unten $\S 10$ Fn. 98). Dagegen aber Sieber/Liesching, MMRBeilage 8/2007, 1 (12); Paal, in: BeckOK Informations- und Medienrecht, TMG $\S 8$ Rn. 25 : bei einem rein technischen Vorgang liege keine menschliche Auswahl vor.

${ }^{154}$ Vgl. in diesem Sinne auch LG Frankfurt/M., Urt. v. 26.10.2017 - 2-03 O 190/16, MMR 2018, 251 (Rn. 37).

${ }^{155} \mathrm{Vgl}$. Ott, in: BeckOK Informations- und Medienrecht, TMG §9 Rn. 27, 29. 


\section{Fazit}

$\mathrm{Zu}$ Beginn dieses Abschnitts wurden zwei Aspekte herausgearbeitet, die das geltende Recht der Haftungsprivilegierungen in Anbetracht der Rolle und Fähigkeiten der Intermediäre als strukturell defizitär erscheinen lässt: erstens die Grundannahme, mit dem Host-Providing seien die Diensteanbieter hinreichend beschrieben und, zweitens, der grundlegende normative Erwartungshorizont der ECRL an die Intermediäre, die deren „Passivität“ bzw. „Neutralität“ in den Vordergrund stellt.

Beide Aspekte verweisen auf ein gemeinsames Motiv, nämlich das fortwährende Abstellen vieler Regulierungsansätze auf eine rein technische Betrachtung ihres Gegenstands. Genau dieser Blickwinkel stellt jedoch nach hier vertretener Auffassung keinen angemessenen Rahmen für einen sachgerechten rechtlichen Zugriff mehr dar. Denn eine solche Perspektive verengt den Blick auf bestimmte, abgrenzbare Aspekte eines Phänomens und blendet insbesondere Funktion und Bedeutung der Diensteanbieter und ihrer Tätigkeit ganz oder teilweise aus. Ein Beharren auf der rein technischen Differenzierung kann damit nur um den Preis der Ignoranz gegenüber den hiermit verbundenen Auswirkungen erfolgen. Diese Einsicht sollte der Gesetzgeber, insbesondere soweit es um eine Reform der ECRL geht, in seine Entscheidungsprozesse mit einfließen lassen.

Mit Blick auf Suchmaschinendienste stellt sich prospektiv ebenfalls die Frage, ob deren explizite Aufnahme in das System der Art. 12 ff. ECRL, $\S \S 7 \mathrm{ff}$. TMG nicht ein lohnenswertes Anliegen darstellen könnte. Dies gilt umso mehr, wenn und weil sich insoweit vergleichbare Machtstrukturen zeigen, die im Kern auf die Vermittlung nicht eigenständig generierter Inhalte verweisen und damit auch hier Sorgfaltspflichten im Einzelfall begründbar sind. 



\section{Perspektiven des Haftungsrechts der Intermediäre: Die Störerhaftung und ihre Weiterentwicklung}

Das vorangegangene Kapitel legte dar, inwiefern das für die Frage der Haftung der Intermediäre relevante Recht strukturelle Defizite aufweist. Im Fokus der Überlegungen rechtlicher Natur lagen bislang die Normen, die den Intermediären eine Privilegierung für den Transport von durch Dritte begangenen Rechtsverletzungen zukommen lassen. Damit stand die Begründung einer möglichen Haftung der Diensteanbieter, der Haftungsgrund, bislang eher am Rande. Dieses Kapitel wendet sich nunmehr den positiven Voraussetzungen einer möglichen Inpflichtnahme der Diensteanbieter im deutschen Recht zu.

Als erstes soll dazu ein analytischer Rahmen aufgezeigt werden, mit dem die Angemessenheit einer Inanspruchnahme von Akteuren beurteilt werden kann, die nicht als Primärverursacher einer Rechtsverletzung gelten können, die aber gleichwohl nicht völlig unbeteiligt zu dieser stehen (§9). Sodann ist der Blick auf die Störerhaftung als maßgebliches Instrument der deutschen Zivilrechtsordnung zu richten. Als primär von der Rechtsprechung entwickeltes Rechtsinstitut liegt es nahe, dass diese womöglich eher in der Lage ist, auf die Herausforderungen einer Inpflichtnahme der Diensteanbieter flexibel zu reagieren. Deshalb ist zu ergründen, inwieweit sie ein geeignetes Instrument des bürgerlichen Rechts zur Spezifizierung der Verantwortung darstellt. Zugleich, und insoweit zeigt sich der Zusammenhang zu den bislang im Fokus stehenden Haftungsprivilegierungen, weist auch die Störerhaftung im Detail problematische Aspekte auf, die es zu benennen gilt (§10).

Ist somit das Panorama der Haftung für von Dritten begangene Rechtsverletzungen für den deutschen Kontext gezeichnet, können Schlussfolgerungen für das gesamte Recht der Haftung der Intermediäre aufgezeigt werden, insbesondere mit Blick auf die Art. 14, 15 ECRL und die $\$ \$ 7$ ff. TMG (§11). Zugleich kann und soll aber nicht ignoriert werden, dass die Entwicklung nicht bei den allgemeinen Regeln zur Inanspruchnahme der Diensteanbieter stehengeblieben ist. Vielmehr hat die Thematik dieser Untersuchung in jüngster Zeit bedeutend an rechtspolitischer Dringlichkeit gewonnen. Gesetzgeber sowohl auf nationaler wie auf europäischer Ebene sind aktiv geworden, um den Intermediären jeweils sektorspezifische Vorgaben machen, die ihre Verantwortung im Kontext dieser Untersuchung unmittelbar betref- 
fen. In Deutschland betrifft dies namentlich das NetzDG. Auf europäischer Ebene geht es hier primär um die Reform des Urheberrechts, bei der insbesondere die Haftungsregel des Art. 17 DSM-RL im Fokus steht. Diese Ausgestaltungen sind zum Abschluss des Kapitels in den Blick zu nehmen (§12). Damit vervollständigt das Kapitel den Blick auf die Haftung der Intermediäre de lege lata und schärft diesen zugleich für die Zukunft. 


\section{Normative Annäherung an das Problem der Inanspruchnahme der Intermediäre als Gatekeeper}

Auf der Suche nach einem analytischen Rahmen, der zu einem besseren Verständnis sowie einer fundierten Kritik der Inanspruchnahme der Intermediäre verhelfen kann, wird man keineswegs nur in spezifisch dieser Thematik gewidmeten Analysen fündig. Mittlerpersonen, wie sie die Intermediäre zweifellos sind, stellen vielmehr schon länger einen geläufigen Anknüpfungspunkt für eine rechtliche Verantwortungszuweisung dar. Dieser Abschnitt hat zum Ziel, das Neue, aber auch das Bekannte des rechtlichen Zugriffs auf solche solch bedeutende Akteure mit einer Mittlerposition hervorzuheben. Mit dem Konzept des Gatekeepers steht hierfür ein tauglicher Anknüpfungspunkt bereit. Dieser ist hier kurz zu umreißen sowie mit Blick auf die Intermediäre zu kontextualisieren (A.). Dies geschieht allerdings nicht zum Selbstzweck. Denn zur sinnvollen Erfassung und Bewertung des rechtlichen Rahmens zur Inanspruchnahme der Intermediäre sowie um das Potenzial zur Weiterentwicklung aufzuzeigen ist es hilfreich, vorab auf das normative Fundament der Inanspruchnahme zur „Abhilfe“ von Rechtsverletzungen einzugehen. Dieses ist keineswegs erst mit den Diensteanbietern des Internets gelegt worden, sondern geht auf Ansätze der ökonomischen Analyse des Rechts zurück. Ein spezifischer Ansatz ist hier vorzustellen (B.).

\section{A. Die Inpflichtnahme der Intermediäre als Gatekeeper}

\section{Gatekeeper}

Der erstmals bei Lewin ${ }^{1}$ entwickelte Begriff Gatekeeper lässt sich mit Schleusen- oder Torwächter übersetzen. Diese entscheiden darüber, wer oder was durch das Tor hindurch gelassen wird oder wer in den Genuss eines Vorteils gelangt. ${ }^{2}$ Dieser Vorteil kann entweder in einer menschlichen Beziehung oder einem Gegenstand liegen. Der Gatekeeper fungiert damit als notwendiges Zwischenglied zwischen demjenigen, der einen Vorteil begehrt und dem Vor-

\footnotetext{
${ }^{1}$ Vgl. Lewin, Human Relations 1, 2 (1947), 143 (145).

${ }^{2}$ Vgl. Corra/Willer, Sociological Theory 20 (2002), 180 (180).
} 
teil selbst. Ohne ihn lässt sich dieser Vorteil nicht erreichen. ${ }^{3}$ Die Funktion eines Gatekeepers kann ein Akteur entweder durch ein bestimmtes Expertenwissen erlangen oder durch die Zuweisung durch den mit Zwangsgewalt ausgestatteten Staat, etwa indem dieser für bestimmte Tätigkeiten Lizenzen vorschreibt. ${ }^{4}$

Der Gatekeeperbegriff wird oft in kommunikationswissenschaftlichen Diskussionen verwendet, um die Rolle der tradierten Medienunternehmen zu beschreiben und zu analysieren, die durch ihr Handeln entscheiden, welche Informationen zu ihren Konsumenten gelangen. ${ }^{5}$ Mit Blick auf das Beispiel der tradierten Medienlandschaft lässt sich insoweit bereits eine erste abstrakte Parallele herstellen. Denn sowohl mit Blick auf den Konsum klassischer Medien als auch die hier im Fokus stehende Nutzung von Diensten des Internets besteht ursprünglich eine direkte Nutzungsbeziehung zwischen Individuen und einem von diesen erstrebten Gut. In dem einem Fall ist dies eine Information, in dem anderen die Dienstleistung. In beiden Fällen entscheidet aber ein „Schleusenwärter" - ein Gatekeeper - über die Weitergabe der Information bzw. über die Gewährung der Dienstleistung. ${ }^{6}$

\section{Das Spezifikum der rechtlichen Inpflichtnahme der Intermediäre als Gatekeeper}

Vergleicht man allerdings den rechtlichen Zugriff auf die so beschriebene Beziehungsdynamik zwischen Individuum, dem begehrten Artefakt und dem Gatekeeper, ergibt sich ein Aspekt, der einen wesentlichen Unterschied begründet. Denn im Kontext der klassischen Medienlandschaft erfolgt der tradierte rechtliche Zugriff mit dem Ziel der Vielfaltssicherung durch Inpflichtnahme weniger, dafür aber sehr wirkmächtiger Akteure, ${ }^{7}$ wie es im Medienund Rundfunkrecht klassischer Prägung geläufig ist. ${ }^{8}$ Die Sicherung der Viel-

${ }^{3}$ Vgl. Corra/Willer, Sociological Theory 20 (2002), 180 (182).

${ }^{4}$ Vgl. Corra/Willer, Sociological Theory 20 (2002), 180 (182f.).

${ }^{5}$ Vgl. Bruns, in: Neuberger/Nuernbergk/Rischke, Journalismus im Internet, S. 107 (107).

${ }^{6}$ Ein wesentlicher Unterschied liegt hier selbstredend in der Beziehung des Gatekeepers zum erstrebten Gegenstand. Medien „gehören“ die von ihnen vermittelten Informationen untechnisch gesprochen nicht, während die Intermediäre durchaus als Verfügungsbefugte des von ihnen geschaffenen Dienstes gelten.

${ }^{7}$ Vgl. umfassend zur Rolle tradierter Medienunternehmen unter den Bedingungen des Internets Lobigs/Neuberger, Meinungsmacht im Internet und die Digitalstrategien von Medienunternehmen, S. $127 \mathrm{ff}$.

${ }^{8}$ Vgl. für den öffentlich-rechtlichen Rundfunk $§ 11$ Abs. 2 RStV; vgl. aus der verfassungsgerichtlichen Judikatur insbesondere BVerfG, Urt. v. 16.06.1981 - 1 BvL 89/78, BVerfGE 57, 295 (320) - 3. Rundfunkentscheidung; Urt. v. 04.11.1986 - 1 BvF 1/84, BVerfGE 73, 118 (159f.) - 4. Rundfunkentscheidung; Beschl. v. 24.03.1987 - 1 BvR 
falt ist zwar auch im Kontext der Intermediäre von besonderer Bedeutung, schon weil diesen aufgrund ihrer faktischen Relevanz als zentrale Informationsquelle großer Teile der Gesellschaft eine herausgehobene Rolle beim Prozess der Meinungsbildung zukommt. ${ }^{9}$

Im wesentlichen Zweck der Inpflichtnahme liegt aber ein erheblicher Unterschied zwischen der Inpflichtnahme klassischer Medienunternehmen und der Intermediäre begründet. Bei Letzteren rückt der Aspekt der Vielfalt, obschon mittelbar ebenfalls von Bedeutung, in den Hintergrund. Denn insbesondere im Kontext der Haftung für von Dritten begangener Rechtsverletzungen geht es nicht um das übergeordnete Vielfaltsideal, sondern steht vielmehr die Durchsetzung subjektiver Rechte im Vordergrund. Entsprechend sind auch die Modi des rechtlichen Zugriffs unterschiedlich ausgestaltet. Denn während die Vielfaltssicherung mit überwiegend organisatorischen Vorgaben grundsätzlich verpflichtender Natur operationalisiert wird, liegt bei der Inpflichtnahme der Intermediäre der Schwerpunkt auf der Durchsetzung subjektiver Rechtspositionen, die lediglich mittelbar auch übergeordneten, kollektiven Zielen zugutekommen soll. Der Zusammenhang zu subjektiven Rechtspositionen bedeutet aber eben auch, dass - jedenfalls im Grundansatz - das Instrumentarium der Inpflichtnahme von der Geltendmachung dieser Rechte abhängt, also in der Regel keinen zwingenden Charakter hat. Über das Risiko einer Haftung instrumentalisiert, dessen Realisierung stets von der Geltendmachung entsprechend zugebilligter Haftungsansprüche abhängt, erweist sich die Inpflichtnahme der Intermediäre damit als mittelbarer rechtlicher Zugriff durch den Gesetzgeber.

\section{B. Analytischer Rahmen zur Bewertung von Gatekeeping-Regimen nach Kraakman}

Die Inanspruchnahme von an einer Rechtsverletzung nicht beteiligten Dritten zu deren Abhilfe bzw. Sanktionierung stellt keineswegs ein neuartiges Phänomen dar. Abstrakt betrachtet stellt der Versuch, die Transaktionskosten einer Rechtsbeziehung in den Mittelpunkt der Aufarbeitung juristischer Konflikte zu stellen, insbesondere wenn sich diese Rechtsbeziehung durch das Vorhandensein einer Vielzahl von Individuen auszeichnet, vielmehr „das

147/86, BVerfGE 74, 297 (324f.) - 5. Rundfunkentscheidung; Urt. v. 05.02.1991 - 1 BvF 1/85, BVerfGE 83, 238 (296f.) - 6. Rundfunkentscheidung; Urt. v. 18.07.2018 - 1 BvR 1675/16, NJW 2018, 3223 (Rn. 77 f.) - Rundfunkbeitrag. Zum privaten Rundfunk vgl. die $\S \S 25 \mathrm{ff}$. RStV.

${ }^{9}$ Vgl. hierzu statt vieler Stark/Magin/Jürgens, Ganz meine Meinung?, passim; Paal, Intermediäre, S. 10 ff.; Schwartmann/Hermann/Mühlenbeck, MMR 2019, 498 (499); Drexl, ZUM 2017, $529 \mathrm{ff}$. 
zentrale Paradigma der ökonomischen Analyse des Rechts" dar. ${ }^{10}$ Ohne sich im Rahmen dieser Untersuchung vollständig der Perspektive der ökonomischen Analyse des Rechts verschreiben zu wollen, kann ein Seitenblick auf einen konkreten Ansatz zur theoretischen Begründbarkeit einer solchen Inanspruchnahme Dritter dennoch lohnenswert sein, um den analytischen Rahmen auf den hiesigen Kontext zu schärfen. Ein solcher Ansatz soll hier Gegenstand einer kursorischen Darstellung und Einordnung im Hinblick auf die Intermediäre sein. Dazu ist dieser zunächst kurz zu erläutern (I.) und mit Blick auf diese Untersuchung zu kontextualisieren (II.).

\section{Darstellung}

Kraakman hat bereits 1986 in einer breit rezipierten, in Deutschland soweit ersichtlich allerdings überwiegend im Bereich des Kapitalmarktrechts ${ }^{11}$ thematisierten Studie darauf hingewiesen, dass die Sekundärhaftung in fast jedem Kontext vor allem dazu diene, den ,primary wrongdoer“ von der Begehung weiterer Rechtsverletzungen durch Indienstnahme seiner „associates and market contacts" abzuschrecken. ${ }^{12}$ Die Sekundärhaftung von Gatekeepern zeichne sich dadurch aus, dass die Haftung diese dazu veranlasse, privates Fehlverhalten durch „,withholding support" zu unterbinden, wobei dieser „support" typischerweise eine besondere Ware oder Dienstleistung darstelle, der der für die Begehung der primären Rechtsverletzung essenziell sei. ${ }^{13}$

Er schlägt vier Kriterien vor, um analytisch den Erfolg bzw. die Legitimität von Gatekeeping-Regimen zu bestimmen:

„(1) serious misconduct that practicable penalties cannot deter; (2) missing or inadequate private gatekeeping incentives; (3) gatekeepers who can and will prevent misconduct reliably, regardless of the preferences and market alternatives of wrongdoers; and (4) gatekeepers whom legal rules can induce to detect misconduct at reasonable cost." "14

Die ersten beiden Kriterien betreffen das Vorhandensein oder Fehlen anderweitiger Alternativen jenseits des Gatekeepings selbst, sind also struktureller Natur, während die beiden letzten die inhaltliche Qualität des Gatekeeping selbst betreffen. ${ }^{15}$

\footnotetext{
${ }^{10}$ Eidenmüller, Effizienz als Rechtsprinzip, S. 65.

${ }^{11}$ Vgl. nur Schantz, Die zivilrechtliche Verantwortlichkeit von Ratingagenturen gegenüber Investoren, S. 163 ff., mwN.

${ }^{12}$ Kraakman, Journal of Law, Economics, and Organization 2 (1986), 53 (53).

${ }^{13}$ Kraakman, Journal of Law, Economics, and Organization 2 (1986), 53 (54).

${ }^{14}$ Kraakman, Journal of Law, Economics, and Organization 2 (1986), 53 (61).

${ }^{15} \mathrm{Vgl}$. Kraakman, Journal of Law, Economics, and Organization 2 (1986), 53 (62).
} 
Grundlegend sei bei der abschreckenden Wirkung des Rechts zwischen ex post- und ex ante-Strategien zu unterscheiden. Den expost-Strategien werden hier die klassischen Rechtsdurchsetzungsmechanismen sowohl gegenüber dem Primärverursacher (straf- und zivilrechtlicher Natur) als auch gegenüber dem Gatekeeper zugeordnet. Die ex-ante-Strategien betreffen etwa Szenarien, in denen die Gatekeeper verpflichtet sind bzw. werden, ihrerseits gegen die Primärverursacher vorzugehen oder das hoheitliche Vorgehen gegen jene zu unterstützen. ${ }^{16} \mathrm{Um}$ aber tatsächlich einen ex ante-Effekt zu erreichen, hat Kraakman bereits darauf hingewiesen, dass hierfür ein präskriptiver Mechanismus, eine „enforceable duty“, erforderlich sei, die den Gatekeeper in die Lage versetze, das Fehlverhalten zu unterbinden. ${ }^{17}$

Zugleich beschreibt Kraakman recht präzise auch die Risiken bzw. die Kosten, die mit Gatekeeping-Regimen entstehen können. Insbesondere die von ihm als „tertiary costs“ bezeichneten Risiken für von dem Regime betroffene Dritte stehen praktisch bei jedem Haftungsregime im Raum, mit dem Gatekeeper zur Kontrolle von Verhalten ihrer Nutzer bzw. Kundinnen angehalten werden. ${ }^{18}$ Sei der Sanktionsmechanismus, mit dem der Staat an den Gatekeeper herantrete, nicht richtig kalibriert, stehe ein „over“- oder „undermonitoring “ $z$ u befürchten, ${ }^{19}$ während „simple operational duties“ die Kosten der von den Gatekeepern abverlangten Handlungen klar begrenzten und den Spielraum für eine „,ungewollte Haftung“ (,inadvertent liability“) eng hielten. ${ }^{20} \mathrm{Zu}$ diesen „simple operational duties“ zählt Kraakman auch jene Regelungen, bei denen eine Handlungspflicht in entscheidender Weise auf den Wissensstand des Gatekeepers abstellt. ${ }^{21}$

\section{Kontextualisierung}

Kraakmans Ansatz bezieht sich zwar auf Fehlverhalten im Finanzsektor bzw. generell im Bereich der Wirtschaftskriminalität. Eine Zuordnung des von ihm vorgeschlagenen Rahmens in den Kontext dieser Untersuchung fällt dennoch nicht schwer.

Abstrakt lässt sich die Differenzierung zwischen ex-ante- und ex-postStrategien der in der deutschen Rechtsordnung in verschiedenen Kontexten

${ }^{16}$ Vgl. Kraakman, Journal of Law, Economics, and Organization 2 (1986), 53 (57).

${ }^{17}$ Kraakman, Journal of Law, Economics, and Organization 2 (1986), 53 (57).

${ }^{18}$ Vgl. Kraakman, Journal of Law, Economics, and Organization 2 (1986), 53 (75 ff.).

${ }^{19}$ Vgl. Kraakman, Journal of Law, Economics, and Organization 2 (1986), 53 (76).

${ }^{20}$ Kraakman, Journal of Law, Economics, and Organization 2 (1986), 53 (75 f.).

${ }^{21} \mathrm{Vgl}$. Kraakman, Journal of Law, Economics, and Organization 2 (1986), 53 (76): , , ...] a scienter standard that looks to the gatekeeper's knowledge or intent is the limiting case, because it restricts liability to misconduct that gatekeepers detect even without a monitoring duty." 
geläufigen Unterteilung der Durchsetzung rechtlicher Pflichten auf die Primär- und Sekundärebene zuordnen. Diese Unterteilung betrifft grob gesprochen die Frage, wann diese Durchsetzung personell oder sachlich einen den Kern der rechtlichen Pflicht unmittelbar betreffenden Aspekt oder eher eine Folgefrage adressieren darf bzw. soll. Hier spielen oftmals Aspekte der Effizienz, der gerechten Verteilung rechtlicher Risiken und damit letztlich das Verhältnismäßigkeitsprinzip eine Rolle - all diese Aspekte sind auch im hiesigen Kontext von entscheidender Bedeutung.

Konkret ist anzumerken, dass die Indienstnahme von Intermediären nach Kraakman sowohl Elemente der ex-ante- als auch der ex-post-Strategie aufgreift. Zum einen kommt die ex-ante-Sicht hier insoweit zur Geltung, als die Regelungen zur Haftung der Intermediäre insbesondere die Handlungsanforderungen als Reaktion auf eine Kenntnisnahme von rechtswidrigen Inhalten betreffen. Als solche sind diese Regelungen bereits prozedural ausgestaltet. Hier lässt sich der Bogen bis hin zu aktuellen Urteilen des BGH schlagen, der in seiner Rechtsprechung immer wieder danach strebt, ein handhabbares Verfahren für den Umgang mit Beschwerden von Nutzern über bestimmte Inhalte durch Diensteanbieter zu etablieren. ${ }^{22}$ Zum anderen ist die ex-ante-Ebene insoweit angesprochen, als die ggf. zulässigen Verhinderungspflichten, die insbesondere im Kontext des Art. 15 ECRL diskutiert werden, nicht mehr auf eine rein reaktive, dem dialektischen Konflikt verhaftete Lösung der Konflikte abstellen, sondern diese Konflikte durch in die Zukunft gerichtete Verhaltensobliegenheiten aktiv zu vermeiden suchen. Hierbei geht es gerade um die Existenz und die Reichweite einer ,enforceable duty" im Sinne von Kraakman.

Das von ihm beschriebene Problem des „over-/undermonitoring“ findet ebenfalls seine Entsprechung im Kontext der Intermediäre. Hier geht es unmittelbar um die richtige oder falsche Anreizsetzung. Liege letztere vor, stehe $\mathrm{zu}$ befürchten, dass der Gatekeeper seine eigenen Risiken auf Kosten seines „client" reduzieren wollen könnte und deshalb zu „over-monitoring“ oder „over-enforcement" verlockt sein könnte. ${ }^{23}$ Dies beschreibt punktgenau den Kern der aktuellen Debatten und der juristischen Kontroversen um das Haftungsregime für Intermediäre.

Einer kritischen Erörterung bedarf allerdings die Behauptung von Kraakman, ein an den Wissensstand des Gatekeepers anknüpfender Haftungsstandard stelle einen eng begrenzten und damit grundsätzlich „kostenarmen" Sorgfaltsmaßstab dar. Das ist einerseits einleuchtend, weil der Gatekeeper nicht mehr zu tun braucht, als sich gleichsam dauerhaft selbst auf eine

${ }^{22}$ Vgl. die Leitsätze in BGH, Urt. v. 01.03.2016 - VI ZR 34/15, NJW 2016, 2106 ff. Ärztebewertungsportal III.

${ }^{23}$ Kraakman, Journal of Law, Economics, and Organization 2 (1986), 53 (77). 
vorhandene Kenntnis zu befragen. Andererseits übergeht diese Auffassung aber, dass der Anreiz hierzu die gegenläufigen Motive eines GatekeepingRegimes ggf. zu unterlaufen droht, weil und soweit er es dem Gatekeeper ermöglicht, die Augen vor der Realität zu verschließen, um eine Kenntnis zu verhindern. ${ }^{24}$ Ferner stellen sich insoweit ebenso Beweisprobleme. Das legt bereits auf dieser abstrakten Ebene nahe, dass eine zu strenge Fokussierung auf einen hohen Kenntnisstandard in diesem Kontext ggf. zu unangemessenen Ergebnissen führen kann. Ferner lassen sich teilweise auch Phänomene beobachten, mit denen Kraakman vermutlich nicht gerechnet hätte. So stellt etwa $§ 230$ CDA die von der Regelung erfassten Diensteanbieter von der Haftung frei, während der Gesetzgeber aber mehr oder minder explizit ein freiwilliges Monitoring erwartete. Das Gesetz installiert damit kein Gatekeeping-Regime, jedenfalls keines im Sinne von Kraakman, sondern stellt eine Kontrolle von Inhalten in das technische bzw. unternehmerisch determinierte Belieben der betroffenen Diensteanbieter. ${ }^{25}$

Kraakmans Ansatz stellt damit insgesamt einen passenden ersten analytischen Zugriff auf das Problem dar, weil er aufgrund seines Abstraktionsniveaus geeignet ist, die Motivlagen und Herausforderungen der Inpflichtnahme der Intermediäre als „Gatekeeper“ aus den konkreten, teilweise recht komplexen Regelungsregimes herauszuarbeiten. Insbesondere ihre Fähigkeit, die Verwirklichung von Fehlverhalten (,misconduct“) zu unterbinden oder nachhaltig zu stören ist bei der Motivlage von besonderer Bedeutung. Auch die intrinsische Motivation zur Unterbindung bzw. Abwehr von Störungen, nämlich zum Schutz der eigenen Reputation, hat Kraakman zutreffend unterstrichen. ${ }^{26}$ Schließlich sind die möglichen Herausforderungen und Implikationen, wie sie sich hier stellen, bei Kraakman ebenfalls benannt. Eine Verortung der im Kontext dieser Untersuchung thematisierten konkreten rechtlichen Fragestellungen in seinem analytischen Rahmen kann damit also lohnenswert sein. Dies wird sich im Verlauf der weiteren Abschnitte noch konkret zeigen.

${ }^{24}$ Zur Konkretisierung dieser Einsicht am Beispiel der Störerhaftung vgl. unten $\S 10$ B. IV. 1. b).

${ }^{25}$ Vgl. Zittrain, Harvard Journal of Law \& Technology 19 (2006), 253 (262 f.).

${ }^{26}$ Vgl. Kraakman, Journal of Law, Economics, and Organization 2 (1986), 53 (56), obschon er diese „reputational concerns“ als ggf. hinreichend beschreibt, um ohne gesetzliche Intervention störendes Verhalten zu unterbinden (zur Relevanz dieses Aspekts für den Wettbewerb zwischen Diensteanbietern vgl. oben $\S 8$ Fn. 44 und den zugehörigen Text). Freilich hängt die Relevanz des (befürchteten) Reputationsschadens immer auch von einer Kosten-Nutzen-Rechnung ab, bei der die Vorteile eines „laissez-faire“-Ansatzes überwiegen können. 


\section{Fazit}

In der übergeordneten, theoretischen Perspektive auf die Bedeutung von Gatekeepern wird deren verstärkte Inpflichtnahme als Symptom einer allgemeineren Entwicklung gesehen. Nach Ansicht mancher Stimmen ist hiernach die Handlungsfähigkeit hoheitlicher Akteure aus verschiedenen Gründen im Niedergang begriffen und stellt die dezentrale Indienstnahme privater Akteure zur Verfolgung hoheitlicher Interessen die logische und zugleich kritikwürdige Konsequenz dieses Umstandes dar. ${ }^{27}$ Insoweit ist unbestritten, dass Gatekeeping-Regimen ein Unsicherheitspotenzial immanent ist, weil sie durch die Indienstnahme eines dritten Akteurs zur Bewältigung von Konflikten, mit denen diese im Ausgangspunkt nichts zu tun haben, rechtliche Irritationen auslösen können. Dieser Umstand sollte aber nicht dazu führen, die Intermediäre als zentrale, die Kommunikation großer Teile der Öffentlichkeit maßgeblich strukturierende Instanzen zu übergehen. Vielmehr ist es gerade die Herausforderung für jede Rechtsordnung, die durchaus mehrdimensionalen Kosten dieses Unsicherheitspotenzials durch juristische Feinsteuerung möglichst klein zu halten. ${ }^{28}$ Genau dies ist die Herausforderung, die mit jedem Regelungsregime verbunden ist, das die Intermediäre als potenzielle Anspruchsgegner für die Verbreitung von Rechtsverletzungen benennt. Dies wird im Blick zu behalten sein, wenn nun im Folgenden der Fokus den konkreten rechtlichen Regelungen und Instrumentarien gilt, die diese Thematik unmittelbar betreffen.

\footnotetext{
${ }^{27}$ Vgl. Black, Current Legal Problems 54 (2001), 103 (105 ff.); Laidlaw, Regulating Speech in Cyberspace, S. 39 f. Der insoweit diagnostizierte Mangel öffentlicher Rechenschaft, der die Legitimität der Tätigkeit der Intermediäre als Gatekeeper in Frage stellt, ist bei Lichte besehen jedoch kein kategorial neues Problem demokratischer Legitimation, sondern verschärft allenfalls die überkommene Perspektive, die der Ausübung öffentlicher Funktionen durch nichtstaatliche Akteure schon grundsätzlich kritisch gegenübersteht. Der von Black präsentierte Gegensatz zwischen der ,decentred regulation“ und dem „,command and control"-Ansatz, dessen sich typischerweise hoheitliche Akteure bedienen (105f.), überzeugt bei der Betrachtung der Regulierung von Kommunikation nicht gänzlich, weil die Beilegung von äußerungsrechtlichen Konflikten durch die unabhängige Judikative institutionell einem solchen Ansatz nicht zugeordnet werden kann.

${ }^{28}$ So auch schon Kraakman, Journal of Law, Economics, and Organization 2 (1986), 53 (78).
} 


\title{
Die Störerhaftung als kontextangemessenes Instrument des bürgerlichen Rechts zur Realisierung der Verantwortung der Intermediäre?
}

\begin{abstract}
Nachdem die Grundlagen für einen kritischen Blick auf die Inpflichtnahme von Intermediären gelegt wurden, soll nun die Störerhaftung als bedeutsames Instrument der deutschen Rechtsordnung hierzu im Fokus stehen. Dieses von der Rechtsprechung geschaffene Rechtsinstitut hat diese in jüngerer Zeit prominent fortentwickelt, um auf Konflikte verschiedener Natur unter Beteiligung von Intermediären flexibel zu reagieren. Dementsprechend zeichnet sich die Störerhaftung auch durch kontextspezifische, teilweise recht unterschiedliche Voraussetzungen und eine kontextabhängig unterschiedliche Handhabung aus.
\end{abstract}

\section{A. Grundlagen der Störerhaftung}

Sofern der Intermediär nicht als Täter haftet, weil er sich etwa keinen Inhalt zu Eigen gemacht hat, und auch keine Teilnehmerhaftung in Betracht kommt, weil es insbesondere am Vorsatz im Hinblick auf die Haupttat fehlt, kommt noch eine Haftung auf Unterlassung ${ }^{1}$ als Störer in Betracht. ${ }^{2}$ Als Störer kann „bei der Verletzung absoluter Rechte auf Unterlassung in Anspruch genommen werden, wer - ohne Täter oder Teilnehmer zu sein - in irgendeiner Weise willentlich und adäquat kausal zur Verletzung des geschützten Rechts beiträgt" “. ${ }^{3}$ Die Störerhaftung ist von der Rechtsprechung in analoger Anwendung des $\S 1004$ BGB entwickelt worden. ${ }^{4}$ Sie stellt eine

\footnotetext{
${ }^{1}$ Vgl. Hofmann, JuS 2017, 713 (715).

${ }^{2}$ Vgl. BGH, Urt. v. 18.10.2001 - I ZR 22/99, GRUR 2002, 618 (619) - Meißner Dekor; Urt. v. 15.10.1998 - I ZR 120/96, NJW 1990, 1960 (1960) - Möbelklassiker.

${ }^{3}$ BGH, Urt. v. 22.07.2010 - I ZR 139/08, GRUR 2011, 152 (Rn. 45) - Kinderhochstühle im Internet I; vgl. ferner Urt. v. 18.10.2001 - I ZR 22/99, GRUR 2002, 618 (619) Meißner Dekor; Urt. v. 06.07.1954 - I ZR 38/53, NJW 1954, 1682 (1683). Das Institut der Störerhaftung reicht bis zu RG, Urt. v. 18.10.1919 - V 97/19, RGZ 97, 25 (26), zurück.

${ }^{4}$ Vgl. Ohly, ZUM 2015, 308 (311).
} 
grundsätzlich eigenständige Beteiligungsform dar, die sich nicht in die tradierten Kategorien der Täterschaft und Teilnahme einpassen lässt. ${ }^{5}$

Zunächst gilt es, den Zweck der Störerhaftung näher zu bestimmen (I.). Sodann ist auf ihren Anwendungsbereich einzugehen (II.). Anschließend können dann ihre genauen Voraussetzungen und die aus der Störerhaftung abgeleiteten Prüfpflichten erörtert werden, die für die Thematik dieser Untersuchung von Bedeutung sind (III.). Schließlich ist der Fokus noch auf die konkreten Rechtsfolgen der Störerhaftung (IV.) und auf ihre Anwendbarkeit auf die Haftungsprivilegierungen (V.) zu lenken.

\section{Zweck}

Es wurde bereits angemerkt, dass die Störerhaftung als eigenes Haftungsinstrument gerade nicht den Kategorien der Täterschaft oder der Teilnahme zuordenbar ist. Dies soll sie nach ihrem Zweck auch gar nicht, da sie gerade darauf ausgelegt ist, den Kreis der möglichen Passivlegitimierten für Rechtsverletzungen, die von Dritten begangen werden, auf jene Akteure auszuweiten, die in einem besonderen Näheverhältnis zu deren Handlungen stehen und deren Inanspruchnahme deshalb als angemessener Weg angesehen wird, um die Rechtsverletzung selbst oder deren Folgen effektiv adressieren zu können. Der Zweck der Störerhaftung wird denn auch oftmals in ihrem ökonomischen Wert gesehen, nicht den Aufwand der Rechtsverfolgung gegenüber dem Primärverursacher als Täter betreiben zu müssen, ${ }^{6}$ der dessen Ermittlung ebenso beinhaltet wie das höhere Risiko dessen Mittellosigkeit. Dieser Mehrwert wird nicht nur von Vertretern der ökonomischen Analyse des Rechts oftmals mit dem Schlagwort „cheapest cost avoider“" verknüpft.?

Nicht ganz eindeutig ist der innere, rechtliche Legitimationsgrund für die Zurechnung des Verhaltens des rechtsverletzenden Dritten zum Dienstean-

${ }^{5}$ Vgl. Schapiro, Unterlassungsansprüche gegen die Betreiber von Internet-Auktionshäusern und Internet-Meinungsforen, S. $32 \mathrm{f}$.

${ }^{6}$ Vgl. Chmelik, Social Network Sites - Soziale Netzwerke, S. $154 \mathrm{f}$.

${ }^{7}$ Der Begriff geht zurück auf Calabresi, The Costs of Accidents, S. $135 \mathrm{ff}$. , der als Mitbegründer der Disziplin „Law and Economics“ gilt; vgl. ferner Hofmann, JuS 2017, 713 (717); Leistner, ZUM 2012, 722 (723); mit Bezug auf Calebresi ferner auch Sylvain, Connecticut Law Review 50 (2018), 203 (244). Die Sinnhaftigkeit dieses Ansatzes wird nicht allseits geteilt; sehr kritisch etwa Hindelang, Freiheit und Kommunikation, S. 317, der diese Einordnung generell mit dem „Overblocking“-Effekt (S. 339 f., 367) bzw. der Einordnung der Intermediäre als ,,private Internetkontroll- und Vollstreckungsinstanz“ (S. 341) gleichzusetzen scheint. Dies trifft allerdings nicht zu, da der Ansatz als rechtsökonomischer (vgl. Ohly, ZUM 2015, 308 (309)) sich schon per se nicht zu diesen materiellen Argumentationsmustern verhält. Diensteanbieter können die „,cheapest cost avoider“ sein und dennoch kann man mit entsprechender Argumentation sich ihrer Inanspruchnahme entgegenstellen. 
bieter im Rahmen der Störerhaftung auf Unterlassen. Denkbar sind sowohl eine Anknüpfung an ein aktives Verhalten des Diensteanbieters selbst, als auch eine Zurechnung aus seiner Beherrschung einer Störungsquelle. ${ }^{8}$ Dabei ist nur auf den ersten Blick die Anknüpfung an ein aktives Verhalten schlechthin undenkbar. Üblicherweise versteht man zwar nur solche Handlungsweisen als tauglichen Anknüpfungspunkt, bei denen die eintretende Störung als eine „typische, zu erwartende Folge des Verhaltens des mittelbaren Störers darstellt. “9 Denn geht es bei der Störerhaftung um den Beitrag, den der in Anspruch genommene zur Verletzung des Rechts leistet, so kann ein solcher bei Intermediären durchaus in der Verbreitung rechtsverletzender Inhalte gesehen werden, die ein solches aktives Tun darstellt. ${ }^{10}$ Letztlich ist es aber ebenso überzeugend, dem Diensteanbieter eine beherrschende Stellung über rechtsverletzende Inhalte als Störungsquelle zuzuschreiben. ${ }^{11}$

${ }^{8}$ Vgl. Sokol, Die Bestimmung der Verantwortlichkeit für die Abwehr und Beseitigung von Störungen im öffentlichen und privaten Recht, S. 48 ff.

${ }^{9}$ Sokol, Die Bestimmung der Verantwortlichkeit für die Abwehr und Beseitigung von Störungen im öffentlichen und privaten Recht, S. 50.

${ }^{10}$ Insoweit bietet sich eine Parallele zur presserechtlich tradierten Verbreiterhaftung an. Die Verbreiterhaftung beruht dort auf der weitgehenden Gleichstellung von Behauptung und Verbreitung im äußerungsrechtlichen Sinne, wie sie in $§ 824$ BGB ebenso zur Geltung kommt wie in den $\S \S 186,187$ StGB, vgl. Soehring, in: Soehring/Hoene, Presserecht, $§ 16$ Rn. 14. Im Prinzip kann der Verbreiter ebenso für den Inhalt zu Verantwortung gezogen werden wie derjenige, der die Behauptung erst aufstellt, vgl. BGH, Urt. v. 03.02.1976 - VI ZR 23/72, NJW 1976, 799 (800) - Alleinimporteur; Urt. v. 26.11.1996 - VI ZR 323/95, NJW 1997, 1148 (1149) - Stern-TV. Bei der Verbreiterhaftung existiert im Grundsatz keine generelle Haftungsprivilegierung, vielmehr operiert die Rechtsprechung hier mit an einzelne Handlungsweisen bzw. Funktionen von Medien anknüpfenden Rechtsfolgen, die sich insbesondere an Gesichtspunkten der Zumutbarkeit orientieren, vgl. Burkhardt/Peifer, in: Wenzel, Das Recht der Wort- und Bildberichterstattung, Kap. 10 Rn. 207. Letztlich gelten bei der Verbreiterhaftung aber ebenso Prüfpflichten, vgl. BGH, Urt. v. 30.06.1972 I ZR 1/71, GRUR 1973, 203 (204) - Badische Rundschau, sowie generelle Haftungseinschränkungen zur „Wahrnehmung berechtigter Interessen“ (vgl. §193 StGB; Urt. v. 30.01.1996 - VI ZR 386/94, NJW 1996, 1131 (1133)) und zur Dokumentation eines Meinungsstands im Sinne eines Pressespiegels (vgl. Urt. v. 30.01.1996 - VI ZR 386/94, NJW 1996, 1131 (1132)). Der entscheidende Unterschied zwischen Verbreiter- und Störerhaftung liegt vor allen Dingen in dem Umstand begründet, dass Presseunternehmen in der Regel alle abgedruckten Inhalte, auch Werbeanzeigen, zur Kenntnis nehmen, bevor sie veröffentlicht werden, während bei der Inanspruchnahme von Intermediären diese Behauptung in der Regel nicht aufgestellt werden kann.

${ }^{11}$ So auch Sokol, Die Bestimmung der Verantwortlichkeit für die Abwehr und Beseitigung von Störungen im öffentlichen und privaten Recht, S. $55 \mathrm{f}$. 


\section{Anwendungsbereich}

\section{Allgemeines}

Generell ist der Anwendungsbereich der Störerhaftung nicht auf einen bestimmten Regelungsbereich beschränkt, sondern als übergreifender Grundsatz anerkannt. ${ }^{12}$ Dennoch verweist sie schon aufgrund ihrer Anknüpfung an die Störung, also die Verletzung rechtlich geschützter Güter, auf sehr verschiedene Interessen- und Konfliktlagen. Für die Bestimmung ihrer konkreten Reichweite macht es einen Unterschied, ob sie gegenüber einer Markenrechts-, Urheberrechts- oder Persönlichkeitsrechtsverletzung in Anschlag gebracht werden soll. ${ }^{13} \mathrm{Im}$ Folgenden liegt der Schwerpunkt auf Persönlichkeitsrechtsverletzungen, wobei Einsichten aus anderen Regelungsbereichen zur Kontrastierung mitunter hilfreich sind. Dieser Schwerpunkt begründet sich vor allem mit der besonderen Interessenlage, die bei Persönlichkeitsrechtsverletzungen um eine bedeutende Facette erweitert ist, wenn und soweit der Äußernde (Primärverursacher) sich auf grundrechtlich verbürgte Kommunikationsfreiheiten berufen kann. ${ }^{14}$

\section{Keine Haftung des Intermediärs als Täter}

Die Störerhaftung greift grundsätzlich nur ein, wenn es sich um fremde Informationen handelt, für die der Intermediär in Anspruch genommen werden soll. Täter- und Störerhaftung schließen sich demnach aus. ${ }^{15}$ Die Rechtsprechungslinie, wonach eine womöglich auch äußerlich erkennbar fremde Information dennoch eine eigene im Sinne von $\S 7$ TMG sein kann, sofern der Betreiber sich diese zu Eigen gemacht hat, ${ }^{16}$ greift auch hier. Die Frage des Zueigenmachens ist bei der Prüfung daher zuerst zu beantworten, bevor die Störerhaftung überhaupt in Betracht kommt. ${ }^{17}$

Dem steht nur scheinbar eine Meinungsverschiedenheit zwischen zwei Zivilsenaten des BGH entgegen, die ein grundsätzlich unterschiedliches Verständnis der Begriffe „Täter“ und „Störer“ vertreten. Während für den I. Zi-

${ }^{12}$ Vgl. nur Klein, Haftung von Social-Sharing-Plattformen, S. 100.

${ }^{13}$ Vgl. Chmelik, Social Network Sites - Soziale Netzwerke, S. 167.

${ }^{14}$ Dies zeigt sich später insbesondere bei prozeduralen Elementen, die der BGH in jüngerer Zeit in die Störerhaftung integriert hat, um auch diese Rechtsposition zu stärken; vgl. dazu noch unten $\S 10$ A. III. 2. c).

${ }^{15}$ Vgl. Hofmann, JuS 2017, 713 (717); überaus kritisch zum Verhältnis von Täter- und Störerhaftung im System des BGB Schapiro, Unterlassungsansprüche gegen die Betreiber von Internet-Auktionshäusern und Internet-Meinungsforen, S. $95 \mathrm{ff}$.

${ }^{16} \mathrm{Vgl}$. oben $\S 4$ D. I. 2.

${ }^{17}$ Vgl. in diesem Sinne KG, Beschl. v. 10.07.2009 - 9 W 119/08, MMR 2010, 203 (203 f.); Hofmann, JuS 2017, 713 (717 f.). 
vilsenat nur der mittelbare Verursacher „Störer“ sein kann,,$^{18}$ verwendet der VI. Zivilsenat einen deutlich weiteren Störerbegriff, der sowohl Fälle der unmittelbaren Verursachung als Täter als auch Fälle der mittelbaren Verursachung unter den Störerbegriff subsumiert. ${ }^{19}$ Der Grund für diese Divergenz ist primär in den in aller Regel zur Verfügung stehenden Anspruchsgrundlagen zu sehen, mit denen die beiden Senate operieren. ${ }^{20}$ Beim VI. Zivilsenat ist dies maßgeblich der $§ 1004$ BGB, der unzweifelhaft sowohl den unmittelbaren als auch den mittelbaren Verursachungsbeitrag unter den Störerbegriff fasst, während der I. Zivilsenat oftmals auf spezialgesetzliche Haftungstatbestände zurückgreifen muss, die der Sache nach ebenfalls „mittelbare“ Verursachungsbeiträge regeln, aber aufgrund ihrer Qualität als Spezialgesetz eine Bezeichnung des Schuldners nur als Täter zulassen.

Im hiesigen Kontext der Störerhaftung geht es ausschließlich um mittelbare Verursachungsbeiträge, weshalb eine Haftung für eigene Inhalte in diesem Kontext insoweit nicht weiter interessiert. Daran ändert auch die bereits angesprochene ${ }^{21}$ Autocomplete-Entscheidung nichts, in der der BGH zwar annimmt, die Vervollständigungen der Suchvorschläge seien eigene Informationen von Google, ${ }^{22}$ dann aber dennoch auf Maßstäbe der Störerhaftung zurückgreift. ${ }^{23}$ Dies erklärt sich in dem dort verhandelten Fall durch die Auffassung des BGH, nicht an eine aktive Handlung des Suchmaschinenbetreibers anknüpfen zu können, da diese nur in dem - grundrechtlich geschützten - Betrieb der Suchmaschine zu sehen sei. Daher könne nur an ein Unterlassen von besonderen Vorkehrungen zur Verhinderung von Rechtsverletzungen angeknüpft werden. ${ }^{24}$ Nur so gelangt der BGH zu der Frage, ob Verhinderungsmaßnahmen möglich und zumutbar sind, die dann den Zugriff auf die Rechtsprechung zu den Prüfpflichten erlaubt, der systematisch bei der Anknüpfung an eine aktive Handlung versperrt gewesen wäre. ${ }^{25}$ Ungeachtet der

${ }^{18}$ Besonders deutlich bei BGH, Urt. v. 19.04.2007 - I ZR 35/04, NJW 2007, 2636 (Rn. 34) - Internet-Versteigerung II; vgl. auch Urt. v. 12.07.2012 - I ZR 18/11, NJW 2013, 784 (Rn. 19) - Alone in the Dark.

${ }^{19}$ Vgl. jüngst BGH, Urt. v. 04.04.2017 - VI ZR 123/16, NJW 2017, 2029 (Rn. 18) klinikbewertungen.de; Urt. v. 01.03.2016 - VI ZR 34/15, NJW 2016, 2106 (Rn. 17, 22) Ärztebewertungsportal III.

${ }^{20}$ So auch Ingendaay, AfP 2011, 126 (127); Pentz, AfP 2014, 8 (16).

${ }^{21} \mathrm{Vgl}$. oben $\S 4$ D. I. 1. b).

${ }^{22}$ Vgl. BGH, Urt. v. 14.05.2013 - VI ZR 269/12, NJW 2013, 2348 (Rn. 17) - Autocomplete.

${ }^{23}$ Vgl. BGH, Urt. v. 14.05.2013 - VI ZR 269/12, NJW 2013, 2348 (Rn. 24) - Autocomplete.

${ }^{24}$ Vgl. BGH, Urt. v. 14.05.2013 - VI ZR 269/12, NJW 2013, 2348 (Rn. 26) - Autocomplete; vgl. auch Pentz, AfP 2014, 8 (16f.).

${ }^{25}$ Vgl. BGH, Urt. v. 14.05.2013 - VI ZR 269/12, NJW 2013, 2348 (Rn. 27 ff.) - Autocomplete. 
Skepsis, die diese Konstruktion über die Haftung wegen eines Unterlassens hervorruft ${ }^{26}$ ist sie insgesamt als Sonderfall einzuordnen und daher hier nicht von weitergehender Bedeutung.

\section{Keine Subsidiarität gegenüber Ansprüchen gegen den Primärverursacher}

Für persönlichkeitsrechtsverletzende Inhalte in Meinungsforen hat der BGH wiederholt festgestellt, dass der Anspruch aus Störerhaftung nicht schon deshalb nicht in Betracht kommt, weil die Identität des Primärverursachers bekannt ist. ${ }^{27}$ Denn im Internet sei eine die Störung beherrschende Stellung eines Diensteanbieters als „Herr des Angebots“ ähnlich wie bei der Verbreiterhaftung der Presse möglich, sodass es auf Ansprüche gegen den Primärverursacher insoweit nicht ankomme. ${ }^{28}$ Andernfalls sei auch ein „,wirksamer und umfassender Schutz" nicht zu erreichen. ${ }^{29}$ Der EGMR scheint dies in der Sache ähnlich zu sehen. ${ }^{30}$

Die zur Störerhaftung des Access-Providers vertretene Auffassung, dass in diesem Szenario tatsächlich ein Subsidiaritätsverhältnis anzunehmen sei, ${ }^{31}$ hat der BGH später explizit als Ausnahme bewertet. ${ }^{32}$ Bemerkenswert erscheint hier aber die Begründung, die Annahme eines Subsidiaritätsverhältnisses sei erforderlich, um das ,von der Rechtsordnung [gebilligte] und in Bezug auf Rechtsverletzungen Dritter [neutrale] Geschäftsmodell“ zu schützen. ${ }^{33}$ Denn diese Argumentation taucht im Zusammenhang mit der Störerhaftung nicht nur betreffend Access-Provider auf, sondern gehört zum übergreifenden Argumentationstopos der Rechtsprechung zur Störerhaftung. ${ }^{34}$ Danach lassen sich diese Eigenschaften auch Kommunikationsdiensten oder

${ }^{26}$ Vgl. insoweit Gounalakis, NJW 2013, 2321 (2323); kritisch auch Volkmann, in: Spindler/Schuster, Recht der elektronischen Medien, BGB § 1004 Rn. 18.

${ }^{27}$ Vgl. BGH, Urt. v. 10.01.2019 - I ZR 267/15, MMR 2019, 522 (Rn. 94) - Cordoba II; Urt. v. 27.02.2018 - VI ZR 489/16, NJW 2018, 2324 (Rn.45) - Internetforum; Urt. v. 27.03.2007 - VI ZR 101/06, NJW 2007, 2558 (Rn. 13).

${ }^{28}$ Vgl. BGH, Urt. v. 27.03.2007 - VI ZR 101/06, NJW 2007, 2558 (Rn. 13).

${ }^{29}$ BGH, Urt. v. 27.02.2018 - VI ZR 489/16, NJW 2018, 2324 (Rn. 45) - Internetforum (zur Haftung eines Suchmaschinenbetreibers).

${ }^{30}$ Vgl. EGMR, Urt. v. 16.06.2015 - 64569/09, NJW 2015, 2863 (Rn. 151) - Delfi.

${ }^{31}$ Vgl. BGH, Urt. v. 26.11.2015 - I ZR 174/14, NJW 2016, 794 (Rn. 81 ff.) - AccessProvider.

${ }^{32}$ Vgl. BGH, Urt. v. 10.01.2019 - I ZR 267/15, MMR 2019, 522 (Rn. 94) - Cordoba II.

${ }^{33}$ BGH, Urt. v. 10.01.2019 - I ZR 267/15, MMR 2019, 522 (Rn. 94) - Cordoba II; vgl. dazu noch sogleich $\S 10$ A. III. 2. b) cc).

${ }^{34}$ Vgl. BGH, Urt. v. 16.05.2013 - I ZR 216/11, MMR 2014, 55 (Rn. 47) - Kinderhochstühle im Internet II; Urt. v. 18.11.2010 - I ZR 155/09, MMR 2011, 459 (Rn. 45) - Sedo; Urt. v. 22.07.2010 - I ZR 139/08, GRUR 2011, 152 (Rn. 38) - Kinderhochstühle im Internet I, mwN. 
Suchmaschinen zuschreiben. ${ }^{35}$ Insoweit leuchtet es nicht ein, die Frage nach der Subsidiarität der Störerhaftung nur auf diese Erwägung zu stützen und gerade hierin den ausschließlich die Access-Provider betreffenden Sonderfall auszumachen.

Der grundsätzlichen Position der Rechtsprechung, dass ein Subsidiaritätsverhältnis zwischen der Inanspruchnahme des Primärverursachers der Störung und der Inpflichtnahme des Diensteanbieters als Störer nicht besteht, ist grundsätzlich beizupflichten. ${ }^{36}$ Angesichts einer den Nutzern von Diensten grundsätzlich zugebilligten Anonymität, ${ }^{37}$ die allerdings durch die Einfügung der §14 Abs. 3-5 TMG im Rahmen der Verabschiedung des NetzDG eine Relativierung erfahren hat ${ }^{38}$ stünden von Rechtsverletzungen betroffene Nutzer bei Annahme eines generellen Subsidiaritätsverhältnisses vor erheblichen Schwierigkeiten bei der effektiven Wahrnehmung ihrer Rechte. Dies scheint sowohl der EuGH, der in der Google Spain-Entscheidung auf das Risiko hinwies, das sich für einen effektiven Grundrechtsschutz ergebe, wenn zuvor oder parallel die Primärverursacher in Anspruch genommen werden müssten, ${ }^{39}$ als auch das BVerfG ähnlich zu sehen. Letzteres weist in seiner jüngeren Entscheidung Recht auf Vergessen II darauf hin, dass die Beurteilung der Rechtmäßigkeit der Handlung des Diensteanbieters nicht in der Rechtmäßigkeit des ursprünglichen Beitrags des „Content-Providers“ aufgehe ${ }^{40}$ und eine Inanspruchnahme des Intermediärs auch unter Gesichtspunkten der Effizienz gleich- bzw. vorrangig sein könne. ${ }^{41}$

${ }^{35}$ Dies sah OLG Schleswig, Beschl. v. 03.07.2017 - 9 U 30/17, MMR 2018, 249 (Rn. 3), genauso.

${ }^{36}$ Anders aber Teile des Schrifttums, wo die Subsidiarität als Ausdruck des Verhältnismäßigkeitsprinzips angesehen wird, vgl. Hindelang, Freiheit und Kommunikation, S. 321 f.; Nolte/Wimmers, GRUR 2014, 16 (24 mit Fn. 123); im Ergebnis auch Chmelik, Social Network Sites - Soziale Netzwerke, S. 166; Peifer, AfP 2015, 193 (199); unklar insoweit Hofmann, ZUM 2017, 102 (106). In der unterinstanzlichen Rechtsprechung wurde teilweise ebenfalls in diesem Sinne entschieden, vgl. OLG Schleswig, Beschl. v. 03.07.2017 - 9 U 30/17, MMR 2018, 249 (Rn. 3). Wie hier hingegen Pille, NJW 2018, 3545 (3546).

${ }^{37} \mathrm{Vgl} . \S 13$ Abs. 6 TMG.

${ }^{38}$ Über deren Sinnhaftigkeit lässt sich jedoch durchaus streiten. Die Kritik entzündet sich u.a. an der fehlenden Pflicht der Diensteanbieter, die Identität der Nutzer zu erfassen, ohne die ein Auskunftsanspruch ins Leere geht, vgl. Pille, NJW 2018, 3545 (3546); Spindler, MMR 2018, 48 (52).

${ }^{39}$ Vgl. EuGH, Urt. v. 13.05.2014 - C-131/12, NJW 2014, 2257 (Rn. 82 ff.) - Google Spain SL.

${ }^{40}$ Vgl. BVerfG, Beschl. v. 06.11.2019 - 1 BvR 276/17, NVwZ 2020, 63 (Rn. 112) - Recht auf Vergessen II.

${ }^{41}$ Vgl. BVerfG, Beschl. v. 06.11.2019 - 1 BvR 276/17, NVwZ 2020, 63 (Rn. 119) - Recht auf Vergessen II. 
Eine für die grundsätzliche Subsidiarität sprechende legislative Entscheidung kann auch nicht $\$ 7$ Abs. 4 TMG entnommen werden. Denn diese Regelung gilt aufgrund ihres Verweises auf $\S 8$ Abs. 3 TMG nur für WLANBetreiber im Falle von Verletzungen des geistigen Eigentums und betrifft damit letztlich den Ausnahmefall der Rechtsprechung, den der BGH auch als solchen bezeichnet hatte. ${ }^{42}$

\section{Voraussetzungen der Störerhaftung unter besonderer Berücksichtigung der Intermediäre}

Sodann sei der Fokus auf die Voraussetzungen der Störerhaftung gelenkt. Zum einen kommt es auf die Identifizierung relevanter Störungshandlungen an (1.). Zum anderen sind hier die richterrechtlich entwickelten Prüfpflichten von besonderer Bedeutung, da diese die Verhaltensanforderungen an die Intermediäre konkretisierend ausgestalten (2.).

\section{Relevante Störungshandlungen}

Wie bereits ausgeführt, kann als Störer auf Unterlassung in Anspruch genommen werden, ,wer - ohne Täter oder Teilnehmer zu sein - in irgendeiner Weise willentlich und adäquat kausal zur Verletzung des geschützten Rechts beiträgt" ". ${ }^{43}$ Als tauglicher Beitrag zählt nach der Rechtsprechung des BGH die „Unterstützung oder die Ausnutzung der Handlung eines eigenverantwortlich handelnden Dritten, sofern der in Anspruch Genommene die rechtliche Möglichkeit zur Verhinderung dieser Handlung hatte". ${ }^{44}$

Die Tätigkeit der Intermediäre erfüllt regelmäßig diese grundlegende Voraussetzung der Störerhaftung, da in dem Bereitstellen der technischen und kommunikativen Infrastruktur ein adäquat-kausaler Beitrag zu einem rechtsverletzenden Verhalten eines Nutzers des Dienstes gesehen werden kann. Dieser ist in der Regel auch als willentlich zu bewerten. Insoweit kommt es nämlich nicht auf den Willen zur konkreten Rechtsverletzung an, ${ }^{45}$

\footnotetext{
${ }^{42}$ Vgl. oben $\S 10$ Fn. 32; kritisch insoweit mit Blick auf die Rechtsprechung des EuGH Spindler, MMR 2018, 48 (51).

${ }^{43}$ BGH, Urt. v. 22.07.2010 - I ZR 139/08, GRUR 2011, 152 (Rn. 45) - Kinderhochstühle im Internet I.

${ }^{44}$ BGH, Urt. v. 28.07.2015 - VI ZR 340/14, NJW 2016, 56 (Rn. 34) - recht§billig, mwN; dort bejahte der BGH dieses Kriterium, nachdem ein Autor wegen eines von ihm auf seiner Website eingestellten und anschließend andernorts weiterverbreiten Beitrags in Anspruch genommen wurde. Hier geht es allerdings genau um den umgekehrten Fall, in dem der Diensteanbieter selbst die Infrastruktur zur Weiterverbreitung stellt und deshalb ggf. in Anspruch genommen wird.

${ }^{45}$ Vgl. auch Spindler, in: Spindler/Schmitz, TMG, § 7 Rn. 38; Chmelik, Social Network Sites - Soziale Netzwerke, S. 159 f.; anders aber OLG Frankfurt/M., Urt. v. 21.12.2017 -
} 
sondern auf den adäquat-kausalen eigenen Beitrag des Störers. Das Bereitstellen der Infrastruktur, die der Begehung der Rechtsverletzung dient, erfolgt unzweifelhaft willentlich. ${ }^{46}$ Dies nimmt zwar noch nicht die weiteren Voraussetzungen einer Inanspruchnahme als Störer vorweg. Gleichwohl kommt es bei der Inanspruchnahme von Diensteanbietern in der Rechtsprechungspraxis in der Regel nicht auf das Vorliegen einer Störungshandlung in diesem Sinne an. Diese scheint in aller Regel als selbstverständlich gegeben angesehen zu werden.

\section{Prüfpflichten im Rahmen der Störerhaftung}

Der BGH hat im Kontext der Störerhaftung in den letzten Jahren ein Prüfprogramm etabliert und kontinuierlich ausgebaut, das den Intermediären spezifische Verhaltensobliegenheiten aufbürdet, um einer Haftung als Störer zu entgehen. Diese Obliegenheiten sind somit für die Verantwortung der Diensteanbieter entscheidend und daher im Folgenden zu erläutern.

\section{a) Bedeutung}

Die Störerhaftung setzt „, die Verletzung zumutbarer Verhaltenspflichten, insbesondere von Prüfungspflichten voraus“, deren Umfang sich nach Umständen des konkreten Falles sowie der Funktion des als Störer in Anspruch genommenen Intermediärs richtet. ${ }^{47}$ Dogmatisch werden die Prüfpflichten überwiegend als Ausprägung der Verkehrspflichten angesehen, die im Rahmen des Deliktsrechts bei lediglich mittelbaren Rechtsgutsverletzungen eine Einschränkung auf Tatbestandsebene vorsehen, ${ }^{48}$ da bei mittelbaren Rechtsgutsverletzungen die Rechtswidrigkeit nicht indiziert ist. Mit dieser Feststellung ist aber noch nichts über die Frage gesagt, ob aus Gründen dogmatischer und systematischer Klarheit die Störerhaftung insgesamt in dem Verkehrspflichtenkonzept aufgehen sollte. ${ }^{49}$

16 U 72/17, NJW 2018, 795 (Rn. 28), das sowohl eine unzutreffende Auffassung zum Verständnis der Willentlichkeit vertritt, als auch die Figur des mittelbaren Störers unzutreffend in zwei dogmatisch unterschiedliche (Unter-)Kategorien mit der Kenntnis des rechtsverletzenden Inhalts als maßgeblichem Differenzierungskriterium aufteilt. Die besonderen Voraussetzungen für Diensteanbieter im Anwendungsbereich des Art. 14 ECRL stellen die Diensteanbieter aber nicht außerhalb des Anwendungsbereichs der Störerhaftung.

${ }^{46}$ So auch Klein, Haftung von Social-Sharing-Plattformen, S. $102 \mathrm{f}$.

${ }^{47}$ BGH, Urt. v. 25.10.2011 - VI ZR 93/10, NJW 2012, 148 (Rn. 22) - Blog-Eintrag.

${ }^{48}$ Vgl. Spindler, in: Spindler/Schmitz, TMG, §7 Rn. 56, § 10 Rn. 99; Holznagel, Notice and Take-Down-Verfahren als Teil der Providerhaftung, S. 112; jeweils mwN; vgl. aber Klein, Haftung von Social-Sharing-Plattformen, S. 119.

${ }^{49}$ Vgl. Klein, Haftung von Social-Sharing-Plattformen, S. 123 ff., mwN; instruktiv insoweit auch Wagner, in: FS Medicus 2009, S. 589 (599 ff.); vgl. dazu noch unten $§ 10$ B. IV.3. 
Rechtlich gesehen sind Prüfpflichten hier nicht im Sinne von Rechtspflichten $\mathrm{zu}$ verstehen. Sie stellen vielmehr lediglich Obliegenheiten gegen sich selbst dar, da deren Nichtbefolgung lediglich mit negativen Konsequenzen in Form des Haftungsrisikos für den Diensteanbieter verbunden ist, darüber hinaus jedoch keine Konsequenzen hat. ${ }^{50}$

\section{b) Übergeordnete Kriterien}

Ausgangspunkt der Bestimmung der Prüfpflichten ist die Frage, inwiefern diese nach den Umständen des Einzelfalls zumutbar ist. ${ }^{51}$ Daraus hat sich eine detailreiche Kasuistik entwickelt, die einzelne Kriterien in das Zentrum der Bestimmung der Prüfpflichten rückt. Diese sollen hier nur kurz skizziert werden..$^{52}$

\section{aa) Grad der Betroffenheit der Rechtsgüter}

Grundsätzlich hat sich die Bestimmung von Prüfpflichten an den jeweils betroffenen, hier also auch zu schützenden Rechtsgütern zu orientieren. So sind im Kontext des Internets etwa der Jugend- $-{ }^{53}$ und der Persönlichkeitsrechtsschutz gewichtige Belange, die es insbesondere gegen die Meinungsfreiheit zu positionieren und abzuwägen gilt. Hierbei kommt es auch auf eine Bewertung von „Intensität und Quantität der Rechtsverletzungen“ an. ${ }^{54}$

\section{bb) Wirtschaftliche Zumutbarkeit bzw. Gefährdung des Geschäftsmodells}

Vielerorts finden sich auch allgemeine Verweise auf die Billigung einzelner Geschäftsmodelle durch die Rechtsordnung, woraus regelmäßig gefolgert wird, dass Prüfpflichten bzw. daraus folgende Kontrollmaßnahmen nicht das verfolgte Geschäftsmodell wirtschaftlich gefährden dürften. ${ }^{55}$ Diese Fest-

${ }^{50}$ Vgl. Holznagel, Notice and Take-Down-Verfahren als Teil der Providerhaftung, S. 131; explizit auch BGH, Urt. v. 07.12.2010 - VI ZR 30/09, NJW 2011, 755 (Rn. 13) Jahrhundert-Mörder.

${ }^{51}$ Vgl. BGH, Urt. v. 26.07.2018 - I ZR 64/17, NJW 2018, 3779 (Rn. 15) - Dead Island; Urt. v. 26.11.2015 - I ZR 174/14, NJW 2016, 794 (Rn. 21) - Access-Provider.

${ }^{52}$ Vgl. umfassend Spindler, in: Spindler/Schmitz, TMG, § 7 Rn. 57 ff.; Wilmer, NJW 2008, 1845 (1849 ff.).

${ }^{53}$ Vgl. BGH, Urt. v. 12.07.2007 - I ZR 18/04, NJW 2008, 758 (Rn. 44) - Jugendgefährdende Medien bei eBay.

${ }^{54}$ Klein, Haftung von Social-Sharing-Plattformen, S. 148.

${ }^{55}$ Vgl. BGH, Urt. v. 27.02.2018 - VI ZR 489/16, NJW 2018, 2324 (Rn. 34) - Internetforum; Urt. v. 26.11.2015 - I ZR 174/14, NJW 2016, 794 (Rn. 26 f.) - Access-Provider; Urt. v. 12.07.2012 - I ZR 18/11, NJW 2013, 784 (Rn. 28) - Alone in the Dark; Urt. v. 19.04.2007 - I ZR 35/04, NJW 2007, 2636 (Rn. 47) - Internet-Versteigerung II; Urt. v. 11.03.2004 - I ZR 304/01, NJW 2004, 3102 (3105) - Internet-Versteigerung I. 
stellung wird häufig auf die Abgrenzung der Prüfpflichten vom Verbot der allgemeinen proaktiven Überwachung von Inhalten gemäß Art. 15 ECRL, $\S 7$ Abs. 2 TMG gemünzt. ${ }^{56}$

\section{cc) Gefahrgeneigtheit des Dienstes}

Erhöhte Prüfpflichten, also eine im Vergleich zum Normalfall der Störerhaftung strengere Haftung, sind insbesondere dann anzunehmen, wenn ein Dienst „von vornherein auf Rechtsverletzungen durch die Nutzer [...] angelegt ist“ oder der Diensteanbieter „durch eigene Maßnahmen die Gefahr einer rechtsverletzenden Nutzung fördert " ${ }^{\text {" }}{ }^{57}$ Unter diese Kategorie lässt sich auch die bereits angesprochene übergeordnete Frage nach der „Billigung“ eines bestimmten Geschäftsmodells durch die Rechtsordnung einordnen, die der BGH bisweilen als Kriterium in Anschlag bringt. ${ }^{58}$ Allgemein geht es hier um eine entsprechend begründungsbedürftige Ausnahme der Störerhaftung. ${ }^{59}$

Allerdings ist der BGH bislang nur im Fall Cybersky in die Nähe der Annahme eines solchen Geschäftsmodells gelangt. ${ }^{60}$ Im Übrigen sind kaum abstrakte Maßstäbe konsentiert, wann ein Geschäftsmodell als nicht mehr von der Rechtsordnung gebilligt angesehen werden kann. ${ }^{61}$ Andererseits wird aber bisweilen in als zu weitgehend empfundenen Prüfungspflichten eine verdeckte „Illegalisierung“ durch weitgehende Verunmöglichung einzelner Geschäftsmodelle gesehen. ${ }^{62}$ Dem ist allerdings zu entgegnen, dass die Begründung von Handlungsanforderungen bzw. die Begründung von Sorgfaltspflichten nach hier vertretener Auffassung nicht schon per se als illegitimes Risiko betrachtet angesehen werden kann. Pragmatisch gesehen ist aber jedenfalls bislang nicht ersichtlich, inwiefern die Annahme von Prüfpflichten bestimmte Geschäftsmodelle, die nicht ersichtlich nur auf rechtswidrige Inhalte angelegt waren, ${ }^{63}$ faktisch zum Erliegen gebracht hätten.

\footnotetext{
${ }^{56} \mathrm{Vgl}$. dazu noch unten $\S 10$ A. IV. 4.

${ }^{57}$ BGH, Urt. v. 12.07.2012 - I ZR 18/11, NJW 2013, 784 (Rn. 22) - Alone in the Dark; vgl. Urt. v. 15.01.2009 - I ZR 57/07, MMR 2009, 625 (Rn. 21 f.) - Cybersky.

${ }^{58}$ Vgl. BGH, Urt. v. 16.05.2013 - I ZR 216/11, MMR 2014, 55 (Rn. 47) - Kinderhochstühle im Internet II; Urt. v. 18.11.2010 - I ZR 155/09, MMR 2011, 459 (Rn. 45) - Sedo; Urt. v. 22.07.2010 - I ZR 139/08, GRUR 2011, 152 (Rn. 38) - Kinderhochstühle im Internet I, mwN.

${ }^{59}$ Vgl. Spindler, in: Spindler/Schmitz, TMG, §7 Rn. 55.

${ }^{60}$ Vgl. BGH, Urt. v. 15.01.2009 - I ZR 57/07, MMR 2009, 625 (Rn. 21 f.) - Cybersky.

${ }^{61}$ Vgl. auch Spindler, in: Spindler/Schmitz, TMG, § 7 Rn. 55.

${ }^{62} \mathrm{Vgl}$. Nolte/Wimmers, GRUR 2014, 16 (23).

${ }^{63}$ Vgl. Danckwerts, GRUR-Prax 2011, 260 (260 f.).
} 


\section{dd) Sozialadäquanz}

Als weiterer Aspekt, der die Bestimmung der Zumutbarkeit zu determinieren vermag, ist die Sozialadäquanz zu nennen. ${ }^{64}$ Danach soll bei der Bestimmung der Zumutbarkeit von Prüfpflichten relevant sein, inwiefern der Dienst soziale Nützlichkeit entfaltet. ${ }^{65}$ Gerade bei diesem Punkt ist allerdings auch stets eine hinreichende Selbstreflexion nötig, ob über den Weg der Bewertung eines Geschäftsmodells als sozialadäquat nicht auch negative gesellschaftliche Effekte durch diesen Aspekt eine Aufwertung erfahren, indem sie die Bestimmung der Prüfpflichten im Einzelfall maßgeblich beeinflussen. Dessen sollte man sich bewusst sein, ohne damit für ein Abstandnehmen von der Sozialadäquanz als zumutbarkeitsrelevantes Kriterium zu plädieren. So mag es zwar grundsätzlich zutreffen, dass Suchmaschinen ,gesellschaftlich erwünscht" sind. ${ }^{66}$ Solch apodiktische Aussagen sind aber vor dem Hintergrund der Gewaltenteilung, insbesondere hinsichtlich der Prärogative des Gesetzgebers, im Bereich des Rechts der Wirtschaft tätig zu werden und damit mittelbar über die Legitimation einzelner Geschäftsmodelle zu entscheiden, nicht vollkommen unbedenklich. ${ }^{67}$

\section{ee) Finanzielle Vorteile?}

Wie schon andernorts festgestellt, ist die ECRL nur auf kommerzielle Dienste ausgerichtet. Daraus leitet der EuGH ab, dass allein finanzielle Vorteile aus dem Angebot eines Dienstes nicht als haftungsauslösendes Kriterium angesehen werden dürfen. ${ }^{68}$ Auf Grundlage dieser Rechtsprechung ist es deshalb zweifelhaft, finanzielle Vorteile als zumutbarkeitsrelevantes Kriterium im Rahmen der Störerhaftung zu werten. ${ }^{69}$ Prospektiv sollte nach hier S. 149 .

${ }^{64}$ Vgl. Spindler, MMR 2018, 48 (49); Klein, Haftung von Social-Sharing-Plattformen,

${ }^{65}$ Vgl. Holznagel, Notice and Take-Down-Verfahren als Teil der Providerhaftung, S. 109; BGH, Urt. v. 01.04.2004 - I ZR 317/01, NJW 2004, 2158 (2160) - Schöner Wetten; sehr deutlich betont beim Urt. v. 19.02.2004 - I ZR 82/01, NJW 2004, 1793 (1793) - kurtbiedenkopf.de.

${ }^{66}$ BGH, Urt. v. 27.02.2018 - VI ZR 489/16, NJW 2018, 2324 (Rn. 34) - Internetforum.

${ }^{67} \mathrm{Vgl}$. auch Zech, in: Gless/Seelmann, Intelligente Agenten und das Recht, S. 163 (183), mit Blick auf neue Technologien; nach hier vertretener Auffassung kann aber im Grundsatz für bereits bekannte Technologien, deren gesellschaftliche oder ökonomische Folgen erst im Lauf der Zeit abgeschätzt werden können, nichts anderes gelten; vgl. ansatzweise auch Hofmann, JZ 2018, 746 (749 f., 753).

${ }^{68}$ Vgl. EuGH, Urt. v. 23.03.2010 - C-236/08, NJW 2010, 2029 (Rn. 116) - Google France und Google.

${ }^{69}$ Dafür aber noch Wilmer, NJW 2008, 1845 (1850f.); Klein, Haftung von SocialSharing-Plattformen, S. 150 f., differenziert zwischen einer allgemeinen, wohl inhaltsneutral verstandenen Finanzierung eines Angebots (z.B. durch Werbung) und der direkten 
vertretener Auffassung der finanzielle Vorteil jedoch als relevantes Kriterium angesehen werden können, denn fragt man nach der Zumutbarkeit bestimmter Maßnahmen im Einzelfall, kann die finanzielle Situation eines Diensteanbieters unter Verhältnismäßigkeitsgesichtspunkten nicht ignoriert werden. ${ }^{70}$ So wird man finanziell schlagkräftigen Unternehmen kostenintensivere Sorgfaltsstandards abverlangen können, als dies bei kleinen oder neuen Unternehmen der Fall sein mag.

\section{c) Prüfprogramm für Kommunikationsdienste bei Persönlichkeitsrechtsverletzungen}

Wie sind nun die Prüfpflichten im Hinblick auf die Intermediäre zu bestimmen? In der jüngeren Rechtsprechung des BGH zur Störerhaftung von Kommunikationsdiensten hat sich ein sehr spezifisches Muster zur Bestimmung der von diesen Unternehmen im Einzelfall erwarteten Prüfpflichten etabliert. Hier wird den Intermediären ein ausdifferenziertes Prüfungsprogramm abverlangt, das ggf. eine Sachverhaltsermittlung unter Beteiligung aller betroffenen Akteure im Falle von Persönlichkeitsrechtsverletzungen, und zwar nicht nur hinsichtlich von ehrenrührigen Tatsachenäußerungen, sondern auch im Falle von Werturteilen erfordert. ${ }^{71}$

Zunächst ist kursorisch darzustellen, welche Sachverhaltskonstellationen den Entscheidungen zugrunde lag, die hier als Ausgangspunkt der Ausgestaltung des Prüfprogramms für Intermediäre angesehen werden können. Die erstmalige Etablierung eines solchen Prüfprogramms in diesem Kontext erfolgte in der Blog-Eintrag-Entscheidung. Hier hatte ein am Rechtsstreit unbeteiligter Dritter auf der von der Beklagten betriebenen Webseite einen Blog eingerichtet, auf dem er sich mehrfach in diffamierender Weise über den als Unternehmer tätigen Kläger äußerte, der daraufhin die Beklagte auf Unterlassung in Anspruch nahm. ${ }^{72}$ In der zweiten hier maßgeblichen Entscheidung Ärztebewertungsportal III ging es um einen Arzt, der die Betreiberin des Ärztebewertungsportals Jameda auf Unterlassung der Verbreitung einer be-

Generierung wirtschaftlicher Vorteile aus rechtswidrigen Inhalten; ähnlich Spindler, in: Spindler/Schmitz, TMG, §7 Rn. 67. Zynisch ist allerdings die Überlegung von Chmelik, Social Network Sites - Soziale Netzwerke, S. 241 ff., Kommunikationsdienste könnten in Zukunft aufgrund weitgehender rechtlicher Vorgaben nicht mehr in der Lage sein, gewinnerzielend tätig zu sein, weshalb etwa die im öffentlichen Interesse handelnde DENIC von Prüfpflichten grundsätzlich freizustellen wäre, wie es etwa BGH, Urt. v. 17.05.2001 - I ZR 251/99, NJW 2001, 3265 (3267) - ambiente.de, annahm.

${ }^{70}$ Dies gilt hingegen nicht für die Frage, ob überhaupt eine entsprechende Pflicht besteht.

${ }^{71}$ Vgl. BGH, Urt. v. 01.03.2016 - VI ZR 34/15, NJW 2016, 2106 (Rn. 24) - Ärztebewertungsportal III.

${ }^{72}$ Vgl. BGH, Urt. v. 25.10.2011 - VI ZR 93/10, NJW 2012, 148 (149) - Blog-Eintrag. 
stimmten Bewertung über seine ärztliche Behandlung in Anspruch nahm. Dies geschah, nachdem er gegenüber Jameda zunächst der Bewertung widersprochen hatte und das Unternehmen daraufhin mitteilte, es habe seinen Widerspruch an den Autor der Bewertung weitergeleitet, woraufhin dieser allerdings seine Bewertung bestätigt hatte. ${ }^{73}$ Beide Entscheidungen betrafen nicht Kommunikationsdienste in dem Sinne, wie sie hier verstanden werden. Dennoch liegt es auf der Hand, diese Rechtsprechung jedenfalls auch auf solche zu übertragen. ${ }^{74}$

\section{aa) Initiierung des Prüfprogramms durch Notifizierung des Diensteanbieters}

Das auf Grundlage dieser Entscheidungen etablierte Prüfprogramm, das der Diensteanbieter zu durchlaufen hat, um eine Haftung als Störer zu vermeiden, wird durch die Notifizierung des Diensteanbieters in Gang gesetzt, womit letztlich ein Notice-and-Takedown-Verfahren etabliert wird. ${ }^{75}$ Insoweit heißt es in der Blog-Eintrag-Entscheidung:

„Ein Hostprovider ist nicht verpflichtet, die von den Nutzern in das Netz gestellten Beiträge vor der Veröffentlichung auf eventuelle Rechtsverletzungen zu überprüfen. Er ist aber verantwortlich, sobald er Kenntnis von der Rechtsverletzung erlangt. Weist ein Betroffener den Hostprovider auf eine Verletzung seines Persönlichkeitsrechts durch den Nutzer eines Blogs hin, kann der Hostprovider als Störer verpflichtet sein, zukünftig derartige Verletzungen zu verhindern“". ${ }^{76}$

Nach der Rechtsprechung genügt aber nicht jedwede Notifizierung. Hier heißt es regelmäßig, dass eine Pflicht zum Tätigwerden nur gegeben ist, wenn diese einen Rechtsverstoß „unschwer“ erkennen lässt. Theoretisch sind danach zwei Szenarien zu unterscheiden, nämlich wenn eine Notifizierung diese Anforderung einhält (1) oder nicht (2).

\section{(1) Szenario I: Keine „unschwere Erkennbarkeit“ der Rechtsverletzung}

In der Blog-Eintrag-Entscheidung heißt es sodann weiter:

„Ist der Provider mit der Beanstandung eines Betroffenen konfrontiert, die richtig oder falsch sein kann, ist eine Ermittlung und Bewertung des gesamten Sachverhalts unter Berücksichtigung einer etwaigen Stellungnahme des für den Blog Verantwortlichen erforderlich. Hiernach ergeben sich für den Provider regelmäßig folgende Pflichten: Ein Tätigwerden des Hostproviders ist nur veranlasst, wenn der Hinweis so konkret gefasst ist, dass der Rechtsverstoß auf der Grundlage der Behauptungen des Betroffenen unschwer - das heißt ohne eingehende rechtliche und tatsächliche Überprüfung - bejaht werden kann. ${ }^{"}{ }^{77}$

${ }^{73}$ Vgl. BGH, Urt. v. 01.03.2016 - VI ZR 34/15, NJW 2016, 2106 (2106f.) - Ärztebewertungsportal III.

${ }^{74}$ So auch Chmelik, Social Network Sites - Soziale Netzwerke, S. 180.

${ }^{75}$ Vgl. Hofmann, JuS 2017, 713 (715).

${ }^{76}$ Vgl. BGH, Urt. v. 25.10.2011 - VI ZR 93/10, NJW 2012, 148 (Rn. 24) - Blog-Eintrag.

${ }^{77}$ BGH, Urt. v. 25.10.2011 - VI ZR 93/10, NJW 2012, 148 (Rn. 25 f.) - Blog-Eintrag. 
Ist eine Rechtsverletzung aufgrund einer Notifizierung nicht „unschwer“ erkennbar, spricht somit auf den ersten Blick einiges dafür, dass der Diensteanbieter nicht als Störer in Haftung genommen werden kann. ${ }^{78}$ Auf den zweiten Blick geht aus der zitierten Passage indes nicht eindeutig hervor, ob der Diensteanbieter bei einer nicht „unschwer“ erkennbaren Rechtsverletzung nicht ggf. doch aktiv werden muss. Für eine Obliegenheit zur Durchführung des Prüfprogramms auch bei Verneinung der unschweren Erkennbarkeit spricht zwar die allgemein gehaltene Aussage zu Beginn der hier zitierten Passage, wonach eine „Ermittlung und Bewertung“ auch dann erforderlich sein kann, wenn eine Beanstandung vorliegt, ,die richtig oder falsch sein kann“. Insoweit ließe sich annehmen, dass zwischen der „Ermittlung und Bewertung“ einerseits und dem „Tätigwerden“ zu differenzieren ist. Die „Ermittlung und Bewertung " wäre demnach schon verpflichtend, wenn eine Beanstandung richtig oder falsch sein kann; das Tätigwerden bedeutete dann nur die Entfernung eines Inhalts. Allerdings heißt es aber eben explizit, dass ein ,Tätigwerden [...] nur veranlasst [ist], wenn [...] der Rechtsverstoß [...] unschwer erkennbar ist. " ${ }^{\text {"79 }}$ Das Tätigwerden kann in diesem Sinne nicht ausschließlich mit der Entfernung eines Inhalts gleichgesetzt werden, sondern beschreibt eben auch die „Ermittlung und Bewertung“ des Sachverhalts. Die spätere Reformulierung durch den BGH bringt es auf den Punkt: „Ist der Provider mit der Beanstandung eines Betroffenen konfrontiert, die so konkret gefasst ist, dass der Rechtsverstoß auf der Grundlage der Behauptung des Betroffenen unschwer bejaht werden kann, ist eine Ermittlung und Bewertung des gesamten Sachverhalts unter Berücksichtigung einer etwaigen Stellungnahme des für den beanstandeten Beitrag Verantwortlichen erforderlich“ ${ }^{80}$ Diese Auffassung wird im Übrigen von einzelnen Mitgliedern des zuständigen VI. Zivilsenats in wissenschaftlichen Äußerungen geteilt. ${ }^{81} \mathrm{Nach}$ dem Verständnis des BGH kommt also eine Obliegenheit jedenfalls mit den ggf. nachteiligen haftungsrechtlichen Konsequenzen nur in Betracht, soweit die Notifizierung eine Rechtsverletzung ,unschwer“" erkennen lässt. $^{82}$

${ }^{78}$ In diesem Sinne Mann/Smid, in: Spindler/Schuster, Recht der elektronischen Medien, Presserecht im Internet Rn. 64; wohl auch Wagner, in: MüKo BGB, § 823 Rn. 876.

${ }^{79}$ BGH, Urt. v. 25.10.2011 - VI ZR 93/10, NJW 2012, 148 (Rn. 26) - Blog-Eintrag (Hervorh. v. Verf.).

${ }^{80}$ Vgl. BGH, Urt. v. 01.03.2016 - VI ZR 34/15, NJW 2016, 2106 (Rn. 24) - Ärztebewertungsportal III; Urt. v. 27.02.2018 - VI ZR 489/16, NJW 2018, 2324 (Rn. 32) - Internetforum.

${ }^{81}$ Vgl. explizit Pentz, AfP 2017, 102 (115): der Provider müsse, ,[...] wenn er von einem Betroffenen auf eine Verletzung seines Persönlichkeitsrechts durch den Nutzer seines Angebots hingewiesen worden ist und der Hinweis so konkret gefasst ist, dass der Rechtsverstoß auf der Grundlage der Behauptung des Betroffenen unschwer bejaht werden kann, in ein Prüfungsverfahren eintreten."

${ }^{82}$ Die Position des EuGH lässt sich, wie schon in anderen Zusammenhang erläutert 
Zwei Ausnahmen zu den hier erläuterten Grundsätzen gelten jedoch bei der wettbewerbsrechtlichen Haftung für Hyperlinks sowie - allem Anschein nach - bei der Inanspruchnahme von Suchmaschinendiensten. Im ersteren Falle sei, so der BGH, auch aufgrund der typischerweise niedrigeren Anzahl an zu prüfenden Verlinkungen der den Link setzende Unternehmer bei Hinweisen auf Rechtsverletzungen stets zur Prüfung verpflichtet, ohne dass es dabei auf eine klare Erkennbarkeit der Rechtsverletzung ankomme. ${ }^{83} \mathrm{Ob}$ diese Differenzierung trägt, ist zweifelhaft, ${ }^{84}$ bedarf aber hier keiner vertieften Erörterung. Allerdings lässt sich aus ihr jedenfalls die soeben getroffene Feststellung validieren, wonach die Rechtsprechung im Übrigen bzw. jedenfalls bei Persönlichkeitsrechtsverletzungen bei nicht aufgrund einer Notifizierung „unschwer“ erkennbaren Rechtsverletzungen gerade kein Tätigwerden des Diensteanbieters für geboten hält. Hinsichtlich der Inanspruchnahme von Suchmaschinendiensten hat der BGH zudem jüngst explizit von dem Erfordernis einer „unschweren Erkennbarkeit“ Abstand genommen ${ }^{85}$ Angesichts der Tatsache, dass das Gericht in der Entscheidung Auslistungsanspruch nicht einmal die Störerhaftung erwähnt, geschweige denn ihre Maßstäbe zur Entfaltung bringt, ist jedoch davon auszugehen, dass diese Einschränkung auf den Fall der Inanspruchnahme von Suchmaschinendiensten beschränkt bleibt. Auf diese ist sogleich gesondert einzugehen. ${ }^{86}$

\section{(2) Szenario II: „Unschwer erkennbare“ Rechtsverletzung auf Grundlage des behaupteten Sachverhalts}

Liegt eine unschwer erkennbare Rechtsverletzung vor, obliegt dem Diensteanbieter nach den vorangegangenen Ausführungen das weitere Prüfprogramm, das ihn zu einer Entscheidung über die Notifizierung führen soll. Dies ist nach den oben zitierten Passagen und deren Diskussion eindeutig. Einem unzutreffenden Verständnis in Bezug auf Persönlichkeitsrechtsverletzungen unterliegen daher jene, die bei „unschwer“ erkennbaren bzw. offensichtlichen Rechtsverletzungen gerade keine Prüfungsobliegenheit für einschlägig erachten, während bei einer unklaren Notifizierung das Prüfprogramm der Rechtsprechung zur Entfaltung zu bringen sei. ${ }^{87}$

wurde, nicht zwingend für die eine oder andere Sichtweise fruchtbar machen, vgl. oben den $\S 4$ Fn. 123 zugehörigen Text.

${ }^{83}$ Vgl. BGH, Urt. v. 18.06.2015 - I ZR 74/14, NJW 2016, 804 (Rn. 27) - Hyperlink.

${ }^{84}$ Vgl. Hofmann, ZUM 2017, 102 (104), mwN.

${ }^{85}$ Vgl. BGH, Urt. v. 27.07.2020 - VI ZR 405/18, NJW 2020, 3436 (Rn. 41) - Auslistungsanspruch.

${ }^{86}$ Vgl. unten $\S 10$ A. III. 2. d).

${ }^{87}$ So Kovacs, Die Haftung der Host-Provider für persönlichkeitsrechtsverletzende Internetäußerungen, S. 287, 291 ff. Ebenso wie sie überträgt die von ihr hierfür referenzierte Entscheidung OLG Frankfurt/M., Urt. v. 10.12.2015 - 6 U 244/14, NJW-RR 2016, 614 


\section{bb) Pflicht zur Sachverhaltsermittlung}

Erfüllt die Notifizierung die vorgenannten Voraussetzungen, „ist eine Ermittlung und Bewertung des gesamten Sachverhalts unter Berücksichtigung einer etwaigen Stellungnahme des für den beanstandeten Beitrag Verantwortlichen erforderlich" ${ }^{88}$ Das LG Hamburg hat insoweit einem Diensteanbieter für die eigene Prüfung zur Entscheidung, wie mit einer Notifizierung umzugehen ist, vier Tage eingeräumt hat. ${ }^{89}$ Das Prüfprogramm erfordert, dass dem für den gerügten Inhalt Verantwortlichen regelmäßig die Notifizierung zur Stellungnahme weiterzuleiten ist. ${ }^{90}$ Das weitere Vorgehen hängt dann von dessen Reaktion ab.

\section{cc) Mögliche Konsequenzen}

„Bleibt eine Stellungnahme innerhalb einer nach den Umständen angemessenen Frist aus, ist von der Berechtigung der Beanstandung auszugehen und der beanstandete Eintrag zu löschen. ${ }^{\text {“91 }}$ Die hierin aufgestellte implizite Vermutung, dass bei Ausbleiben einer Reaktion an der Notifizierung „etwas dran" ist, überzeugt allerdings nur im Falle der ausbleibenden Verteidigung trotz Erreichbarkeit des Verantwortlichen. ${ }^{92}$

Ist der Verantwortliche dagegen nicht erreichbar, ist fraglich, inwieweit die fehlende Reaktion eine solche Vermutung tragen kann. Hier ist jedenfalls auf tatsächlicher Ebene zwischen der Nichterreichbarkeit aufgrund der Anonymität des Verantwortlichen und sonstigen Gründen zu unterscheiden. Im ersteren Falle überzeugt eine strikte Vermutung im beschriebenen Sinne nicht. Allerdings ist es auch nicht undenkbar, hier den Maßstab an der inhaltlichen Bewertung des Inhalts und der Überzeugungskraft einer substanziierten Notifizierung auszurichten. Danach können die Umstände des Einzelfalls auch in diesem Szenario zur Löschung eines Inhalts führen, während im letzteren Fall, wenn also eine Person schlicht nicht erreichbar ist, eine Vermutung im beschriebenen Sinne in aller Regel nicht überzeugt.

Reagiert der Verantwortliche in substanziierter Weise, die Zweifel an der Berechtigung der Notifizierung weckt, soll der Diensteanbieter dies der No-

(Rn. 23) - Kritischer Thread, unzutreffend Maßgaben, die gerade nicht für Persönlichkeitsrechtsverletzungen gelten, auf diese; insoweit missverständlich auch Chmelik, Social Network Sites - Soziale Netzwerke, S. 176.

${ }^{88}$ BGH, Urt. v. 01.03.2016 - VI ZR 34/15, NJW 2016, 2106 (Rn. 24) - Ärztebewertungsportal III; vgl. Urt. v. 25.10.2011 - VI ZR 93/10, NJW 2012, 148 (Rn. 26) - BlogEintrag.

\footnotetext{
${ }^{89}$ Vgl. LG Hamburg, Urt. v. 24.03.2017 - 324 O 148/16, NJW-RR 2017, 1323 (Rn. 44).

${ }^{90}$ Vgl. BGH, Urt. v. 25.10.2011 - VI ZR 93/10, NJW 2012, 148 (Rn. 27) - Blog-Eintrag.

${ }^{91}$ BGH, Urt. v. 25.10.2011 - VI ZR 93/10, NJW 2012, 148 (Rn. 27) - Blog-Eintrag.

${ }^{92}$ So auch Holznagel, GRUR Int 2014, 105 (108).
} 
tifizierenden mitteilen und ggf. weitere Nachweise verlangen.${ }^{93}$ Erfolgt dann keine Stellungnahme der Notifizierenden oder legt sie die erforderlichen Nachweise nicht vor, ist der Diensteanbieter zur Entfernung nicht verpflichtet. ${ }^{94}$ Diese Konstellation, in der eine zumindest nicht völlig unsubstanziierte Reaktion des Verantwortlichen erfolgt, birgt wohl die größte Herausforderung, denn hier obliegt es dem Diensteanbieter, zwischen zwei konträren Stellungnahmen zu entscheiden. ${ }^{95}$

\section{d) Suchmaschinen}

Einer ggf. modifizierten Sichtweise bedarf es im Hinblick auf die Störerhaftung von Suchmaschinen. In der Entscheidung Internetforum hatte der BGH sich mit der Übertragbarkeit der soeben skizzierten Grundsätze auf die Inanspruchnahme von Suchmaschinenbetreibern wegen Persönlichkeitsrechtsverletzungen zu befassen. Hier hatten die Kläger ein Internetforum betrieben, dessen Nutzer mit denen eines anderen Internetforums „verfeindet“ waren. Im Zuge dieser Auseinandersetzung wurden Äußerungen über die Kläger getätigt. Die Kläger monierten die Auffindbarkeit dieser Äußerungen gegenüber Google als persönlichkeitsrechtsverletzend und verlangten, entsprechende Verknüpfungen mit ihnen zu löschen. ${ }^{96}$

\section{aa) Anspruchsgrundlage}

Zunächst ist auf die Frage einzugehen, welcher Rechtsnorm ein Anspruch auf eine Entfernung von Suchergebnissen zu entnehmen ist. Das hängt davon ab, ob man in einem solchen Anspruch ein Begehren auf Unterlassung oder auf die Löschung von personenbezogenen Daten erblickt. Im ersten Fall handelt es sich um die Anwendung von $\$ 1004$ BGB in seinen verschiedenen Spielarten, eben u.a. auch in Form der Störerhaftung. Im zweiten Fall ginge es um die Anwendung des materiellen Datenschutzrechts. Der BGH schien sich in seinen Entscheidungen hierzu bislang auf die Störerhaftung zu stützen. ${ }^{97}$ Manche Instanzgerichte ordnen das Begehren dagegen dem Datenschutzrecht, nach Geltungsbeginn der DS-GVO deren Art. 17 zu, ${ }^{98}$ integrie-

\footnotetext{
${ }^{93}$ Vgl. BGH, Urt. v. 25.10.2011 - VI ZR 93/10, NJW 2012, 148 (Rn. 27) - Blog-Eintrag.

${ }^{94}$ Vgl. BGH, Urt. v. 25.10.2011 - VI ZR 93/10, NJW 2012, 148 (Rn. 27) - Blog-Eintrag.

${ }^{95}$ Vgl. Holznagel, GRUR Int 2014, 105 (108); Hofmann, ZUM 2017, 102 (106f.); vgl. dazu noch unten $\S 10$ B. III. 3.

${ }^{96}$ Vgl. BGH, Urt. v. 27.02.2018 - VI ZR 489/16, NJW 2018, 2324 (2324) - Internetforum.

${ }^{97}$ Vgl. BGH, Urt. v. 27.02.2018 - VI ZR 489/16, NJW 2018, 2324 (Rn. 25) - Internetforum; Urt. v. 24.07.2018 - VI ZR 330/17, ZUM-RD 2019, 203 (Rn. 30).

${ }^{98}$ Vgl. OLG Frankfurt/M., Urt. v. 06.09.2018 - 16 U 193/17, ZD 2019, 78 (Rn. 44); vgl. ferner OLG Celle, Urt. v. 01.06.2017 - 13 U 178/16, ZD 2017, 428 (Rn. 7 f.).
} 
ren aber das vom BGH entwickelte Prüfprogramm in die Abwägung, die aufgrund des Kriteriums der Erforderlichkeit in Art. 17 Abs. 3 lit. a DS-GVO geboten sei. ${ }^{99}$ Dies scheint der BGH für ein sog. Auslistungsbegehren gegen einen Suchmaschinenbetreiber nunmehr ebenfalls als den gebotenen Weg zu betrachten. Dieses stützt er nämlich ausschließlich auf Art. 17 DS-GVO, ${ }^{100}$ nimmt aber in der Sache lediglich eine Grundrechtsabwägung vor. ${ }^{101}$ Die materiellen Vorgaben des Prüfpflichtenprogramms für Störer greift er allenfalls implizit in Form einer Referenzierung der Entscheidung Internetforum auf, ohne von der Störerhaftung als solcher zu sprechen. ${ }^{102}$

Dieser integrative Ansatz bleibt nicht ohne Zweifel, denn das prozeduralisierende Prüfprogramm fügt sich nahtlos in den Grundansatz der Störerhaftung ein, während bei der Aufnahme prozeduraler Elemente in den Abwägungsvorgang Reibungsverluste oder die Zunahme materieller Unschärfen nicht ausgeschlossen sind. Aus dieser Einsicht ergibt sich auch die Vorzugswürdigkeit der Anknüpfung solcher Begehren gegen Suchmaschinenbetreiber an den $\$ 1004$ BGB, der materiell ohnehin über einen Löschungsanspruch hinausgeht. ${ }^{103}$ Systematisch gelingt so die Integration der Maßgaben des EuGH aus der Google Spain-Entscheidung, die unter dem Stichwort „Recht auf Vergessenwerden“ geläufig sind, besser, da die entsprechend interpretierten Normen des Datenschutzrechts ebenfalls als deliktsrechtliche Schutzgesetze verstanden werden können. Deren fortdauernde Verletzung durch Auffindbarkeit bestimmter Suchergebnisse kann ebenfalls mittels der Störerhaftung adressiert werden. ${ }^{104}$ Dogmatisch ist der Weg über die Störerhaftung ebenfalls vorzugswürdig, weil so die Kategorie des datenschutzrechtlich Verantwortlichen nicht überstrapaziert werden braucht, was insbesondere bei Fehlen einer existierenden Sonderbeziehung zur betroffenen Person im datenschutzrechtlichen Sinne von Bedeutung ist.

\section{bb) Grundsätzliche Wertungen}

Die Übertragbarkeit der Störerhaftung auf die Inanspruchnahme von Suchmaschinendiensten ist nach den jüngsten Äußerungen des BGH offen. In der Entscheidung Internetforum hält das Gericht die Übertragung der Grund-

\footnotetext{
${ }^{99}$ Vgl. OLG Frankfurt/M., Urt. v. 06.09.2018 - 16 U 193/17, ZD 2019, 78 (Rn. 57 ff.).

${ }^{100}$ Vgl. BGH, Urt. v. 27.07.2020 - VI ZR 405/18, NJW 2020, 3436 (Rn. 17) - Auslistungsanspruch.

${ }^{101}$ Vgl. BGH, Urt. v. 27.07.2020 - VI ZR 405/18, NJW 2020, 3436 (Rn. 23) - Auslistungsanspruch; Verständnis hierfür äußert Mörsdorf, NJW 2020, 3417 (3418).

${ }^{102}$ Vgl. BGH, Urt. v. 27.07.2020 - VI ZR 405/18, NJW 2020, 3436 (Rn. 19) - Auslistungsanspruch.

${ }^{103}$ So auch Cordes, GRUR-Prax 2018, 475 (475).

${ }^{104}$ Vgl. BGH, Urt. v. 27.02.2018 - VI ZR 489/16, NJW 2018, 2324 (Rn. 42 f.) - Internetforum.
} 
sätze der Störerhaftung auf die Inanspruchnahme von Suchmaschinen grundsätzlich für möglich. An die Prüfpflichten von Google als Suchmaschinenbetreiber seien aber geringere Anforderungen zu stellen. ${ }^{105}$ Das Gericht hält hier zwar einen erhöhten Begründungsaufwand für geboten, um eine im Rahmen seiner Rechtsprechung eigentlich etablierte Position erneut zu begründen, nämlich weshalb allgemeine bzw. „proaktive“ Prüfpflichten von Suchmaschinenbetreibern nicht zu fordern seien. ${ }^{106}$ Als entscheidenden Unterschied zum soeben skizzierten Modell der Prüfpflichten für Kommunikationsdienste stellt das Gericht aber zutreffend fest, dass der Suchmaschinenbetreiber regelmäßig in keinem rechtlich qualifizierbaren Verhältnis zu den von ihm auffindbar gemachten Informationen stehe. ${ }^{107}$ Die Kontaktierung von für einzelne gerügte Inhalte Verantwortlichen sei deshalb bedeutend schwieriger, sodass im Gegensatz zu Kommunikationsdiensten keine Vermutung für ausgebliebene Stellungnahmen der Verantwortlichen begründbar sei. ${ }^{108}$

\section{cc) Spezifische Prüfpflichten für Suchmaschinenbetreiber}

Wann einen Suchmaschinenbetreiber aber „spezifische Verhaltenspflichten“ treffen, ist aber nach der jüngsten Entscheidung Auslistungsanspruch unklar. In der Entscheidung Internetforum hieß es noch, den Suchmaschinenbetreiber träfen „,spezifische Verhaltenspflichten“ erst dann, „,wenn er durch einen konkreten Hinweis Kenntnis von einer offensichtlichen und auf den ersten Blick klar erkennbaren Rechtsverletzung erlangt hat ". ${ }^{109}$ In den Worten einer dem maßgeblich zuständigen VI. Zivilsenat angehörigen Richterin müsse der Rechtsverstoß ,gewissermaßen auf der Hand liegen. “ ${ }^{110}$ Solch ein klarer Fall eines Rechtsverstoßes, so der BGH weiter, sei bei „bei Kinderpornografie, Aufruf zur Gewalt gegen Personen, offensichtlichen Personenverwechslun-

${ }^{105}$ Vgl. BGH, Urt. v. 27.02.2018 - VI ZR 489/16, NJW 2018, 2324 (Rn. 33) - Internetforum; grundsätzlich bestätigt durch Urt. v. 24.07.2018 - VI ZR 330/17, ZUM-RD 2019, 203 (Rn. 37).

${ }^{106}$ Vgl. BGH, Urt. v. 27.02.2018 - VI ZR 489/16, NJW 2018, 2324 (Rn. 34) - Internetforum; der erhöhte Begründungsaufwand lässt sich wohl eher mit dem gerichtlichen Bedürfnis begründen, die tatsächliche Bedeutung von Suchmaschinen in den Vordergrund zu stellen, als mit der Erforderlichkeit, in rechtlicher Hinsicht neues Terrain zu erkunden. Denn auch für Kommunikationsdienste hat das Gericht ja nie von einer evtl. bestehenden „proaktiven“ Prüfpflicht gesprochen.

${ }^{107}$ Vgl. BGH, Urt. v. 27.02.2018 - VI ZR 489/16, NJW 2018, 2324 (Rn. 35) - Internetforum.

${ }^{108}$ Vgl. BGH, Urt. v. 27.02.2018 - VI ZR 489/16, NJW 2018, 2324 (Rn. 35) - Internetforum; vgl. auch Urt. v. 24.07.2018 - VI ZR 330/17, ZUM-RD 2019, 203 (Rn. 37).

${ }^{109}$ BGH, Urt. v. 27.02.2018 - VI ZR 489/16, NJW 2018, 2324 (Rn. 36) - Internetforum.

${ }^{110}$ Pentz, AfP 2019, 113 (123). 
gen, Vorliegen eines rechtskräftigen Titels gegen den unmittelbaren Störer, Erledigung jeglichen Informationsinteresses durch Zeitablauf [...] oder eindeutiger Schmähkritik" denkbar. ${ }^{111} \mathrm{Da}$ aber insbesondere bei äußerungsrechtlichen Konflikten eine „Validierung des Vortrags der Betroffenen regelmäßig nicht möglich" sei, führe dieser Maßstab jedoch nur ausnahmsweise zu einem solch klaren Fall. ${ }^{112}$

In der Entscheidung Auslistungsanspruch, in der das Gericht sich auf Grundlage einer Grundrechtsabwägung im Rahmen von Art. 17 DS-GVO zu den Pflichten eines Suchmaschinenbetreibers äußert, hält der BGH an seiner Linie zur „unschweren Erkennbarkeit“ jedoch nicht mehr fest. Da sich hier Meinungsfreiheit der Inhalteanbieter, die von einer Auslistung betroffen wären, und das Persönlichkeitsrecht von Betroffenen gleichrangig gegenüber stünden, müsse der Suchmaschinenbetreiber ,nicht erst dann tätig werden [...], wenn er von einer offensichtlichen und auf den ersten Blick klar erkennbaren Rechtsverletzung des Betroffenen Kenntnis erlangt." "113 Weitere Hinweise, welche konkrete Anforderungen an eine die Verhaltensobliegenheit des Suchmaschinenbetreibers auslösende Notifizierung zu stellen sind, erfolgen jedoch nicht.

\section{dd) Einordnung}

Danach bleibt es völlig offen, ob und in welcher Art und Weise sich die Inanspruchnahme von Suchmaschinenbetreibern von Kommunikationsdiensten unterscheidet. Zum einen ist schon unklar, ob die Störerhaftung und das originär mit ihr verbundene Prüfprogramm noch über einen eigenständigen Anwendungsbereich betreffend Suchmaschinendiensten verfügen oder ob der BGH fortan allein auf Grundlage einer Grundrechtsabwägung im Kontext des Art. 17 DS-GVO entsprechende Ansprüche prüfen wird. Vom Anspruch des BGH, sein etabliertes Prüfprogramm im Fall von Persönlichkeitsrechtsverletzungen diensteübergreifend zur Entfaltung bringen zu können, ${ }^{114}$ bleibt jedenfalls nicht mehr viel übrig. Ob sich auf dieser Grundlage eine überzeugende Dogmatik für die Inanspruchnahme von Suchmaschinendiensten begründen lässt, ist vielmehr offen.

Zum anderen bleiben die materiellen Maßstäbe für die Prüfung eines Anspruchs der Sache nach ebenfalls unklar. Zunächst zog der BGH die Stell-

${ }^{111}$ BGH, Urt. v. 27.02.2018 - VI ZR 489/16, NJW 2018, 2324 (Rn. 36) - Internetforum; vgl. auch Urt. v. 24.07.2018 - VI ZR 330/17, ZUM-RD 2019, 203 (Rn. 37).

${ }^{112}$ BGH, Urt. v. 27.02.2018 - VI ZR 489/16, NJW 2018, 2324 (Rn. 36) - Internetforum.

${ }^{113}$ BGH, Urt. v. 27.07.2020 - VI ZR 405/18, NJW 2020, 3436 (Rn. 41) - Auslistungsanspruch.

${ }^{114}$ Vgl. BGH, Urt. v. 27.02.2018 - VI ZR 489/16, NJW 2018, 2324 (Rn. 33) - Internetforum. 
schrauben so weit an, dass eine erfolgreiche Inanspruchnahme von Suchmaschinenbetreibern wohl kaum zu erwarten war, soweit es um eine Inanspruchnahme für fremde Inhalte geht. Trotz des nominellen Festhaltens an dem Prüfprogramm der Störerhaftung, das insbesondere eine Sachverhaltsermittlung und die Einräumung der Gelegenheit zur Stellungnahme für beteiligte Personen beinhaltet, ${ }^{115}$ waren zumindest manche der vom Gericht genannten konkreten Beispiele, bei denen eine Rechtsverletzung hinreichend klar erkennbar sei (z.B. Kinderpornografie oder Aufrufe zur Gewalt gegen Personen), so eindeutig, dass wohl auch die Durchführung des Prüfprogramms in diesen Fällen nicht bzw. nicht im Regelfall notwendig wäre. Mit der jüngsten Entscheidung Auslistungsanspruch bricht der BGH aber unvermittelt mit seiner bislang formulierten Position, nach der an die Inanspruchnahme von Suchmaschinendiensten höhere Anforderungen zu stellen sind. $\mathrm{Ob}$ und in welcher Art und Weise eine erfolgreiche Inanspruchnahme von einer hinreichenden Notifizierung abhängt, bleibt jedenfalls hier offen.

Insgesamt ist damit festzuhalten, dass die augenscheinliche und allmähliche Abkopplung von den Anforderungen der Störerhaftung zu einem Verlust an klaren Maßstäben für die Haftung von Suchmaschinendiensten für Inhalte Dritter führt. Unter dem Regime des Art. 17 DS-GVO scheint die Rechtsprechung jedenfalls zunehmend dem Instrument der Grundrechtsabwägung zuzuneigen. Abstrakte, aber zugleich hinreichend prognostizierbare Maßstäbe sind demgegenüber bislang nicht zu erkennen. Ob dies sich in Zukunft ändern könnte, bleibt abzuwarten.

\section{Rechtsfolgen der Störerhaftung}

Im Folgenden sind die Rechtsfolgen der Störerhaftung darzustellen. Zunächst ist darauf einzugehen, wie sich die Anspruchsziele „Beseitigung“ und „Unterlassung“" voneinander unterscheiden (1.-2.). Erst dies ermöglicht eine Erläuterung der Gefahr der ersten Begehung bzw. der Wiederholung einer Störung, wie sie in Entsprechung von § 1004 BGB auch im Rahmen der Störerhaftung stets erforderlich ist (3.). Als äußere materielle Grenze der Inpflichtnahme der Intermediäre wurde bereits das Verbot allgemeiner Überwachungspflichten benannt, das auch mit Blick auf die Störerhaftung zu thematisieren ist (4.). Die Kosten der Inanspruchnahme runden den Blick auf die Rechtsfolgen der Störerhaftung ab (5.).

${ }^{115}$ Vgl. BGH, Urt. v. 27.02.2018 - VI ZR 489/16, NJW 2018, 2324 (Rn. 37) - Internetforum; Urt. v. 24.07.2018 - VI ZR 330/17, ZUM-RD 2019, 203 (Rn. 39). 


\section{Beseitigung}

\section{a) Allgemeine Bedeutung}

Ausgangspunkt des Anspruchsziels, das die Störerhaftung in den Blick nimmt, ist $\$ 1004$ S. 1 BGB. Danach kann grundsätzlich die Beseitigung einer gegenwärtigen Störung für die Zukunft verlangt werden. ${ }^{116}$ Seiner Bedeutung im Wortsinne nach setzt die Beseitigung die Veränderung eines gegenwärtigen, also noch andauernden Zustands voraus. Insoweit ist bei §1004 BGB von einem um das Verschulden gekürzten Tatbestand des Deliktsrechts die Rede. ${ }^{117}$

In der Dogmatik des bürgerlichen Rechts ist diese eigentlich simple Feststellung jedoch insofern nicht unproblematisch, als sie die Grenze zur Wiederherstellung des status quo ante leicht zu verwischen vermag, die auch das Ziel des $§ 249$ BGB ist. Diese Norm erfordert in Zusammenhang mit dem deliktischen Schadensersatzanspruch gemäß $\S 823$ BGB jedoch stets ein Verschulden. ${ }^{118}$ Trotz der damit in aller Kürze beschriebenen problematischen Abgrenzung sieht der BGH auch im Rahmen des $§ 1004$ BGB den Störer in der Pflicht zur Wiederherstellung des Zustands vor der Beeinträchtigung. ${ }^{119}$ Voraussetzung des Beseitigungsanspruchs auch im Rahmen der Störerhaftung ist das Andauern eines rechtswidrigen Zustands. ${ }^{120}$ Auch hier ist die Abgrenzung zum Schadensersatzanspruch theoretisch heikel, weil bei der Inanspruchnahme des Diensteanbieters zumindest in vielen Fällen kein Verschulden hinsichtlich der von Dritten begangenen Rechtsverletzungen anzunehmen sein wird. Deshalb ließe sich theoretisch bei jeder extensiven Interpretation der Störerhaftung der Vorwurf erheben, es würden die schärferen Voraussetzungen eines deliktischen Anspruchs gegen den Primärverursacher umgangen. Die Abgrenzung zum verschuldensabhängigen deliktischen Anspruch verliert allerdings an Schärfe, weil die Naturalrestitution im Sinne des $\S 249$ BGB etwa bei begangenen Persönlichkeitsrechtsverletzungen, deren (untechnisch gesprochen) immaterielle Folgen kaum reversibel sind, ${ }^{121}$ ohnehin selten in Betracht kommt.

${ }^{116}$ Vgl. Volkmann, in: Spindler/Schuster, Recht der elektronischen Medien, BGB $§ 1004$ Rn. 64, mwN.

${ }^{117}$ Vgl. Wagner, in: FS Medicus 2009, S. 589 (590), mwN.

${ }^{118}$ Vgl. zu der Problematik Wagner, in: FS Medicus 2009, S. 589 ff.; Raff, in: MüKo BGB, § 1004 Rn. $229 \mathrm{ff}$.

${ }^{119}$ Vgl. nur BGH, Urt. v. 18.04.1997 - V ZR 28/96, NJW 1997, 2234 (2235), wo das Gericht eine Pflicht zur Wiederherstellung eines Tennisplatzes infolge dessen Beschädigung durch den Wurzelwuchs eines Nachbargrundstücks bejahte.

${ }^{120}$ Vgl. BGH, Urt. v. 28.07.2015 - VI ZR 340/14, NJW 2016, 56 (Rn. 13) - recht§billig, mwN.

${ }^{121}$ Dies gilt zumindest, soweit man vom Widerrufsanspruch bei ehrenrührigen Tatsachenbehauptungen absieht, vgl. insoweit Oetker, in: MüKo BGB, §249 Rn. 355. 


\section{b) Varianten der Beseitigung}

Im Verlauf ihrer judikativen Entwicklung wurden der Störerhaftung verschiedene Erscheinungsformen bzw. Anspruchsziele zugeordnet, die jeweils den Bedingungen ihrer gebietsspezifischen Eigenheiten geschuldet sind. Im Falle von Persönlichkeitsrechtsverletzungen durch die Presse hat die Rechtsprechung etwa einen Berichtigungsanspruch konstruiert, der aus dem Beseitigungsanspruch folgt. ${ }^{122}$ Im Kontext des Internets und damit für diese Untersuchung von besonderer Bedeutung ist eine weitere Ausprägung des Beseitigungsanspruchs, nämlich die Löschung bzw. das Hinwirken auf Löschung der rechtsverletzenden Inhalte, soweit dies für den Schuldner möglich und zumutbar ist. ${ }^{123}$

\section{Unterlassung}

Die Unterlassung einer Störung kann gemäß § 1004 Abs. 1 S. 2 BGB (analog) verlangt werden. Dies betrifft die Fälle der zu „,besorgenden“ künftigen Störung. ${ }^{124}$ Für Persönlichkeitsrechtsverletzungen bei Kommunikationsdiensten spricht der BGH von einer ggf. bestehenden Pflicht, „künftige [...] Störungen zu verhindern. " 125 Die mitunter nicht ganz einfache Abgrenzung von Beseitigungs- und Unterlassungsanspruch führt zu einer im Schrifttum verbreiteten Annahme, letztlich seien diese beiden Spielarten des §1004 BGB deckungsgleich. ${ }^{126}$ Dies ist jedoch nicht zwingend die Regel. Eine fehlende Deckungsgleichheit lässt sich für die Inanspruchnahme von Suchmaschinenbetreibern wegen persönlichkeitsrechtsverletzenden Suchergebnissen beschreiben. Die bestehende Auffindbarkeit solcher Inhalte gilt es aus Sicht des Betroffenen zu beseitigen; vom Suchmaschinenbetreiber verlangt sie zudem, die Auffindbarkeit in der Zukunft auch weiterhin zu unterlassen. ${ }^{127}$

${ }^{122}$ Vgl. BGH, Urt. v. 18.11.2014 - VI ZR 76/14, NJW 2015, 778 (Rn. 13), mwN.

${ }^{123}$ Vgl. BGH, Urt. v. 18.09.2014 - I ZR 76/13, MMR 2015, 262 (Rn. 66 f.)-CT-Paradies (für die Störerhaftung im Rahmen des Urheberrechts); Urt. v. 28.07.2015 - VI ZR 340/14, NJW 2016, 56 (Rn. 13) - recht§billig, überträgt dies auf Persönlichkeitsrechtsverletzungen und unterlegt einen solchen Anspruch auch dem - damals noch im Entwurfsstadium befindlichen - Art. 17 DS-GVO als Kodifizierung einer der Grundaussagen von EuGH, Urt. v. 13.05.2014 - C-131/12, NJW 2014, 2257 ff. - Google Spain SL.

${ }^{124}$ Dies betrifft nicht nur den im Gesetz explizit angesprochenen Fall der Wiederholungsgefahr, sondern auch die Erstbegehungsgefahr, vgl. Volkmann, in: Spindler/Schuster, Recht der elektronischen Medien, BGB § 1004 Rn. 66.

${ }^{125}$ BGH, Urt. v. 01.03.2016 - VI ZR 34/15, NJW 2016, 2106 (Rn. 23) - Ärztebewertungsportal III; vgl. Urt. v. 25.10.2011 - VI ZR 93/10, NJW 2012, 148 (Rn. 24) - BlogEintrag.

${ }^{126}$ Vgl. Chmelik, Social Network Sites - Soziale Netzwerke, S. 255.

${ }^{127}$ Vgl. für einen solchen Fall BGH, Urt. v. 27.02.2018 - VI ZR 489/16, NJW 2018, 2324 ff. - Internetforum. 


\section{Erstbegehungs- und Wiederholungsgefahr; Verhältnis von Kenntnisnahme} und Prüfpflicht

Voraussetzung einer Inanspruchnahme im Rahmen der Störerhaftung ist, wie auch in ihrer positiv-rechtlichen Anknüpfung in $\S 1004$ BGB mittelbar geregelt, eine Gefahr der ersten Begehung einer Rechtsverletzung oder die Gefahr der Wiederholung einer solchen. ${ }^{128}$ Diese alternativen Voraussetzungen sind beides materielle Anspruchsvoraussetzungen und nicht lediglich prozessuale Anforderungen. ${ }^{129}$

Nach der Rechtsprechung des BGH ist der Diensteanbieter als Störer grundsätzlich ,verantwortlich, sobald er Kenntnis von einer Rechtsverletzung [...] erlangt. " ${ }^{30}$ Dann schuldet er auch die Verhinderung ,derartiger" Verletzungen. ${ }^{131}$ Sodann heißt es aber in der Entscheidung Stiftparfüm weiter, dass eine Wiederholungsgefahr als Merkmal der Störerhaftung nicht schon nach erstmaliger Kenntnisnahme des Diensteanbieters gegeben sei. „Für die Annahme von Wiederholungsgefahr ist vielmehr eine vollendete Verletzung nach Begründung der Pflicht zur Verhinderung weiterer derartiger Rechtsverletzungen erforderlich“. ${ }^{132}$ Demgegenüber setze die Annahme einer Erstbegehungsgefahr als alternatives Szenario für einen Unterlassungsanspruch voraus, dass ,ernsthafte und greifbare tatsächliche Anhaltspunkte dafür vorhanden sind, der [Diensteanbieter] werde sich in naher Zukunft in der fraglichen Weise rechtswidrig verhalten. "133 Auf diese Voraussetzungen verweist der BGH auch bei der Inanspruchnahme von Diensteanbietern im Kontext dieser Untersuchung. ${ }^{134}$

Danach ist eine Inanspruchnahme auf Unterlassung erst nach Inkenntnissetzung bzw. anderweitiger Kenntnisnahme sowie einer hierauf folgenden Verletzung von Prüfpflichten möglich, ${ }^{135}$ während die Verhinderungspflicht

${ }^{128}$ Vgl. Raff, in: MüKo BGB, § 1004 Rn. 302.

${ }^{129}$ Vgl. nur Raff, in: MüKo BGB, § 1004 Rn. 306.

${ }^{130}$ BGH, Urt. v. 17.08.2011 - I ZR 57/09, MMR 2012, 178 (Rn. 26) - Stiftparfüm.

${ }^{131}$ BGH, Urt. v. 17.08.2011 - I ZR 57/09, MMR 2012, 178 (Rn. 26) - Stiftparfüm; zur Kerntheorie vgl. sogleich $\S 10$ A. IV. 4.

${ }^{132}$ BGH, Urt. v. 17.08.2011 - I ZR 57/09, MMR 2012, 178 (Rn. 39) - Stiftparfüm (Hervorh. v. Verf.); vgl. Urt. v. 19.03.2015 - I ZR 94/13, MMR 2015, 726 (Rn. 41) Hotelbewertungsportal; Urt. v. 15.08.2013 - I ZR 80/12, NJW 2013, 3245 (Rn. 45) - RapidShare; Urt. v. 12.07.2007 - I ZR 18/04, NJW 2008, 758 (Rn. 53) - Jugendgefährdende Medien bei eBay.

${ }^{133}$ BGH, Urt. v. 17.08.2011 - I ZR 57/09, MMR 2012, 178 (Rn. 44) - Stiftparfüm.

${ }^{134}$ Vgl. BGH, Urt. v. 25.10.2011 - VI ZR 93/10, NJW 2012, 148 (Rn. 32) - Blog-Eintrag.

${ }^{135}$ Vgl. Lehment, WRP 2012, 149 (152); Frey, Die Haftung von Host-Providern für Immaterialgüterrechtsverletzungen, S. 106; jüngst bekräftigt in $\mathrm{BGH}$, Beschl. v. 13.09.2018 - I ZR 140/15, GRUR 2018, 1132 (Rn. 50 ff.) - YouTube. Dem stellt sich allerdings für den Anwendungsbereich von Art. 8 Abs. 3 InfoSoc-RL GA Øe, Schlussantrag v. 16.07.2019 - C-682/18, juris, Rn. 205 ff. - YouTube, entgegen. Damit sei nicht die 
unmittelbar nach Kenntnis greift. Das bedeutet, dass die Verhinderungspflicht und ein materiell durchsetzbarer Anspruch auf Unterlassung nicht miteinander korrespondieren. ${ }^{136}$ Einer ,haftungskonkretisierenden Erstabmahnung", ${ }^{137}$ die ohne Weiteres eine Unterlassungshaftung begründen könnte, ist damit die Grundlage entzogen. ${ }^{138}$

\section{Begrenzung der Störerhaftung durch das Verbot allgemeiner Überwachungspflichten}

\section{a) Bedeutung}

Auch im Rahmen der Störerhaftung gilt das Verbot ,allgemeiner Überwachungspflichten“ nach §7 Abs. 2 TMG, der den Art. 15 Abs. 1 ECRL umsetzt. Gerade mit Blick auf Kommunikationsdienste betont der BGH in seiner jüngeren Rechtsprechung, dass keine grundsätzliche Pflicht bestehe, „die von den Nutzern in das Netz gestellten Beiträge vor der Veröffentlichung auf eventuelle Rechtsverletzungen zu überprüfen." "139 Wagner erklärt dies gar zum „Grundprinzip“ der Haftung der Diensteanbieter als Host-Provider. ${ }^{140}$ Dieses markiert somit nach geltendem Recht eine rigide Begrenzung der Verantwortung der Intermediäre. Das Verhältnis dieses in § 7 Abs. 2 TMG niedergelegten Grundsatzes zur Störerhaftung ist allerdings insoweit konfliktträchtig, als eine Geltendmachung eines Unterlassungsanspruchs gegenüber dem Diensteanbieter diesen immer zu einem bestimmten Verhalten in der Zukunft verpflichtet, nämlich eine weitere Verbreitung von Inhalten zu verhindern. ${ }^{141}$ Das betrifft insbesondere die Frage, ob über die Entfernung des ursprünglich bemängelten Inhalt hinaus der Diensteanbieter verpflichtet ist, ähnliche Informationen zu entfernen.

\section{b) Allgemeine vs. „spezifische“ Überwachungspflichten}

Nach dem BGH sind - parallel zur EuGH-Rechtsprechung ${ }^{142}$ - unzulässige „Überwachungspflichten allgemeiner Art“, die das Gericht unter Beachtung

Störerhaftung an sich in Frage gestellt, sondern das Fehlen eines anderen Rechtsbehelfs, der eine gerichtliche Anordnung bereits nach erstmaliger Rechtsverletzung ermöglicht, aus unionsrechtlicher Sicht fehlerhaft (Rn. 212).

${ }^{136}$ Vgl. Wiebe, WRP 2012, 1182 (1185); Chmelik, Social Network Sites - Soziale Netzwerke, S. 182 f.; Frey, Die Haftung von Host-Providern für Immaterialgüterrechtsverletzungen, S. 106.

${ }^{137}$ Lehment, WRP 2012, 149 (150 ff.).

${ }^{138}$ Vgl. Wiebe, WRP 2012, 1182 (1185); vgl. dazu noch unten $\S 10$ B. IV. 2.

${ }^{139}$ BGH, Urt. v. 27.02.2018 - VI ZR 489/16, NJW 2018, 2324 (Rn. 32) - Internetforum.

${ }^{140}$ Wagner, in: MüKo BGB, $§ 823$ Rn. 869.

${ }^{141}$ Vgl. auch Spindler, in: Spindler/Schmitz, TMG, §7 Rn. 49.

${ }^{142}$ Vgl. oben $\$ 4$ C. IV. 
von Erwägungsgrund 47 dem Art. 15 ECRL zuordnet, ${ }^{143}$ von zulässigen Überwachungspflichten in „spezifischen Fällen“ abzugrenzen. ${ }^{144}$ Das Gericht stützt sich hier auch auf Erwägungsgrund 48 ECRL, demzufolge es den Mitgliedstaaten unbenommen bleibt, Diensteanbietern Sorgfaltspflichten aufzuerlegen, ,um bestimmte Arten rechtswidriger Tätigkeiten aufzudecken und $\mathrm{zu}$ verhindern. " 145 Es geht damit stets um anlassbezogene Überwachungspflichten, wo also ein ,eine spezifische Verletzungshandlung Anlass für die Verhinderungspflichten ist" ${ }^{146}$ Die materielle Grenze solcher Überwachungspflichten ist dabei stets das Kriterium der Zumutbarkeit. ${ }^{147}$ Wie im europäischen ist auch im nationalen Kontext im Rahmen des Verbots allgemeiner Überwachungspflichten insbesondere die Frage von Bedeutung, ob über die konkret in Rede stehende Rechtsverletzung hinaus auch weitere Inhalte zu sperren bzw. entfernen sind.

\section{aa) Die „Kerntheorie“}

Im nationalen Kontext ist die Frage nach der Reichweite des Verbots allgemeiner Überwachungspflichten insoweit unter dem Stichwort „Kerntheorie“ bekannt, die - der Sache nach dem Unionsrecht vergleichbar - darauf abstellt, ob eine „Gleichartigkeit“ besteht bzw. die weiteren Inhalte ,entsprechend" dem ursprünglich beanstandeten Inhalt sind. ${ }^{148}$ Ansatzpunkt in der Rechtsprechung ist die häufig verwendete Formulierung des BGH, im Rahmen der Störerhaftung sei der Störer bei Kenntnis der Rechtsverletzung verpflichtet, „künftig derartige Störungen zu verhindern“. ${ }^{149}$ Entscheidend ist die Frage, ob in Handlungen, die über die konkrete Verletzungshandlung hinausgehen, „das Charakteristische der Verletzungshandlung zum Ausdruck kommt. " ${ }^{150}$ Ratio dieser Erweiterung der Unterlassungspflichten ist

${ }^{143}$ Vgl. BGH, Urt. v. 18.11.2010 - I ZR 155/09, MMR 2011, 459 (Rn. 40) - Sedo.

${ }^{144}$ Vgl. BGH, Urt. v. 26.11.2015 - I ZR 3/14, ZUM-RD 2016, 156 (Rn. 20, 26); Urt. v. 19.03.2015 - I ZR 94/13, MMR 2015, 726 (Rn. 31) - Hotelbewertungsportal; Urt. v. 05.02.2015 - I ZR 240/12, MMR 2015, 674 (Rn. 51) - Kinderhochstühle im Internet III.

${ }^{145}$ Vgl. BGH, Urt. v. 05.02.2015 - I ZR 240/12, MMR 2015, 674 (Rn. 51) - Kinderhochstühle im Internet III; Urt. v. 18.11.2010 - I ZR 155/09, MMR 2011, 459 (Rn. 40) - Sedo.

${ }^{146}$ Holznagel, ZUM 2019, 910 (910).

${ }^{147}$ Vgl. BGH, Urt. v. 12.07.2007 - I ZR 18/04, NJW 2008, 758 (Rn. 43) - Jugendgefährdende Medien bei eBay; Urt. v. 16.05.2013 - I ZR 216/11, MMR 2014, 55 (Rn. 52) Kinderhochstühle im Internet II.

${ }^{148}$ Überblick, allerdings noch ohne Berücksichtigung der Entscheidung EuGH, Urt. v. 03.10.2019 - C-18/18, NJW 2019, 3287 ff. - Glawischnig-Piesczek, bei Schapiro, Unterlassungsansprüche gegen die Betreiber von Internet-Auktionshäusern und Internet-Meinungsforen, S. $246 \mathrm{ff}$;; Chmelik, Social Network Sites - Soziale Netzwerke, S. $255 \mathrm{ff}$.

${ }^{149}$ BGH, Urt. v. 27.02.2018 - VI ZR 489/16, NJW 2018, 2324 (Rn. 32) - Internetforum, mwN (Hervorh. v. Verf.); erstmals wohl beim Urt. v. 11.03.2004 - I ZR 304/01, NJW 2004, 3102 (3105) - Internet-Versteigerung I.

${ }^{150}$ BGH, Urt. v. 04.04.2018 - VI ZR 128/18, NJW 2019, 1142 (Rn. 18). 
die Einsicht, dass die rechtsverletzende Handlung eine Wiederholungsgefahr jenseits dieser konkreten Verletzungshandlung mit sich bringen kann. ${ }^{151}$ Die Kerntheorie stellt damit der Sache nach eine dogmatisch konsistente Fortschreibung des $\S 1004$ Abs. 1 S. 2 BGB dar und ist als solche nach Auffassung des BVerfG verfassungsrechtlich unbedenklich. ${ }^{152}$

Fraglich ist allerdings, wie weit die so beschriebene Pflicht reicht. Der BGH hat erstmals in der wettbewerbsrechtlich eingekleideten Entscheidung Jugendgefährdende Medien bei eBay die Meinung vertreten, von der Pflicht zur Verhinderung ,derartiger" Rechtsverletzungen seien nicht nur identische Schutzrechtsverletzungen durch denselben „Täter“ erfasst, sondern auch Verstöße durch andere Nutzer eines Dienstes. ${ }^{153}$ Diese Auffassung hat das Gericht dann auch im Urheberrech $t^{154}$ und im Markenrecht ${ }^{155}$ wiederholt. Bei Persönlichkeitsrechtsverletzungen ist zwar einerseits zu berücksichtigen, dass es bei deren Prüfung oftmals auf den gesamten Kontext der getroffenen Äußerung ankommt, ${ }^{156}$ eine Gleichartigkeit und eine hieraus folgende Erweiterung der Pflichten des Diensteanbieters also nicht einfach zu begründen ist. Der BGH verweist jedoch in seinen jüngeren Entscheidungen im persönlichkeitsrechtlichen Kontext immer wieder auf die entsprechende Passage der Entscheidung Jugendgefährdende Medien bei eBay und gibt so zu erkennen, dass er auch bei Persönlichkeitsrechtsverletzungen im Grundsatz eine erweiterte Pflicht der Diensteanbieter befürwortet. ${ }^{157}$

Diese Auffassung wird im Schrifttum teilweise kritisiert. Zum einen widerspreche diese Position dem in $\S 10$ TMG niedergelegten und damit unionsrechtlich verankerten Grundsatz, dass stets bezüglich eines spezifischen

${ }^{151}$ Vgl. BGH, Urt. v. 04.04.2018 - VI ZR 128/18, NJW 2019, 1142 (Rn. 18).

${ }^{152}$ Vgl. BVerfG, Beschl. v. 04.12.2006 - 1 BvR 1200/04, GRUR 2007, 618 (Rn. 20) Organisationsverschulden; Beschl. v. 09.07.1997 - 1 BvR 730/97, BeckRS 1997, 9994 (Rn. 12).

${ }^{153}$ Vgl. BGH, Urt. v. 12.07.2007 - I ZR 18/04, NJW 2008, 758 (Rn. 43) - Jugendgefährdende Medien bei eBay, wo es um die Bewertung des Versandhandels mit jugendgefährdenden Medien ging.

${ }^{154}$ Vgl. BGH, Urt. v. 12.07.2012 - I ZR 18/11, NJW 2013, 784 (Rn. 32) - Alone in the Dark.

${ }^{155}$ Vgl. BGH, Urt. v. 30.04.2008 - I ZR 73/05, GRUR 2008, 702 (Rn. 55) - InternetVersteigerung III, wobei im Markenrecht die Besonderheit gilt, dass Produkte nicht selten durch mehrere Markenrechte geschützt sind, vgl. Chmelik, Social Network Sites - Soziale Netzwerke, S. 256.

${ }^{156}$ Vgl. BGH, Urt. v. 30.04.2008 - I ZR 73/05, GRUR 2008, 702 (Rn. 24) - InternetVersteigerung III; kritisch, wenngleich ohne Stellungnahme zur Rechtsfrage das Urt. v. 04.04.2018 - VI ZR 128/18, NJW 2019, 1142 (Rn. 19 f.).

${ }^{157}$ Vgl. BGH, Urt. v. 01.03.2016 - VI ZR 34/15, NJW 2016, 2106 (Rn. 23) - Ärztebewertungsportal III; Urt. v. 25.10.2011 - VI ZR 93/10, NJW 2012, 148 (Rn. 24) - BlogEintrag. 
Inhalts Kenntnis vermittelt werden müsse. ${ }^{158}$ Zum anderen höhle der BGH das Verbot allgemeiner Überwachungspflichten aus. ${ }^{159}$ Rechtssystematisch sei eine Verortung des Problems im Vollstreckungsrecht ${ }^{160}$ fehl am Platz. ${ }^{161}$ Hinsichtlich des $\$ 10 \mathrm{TMG}$ hat der der BGH allerdings explizit zugestanden, den Pflichtenkreis weiter zu ziehen, als es diese Bestimmung vorsehe. ${ }^{162}$

Für die Einbeziehung von Rechtsverletzungen, die durch andere als diejenigen Personen begangen wurden, die im geltend gemachten Unterlassungsanspruch bezeichnet sind, spricht im Ausgangspunkt die Einsicht, dass es bei der Störerhaftung gerade darum geht, den aus ihr Berechtigten eine Erleichterung hinsichtlich der Auswahl des Schuldners in Bezug auf eine einzelne Rechtsverletzung zur Verfügung zu stellen. Dies würde konterkariert, wenn die Berechtigte verpflichtet wäre, immer wieder neue Primärverursacher gegenüber dem Diensteanbieter zu benennen. ${ }^{163}$ Außerdem könne, so immerhin das BVerfG, ansonsten die Unterlassungspflicht letztlich einfach umgangen werden. ${ }^{164}$ Gegen das rechtssystematische Argument, das die Verortung im Kern materiell-rechtlicher Fragen im Vollstreckungsrecht bemängelt, spricht schließlich, dass es dem Unterlassungsschuldner freisteht, bereits im Erkenntnisverfahren auf eine sachgerechte und begrenzte Formulierung des Tenors hinzuwirken. ${ }^{165}$ Für Persönlichkeitsrechtsverletzungen ist damit ein Spielraum auszumachen, innerhalb dessen die Prüfung der Diensteanbieter sich auch auf weitere kerngleiche Rechtsverletzungen beziehen kann. ${ }^{166}$ Das ist insbesondere bei Wiederholungen des identischen oder

${ }^{158}$ Vgl. Spindler, in: Spindler/Schmitz, TMG, §7 Rn. 51.

${ }^{159}$ Vgl. Paal, in: BeckOK Informations- und Medienrecht, TMG § 7 Rn. 65.

${ }^{160}$ Der verfahrensrechtliche Vorteil der Kerntheorie liegt gerade darin, aus einem tenorierten Unterlassungsanspruch auch bei kerngleichen Äußerungen vollstrecken zu können.

${ }^{161}$ Vgl. Spindler, in: Spindler/Schmitz, TMG, § 7 Rn. 52; kritisch auch Fischer, Die Einbindung von Providern in die Durchsetzung von Urheberrechten, S. $112 \mathrm{f}$.

${ }^{162}$ Für § 11 TDG a.F. BGH, Urt. v. 11.03.2004 - I ZR 304/01, NJW 2004, 3102 (3105) Internet-Versteigerung I: „Dies bedeutet, dass die Bekl. immer dann, wenn sie auf eine klare Rechtsverletzung hingewiesen worden ist, nicht nur das konkrete Angebot unverzüglich sperren muss ( $\$ 11 \mathrm{~S} .1 \mathrm{Nr} .2$ TDG n.F.), sie muss vielmehr auch Vorsorge treffen, dass es möglichst nicht zu weiteren derartigen Markenverletzungen kommt."

${ }^{163}$ Überzeugend Klatt, ZUM 2009, 265 (273).

${ }^{164}$ Vgl. BVerfG, Beschl. v. 09.07.1997 - 1 BvR 730/97, BeckRS 1997, 9994 (Rn. 12).

${ }^{165}$ Vgl. BVerfG, Beschl. v. 04.12.2006 - 1 BvR 1200/04, GRUR 2007, 618 (Rn. 20) Organisationsverschulden; hieran hat der Unterlassungsschuldner ohnehin ein prozesstaktisches Interesse, weil die gerichtliche Beschränkung des Tenors (im Vergleich zum Antrag) zu einer Teilung der Kosten führt, §92 Abs. 1 ZPO.

${ }^{166}$ Für eine grundsätzliche Anwendbarkeit der Kerntheorie bei Inanspruchnahme von Suchmaschinenbetreibern wegen Persönlichkeitsrechtsverletzungen vgl. BGH, Urt. v. 24.07.2018 - VI ZR 330/17, ZUM-RD 2019, 203 (Rn. 44); grundsätzlich auch Urt. v. 23.06.2009 - VI ZR 232/08, NJW 2009, 2823 (Rn. 11); für eine Anwendung im Bereich 
nur unwesentlich veränderten Wortlauts durch mehrere Personen denkbar, wenngleich die Umstände des Einzelfalls hier Vorrang vor einer abstrakten Festlegung genießen sollten.

Zusammenfassend lässt sich damit festhalten, dass auch bei Persönlichkeitsrechtsverletzungen eine eher weit zu verstehende Pflicht des Diensteanbieters besteht, „kerngleiche“ Rechtsverletzungen zu verhindern. Dies umfasst auch kerngleiche Rechtsverletzungen durch weitere Nutzer. Solange eine hinreichend spezifische Information Gegenstand des Unterlassungsbegehrens ist, stellt sich dies auch unter Art. 15 ECRL als nicht per se problematisch dar, wie der EuGH jüngst erneut bestätigte. ${ }^{167}$

\section{bb) Filtersysteme}

Trotz des allgemeinen Verbots der proaktiven Überwachung als Grenze der Störerhaftung hat die Rechtsprechung bisweilen auch die Entfernung einzelner legaler Inhalte durch Filtersysteme für gerechtfertigt erachtet. ${ }^{168}$ Den $S A B A M$-Entscheidungen stand dies nach Auffassung des $\mathrm{BGH}^{169}$ nicht entgegen, da diese eine generelle, umfassende Filterung zum Gegenstand hatten, während es bei den Entscheidungen des BGH um Inanspruchnahmen ging, bei denen die Rechtsverletzung konkret identifiziert und dem Diensteanbieter zur Kenntnis gebracht worden war. ${ }^{170}$

der Wortberichterstattung plädiert auch Burkhardt, in: Wenzel, Das Recht der Wort- und Bildberichterstattung, Kap. 12 Rn. 158.

${ }^{167}$ Vgl. EuGH, Urt. v. 03.10.2019 - C-18/18, NJW 2019, 3287 ff. - Glawischnig-Piesczek, insbesondere Rn. 37: Verhinderungspflicht, ,ganz gleich, wer den Auftrag zur Speicherung dieser Information gegeben hat." Damit scheint der EuGH sich auch von einer früheren Auffassung zu distanzieren, als er die Pflicht zum Auffinden „derartiger“ Verletzungen angesprochen und sich eher dahingehend positioniert hatte, dass eine Identität des Verletzers und des verletzten Schutzrechts erforderlich sei, vgl. insoweit den Verweis im Urt. v. 12.11.2011 - C-324/09, MMR 2011, 596 (Rn. 141) - L'Oréal SA, auf GA Jääskinen, Schlussantrag v. 09.12.2010 - C-324/09, juris, Rn. 182 - L'Oréal SA, wo eine „doppelte Identität" gefordert wird. Dies hatte auch GA Szpunar, Schlussantrag v. 04.06.2019 C-18/18, BeckRS 2019, 10236 (Rn. 72 ff.) - Glawischnig-Piesczek, in seinem Schlussantrag gefordert.

${ }^{168}$ Vgl. BGH, Urt. v. 12.07.2012 - I ZR 18/11, NJW 2013, 784 (Rn. 35, 45) - Alone in the Dark; Urt. v. 22.07.2010 - I ZR 139/08, GRUR 2011, 152 (Rn. 38) - Kinderhochstühle im Internet I; Urt. v. 19.04.2007 - I ZR 35/04, NJW 2007, 2636 (Rn. 47) - Internet-Versteigerung II; Urt. v. 11.03.2004 - I ZR 304/01, NJW 2004, 3102 (3105) - Internet-Versteigerung I; zur Kritik vgl. nur Kastl, GRUR 2016, 671 (674 ff.).

${ }^{169}$ Vgl. BGH, Urt. v. 05.02.2015 - I ZR 240/12, MMR 2015, 674 (Rn. 59) - Kinderhochstühle im Internet III; Urt. v. 16.05.2013 - I ZR 216/11, MMR 2014, 55 (Rn. 52) - Kinderhochstühle im Internet II.

${ }^{170}$ Dies ist freilich stark kritisiert worden, vgl. Paal, in: BeckOK Informations- und Medienrecht, TMG § 7 Rn. 65; Sieber/Höfinger, in: Hoeren/Sieber/Holznagel, Handbuch Multimedia-Recht, Teil 18.1 Rn. 57. 
Der entscheidende Unterschied zur nunmehrigen Auffassung des EuGH liegt allerdings in der Frage begründet, ob manuelle Nachkontrollen Bestandteil der Pflichten der Diensteanbieter darstellen sollen oder nicht. Der BGH hatte es bislang, allerdings nicht in Bezug auf Persönlichkeitsrechtsverletzungen, oftmals als Teil der zumutbaren Prüfpflichten bezeichnet, den Diensteanbietern die Durchführung von Filterverfahren einschließlich einer manuellen Nachkontrolle aufzuerlegen. ${ }^{171}$ Wie bereits ausgeführt, hat der EuGH aber nun dezidiert eine ,autonome Beurteilung“ als mit Art. 15 Abs. 1 ECRL unvereinbar erklärt. ${ }^{172}$ Damit ist die rechtliche Pflicht zur Durchführung manueller Nachkontrollen aus unionsrechtlicher Sicht unzulässig. Im Hinblick hierauf wird der BGH dies nunmehr in seiner Rechtsprechung berücksichtigen müssen, soweit es nicht zu einer anderweitigen legislativen Entscheidung kommt oder der EuGH seine Auffassung noch weiterentwickeln sollte. Für den Einsatz von Filtersystemen, die die Anforderungen der Rechtsprechung einhalten, bedeutet dies, dass diese so programmiert sein müssen, dass eine manuelle Nachkontrolle nicht erforderlich ist. Für die Zuverlässigkeit solcher Systeme ist dies freilich eine hohe Hürde.

\section{Kosten der Inanspruchnahme des Störers}

Die Geltendmachung der Störerhaftung beinhaltet theoretisch auch die Kosten der Rechtsverfolgung, insbesondere die Kosten einer anwaltlichen Tätigkeit, soweit diese erstattungsfähig sind. ${ }^{173} \mathrm{Zu}$ beachten ist insoweit, dass solche Kosten jedoch erst ab dem Zeitpunkt des materiellen Bestehens einer rechtlichen Pflicht des Schuldners bestehen. Da dies unter dem geltenden Recht in diesem Kontext stets die Kenntnisnahme und die Verletzung von Prüfpflichten erfordert, die die Störereigenschaft in diesem Sinne erst begründen, kommt eine Erstattung von Kosten, die zur Inkenntnissetzung erforderlich sind, in der Regel nicht in Betracht, da dies ansonsten auf eine allgemeine Überwachungspflicht hinausliefe. ${ }^{174}$ Insoweit wird das Kostenrisiko gar als wesentlicher Grund für die Etablierung einer Prüfpflichtverletzung gesehen, die eigentlich eine systemwidrige Anomalie im Kontext der verschuldensunabhängigen Haftung als Störer darstelle. ${ }^{175}$ Damit entstehen

${ }^{171}$ So BGH, Urt. v. 16.05.2013 - I ZR 216/11, MMR 2014, 55 (Rn. 47) - Kinderhochstühle im Internet II; Urt. v. 12.07.2012 - I ZR 18/11, NJW 2013, 784 (Rn. 35, 39) - Alone in the Dark; Urt. v. 22.07.2010 - I ZR 139/08, GRUR 2011, 152 (Rn. 38) - Kinderhochstühle im Internet I; vgl. auch Holznagel, ZUM 2018, 350 (352).

${ }^{172}$ EuGH, Urt. v. 03.10.2019 - C-18/18, NJW 2019, 3287 (Rn. 45 f.) - GlawischnigPiesczek; vgl. dazu auch schon oben $\S 4$ C. IV. 2. a) bb).

${ }^{173} \mathrm{Zu}$ gesetzlichen Sonderregeln vgl. insbesondere die $\S 97 \mathrm{a}$ UrhG, $\S 12$ Abs. 1 S. 2 UWG.

${ }^{174}$ Vgl. Chmelik, Social Network Sites - Soziale Netzwerke, S. 86 f.

${ }^{175}$ Vgl. Diesterhöft, Das Recht auf medialen Neubeginn, S. $277 \mathrm{f}$. 
Kostenerstattungsansprüche unter dem geltenden Recht erst ab der zweiten Abmahnung. ${ }^{176}$

\section{Sperrung durch die Haftungsprivilegierungen?}

Für die Zwecke dieser Untersuchung ist schließlich noch von Bedeutung, in welchem Verhältnis die Störerhaftung und die Haftungsprivilegierungen stehen. Denn soweit man annimmt, dass die Privilegierungen Unterlassungsansprüche und damit die Störerhaftung prinzipiell erfassen können, droht die Störerhaftung durch den EuGH überformt und dadurch womöglich ihrer Flexibilität beraubt werden zu können.

\section{Unionsrechtliche Ausgangslage}

Hier ist zunächst zu berücksichtigen, dass Art. 14 Abs. 3 ECRL den Mitgliedstaaten offenkundig eine nur durch Art. 15 ECRL beschränkte Autonomie einräumt, verwaltungsbehördlich oder gerichtlich das „Abstellen“ oder „Verhindern“ der Rechtsverletzung anzuordnen. ${ }^{177}$ Mit „Verhinderung“ ist der in die Zukunft gerichtete Unterlassungsanspruch gemeint. ${ }^{178}$ Damit ist die Rechtsfrage nach der Reichweite der Haftungsprivilegierung im Hinblick auf Unterlassungsansprüche im Ausgangspunkt eine mitgliedstaatliche.

\section{2. Übertragung auf den mitgliedstaatlichen Kontext}

Nach einer schon älteren Rechtsprechungslinie des BGH soll die Privilegierung des §10 TMG nicht auf Unterlassungsansprüche anwendbar sein, was damit auch entsprechend für die Störerhaftung gelten soll. ${ }^{179}$ Dafür spreche bereits der Wortlaut von $\S 10$ TMG, da mit „Verantwortlichkeit“ nur die

${ }^{176}$ Vgl. auch Spindler, in: Spindler/Schmitz, TMG, § 10 Rn. 115; Klein, Haftung von Social-Sharing-Plattformen, S. 173.

${ }^{177}$ Vgl. auch Schapiro, Unterlassungsansprüche gegen die Betreiber von InternetAuktionshäusern und Internet-Meinungsforen, S. $373 \mathrm{f}$.

${ }^{178}$ Vgl. nur Schapiro, Unterlassungsansprüche gegen die Betreiber von Internet-Auktionshäusern und Internet-Meinungsforen, S. 366 ff.; Frey, Die Haftung von Host-Providern für Immaterialgüterrechtsverletzungen, S. $153 \mathrm{ff}$. Unzutreffend dagegen Berger/Janal, CR 2004, 917 (920 f.), die die „Entfernung einer Information“ oder die Zugangssperrung als einzige von Art. 14 Abs. 3 ECRL in das mitgliedstaatliche Regelungsermessen gestellte Verhaltenspflicht (im Sinne eines Beseitigungsanspruchs) ansehen und dabei der „Abstellung“ bzw. „Verhinderung“ (im Sinne eines echten Unterlassungsanspruchs) demgegenüber keine eigenständige Bedeutung beimessen.

${ }^{179}$ Soweit ersichtlich erstmals (noch für $\S 11$ TDG in der Fassung vom 21.12.2001) BGH, Urt. v. 11.03.2004 - I ZR 304/01, NJW 2004, 3102 (3103 f.) - Internet-Versteigerung I; vgl. Urt. v. 25.10.2011 - VI ZR 93/10, NJW 2012, 148 (Rn. 19) - Blog-Eintrag, mwN. 
strafrechtliche Verantwortung und die Schadensersatzhaftung, mithin verschuldensabhängige Rechtsfolgen gemeint seien. ${ }^{180}$ Hierfür spreche auch die Klarstellung in $\S 7$ Abs. 3 S. 1 TMG, wonach „Verpflichtungen zur Entfernung von Informationen oder zur Sperrung der Nutzung von Informationen nach den allgemeinen Gesetzen aufgrund von gerichtlichen oder behördlichen Anordnungen [...] auch im Falle der [...] nach den $\S \S 8$ bis 10 unberührt “ bleiben. ${ }^{181}$ Dem stimmen viele Stimmen im Schrifttum zu. ${ }^{182}$ Denn mit Entfernung und Sperrung seien letztlich auch Unterlassungsansprüche gemeint. Noch deutlicher ist insoweit Art. 14 ECRL, der von dem Abstellen bzw. der Verhinderung der Rechtsverletzung spricht. Auch der Verweis auf die ,allgemeinen Gesetze“ in $§ 7$ Abs. 3 TMG legt eine Geltung der sich aus den allgemeinen Regeln ergebenden Rechtsinstitute nahe, zu der auch die Störerhaftung als Ausprägung des Gedankens von $§ 1004$ BGB gehört. Die Auffassung des BGH wird allerdings verschiedentlich aufgrund ihrer - so jedenfalls die Gegenauffassung - Unvereinbarkeit mit der Rechtsprechung des EuGH zu den Art. 12 ff. ECRL kritisiert. ${ }^{183}$ In jüngerer Zeit hatte der BGH auch seine Auffassung jedenfalls teilweise nicht mehr wiederholt, sondern vielmehr seine mittels der Störerhaftung gefundenen Ergebnisse mit den Art. 14, 15 ECRL explizit abgeglichen. ${ }^{184}$ Das sprach für eine Konvergenz der $\S \S 7,10 \mathrm{TMG}$ und der Störerhaftung jedenfalls in den Grundzügen. ${ }^{185}$

${ }^{180}$ Vgl. BGH, Urt. v. 11.03.2004 - I ZR 304/01, NJW 2004, 3102 (3103) - InternetVersteigerung I; ob dieses Wortlautargument überzeugt ist allerdings zweifelhaft, da jedenfalls im bürgerlichen Recht der Begriff keineswegs auf verschuldensabhängige Maßstäbe beschränkt ist, vgl. Hollenders, Mittelbare Verantwortlichkeit von Intermediären im Netz, S. 46 f., 274; im Ergebnis ebenso Chmelik, Social Network Sites - Soziale Netzwerke, S. 30 f., 273, mwN; anders dagegen Volkmann, Der Störer im Internet, S. 101.

${ }^{181}$ Vgl. BGH, Urt. v. 25.10.2011 - VI ZR 93/10, NJW 2012, 148 (Rn. 19) - Blog-Eintrag; für Access-Provider vgl. auch Urt. v. 26.07.2018 - I ZR 64/17, NJW 2018, 3779 (Rn. 20) Dead Island.

${ }^{182}$ Vgl. Spindler, in: Spindler/Schmitz, TMG, TMG § 7 Rn. 46 ff.; Hoffmann/Volkmann, in: Spindler/Schuster, Recht der elektronischen Medien, TMG $\$ 7$ Rn. 37 f.; Klein, Haftung von Social-Sharing-Plattformen, S. 110 f.; sowie die Nachweise bei Paal, in: BeckOK Informations- und Medienrecht, TMG $\S 7$ Rn. 54.

${ }^{183} \mathrm{Vgl}$. Paal, in: BeckOK Informations- und Medienrecht, TMG $\S 7$ Rn. 56; Specht, ZUM 2017, 114 (116f.); Ohly, ZUM 2015, 308 (310, 312); Ehret, CR 2003, 754 (759 f.); vgl. auch OLG Hamburg, Urt. v. 01.07.2015 - 5 U 87/12, MMR 2016, 269 (Rn. 130) - YouTube, allerdings unter Verweis auf eine BGH-Entscheidung, die explizit nicht den HostProvidern galt; KG, Urt. v. 16.04.2013 - 5 U 63/12, MMR 2014, 46 (48) - Hotelbewertungsportal; offen gelassen von LG Würzburg, Urt. v. 07.03.2017 - 11 O 2338/16 UVR, ZUM 2017, 437 (446).

${ }^{184}$ Vgl. BGH, Urt. v. 17.08.2011 - I ZR 57/09, MMR 2012, 178 (Rn. 22) - Stiftparfüm; siehe auch Nolte/Wimmers, GRUR 2014, 16 (20).

${ }^{185}$ Skeptisch aber OLG Köln, Urt. v. 13.10.2016 - 15 U 189/15, BeckRS 2016, 18916 (Rn. 59). 
Die hier dargestellten konträren Auffassungen gehen auf eine über längere Zeit nicht weiter zwischen Schadensersatz- und Unterlassungshaftung differenzierende Rechtsprechung des EuGH zurück. Dies lässt sich damit erklären, dass für den Gerichtshof die Inanspruchnahme von Diensteanbietern konzeptionell eine Haftung von „Nichtverantwortlichen“ darstellte, weshalb Art. 14 ECRL ohnehin nicht von Bedeutung sei. ${ }^{186}$ In der Entscheidung McFadden nimmt der EuGH jedoch erstmals eine Haltung ein, die unter den Art. 12 ff. ECRL zwischen zulässigen Unterlassungsansprüchen und im Grundsatz privilegierten Schadensersatzansprüchen differenziert. ${ }^{187}$ Für Host-Provider und Art. 14 ECRL wiederholt der Gerichtshof dies in einer jüngeren Entscheidung recht deutlich. ${ }^{188}$

Damit scheint die ältere Rechtsprechungslinie des BGH bestätigt und die Rechtslage insoweit unionsrechtlich geklärt. Insgesamt sprechen auch die besseren Argumente für einen zumindest teilweisen Ausschluss der Haftungsprivilegierungen im Anwendungsbereich der Störerhaftung. ${ }^{189}$ Es ist allerdings auch nicht von der Hand zu weisen, dass die Störerhaftung mitunter auch über Art. 14 ECRL hinausgeht. Dies betrifft zum einen vorbeugende Unterlassungsklagen im gewerblichen Rechtsschutz. ${ }^{190}$ Zum anderen ist damit die mögliche Verpflichtung des Störers zur Entfernung und Verhinderung des Hochladens von rechtswidrigen Inhalten jenseits spezifizierter, dem Diensteanbieter im Sinne von Art. 14 ECRL zur Kenntnis gebrachter Inhalte angesprochen. ${ }^{191}$ Denn erstens standen bislang eher die reaktiven Ansprüche auf Entfernung von Informationen im Sinne des „Abstellens" nach Art. 14 Abs. 3 ECRL im Vordergrund. Zweitens hat der EuGH die vom BGH entwickelte Kategorie der „Kerngleichheit“, die den Kreis der von der Störerhaftung erfassten Pflichten erweitert, zwar jüngst in der Entscheidung Glawischnig-Piesczek aufgegriffen, dabei aber so eng geführt, ${ }^{192}$ dass jedenfalls bislang von einer vollständigen Übereinstimmung mit der Störerhaftung

${ }^{186}$ Nach Holznagel, jurisPR-WettbR 10/2016, Anm. 1 (II.), habe der EuGH die deutsche Störerhaftung implizit auch einer solchen „Nichtverantwortlichkeit“ zugeordnet.

${ }^{187}$ Vgl. EuGH, Urt. v. 15.09.2016 - C-484/14, NJW 2016, 3503 (Rn. 76) - McFadden, allerdings in Bezug auf Access-Provider (Art. 12 ECRL); vgl. auch Hofmann, ZUM 2019, 617 (624).

${ }^{188}$ Vgl. EuGH, Urt. v. 07.08.2018 - C-521/17, NJW 2018, 2781 (Rn. 51) - Deepak Mehta.

${ }^{189}$ In systematisch unklarer Weise fordert aber Volkmann, Der Störer im Internet, S. 103, die Begrenzung der Verantwortlichkeit auch unter dem Institut der Störerhaftung. Diese Begrenzung macht er dann allerdings in dem Verbot allgemeiner Überwachungspflichten aus.

${ }^{190} \mathrm{Vgl}$. Paal, in: BeckOK Informations- und Medienrecht, TMG § 7 Rn. 57.

${ }^{191}$ In diesem Sinne Paal, in: BeckOK Informations- und Medienrecht, TMG § 7 Rn. 56; Chmelik, Social Network Sites - Soziale Netzwerke, S. 272.

${ }^{192}$ Vgl. dazu oben §4 C. IV. 2 a) bb); ergänzend Specht, MMR 2019, 801 (801 f.). 
noch nicht die Rede sein kann. ${ }^{193}$ Angesichts dieses Befundes lässt sich auch nicht leugnen, dass die vom BGH entwickelten Lösungsansätze im Rahmen der Störerhaftung nach wie vor einer potenziellen Einflussnahme durch den EuGH unterliegen können. Eine auf Unionsebene gesetzgeberisch angestoBene weitere Harmonisierung könnte als positiv bewertete, vom BGH entwickelte Kriterien und Maßstäbe jedoch aufgreifen. ${ }^{194}$

\section{B. Bewertung der Störerhaftung im Lichte der Verantwortung der Intermediäre}

Im Folgenden soll eine Bewertung der dargestellten Figur der Störerhaftung im Kontext des Gegenstands dieser Untersuchung erfolgen. Deshalb erfolgt nur ein kurzer Verweis auf die dogmatischen Einwände, die gegen die Störerhaftung erhoben werden (I.), bevor sodann auf einen gewichtigen Einwand einzugehen ist, nämlich die behauptete und problematisierte Übertragung hoheitlicher Funktionen auf Private (II.). Die Störerhaftung erweist sich als grundsätzlich geeignet, den vielfältigen Problemen beim Umgang mit den Intermediären zu begegnen (III.), weist aber zugleich im Detail auch Herausforderungen auf, die es anzugehen gilt (IV.).

\section{Dogmatische Einwände gegen die Störerhaftung}

Die Figur der Störerhaftung ist nicht frei von kritischen Stellungnahmen dogmatischer Natur geblieben. So wird ihr vorgeworfen, überwiegend konturenlos bzw. ohne dogmatische Konsistenz primär in den Dienst einzelfallgerechter Entscheidungen gestellt zu werden. ${ }^{195}$ Ein Hauptaugenmerk der dogmatisch konnotierten Kritik liegt auf der Unterscheidung zwischen der Täterhaftung einerseits und der Störerhaftung andererseits. Diese überspiele die Tatsache, dass auch der Störer in der Rechtsprechung der Sache nach stets Täter eines Delikts sei. ${ }^{196}$ Die Erweiterung des Anwendungsbereichs von Unterlassungsansprüchen auf solche Schuldner, die gegen kein Schutzgesetz verstießen, stelle eine systemwidrige Erweiterung des Anwendungsbereichs von $\S 1004$ BGB dar. ${ }^{197}$ Auch hinsichtlich der Konkretisierung der Reichweite

\footnotetext{
${ }^{193}$ Vgl. zur Divergenz zwischen Störerhaftung und Art. 14 ECRL bzw. §10 TMG insoweit schon die Ausführungen oben unter $§ 10$ A. IV.4. b) aa).

${ }^{194} \mathrm{Vgl}$. dazu noch unten $\S 11$.

${ }^{195}$ Vgl. Volkmann, in: Spindler/Schuster, Recht der elektronischen Medien, BGB $§ 1004$ Rn. 20.

${ }^{196} \mathrm{Vgl}$. Schapiro, Unterlassungsansprüche gegen die Betreiber von Internet-Auktionshäusern und Internet-Meinungsforen, S. 96.

${ }^{197}$ Statt vieler Schapiro, Unterlassungsansprüche gegen die Betreiber von InternetAuktionshäusern und Internet-Meinungsforen, S. 97.
} 
der Störerhaftung durch die Prüfpflichten wird immer wieder bemerkt, diese ließen sich nicht dogmatisch überzeugend in den Anspruchsaufbau einpassen. ${ }^{198}$

Die Kritiker plädieren stattdessen für ein erweitertes Konzept der Verkehrspflichten, in das die Diensteanbieter zu integrieren seien, ${ }^{199}$ oder aber für eine Anwendung der Gehilfenhaftung entsprechend $\S 830$ Abs. 2 BGB..$^{200}$ Aus dogmatischer Perspektive überzeugt dies nicht vollends, denn während der Gleichlauf deliktischer und negatorischer Haftung zwar die gleichen Ergebnisse produziert, ${ }^{201}$ liefe eine materielle Vereinheitlichung auf die Nivellierung der Differenzierung zwischen Täter und Störer hinaus, die nicht nur aufgrund der textlichen Differenzierung zwischen $\S 823$ BGB und $\S 1004$ BGB zu beachten ist, sondern aufgrund des Verweisungscharakters der negatorischen Haftung auf viele spezialgesetzliche Regelungen für den Störerbegriff einen deutlich weiteren Spielraum erfordert. ${ }^{202}$ Hinsichtlich des Vorschlags, die Gehilfenhaftung für die hiesige Problematik fruchtbar zu machen, mag es zwar nach Auffassung mancher Stimmen zutreffen, dass beim zivilrechtlichen Fahrlässigkeitsdelikt der Einheitstäterbegriff gilt, sodass ein doppelter Gehilfenvorsatz nicht notwendig ist. ${ }^{203}$ Freilich sieht die Rechtsprechung dies bislang anders. ${ }^{204}$ Eine Heranziehung der Gehilfenhaftung verspricht demnach keine über jeden Zweifel erhabene Befreiung von dogmatischen Bedenken bei dem von der Störerhaftung verfolgten Anliegen.

Den hier dargestellten Bedenken zum Trotz ist die Bewertung der Inpflichtnahme der Intermediäre unter der Störerhaftung geltendes Recht. Anliegen des nachfolgenden Abschnitts ist es daher, ihre Vorzüge wie auch

${ }^{198}$ Vgl. Spindler/Volkmann, WRP 2003, 1 (6); Volkmann, in: Spindler/Schuster, Recht der elektronischen Medien, $§ 1004$ Rn. 20.

${ }^{199}$ Statt vieler Volkmann, in: Spindler/Schuster, Recht der elektronischen Medien, $\S 1004$ Rn. 20; kritisch mit Blick auf das Deliktsrecht Sokol, Die Bestimmung der Verantwortlichkeit für die Abwehr und Beseitigung von Störungen im öffentlichen und privaten Recht, S. 55; Frey, Die Haftung von Host-Providern für Immaterialgüterrechtsverletzungen, S. 102.

${ }^{200}$ Vgl. Jaworski/Nordemann, GRUR 2017, 567 ff. Für einen beide Elemente kombinierenden Ansatz vgl. Schapiro, Unterlassungsansprüche gegen die Betreiber von InternetAuktionshäusern und Internet-Meinungsforen, passim.

${ }^{201}$ Vgl. Wagner, in: MüKo BGB, § 823 Rn. 855.

${ }^{202}$ Zur Erklärung der begrifflichen Differenzierung zwischen Tätern und Störern in der Rechtsprechung des BGH vgl. oben $§ 10$ A. II. 2. In diese Richtung wohl auch Fischer, Die Einbindung von Providern in die Durchsetzung von Urheberrechten, S. 338.

${ }^{203}$ Vgl. Wagner, GRUR 2020, 329 (334).

${ }^{204}$ Vgl. BGH, Urt. v. 19.04.2007 - I ZR 35/04, NJW 2007, 2636 (Rn. 31) - InternetVersteigerung II; Urt. v. 11.03.2004 - I ZR 304/01, NJW 2004, 3102 (3105) - InternetVersteigerung I, mwN; OLG Hamburg, Urt. v. 01.07.2015 - 5 U 87/12, MMR 2016, 269 (Rn. 277) - YouTube; in diesem Sinne auch Spindler, in: Spindler/Schmitz, TMG, §7 Rn. 40. 
Schwächen mit Blick auf den Kontext dieser Untersuchung darzulegen und mögliche Lösungsansätze ausgehend von der Störerhaftung aufzuzeigen. Diese erweist sich danach im Grundsatz als geeignetes Instrument zur Adressierung der Verantwortung der Intermediäre. ${ }^{205}$

\section{Problematische Übertragung richterlicher Aufgaben?}

\section{Der Vorwurf}

Zuvor gilt es allerdings, ein häufig anzutreffendes Argument zu entkräften, das zwar nicht die Dogmatik der Störerhaftung im engeren Sinne betrifft, aber doch an ihrem Wesenskern rührt. Immer wieder wird der Vorwurf gegenüber der Störerhaftung, aber auch gegenüber gesetzlichen Versuchen der Ausgestaltung der Haftung von Intermediären geäußert, dass private Unternehmen durch solche Regelungen staatlicherseits in eine Position gerückt würden, die ihrer Rolle nicht gerecht werde. Erwähnt seien hier allein die jüngst besonders konfliktträchtigen Maßnahmen wie das NetzDG oder die unionsrechtliche Reform des Urheberrechts. Konkret stelle jede staatliche Verpflichtung der Intermediäre, Inhalte strenger oder in einer bestimmten Art und Weise zu kontrollieren und die Sanktionsbewehrung von Verstößen gegen diese Vorgaben eine „Privatisierung“ der Rechtsdurchsetzung dar. ${ }^{206}$ Der Staat verpflichte Private zur Ausübung von Kontrollbefugnissen, übe aber mittelbar weiterhin die „Kontrolle“ über das Verhalten der Dienstenutzer aus. ${ }^{207}$ Für die Rechtsdurchsetzung sei die Justiz, also die Staatsanwaltschaften und die Gerichte zuständig. Solchen oder verwandten Argumenten

${ }^{205}$ Die wenigsten ihrer Kritiker zweifeln an dem grundsätzlichen Anliegen der Störerhaftung, sondern verweisen „lediglich“ auf dogmatische Einzelheiten als Grundlage ihrer Kritik. Dementsprechend ist es auch nicht Ziel der Kritik, eine Abkehr der von der Rechtsprechung unter Anwendung der Störerhaftung erzielten Ergebnisse zu veranlassen, vgl. Wagner, in: MüKo BGB, $\S 823$ Rn. 857.

${ }^{206}$ Für die Störerhaftung vgl. Specht, ZUM 2017, 114 (121); dies., in: Röhricht/Westphalen/Haas, HGB: Plattformnutzungsverträge, Rn. 73; mit moderat-kritischem Anklang auch Holznagel, GRUR Int 2014, 105 (108); für die DSM-RL statt vieler Pravemann, GRUR 2019, 783 (787); für das NetzDG vgl. Wimmers/Heymann, AfP 2017, 93 (97f.); Schütz, MMR 2018, 36 (37); Lüdemann, in: Eifert/Gostomzyk, Netzwerkrecht, S. 153 (163 f.) („,Fundamentalproblem“); eine andere Perspektive betont insoweit Lembke, Kollektive Rechtsmobilisierung gegen Digitale Gewalt, S. 13, die am NetzDG kritisiert, dass nach dessen Konzeption die Intermediäre nur aufgrund von Beschwerden tätig werden müssten und die „wesentliche Mobilisierungslast“ daher im zivilgesellschaftlichen Bereich verbleibe und nicht auf die Unternehmen übergehe.

${ }^{207}$ Vgl. allgemein Kuczerawy, Intermediary Liability and Freedom of Expression in the EU, S. 40. In der Diskussion um das NetzDG waren bisweilen ähnliche Stellungnahmen anzutreffen, vgl. Guggenberger, ZRP 2017, 98 (100): „NetzDG [...] verwandelt die Anbieter sozialer Netzwerke in Hilfspolizisten." 
begegnet man immer wieder, wenn es um die Frage geht, inwieweit eine Rechtsordnung den Intermediären direkt oder vermittelt durch Haftungsrecht Handlungspflichten auferlegt.

\section{Stellungnahme $e^{208}$}

Hinter der skizzierten Kritik scheint die implizite Grundannahme auf, dass die Verantwortlichkeit für die Rechtsdurchsetzung im Normalfall nur beim Staat liege. Nur ausnahmsweise - beispielsweise im Rahmen der Notwehr bzw. Selbsthilfe - könne und dürfe der Bürger das Recht unmittelbar selbst durchsetzen.

Diese Kritik überzeugt schon aus theoretischer Perspektive nicht. ${ }^{209}$ Denn ganz grundsätzlich geht kaum eine Verfassungsrechtsordnung, jedenfalls nicht die deutsche, davon aus, dass die Kontrolle über den Normvollzug ausschließlich beim Staat liegt. Entsteht zwischen zwei Privaten ein rechtlicher Konflikt, so liegt die Entscheidung über die Anwendung juristischer Maßstäbe als Prinzip zunächst bei derjenigen Partei eines Konflikts, die sich einer Forderung der anderen ausgesetzt sieht. Sie hat gewissermaßen den „Erstzugriff“ auf die rechtliche Beurteilung des Konflikts. Dies reflektiert zuvörderst das Prozessrecht. Wer eine zivilrechtliche Klage erhebt, ohne dass die Gegenseite dafür Anlass gegeben hat, muss beim sofortigen Anerkenntnis des Beklagten trotz Obsiegens ausweislich $\S 93$ ZPO die Prozesskosten zahlen. ${ }^{210}$ Auch materiell-rechtlich ist dies reflektiert. ${ }^{211}$ Unter der Störerhaftung schuldet der Störer die Abmahnkosten der zweiten Abmahnung, ${ }^{212}$ bevor er gerichtlich zur Unterlassung verurteilt werden kann. Materiell bringt das Recht damit eine Pflicht des Schuldners gerade jenseits der gerichtlichen Auseinandersetzung zum Ausdruck. ${ }^{213}$ Das Prozessrecht erwartet also ebenso wie das materielle Recht die „private“ Konfliktlösung vor Einschaltung der Gerichte. Bereits deshalb ist der immer wieder anzutreffende Standpunkt, die Beurteilung der Rechtmäßigkeit von Inhalten sei generell mit dem Rechtsstaatsprinzip unvereinbar, ${ }^{214}$ unzutreffend. Die formalistische Per-

${ }^{208}$ Vgl. insoweit schon die Vorüberlegungen im Kontext des NetzDG, die ich unter Schiff, MMR 2018, 366 (368 f.), veröffentlicht habe und die ich im Folgenden aufgreife.

${ }^{209}$ Vgl. auch Eifert, NJW 2017, 1450 (1451); Hofmann, ZUM 2017, 102 (108).

${ }^{210}$ Vgl. Hegemann/Amelung, in: Raue/Hegemann, Münchener Anwalts-Handbuch Urheber- und Medienrecht, $\S 15$ Rn. 26.

${ }^{211}$ So ist zentrales Steuerungsmittel aller Vertragsbeziehungen nach Leistungsstörung die Fristsetzung gemäß $§ 281$ BGB; für Individualrechtsgüter lässt sich ein solcher Rechtsgedanke dem polizeirechtlichen Subsidiaritätsprinzip entnehmen.

${ }^{212}$ Vgl. Spindler, in: Spindler/Schmitz, TMG, § 10 Rn. 115.

${ }^{213}$ Nach Husovec, Injunctions against Intermediaries in the European Union, S. 117, ist gerade dieser Umstand aber eine Verletzung des Prinzips, das den Haftungsprivilegierungen zugrunde liegt.

${ }^{214}$ Vgl. etwa Pille, NJW 2018, 3545 (3549). 
spektive auf den „privaten Normvollzug“, die lediglich die gerichtliche Auseinandersetzung und Streitentscheidung unter Beteiligung ausschließlich privater Akteure sowie die Notwehr bzw. Selbsthilfe als privaten Normvollzug versteht,$^{215}$ greift dagegen in diesem Kontext zu kurz, weil sie die Realität der vorgerichtlichen Auseinandersetzung nicht mit berücksichtigt.

Um auf den Kontext dieser Untersuchung zurückzukommen, werden etwa im Fall der Kontaktierung von Diensteanbietern zur Beschwerde über bestimmte Inhalte sich nicht wenige Dienstenutzer mit der Antwort der Gegenseite zufriedengeben. Dies kann der Fall sein, weil die Diensteanbieter ihrer Beschwerde nachkommen, indem sie etwa die Unterlassungserklärung abgeben, den Inhalt beseitigen oder nachvollziehbar begründen, warum kein Rechtsverstoß vorliegt. Die innere Logik dieses Befunds liegt auf der Hand. Gerichte werden nicht überflüssigerweise in Anspruch genommen, ${ }^{216}$ der konsensuale Ausgleich unter Privaten gefördert. ${ }^{217}$ Vielfach bleibt es also mit guten Gründen bei der Entscheidung des Intermediärs. ${ }^{218}$ Wollte man dagegen die Befugnis zur Regulierung der Netzkommunikation ausschließlich bei staatlichen Institutionen verorten, wie es in den kritischen Stellungnahmen oftmals durchscheint, wäre dagegen ein rein staatlicher Kontrollapparat für Kommunikation die Folge. Die Verfassungswidrigkeit stünde einem solchen System auf die Stirn geschrieben. ${ }^{219}$ Die Rechtsordnung kennt also kein „Justizmonopol“ für die Rechtsdurchsetzung. Als Zwischenergebnis lässt sich damit festhalten, dass eine Zuweisung von rechtlich relevanten und determinierten Entscheidungen ausschließlich an staatliche Organe weder theoretisch noch praktisch überzeugt. Die oftmals sehr pauschale Kritik an Maßnahmen wie dem NetzDG oder Art. 17 DSM-RL ${ }^{220}$ hier würden richterliche Aufgaben an Private übertragen und damit ,rechtliche Entscheidungen in die Hände von privaten Unternehmen gelegt" "221 überzeugt daher nicht.

Nach hier vertretener Auffassung erweist sich damit jener Ansatz als zutreffend, der die Verantwortung für die Durchsetzung des Rechts nicht ausschließlich beim privaten Intermediär oder beim Staat bzw. einer öffentlichen Institution ansieht, sondern ein aufeinander Bezug nehmendes Verhältnis

${ }^{215}$ Vgl. etwa Dorn, Private und administrative Rechtsdurchsetzung im europäischen Beihilfenrecht, S. $55 \mathrm{ff}$.

${ }^{216}$ Dies wäre aber die Konsequenz, würden sich Vorschläge wie jener von Pille, NJW 2018, 3545 (3549), durchsetzen.

${ }^{217}$ Vgl. Schulz, in: MüKo ZPO, §93 Rn. 1.

${ }^{218}$ Dies kann allerdings auch daran liegen, dass Dienstenutzer das Kostenrisiko eines Rechtsstreits scheuen, was allerdings mit Blick auf die Dispositionsmaxime des Zivilverfahrensrechts nicht per se zu kritisieren wäre.

${ }^{219}$ Vgl. Hong, zitiert bei Kurz, Netzpolitik.org (18.02.2018), netzpolitik.org/2018/diemeinungsfreiheit-und-das-netzdg-schwerwiegender-verstoss-gegen-grundrecht.

${ }^{220}$ Vgl. dazu und wie hier auch Hofmann, GRUR 2019, 1219 (1223).

${ }^{221}$ Pravemann, GRUR 2019, 783 (787). 
zwischen beiden Ebenen annimmt. Materiell ist der Intermediär bereits aus Subsidiaritätsgesichtspunkten der richtige Akteur, um den ersten „Zugriff“ auf ein rechtlich streitiges Verhältnis vorzunehmen. Der Geltungsanspruch der Rechtsordnung verlangt daher auch, dass sich Intermediäre ihrer Rolle insoweit bewusst sind. Das bedeutet nicht, dass sie in jedem Fall zwingend die rechtlich unzweifelhaft richtige Entscheidung treffen müssten, um ihrerseits rechtliche Sanktionen zu vermeiden. Dies kann schon deshalb nicht die Erwartung sein, weil es kaum je rechtlich „einfache“ Fälle gibt bzw. die einfachsten Fälle oft gerade diejenigen sind, in denen nicht primär das Recht zu ihrer Lösung herangezogen werden muss. Das Recht kann aber auch und gerade in diesen ,schwierigen Fällen“ erwarten, dass auch die Rechtsunterworfenen versuchen, seine normativen Standards zu erfüllen. ${ }^{222}$

Abschließend lassen sich die hier getroffenen Feststellungen sogar an der Ausgestaltung der Störerhaftung validieren. Denn in der Inpflichtnahme der Diensteanbieter nur in den Fällen, in denen die Nutzer eine Beschwerde an den Intermediär richten, die so konkret ist, dass die Rechtsverletzung „unschwer bejaht werden kann", ${ }^{223}$ bringt die Störerhaftung die implizite Erwartung zum Ausdruck, dass private Akteure zunächst eigenständig versuchen sollen, einen entstandenen Konflikt unter Beachtung rechtlicher Maßstäbe zu verarbeiten. Dies gilt sowohl für die Nutzer, die diesen Erwartungshorizont erfüllen müssen, um sich erfolgreich an den Intermediär zu wenden, als auch für die Intermediäre, die die Erfüllung dieses Erwartungshorizonts für sich selbst typischerweise nachvollziehen müssen, um das Realisierungsrisiko der soeben dargelegten Haftungsvoraussetzungen abschätzen zu können.

\section{Die grundsätzlichen Vorzüge der Störerhaftung}

Ist dieser gegenüber der Störerhaftung und weiteren Verantwortungszuweisungen geltend gemachte Vorwurf auf theoretischer Ebene aus dem Weg geräumt, soll im Folgenden herausgearbeitet werden, inwiefern die Störerhaftung die Rolle und Bedeutung der Intermediäre angemessen adressiert und deren Verantwortung konturiert.

${ }^{222} \mathrm{Zu}$ den theoretischen Defiziten der kontraintuitiven, aber vielfach vertretenen Auffassung, dass gerade schwierige Fälle (,hard cases") sich der Lösung durch originär rechtliche Maßstäbe entzögen, vgl. Poscher, in: Giltrow/Stein, The Pragmatic Turn in Law, S. 307 (307 ff.).

${ }^{223} \mathrm{Vgl}$. dazu oben $\S 10$ A. III. 2. c) aa). 


\section{Flexible und kontextangemessene Ausformung der Verantwortung der Intermediäre}

Mit dem Modell der Störerhaftung ist die Rechtsprechung grundsätzlich in der Lage, der theoretisch begrüßenswerten, aber praktisch mit enormen Herausforderungen verbundenen Abstraktion horizontal anwendbarer rechtlicher Erwartungen an die Diensteanbieter in Gestalt des Haftungsrechts ${ }^{224}$ ein Stück weit zu entkommen. So liegt für Persönlichkeitsrechtsverletzungen ein spezifisches und in der Sache überwiegend überzeugendes Modell vor, das die Verantwortung der Intermediäre grundsätzlich angemessen reflektiert, während für sonstige Schutzrechtsverletzungen andere Maßstäbe und Verhaltenserwartungen an Diensteanbieter gelten ${ }^{225}$ Flexibilität ist aus übergeordneter Perspektive ein zentraler Wert und Maßstab zur Indienstnahme der Intermediäre, denn mit der starken Einzelfallbezogenheit der Anknüpfung an rechtswidriges Verhalten wäre jede im Vergleich hierzu abstraktere Modellierung einer Verantwortlichkeit nur schwerlich zu vereinbaren. Ihre Flexibilität verdankt die Störerhaftung nicht zuletzt ihrer richterlichen Herkunft. Dieser Umstand erzeugt freilich nicht nur positive Eigenschaften. Die „richterrechtliche Methode“"226 entzieht den Gegenstand etwa einem ggf. wünschenswerten Zugriff durch den EU-Gesetzgeber, sollten auf dieser Ebene Harmonisierungsbestrebungen verfolgt werden. Auch auf nationaler Ebene kann aus institutioneller Perspektive eine reiche Kasuistik den gesetzgeberischen Zugriff erschweren. ${ }^{227}$ In der Sache ändert dies aber hier nicht die positive Bewertung des Flexibilitätsmoments.

\section{Der Gedanke der Prozeduralisierung}

Die Entscheidungen Blog-Eintrag und Ärztebewertungsportal III stellen den Gedanken der Prozeduralisierung in Form der Übermittlung entsprechender Notifizierungen an den Äußernden und die Einräumung der Gelegenheit zur Stellungnahme konsequent in den Vordergrund. Dies ist grundsätzlich zu begrüßen. ${ }^{228}$ Sie finden im Dilemma zwischen dem Verbot der allgemeinen Überwachungspflicht und dem Belassen rechtlicher Konflikte im Verhältnis zwischen dem Verletzten und dem Primärverursacher einen prinzipiell sinnvollen Ansatz. ${ }^{229}$

${ }^{224} \mathrm{Vgl}$. dazu oben $\S 4$ E. II.

${ }^{225}$ Die Flexibilität lobt Ohly, ZUM 2015, 308 (312f.); Zustimmung zu BGH, Urt. v. 25.10.2011 - VI ZR 93/10, NJW 2012, 148 ff. - Blog-Eintrag, äußert auch Kersten, Schwarmdemokratie, S. 248 (die Entscheidung weise ,in die verfassungsrechtlich richtige Richtung").

${ }^{226}$ Ohly, ZUM 2015, 308 (314).

${ }^{227} \mathrm{Vgl}$. schon oben $\S 4$ E. II. 2.

${ }^{228}$ Zustimmung in Bezug auf Persönlichkeitsrechtsverletzungen auch bei Spindler, in: Spindler/Schmitz, TMG, §7 Rn. 71. 
Durch die grundsätzliche Pflicht, beide Parteien des Konflikts zu Wort kommen zu lassen, greift die Störerhaftung dialektische Elemente von Rede und Gegenrede auf. Dies ist zum einen angesichts der äußerungsrechtlichen Implikationen naheliegend, weil die Kontextualisierung angegriffener ÄuBerungen dort schon aus grundrechtlicher Perspektive in der Regel unabkömmlich ist. ${ }^{230}$ Damit ist auf den tradierten Argumentationstopos „Grundrechtsschutz durch Verfahren" verwiesen. ${ }^{231}$ Dieser prozedurale Grundrechtsschutz lässt sich auch als ,grundrechtliches Vorverfahren“ bezeichnen, das - dogmatisch abgeleitet aus der Kollision zweier Grundrechte - vorgerichtlichen Konfliktlösungs- bzw. Konfliktvermeidungsstrategien eine freiheitsermöglichende, wenngleich nicht von materiellen Wertungen vollständig befreiende Funktion zuschreibt. ${ }^{232}$ Eine ähnliche Haltung deutet neuerdings auch das BVerfG an, wenn es darauf hinweist, dass die „Eigenständigkeit der Grundrechtsabwägung" im zivilrechtlichen Rechtsstreit sich eben auch im Maßstab der Störerhaftung materialisiere, unter dem die Fachgerichte den sich gegenüberstehenden Grundrechten Rechnung trügen. ${ }^{233}$ In diesem $\mathrm{Zu}$ sammenhang spricht das Gericht dem Grundsatz des Notice-and-Takedown-Verfahrens, wonach erst aufgrund eines entsprechenden Nutzerbegehrens der Intermediär zur Entfernung eines Inhalts verpflichtet ist, einen verfassungsrechtlichen Wert zu, ${ }^{234}$ wobei unklar bleibt, ob das Erfordernis einer

${ }^{229}$ Vgl. Hofmann, ZUM 2017, 102 (104).

${ }^{230}$ Den Zusammenhang zu Art. 5 GG betont auch Holznagel, Notice and Take-DownVerfahren als Teil der Providerhaftung, S. 235. Unzutreffend ist daher die Behauptung von Wagner, GRUR 2020, 447 (452), die Störerhaftung konstruiere ausschließlich ein bilaterales Rechtsverhältnis zwischen Rechtsinhaber und Intermediär.

${ }^{231}$ Verfassungsgerichtlich im abwehrrechtlichen Kontext konnotiert seit BVerfG, Beschl. v. 20.12.1979 - 1 BvR 385/77, BVerfGE 53, 30 (62 ff.) - Mülheim-Kärlich. Zur historischen Entwicklung vgl. Schmidt-Aßmann, AöR 142 (2017), 325 (339ff.). Mit Blick auf die Drittwirkungsdimension im Kontext der Meinungsfreiheit aktualisiert durch BVerfG, Beschl. v. 11.04.2018 - 1 BvR 3080/09, NVwZ 2018, 813 (Rn. 46 ff.) - Stadionverbot.

${ }^{232}$ Vgl. Britz, Der Staat 42 (2003), $35 \mathrm{ff}$., die einerseits auf den hier besonders relevanten Konflikt von Meinungsfreiheit und Persönlichkeitsschutz verweist (40 f.), andererseits allerdings die Eignung dieses Ansatzes als dogmatische Argumentationsfigur in der Rechtsprechung bezweifelt (45ff.). Ihre Position, dass solche Verfahrenspflichten aus Gründen der Vorhersehbarkeit aber nur bei gesetzlicher Regelung zulässig seien (47ff.), überzeugt allerdings nicht restlos, denn letztlich entspricht es dem Wesen (höchst-)richterlicher Rechtsfortbildung, innerhalb der durch Gesetz gesetzten Grenzen Entscheidungen zu treffen, die notwendig für mindestens eine Partei eines Rechtsstreits nicht vollständig vorhersehbar waren.

${ }^{233}$ BVerfG, Beschl. v. 06.11.2019 - 1 BvR 276/17, NVwZ 2020, 63 (Rn. 113) - Recht auf Vergessen II.

${ }^{234}$ Vgl. BVerfG, Beschl. v. 06.11.2019 - 1 BvR 276/17, NVwZ 2020, 63 (Rn. 113) - Recht auf Vergessen II. 
Notifizierung als verfassungsrechtlichen Minimalstandard erachtet. Die insoweit verfassungsrechtlich jedenfalls anerkannten Prüfpflichten konkretisieren hier also die Pflicht zur „mehrseitigen grundrechtskonformen $\mathrm{Zu}$ gangsregulierung ${ }^{“{ }^{6235}}$ und gestalten diese aus. Zum anderen und hiermit zusammenhängend entwickelt dieser Ansatz auch das Potenzial, zur inhaltlichen Legitimität gefundener Entscheidungen gerade gegenüber den Beteiligten beizutragen. Schließlich ist das Stellungnahmeverfahren von der Absicht getragen, Beurteilungsrisiken der Diensteanbieter zu reduzieren, indem diese nicht unmittelbar nach einer Notifizierung zu einer Entscheidung gezwungen werden. ${ }^{236}$

Der Gedanke der Prozeduralisierung findet auch im Unionsrecht Anklang. Dies gilt insbesondere für Erwägungsgrund 46 S. 3 ECRL, wo von „spezifischen Anforderungen“ vor der Entfernung eines Inhalts die Rede ist, die die Mitgliedstaaten vorsehen können. Dies betrifft insbesondere verfahrensrechtliche Anforderungen vor Entfernungen eines Inhalts. ${ }^{237}$ In der Entscheidung UPC Telekabel, die eine Sperrverfügung gegenüber einem AccessProvider aufgrund des von ihm vermittelten Zugangs zu urheberrechtsverletzenden Inhalten zum Gegenstand hatte, forderte der EuGH außerdem, dass die „nationalen Verfahrensvorschriften die Möglichkeit für die Internetnutzer vorsehen, ihre Rechte vor Gericht geltend zu machen, sobald die vom Anbieter von Internetzugangsdiensten getroffenen Durchführungsmaßnahmen bekannt sind." ${ }^{\text {"238 }}$ Damit ist primär nur der gerichtliche Rechtsschutz angesprochen. Gleichwohl ist hier eine Anerkennung prozeduraler Pflichten als Folge der möglichen Betroffenheit der Dienstenutzer festzustellen. Letztlich ist dem Unionsrecht also bereits jetzt eine prozedurale Sichtweise auf Verhaltensobliegenheiten der Diensteanbieter eingeschrieben, wenngleich dies aktuell nur punktuell zur Geltung kommt. Freilich wäre es ratsam und vor dem Hintergrund der Rechtsklarheit vorzugswürdig, eine ansatzweise Ausgestaltung dieses Gedankens auch im rechtsverbindlichen Teil eines Sekundärrechtsaktes vorzunehmen.

Eine Begrüßung prozeduraler Komponenten unter gleichzeitiger Betonung deren Grenzen findet sich auch in der Rechtsprechung des EGMR. Der Fall Delfi betraf die Verurteilung des gleichnamigen estnischen Nachrichtenportals auf Zahlung von Schadensersatz. Auf diesem Portal erscheinen Nachrichtenartikel, die in einer Art Kommentarspalte von Leserinnen kommentiert werden können. Es existiert ein Verfahren zur Löschung von Kommentaren, wenn diese dem Nachrichtenportal mitgeteilt werden. Ein Unternehmer, der sich von Kommentaren unter einem sich mit seinem Geschäfts-

\footnotetext{
${ }^{235}$ Wielsch, in: Eifert/Gostomzyk, Netzwerkrecht, S. 61 (89).

${ }^{236}$ So auch Hofmann, ZUM 2017, 102 (105).

${ }^{237}$ Vgl. Spindler, ZUM 2017, 473 (479).

${ }^{238}$ EuGH, Urt. v. 27.03.2014 - C-314/12, GRUR 2014, 468 (Rn. 57) - UPC Telekabel.
} 
gebaren befassenden Artikel beleidigt und bedroht fühlte, nahm das Portal in Anspruch, die beleidigenden Kommentare zu entfernen und einen Nichtvermögensschaden zu ersetzen. Der letztere Anspruch blieb streitig und wurde von den estnischen Gerichten bejaht, weshalb Delfi in seiner Beschwerde beim EGMR die Verletzung seines Rechts auf freie Meinungsäußerung gemäß Art. 10 EMRK rügte. ${ }^{239}$ Der EGMR bejahte einen gesetzlich vorgesehenen Eingriff im Sinne von Art. 10 Abs. 2 EMRK $^{240}$ und die VerhältnismäBigkeit der Verurteilung von Delfi. ${ }^{241}$ Zwar habe Delfi ein Filtersystem zum automatischen Auffinden bestimmter Inhalte verwendet sowie die Möglichkeit zur Notifizierung entsprechender Inhalte vorgesehen und schließlich mitunter auch eigenständig Kommentare entfernt. ${ }^{242}$ Weil es sich bei den streitgegenständlichen Äußerungen aber um ,unerträgliche Hassreden und Aufforderungen zu Gewalt" handelte, ${ }^{243}$ sei die Verurteilung des Nachrichtenportals, das nicht alles in seiner Macht Stehende unternommen hatte, um all diese Inhalte unverzüglich zu entfernen, im Ergebnis verhältnismäßig. ${ }^{244}$ Wenn ein System zur Löschung rechtswidriger Kommentare installiert sei, treffe das Nachrichtenportal auch die Pflicht, ,,eindeutig rechtswidrige Kommentare unverzüglich zu entfernen, auch ohne Mitteilung des Opfers oder Dritter.“245 Dies folge auch aus seiner „Pflicht, Schaden von Dritten abzuwenden" ${ }^{246}$ Dennoch betont der Gerichtshof, ein vom Diensteanbieter etabliertes „System des Löschens auf Mitteilung“, womit letztlich Notice-andTakedown-Verfahren gemeint sind, könne ,in vielen Fällen als angemessenes Werkzeug zum Ausgleich der Rechte und Interessen aller Beteiligten funktionieren“, wenn es „,von einem wirksamen Verfahren begleitet wird, das eine schnelle Antwort ermöglicht" “. ${ }^{247}$ Den letzteren Aspekt bestätigte der EGMR in einem weiteren, ähnlich gelagerten Fall. ${ }^{248}$ Damit unterstreicht der EGMR in deutlicher Weise den Wert von Systemen, die maßgeblich auf Notifizie-

${ }^{239}$ Vgl. EGMR, Urt. v. 16.06.2015 - 64569/09, NJW 2015, 2863 (2863) - Delfi.

${ }^{240}$ Das Unternehmen hätte sich ,als professioneller Herausgeber" über die anwendbaren Gesetze informieren müssen, vgl. EGMR, Urt. v. 16.06.2015 - 64569/09, NJW 2015, 2863 (Rn. 129) - Delfi; sehr kritisch dazu allerdings Schapiro, ZUM 2014, 201 (203 f.).

${ }^{241}$ Insoweit kommt es dem Gerichtshof insbesondere auf die bewusste, wirtschaftlich motivierte Entscheidung des Nachrichtenportals an, Kommentare zuzulassen bzw. sogar hierzu aufzufordern, vgl. EGMR, Urt. v. 16.06.2015 - 64569/09, NJW 2015, 2863 (Rn. 144) - Delfi; dagegen wiederum Schapiro, ZUM 2014, 201 (204 f.).

${ }^{242}$ Vgl. EGMR, Urt. v. 16.06.2015 - 64569/09, NJW 2015, 2863 (Rn. 155) - Delfi.

${ }^{243}$ EGMR, Urt. v. 16.06.2015 - 64569/09, NJW 2015, 2863 (Rn. 156) - Delfi.

${ }^{244}$ Vgl. EGMR, Urt. v. 16.06.2015 - 64569/09, NJW 2015, 2863 (Rn. 156 ff.) - Delfi; kritisch dagegen Schapiro, ZUM 2014, 201 (204).

${ }^{245}$ EGMR, Urt. v. 16.06.2015 - 64569/09, NJW 2015, 2863 (Rn. 159) - Delfi.

${ }^{246}$ EGMR, Urt. v. 16.06.2015 - 64569/09, NJW 2015, 2863 (Rn. 156) - Delfi.

${ }^{247}$ EGMR, Urt. v. 16.06.2015 - 64569/09, NJW 2015, 2863 (Rn. 159) - Delfi.

${ }^{248}$ Vgl. EGMR, Urt. v. 02.02.2016 - 22947/13, NJW 2017, 2091 (Rn. 91) - Magyar T.E. 
rungen aufbauen. ${ }^{249}$ Der Prozeduralisierungsgedanke tritt hier deutlich zutage. Zugleich zeigt der Gerichtshof allerdings auch die Grenzen solcher „Systeme“ auf, nämlich wenn es um „Hassreden“ oder direkte körperliche Bedrohung von Personen geht. Dann sollen die Konventionsstaaten eine Haftung der Diensteanbieter ohne Weiteres, insbesondere „auch ohne Mitteilung des Opfers oder Dritter" vorsehen können. ${ }^{250}$

Mit der Betonung der prozeduralen Komponente verbindet sich auch die allgemeine Einsicht, sozio-technische Komplexität nicht allein mithilfe materiell-rechtlicher Regeln erfassen zu können. ${ }^{251}$ Das Paradigma des „Grundrechtsschutzes durch Verfahren“ stellt hier also gleichsam eine „Meta-Perspektive“252 dar. Das Recht setzt materielle Standards und ergänzt diese durch eine zurückgenommene Kontrollfunktion hinsichtlich der von Privaten etablierten Verfahren zur Durchsetzung dieser Standards. Freilich ist dieser Ansatz voraussetzungsvoll. Denn er sieht die Obliegenheit beim Betroffenen einer Persönlichkeitsrechtsverletzung, sich auf einen nicht unkomplizierten Konflikt einzulassen. Ferner setzt er grundsätzlich einen „,zuverlässigen Kommunikationskanal" zum Primärverursacher voraus, ${ }^{253}$ der aus verschiedenen Gründen nicht immer gegeben sein muss. ${ }^{254}$ Insofern sind die Vermutungsregeln, die der BGH aufstellt, ohne sie als solche zu bezeichnen, grundsätzlich geeignet, einen pragmatischen Umgang mit entsprechenden Notifizierungen zu ermöglichen. Neben diesen praktischen Fragen ist auch die Herleitung des nunmehr als etabliert zu bezeichnenden Prüfprogramms nach wie vor offen. ${ }^{255}$ Die Entscheidungen des BGH schweigen hierzu bislang. Hier dürften pragmatische Gründe eher eine Rolle spielen als eine dogmatisch saubere Ableitung. Ist der Diensteanbieter oftmals der einzige Akteur, der in der Lage ist, eine Klärung voranzubringen, ${ }^{256}$ ist seine Heranziehung im Rahmen der ohnehin weit formulierten Grundsätze der Störerhaftung und in den Grenzen der Verhältnismäßigkeit nicht nur rechtlich zulässig, sondern auch sinnvoll. Dies gilt nicht zuletzt im Sinne des bereits dargestellten Zugriffs von Kraakman, der die ,reasonable costs“ der Verhinderung von „serious misconduct“ durch den Gatekeeper als relevantes Kriterium bezeichnet hatte. ${ }^{257}$

${ }^{249}$ Vgl. auch Spano, Human Rights Law Review 2017, 665 (678).

${ }^{250}$ Eine ähnliche Position bezieht auch Eifert, in: Eifert/Gostomzyk, Netzwerkrecht, S. 9 (37), mit Blick auf (,hinreichend eng“ verstandene) evident rechtswidrige Inhalte.

${ }^{251}$ Vgl. Wielsch, in: Eifert/Gostomzyk, Netzwerkrecht, S. 61 (93 f.).

${ }^{252}$ Wielsch, in: Eifert/Gostomzyk, Netzwerkrecht, S. 61 (93).

${ }^{253}$ Holznagel, Notice and Take-Down-Verfahren als Teil der Providerhaftung, S. 142.

${ }^{254} \mathrm{Vgl}$. oben $\S 10$ Fn. 37, 38 und den jeweils zugehörigen Text.

${ }^{255}$ Vgl. Eifert, in: Eifert/Gostomzyk, Netzwerkrecht, S. 9 (37).

${ }^{256}$ So auch Kriegesmann, CR 2016, 394 (396).

${ }^{257} \mathrm{Vgl}$. dazu oben $\$ 9$ A. 


\section{Grenzen des Prozeduralisierungsgedankens und ihre mögliche Bewältigung}

An ihre Grenzen gelangen die skizzierten, die Stellungnahmerechte der Beteiligten flankierenden Vermutungsregeln wie bereits angemerkt jedoch, wenn der Diensteanbieter mit zwei konträren, jeweils der Substanziierungslast hinreichend Rechnung tragenden Stellungnahmen konfrontiert ist. Hier ist es zum einen denkbar, dem Diensteanbieter entweder eine „volle“ inhaltliche Prüfung abzuverlangen. Im Anschluss hieran wären allerdings Rechtsschutzmöglichkeiten zugunsten des von dieser Entscheidung nachteilig Betroffenen zu gewähren, die aus Gründen der Rechtsklarheit gesetzlich vorgesehen werden sollten. Zum anderen wäre es denkbar, das weitere Vorgehen von einzelnen Indizien abhängig zu machen. So wäre es möglich, die Aufgabe der Anonymität durch den Inhaltsverantwortlichen bei seiner (substanziierten) Reaktion als gewichtige Erschütterung der Beanstandung des Notifizierenden anzusehen, die dann entsprechend bei der Entscheidung des Diensteanbieters zu berücksichtigen wäre. ${ }^{258}$

Gerade mit Blick auf den „schwierigen“ Fall plädieren Hofmann ${ }^{259}$ und Holznage ${ }^{260}$ insoweit für die Einführung des bereits oben im Zusammenhang mit §512 DMCA erläuterten „counter-notice and put back“-Verfahrens. ${ }^{261}$ Danach sei es nach Entfernung eines Inhalts dem Urheber möglich, eine "Gegennotifizierung“ beim Diensteanbieter zu hinterlegen, die diesen verpflichte, den Inhalt wiederherzustellen und ihn zugleich aus der Haftung entlassen, sodass der Konflikt nunmehr zwischen den beiden Nutzern auszufechten sei. Es wurde aber bereits darauf hingewiesen, dass auch dieses Verfahren nicht frei von Mängeln wäre. Insbesondere ist bei der Einführung eines solchen Verfahrens nicht auszuschließen, dass der Anreiz der Diensteanbieter, sich dem Konflikt gänzlich entziehen zu können, strukturelle Auswirkungen auf die vertragliche Gestaltung und technische Ausführung solcher „counter-notices“ haben könnte. Dies könnte sich etwa darin äußern, dass die Einreichung einer ,counter-notice“ betont einfach gestaltet würde, um das „Patt" von Notifizierung und Stellungnahme zu erreichen und damit haftungsrechtlich auf der sicheren Seite zu sein. Der Diensteanbieter könnte es sich auch bei der Beurteilung ${ }^{262}$ der "counter-notice" hinsichtlich ihrer Substanziierung sehr einfach machen. Es ist ferner nicht unwahrscheinlich, dass Diensteanbieter bei expliziter Regelung eines solchen Verfahrens geneigt wären, Inhalte vorschnell aus Kulanz zu entfernen, um den Weg zur dann

${ }^{258}$ So etwa Holznagel, GRUR Int 2014, 105 (108).

${ }^{259}$ Hofmann, ZUM 2017, 102 (107 f.).

${ }^{260}$ Vgl. Holznagel, GRUR Int 2014, 105 (112).

${ }^{261}$ Vgl. oben $\$ 3$ B. II. 4.

${ }^{262}$ Sofern eine solche vorgesehen wäre; das Gegenteil wäre allerdings auch nicht unproblematisch. 
gesetzlich normierten ,counter-notice“ zu versperren. ${ }^{263} \mathrm{Nicht}$ zuletzt stellten sich bei der Übermittlung von Notifizierung und Gegennotifizierung auch datenschutzrechtliche Bedenken ein. ${ }^{264}$ Für den Inhaltsverantwortlichen wäre die Aufgabe seiner Anonymität dann eben der Preis, den er ggf. zu zahlen bereit wäre. ${ }^{265}$ Für die Notifizierende wäre die Einleitung eines gerichtlichen Verfahrens ebenfalls mit der Aufgabe ihrer Anonymität verbunden, wozu sie zwar ebenso ggf. bereit sein könnte. Dies würde ihr allerdings nicht darüber hinweghelfen, dass sie auf das ggf. effektivere Mittel der auf kerngleiche Verstöße erweiterten Unterlassungspflicht des Diensteanbieters nicht mehr zurückgreifen könnte.

Alles in allem ist das hier diskutierte ,counter-notice and put back“-Verfahren nicht frei von kritischen Aspekten, kann aber zumindest dabei helfen, das Dilemma des „schwierigen Falles“, bei dem der Prozeduralisierungsgedanke an seine Grenzen stößt, aufzulösen. Insoweit sollte es in Zukunft durchaus rechtspolitisch in Erwägung gezogen werden, dieses Verfahren als Modifikation der Verhaltensobliegenheiten der Diensteanbieter vorzusehen. ${ }^{266}$ Dabei sollte allerdings eine ausgewogene Anreizstruktur höchste Priorität unter möglichst umfassender Berücksichtigung der hier geäußerten Bedenken genießen, um weder ein ,underenforcement" noch eine Erfassung von Falschpositiven, also ein Overblocking, zu verursachen sowie die praktische Zugänglichkeit einer „counter-notice“ für Rechteinhaber zu gewährleisten. In der Praxis dürfte die hier diskutierte Konstellation jedenfalls nicht die Mehrheit aller Fälle ausmachen. Die meisten Fälle dürften demgegenüber mit den weiter oben skizzierten Grundsätzen ${ }^{267}$ lösbar sein.

\section{Offene Flanken der Störerhaftung}

Trotz der positiven Eigenschaften der Störerhaftung in ihrer skizzierten Ausprägung sind auch einige negative Aspekte sichtbar, die sich insbesondere auf die richtige Anreizstruktur der Störerhaftung beziehen und hierbei vor allen Dingen die Betroffenen einer möglichen Rechtsverletzung in Nöte bringen können.

${ }^{263} \mathrm{Vgl}$. Maier, Remixe auf Hosting-Plattformen, S. $187 \mathrm{f}$.

${ }^{264}$ Vgl. Specht, ZUM 2017, 114 (121 f.).

${ }^{265}$ Holznagel, GRUR Int 2014, 105 (112).

${ }^{266}$ Für eine richterrechtliche Einführung eines solchen Verfahrens bieten die derzeit in der EU geltenden Vorschriften jedenfalls nicht allzu viel Spielraum.

${ }^{267} \mathrm{Vgl}$. oben $\S 10$ A. III. 2. c). 


\section{Die Unangemessenheit der Anforderungen an eine die Prüfpflicht auslösenden Notifizierung}

Ein erster solcher Aspekt betrifft die Anforderungen, die die Rechtsprechung bei der Ausgestaltung der Störerhaftung an den Inhalt der Notifizierung stellt und die es zu beachten gilt, um die Erfolgsaussichten der Inpflichtnahme des Diensteanbieters nicht zu gefährden. Mag man die Notifizierung zwar grundsätzlich als ein Instrument ansehen, um Sorgfaltspflichten der Intermediäre im Einzelfall sinnvoll zu konkretisieren, ${ }^{268}$ stellen die von der Rechtsprechung aufgestellten Voraussetzungen an eine taugliche Notifizierung im Einzelnen kritikwürdige Anreize dar.

\section{a) Unangemessene Begründungslasten}

So wird die Ausgestaltung der Begründungslasten im Rahmen der Prüfpflichten der Störerhaftung sowohl der Rolle der Nutzer von intermediären Diensten, als auch der Rolle der Diensteanbieter selbst kaum gerecht.

Zur erfolgreichen Inanspruchnahme als Störer muss der Diensteanbieter - ebenso wie unter Art. 14 ECRL und § 10 TMG - über die Rechtsverletzung in Kenntnis gesetzt werden. ${ }^{269}$ Sofern dies mit einem Hinweis durch einen Nutzer erfolgt, muss dieser Hinweis - im Urheberrecht - dem Intermediär „über die urheberrechtliche Berechtigung [...] hinreichende Klarheit verschaffen. ${ }^{\text {"270 }}$ Es erscheint jedoch unangemessen, die materielle Begründungslast zumindest in dem Ausmaß, wie es aktuell der Fall ist, demjenigen aufzuerlegen, der institutionell in der Regel am schlechtesten in der Lage sein dürfte, einen solchen Begründungsaufwand zu leisten. Die Fälle von Urheberrechtsverletzungen sind hier immerhin noch weniger kritisch, soweit es um Beschwerden von Rechteinhabern geht, die in aller Regel besser organisiert und strukturell stärker ausgestattet sind als sonstige natürliche Personen ohne Erfahrungen und Kenntnisse im entsprechenden Bereichen. Geht es um Persönlichkeitsrechtsverletzungen, ist die rechtliche Bewertung ungleich schwieriger ${ }^{271}$ Dies reflektiert die Rechtsprechung zwar insoweit, als sie eine bestimmte Vorgehensweise des Intermediärs verlangt, die ihn in eine Art Schiedsrichterrolle im eben skizzierten Sinn bewegt. ${ }^{272}$ Auch hier muss die

${ }^{268}$ Vgl. Wagner, GRUR 2020, 447 (448).

${ }^{269}$ Vgl. BGH, Urt. v. 27.02.2018 - VI ZR 489/16, NJW 2018, 2324 (Rn. 32) - Internetforum; Urt. v. 01.03.2016 - VI ZR 34/15, NJW 2016, 2106 (Rn. 23) - Ärztebewertungsportal III; Urt. v. 25.10.2011 - VI ZR 93/10, NJW 2012, 148 (Rn. 24) - Blog-Eintrag.

${ }^{270}$ Vgl. BGH, Urt. v. 29.04.2010 - I ZR 69/08, NJW 2010, 2731 (Rn. 39) - Vorschaubilder I.

${ }^{271}$ Nach der abzulehnenden Auffassung von Nolte/Wimmers, GRUR 2014, 16 (24), soll hier eine „unschwere“ Erkennbarkeit gänzlich ausgeschlossen sein.

${ }^{272}$ Vgl. BGH, Urt. v. 27.02.2018 - VI ZR 489/16, NJW 2018, 2324 (Rn. 32) - Internet- 
Beschwerde des Betroffenen den Intermediär jedoch in die Lage versetzen, „auf der Grundlage der Behauptungen des Betroffenen unschwer - das heißt ohne eingehende rechtliche und tatsächliche Überprüfung [...]" den Rechtsversto $\beta$ zu bejahen.$^{273}$ Erstens handelt es sich hier um eine Tautologie, denn die Feststellung eines Rechtsverstoßes „ohne rechtliche Prüfung“ dürfte selten praktisch durchführbar sein. Man könnte zwar dem Wort „eingehend“ eine entscheidende Bedeutung zumessen, weil also gerade die eingehende Prüfung, nicht aber eine „einfache“ oder oberflächliche Prüfung dem Diensteanbieter nicht zumutbar sein soll. Es wäre dann aber schon völlig unklar, wo genau die Grenze zu ziehen wäre. Das bislang in der Rechtsprechung thematisierte Fallmaterial hilft insoweit bei der Abstraktion dieser Maßstäbe nicht entscheidend weiter. Zweitens erscheint es widersinnig, einerseits die Substanziierungslast so „hoch“ zu ziehen, um dann aber den Diensteanbieter zur „Ermittlung und Bewertung“ zu verpflichten. Denn was soll mit Bewertung anderes gemeint sein als die Nachvollziehbarkeit der Beanstandung auch im rechtlichen Sinne? Soll diese Bewertungspflicht durch den Diensteanbieter einen eigenen Wert in sich tragen, muss diese zumindest gegenüber der ersten Notifizierung einen vergleichbaren Umfang haben.

Unter der aktuell geltenden Rechtslage steigen außerdem die Begründungslasten in dem Maße, in dem der Betroffene nicht nur die ursprüngliche Rechtsverletzung angreifen, sondern den Intermediär auch zur Verhinderung der wiederholten Begehung verpflichten will. Denn nach jüngst geäußerter Auffassung des EuGH muss die Verfügung alle ,spezifische[n] Einzelheiten umfassen“ und von der die Verfügung erlassenden Instanz „gebührend identifiziert" werden, damit der Diensteanbieter seiner Verhinderungspflicht nachkommen kann. ${ }^{274}$ Für den Erlass der Verfügung in diesem Sinne ist zwar in erster Linie das mitgliedstaatliche Gericht zuständig. Mittelbar fallen diese Spezifizierungspflichten allerdings primär dem Betroffenen zur Last, denn in der Regel kann das Gericht nur auf Grundlage der Angaben der Betroffenen eine entsprechende Verfügung erlassen. In Zukunft werden die Notifizierenden also bereits entsprechende Überlegungen anstellen und Kriterien für die Identifizierung „entsprechender“ Inhalte benennen müssen, bevor sie den

forum, zu Suchmaschinen; Urt. v. 01.03.2016 - VI ZR 34/15, NJW 2016, 2106 (Rn. 24) Ärztebewertungsportal III; Urt. v. 25.10.2011 - VI ZR 93/10, NJW 2012, 148 (Rn. 25 ff.) Blog-Eintrag.

${ }^{273}$ BGH, Urt. v. 25.10.2011 - VI ZR 93/10, NJW 2012, 148 (Rn. 26) - Blog-Eintrag. Generell hierzu auch Pille, NJW 2018, 3545 (3548), der allerdings unzutreffend kritisiert, der Diensteanbieter werde ,in eine rechtliche Auseinandersetzung zwischen dem Täter und dem Betroffenen hineingezogen“ (3548). Generell begrüßt von Spindler, MMR 2018, 48 (52).

${ }^{274}$ EuGH, Urt. v. 03.10.2019 - C-18/18, NJW 2019, 3287 (Rn. 45) - Glawischnig-Piesczek. 
Diensteanbieter in Anspruch nehmen. ${ }^{275}$ Haftungsrechtlich spielt dies den Diensteanbietern gewiss in die Hände, denn so sinkt die Wahrscheinlichkeit in erheblichem Ausmaß, dass sie gerichtlich zu entsprechenden Maßnahmen verpflichtet werden können.

\section{b) Anreiz zum Blindflug}

Die Auferlegung übermäßig hoher Begründungslasten ermöglicht es außerdem dem Intermediär, sich in einer Art „,rechtlichem Vakuum“ zu schützen. Denn einerseits darf ihm die Rechtsordnung keine Eigeninitiative bei der Prüfung von Inhalten abverlangen, wie aus Art. 15 ECRL, § 7 Abs. 2 TMG hervorgeht, ${ }^{276}$ andererseits besteht eine Reaktionspflicht erst bei der hinreichenden Substanziierung des Rechtsverstoßes. Das „Vakuum“ ermöglicht es dem Diensteanbieter, bereits das Vorliegen einer hinreichenden Beschwerde zu bestreiten und sich so nicht in der Pflicht zu sehen, im Sinne der Rechtsprechung aktiv zu werden und den ihm abverlangten eigenen Ermittlungsaufwand zu leisten. Geradezu paradigmatisch erscheint insoweit wiederum die Entscheidung Glawischnig-Piesczek, in der der EuGH zwar Verhinderungspflichten für sinngleiche (persönlichkeits-)rechtswidrige Inhalte annimmt, diese aber den Diensteanbieter nicht dazu zwingen dürfen, ,eine autonome Beurteilung dieses Inhalts vorzunehmen. "“277 Stattdessen dürfe nur eine ,automatisierte Technik“ für die Ausführung einer Verhinderungspflicht eingesetzt werden, während manuelle Nachkontrollen ${ }^{278}$ explizit ausgeschlossen sein sollen. Hier verstärkt sich der Eindruck, dass die Diensteanbieter jedenfalls in Form ihres handlungsfähigen Personals zunehmend in den Hintergrund und in eine „passive“ Stellung gerückt werden sollen, die sich um die Konflikte der hier relevanten Natur nicht zu kümmern brauchen. Damit scheint der Gerichtshof zudem in problematischer Weise erste Schritte in Richtung einer Behandlung ,automatisierter Techniken“ als autonome Akteure zu gehen, worauf hier aber nicht näher eingegangen werden kann.

Insgesamt ist jedenfalls, wie auch schon mit Blick auf das von Kraakman formulierte Ideal eines Gatekeeping-Regime erörtert, ${ }^{279}$ eine problematische

${ }^{275} \mathrm{Ob}$ das darauf hinausläuft, dass „Ähnlichkeits-Indikatoren“ dargelegt werden müssen, wie es Holznagel, ZUM 2019, 910 (912), befürchtet, ist zwar noch nicht abzusehen. In jedem Falle ist damit aber eine erhebliche Steigerung der Substanziierungslasten verbunden.

${ }^{276}$ Kritisch insoweit auch Valcke/Kuczerawy/Ombelet, in: Taddeo/Floridi, The Responsibilities of Online Service Providers, S. 101 (114).

${ }^{277}$ EuGH, Urt. v. 03.10.2019 - C-18/18, NJW 2019, 3287 (Rn. 45) - Glawischnig-Piesczek.

${ }^{278}$ Die Zulässigkeit bzw. Anordnung solcher hatte sich auch Holznagel, ZUM 2018, 350 (353), von der Entscheidung des EuGH erhofft.

${ }^{279} \mathrm{Vgl}$. oben $\S 9 \mathrm{~B}$. 
Anreizstruktur festzustellen, die es für die Intermediäre äußerst attraktiv macht, sich gegenüber einer Kenntnisnahme abzuschirmen und sich damit gleichsam „blind“ zu stellen. Diese Einsicht wird verstärkt durch die vereinzelt vom BGH geäußerte Auffassung, ,die Hinzuziehung eines mit der Materie vertrauten Juristen" sei Diensteanbietern nicht zuzumuten. ${ }^{280}$ Auch in der Literatur scheint - jenseits des bereits thematisierten Arguments der „Privatisierung der Rechtsdurchsetzung", wenngleich durchaus inhaltlich an dieses anknüpfend - bisweilen die Position auf, private Unternehmen verfügten nicht per se über „spezifische Rechtskenntnisse“. ${ }^{281}$ Diese Perspektive entbehrt nicht eines gewissen Zynismus und übergeht die Realität der Rechtspraxis, bei der keinerlei unternehmerische Tätigkeit ohne Heranziehung juristischer Expertise möglich ist. Dies gilt gerade mit Blick auf Haftungsfragen.

\section{c) Implikationen rechtlicher Bewertungen}

Auch jenseits dieser übergeordneten Bedenken fällt eine Annäherung an die „unschwere Erkennbarkeit“ eines Rechtsverstoßes im Sinne des BGH schwer. ${ }^{282}$ Die aufgeführten Beispiele - „Kinderpornografie, Aufruf zur Gewalt gegen Personen, offensichtlichen Personenverwechslungen, Vorliegen eines rechtskräftigen Titels gegen den unmittelbaren Störer, Erledigung jeglichen Informationsinteresses durch Zeitablauf ${ }^{\text {"2 } 283}$ - sind an sich schon nicht, jedenfalls nicht durchweg eindeutig zuordenbar. ${ }^{284}$ Anders als nach ähnlichen kritischen Stellungnahmen geht es nach hier vertretener Auffassung aber nicht so sehr um die Herausforderung, die eine Operationalisierung von Prüfpflichten mit unbestimmten Rechtsbegriffen wie „unschwer“ oder „offensichtlich" darstellt - dies ist unbestritten -, sondern darum, dass die Rechtsprechung einerseits mit der Formulierung recht hoher Hürden zur Initiierung des Prüfprogramms den Intermediären entgegenzukommen scheint, andererseits aber die Frage nach der Offensichtlichkeit eines behaup-

${ }^{280}$ BGH, Urt. v. 22.07.2010 - I ZR 139/08, GRUR 2011, 152 (Rn. 48) - Kinderhochstühle im Internet I (hier hält das Gericht die „Hinzuziehung eines mit der Materie vertrauten Juristen" durch eBay für unzumutbar); die in den vorangehenden Fußnoten referenzierten Entscheidungen zum Prüfprogramm von Kommunikationsdiensten verweisen zwar nicht auf dieses Urteil, der sachliche Zusammenhang lässt sich allerdings nicht bezweifeln, wie auch bei Spindler, ZUM 2017, 473 (481), deutlich wird.

${ }^{281}$ Fischer, Die Einbindung von Providern in die Durchsetzung von Urheberrechten, S. 360 .

${ }^{282}$ Kritisch auch Pille, NJW 2018, 3545 (3547).

${ }^{283}$ BGH, Urt. v. 27.02.2018 - VI ZR 489/16, NJW 2018, 2324 (Rn. 36) - Internetforum.

${ }^{284}$ Vgl. auch Gounalakis/Muer, NJW 2018, 2299 (2301), die allerdings das Petitum ableiten, der Konflikt sei ohne explizite legislative Ausformung stets zwischen dem Primärverursacher und dem Betroffenen zu klären. 
teten Rechtsverstoßes schon generell nur mittels einer Abwägung zu erreichen ist. ${ }^{285}$ Akzeptiert man, dass eine rechtliche Wertung durch den Diensteanbieter in diesen Fällen möglich und geboten sein kann, ist in dem Versuch der Hochzonung des Auslösers einer solchen Wertung eine Erschwernis für alle betroffenen Akteure zu sehen. Nicht zu Unrecht wird allenthalben betont, dass die Diensteanbieter entsprechende Probleme bei der Anwendung dieser Maßstäbe treffen ${ }^{286}$ Seltener wird hingegen thematisiert, dass auch den betroffenen Nutzern kaum geholfen ist, wenn deren Substanziierungslast mit den hier dargestellten Maßstäben so hoch angesetzt ist. ${ }^{287}$

\section{Ineffizienz wegen zu strenger Anforderungen an die Erstbegehungs- bzw.} Wiederholungsgefahr?

\section{a) Der Ansatzpunkt der Kritik}

Ein weiterer zentraler Vorwurf gegenüber der konkreten Ausgestaltung der Störerhaftung durch den BGH betrifft die Differenzierung zwischen der Kenntnisnahme eines rechtsverletzenden Inhalts und der hieraus folgenden Verletzung von Prüfpflichten. ${ }^{288}$ Durch das Anlegen sehr strenger Voraussetzungen an die Erstbegehungsgefahr, die klare Differenzierung zwischen der Kenntnisnahme und der Verletzung von Prüfpflichten und der Annahme einer Wiederholungsgefahr erst im letzteren Falle wird im Schrifttum namentlich der Vorwurf erhoben, der BGH habe die Störerhaftung zu einer Verschuldenshaftung ausgebaut. Ohne die Möglichkeit der Unterlassungshaftung infolge einer „haftungsbegründenden Erstabmahnung“ werde die Effizienz des Instruments, das den von Rechtsverletzungen Betroffenen an die Hand gegebenen ist, in erheblicher Weise unterminiert. ${ }^{289}$

In der Tat stellt die so dargestellte Rechtslage für Betroffene eine Beeinträchtigung der Effektivität der Störerhaftung dar, weil die nach den bisherigen Ausführungen stets notwendige Notifizierung des Diensteanbieters soweit keine vom Gläubiger der Störerhaftung zu beweisende ${ }^{290}$ anderweitige

${ }^{285}$ Genau das scheint aber BGH, Urt. v. 01.03.2016 - VI ZR 34/15, NJW 2016, 2106 (Rn. 24) - Ärztebewertungsportal III, zumindest rhetorisch in Abrede zu stellen, wenn als exklusive Alternativen die Abwägung der betroffenen Rechtspositionen oder die „unschwere Erkennbarkeit" genannt werden.

${ }^{286} \mathrm{Vgl}$. etwa Schapiro, ZUM 2014, 201 (209).

${ }^{287}$ Vgl. in Ansätzen etwa Holznagel, GRUR Int 2014, 105 (108).

${ }^{288}$ Vgl. oben $\$ 10$ A. IV. 3. In dem Vorlagebeschluss BGH, Beschl. v. 13.09.2018 - I ZR 140/15, GRUR 2018, 1132 (Rn. 50 ff.) - YouTube, gibt dieser dem EuGH Gelegenheit, hierzu Stellung zu beziehen; vgl. zur diesbezüglichen Position des Generalanwalts oben $\S 10$ Fn. 135 .

${ }^{289}$ Vgl. Lehment, WRP 2012, 149 (152 ff.); jüngst auch Fischer, ZGE 2020, 272 (301), sowie Frey, Die Haftung von Host-Providern für Immaterialgüterrechtsverletzungen, S. $104 \mathrm{ff} ., 278 \mathrm{ff}$., mwN.

${ }^{290} \mathrm{Vgl}$. oben $\S 4$ D. II. 3. 
Kenntnisnahme des Diensteanbieters zu bejahen ist - in der Regel nicht genügt, um eine Unterlassungshaftung zu begründen.

\section{b) Differenzierung zwischen Urheberrecht und Persönlichkeitsrecht}

Diese Handhabung betrifft allerdings eher Urheberrechtsverletzungen als Persönlichkeitsrechtsverletzungen. Denn die nicht selten festzustellende Häufigkeit der Verletzung von urheberrechtlichen Schutzrechten stellt ein zusätzliches Problem dar, welches das Urheberrecht insoweit von persönlichkeitsrechtlichen Schutzrechten strukturell unterscheidet. Das illegale Hochladen von Musikvideos bei YouTube erweist sich etwa strukturell als wiederholungsanfälliger, weil es schlicht sehr viele Personen geben mag, die ein und dasselbe Werk dort einstellen. Demgegenüber können Persönlichkeitsrechtsverletzungen zwar - insbesondere bei in der Öffentlichkeit stehenden Personen - durchaus auch massenhaft auftreten. Strukturell geht es hier aber nicht in gleicher Weise um die Wiederholungsanfälligkeit. Die Tatsache, dass eine Person in der Öffentlichkeit steht und etwa in sozialen Medien aktiv ist, erhöht wohl nicht schon strukturell die Wahrscheinlichkeit, dass es ihr gegenüber zu Persönlichkeitsrechtsverletzungen kommt. Damit bestehen aber hinsichtlich der Effizienz als Leitgedanken der Inanspruchnahme der Diensteanbieter zwischen dem Urheberrecht bzw. verwandten Schutzrechten und dem Persönlichkeitsrecht wesentliche Unterschiede.

Außerdem ist im Urheberrecht die sekundärrechtliche Rechtslage noch etwas klarer ausgestaltet, insoweit der EuGH aus den Art. 8 Abs. 3 InfoSocRL, Art. 11 S. 3 Enforcement-RL explizit ableitet, dass ein Diensteanbieter „unabhängig von seiner etwaigen eigenen Verantwortlichkeit [...] gezwungen werden kann, [...] Maßnahmen zur Vorbeugung gegen erneute derartige Verletzungen zu treffen. ${ }^{\text {“291 }}$ Daraus wird geschlussfolgert, dass es für einen Unterlassungsanspruch gerade nicht auf die Verletzung von Prüfpflichten ankommen könne. ${ }^{292}$ Unter der ECRL sind die Gerichte der Mitgliedstaaten hingegen etwas freier, die Verantwortlichkeit der Intermediäre zu regeln, wozu eben auch prozedurale Elemente gehören. Im Urheberrecht erweist sich die Störerhaftung damit aus unionsrechtlicher Sicht als grundsätzlich problematischer als in anderen Regelungsbereichen. ${ }^{293}$

${ }^{291}$ EuGH, Urt. v. 12.11.2011 - C-324/09, MMR 2011, 596 (Rn. 127) - L'Oréal SA (Hervorh. v. Verf.).

${ }^{292}$ Vgl. Nordemann, GRUR 2011, 977 (979); anders Chmelik, Social Network Sites Soziale Netzwerke, S. 184.

${ }^{293}$ So wohl auch Ohly, ZUM 2015, 308 (313), der mit Blick auf die mitgliedstaatliche Pflicht zur Eröffnung der Möglichkeit, eine Unterlassungsanordnung ohne vorherige Pflichtverletzung erwirken zu können, eine solche lediglich auf die Sonderregeln des europäischen Urheberrechts bezieht; in diesem Sinne auch Czychowski/Nordemann, GRUR 2013, 986 (989 f.); Nordemann, GRUR 2011, 977 (979). 


\section{c) Schlussfolgerungen}

Damit ist festzuhalten, dass die fehlende Möglichkeit der „,haftungsbegründenden Erstabmahnung" im Urheberrecht deutlich problematischer ist als bei der Anwendung der Störerhaftung auf Persönlichkeitsrechtsverletzungen. Allerdings ist nach der Entscheidung Glawischnig-Piesczek nicht ausgeschlossen, dass der EuGH auch im Bereich von Persönlichkeitsrechtsverletzungen die Rechtsprechung des BGH zur Störerhaftung für unionsrechtswidrig erachten könnte, da die Realisierung der Unterlassungshaftung auch dort von einer separaten Verletzung von Prüfpflichten nach erfolgter Kenntnisnahme abhängt. Denn in diesem Fall hatte Facebook zunächst auf eine Notifizierung der Betroffenen nicht reagiert, die das Unternehmen daraufhin auf Unterlassung in Anspruch nahm..$^{294}$ Die begehrte Verurteilung von Facebook bezog sich hierbei auf die Verbreitung von „wörtlichen und/oder sinngleichen Behauptungen" “. ${ }^{295}$ Wie bereits ausgeführt, sieht der EuGH eine solche Verpflichtung unter bestimmten Voraussetzungen als zulässig an. ${ }^{296}$ Damit kann die Verpflichtung zur Unterlassung wortgleicher wie auch sinngleicher Wiederholungen gerade bereits nach der „Erstabmahnung“ ausgesprochen werden. Mithin geht der EuGH insoweit über das hinaus, was der BGH im Rahmen seiner Rechtsprechung zur Störerhaftung demjenigen zugestehen würde, der lediglich eine „Erstabmahnung" an den Diensteanbieter richtet.

$\mathrm{Zu}$ berücksichtigen ist hier allerdings, dass es bei Glawischnig-Piesczek um einen Fall evidenter Rechtswidrigkeit ging. ${ }^{297}$ Die in Rede stehenden Äußerungen waren vom vorlegenden Gericht bereits als rechtswidrig beurteilt worden, sodass es gerade nicht um den Umgang mit solchen Fällen ging, die Gegenstand des soeben skizzierten Prüfpflichtenprogramms sind. Wie in jenen Fällen vorzugehen ist, bei denen die Rechtswidrigkeit von einer äußerungsrechtlichen Kontextualisierung abhängt, die eine Stellungnahme des Äußernden erfordert, hatte der EuGH nicht zu entscheiden. In der Sache spricht viel dafür, dass es in jenen Fällen, bei denen die Rechtswidrigkeit des gerügten Inhalts gerade nicht evident ist, bei dem soeben skizzierten Prüfpflichtenprogramm bleiben sollte, da nur so die erforderliche grundrechtliche Kontextualisierung durchführbar ist. Auch insoweit wäre die Möglichkeit zur „haftungsbegründenden Erstabmahnung“ jenseits der Beseitigung

\footnotetext{
${ }^{294}$ Vgl. EuGH, Urt. v. 03.10.2019 - C-18/18, NJW 2019, 3287 (Rn. 27) - GlawischnigPiesczek.

${ }^{295}$ EuGH, Urt. v. 03.10.2019 - C-18/18, NJW 2019, 3287 (3287) - Glawischnig-Piesczek.

${ }^{296}$ Vgl. EuGH, Urt. v. 03.10.2019 - C-18/18, NJW 2019, 3287 (Rn. 37 ff.) - Glawischnig-Piesczek. Dort geht es maßgeblich um die Reichweite des Art. 15 ECRL. Damit ist die Frage nach der Pflicht in der Sache jedoch bejaht.

${ }^{297}$ Das heben auch Holznagel, ZUM 2019, 910 (911), und Paal, JZ 2020, 92 (94), hervor.
} 
der konkret bemängelten Äußerung ggf. nur auf Kosten der Kommunikationsrechte des Inhaltsverantwortlichen zu erreichen. Bis zu einer endgültigen Klärung ist mithin nicht davon auszugehen, dass der EuGH sich dem Prüfpflichtenmodell des BGH entgegenstellen wollte. ${ }^{298}$

\section{Ausschluss einer Schadensersatzhaftung; Verhältnis \\ zum Verkehrspflichtenkonzept}

Ein weiterer kritischer Aspekt der Störerhaftung liegt ferner im Verhältnis der Störerhaftung zum zivilrechtlichen Verkehrspflichtenkonzept begründet, ${ }^{299}$ das insbesondere bei der Frage nach der Schadensersatzhaftung des Störers virulent wird. Die Rechtsprechung schließt nämlich bislang Schadensersatzansprüche kategorial vom Anwendungsbereich der Störerhaftung aus. ${ }^{300}$ Zur Begründung heißt es, für eine Anerkennung eines Schadensersatzes fehle es an einer gesetzlichen Grundlage, da die Störerhaftung ihre Grundlage nicht im Deliktsrecht, sondern im Recht der Besitz- und Eigentumsstörung finde und damit lediglich Abwehransprüche vermittele. ${ }^{301}$

Der pauschale Ausschluss von Schadensersatzansprüchen aus dem Rechtsfolgenkreis der Störerhaftung leuchtet jedoch dogmatisch nicht ohne Weiteres ein. Denn die Regelung des $\S 1004$ BGB ist unzweifelhaft ein Schutzgesetz im Sinne des $\S 823$ Abs. 2 BGB. ${ }^{302}$ Nach der Analyse von Schapiro ist die Inkonsequenz der Rechtsprechung mit ihrem grundsätzlichen dogmatischen Verständnis der Störerhaftung zu erklären. Da diese als eigene Kategorie jenseits der Täter- und Teilnehmerhaftung konstruiert sei, könne die Rechtsprechung keinen Schadensersatzanspruch nach $\S 823$ Abs. 2 BGB zuerkennen, ohne dieses Konzept ad absurdum zu führen. ${ }^{303}$ Auch die umgekehrte These ist nicht unplausibel. Gerade weil eine Schadensersatzhaftung

${ }^{298}$ Wenn dies eines Tages eintreten sollte, wären dann allerdings auch die sehr strengen Anforderungen an die Konkretisierung solcher Verhinderungspflichten zu beachten, die nach der Entscheidung des EuGH primär dem Notifizierenden aufgebürdet sind, vgl. den Nachweis oben in $§ 10$ Fn. 275 und den zugehörigen Text.

${ }^{299}$ Das Konzept ist im Wesentlichen durch richterliche Rechtsfortbildung geprägt und bezieht sich auf die Feststellung des Pflichtenkreises, dessen (zumeist fahrlässige) Verletzung „Kernstück der Haftungsbegründung“ im deliktischen Schadensersatzanspruch nach $\S 823$ Abs. 1 BGB darstellt, vgl. Wagner, in: MüKo BGB, §823 Rn. 433, $441 \mathrm{ff}$.

${ }^{300}$ Vgl. BGH, Urt. v. 18.10.2001 - I ZR 22/99, GRUR 2002, 618 (619) - Meißner Dekor; Urt. v. 11.03.2004 - I ZR 304/01, NJW 2004, 3102 (3105) - Internet-Versteigerung I; Urt. v. 17.08.2011 - I ZR 57/09, MMR 2012, 178 (Rn. 47) - Stiftparfüm.

${ }^{301}$ Vgl. BGH, Urt. v. 18.10.2001 - I ZR 22/99, GRUR 2002, 618 (619) - Meißner Dekor.

${ }^{302}$ Vgl. BGH, Urt. v. 16.03.1988 - VIII ZR 184/87, NJW 1988, 1778 (1780); Wagner, in: MüKo BGB, §823 Rn. 595; Schapiro, Unterlassungsansprüche gegen die Betreiber von Internet-Auktionshäusern und Internet-Meinungsforen, S. 99 f., mwN.

${ }^{303} \mathrm{Vgl}$. Schapiro, Unterlassungsansprüche gegen die Betreiber von Internet-Auktionshäusern und Internet-Meinungsforen, S. $100 \mathrm{f}$. 
für zu weitgehend erachtet wird, halte der BGH an der Störerhaftung fest, ${ }^{304}$ anstatt auf einen einheitlichen deliktischen Tatbestand hinzuwirken. Schließlich ist auch die hemmende Funktion eines monetären Anspruchs mit Blick auf die Ermöglichung von Rechtsverletzungen ein Umstand, der gegenüber dem Ausschluss der Schadensersatzhaftung kritisch gesehen wird. ${ }^{305}$

Im Schrifttum sind deshalb nicht gerade selten Versuche zu erkennen, eine Schadensersatzhaftung zu begründen. ${ }^{306}$ Insbesondere die Integration der Störerhaftung mit dem bereits erwähnten Verkehrspflichtenkonzept spielt hier eine herausragende Rolle. ${ }^{307}$ So ist Hofmann der Auffassung, dass durch eine Koppelung von Haftung auf Schadensersatz mit dem Konzept der Verkehrspflichten für die Intermediäre keine unangemessenen Haftungsrisiken $\mathrm{zu}$ befürchten stünden. ${ }^{308}$ Zur Untermauerung dieser Sichtweise verweist er auf die Morpheus-Entscheidung des BGH, in der das Gericht die klassische Verkehrspflichtendogmatik - nämlich die Haftung von Eltern für ihre Kinder $^{309}$ - mit der Prüfpflicht im Rahmen der Störerhaftung für Urheberrechtsverstöße in Gleichlauf gebracht hatte. ${ }^{310}$ Ensthaler und Heinemann begründen einen solchen Schadensersatzanspruch - ungeachtet des §10 TMG - mit der Annahme einer zivilrechtlichen Sonderbeziehung zwischen Schuldner und Gläubiger eines aus der Störerhaftung abgeleiteten Unterlassungsanspruchs. ${ }^{311}$ Ohly plädiert hingegen ohnehin für eine Abkehr von der Störerhaftung und eine Hinwendung zur deliktischen Haftung wegen der Verletzung von Verkehrspflichten. Dies erlaubt es ihm, im Prinzip für eine Schadensersatzpflicht einzutreten, die es dann aber im Hinblick auf den Sorgfaltsmaßstab restriktiv handzuhaben, konkret auf grob fahrlässige Pflichtverletzungen zu beschränken gelte. ${ }^{312}$

Diesen Vorschlägen ist insgesamt insoweit zuzustimmen, als sie auf der begrüßenswerten Forderung beruhen, die Diensteanbieter für die Realisierung von durch sie hervorgerufenen Risiken in (Mit-)Haftung zu nehmen. ${ }^{313}$ Dies entspräche dem Prinzip des Haftungsrechts im Allgemeinen, einen An-

${ }^{304} \mathrm{Vgl}$. Hofmann, JuS 2017, 713 (719).

${ }^{305}$ Vgl. Specht/Eickhoff, CR 2016, 740 (743).

${ }^{306}$ Kritisch aber Koreng/Feldmann, in: Hoeren/Bensinger, Haftung im Internet, S. 461 (490), wonach dies auf eine Gefährdungshaftung hinauslaufe.

${ }^{307}$ Vgl. Wagner, in: MüKo BGB, §823 Rn. 867; Leistner, GRUR-Beilage 2010, 1 (18 ff.); von Ohly, ZUM 2017, 793 (801), als „,wohl herrschende Lehre“ bezeichnet.

${ }^{308} \mathrm{Vgl}$. Hofmann, JuS 2017, 713 (719).

${ }^{309}$ Vgl. $§ 832$ BGB.

${ }^{310}$ Vgl. BGH, Urt. v. 15.11.2012 - I ZR 74/12, NJW 2013, 1441 (Rn. 42, 22 ff.) - Morpheus.

${ }^{311}$ Vgl. Ensthaler/Heinemann, GRUR 2012, 433 (439); kritisch insoweit aber Holznagel, Notice and Take-Down-Verfahren als Teil der Providerhaftung, S. $130 \mathrm{ff}$.

${ }^{312}$ Vgl. Ohly, ZUM 2015, 308 (315f.); vgl. auch Krüger/Apel, MMR 2012, 144 (148ff.).

${ }^{313}$ Vgl. Ohly, ZUM 2015, 308 (315); Czychowski/Nordemann, GRUR 2013, 986 (990 f.). 
reiz zur Internalisierung externer Effekte zu setzen, ${ }^{314}$ die hier jedenfalls auch durch die Tätigkeit der Intermediäre gesetzt sein können. Gelingen könnte dies allerdings nur um den Preis einer dogmatischen Neubestimmung der Störerhaftung, zumindest falls man den Weg über eine Integration mit dem Verkehrspflichtenkonzept wählt. Denn damit müsste die in der Effizienz der Inanspruchnahme des Diensteanbieters als „cheapest cost avoider“"315 liegende Ratio dem Grundsatz der persönlichen Vorwerfbarkeit der Außerachtlassung der verkehrsüblichen Sorgfalt nach $\$ 276$ BGB weichen. ${ }^{316}$ Es wurde insoweit bereits darauf hingewiesen, dass allerdings gerade im Urheberrecht das mit dem Verschuldensmaßstab jedenfalls prinzipiell in Widerstreit stehende Ziel einer weitgehenden Effektivität bei der Inanspruchnahme nach der Erstabmahnung eine Rolle spielt, während im Persönlichkeitsrecht dieser Gedanke zwar auch von Bedeutung ist, dort allerdings der äußerungsrechtliche Kontext eine Facette neuer Qualität darstellt. ${ }^{317}$ Konzeptionell stehen damit die Anforderungen, die der BGH durch sein Prüfpflichtenprogramm den Diensteanbietern grundsätzlich zu Recht auferlegt, dem Verkehrspflichtenkonzept im Persönlichkeitsrecht etwas näher als es im Urheberrecht der Fall ist. ${ }^{318}$ Praktisch spricht diese Einsicht in der Tat dafür, prospektiv Schadensersatzansprüche in die Störerhaftung einzubeziehen.

Das Verkehrspflichtenkonzept wäre ohne Weiteres in der Lage, solche Ansprüche sinnvoll zu verarbeiten und in geordnete Bahnen zu lenken, sodass eine prohibitive Wirkung nicht zu befürchten stünde. Die Entstehung von Verkehrspflichten wäre insoweit an die Schaffung einer Gefahrenquelle zu binden, die in der Eröffnung eines Dienstes durch dessen Anbieter in diesem Kontext zu bejahen wäre. ${ }^{319}$ Für die konkrete Ausgestaltung der Verkehrspflichten ließe sich unmittelbar an das soeben skizzierte Prüfpflichtenprogramm anknüpfen. ${ }^{320}$ Insbesondere bei fehlender Evidenz der Rechtsver-

${ }^{314}$ Vgl. Hoffmann-Riem, in: Röhl, Wissen, S. 160 (187): Haftungsregeln als „RisikoAllokation“; ähnlich mit Blick auf Intermediäre Wagner, GRUR 2020, 329 (329). Für eine positive Deutung der Haftung im Technologiebereich vgl. Spiecker gen. Döhmann, in: Hill/ Schliesky, Die Vermessung des virtuellen Raums, S. 137 (152 f.): weil die Haftung per se nur die Sekundärebene betreffe, während auf Primärebene eine Tätigkeit (implizit) als rechtlich erlaubt angesehen werde, setze sich auf Primärebene die „Chance der Weiterentwicklung des Wissensbestandes" durch.

${ }^{315} \mathrm{Vgl}$. oben $\S 10 \mathrm{Fn} .7$.

${ }^{316}$ In der Sache schon Lehment, WRP 2012, 149 (152 f.).

${ }^{317} \mathrm{Vgl}$. soeben $\S 10$ B. IV. 2.

${ }^{318}$ Damit ist allerdings nicht bestritten, dass auch im Urheberrecht äußerungsrechtliche Implikationen eine herausgehobene Rolle spielen können.

${ }^{319} \mathrm{Vgl}$. Frey, Die Haftung von Host-Providern für Immaterialgüterrechtsverletzungen, S. 313, mit dem Hinweis auf die Funktion der Diensteanbieter als „Multiplikatoren von Inhalten", die deren Verbreitung bewirke.

${ }^{320}$ Letztlich knüpft der BGH auch im Urheberrecht an ähnliche Maßstäbe an, insoweit 
letzung wären die entsprechenden Schritte durchzuführen. Über eine Begrenzung des Schadensersatzes auf grob fährlässig verursachte Verstöße gegen eine begründete Prüfpflichtließe sich ebenfalls nachdenken ${ }^{321}$ In jedem Falle käme eine Haftung auf Schadensersatz nur beim „zweiten“ Verstoß in Betracht, wie sich schon aus Art. 14 ECRL ergibt. ${ }^{322}$

\section{Zweifelhafte grundrechtliche Verortung}

Schließlich ist noch auf einen zentralen Schwachpunkt im Begründungsmuster der Rechtsprechung hinzuweisen. In seinen Ausführungen zur „unschweren Erkennbarkeit" einer Rechtsverletzung und der hiernach ggf. erforderlichen Vornahme des oben skizzierten Prüfprogramms sieht der BGH nämlich stets eine Abwägung zwischen dem Persönlichkeitsrecht des Notifizierenden und der „Meinungs- und Medienfreiheit“ „,jedenfalls des Providers" vor. ${ }^{323}$ Die hier zutage tretende Einschätzung zur grundrechtlichen Einordnung der Tätigkeit von Intermediären überzeugt nicht ohne Weiteres. ${ }^{324}$ Diesen räumt der BGH regelmäßig eine Teilnahme am Schutz der Meinungsfreiheit gemäß Art. 5 Abs. 1 S. 1 GG ein, da sie ,unverzichtbare Mittlerpersonen ${ }^{\text {“625 }}$ seien. ${ }^{326}$ Häufig rekurriert diese Perspektive auf den PressespiegelBeschluss des BVerfG, ${ }^{327}$ in dem dieses gegenüber der Verurteilung zur Unterlassung der Veröffentlichung eines Pressespiegels die Meinungsfreiheit selbst in Anschlag brachte und hierzu ausführte, zum von Art. 5 Abs. 1 S. 1 GG geschützten Kommunikationsprozess könne

„auch die Mitteilung einer fremden Meinung oder Tatsachenbehauptung zählen, und zwar auch dann, wenn der Mitteilende sich diese weder zu eigen macht noch sie in eine eigene Stellungnahme einbindet, sondern die fremde Äußerung lediglich verbreitet. Es ist Teil des meinungsbildenden Diskussionsprozesses, dessen Schutz Art. 5 Abs. 1 S. 1 GG im Sinn hat, sich und andere auch über Stellungnahmen Dritter zu informieren“. 328

er bei Zweifeln über das Vorliegen einer Rechtsverletzung den Diensteanbieter in der Pflicht sieht, ,,auf diese Zweifel hinzuweisen und nach den Umständen angemessene Belege für die behaupteten [...] Rechtsverletzungen [...] zu verlangen“, BGH, Urt. v. 17.08.2011 I ZR 57/09, MMR 2012, 178 (Rn. 32) - Stiftparfüm.

${ }^{321}$ So Ohly, ZUM 2017, 793 (801).

${ }^{322}$ Vgl. Krüger/Apel, MMR 2012, 144 (149 f.); Chmelik, Social Network Sites - Soziale Netzwerke, S. 264.

${ }^{323}$ BGH, Urt. v. 01.03.2016 - VI ZR 34/15, NJW 2016, 2106 (Rn. 24) - Ärztebewertungsportal III; ohne die Relativierung, ,jedenfalls“ Urt. v. 25.10.2011 - VI ZR 93/10, NJW 2012, 148 (Rn. 25) - Blog-Eintrag.

${ }^{324}$ Vgl. zum Folgenden Schiff, ZD 2018, $534 \mathrm{ff}$.

${ }^{325}$ Schröder, Verwaltungsarchiv 101 (2010), 205 (214).

${ }^{326}$ Vgl. nur BGH, Urt. v. 23.09.2014 - VI ZR 358/13, NJW 2015, 489 (Rn. 28) - Ärztebewertungsportal II.

${ }^{327}$ BVerfG, Beschl. v. 25.06.2009 - 1 BvR 134/03, NJW-RR 2010, 470 ff. - Pressespiegel.

${ }^{328}$ BVerfG, Beschl. v. 25.06.2009 - 1 BvR 134/03, NJW-RR 2010, 470 (Rn. 58) - Pressespiegel. 
Daraus leitet das BVerfG ab, dass bei einer Untersagung der Verbreitung fremder Meinungen ein Rückgriff auf die Pressefreiheit nur erforderlich sei, wenn die Veröffentlichung ,sich auf die bloße technische Verbreitung der

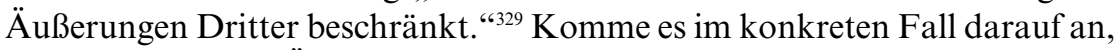
ob eine bestimmte Äußerung zulässig sei, sei „ungeachtet des Verbreitungsmediums Art. 5 Abs. 1 S. 1 GG einschlägig““. ${ }^{330}$

Daraus kann allerdings nach hier vertretener Auffassung nicht geschlussfolgert werden, dass beispielsweise Kommunikationsdienste wie Facebook pauschal dem Schutz der Meinungsfreiheit zu unterstellen wären, wie es die Rechtsprechung aber insinuiert. ${ }^{331}$ Denn erstens bildet der angesprochene Pressespiegel-Beschluss des BVerfG die Sachlage für Intermediäre nicht vollständig ab, da es bei diesen in der Regel an der pressetypischen Selektivität bei der Auswahl fremder Beiträge fehlt. Zweitens ist die verfassungsgerichtliche Anknüpfung an die lediglich „technische Verbreitung" von Meinungen Dritter auch mit seiner früheren Entscheidung Benetton $I^{32} \mathrm{zu}$ erklären. Hier hatte das BVerfG eine nachteilige Sanktion für den Abdruck einer Werbeanzeige nur an der Pressefreiheit gemessen. Die Meinungsfreiheit hatte es daneben nicht zur Anwendung kommen lassen. ${ }^{333}$ Auf diese konnte sich das Presseunternehmen nur akzessorisch berufen. Daraus lässt sich nur der Schluss ziehen, dass die rein organisationsrechtliche Absicherung der kommunikativen Rahmenbedingungen des öffentlichen Diskurses primär gerade nicht, jedenfalls nicht notwendig ausschließlich, von der Meinungsfreiheit selbst abhängt. Anders gewendet: das Fehlen einer spezifischen Grundrechtsverbürgung für den institutionellen Rahmen des Meinungsdiskurses über Art. 5 Abs. 1 S. 2 GG hinaus rechtfertigt es noch nicht, diesen institutionellen Schutz in der Meinungsfreiheit selbst zu verorten. ${ }^{334}$ Drittens ist die vom BVerfG vertretene Auffassung, nur bei einer „,technischen Verbreitung“ stehe der Rekurs auf die Pressefreiheit offen, nicht ohne Weiteres mit der überkommenen Differenzierung zwischen ,aktiver“ und „passiver“ bzw. „neutraler“ Rolle kurzzuschließen, die hier bereits kritisiert wurde. ${ }^{335}$ Denn die vom BVerfG verwendete Redewendung der "technischen Verbreitung"

${ }^{329}$ BVerfG, Beschl. v. 25.06.2009 - 1 BvR 134/03, NJW-RR 2010, 470 (Rn. 59) - Pressespiegel.

${ }^{330}$ BVerfG, Beschl. v. 25.06.2009 - 1 BvR 134/03, NJW-RR 2010, 470 (Rn. 59) - Pressespiegel.

${ }^{331}$ Kritisch auch Spindler, in: Spindler/Schmitz, TMG, §7 Rn. 60; Hindelang, Freiheit und Kommunikation, S. 318 ff., der seine Kritik aber zu einem generellen Bezweifeln der Sinnhaftigkeit der Störerhaftung entwickelt.

${ }^{332}$ BVerfG, Urt. v. 12.12.2000 - 1 BvR 1762/95, BVerfGE 102, 347 ff. - Benetton I.

${ }^{333}$ Vgl. BVerfG, Urt. v. 12.12.2000 - 1 BvR 1762/95, BVerfGE 102, 347 (359) - Benetton I.

${ }_{334}$ Ähnlich der Sache nach Buchheim, JuS 2018, 548 (550 f.).

${ }^{335} \mathrm{Vgl}$. oben $\S 8 \mathrm{~B}$. 
erscheint hier eher als funktionaler Platzhalter für die wesentlich bedeutsamere Frage, ob in dem Geschäftsmodell bzw. in dessen technischer Konfiguration konkret eine eigene Meinung des Diensteanbieters zum Ausdruck kommt. ${ }^{336}$ Das aber ist keineswegs offensichtlich, sondern in aller Regel zweifelhaft ${ }^{337}$ Schließlich scheint das BVerfG in einer jüngeren Entscheidung zu einem gegenüber Google geltend gemachten Anspruch auf Entfernung eines Suchergebnisses dies ähnlich zu sehen. ${ }^{338}$

Nach dem hier dargestellten Modell des Prüfprogramms besteht für eine grundrechtliche Zuordnung unter die Meinungsfreiheit allerdings auch kein normativ überzeugender Grund, da den Intermediären zugeordnete Handlungsanforderungen jedenfalls von ihren grundrechtlich verbürgten unternehmerischen Freiheiten abgebildet werden können, wie sie insbesondere in Art. 12 GG und Art. 16 GRCh niedergelegt sind. ${ }^{339}$

\section{Fazit}

Die Ausgestaltung der Störerhaftung erweist sich nach diesen Überlegungen als grundsätzlich geeignete Figur, um die Haftung der Intermediäre flexibel auszugestalten und hierbei die Besonderheiten der jeweils betroffenen Geschäftsmodelle hinreichend zu berücksichtigen. Insbesondere der Aspekt der Prozeduralisierung reflektiert in geeigneter Weise das Geflecht verschiedener freiheitsrechtlicher Interessen, die sich bei der Inanspruchnahme der Intermediäre als Störer gegenüberstehen. Seit neuestem ist dieser Befund auch verfassungsgerichtlich bestätigt.

Allerdings zeigen sich bei der Störerhaftung auch diverse kritische Eigenschaften bzw. Implikationen bei der Handhabung durch die Rechtsprechung. Generell scheint dies an einer Position zu liegen, die nach hier vertretener Auffassung den Schwerpunkt immer noch zu oft auf eine haftungsrechtliche Absicherung der Diensteanbieter legt. Das betrifft zwar nicht die Grundlagen der Störerhaftung per se, sondern - neben dem pauschalen Ausschluss von Schadensersatzansprüchen - konkrete Details ihrer Ausgestaltung, die bisweilen ein nicht unerhebliches kritisches Potenzial offenbaren. Insbesondere die zu strengen Anforderungen an die Substanziierungsobliegenheiten stehen hier im Kern der Kritik und tangiert die von möglichen Rechtsverletzungen Betroffenen potenziell in erheblicher Weise.

\footnotetext{
${ }^{336}$ Pille, Meinungsmacht sozialer Netzwerke, S. 178 f., verlangt hierfür zu Recht einen „Auswahl- oder Wertungsprozess".

${ }^{337}$ Wie hier zweifelnd Franzius, JZ 2016, 650 (654); anders aber Milstein/Lippold, NVwZ 2013, 182 ff. Zur Debatte in den USA vgl. oben $§ 7$ Fn. 12.

${ }^{338}$ Vgl. BVerfG, Beschl. v. 06.11.2019 - 1 BvR 276/17, NVwZ 2020, 63 (Rn. 105) - Recht auf Vergessen II.

${ }^{339}$ Zur GRCh vgl. auch Chmelik, Social Network Sites - Soziale Netzwerke, S. 202.
} 
Konkret ergibt sich danach das Petitum an die Rechtsprechung, an entscheidenden Stellschrauben die hier erläuterten Wertungen einfließen zu lassen. So sollte von dem Kriterium der „unschweren“ Erkennbarkeit bzw. ähnlichen Maßstäben für die Notifizierungen an Diensteanbieter Abstand genommen werden. Ferner sollte in Erwägung gezogen werden, den Ausschluss von Schadensersatzansprüchen aus dem Anwendungsbereich der Störerhaftung zu revidieren. Dies ließe sich mit Art. 14 ECRL durchaus vereinbaren und könnte überdies einen Beitrag zur angemessenen Kompensation der von Rechtsverletzungen Betroffenen liefern. Nicht zuletzt wäre damit ein Schritt in Richtung einer Klärung der dogmatischen Verortung der Störerhaftung verbunden. Denn neben dem wichtigen und stets zu berücksichtigen Prinzip der Effektivität der Inanspruchnahme der Diensteanbieter als „,cheapest cost avoider" spielen insbesondere mit Blick auf Persönlichkeitsrechtsverletzungen konkrete Verhaltenspflichten eine Rolle, die einen sehr starken Zusammenhang zum zivilrechtlichen Konzept der Verkehrspflichten aufweisen. 



\title{
$\S 11$ \\ Folgerungen für das Recht der Haftung der Intermediäre
}

\begin{abstract}
Nachdem die Störerhaftung in ihren Grundlagen dargestellt und bewertet wurde, sollen nunmehr die bisherigen Erkenntnisse dieser Untersuchung zur Rolle der Diensteanbieter auf das Recht der Haftungsprivilegierungen rückbezogen werden. Hierbei sind die problematischen Aspekte der Störerhaftung ebenfalls zu berücksichtigen. Zunächst ist kurz auf die Bedeutung einzugehen, die dem Recht der Haftung der Intermediäre in Form der Haftungsprivilegierungen noch zukommt (A.). Sodann ist eine teleologische Weiterentwicklung dieses Regelungsbereichs vorzunehmen (B.). Im Anschluss ist auf konkrete Folgerungen der bisherigen Überlegungen einzugehen, die mit Blick auf die Art. 14 ECRL, §10 TMG wie auch auf die Störerhaftung ausbuchstabiert werden (C.). Schließlich sind die Erkenntnisse bezüglich des Verbots der Auferlegung allgemeiner Überwachungspflichten gemäß Art. 15 ECRL, § 7 Abs. 2 TMG in konkrete Folgerungen umzuformulieren (D).
\end{abstract}

\section{A. Die Bedeutung der Haftungsprivilegierungen für die Haftung der Intermediäre}

Wie zu Beginn dieser Untersuchung bereits dargestellt wurde, stammen die Haftungsprivilegierungen noch aus der Anfangszeit des Internet als Technologie der Massen. 25 Jahre später ist die Frage angezeigt, ob die Entwicklung über diese, überwiegend immer noch in ihrem Ausgangszustand verharrenden Regelungen hinweggegangen ist und ob für die damals adressierten Interessenkonflikte mittlerweile nicht neue Antworten technischer oder rechtlicher Natur formuliert werden könnten. In diesem Zusammenhang meinen allerdings manche Stimmen, dass dem Recht der Haftungsprivilegierungen keine besondere Bedeutung mehr zukäme. Insbesondere Hofmann vertritt die Auffassung, dass die Haftungsbeschränkungen aufgrund des Art. 14 ECRL bzw. § 10 TMG keine größere Rolle mehr spielten. Er begründet dies mit der Unanwendbarkeit der Haftungsprivilegierungen auf Unterlassungsansprüche. Außerdem sei die Wirkmacht der Haftungsprivilegierungen angesichts ihrer richterrechtlichen Beschränkung auf „passive“ Intermediäre ohnehin beschränkt. Schließlich seien die für die Diensteanbieter 
relevanten Haftungsbeschränkungen primär aus den durch den EuGH verstärkt in Stellung gebrachten Grundrechten sowie aus dem Verbot allgemeiner Überwachungspflichten zu folgern. ${ }^{1}$

Diese weitgehende Relativierung der Haftungsbeschränkungen der ECRL überzeugt nicht vollumfänglich. Zur Frage der Anwendbarkeit der Art. 14 ECRL bzw. des 10 TMG auf Unterlassungsansprüche wurde bereits angemerkt, dass die Störerhaftung teilweise immer noch über den Rahmen der ECRL hinausweist und insoweit das unionsrechtliche Konfliktpotenzial noch nicht abschließend geklärt ist. ${ }^{2}$ Die von Hofmann betonte Beschränkung der Haftungsprivilegierungen auf ,passive“ Intermediäre mag dem status quo der Rechtspraxis entsprechen. Es wurde jedoch schon begründet, inwieweit diese Beschränkung auf theoretischer und praktischer Ebene nicht überzeugt. ${ }^{3}$ Die Betonung der grundrechtlichen Perspektive, wie sie vom EuGH insbesondere in den $S A B A M$-Entscheidungen und in ähnlichen Fragestellungen in jüngerer Zeit zum Ausdruck kommt, ${ }^{4}$ weist zwar einerseits auf einen richtigen Punkt hin. Dass der EuGH in letzter Zeit immer deutlicher wahrnehmbar zum Grundrechtsgericht avanciert, ${ }^{5}$ ist als teilweise Abkehr von seiner Rolle als marktliberaler Motor der „Integration durch Recht" zu begrüßen, die die Europäischen Gemeinschaften in ihren ersten Jahrzehnten entscheidend prägte. ${ }^{6}$ Dennoch übergeht die Betonung der Grundrechte in diesem Kontext, dass die Haftung der Intermediäre immer noch in erster Linie durch Sekundär- bzw. einfaches Recht geprägt ist und die sich daraus ergebenden Konflikte zuerst mit dem Blick hierauf zu beantworten sind. ${ }^{7}$ Eine übermäßige Aufladung der Grundrechte gerade angesichts der Existenz recht detaillierter einfachrechtlicher Ausformungen intermediärer Sorgfaltsstandards droht demgegenüber, diese demokratisch gesetzten Standards zu unterminieren. Zuletzt spricht außerdem viel für die bereits erwähnte Integration der Haftungsprivilegierungen in das Verkehrspflichtenkonzept, soweit in ihnen konkrete Sorgfaltspflichten zum Ausdruck kommen. ${ }^{8}$ Mit einer solchen integrativen Lösung ${ }^{9}$ verträgt sich eine Entwertung

\footnotetext{
${ }^{1}$ Hofmann, ZUM 2019, 617 (624 f.); vgl. auch ders., GRUR 2019, 1219 (1222f.).

${ }^{2}$ Vgl. oben $\S 10$ A. V. 2 .

${ }^{3}$ Vgl. oben $\S 8$ B.

${ }^{4}$ Vgl. oben $\$ 4$ C. IV. 2. a) cc).

${ }^{5}$ Vgl. dazu die Nachweise oben in $\S 4$ Fn. 280.

${ }^{6}$ Vgl. dazu instruktiv Scharpf, Socio-Economic Review 8 (2010), 211 (221 ff.).

${ }^{7}$ Daran ändert auch die durchaus bemerkenswerte verfassungsrechtliche Aufwertung der Störerhaftung in BVerfG, Beschl. v. 06.11.2019 - 1 BvR 276/17, NVwZ 2020, 63 (Rn. 113) - Recht auf Vergessen II, nichts.

${ }^{8}$ Vgl. in diesem Sinne Wagner, in: MüKo BGB, $\S 823$ Rn. 867; ansatzweise wohl auch Spindler, in: Spindler/Schmitz, TMG, TMG $\$ 10$ Rn. 100.

${ }^{9}$ Für diese spricht sich nicht zuletzt auch Hofmann, GRUR 2019, 1219 (1222), selbst
} aus. 
des einfachen Rechts zulasten von Maßstäben grundrechtlicher Provenienz nur schlecht.

Die Tatsache, dass im rechtspolitischen Raum in jüngerer Zeit wiederholt um eine gesetzliche Fortentwicklung des Haftungsregimes gerungen wurde und wird, mag hier zwar nur anekdotischen Charakter haben, unterstreicht aber die Vermutung, dass das Haftungsrecht von zentraler Bedeutung ist, wenn es um die Aushandlung richtiger regulativer Zugriffe auf diesen Lebensbereich geht und unterstreicht zugleich dessen demokratische Relevanz. Zusammenfassend entfaltet das Recht der Haftungsprivilegierungen der Intermediäre also durchaus eine eigenständige Bedeutung, die es für die Fortentwicklung der Sorgfaltsmaßstäbe für die Intermediäre zu einem wichtigen Anknüpfungspunkt machen.

\section{B. Die Zwecke des Rechts der Haftungsprivilegierungen}

Zunächst ist auf die Zwecksetzungen einzugehen, die die Gesetzgeber beim Erlass der Haftungsprivilegierungen verfolgten. Soweit diese Zwecke nicht, nicht mehr oder nicht vollständig verwirklicht sind, sollte über deren Präzisierung nachgedacht werden. Dies ist Anliegen des nachfolgenden Abschnitts.

\section{Blick zurück in die USA}

Wie oben ausführlich dargelegt wurde, war es das grundlegende Ziel des USGesetzgebers, mit § 230 CDA den Intermediären eine Immunisierung als Belohnung für deren ernsthafte Bemühungen gewähren, eigenständig ihre Machtsphäre und die Inhalte ihrer Nutzer zu regulieren..$^{10}$ In ihrer ursprünglichen Intention verlangt die Norm in den USA den Diensteanbietern damit sogar mehr freiwillige Überwachungsmaßnahmen ab bzw. lässt das Privileg trotz der Ausführung solcher Maßnahmen unberührt. Unter den Art. $14 \mathrm{f}$. ECRL ist dagegen jede Maßnahme, die den Diensteanbieter nicht mehr als „neutral“" erscheinen lässt, für diesen ein Haftungsrisiko. Insoweit lässt sich also festhalten, dass diese Regelung die rechtliche Auferlegung einer Pflicht zu dem Verhalten verbietet, zu dem \$230 CDA gerade einen Anreiz liefern soll. ${ }^{11}$ Diese Anreizwirkung wurde zwar bislang, wie zu Beginn dieser Untersuchung dargelegt wurde, aufgrund einer sehr unternehmensfreundlichen Handhabung durch die Gerichte in den USA faktisch unterlaufen. Dennoch

\footnotetext{
${ }^{10}$ Vgl. oben $\S 3$ Fn. 16 und den zugehörigen Text.

${ }^{11}$ Anders insoweit Beckmann, Verantwortlichkeit von Online-Diensteanbietern in Europa und den Vereinigten Staaten von Amerika, S. 274 f.
} 
muss die Frage erlaubt sein, inwiefern sich der in Art. 15 ECRL niedergelegte Grundsatz im Vergleich zu dieser Regelung behaupten kann, die prinzipiell einen „aktiveren“ Part der Intermediäre fordert. Berücksichtigt man die Bedeutung und die Einflussmöglichkeiten gerade der etablierten, wirkmächtigen Intermediäre, spricht viel dafür, mit rechtlichen Mitteln verstärkte Anreize zu einem freiwilligen Engagement bezüglich der Verhinderung rechtswidriger Inhalte zu setzen. Das ebenfalls auf die US-amerikanische Regelung zurückgehende Prinzip des ,guten Samariters“ wäre hierfür geeignet, wie im Folgenden zu erläutern ist.

\section{Modifizierung der Zwecke des Rechts der Haftungsprivilegierung durch Einführung einer ,, Good Samaritan“-Regelung}

Die Haftungsprivilegierungen werden gemeinhin mit dem Geschäftsmodell der von den Intermediären erbrachten Dienstleistungen gerechtfertigt. Dieses beruht in diesem Kontext auf dem Fehlen einer inhaltlichen Beziehung zu den von ihnen transportierten Inhalten, mit deren Erstellung sie in der Regel nicht unmittelbar zu tun haben. ${ }^{12}$ Diese Sichtweise mag zwar als Ausgangspunkt für die Betrachtung von Bedeutung sein. Es genügt jedoch nach hier vertretener Auffassung nicht, bei der Betonung des Fehlens einer Beziehung zum Inhalt stehen zu bleiben. Denn mag ein konkretes Interesse an dem Inhalt im Sinne der hierin zutage tretenden Positionen, Meinungen etc. in der Tat nicht vorhanden sein, liegt ein hohes wirtschaftliches Interesse an der Verbreitung der Inhalte aufseiten der Diensteanbieter umso näher. ${ }^{13}$ Die Fixierung auf den „,bloßen Vermittlungsvorgang ${ }^{\text {“14 }}$ genügt nach hier vertretener Auffassung als teleologische Rechtfertigung einer Privilegierung jedoch nicht.

Es bedarf vielmehr weiterer Zwecke, um eine Haftungsprivilegierung im Grundsatz - über Ausnahmen ist damit freilich noch nichts gesagt - zu rechtfertigen. Eine solche Zweckerweiterung könnte gerade darin bestehen, freiwilligen Kontrollmaßnahmen und Haftungsfreistellungen miteinander zu verknüpfen. Insoweit wurde bereits der Vorschlag unterbreitet, die Privilegierungen des Art. 14 ECRL jenseits bereits geltender reaktiver Pflichten aufgrund von Hinweisen auf konkrete rechtswidrige Inhalte an die unternehmensseitige Einführung von Überwachungsinstrumenten im Sinne einer in den USA bekannten und hier bereits angesprochenen „Good Samaritan“Regelung zu binden. ${ }^{15}$ Bei dieser ginge es darum, im Rahmen des Haftungs-

${ }^{12}$ Vgl. Frey, Die Haftung von Host-Providern für Immaterialgüterrechtsverletzungen, S. 119 .

${ }^{13}$ Vgl. oben die Nachweise in den $\S 8$ Fn. 37, 87 und die zugehörigen Ausführungen.

${ }^{14}$ Paal, in: BeckOK Informations- und Medienrecht, TMG § 10 Rn. 3.

${ }^{15}$ Vgl. Spindler, MMR 2018, 48 (52); ders., in: Eifert/Hoffmann-Riem, Innovation, 
maßstabs bzw. der Reichweite der Haftung die aktiven „Eigenbemühungen der Provider ${ }^{116}$ zu berücksichtigen. ${ }^{17}$ Das könnte von einer beschränkten Haftung lediglich im Innenverhältnis zum inhaltsverantwortlichen Nutzer, wie es etwa im US-amerikanischen Modell unter $\$ 230$ (c)(2)(A) CDA oder $\S 512(\mathrm{~g})$ DMCA der Fall ist, ${ }^{18}$ bis hin zu einer Privilegierung gegenüber Betroffenen einer Rechtsverletzung gleichsam im Außenverhältnis reichen. ${ }^{19} \mathrm{Im}$ deutschen Recht erscheint das erstere Modell allerdings schwer realisierbar, soweit den von einer Entfernung eines Inhalts Betroffenen ein Anspruch auf Wiederherstellung zusteht. ${ }^{20}$ Denn die Annahme eines solchen Wiederherstellungsanspruchs ist mit einer Privilegierung für „takedowns“ im Innenverhältnis kaum widerspruchsfrei in Einklang zu bringen. Deshalb spricht mehr dafür, eine Privilegierung für die redlichen Eigenbemühungen im AuBenverhältnis wirken zu lassen. Nicht zuletzt für die Diensteanbieter wäre dies auch die attraktivere Lösung, weil das Haftungsrisiko für eingetretene bzw. behauptete Rechtsverletzungen im Außenverhältnis für sie höhere organisatorische und finanzielle Kosten $\left(\mathrm{Kraakman}^{21}\right)$ verursacht, vor denen sie eine entsprechende Privilegierung bewahren könnte. Eine solche Modifizierung der Haftungsprivilegierungen wäre insbesondere geeignet, den Wertungswiderspruch zu beseitigen bzw. abzumildern, der in der Benachteiligung jener Diensteanbieter liegt, die eigeninitiativ ein Monitoring durchfüh-

Recht und öffentliche Kommunikation, S. 67 (79f.); kritisch auch Holznagel, Notice and Take-Down-Verfahren als Teil der Providerhaftung, S. 119.

${ }^{16}$ Leistner, ZUM 2016, 580 (585).

${ }^{17}$ Vgl. in diesem Sinne auch Fischer, Die Einbindung von Providern in die Durchsetzung von Urheberrechten, S. $358 \mathrm{f}$.

${ }^{18} \mathrm{Vgl}$. dazu oben $\S 3$ A. sowie Text zu §3 Fn. 167.

${ }^{19}$ Vgl. etwa den Vorschlag zur Erweiterung von $\S 10$ TMG bei Holznagel, Notice and Take-Down-Verfahren als Teil der Providerhaftung, S. 249 f., der sich tatbestandlich an $\S 512$ DMCA orientiert, die Privilegierung insoweit durch die Verankerung in $§ 10$ TMG aber in das Außenverhältnis kehrt.

${ }^{20}$ Insbesondere in grundrechtlicher Hinsicht berührt dieser Aspekt aber grundlegende Abgrenzungsfragen bei der Reichweite der mittelbaren Drittwirkung der Grundrechte. Für eine in diese Richtung weisende Bindung Privater an die Meinungsfreiheit OLG München, Beschl. v. 24.08.2018 - 18 W 1294/18, NJW 2018, 3115 ff. - Hassrede; LG Frankfurt/M., Beschl. v. 14.05.2018 - 2-03 O 182/18, MMR 2018, 545 (Rn. 17 ff.); OLG Oldenburg, Urt. v. 01.07.2019 - 13 W 16/19, MMR 2020, 41 (Rn.9); LG Bamberg, Urt. v. 18.10.2018 - 2 O 248/18, MMR 2019, 56 (Rn. 51); KG, Beschl. v. 22.03.2019 - $10 \mathrm{~W}$ 172/18, NJW-RR 2019, 1260 (Rn. 17); dafür auch Raue, JZ 2019, 961 (964ff.); vgl. ferner Holznagel, CR 2018, 369 (371); dagegen etwa OLG Stuttgart, Beschl. v. 06.09.2018 - 4 W 63/18, MMR 2019, 110 (Rn. 29f.); OLG Dresden, Beschl. v. 08.08.2018 - 4 W 577/18, NJW 2018, 3111 (Rn. 20); OLG Karlsruhe, Beschl. v. 25.06.2018 - 15 W 86/18, NJW 2018, 3110 (Rn. 18); vgl. Lüdemann, MMR 2019, 279 (280 ff.).

${ }^{21}$ Vgl. oben unter $\$ 9 \mathrm{~B}$. 
ren (wollen). ${ }^{22}$ Sie käme im Übrigen auch keineswegs einer Revolution des Haftungsrechts gleich, denn die Erwartung zur aktiven, eigeninitiativen Bemühung der Diensteanbieter zur Kontrolle des eigenen Einflussbereichs äußert, wenngleich nur ansatzweise, auch die ECRL. So heißt es in deren Erwägungsgrund 40 S. 6, dass die in der ECRL statuierten Bestimmungen über die Verantwortlichkeit die Diensteanbieter ,nicht daran hindern, innerhalb der von den Richtlinien 95/46/EG und 97/66/EG gezogenen Grenzen technische Schutz- und Erkennungssysteme und durch die Digitaltechnik ermöglichte technische Überwachungsgeräte zu entwickeln und wirksam anzuwenden." Diese bislang selten beachtete Aussage, die eher programmatischer als normativer Natur ist, dient hier als Beleg, dass die Grundtendenz des Gesetzgebers bereits in der ECRL in diese Richtung weist. Dies gilt es, in moderater und zugleich das Verhältnismäßigkeitsprinzip achtender Weise fortzuentwickeln.

Die genauen Voraussetzungen einer solchen „Good Samaritan“-Regel sind mit nicht gerade einfachen Wertungen verbunden, ${ }^{23}$ die letztlich mitten ins Herz der Frage nach den Qualitäten bzw. Eigenschaften eines ,idealen“ Intermediärs zielen. Diese Frage abstrakt, also diensteübergreifend beantworten zu wollen, dürfte jedoch ein schwieriges Unterfangen darstellen. Eine Typisierungsbefugnis lässt sich dem Gesetzgeber jedoch kaum aus der Hand schlagen. Eine Orientierung an den Kriterien des $§ 512$ DMCA als erster Anhaltspunkt, verbunden mit dem Abschied von der geltenden, nur sehr grob zwischen verschiedenen Dienstetypen differenzierenden Einteilung, bei der insbesondere das Konzept des „Host-Providers“ nur wenig Differenzierungspotenzial aufweist,${ }^{24}$ kann hier den Weg weisen. ${ }^{25}$

\section{Elemente einer Reform des Haftungsrechts der Intermediäre}

Für die konkreten Regelungen zur Privilegierung von Intermediären im Kontext dieser Untersuchung, also insbesondere den Art. 14 ECRL, §10 TMG, sind ebenfalls Ansatzpunkte zu erkennen und zu benennen, um die hier dargestellten Bedenken aufzugreifen. Diese betreffen eine grundlegende Neukonzeption der von Haftungsprivilegien grundsätzlich profitierenden

${ }^{22} \mathrm{Vgl}$. Hoffmann/Volkmann, in: Spindler/Schuster, Recht der elektronischen Medien, TMG $\$ 10$ Rn. 2.

${ }^{23}$ Vgl. Holznagel, Notice and Take-Down-Verfahren als Teil der Providerhaftung, S. 250 .

${ }^{24} \mathrm{Vgl}$. schon oben $\S 8 \mathrm{~A}$.

${ }^{25}$ Vgl. den Vorschlag von Holznagel, Notice and Take-Down-Verfahren als Teil der Providerhaftung, S. 249. 
Dienste (I.), die Konsequenzen, die sich aus der mangelnden Eignung von „Neutralität" als Leitbild für die Intermediäre ergeben (II.), sowie einzelne Elemente zur Modifizierung der durch die Haftungsprivilegien vermittelten Anreizstrukturen (III.).

\section{Neukonzeption der Einteilung in spezifische Dienste}

Es wurde bereits dargelegt, weshalb die aktuelle Einteilung der Dienste in den Art. 12-14 ECRL insbesondere hinsichtlich der Privilegierung des „Hostings" nicht überzeugt. ${ }^{26}$ Auch übergreifend wurde bereits auf die Probleme eines materiell horizontalen Ansatzes hingewiesen. ${ }^{27}$ Dementsprechend sollte eine Reform der ECRL von dem Konzept des Host-Providers als einzige Kategorie der Privilegierung der hier in den Blick genommenen Diensteanbieter Abstand nehmen. Stattdessen sollte sich die gesetzliche Regelung von einer genaueren funktionalen Zuordnung leiten lassen. ${ }^{28}$ Diese kann weiterhin in einer Speicherung liegen, wenn es im Kern des jeweils betroffenen Geschäftsmodells eben wirklich um eine Speicherung geht, wie es etwa bei Filesharing-Diensten der Fall ist. Liegt der Kern des Geschäftsmodells in einer funktionalen Betrachtung dagegen auch in der Verbreitung von Inhalten, sollte eine Privilegierungsnorm dies in den Vordergrund stellen und $\mathrm{Pa}-$ rameter entsprechender Sorgfaltsstandards vorgeben. Auch weitere Kriterien wie insbesondere die Gefahrgeneigtheit eines Dienstes könnten insoweit zusätzlichen Differenzierungsspielraum eröffnen, der sich etwa in differenzierenden Sorgfaltsstandards niederschlagen könnte. Ferner wäre eine Einteilung der verschiedenen, bereits geläufigen Geschäftsmodelle anzudenken, bei der dann eine deutlich differenziertere Ausformung von Sorgfaltsstandards denkbar wäre. ${ }^{29}$ Umrahmt werden könnte ein solcher differenzierender Ansatz immer noch von einer Art Generalklausel, die dann im Vergleich zu spezifizierten Geschäftsmodellen eben nur ein Mindestmaß an objektiven und/oder subjektiven Anforderungen voraussetzen könnte, um die Privilegierung zu gewähren. Unter dem geltenden System fallen schlichtweg zu viele Geschäftsmodelle unter ein und dieselbe Haftungsprivilegierung, sodass das Fehlen eines solchen hinreichenden Spielraums zur Differenzierung und Kategorisierung im Grunde nicht einmal sonderlich überrascht.

\footnotetext{
${ }^{26} \mathrm{Vgl}$. oben $\S 8 \mathrm{~A}$.

${ }^{27} \mathrm{Vgl}$. oben $\S 4$ E. I.

${ }^{28} \mathrm{Im}$ Urheberrecht ist die Kritik der unzureichenden Differenzierung zwischen verschiedenen Diensten keineswegs neu, vgl. Leistner, ZUM 2016, 580 (587); Czychowskil Nordemann, GRUR 2013, 986 (991 f.); vgl. auch Stieper, ZUM 2017, 132 (138).

${ }^{29}$ So wäre etwa beispielsweise bei Kinderpornographie zwar weiterhin die Möglichkeit einer Beschwerde zwingend vorzusehen. Sie ist jedoch zweifelsfrei nicht notwendig, um dem Diensteanbieter das Haftungsprivileg abzusprechen, vgl. Holznagel, Notice and Take-Down-Verfahren als Teil der Providerhaftung, S. $91 \mathrm{f}$.
} 
Dass eine solche funktionale Abschichtung wünschenswert und erforderlich ist, lässt sich indiziell auch der Rechtspraxis entnehmen. Allein ein Blick auf ausführlichere Kommentierungen des $\S 10 \mathrm{TMG}$, die in substanziellen Teilen auf eine Vielzahl von funktional unterschiedenen Einzelfällen zurückgreifen, um die Anwendung dieser Norm handhabbar zu machen, verdeutlicht, dass das horizontale, sektorübergreifende Modell dieser Regelung womöglich überholt ist. ${ }^{30}$ Jedenfalls trägt ein solcher Zustand nicht zu einer für die Rechtspraxis sicheren und prognostizierbaren Handhabung bei.

Eine funktionale Abschichtung hätte hier außerdem den Vorteil, ein Argument entkräften zu können, das des Öfteren Versuchen eines regulativen Zugriffs entgegengebracht wird, nämlich die unangemessenen Konsequenzen bei kleineren Diensten (Start-ups), die nicht über die finanziellen und technischen Ressourcen verfügten, um rechtliche Vorgaben im gleichen Ausmaß umzusetzen, wie es bei den großen Konzernen der Fall ist. Als Blaupause kann insoweit auf die Idee hinter der Beschränkung des Anwendungsbereichs neuerer gesetzgeberischer Vorhaben verwiesen werden. So werden sowohl in $\S 1$ Abs. 2 NetzDG ${ }^{31}$ als auch in der DSM-RL schon durch eine beschränkte Definition der von der Richtlinie anvisierten Akteure in Art. 2 Nr. 6 UAbs. 2 DSM-RL viele Dienste nicht erfasst. ${ }^{32}$ Ferner werden Teile der eigentlich von diesen Regelwerken erfassten Akteure von bestimmten Pflichten ausgenommen, wie Art. 17 Abs. 6 DSM-RL festhält. ${ }^{33}$ Ungeachtet etwaiger Auslegungsschwierigkeiten bei diesen Regelungen ${ }^{34}$ sind in diesem Sinne abgestufte Compliance- und Haftungskonzeptionen nicht allein den Realitäten rechtspolitischer Kompromissfindung geschuldet, sondern stellen nach hier vertretener Auffassung prinzipiell Schritte in die richtige Richtung dar, um die Potenziale differenzierter Lösungen der Haftungsfrage im Allgemeinen auszuloten. Allerdings ist eine Beschränkung allein auf den wirtschaftlichen bzw. kommerziellen Erfolg und das Alter von Diensten, wie es bei den genannten Beispielen der Fall ist, nicht hinreichend. Ginge es um eine Reform der Art. 12-14 ECRL, wäre alternativ oder kumulativ eine Abstufung nach den jeweils angebotenen Diensten erforderlich, um das Maß an

\footnotetext{
${ }^{30}$ Vgl. etwa die von Spindler, in: Spindler/Schmitz, TMG, § 10 Rn. 77 ff., aufgeführten „Einzelfälle“.

${ }^{31}$ Wobei hier trotz dieser akteurspezifischen Ausnahme gleichwohl die Störerhaftung weiterhin greift.

${ }^{32} \mathrm{Vgl}$. dazu oben $\S 7$ C. I. 2.

${ }^{33}$ Vgl. zu Art. 17 DSM-RL noch unten $§ 12$ B. Vgl. auch den von den Regierungschefinnen der Länder verabschiedeten Medienstaatsvertrag (vgl. oben $\S 6$ Fn. 28), der in $\S 91$ einen ähnlichen Ansatz bei der Definition von „Medienintermediären“ verfolgt; kritisch dazu aber Ladeur/Gostomzyk, K\&R 2018, 686 (689f.).

${ }^{34} \mathrm{Zu} \S 1$ Abs. 2 NetzDG vgl. Spindler, GRUR 2018, 365 (368); zu Art. 17 DSM-RL vgl. ders., CR 2019, 277 (288).
} 
Kontrolle, das die jeweiligen Diensteanbieter im Hinblick auf ein bestimmtes Geschäftsmodell bezüglich der von ihnen transportierten Inhalte haben und den potenziellen Nutzen aus der Verbreitung oder Speicherung dieser Inhalte angemessen berücksichtigen zu können.

Zur Klarstellung sei hier betont, dass es mit dem hier vorgeschlagenen Ansatz nicht darum geht, einer materiellen Gleichbehandlung von ContentProvidern und sonstigen Diensten das Wort zu reden. Denn es bleibt richtig und wichtig, im Sinne des Prinzips der Eigenverantwortung tatsächlich eigene bzw. selbst produzierte Informationen materiell stärker zuzurechnen, als es bei den Diensteanbietern der Fall ist. Nach den bisherigen Überlegungen dieser Untersuchung ist dies allerdings auch kein überzeugendes Argument, um sich für eine legislative Untätigkeit einzusetzen.

\section{Aufgabe der Unterscheidung zwischen aktiver und passiver Rolle}

Ein weiterer Ansatzpunkt für eine Reform wäre eine Befreiung des Art. 14 ECRL und seiner Umsetzungsvorschriften von der Fixierung auf die Differenzierung zwischen „aktiven“ und „passiven“ Diensten. Denn eine solche Binarität verhindert die Kontextualisierung der Funktionen der jeweils betroffenen Diensteanbieter und zwingt stattdessen zu schematischen Lösungen. ${ }^{35}$ Die Rechtsprechung des EuGH sucht zwar in diesem Rahmen Kompromisse im Rahmen der weiteren Handhabung von Art. 14 f. ECRL, ${ }^{36}$ vermag sich jedoch nicht von den genannten Attributen zu befreien. ${ }^{37}$

Eine positive Deutung bzw. Neukonzeption des Neutralitätsprinzips befürwortet allerdings Husovec. ${ }^{38}$ Während eine zu breite Interpretation der Passivität den praktischen Anwendungsbereich der Privilegierungen zu entleeren drohe, sei auch eine Lesart der Neutralität denkbar, die das Regelungsregime positiv fortentwickeln könne. Namentlich sei die Frage der Passivität auch als Kriterium zur Abgrenzung von „third-party information“, also von Nutzern eingegebenen Informationen im Sinne von Art. 14 Abs. 1 ECRL, gegenüber diensteeigenen Informationen nutzbar..$^{39}$ De lege lata wäre damit allerdings eine Reduktion des Neutralitätsprinzips verbunden, die in der Haltung des EuGH keinen Niederschlag findet. Denn dieser betrachtet

\footnotetext{
${ }^{35}$ Vgl. dazu schon oben $\S 8$ B; ferner Ohly, ZUM 2015, 308 (313); Spindler, MMR 2018, 48 (52).

${ }^{36}$ Dies betont u.a. Husovec, Injunctions against Intermediaries in the European Union, S. 57.

${ }^{37}$ Vgl. paradigmatisch EuGH, Urt. v. 23.03.2010 - C-236/08, NJW 2010, 2029 (Rn. 113 f.) - Google France und Google; Urt. v. 12.11.2011 - C-324/09, MMR 2011, 596 (Rn. 113) - L'Oréal SA.

${ }^{38}$ Vgl. Husovec, Injunctions against Intermediaries in the European Union, S. $55 \mathrm{ff}$.

${ }^{39}$ Husovec, Injunctions against Intermediaries in the European Union, S. $55 \mathrm{f}$.
} 
die Passivität bzw. Neutralität als essenzielle Voraussetzung, um überhaupt $\mathrm{zu}$ einer Anwendung der Privilegierungen im Einzelnen zu gelangen ${ }^{40}$ Eine doppelte Relevanz sowohl in dieser Hinsicht als auch mit Blick auf die Abgrenzung von nutzer- und dienstegenerierten Inhalten wäre indes systematisch kaum überzeugend. Auch de lege ferenda wäre das Abstellen auf Neutralität zum Zwecke dieser Abgrenzung nicht vorzugswürdig. Denn sofern es bei einer rein technischen Abgrenzung der Eingabe von Informationen durch verschiedene Akteure bliebe, hätte die Neutralität als in erheblichem Ausmaß auf Wertungen abstellendes Prinzip eher den Effekt einer Nivellierung prinzipiell klar trennbarer Kategorien. Wollte man dagegen die Abgrenzung eigener und fremder Inhalte selbst stärker von Wertungen abhängig machen, wie es etwa bei der Kategorie des Zueigenmachens in der deutschen Rechtsordnung der Fall ist, ${ }^{41}$ führte eine Aufladung dieser Wertungsentscheidung mit dem Gedanken der Neutralität indes ebenfalls nicht zu einer verbesserten Abgrenzbarkeit, sondern im Gegenteil eher zu einer Einbuße an abstraktionsfähigen Kriterien, an denen die Diensteanbieter ihre Praxis ausrichten könnten. Gewonnen wäre damit also wenig. Stattdessen sollte bei einer Reform verstärkt auf die materiellen Funktionen des Diensteanbieters abgestellt werden. So sollte insbesondere seine redaktionelle Funktion eine Rolle spielen, die nicht allein auf die technische Kontrolle von Inhalten abstellt, sondern auch Gestaltungsfunktionen im Umfeld von Inhalten als „,kenntnisähnliches" oder der Kenntnis nahekommendes Element in den Blick nimmt. Eine sinnvolle Eingrenzung der Haftungsprivilegierung könnte dann immer noch an einer Zuordnung des jeweils transportierten Inhalts erfolgen. ${ }^{42}$

Der Weg zu einer Aufgabe des Neutralitätsprinzips könnte ferner auch darin liegen, sich auf eine teilweise bereits vertretene Interpretation des Erwägungsgrundes 42 der ECRL zu berufen, der zufolge die Beschränkung der Haftungsprivilegien auf Dienste „rein technischer, automatischer und passiver Art" nur Dienste im Sinne von Art. 12 und 13 ECRL erfassen soll. ${ }^{43}$ Freilich könnte es aber damit nicht sein Bewenden haben, da auch eine Aufgabe des Neutralitätskriteriums in diesem Sinne noch keine positive Formulierung der Funktionen oder der Pflichten der Diensteanbieter beinhalten würde, die nach hier vertretener Auffassung zentraler Bestandteil einer Reformulierung der Haftungsprivilegien darstellen sollte. Die Aufgabe des Neutralitätsdogmas ist damit notwendiger, aber noch nicht hinreichender

${ }^{40} \mathrm{Vgl}$. schon oben $\S 4$ C. II. 3.

${ }^{41}$ Vgl. dazu oben $\$ 4$ D. I. 2.

${ }^{42}$ Nach Stalla-Bourdillon, in: Taddeo/Floridi, The Responsibilities of Online Service Providers, S. 275 (288), soll hier eine grundrechtliche Verortung ein entscheidendes Kriterium bilden.

${ }^{43}$ Vgl. dazu schon oben $§ 4$ Fn. 84; ferner Valcke/Kuczerawy/Ombelet, in: Taddeo/Floridi, The Responsibilities of Online Service Providers, S. 101 (107 f.). 
Bestandteil einer Anerkennung der Verantwortung der Intermediäre. Vielmehr kommt es auch auf die weiteren Anreizstrukturen an, die im Recht der Haftungsprivilegierungen eingewoben sind.

\section{Elemente verbesserter Anreizstrukturen}

Auf diese Anreizstrukturen ist im Folgenden einzugehen. Unter Rückgriff auf bereits herausgearbeitete Einsichten lassen sich so auch Perspektiven de lege ferenda formulieren. Dabei lassen sich die soeben ${ }^{44}$ im Rahmen der Störerhaftung problematisierten Anreizstrukturen ${ }^{45}$ in Folge der Begründungslasten und deren Konsequenzen im Grunde genommen auch als Problem der Haftungsprivilegierung nach Art. 14 ECRL, §10 TMG beschreiben. Denn deren Wertungen sind - trotz ihrer eigentlichen Unanwendbarkeit - auch im Rahmen der Störerhaftung selbst zu berücksichtigen. ${ }^{46} \mathrm{Im}$ Folgenden soll daher auf die Besonderheiten dieser Regelungen eingegangen und es sollen mögliche Ansätze für eine Reform aufgezeigt werden, die die bereits beschriebenen problematischen Aspekte der geltenden Rechtslage und -praxis berücksichtigen.

\section{Die Kenntnisnahme als Anknüpfungspunkt intermediärer Verantwortung im Haftungsrecht}

Zuerst ist der Blick auf die Kenntnisnahme zu richten, die in Art. 14 Abs. 1 lit. a ECRL, § 10 TMG sowie unter der Störerhaftung jeweils eine entscheidende Rolle bei der Bestimmung der Haftung eines Diensteanbieters im konkreten Fall spielt. Die rechtliche Ausgestaltung der Anforderungen an die Kenntnisnahme kann danach einen zentralen Baustein einer angemessenen Anreizstruktur darstellen, worauf - daran sei hier noch einmal erinnert Kraakman bereits hingewiesen hat. ${ }^{47}$

Das Abstellen auf die Kenntnis im Rahmen der Inpflichtnahme eines Störers lässt sich mit Wagner verhaltensökonomisch grundlegend dadurch begründen, dass die Kenntniserlangung den „Informationsstand [...] verändert, so dass die Kosten für die Vermeidung der Rechtsverletzung häufig sinken. ${ }^{“ 48}$ Diese Einsicht kann hier den Ausgangspunkt für die konkrete Aus-

${ }^{44} \mathrm{Vgl}$. oben $\S 10$ B. IV. 1.

${ }^{45}$ Vgl. zu Anreizstrukturen in diesem Zusammenhang auch Eifert, in: Hermstrüwer/ Lüdemann, Der Schutz der Meinungsbildung im digitalen Zeitalter, S. 189 (199f.).

${ }^{46}$ So OLG Hamburg, Urt. v. 14.01.2009 - 5 U 113/07, MMR 2009, 631 (636) - Usenet I; Kartal-Aydemir/Krieg, MMR 2012, 647 (649); Brock/Schmittmann, in: Schwartmann, Praxishandbuch Medien-, IT- und Urheberrecht, Kap. 10 Rn. 81; Kovacs, Die Haftung der Host-Provider für persönlichkeitsrechtsverletzende Internetäußerungen, S. 196.

${ }^{47}$ Vgl. Kraakman, Journal of Law, Economics, and Organization 2 (1986), 53 (76).

${ }^{48}$ Wagner, in: FS Medicus 2009, S. 589 (606), dort bezogen auf die Geltendmachung des Unterlassungsbegehrens, das aber einer Kenntnis insoweit gleichkommt. 
formung spezifischer normativer Erwartungen an die Intermediäre markieren. Denn sie bringt bei der Frage nach den Kosten der Vermeidung von Rechtsverletzungen zu Recht zum einen den Effektivitätsgedanken zum Ausdruck, der einer Zuweisung von Verantwortung zur Vermeidung und Beseitigung von rechtlich relevanten Rechtsgutbedrohungen immanent ist (,cheapest cost avoider ${ }^{\text {“49 }}$ ). Zum anderen negiert sie aber auch nicht den Grundsatz der Eigenverantwortung, demzufolge der Primärverursacher als Adressat rechtlicher Pflichten stets in den Blick zu nehmen ist, insoweit bei der Frage nach den „Kosten“ auch auf die Aufwände zur Vermeidung der Rechtsverletzung durch den Primärverursacher abzustellen sein kann. Die Auslegung von $\$ 10$ TMG und Art. 14 ECRL spiegelt, wie schon in den vorangegangenen Abschnitten teilweise sichtbar wurde, diese Einsichten indes in mehreren Aspekten nicht vollständig wieder bzw. wird diesen Maßstäben nicht vollumfassend gerecht.

\section{a) Vermittlung eines Wissensstandes unterhalb positiver Kenntnis}

Denn die angesprochenen Kosten der Vermeidung von Rechtsverletzungen sinken nicht erst, wenn eine sichere positive Kenntnis im oben beschriebenen Sinne vorliegt und bewiesen ist, sondern bereits bei einem vorsätzlichen Verschließen der Augen vor den Fakten. Das soll aber zum einen unter § 10 TMG gerade nicht erfasst werden. ${ }^{50} \mathrm{Zum}$ anderen eröffnet die Handhabung der Störerhaftung insoweit ebenfalls das Risiko, dass Diensteanbieter gerade versuchen, die Kenntnisnahme entweder aktiv zu verhindern, indem etwa das Design entsprechender Mitteilungsoptionen in diesem Sinne gewählt wird ${ }^{51}$ oder aber sich auf die Anforderungen an eine entsprechende Notifizierung berufen, mit denen die Rechtsprechung ihnen entgegenkommt. ${ }^{52} \mathrm{Je}-$ denfalls wenn infolge einer Notifizierung die Wahrscheinlichkeit einer tatsächlichen Rechtsverletzung als überwiegend erscheint, sollte eine Inanspruchnahme bei Untätigkeit des Diensteanbieters haftungsrechtliche Konsequenzen zeitigen. Der Maßstab der Kenntnis als solcher ist demgegenüber zu hoch angesetzt, weil er Beweisprobleme mit sich bringt, die bei einer hier vorgeschlagenen Absenkung des Standards so nicht mehr gegeben wären.

Fraglich ist, ob sich insoweit an die bereits diskutierte Aussage des EuGH aus der Entscheidung L'Oréal anknüpfen ließe, der mit Blick auf die Privilegierung der Diensteanbieter festgehalten hatte, dass es bei der insoweit erforderlichen fahrlässigen Unkenntnis auf die Sichtweise eines „sorgfältigen Wirtschaftsteilnehmers" ankomme. ${ }^{53}$ Das hängt davon ab, worauf diese Hal-

\footnotetext{
${ }^{49} \mathrm{Vgl}$. oben $\S 10$ Fn. 7.

${ }^{50}$ Vgl. oben $\S 4$ D. II. 2. b) aa).

${ }^{51}$ Vgl. insoweit oben $\S 8$ A. II. 2. a).

${ }^{52} \mathrm{Vgl}$. oben $\S 10$ B. IV. 1.

${ }^{53}$ Vgl. EuGH, Urt. v. 12.11.2011 - C-324/09, MMR 2011, 596 (Rn. 120) - L'Oréal SA.
} 
tung des Gerichtshofs letztlich abzielt. Versteht man sie als Erweiterung des Pflichtenkreises gegenüber der üblichen Kenntnis, wie sie durch Art. 14 Abs. 1 lit. a Var. 1 ECRL geregelt ist, wäre eine Erweiterung der Möglichkeiten der Kenntnisnahme in diese Richtung denkbar. Sieht man dagegen das Kriterium des „sorgfältigen Wirtschaftsteilnehmers“ schon jetzt als mit dem Kenntnismaßstab der Störerhaftung identisch an, ${ }^{54}$ ist dieses für das hiesige Anliegen unbrauchbar. Denn dann gälte schon unter Art. 14 ECRL, was hier bereits mit Blick auf die Störerhaftung kritisiert wurde, nämlich die unangemessen hohe Substanziierungslast, wenn der Notifizierende die rechtliche Subsumtionsarbeit selbst leisten muss. ${ }^{55}$ Es wurde aber bereits festgehalten, dass das Kriterium des ,sorgfältigen Wirtschaftsteilnehmers" richtig verstanden nur auf Schadensersatzansprüche gemünzt ist. ${ }^{56}$ Hinsichtlich der Unterlassungsansprüche erfordert Art. 14 ECRL dagegen die gewöhnliche positive Kenntnis. Dies wäre aber nach hier vertretener Auffassung aber gerade der Ansatzpunkt zur Modifizierung des Haftungsrechts der Intermediäre, die sich dann konsequenterweise auch auf $\S 10$ TMG beziehen müsste.

\section{b) Bezugspunkt der Kenntnis}

Die hier eingenommene kritische Haltung gegenüber einem strengen Kenntnismaßstab gilt insbesondere, soweit die Kenntnis unter Art. 14 ECRL, § 10 TMG sich nach dem heutigen Stand auch auf die Rechtswidrigkeit der Information beziehen muss und dementsprechend an eine Notifizierung durch einen Nutzer entsprechende Hürden gestellt werden. ${ }^{57}$ Gerade diese Hürden hinsichtlich der Kenntnisverschaffung und auch hinsichtlich der Rechtswidrigkeit entsprechen ebenso wenig dem Maßstab des „kostensenkenden“ Informationsstandes. Die Kosten der Vermeidung von Rechtsverletzungen sind nämlich bei Lichte betrachtet ebenfalls gering, wenn die Kenntnis allein hinsichtlich der Tatsachengrundlage vermittelt wird und gerade nicht erst dann, wenn die Kenntnis auch hinsichtlich der Rechtswidrigkeit vermittelt wird, wie es aber die überwiegende Auffassung bislang verlangt. ${ }^{58}$ Eine solche Interpretation bevorteilt jene Diensteanbieter, die bewusst keine Anstrengungen unternehmen, juristischen Sachverstand vorzuhalten ${ }^{59}$ während Nutzerinnen gerade zu dessen Einsatz gezwungen werden, wollen sie eine rechtssichere Notifizierung an einen Diensteanbieter übermitteln.

${ }^{54}$ So Frey, Die Haftung von Host-Providern für Immaterialgüterrechtsverletzungen, S. 282.

${ }^{55} \mathrm{Vgl}$. oben $\S 10$ B. IV. 1.

${ }^{56}$ Vgl. oben $\$ 4$ C. III. 1. e), insb. §4 Fn. 136.

${ }^{57}$ Vgl. dazu schon oben $\S 10$ B. IV. 1.

${ }^{58} \mathrm{Vgl}$. dazu oben $\S 4$ D. II. 2. b) bb).

${ }^{59}$ Deutlich auch Spindler, in: Spindler/Schmitz, TMG, § 10 Rn. 30; vgl. zur übergeordneten Frage nach dem Vorhalten juristischen Sachverstands oben $\S 10$ B. IV. 1. b). 


\section{Fortentwicklung der Substanziierungslasten im Rahmen der Störerhaftung}

Zur Störerhaftung wurde bereits festgestellt, dass die konkreten Anforderungen an eine Notifizierung, die eine Inanspruchnahme begründen soll, zu einem unangemessenen Kräfteverhältnis zwischen Diensteanbieter und den Nutzern bzw. den Notifizierenden führen. Die „unschwere“ Erkennbarkeit einer Rechtsverletzung ist in diesem Sinne sowohl für die Diensteanbieter als auch für die Notifizierenden nachteilhaft, weil einerseits hiermit schwierige Subsumtionsfragen verbunden sind, andererseits die Begründungslast unangemessen hoch ist, sodass die „Kosten für die Vermeidung der Rechtsverletzung" $\left(\right.$ Wagner $\left.^{60}\right)$ insgesamt zu hoch ausfallen.

Eine angemessenere Verteilung der Substanziierungslasten sollte also dementsprechend den Maßstab, ab wann die im Rahmen des Prüfprogramms ohnehin zur rechtlichen Wertung ,verpflichteten“" ${ }^{\text {“1 }}$ Diensteanbieter diese Prüfung durchführen müssen, absenken. Insbesondere der Maßstab der „unschwer" erkennbaren Rechtsverletzungen sollte insoweit eine Präzisierung erfahren, die die hier angesprochenen Punkte aufgreift. Dementsprechend sollte hierzu eine Obliegenheit des Diensteanbieters in Erwägung gezogen werden, im Falle nicht „unschwer" erkennbarer Rechtsverletzungen dem Notifizierenden Gelegenheit zu geben, seinen Hinweis zu konkretisieren. ${ }^{62}$ Denn liegt eine Notifizierung vor, aus der Anhaltspunkte für ein womöglich berechtigtes Anliegen erkennbar hervorgehen, wäre die gleichsam „blinde“ Berufung auf die fehlende unschwere Erkennbarkeit problematisch. Ist eine zivilrechtliche Sonderbeziehung zwischen der Notifizierenden und dem Diensteanbieter nicht gegeben - im Falle der Verletzung von Urheberrechten oder verwandten Schutzrechten dürfte dies eher die Regel sein - ließe sich dies in zweierlei Weise begründen. Entweder wäre die fehlende Rückfrage als Versto $ß$ gegen das Verbot treuwidrigen Verhaltens zu bewerten, wie es der BGH in der Entscheidung Stiftparfüm tat. ${ }^{63}$ Oder aber die Diensteanbieter unterfallen insoweit dem in diesem Sinne erweiterten Konzept der Prüfpflichten, wonach insbesondere Zumutbarkeitsaspekte die Pflicht zur

\footnotetext{
${ }^{60}$ Vgl. oben $\S 11$ Fn. 48 und den zugehörigen Text.

${ }^{61}$ Tatsächlich handelt es sich um eine Obliegenheit gegen sich selbst, vgl. oben $\S 10$ Fn. 50 .

${ }^{62}$ Vgl. auch Chmelik, Social Network Sites - Soziale Netzwerke, S. 176 mit Fn. 672; Holznagel, Notice and Take-Down-Verfahren als Teil der Providerhaftung, S. 142.

${ }^{63}$ Vgl. BGH, Urt. v. 17.08.2011 - I ZR 57/09, MMR 2012, 178 (Rn. 32 f.) - Stiftparfüm, der die Treuepflicht aus dem sich durch die Abmahnung ergebenden Verhältnis ableitet; freilich lag dort insofern eine Sonderkonstellation vor, als eine - vorgeblich - nicht hinreichend begründete Notifizierung vom Diensteanbieter eBay nicht nur nicht bestritten, sondern ausdrücklich zum Anlass genommen wurde, eine beanstandete Auktion zu beenden; kritisch dazu Holznagel, Notice and Take-Down-Verfahren als Teil der Providerhaftung, S. 131 .
} 
Rückfrage begrenzen..$^{64}$ Besteht hingegen eine Sonderbeziehung, wie es etwa bei Persönlichkeitsrechtsverletzungen öfters der Fall sein kann, weil die Notifizierenden etwa ebenfalls registrierte Nutzer eines Kommunikationsdienstes sind, lässt sich erst recht eine solche Treuepflicht begründen. Lässt die Beschwerde dagegen keinerlei Anhaltspunkte dafür erkennen, dass ihr ein berechtigtes Anliegen zugrunde liegt oder ist sie gar nicht begründet, dürfte eine solche Einräumung zur Präzisierung durch den Diensteanbieter jedenfalls rechtlich nicht gefordert sein. Räumt der Diensteanbieter aber diese Möglichkeit zur Präzisierung ein, muss er sich hieran auch festhalten lassen.

Die hier vorgeschlagene Modifizierung der Störerhaftung würde den Spielraum der Intermediäre, sich absichtlich einem Tätigwerden im soeben beschriebenen Sinne zu entziehen, bedeutend einengen. ${ }^{65}$ Zugleich ließe dies das grundlegende Prinzip, demzufolge eine nachlaufende gerichtliche Kontrolle rechtlich möglich bliebe ${ }^{66}$ unberührt. Letzterer Punkt setzt freilich voraus, dass den ggf. betroffenen Primärverursachen einer Rechtsverletzung entsprechende rechtliche Mittel eingeräumt werden. ${ }^{67}$ In diese Richtung weisen in der Tat viele Gerichtsentscheidungen der jüngeren Vergangenheit, die Dienstenutzern einen Anspruch auf Wiederherstellung von Inhalten einräumen, deren Entfernung sich als rechtswidrig erweist. ${ }^{68}$

\section{Umgang der Diensteanbieter mit Notifizierungen}

In der Perspektive auf das Recht als Anreizstruktur ist im Kontext der Haftungsprivilegierungen von maßgeblicher Bedeutung, welche Anforderungen an den Umgang mit einer Notifizierung durch den Diensteanbieter gestellt werden. In diesem Zusammenhang ist entscheidend, zu welchen Handlungen das Recht den Diensteanbieter motiviert, der eine Notifizierung über eine Rechtsverletzung erhalten oder anderweitig Anhaltspunkte für eine solche erlangt hat. Im Kontext der Störerhaftung wurde herausgearbeitet, inwiefern das von der Rechtsprechung entwickelte Prüfprogramm geeignet ist, insbesondere durch das dort beschriebene Stellungnahmeverfahren eine Wahrung der Interessen nicht nur der von einer potenziellen Rechtsverletzung Betroffenen, sondern auch der mutmaßlichen Primärverursacher herzustellen. ${ }^{69}$

\footnotetext{
${ }^{64}$ So der Sache nach LG München I, Urt. v. 11.01.2006 - 21 O 2793/05, MMR 2006, 332 (334).

${ }^{65}$ Vgl. aber Mayen, ZHR 2018, 1 (5f.), der unzutreffend in dem Kriterium der „unschweren Erkennbarkeit" ein Spezifikum der mittelbaren Grundrechtsbindung sieht (und einen materiellen Übergang zur unmittelbaren Bindung teilweise für geboten zu sehen scheint).

${ }^{66}$ Für den Rechtsschutz des Notifizierenden ausdrücklich BGH, Urt. v. 23.09.2014 - VI ZR 358/13, NJW 2015, 489 (Rn. 36) - Ärztebewertungsportal II.

${ }^{67}$ So auch das Petitum von Hofmann, ZUM 2017, 102 (108).

${ }^{68} \mathrm{Vgl}$. die Nachweise oben in $§ 11$ Fn. 20.

${ }^{69} \mathrm{Vgl}$. oben $\S 10$ B. III. 1.-2.
} 
Eine der Rechtslage in Deutschland ähnliche Entwicklung ist auf der unionsrechtlichen Ebene bislang nur in Ansätzen zu erkennen. In diesem Sinne scheint die ECRL ein Notice-and-Takedown-Verfahren vorauszusetzen, dieses aber nicht explizit zu regeln. ${ }^{70}$ Ansatzweise scheint der EuGH Elemente einer Pflicht zur Berücksichtigung der verschiedenen betroffenen Akteure und ihrer Interessen in der Entscheidung UPC Telekabel zwar anzudeuten. Dort betont der Gerichtshof, dass die vom Schuldner einer Untersagungsverfügung, hier einem Access-Provider, getroffenen Maßnahmen nicht nur „hinreichend wirksam“ im Hinblick auf die Interessen des Inhabers eines Schutzrechts sein müssten, sondern auch die Grundrechte der Internetnutzer in den Blick zu nehmen hätten, die von einer solchen Untersagungsverfügung betroffen sein könnten. ${ }^{71}$ Das entspricht allerdings nicht dem Schutzniveau, das der BGH im Rahmen der Störerhaftung hinsichtlich der grundrechtlichen Interessen der von einer Inanspruchnahme betroffenen Nutzer insbesondere durch spezifische Stellungnahmerechte entfaltet. ${ }^{72} \mathrm{Au}-$ Berdem ist es auch keineswegs gesichert, dass der EuGH auf Grundlage des geltenden Rechtsrahmens zu einer ähnlichen Ausgestaltung kommen könnte, insbesondere da Erwägungsgrund 46 S. 2 ECRL es gerade den Mitgliedstaaten überlässt, ,spezifische Anforderungen vorzuschreiben“, die vor der Entfernung bzw. Sperrung eines Inhalts zu erfüllen sind.

Damit ergibt sich als weiteres Petitum, entsprechende Anreize insbesondere in Zusammenhang mit Art. 14 ECRL und § $10 \mathrm{TMG}$ zu positivieren, die zu einer Absicherung der Interessen aller betroffenen Nutzer führen können. Das ließe sich im Bereich des Persönlichkeitsrechts, das nach wie vor primär in der Regelungszuständigkeit der Mitgliedstaaten liegt, durch die Pflicht zur Berücksichtigung der Interessen aller Beteiligten bei der Ermittlung der Rechtswidrigkeit eines Inhalts bewerkstelligen, wozu explizit die Position des von einer Entfernung oder Sperrung Betroffenen zu zählen wäre. Dadurch wäre einerseits die Zuständigkeit der Mitgliedstaaten für die Regelung des Haftungsgrundes materiell abgesichert, während andererseits die haftungsrechtliche Regelung für Diensteanbieter auf der Ebene des Unionsrechts ein entsprechendes Mindestmaß garantieren könnte.

Abschließend erwähnt sei die Frage nach den Konsequenzen einer unberechtigten Notifizierung. Mit Blick auf Kommunikationsrechte kann sich diese als potenziell problematischer Faktor erweisen. Insbesondere soweit

\footnotetext{
${ }^{70} \mathrm{Vgl}$. auch Kuczerawy, Intermediary Liability and Freedom of Expression in the EU, S. 99.

${ }^{71}$ EuGH, Urt. v. 27.03.2014 - C-314/12, GRUR 2014, 468 (Rn. 57, 62 f.) - UPC Telekabel.

${ }^{72}$ Vgl. aber Spindler, MMR 2018, 48 (52), der bereits von einer „,verfahrensrechtlichen Absicherung" spricht, dies allerdings ersichtlich nicht auf ein dem Stellungnahmeverfahren des BGH äquivalentes Schutzniveau münzt.
} 
man annimmt oder sogar belegen kann, dass unberechtigte Notifizierungen überdurchschnittlich häufig zu einer unberechtigten Entfernung führen können, liegt hier die Gefahr von „, chilling effects“ für die Meinungsfreiheit nicht ganz fern. ${ }^{73}$ Die Erörterung der vielfältigen Anspruchsgrundlagen, um einen Anspruch des Inhaltsverantwortlichen gegen eine unberechtigte Notifizierung zu begründen, würde den Rahmen dieser Untersuchung sprengen. Grundsätzlich denkbar und insbesondere in Konstellationen mit äußerungsrechtlichem Hintergrund relevant sind deliktische Ansprüche nach den $\S \S 823$ ff. BGB. ${ }^{74}$ Insoweit bleibt hier nur Raum für zwei Bemerkungen. Erstens können potenzielle Ansprüche gegenüber den unberechtigt Notifizierenden nur dann in Betracht kommen, wenn die Beschwerde in bewusster bzw. missbräuchlicher Weise unberechtigt an den Diensteanbieter gerichtet wurde. Denn nach der hier dargelegten Auffassung muss gerade im Falle von möglichen Anhaltspunkten für ein berechtigtes Anliegen eine Notifizierung mit entsprechenden Reaktionsobliegenheiten der Diensteanbieter möglich sein. Gibt es tatsächliche Anhaltspunkte dafür, dass der Notifizierung ein berechtigtes Anliegen zugrunde liegt, kann eine Haftung des Notifizierenden damit nicht überzeugen. Zweitens bedarf es besonderer Zurückhaltung bei der Gewährung eines entsprechenden Anspruchs gegen den Notifizierenden. ${ }^{75}$ Dies gilt insbesondere, soweit es um die bewusst unberechtigte Notifizierung in äußerungsrechtlichen Konstellationen geht, bei denen vor allen Dingen das Allgemeine Persönlichkeitsrecht eine Rolle spielt.

\section{Die Zukunft des Verbots allgemeiner Überwachungspflichten}

Die Entwicklung eines Reformpotenzials für das Haftungsrecht der Intermediäre wäre nicht vollständig, ohne die Konsequenzen mitzudenken, die sich durch diese Fortentwicklung für das Verbot allgemeiner Überwachungspflichten nach Art. 15 ECRL bzw. §7 Abs. 2 TMG ergeben. Insbesondere stellt sich die Frage, wie sich eine explizite Verankerung einer „Good Samaritan"-Regel auf das in den genannten Normen niedergelegte Prinzip verhielte (1.). De lege ferenda sind ferner Modifizierungen der geltenden Rechtslage sinnvoll, um das Verbot allgemeiner Überwachungspflichten rechtssi-

${ }^{73}$ Vgl. Holznagel, Notice and Take-Down-Verfahren als Teil der Providerhaftung, S. 170; Kuczerawy, Intermediary Liability and Freedom of Expression in the EU, S. $100 \mathrm{f}$.

${ }^{74}$ Vgl. umfassend zu möglichen Anspruchsgrundlagen Holznagel, Notice and TakeDown-Verfahren als Teil der Providerhaftung, S. $183 \mathrm{ff}$.

${ }^{75}$ Vgl. BGH, Urt. v. 12.12.2006 - VI ZR 224/05, NJW 2007, 1458 (Rn. 17): „Der Auffassung, die unberechtigte Geltendmachung einer Forderung stelle regelmäßig eine Verletzung des allgemeinen Persönlichkeitsrechts dar [...], kann nicht gefolgt werden.“ 
cher und mit Blick auf die Bedeutung und Funktion der Intermediäre angemessen fortzuentwickeln (2.-3.).

\section{Auswirkungen eines verstärkten Anreizes zur privaten Kontrolle im Sinne einer „, Good Samaritan“-Regel}

Es wurde soeben bereits darauf hingewiesen ${ }^{76}$ dass die Aufnahme einer Anreizstruktur zur besseren unternehmensseitigen Kontrolle der von den Diensteanbietern transportierten Inhalte durch eine „Good Samaritan“Regelung sich sinnvoll in eine modifizierte Zwecksetzung der Haftungsprivilegien integrieren ließe. Hinsichtlich des Grundsatzes, dass den Diensteanbietern keine allgemeinen Überwachungspflichten auferlegt werden dürfen, entstünde durch eine solche Regelung auch kein Zielkonflikt. Zwar ließe sich mit Blick auf den Aspekt des Schutzes der Kommunikationsfreiheiten durch das Verbot zu weitreichender Überwachungspflichten ${ }^{77}$ argumentieren, dass ein entsprechender Anreiz eine womöglich ebenso problematische mittelbare Verkürzung grundrechtlich verbürgter Freiheit darstellte. Allerdings ginge es insoweit eben nur um einen Anreiz, keine gesetzliche Pflicht. Zudem ist zu berücksichtigen, dass die Überwachung aus eigenem Antrieb bereits aktuelle Praxis vieler Diensteanbieter ist, die unter dem Stichwort „Content Moderation“ diskutiert wird. Der EuGH hält es mit Blick auf Art. 14 ECRL jedoch für unproblematisch, dass der Diensteanbieter „auf Grund einer aus eigenem Antrieb vorgenommenen Prüfung" Kenntnis von einem rechtswidrigen Inhalt erlangt. ${ }^{78}$ Ohne eine „Good Samaritan“-Regelung bewegen sich Diensteanbieter mit einem ,aktiven“ Einsatz von Überwachungsinstrumenten aktuell in einer heiklen Grauzone, in der ihnen eine Haftung aufgrund dann zu bejahender Kenntnis von einzelnen Rechtsverletzungen droht. Dies ist kein befürwortenswerter Zustand, weil er einen Anreiz zum „Blindflug“ darstellt. ${ }^{79}$ Die Einführung einer „Good Samaritan“Regelung sollte also idealiter negative Konsequenzen für einen konsequenteren und offenen Einsatz von Überwachungsinstrumenten beseitigen. Die Anerkennung von Wiederherstellungsansprüchen bei zu Unrecht entfernten Inhalten reflektiert zugleich die Freiheitssphäre der betroffenen Nutzer, sodass diese auch bei entsprechend erfolgten Entfernungen nicht schutzlos gestellt sind. ${ }^{80}$

\footnotetext{
${ }^{76} \mathrm{Vgl}$. oben $\S 11$ B. II.

${ }^{77}$ Vgl. Directorate General of Human Rights and Legal Affair of the Council of Europe, Human rights Guidelines for Internet Service Providers, S. 3; Kuczerawy, Intermediary Liability and Freedom of Expression in the EU, S. 63.

${ }^{78}$ Vgl. EuGH, Urt. v. 12.11.2011 - C-324/09, MMR 2011, 596 (Rn. 122) - L'Oréal SA (Hervorh. v. Verf.).

${ }^{79}$ Vgl. oben $\S 10$ B. IV. 1. b).

${ }^{80}$ Vgl. die Nachweise oben in $§ 11$ Fn. 20.
} 
$\mathrm{Zu}$ spezifizieren ist allerdings noch, in welchem Verhältnis eine solche „Good Samaritan“-Regel zu zulässigen Verhinderungspflichten betreffend kern- bzw. sinngleicher Inhalte infolge bereits zur Kenntnis gebrachter rechtswidriger Inhalte stünde. Insoweit besteht zwar aktuell kein Zielkonflikt mit dem Verbot der allgemeinen Überwachungspflichten, wie es sich derzeit aus der dargestellten Rechtsprechung ergibt. Beließe man es aber bei einer pauschalen „Belohnung“ redlicher Eigenbemühungen, differenzierte man also nicht zwischen präventiven Eigenbemühungen und solchen Maßnahmen, die zur Verhinderung kern- oder sinngleicher Inhalte infolge bereits spezifizierter und zur Kenntnis gebrachter rechtswidriger Inhalte vorgenommen werden, ${ }^{81}$ würde eine „Good Samaritan“-Regel die bereits bestehende, mit Art. 15 ECRL vereinbare Pflicht unterlaufen, zur Kenntnis gebrachte Inhalte zu entfernen. Damit verbunden wäre eine Absenkung des Schutzniveaus de lege lata, da der entsprechende Anspruch der Dienstenutzer nunmehr verneint werden müsste. Dies ist deshalb abzulehnen. Eine Belohnung von redlichen Eigenbemühungen mit einer Haftungsfreistellung sollte sich sinnvoller Weise nur auf die Privilegierung gegenüber solchen rechtswidrigen Inhalten beziehen, die nicht durch einen Dienstenutzer spezifiziert zur Kenntnis gebracht wurden. Damit geht es ausschließlich um solche Inhalte, die der Diensteanbieter nur durch eigene Bemühungen auffindet. In diesem Umfang wäre ein verbessertes Maß an Rechtsdurchsetzung erreicht, das zugleich mit der Rechtsprechung des EuGH zu Art. 15 ECRL vereinbar wäre.

\section{Ausgestaltung der Reichweite des Verbots}

Ferner wäre es sinnvoll, die materielle Reichweite eines Verbots im Sinne von Art. 15 Abs. 1 ECRL genauer zu spezifizieren, die eine bessere Handhabung der Unterscheidung von ,allgemeinen“ und „spezifischen“ Überwachungspflichten ermöglichte. Dabei wäre zum einen zu klären, inwiefern die Überwachung ,aller" Inhalte, wie sie der EuGH bislang pauschal einem Verbot zu unterstellen scheint ${ }^{82}$ in gewissen Fällen nicht doch auch zulässig sein könnte. Denn nicht zuletzt in den SABAM-Entscheidungen ging es um eine rein präventive und pauschale Filterung, während in anderen Fallgestaltungen ggf. eine reaktive, aber dennoch recht breite Verhinderungspflicht angenommen werden könnte. ${ }^{83}$ Holznagel argumentiert insoweit überzeugend, dass es „trotz Art. 15 [ECRL] bei Pflichten zum Auffinden spezifischer Verletzungen zu einer Prüfung aller Inhalte/Nutzer kommen darf", da schon

\footnotetext{
${ }^{81}$ Auf diese Konsequenz liefe der Vorschlag von Holznagel, Notice and Take-DownVerfahren als Teil der Providerhaftung, S. 249, wohl hinaus.

${ }^{82}$ Vgl. EuGH, Urt. v. 12.11.2011 - C-324/09, MMR 2011, 596 (Rn. 139) - L’Oréal SA; Urt. v. 16.02.2012 - C-360/10, MMR 2012, 334 (Rn. 34) - SABAM/Netlog.

${ }^{83}$ Vgl. auch Kastl, GRUR 2016, 671 (676).
} 
begrifflich nicht klar zwischen einer Prüfung sämtlicher bzw. aller und weniger als aller Inhalte zu unterscheiden sei. ${ }^{84}$ In der Tat hängt dies maßgeblich von der jeweiligen Fallgestaltung und den konkret betroffenen Rechtsgütern $\mathrm{ab}$, die es zu beurteilen gilt. Gerade die Entscheidung Glawischnig-Piesczek zeigt, dass auch eine recht detailreiche Spezifizierung von bestehenden Unterlassungspflichten durch das vorlegende Gericht den EuGH nicht davon abhält, in der Sache recht weitgehende Verhinderungspflichten zur Ermittlung von Inhalten mit „im Wesentlichen“ gleichen Aussagen vorzusehen. Nach hier vertretener Auffassung ändern die vom Gerichtshof vorgenommenen Einschränkungen ${ }^{85}$ nichts an diesem Eindruck. Es ergibt sich damit eine recht komplexe Rechtslage schon auf der Ebene des Unionsrechts. Bei einer Reform der ECRL wäre eine Vereinfachung bzw. klarere Abgrenzung daher erforderlich. Unabhängig davon wäre auch eine genauere sekundärrechtliche Eingrenzung der von Art. 15 ECRL erfassten Sachverhalte hilfreich, um den Erwartungshorizont für Diensteanbieter wie auch -nutzer verständlicher zu machen.

Zum anderen und in Zusammenhang mit den bisherigen Einsichten kommt eine sachangemessene, vor allen Dingen die Realität der technischen Machbarkeit von Filtersystemen achtende Handhabung von Verhinderungspflichten jedenfalls nicht umhin, den normativen Maßstab solcher Pflichten auch anhand sachverständiger Beratung über den Stand und die Entwicklungsperspektiven entsprechender Systeme auszurichten. ${ }^{86}$ Vor diesem Hintergrund überzeugt insbesondere die in Glawischnig-Piesczek erfolgte Konkretisierung der Reichweite von Art. 15 ECRL nicht. Zu begrüßen wäre bei einer Reform dieser Regelung eine Pflicht zur Berücksichtigung von einzelfallbezogenen Faktoren bzw. jedenfalls die Spezifizierung seiner Anforderungen, die unter bestimmten Geschäftszweigen differenzieren könnte, sowie eine Ausrichtung entsprechender Pflichten an dem Stand der Technik.

\section{Zulässigkeit manueller Kontrollen}

Eine weitere Forderung ergibt sich nach den bisherigen Ausführungen zur Reichweite des Verbots allgemeiner Überwachungspflichten hinsichtlich deren konkreter Ausgestaltung. Der EuGH vollzieht in Glawischnig-Piesczek nämlich eine problematische ${ }^{87}$ Wendung hin zu ausschließlich automatisierten Kontrollen auf wort- und sinngleiche Wiederholungen von Rechtsverletzungen. Insoweit scheint der EuGH der Auffassung zu sein, dass nur mit-

${ }^{84}$ Holznagel, ZUM 2018, 350 (355).

${ }^{85}$ Vgl. EuGH, Urt. v. 03.10.2019 - C-18/18, NJW 2019, 3287 (Rn. 45) - GlawischnigPiesczek.

${ }^{86}$ Vgl. Holznagel, ZUM 2018, 350 (356).

${ }^{87} \mathrm{Vgl}$. dazu schon oben $\S 4$ C. IV. 2. a). 
tels eines strengen Verbots der manuellen Nachkontrolle ein Overblocking verhindert werden kann, ohne dies ausdrücklich auszusprechen. ${ }^{88}$ Das mag zwar ein im Prinzip nachvollziehbares Motiv sein. Damit nimmt der EuGH aber den Gerichten der Mitgliedstaaten ein wesentliches Mittel aus der Hand, um Verhinderungspflichten angemessen auszugestalten und die bislang jedenfalls noch nicht vollständig vorhersehbare und beherrschbare Funktionalität von Filtersystemen noch einer manuellen Nachkontrolle zu unterstellen. Insbesondere wenn es um Persönlichkeitsrechtsverletzungen geht, kann eine automatisierte Kontrolle jenseits von klaren, allein anhand der verwendeten Begriffe erkennbaren Fälle nach bisherigem Kenntnisstand eine solche Einordnung noch nicht eigenständig vornehmen. Die „Streubreite“ solcher automatisierten Techniken im Hinblick auf die Erfassung von Falschpositiven, also unzutreffend als rechtswidrig bewerteten Inhalten, kann damit jedenfalls noch aktuell als relevanter Faktor angesehen werden. ${ }^{89}$ Insoweit liegt Holznagel auch richtig, wenn er als zweites denkbares Szenario, bei dem keine ,autonome Beurteilung" im Sinne einer Nachkontrolle notwendig ist, die rechtliche Zulässigkeit der Erfassung von rechtmäßigen Inhalten im Sinne eines Overblockings in Erwägung zieht. ${ }^{90}$ Denn auch in diesem Szenario ist eine autonome Beurteilung nicht denklogisch notwendig, obwohl es praktisch gedacht unwahrscheinlich erscheint, dass eine solche rechtliche Zulässigkeitserklärung ohne einen Vorbehalt der menschlichen Nachprüfung ergehen könnte. Selbst wenn man ein solches Overblocking in geringem Umfang noch für akzeptabel halten sollte, was derzeit offen ist, ${ }^{91}$ kann ein prinzipieller Ausschluss manueller Nachkontrollen allerdings unter dem aktuellen Sach- und Kenntnisstand nicht überzeugen, weil er eine einzelfallbezogene Differenzierung eher verhindert als ermöglicht. ${ }^{92}$ Eine künf-

${ }^{88}$ Insoweit muss die Entscheidung vor dem Hintergrund der aktuellen Debatte gelesen werden, in der autonomen Systemen eine nur unzuverlässige Prüfungskompetenz hinsichtlich der Rechtmäßigkeit von Inhalten unterstellt wird. Dies meint wohl auch Holznagel, ZUM 2019, 910 (911).

${ }^{89}$ Vgl. aber zur Entwicklungsoffenheit dieses Arguments Rademacher, JZ 2019, 702 (706f.).

${ }^{90}$ Vgl. Holznagel, ZUM 2019, 910 (911).

${ }^{91}$ Vgl. BGH, Urt. v. 12.07.2012 - I ZR 18/11, NJW 2013, 784 (Rn. 45) - Alone in the Dark, mit dem Verweis auf die Möglichkeit der Diensteanbieter, sich insoweit vertragsrechtlich gegenüber betroffenen Nutzern abzusichern; deutlich kritischer allerdings das Urt. v. 27.02.2018 - VI ZR 489/16, NJW 2018, 2324 (Rn. 35) - Internetforum. Vgl. noch unten $\S 12$ Fn. 48 und den zugehörigen Text.

${ }^{92}$ So auch Holznagel, ZUM 2019, 910 (912); zu den Vorteilen „hybrider“ Entscheidungssysteme, bei denen der menschlichen Bewertung weiterhin eine wichtige Rolle zukomme, vgl. auch Wu, Columbia Law Review 119 (2019), 2001 (2020 ff.) (der allerdings unter den menschlichen Aspekt nur die judikative Perspektive fasst, was hier nicht geteilt wird). 
tige Neuregelung sollte also in Abhängigkeit der konkret zu regelnden Materie und unter Berücksichtigung des jeweils geltenden Standes der Technik eine manuelle Nachkontrolle vorsehen bzw. jedenfalls explizit zulassen.

\section{F. Fazit}

Zusammenfassend lässt sich festhalten, dass das Recht der Haftung der Intermediäre in vielerlei Hinsicht Reformpotenzial in sich birgt, um eine angemessenere Berücksichtigung aller von einer Inanspruchnahme eines Diensteanbieters betroffenen Interessen zu erreichen. Im Kontext der hier vertretenen Position zur Rechtsprechung des BGH erweisen sich dabei wenig überraschend vor allen Dingen die zur Störerhaftung entwickelten Kriterien als im Ansatz geeignet, um das Regime der ECRL und des TMG zu ergänzen. Betonung verdient hier die übergeordnete Einsicht, dass Telos und Anreizstrukturen des Telemedienrechts die Rolle der Intermediäre angemessener berücksichtigen könnten, als es heute der Fall ist.

Die hier vorgeschlagenen Anpassungen im Bereich der Art. 14 ECRL, § 10 TMG gewinnen an Dringlichkeit, wenn man sich die Wahrscheinlichkeit vor Augen führt, dass der BGH aus unionsrechtlichen Gründen gehalten sein könnte, seine Rechtsprechung zur Störerhaftung zu überdenken.${ }^{93}$ Es ist danach insbesondere nicht ausgeschlossen, dass der EuGH die Störerhaftung in ihrer oben erläuterten Ausprägung mit Blick auf die Harmonisierungsfunktion der ECRL in Zweifel ziehen oder in ihrer Wirksamkeit beschränken könnte, um ein einheitliches Haftungsregime herzustellen. Selbst wenn man dies anders sehen wollte, wäre eine Verankerung der hier vorgetragenen Vorschläge im positiven Recht aus Gründen der Rechtsklarheit und Transparenz zu befürworten.

Die hier betonten Potenziale einer präziseren Fassung der Haftungsprivilegierungen sollten aber nicht den Eindruck vermitteln, es ginge nur um eine schärfere Haftung der Diensteanbieter. Es lässt sich zwar nicht abstreiten, dass die Haftungsprivilegierungen insoweit relativiert sind, als Unterlassungsansprüche in immer größerem Ausmaß von einer Privilegierung ausgenommen sind. ${ }^{94}$ Es obliegt allerdings ebenso der rechtspolitischen Entscheidungshoheit des europäischen Gesetzgebers, die Privilegierung bestimmter Dienste in bestimmten Situationen positiv zu reglementieren ${ }^{95}$ Sinnvoll umgesetzt kann dies ebenso zu einer verbesserten Anreizstruktur und damit

${ }^{93}$ Vgl. dazu schon oben $\S 10$ A. IV. 4. b) aa), V. 2.

${ }^{94} \mathrm{Vgl}$. kritisch zu dieser Perspektive aber oben $\S 11 \mathrm{~A}$.

${ }^{95}$ Vgl. Holznagel, jurisPR-WettbR 10/2016, Anm. 1 (D.), mit ähnlichem Petitum wie hier. 
verbunden zu einer klareren normativen Erwartung gegenüber den Intermediären führen. 



\section{Neuere sektorspezifische Zuschreibungen von Verantwortlichkeit als Modifikation der Störerhaftung}

Die bislang diskutierte Störerhaftung und die Haftungsprivilegierungen in den Art. $12 \mathrm{ff}$. ECRL und $\S \S 7 \mathrm{ff}$. TMG sind überwiegend sektorübergreifend und damit als grundsätzlich rechtsgebietsübergreifend anwendbare Instrumente konzipiert. In den letzten Jahren ist allerdings die Entwicklung dieser Grundsätze nicht auf deren Anpassung durch die Rechtsprechung beschränkt geblieben. Vielmehr haben Entwicklungen auf nationaler und auf europäischer Ebene die Gesetzgebungsorgane dazu veranlasst, Sonderregeln zu erlassen, die die Inpflichtnahme der Intermediäre unmittelbar betreffen. Bislang sind diese allerdings auf abgegrenzte Teilbereiche möglicher Rechtsverletzungen beschränkt, die durch Diensteanbieter verbreitet werden. Zum einen stellt das NetzDG in Deutschland zusätzliche Anforderungen an den Umgang mit Nutzerbeschwerden im Bereich spezifisch definierter Rechtsverstöße auf (A.). Zum anderen hat die EU bei der Reform des Urheberrechts ebenfalls neue Regelungen zur Haftung der Diensteanbieter geschaffen (B.). Um die kritische Analyse dieser Komplexe und deren Einordnung in den Kontext dieser Untersuchung soll es in diesem Abschnitt gehen.

\section{A. Die Modifizierung der Störerhaftung zur Durchsetzung des Persönlichkeitsrechts durch das NetzDG}

Zunächst soll der Hintergrund des Erlasses des NetzDG erläutert werden (I.). Sodann folgt eine Darstellung der hier entscheidenden gesetzlichen Regelungen (II.). Diese werden im Anschluss mit Blick auf den Kontext dieser Untersuchung bewertet (III.).

\section{Hintergrund}

Der rechtspolitische Entstehungsprozess zum NetzDG nahm bereits im Jahr 2015 seinen Lauf, als der Umgang mit einer Zunahme von Flucht- und Migrationsbewegungen in Deutschland und Europa die politische und gesellschaftliche Stimmung in dem Zeitraum prägte. Verstärkt nahm man gesell- 
schaftlich und politisch die aufgeheizte Debattenkultur im Netz kritisch wahr, was auch an einem immer größer werdenden Anteil der medien- und technikaffinen Bevölkerung gelegen haben mag. Der zunehmend als verroht wahrgenommene Diskurs veranlasste in Deutschland einerseits das Bundesministerium der Justiz und für Verbraucherschutz zur Einberufung einer sog. Task Force „Umgang mit rechtswidrigen Hassbotschaften im Internet“.' Andererseits handelte die Europäische Kommission im Jahr 2016 mit Facebook, Microsoft, Twitter und YouTube den „EU Code of conduct on countering illegal hate speech online“ aus, der als „soft law" diese Unternehmen zur konsequenteren Verfolgung von Beschwerden über ,illegal hate speech“ und zur Stärkung der Transparenz bei diesen Bemühungen verpflichtete. ${ }^{2}$ Beide Initiativen hatten jedoch nach Auffassung der damaligen Bundesregierung, insbesondere des damaligen Bundesministers der Justiz und für Verbraucherschutz, nicht die gewünschten Verbesserungen zur Folge, weshalb dieser den Entwurf für das NetzDG am 5. April 2017 vorlegte. ${ }^{3}$ Nach einem auch durch den Bundestagswahlkampf im Herbst desselben Jahres geprägten kontroversen Gesetzgebungsprozess wurde das NetzDG durch den Bundestag verabschiedet und trat am 1. Oktober 2017 in Kraft.

\section{Konkrete gesetzliche Regelung}

\section{Allgemeines}

Das insgesamt sechs Paragraphen umfassende NetzDG sieht neben der Regelung seines Anwendungsbereichs eine Berichtspflicht der erfassten Diensteanbieter vor, statuiert konkrete Pflichten der Netzwerkbetreiber zum Umgang mit Beschwerden über rechtswidrige Inhalte, beinhaltet Bußgeldvorschriften und verpflichtet die Anbieter zur Benennung inländischer Zustellungsbevollmächtigter für Angelegenheiten in seinem Anwendungsbereich. Dieser ist beschränkt auf Netzwerke mit mehr als zwei Millionen registrierten Nutzern $^{4}$ und definiert für das NetzDG als rechtswidrig alle Inhalte, die den Tatbestand einer in $\S 1$ Abs. 3 NetzDG aufgeführten Strafbarkeitsvorschrift des StGB ,erfüllen und nicht gerechtfertigt sind.“

\footnotetext{
${ }^{1}$ Vgl. www.bmjv.de/SharedDocs/Downloads/DE/News/Artikel/12152015_TaskForce Ergebnispapier.html.

${ }^{2}$ Vgl. ec.europa.eu/info/policies/justice-and-fundamental-rights/combattingdiscrimination/racism-and-xenophobia/eu-code-conduct-countering-illegal-hate-speechonline.

${ }^{3}$ Vgl. www.bmjv.de/SharedDocs/Gesetzgebungsverfahren/DE/NetzDG.html.

${ }^{4} \S 1$ Abs. 2 NetzDG.
} 


\section{Organisationspflichten}

Im Kontext dieser Untersuchung ist der $\S 3$ NetzDG, der den Diensteanbietern Vorgaben zum Umgang mit Beschwerden macht, von besonderem Interesse. Nach $\S 3$ Abs. 1 NetzDG müssen Netzwerkbetreiber ein „wirksames und transparentes Verfahren" zum Umgang mit Nutzerbeschwerden vorhalten. Die weiteren Absätze konkretisieren die genauen Anforderungen an dieses Verfahren. Maßgeblich ist insbesondere die Pflicht, „unverzüglich“ von Beschwerden Kenntnis zu nehmen und zu prüfen, ob die gemeldeten Inhalte rechtswidrig und deshalb zu entfernen sind bzw. ob „der Zugang zu ihnen zu sperren ist" ${ }^{5}$ Handelt es sich um einen „offensichtlich rechtswidrigen Inhalt" muss er als Grundregel innerhalb von 24 Stunden entfernt bzw. der Zugang zu ihm gesperrt werden, ${ }^{6}$ soweit nicht das „Netzwerk mit der zuständigen Strafverfolgungsbehörde einen längeren Zeitraum für die Löschung oder Sperrung des offensichtlich rechtswidrigen Inhalts vereinbart hat". . Ist der Inhalt nicht offensichtlich, sondern „lediglich" rechtswidrig, beläuft sich die Frist auf ,in der Regel“ sieben Tage nach Eingang der Beschwerde. ${ }^{8}$ Diese Frist kann auch überschritten werden, soweit es für die Bewertung der Rechtswidrigkeit auf die Unwahrheit einer Tatsachenbehauptung oder auf andere tatsächliche Umstände ankommt. In diesem Fall „kann“ der Diensteanbieter dem Nutzer - gemeint ist hier der Urheber des relevanten Inhalts ${ }^{9}$ - Gelegenheit zur Stellungnahme geben, muss dies aber nach dem Wortlaut des Gesetzes nicht. ${ }^{10}$

\section{Bußgelder}

Die organisatorischen Vorgaben zum Umgang mit Beschwerden werden in $\S 4$ NetzDG mit Bußgeldandrohungen „scharf“" gestellt. Maßgeblich ist in diesem Kontext insbesondere $\S 4$ Abs. 1 Nr. 2 NetzDG, der die Möglichkeit der Verhängung eines Bußgelds vorsieht, wenn das in $\S 3$ Abs. 1 S. 1 ,nicht, nicht richtig oder nicht vollständig" vorgehalten wird. Die erste Alternative betrifft damit die Existenz eines Verfahrens im Sinne des $\S 3$ NetzDG überhaupt. Die zweite Alternative betrifft das Abweichen von den in $\S 3$ vorgesehenen materiellen Vorgaben. Die dritte Alternative sanktioniert das Fehlen einzelner in $\S 3$ NetzDG vorgesehener Verfahrensschritte. ${ }^{11}$ Alle Alternativen

${ }^{5} \S 3$ Abs. 2 Nr. 1 NetzDG.

${ }^{6} \S 3$ Abs. 2 Nr. 2 Hs. 1 NetzDG.

${ }^{7} \S 3$ Abs. 2 Nr. 2 Hs. 2 NetzDG.

${ }^{8} \S 3$ Abs. 2 Nr. 3 Hs. 1 NetzDG.

${ }^{9}$ Vgl. Liesching, in: Spindler/Schmitz, TMG, NetzDG $§ 3$ Rn. 23, der darauf hinweist, dass sich das Recht des „Beschwerdeführers“ zur Reaktion auf diese Stellungnahme in der Regel aus der Rechtsprechung des BGH zur Störerhaftung ergeben dürfte.

${ }^{10} \S 3$ Abs. 2 Nr. 3 Hs. 2 lit. a NetzDG.

${ }^{11}$ Vgl. Schiff, MMR 2018, 366 (369). 
dieses Bußgeldtatbestands erfassen damit nur die systemische „non-compliance" und sanktionieren gerade nicht das Verhalten eines Diensteanbieters in einem bestimmten Fall einer Nutzerbeschwerde. ${ }^{12}$

\section{Bewertung vor dem Hintergrund der Verantwortung der Intermediäre}

Im Folgenden soll eine Bewertung des NetzDG vorgenommen werden. Angesichts der Breite der Diskussion bleibt diese auf die im Kontext dieser Untersuchung zentralen Punkte beschränkt. ${ }^{13}$ Ein besonderes Augenmerk verdienen die Verhaltenspflichten der Diensteanbieter, die eine neue Ausgestaltung erfahren haben. Auf die insbesondere in der Frühphase der Diskussion vor und nach Verabschiedung besonders heftig formulierte Kritik ${ }^{14}$ wird nur eingegangen, soweit die Zwecke der Untersuchung dazu Anlass geben.

\section{Positivierung der Verantwortung der Intermediäre durch Anknüpfung an die Störerhaftung}

Mit dem NetzDG hat der Bund Regelungen zum Umgang mit Beschwerden von Nutzern über bestimmte Inhalte erlassen, die im Grunde nahtlos an die durch die Rechtsprechung ausgeformte, hier bereits ausführlich besprochene Störerhaftung ${ }^{15}$ und insbesondere deren Prüfpflichten anknüpfen. ${ }^{16} \S 3$ NetzDG trifft für die Ausgestaltung dieses Verfahrens - dessen Anwendbarkeit per se durch das NetzDG nicht berührt wird ${ }^{17}$ - neue organisatorische

${ }^{12}$ Vgl. Eifert, in: Eifert/Gostomzyk, Netzwerkrecht, S. 9 (20). Zwar scheint die Begründung des Regierungsentwurfs hinsichtlich $\S 4$ Abs. 5 NetzDG offenbar die Möglichkeit der Sanktionierung auch in einzelnen Fällen vorzusehen, weil dort von dem ,gemeldeten Inhalt nebst dem Kontext, in dem er verfasst worden ist" (BT-Drucks. 18/12356, S. 26) die Rede ist. Das ändert aber nichts an dem vorrangig zu berücksichtigenden Wortlaut und der übergeordneten Pflicht der Bußgeldbehörde, ihr Ermessen pflichtgemäß (§47 OWiG), also auch verhältnismäßig auszuüben, vgl. Schiff, MMR 2018, 366 (369).

${ }^{13}$ Vgl. insoweit die Überlegungen zur verfassungsrechtlichen Bewertung des NetzDG, die ich unter Schiff, MMR 2018, 366 ff., veröffentlicht habe und im Folgenden aufgreife.

${ }^{14}$ Vgl. unter anderen Liesching, in: Spindler/Schmitz, TMG, NetzDG $§ 1$ Rn. 6 ff.; Kalscheuer/Hornung, NVwZ 2017, 1721 ff.; Wimmers/Heymann, AfP 2017, 93 ff.; Guggenberger, ZRP 2017, 98 ff.; Gersdorf, MMR 2017, 439 ff.; Nolte, ZUM 2017, 552 (553 ff.); Lüdemann, in: Eifert/Gostomzyk, Netzwerkrecht, S. 153 ff.; Hong, in: Albers/Katsivelas, Recht \& Netz, S. 59 (80 ff.).

${ }^{15} \mathrm{Vgl}$. oben $\S 10$.

${ }^{16}$ Vgl. Hofmann, WRP 9/2017, Editorial: ,[...] § 3 NetzDG bringt im Grundsatz [...] wenig Neues"; ,[es] erinnert eher an die „Blog-Eintrag"-Rechtsprechung denn an einen Paradigmenwechsel"; vgl. auch Eifert, in: Eifert/Gostomzyk, Netzwerkrecht, S. 9 (14); Spindler, GRUR 2018, 365 (372); Kubiciel, jurisPR-StrafR 7/2017, Anm. 1 (III. 1.).

${ }^{17} \mathrm{Vgl}$. die vorangehenden Hinweise sowie den den Hinweis in BT-Drucks. 18/12356, S. 22, auf BGH, Urt. v. 25.10.2011 - VI ZR 93/10, NJW 2012, 148 (Ls. 2) - Blog-Eintrag. 
Anforderungen. ${ }^{18}$ Die Regelung diktiert insbesondere, wie mit rechtswidrigen Inhalten umzugehen ist und ergänzt diese Regelungen um einen Bußgeldtatbestand. Dadurch wird das von der Rechtsprechung rein zivilrechtlich konzipierte Verhältnis zwischen Intermediären und ihren Nutzern nunmehr ordnungsrechtlich umrahmt. Die Besonderheit liegt also nicht so sehr im Bestehen entsprechender Verhaltenspflichten überhaupt, sondern in der positiven Konturierung dieser durch den Gesetzgeber, der damit das gesetzlich positivierte Dogma einer grundsätzlichen Privilegierung zugunsten der Anerkennung einer bestehenden Verantwortung der Diensteanbieter teilweise aufgibt. ${ }^{19}$ Jenseits der konkret getroffenen Wertungen ist es jedenfalls zu begrüßen, dass eine solche positive Ausformung der Pflichten der Diensteanbieter unzweifelhaft den Grad der Transparenz insbesondere gegenüber den Nutzern solcher Dienste erhöhen und dadurch zu deren Selbstermächtigung beitragen kann. Insofern spricht das NetzDG eine klarere und deutlichere Sprache als es die Rechtsprechung vermag.

Die gesetzgeberische Konkretisierung und Weiterentwicklung der von der Rechtsprechung etablierten Verhaltenspflichten birgt allerdings auch rechtspraktische Risiken. So ist nicht ausschließbar, dass die Regelungen zum Beschwerdeverfahren in $\S 3$ NetzDG die in hohem Maße einzelfallorientierte und im Grundansatz eine enorme Flexibilität gewährende Rechtsprechungslinie des BGH zur Störerhaftung ein Stück weit an Flexibilität berauben könnten. Denn es dürfte dem Gericht zunehmend schwerfallen, eine Fortentwicklung der Störerhaftung dort zu rechtfertigen, wo eine solche den im NetzDG niedergelegten Grundsätzen widerspräche. Ein solches Risiko muss auch nicht auf Fälle beschränkt bleiben, in denen das NetzDG explizit Anwendung findet. Denn auch ansonsten wäre eine diskursive Anknüpfung juristischer Konflikte am NetzDG zu vermuten, soweit es sich in der Sache zu einer Rechtsfrage explizit oder implizit verhält. Dies ändert allerdings nichts daran, dass es dem demokratisch legitimierten Gesetzgeber freistand und weiterhin freisteht, auch durch richterliche Rechtsfortbildung geprägte Verhaltenspflichten der Intermediäre stärker zu konturieren oder gar weiterzuentwickeln. Die Judikatur zur Störerhaftung hat nicht durchweg zu einer systematischen Befolgung durch die Diensteanbieter geführt. Diese waren stets in der Lage, ihre „Compliance“ ausschließlich an Prozessrisiken auszurichten und insoweit rechtliche Standards teilweise zu relativieren. Dies spricht dafür, dass eine zusätzliche, aber gerade nicht auf die „blinde“ Rechtsdurchsetzung zielende Anreizwirkung durch die in $\$ 4$ NetzDG eingeführten Bußgelder für die systemische Missachtung einzelner Verhaltenspflichten jedenfalls prinzipiell sinnvoll gewesen sein könnte.

${ }^{18}$ Insbesondere kennt $§ 3$ NetzDG keine Zumutbarkeitsschwelle mehr, vgl. Gersdorf, MMR 2017, 439 (446).

${ }^{19}$ Vgl. Eifert, in: Eifert/Gostomzyk, Netzwerkrecht, S. 9 (14). 


\section{Keine „Privatisierung“ der Rechtsdurchsetzung}

Auf den Vorwurf der Übertragung richterlicher bzw. staatlicher Aufgaben an private Akteure wurde bereits im Kontext der Störerhaftung in grundlegender Weise eingegangen. ${ }^{20} \mathrm{Da}$ das Recht insbesondere in den hier relevanten Konfliktfällen zwischen einem Diensteanbieter und seinen Nutzern kein Justizmonopol für die Durchsetzung des Rechts kennt, haben erstere in aller Regel den „Erstzugriff“ auf die Beurteilung eines gerügten Inhalts. Dies war schon die geltende Rechtslage vor Erlass des NetzDG, das mithin nichts Grundlegendes hieran geändert hat. Der Einwand, dass das NetzDG den Nutzern die Möglichkeit entziehe, Abwehrrechte gegen den Staat geltend zu machen, ${ }^{21}$ geht daher an der Sache vorbei. Denn er impliziert, dass es vor dem Erlass des NetzDG solche Abwehrrechte gegeben habe. Woraus sich diese bei einem Vertragsverhältnis zwischen Nutzer und Plattform aber ergeben sollen, bleibt unklar. Ein „Anspruch“ auf Geltendmachung von Abwehrrechten ist deren Konzeption fremd. Nach dieser Argumentation müsste jeder Fall privat ausgeübter Kontrolle über Freiheitsrechte, die grundrechtsdogmatisch der der mittelbaren Drittwirkung im Privatrechtsverhältnis zuzuordnen wäre, verfassungswidrig sein, weil Abwehrrechte gegen den Staat ,entzogen“ werden. Das kann offensichtlich nicht zutreffen. ${ }^{22}$

\section{Verkürzung der Rechte Einzelner durch Ausgestaltung des Beschwerdeverfahrens}

Die Ausformung des Beschwerdeverfahrens in $\S 3$ NetzDG sieht sich jedoch insoweit einer berechtigten Kritik ausgesetzt, als dass Nutzer nach Beanstandung ihres Inhalts nicht angehört werden und zu deren Gunsten auch keine Rechtsbehelfe vorgesehen sind, mittels derer sie sich gegen Entscheidungen der Netzwerkbetreiber zur Wehr setzen können. Insbesondere im Rahmen von Ehrschutzdelikten kommt es aber maßgeblich auf eine Abwägung widerstreitender grundrechtlicher Positionen an. ${ }^{23}$ Bei gerügten Persönlich-

\footnotetext{
${ }^{20} \mathrm{Vgl}$. oben $\S 10$ B. II.

${ }^{21}$ So immerhin Wissenschaftlicher Dienst des Deutschen Bundestages, Entwurf eines Netzwerkdurchsetzungsgesetzes, WD 10-3000-037/17, S. 16, und Deutscher Anwaltverein, Stellungnahme zum Entwurf eines Gesetzes zur Verbesserung der Rechtsdurchsetzung in sozialen Netzwerken, Stellungnahme 41/2017, S. 17.

${ }^{22}$ Will man das Argument so verstehen, dass der Staat vermittelt über Private etwas anordnet, was ihm selbst verwehrt ist, unterschlägt diese Sichtweise aber, dass die Beurteilungsperspektive sich entscheidend verschiebt. Denn der Private darf im ,ersten Zugriff“ eben willkürgeleitet entscheiden, bevor staatliche Gerichte angerufen werden und Grundrechte (,lediglich“) im Rahmen der mittelbaren Drittwirkung geltend gemacht werden können. Jedenfalls trifft das Argument aber auch so verstanden in der Sache nicht zu, weil auch der Staat selbst kommunikationsregulierend tätig werden darf.

${ }^{23}$ Vgl. BVerfG, Beschl. v. 08.02.2017 - 1 BvR 2973/14, NJW 2017, 1460 (Rn. 13) Obergauleiter.
} 
keitsrechtsverletzungen verlangt auch der BGH im Rahmen der Störerhaftung $^{24}$, wie bereits erläutert, die Einräumung einer Gelegenheit zur Stellungnahme. Die Regelung in $\S 3$ Abs. 2 Nr. 3a Hs. 2 NetzDG wird diesen Maßstäben wegen ihres fakultativen Charakters indes nicht gerecht. Zur Realisierung der gebotenen Internalisierung aller Abwägungsparameter ist eine obligatorische Beteiligung erforderlich. ${ }^{25} \mathrm{Zwar}$ liegt es durchaus nahe, dass neben dem NetzDG die Störerhaftung weiterhin Anwendung findet und deshalb die entsprechenden Obliegenheiten der Diensteanbieter einschlägig sind ${ }^{26}$ Aber schon aus Gründen der rechtsnormativen Transparenz kann auf ein solches Ergänzungsverhältnis allein nicht abgestellt werden. Denn neben den mit juristischem Sachverstand ausgestatteten Intermediären geht es im NetzDG bewusst auch um eine entsprechende Ermächtigung der Dienstenutzer, von den ihnen eingeräumten Rechten effektiv Gebrauch machen zu können. Bei einem systematisch zwar naheliegenden Ergänzungsverhältnis zwischen NetzDG und Störerhaftung wäre ohne eine entsprechende Verankerung der Rechte der Dienstenutzer im NetzDG diesem Anliegen nicht hinreichend Rechnung getragen. Dies gilt auch für das Bußgeldverfahren nach $\S 4$ Abs. 5 NetzDG. Zwar betrifft dieses wegen der Anknüpfung an systemische Mängel bei der Umsetzung im rechtlichen Sinne nicht mehr die Dienstenutzer. Dennoch ist impliziter Gegenstand dieses Verfahrens die hoheitliche Feststellung über die Rechtmäßigkeit von Meinungsäußerungen, sodass auch hier aus grundrechtlicher Perspektive eine Stellungnahme erforderlich ist. Konzeptionell wäre eine Beteiligung auch umsetzbar, etwa durch Erstellung einer „Fallsammlung“ zur Vorlage beim Gericht im Verfahren nach $\S 4$ Abs. 5 NetzDG, die die Stellungnahmen der Autoren inkludiert.

Auf der Sekundärebene sollten ferner Rechtsbehelfe der betroffenen Personen vorgesehen werden. ${ }^{27}$ Zwar bestehen auch schon jetzt auf Grundlage einer verfassungskonformen Interpretation der AGB einzelner Diensteanbieter gerichtlich durchsetzbare Ansprüche auf Wiederherstellung entfernter rechtmäßiger Inhalte. ${ }^{28}$ Mit einer verbindlichen Klarstellung wäre jedoch zum einen sichergestellt, dass sich die Plattformen einer gerichtlichen Kontrolle nicht entziehen können. ${ }^{29}$ Zum anderen ließe sich so auch schon vor einer endgültigen verfassungsrechtlichen Klärung der Reichweite solcher Wiederherstellungsansprüche ein entsprechendes Schutzniveau erreichen.

\footnotetext{
${ }^{24}$ Vgl. BGH, Urt. v. 25.10.2011 - VI ZR 93/10, NJW 2012, 148 (Rn. 25 ff.) - BlogEintrag; Urt. v. 01.03.2016 - VI ZR 34/15, NJW 2016, 2106 (Ls. 2) - Ärztebewertungsportal III.

${ }^{25}$ Vgl. Steinbach, JZ 2017, 653 (660 f.).

${ }^{26}$ Vgl. die Nachweise oben in $\S 12$ Fn. 17 sowie den zugehörigen Text.

${ }^{27}$ So auch Löber/Roßnagel, MMR 2019, 71 (75).

${ }^{28} \mathrm{Vgl}$. dazu oben die Nachweise in $\S 11$ Fn. 20.

${ }^{29} \mathrm{Zu}$ einem entsprechenden Vorschlag vgl. Peukert, MMR 2018, 572 (574, 577).
} 
Diese Rechtsbehelfsmöglichkeiten könnten sich an die Entscheidung nach $\S 4$ Abs. 5 NetzDG anschließen, sollten aber nicht hierauf beschränkt sein. $\mathrm{Zu}$ beachten gilt es, dass ein Rechtsbehelf nur zur Durchsetzung subjektiver Rechte existieren kann. Sofern kollektive Rechtsgüter in § 1 Abs. 3 NetzDG betroffen sind, verbleibt Individuen nach einer Entscheidung des sozialen Netzwerks nur der Hinweis an die Polizei- bzw. Justizbehörden. ${ }^{30}$

\section{Verantwortungsbezogene Aspekte der Verhältnismäßigkeit}

Die Diskussion um das NetzDG kreist vor allen Dingen um dessen Vereinbarkeit mit dem Verfassungsrecht, insbesondere mit der Meinungsfreiheit gemäß Art. 5 Abs. 1 S. 1 GG. Hier soll nur auf die zentralen Aspekte eingegangen werden, die die Frage nach der Verhältnismäßigkeit des NetzDG entscheidend prägen.

\section{a) Gemeinwohlsicherung als legitimes Ziel}

Zunächst stellt es eine verfassungsrechtlich legitime Zielsetzung dar, gemeinwohlsichernde Regeln für Geschäftsmodelle vorzusehen, die Relevanz für den öffentlichen Diskurs haben können. Ziel ist es gerade nicht, ein Medienpolizeirecht zu schaffen, das auf die Erhaltung der verfassungsmäßigen Ordnung in den Medien ausgerichtet ist, ${ }^{31}$ sondern im Hinblick auf bestimmte Minimalziele den Diensteanbietern Vorgaben für ihr Geschäftsmodell zu machen. Auch die Konkretisierung solcher Anforderungen durch Anknüpfung an strafrechtswidrige Inhalte ist kein verfassungsrechtlich illegitimes Ziel. ${ }^{32}$

\section{b) Effektivität der Heranziehung der Intermediäre und Alternativen}

Die materiell abgestufte, hinsichtlich der Offensichtlichkeit differenzierte Heranziehung der Plattformen erweist sich als mildestes unter den zur Verfügung stehenden Mitteln, um auf eine konsequentere Sanktionierung strafrechtswidriger Inhalte in Netzwerken hinzuwirken. Insbesondere überlässt es das NetzDG den Plattformen, über die Modalitäten der Beschwerdesysteme $\mathrm{zu}$ entscheiden, geht also über ein geringes $\mathrm{Ma}$ organisatorischer Anforderungen an den Umgang mit Beschwerden, die die Unternehmensfreiheit beeinträchtigen, nicht hinaus. Häufig ist in diesem Kontext die Auffassung anzutreffen, die Stärkung der Strafverfolgung sei das probate Mittel zur Adressierung des vom NetzDG in den Blick genommenen Problems. ${ }^{33}$ Die

\footnotetext{
${ }^{30}$ Das Risiko des $\S 164$ StGB wäre einzukalkulieren.

${ }^{31}$ Vgl. Kubiciel, jurisPR-StrafR 7/2017, Anm. 1 (III. 2.).

${ }^{32}$ Vorauszusetzen ist hier natürlich stets, dass die jeweils betroffene Strafnorm verfassungsgemäß ist.

${ }^{33}$ Vgl. statt vieler Guggenberger, ZRP 2017, 98 (101).
} 
Verfechter dieser Position verkennen allerdings, dass zur Ahndung von Straftaten die Justizorgane ohnehin allein berufen sind. ${ }^{34}$ Dem NetzDG geht es dagegen allein um die effektive Beseitigung von rechtswidrigen Inhalten im Privatrechtsverhältnis, ${ }^{35}$ eine strafrechtliche Ahndung ist oftmals nicht das primäre Ziel von betroffenen Personen. ${ }^{36}$

\section{c) Anreizstrukturen und Overblocking}

Bei der Angemessenheit ist zu berücksichtigen, dass die vom Schutzbereich erfasste Freiheit, strafrechtswidrige Äußerungen zu tätigen, nicht als sonderlich hochrangig anzusehen ist. Demgegenüber besteht ein eminent hohes Interesse daran, rechtmäßige Inhalte äußern zu können. Hier kommt es entscheidend darauf an, ob die Anreizstruktur des NetzDG zu Kollateralschäden bei grundrechtlich verbürgten Freiheiten führt, weil etwa der Anreiz zum Löschen höher ist als zum Nichtlöschen. Insbesondere darf die „Vermutung für die Zulässigkeit der freien Rede ${ }^{\text {“37 }}$ in Zweifelsfällen nicht eingeschränkt werden. ${ }^{38}$ Rein theoretisch ist mit der Feststellung eines Overblockings allerdings noch nichts über dessen (Verfassungs-)Rechtswidrigkeit gesagt. So hat der BGH in Bezug auf urheberrechtliche Konstellationen früher bereits mehrfach darauf hingewiesen, dass „Falschpositive“ zugunsten eines wirksamen Rechtsschutzes ggf. auch hinzunehmen sein könnten. ${ }^{39}$ In der jüngeren

${ }^{34}$ Diese stehen allerdings durchaus vor der Herausforderung, mit der vielfach als verfassungsrechtlich geschützt bzw. schützenswert bewerteten Anonymität im Netz umzugehen. Hier ist einerseits von der „vorherrschende[n] Anonymitätskultur im Netz“ die Rede (Di Fabio, Grundrechtsgeltung in digitalen Systemen, S. 51), die vom Allgemeinen Persönlichkeitsrecht (Art. 2 Abs. 1 iVm Art. 1 Abs. 1 GG) und der Meinungsfreiheit (Art. 5 GG) grundsätzlich geschützt sei, da sonst „chilling effects“ für die politische Auseinandersetzung zu befürchten seien, vgl. Kersten, JuS 2017, 193 (195 ff.). Andererseits werden auch die Risiken einer solchen Anonymität im Hinblick auf „die Achtung der Subjektstellung des jeweils anderen" betont, die Fundament jeder Rechtsordnung sei (wiederum Di Fabio, Grundrechtsgeltung in digitalen Systemen, S. 53). Aus einer übergeordneten Perspektive kritisch zur rechtlichen Fixierung der Anonymität etwa Thiel, Zeitschrift für Menschenrechte 10 (2016), 9 (19ff.). Dass gerade im Lichte des politischen Aktivismus Anonymität jedoch kein Trumpf darstellt, sondern auch von politisch engagierten Bürgern erwartet werden darf, zu ihren Anliegen ,zu stehen“, legt RhPfVerfGH, Beschl. v. 27.10.2017 - VGH B 37/16, NVwZ 2018, 492 (Rn. 18), für den Fall eines Informationszugangsanspruchs überzeugend dar.

${ }^{35}$ Die Erfassung von Kollektivrechtsgütern in $§ 1$ Abs. 3 NetzDG ist nicht unproblematisch, sprengt sie doch die Grenzen des Schutzes subjektiver Rechte im Privatrechtsverkehr. Es erscheint aber legitim, die Bevölkerung zur praktischen Durchsetzung solcher Normen wie auch mit Strafanzeigen ( 1158 StPO) zu mobilisieren.

${ }^{36} \mathrm{Vgl}$. Höch, K\&R 2017, 289 (289 f.).

${ }^{37}$ BVerfG, Urt. v. 15.01.1958 - 1 BvR 400/51, BVerfGE 7, 198 (212) - Lüth.

${ }^{38}$ Eine Verletzung dieses Grundsatzes im Fall des NetzDG erkennt Hong, in: Albers/ Katsivelas, Recht \& Netz, S. 59 (82 ff.).

${ }^{39}$ Vgl. BGH, Urt. v. 15.08.2013 - I ZR 80/12, NJW 2013, 3245 (Rn. 62) - RapidShare; 
Entscheidung Internetforum, in der es um die Haftung eines Suchmaschinendienstes wegen persönlichkeitsrechtsverletzender Suchergebnisse ging, scheint der BGH indes eine Kehrtwende bzw. eine Differenzierung zwischen Persönlichkeitsrechten und sonstigen Schutzrechten anzudeuten, indem er nunmehr das Risiko des Overblockings betont und insoweit zu erkennen gibt, ein solches für unzulässig zu halten..$^{40}$ Auch der EuGH äußerte sich in den $S A B A M$-Entscheidungen schon ähnlich. ${ }^{41}$ Hingewiesen sei hier auf das Risiko, dass durch die Überbetonung der negativen Implikationen des Overblockings für die Meinungsfreiheit aus dem Blick geraten kann, dass bei der zunehmenden technischen Feinsteuerung entsprechender Konflikte auch der gegenteilige Effekt, das „Underblocking“, aus grundrechtlicher Perspektive ebenso problematisch ist. ${ }^{42}$ Deutlich wird das rechtliche Risiko des Overblockings jedenfalls nur, wenn dieses Risiko systemischer Natur ist. ${ }^{43}$ Ist die Anreizstruktur hingegen ausgewogen oder in ihrem Ansatz jedenfalls auf ein Mindestma $\beta$ falschpositiv erfasster Inhalte beschränkt, ist der Vorwurf nicht überzeugend.

Vor diesem Hintergrund könnte sich die Anreizstruktur im NetzDG, namentlich insbesondere die Bußgeldandrohung für das „nicht richtige“ Vorhalten des in $\S 3$ NetzDG vorgesehenen Verfahrens, insoweit als heikel erweisen, als ein Anreiz gesetzt wird, auch rechtmäßige Inhalte zu löschen, weil es den Diensten darum gehen könnte, „um jeden Preis“ keine finanziellen Nachteile zu erleiden. Daran lässt sich einerseits zweifeln, weil sich das NetzDG, also auch die angesprochene Bußgeldandrohung, auf das „Vorhalten" eines Beschwerdeverfahrens bezieht und damit gerade nicht am Einzelfall ansetzt, sondern das generelle Vorhalten eines den Vorgaben des $\S 3$ Abs. 2-3 NetzDG entsprechenden Verfahrens erzwingen will und daher nur

Urt. v. 12.07.2012 - I ZR 18/11, NJW 2013, 784 (Rn. 45) - Alone in the Dark; Urt. v. 12.07.2007 - I ZR 18/04, NJW 2008, 758 (Rn. 60) - Jugendgefährdende Medien bei eBay.

${ }^{40}$ Vgl. BGH, Urt. v. 27.02.2018 - VI ZR 489/16, NJW 2018, 2324 (Rn. 35) - Internetforum.

${ }^{41}$ Vgl. EuGH, Urt. v. 24.11.2011 - C-70/10, MMR 2012, 174 (Rn. 52) - Scarlet Extended/SABAM; Urt. v. 16.02.2012 - C-360/10, MMR 2012, 334 (Rn. 50) - SABAM/Netlog.

${ }^{42}$ Vgl. Lang, AöR 143 (2018), 220 (232 f.); ansatzweise auch Rademacher, JZ 2019, 702 (706f.).

${ }^{43}$ Vgl. Eifert, in: Eifert/Gostomzyk, Netzwerkrecht, S. 9 (25, 33 f.). Eine abwägende Position nimmt auch Kastl, Automatisierung im Internet, S. 437, ein; vgl. für das Urheberrecht Leistner, ZUM 2012, 722 (729f.), anders Maier, Remixe auf Hosting-Plattformen, S. 151 ff., die die Schwierigkeit der tatsächlichen Feststellung konzediert, aber dennoch ,in absoluten Zahlen“ ein relevantes Overblocking konstatiert (153); für das NetzDG dezidiert anderer Auffassung (bereits die Löschung eines einzigen rechtmäßigen Inhalts sei verfassungsrechtlich „hochproblematisch“) Hong, Verfassungsblog (09.01.2018), verfassungsblog.de/das-netzdg-und-die-vermutung-fuer-die-freiheit-der-rede; ergänzend ders., in: Albers/Katsivelas, Recht \& Netz, S. 59 (80 ff.). 
auf systemische Mängel im Beschwerdeverfahren ausgerichtet ist. Auf Rechtsfolgenseite zwingt $\S 47 \mathrm{OWiG}$ die zuständige Behörde sogar dazu, ihr Ermessen den Umständen des Einzelfalls entsprechend auszuüben und dem Verhältnismäßigkeitsprinzip Rechnung zu tragen. ${ }^{44}$ Schließlich erscheint auch eine Auslegung dahingehend, dass auch die Löschung rechtmäßiger Inhalte sanktioniert ist, vor dem Hintergrund der verfassungsrechtlichen Relevanz der Anreizstruktur und dem hieraus ableitbaren Gebot der verfassungskonformen Auslegung denkbar. ${ }^{45}$ Andererseits ist allerdings nicht ausschließbar, dass eine Sanktionsdrohung unternehmensintern eine Logik in Gang setzt, die tatsächlich zur Löschung rechtmäßiger Inhalte führen könnte. Das von Balkin als „Kollateralzensur“ bezeichnete Phänomen unterstellt dem Intermediär einen Anreiz zur privaten „Zensur“ ${ }^{46}$ weil bzw. wenn es sich nicht um seine eigene Meinungsäußerung handelt und er einer Haftung entgehen will. Insbesondere wenn es sich nicht um eine eigene Meinungsäußerung handelt, habe er keinen intrinsischen Anreiz, für fremde ÄuBerungen rechtlich negative Folgen zu riskieren. ${ }^{47}$ Mit Blick auf das Gebot der ausgewogenen Anreizstruktur gewinnt aber in diesem Kontext die subjektive, unternehmensseitige Einschätzung der Sanktionsdrohung an Relevanz. An einer nüchternen, nicht auf die strategischen Eigeninteressen schielende Bewertung durch die betroffenen Unternehmen mag man hier zwar zweifeln. ${ }^{48}$ Begreift man die Figur der „Kollateralzensur“" als verfassungsrechtlich relevant, was mit dem modernen Eingriffsbegriff nach dem $\mathrm{BVerfG}^{49}$ nahe liegt, muss die Perspektive der Normadressaten dennoch eine gewichtige Rolle spielen, nicht allein diejenige einer vernünftigen, die legislative Intention kennenden Beobachterin. Dass also die Möglichkeit einer durch das NetzDG gesetzten, unternehmensintern nicht ausgewogen umgesetzten Anreizstruktur besteht, begründet hier einen verfassungsrechtlichen Mangel des NetzDG.

Dieser ließe sich allerdings recht einfach aus der Welt schaffen. Denn insbesondere die bereits angesprochene erforderliche Stärkung der Nutzerrechte könnte einen entscheidenden Baustein darstellen, um zu einer ange-

${ }^{44}$ BT-Drucks. 18/12356, S. 24 f.

${ }^{45}$ Näher dazu Schiff, MMR 2018, 366 (369).

${ }^{46} \mathrm{Im}$ verfassungsrechtlichen Sinne geht es hier selbstredend nicht um eine Zensur im Sinne von Art. 5 Abs. 1 S. 3, vgl. Kühling, in: BeckOK Informations- und Medienrecht, GG Art. 5 Rn. 131; vgl. aber noch unten $\S 12$ Fn. 55.

${ }^{47}$ Vgl. Balkin, Columbia Law Review 99 (1999), 2295 (2298 f.); ders., Harvard Law Review 127 (2014), 2296 (2308 f.).

${ }^{48}$ Näher Schiff, MMR 2018, 366 (369f.); ähnlich Eifert, in: Eifert/Gostomzyk, Netzwerkrecht, S. 9 (35 f.).

${ }^{49}$ Vgl. nur BVerfG, Beschl. v. 26.06.2002 - 1 BvR 670/91, BVerfGE 105, 279 (303 f.) Osho. 
messeneren Ausgestaltung der Anreizstruktur zu kommen. Hierzu zählt zum einen eine obligatorische Einbeziehung der Stellungnahmen aller an einem Konflikt beteiligten Personen, zum anderen eine explizite Regelung von Ansprüchen zur Wiederherstellung rechtmäßiger Inhalte. Für eine ausbalancierte Anreizstruktur wäre ferner die gesetzliche Klarstellung erforderlich, dass auch die systemisch relevante Löschung rechtmäßiger Inhalte sanktionsbewehrt ist. Denn so wäre es den betroffenen Diensteanbietern nicht mehr möglich, sich auf eine einseitige Anwendung des Gesetzes zurückzuziehen.

\section{Fazit zum NetzDG}

Als Fazit ergibt sich für das NetzDG damit ein gemischtes Bild..$^{50}$ Die Struktur der im NetzDG angelegten Sanktionen leidet unter der dargestellten Möglichkeit eines ungleichgewichtigen Anreizes, der es möglich erscheinen lässt, dass nicht nur rechtswidrige Inhalte gelöscht werden. In einem derart grundrechtssensiblen Bereich sind die richtigen Anreizwirkungen aber entscheidend, um eine ausgewogene Anwendung durch die Diensteanbieter zu ermöglichen und ihnen keinen einfachen Weg aus dem äußerungsrechtlichen Dilemma zu ermöglichen. Hier besteht also Verbesserungspotenzial. ${ }^{51}$ Allerdings sind bislang keine Anhaltspunkte erkennbar, dass es gegenwärtig zu einem systemischen Overblocking in größerem Ausmaß kommt. ${ }^{52}$

Die grundsätzliche Intention und der gewählte Ansatz des NetzDG sind hingegen zu begrüßen, da es auf einem prinzipiell sinnvollen Instrument, der Störerhaftung, aufbaut und dieses ordnungsrechtlich überformt. Das ordnungsrechtliche Tätigwerden, dem eine grundsätzlich legitime, jedenfalls nicht unvertretbare Feststellung eines Durchsetzungsdefizits im Bereich des Umgangs der Netzwerkbetreiber mit Beschwerden zugrunde liegt, bot sich dem Gesetzgeber hierbei als naheliegendes Mittel an, das strukturell gegen-

\footnotetext{
${ }^{50}$ Nicht unerwähnt sollten die unionsrechtlichen Bedenken, insbesondere die fragliche Vereinbarkeit mit dem Herkunftslandprinzip der ECRL, vgl. dazu Spindler, ZUM 2017, 473 (474 ff.); positiver hingegen Nölscher, ZUM 2020, 301 ff.; Eifert, in: Eifert/Gostomzyk, Netzwerkrecht, S. 9 (23 f.), erkennt die Problematik an, spricht aber von einem Fall ,politisch akzeptabler Provokation".

${ }^{51}$ Die Gesetzesbegründung zum NetzDG sieht eine derzeit durchgeführte Evaluierung vor, vgl. BT-Drucks. 18/12356, S. 18. Hier nicht von weiterer Bedeutung ist die Änderung des NetzDG durch Art. 6 des Gesetzes zur Bekämpfung des Rechtsextremismus und der Hasskriminalität (BT-Drucks. 19/17741), das der Bundestag am 18.06.2020 beschlossen hat, vgl. www.zeit.de/digital/internet/2020-06/strafverfolgung-netz-bundestag-hass-hetzedrohungen-haertere-strafen-gesetz. Denn die maßgeblichen Bestimmungen des NetzDG zur Verantwortung Diensteanbieter hat dieses nicht geändert.

${ }^{52} \mathrm{Vgl}$. Eifert et al., Netzwerkdurchsetzungsgesetz in der Bewährung, S. $76 \mathrm{ff}$.; Löberl Roßnagel, MMR 2019, 71 (73).
} 
über einem gesetzlichen Eingriff in die dogmatischen Strukturen des bürgerlichen Rechts vorzugswürdig ist. Der Gewinn an Transparenz ist dabei ein doppelter. Zum einen sind die rechtlichen Standards für den Umgang mit Beschwerden von Nutzern erstmals gesetzlich markiert und damit für die Allgemeinheit besser nachvollziehbar. Zum anderen sind die Berichtspflichten ebenfalls ein transparenzerhöhendes Merkmal, das eine aufgeklärtere Diskussion über die konkrete Praxis der Diensteanbieter in einem wichtigen gesellschaftlichen Kontext ermöglichen kann. Es ist daher auch kein Zufall, dass der erste durch das Bundesamt für Justiz erlassene Bußgeldbescheid nach $\S 4$ NetzDG nicht wegen eines systemischen Verstoßes gegen die Vorgaben zum Umgang mit Beschwerden erfolgt ist, sondern eine Verletzung der Transparenzpflichten durch Facebook rügt. ${ }^{53}$

In einer übergeordneten Perspektive stellt das NetzDG trotz der aufgezeigten Mängel einen grundsätzlich sinnvollen Versuch der Rechtsordnung dar, auf immer weiter zunehmende Vermachtungstendenzen im Bereich der Intermediäre zu reagieren. Die faktische Verlagerung von Potenzialen effektiver Normsetzung bzw. Letztentscheidung hin zu diesen Intermediären und die hierin zutage tretende Zurückdrängung von Normen kollektiver Selbstbestimmung zugunsten von oder deren Überlagerung durch privat gesetzte Standards ist aus demokratietheoretischer Perspektive prekär, ${ }^{54}$ gerade weil immer weniger Raum für kollektive Selbstbestimmung von Kommunikationssphären verbleibt. ${ }^{55}$ Insoweit kann das NetzDG auch als Versuch einer demokratisch verfassten Gesellschaft gesehen werden, die Deutungshoheit über öffentliche bzw. als öffentlich konzipierte Kommunikationssphären

${ }^{53}$ Vgl. Bundesamt für Justiz, Pressemitteilung vom 03.07.2019, www.bundesjustizamt.d e/DE/Presse/Archiv/2019/20190702.html?nn=3451904. Gegen den Bußgeldbescheid setzt Facebook sich aktuell allerdings noch zur Wehr, vgl. www.heise.de/newsticker/meldung/F acebook-wehrt-sich-gegen-NetzDG-Bussgeld-4475699.html.

${ }^{54}$ Ingold, Der Staat 56 (2017), 491 (526 f.), beklagt allerdings mit Blick auf das NetzDG eine „Skandalisierung“ von als Demokratiegefährdung empfundenen Phänomena; es fehle das Wissen „über die komplexen Wechselwirkungen des kommunikativen Settings und damit über die Eignung der Maßnahmen [...]“; eine Regelung sei erst möglich, wenn über die „konkrete normative Gestalt und Verortung sowie [...] Bedeutung [der demokratischen Öffentlichkeit] Klarheit herrsche. Mit einem solch voraussetzungsvollen Maßstab wäre dem Gesetzgeber allerdings kaum jemals ein rechtlicher Zugriff auf Intermediäre möglich.

${ }^{55}$ Vgl. Hoffmann-Riem, in: Denninger et al., AK-GG, Art. 5 Abs. 1, 2 Rn. 45: „Besondere Vorkehrungen sind angezeigt, wenn Träger hervorgehobener gesellschaftlicher und ökonomischer Macht Filterfunktionen innehaben. “Zwar vertritt Hoffmann-Riem ,[a]ngesichts moderner Gefährdungen freier Kommunikation" (Rn. 91) einen gegenüber der tradierten Auffassung erweiterten Zensurbegriff, der insbesondere eine Verschärfung des Haftungsrechts der Diensteanbieter erfassen solle. Allerdings sieht auch er eine ,anlassbezogene Handlungspflicht der Diensteanbieter“ als „unproblematisch“ an (Rn. 94), um die es beim NetzDG ja gerade geht. 
teilweise zurückzugewinnen. Hier dürfte das letzte Wort mit Blick auf Gesetzgebungsvorhaben auf nationaler und unionaler Ebene indes noch nicht gesprochen sein.

\section{B. Die Modifizierung der Wertungen der ECRL und der Störerhaftung zur Durchsetzung des Urheberrechts durch die DSM-RL}

Der zweite große Bereich, in dem sich in jüngerer Zeit ein Umbruch mit Blick auf die Thematik dieser Untersuchung anzudeuten scheint, ist das unionsrechtliche Urheberrecht. Um dieses soll es im Folgenden gehen.

\section{Hintergrund}

Das unionsrechtliche Urheberrecht wurde vor Erlass der DSM-RL primär durch die InfoSoc-RL und die Enforcement-RL geprägt. Diese beiden Instrumente erwiesen sich allerdings aufgrund ihres Alters zunehmend als nicht mehr geeignet, den Aufstieg von Diensten zum Teilen und Verbreiten von urheberrechtlich geschützten Inhalten sinnvoll zu erfassen. Die zunehmenden monetären Einbußen, die die Kreativbranchen in den letzten Jahren erlitten, wurden überwiegend der lizenzlosen und oftmals illegalen Wiedergabe von geschützten Werken zugeordnet (sog. „Value Gap“). ${ }^{56}$ Eine solche Perspektive wurde allerdings auch immer wieder als empirisch nicht belegbar kritisiert. ${ }^{57}$ In diesem Kontext wurde die Kommission Juncker aktiv, die den „Digitalen Binnenmarkt“ zu einer Priorität ihres Mandats erklärt hatte, ${ }^{58}$ und veröffentlichte am 14. September 2016 ersten Entwurf für eine Modernisierung des Urheberrechts. ${ }^{59}$

Dieser sollte die beiden bestehenden Regelungsinstrumente des Sekundärrechts nicht gänzlich ersetzen, sondern nur ergänzen. Insbesondere die Haftungsfrage wurde als elementar für eine Verbesserung der Lage der Kreativbranchen bei einer zeitgleichen Positivierung der Verantwortung der Intermediäre angesehen. Dementsprechend geriet recht früh im Laufe des Ge-

\footnotetext{
${ }^{56}$ Vgl. Leistner/Metzger, FAZ, 04.01.2017; Hornschuh, ZUM 2019, 222 (224 f.).

${ }^{57}$ Vgl. Wimmers/Barudi, GRUR 2017, 327 (328 ff.); relativierend aber Metzger, ZUM 2018, 233 (237 f.), der zutreffend auf die Bigotterie auf beiden Seiten der Diskussion verweist, aber zugleich und zu Recht die politische Dimension des Argumentationstopos „Value Gap“ betont, die nicht auf eine empirische Validierung angewiesen sei, um eine gesetzliche Reaktion zu rechtfertigen.

${ }^{58} \mathrm{Vgl}$. COM(2015) 192 final.

${ }^{59} \mathrm{COM}(2016) 593$ final.
} 
setzgebungsprozesses der damalige Art. 13 (heute Art. 17) des Entwurfs, der in seiner nunmehrigen Form Hauptbetrachtungsgegenstand der folgenden Ausführungen ist, in den Fokus der Öffentlichkeit und stellte zum Ende des Prozesses zumindest in interessierten Kreisen der Öffentlichkeit ebenso wie dessen unterstellte Folge, die Einführung von „Upload-Filtern“, fast ein geflügeltes Wort dar. ${ }^{60}$

\section{Konkrete gesetzliche Regelung}

Im Folgenden sollen die in diesem Kontext wesentlichen Regelungen zur Haftung von Diensteanbietern dargestellt werden. ${ }^{61}$

\section{Etablierung der Primärhaftung über das Instrument der öffentlichen Wiedergabe}

Die erste Grundaussage der Regelung in Art. 17 DSM-RL ist die Feststellung in Abs. 1 UAbs. 1, dass „Diensteanbieter für das Teilen von Online-Inhalten eine Handlung der öffentlichen Wiedergabe" vornehmen. ${ }^{62}$ Damit wird im Grundsatz eine täterschaftliche Haftung der Diensteanbieter etabliert, ${ }^{63}$ weil die Handlung der öffentlichen Wiedergabe einen eigenen urheberrechtlichen Tatbestand begründet. Konsequenterweise verpflichtet Art. 17 Abs. 1 UAbs. 2 DSM-RL den Diensteanbieter deshalb auch, für eine solche Wiedergabe eine Erlaubnis des Rechteinhabers einzuholen, ${ }^{64}$ ohne dass aber damit ein Kontrahierungszwang für eine der beiden betroffenen Seiten verbunden wäre. ${ }^{65}$ Wird die Erlaubnis nicht erteilt, unterliegt der Diensteanbieter damit für jede rechtswidrige Nutzung grundsätzlich einem Haftungsrisiko. Wird sie erteilt, muss sie gemäß Art. 17 Abs. 2 DSM-RL auch für die Wiedergabehandlung der Nutzer der Dienste selbst gelten. Damit ist das Risiko der Eigenhaftung der Nutzer dieser Dienste, das zuvor im Hinblick auf den Wiedergabecharakter des Hochladens geschützter Werke gegeben war, ${ }^{66}$ deutlich reduziert.

${ }^{60}$ Vgl. Hurtz, Süddeutsche Zeitung, 07.03.2019.

${ }^{61}$ Der Anwendungsbereich der DSM-RL wurde bereits in anderem Zusammenhang angesprochen; vgl. oben § 7 C. I. 2.; vgl. dazu ferner Gielen/Tiessen, EuZW 2019, 639 (641).

${ }^{62}$ Zum Konzept der öffentlichen Wiedergabe vgl. Art. 3 InfoSoc-RL; zum Verhältnis zur DSM-RL vgl. deren Erwägungsgrund 64 S. 3.

${ }^{63}$ Vgl. Spindler, GRUR 2020, 253 (254); Hofmann, ZUM 2019, 617 (620 f.). Dogmatisch dürfte es sich um ein Tätigkeitsdelikt einschließlich der Regelung konkreter Verkehrspflichten handeln, vgl. Peifer, GRUR-Prax 2019, 403 (404); ähnlich Sesing, MMR 2019, 788 (789).

${ }^{64}$ Spindler, CR 2019, 277 (285), spricht von einer Selbstverständlichkeit.

${ }^{65}$ Vgl. Hofmann, ZUM 2019, 617 (620); Volkmann, CR 2019, 376 (380).

${ }^{66}$ Vgl. EuGH, Urt. v. 08.09.2016 - C-160/15, NJW 2016, 3149 (Rn. 35) - GS Media; Urt. v. 07.08.2018 - C-161/17, NJW 2018, 3501 (Rn. 24, 30, 35, 44, 47) - Renckhoff. 
Diese in der DSM-RL getroffene Grundsatzentscheidung nimmt damit im Grunde die Entscheidung vorweg, die der EuGH unter der InfoSoc-RL ebenfalls zu treffen gehabt hätte. Entsprechende Vorlagen zur Vorabentscheidung hatte der BGH indes bereits beschlossen ${ }^{67}$ sodass der EuGH für das nunmehr alte Sekundärrecht eine nicht minder grundlegende eine Entscheidung zu treffen haben wird. Nach dem Schlussantrag in dieser Sache ist es zumindest denkbar, dass der EuGH für Art. 3 InfoSoc-RL verneinen wird ${ }^{68}$ was der Gesetzgeber mit Art. 17 Abs. 1 UAbs. 1 DSM-RL bejaht hat.

\section{Einschränkungen der Haftung}

\section{a) Obliegenheiten des Diensteanbieters}

Das durch Art. 17 Abs. 1 UAbs. 1 DSM-RL etablierte Haftungsrisiko wird allerdings von Art. 17 Abs. 4 DSM-RL bedeutend eingeschränkt. ${ }^{69}$ Danach muss der Diensteanbieter „alle Anstrengungen“ unternehmen, um die Erlaubnis doch zu erhalten (lit. a). „Nach Maßgabe hoher branchenüblicher Standards für die berufliche Sorgfalt" muss er sich redlich bemühen, bestimmte Schutzgegenstände, zu denen er vom Rechteinhaber bestimmte Informationen bzw. eine Referenzdatei erhalten hat, unverfügbar zu halten (lit. b). Schließlich muss nach Erhalt eines „hinreichend begründeten Hinweises von den Rechteinhabern" der Zugang zu entsprechenden Schutzgegenständen gesperrt bzw. müssen diese entfernt und wiederum redliche Bemühungen unternommen werden, um die erneute Rechtsverletzung künftig zu verhindern (lit. c).

\section{aa) Anstrengungen zur Einholung einer Erlaubnis}

Das redliche Bemühen im Sinne von Art. 17 Abs. 4 lit. a DSM-RL ist unter dem Verhältnismäßigkeitsprinzip auszulegen, wie es in Art. 17 Abs. 5 DSMRL geregelt ist, und soll so eine flexible Handhabung unter Beachtung des jeweils in Rede stehenden Diensteanbieters ermöglichen. ${ }^{70}$ Jedenfalls dürften seitens der Diensteanbieter zumindest „Kontaktaufnahmen und [ernsthafte] Verhandlungsbereitschaft" gefordert werden können, ${ }^{71}$ um redliche Bemü-

${ }^{67}$ Vgl. BGH, Beschl. v. 13.09.2018 - I ZR 140/15, GRUR 2018, 1132 (1. Frage) - YouTube; Beschl. v. 20.09.2018 - I ZR 53/17, ZUM 2018, 870 (1. Frage) - uploaded.

${ }^{68}$ Vgl. GA Øe, Schlussantrag v. 16.07.2019 - C-682/18, juris, Rn. 66 ff. - YouTube.

${ }^{69}$ Theoretisch ist dies bereits eine grundlegende Abkehr von dem in Art. 14 ECRL statuierten Prinzip der Nichthaftung als Grundregel. Konsequenterweise stellt Art. 17 Abs. 3 ECRL genau dies auch fest. Es wäre deshalb denkbar, die Umsetzung statt in den $\S \S 97 \mathrm{ff}$. UrhG in $\S 10 \mathrm{TMG}$ als weiteren „Haftungsfilter“ vorzunehmen, vgl. Peifer, GRUR-Prax 2019, 403 (405); Sesing, MMR 2019, 788 (791 ff.).

${ }^{70}$ Vgl. Gielen/Tiessen, EuZW 2019, 639 (643), die allerdings ebenso wie Gerpott, MMR 2019, 420 (424), die damit verbundene Rechtsunsicherheit der Diensteanbieter betonen.

${ }^{71}$ Spindler, CR 2019, 277 (285). 
hungen im Sinne dieser Bestimmung annehmen zu können. ${ }^{72}$ Die Zulässigkeit eines Kontrahierungszwangs bei der mitgliedstaatlichen Umsetzung liegt dagegen schon angesichts des Wortlauts der Norm eher fern. ${ }^{73}$ Für professionell organisierte Rechteinhaber, die insbesondere mit wirtschaftlich wertvollen Werken agieren, stellt sich dieses Erfordernis nicht ganz so problematisch dar wie bei solchen Inhalten, die von nicht professionell agierenden und deshalb nicht selten schwer erreichbaren Personen hochgeladen werden und welche auch bei Bearbeitungen im Sinne von „User-generated content“ urheberrechtlichen Schutz genießen können. ${ }^{74}$ Unter Beachtung des VerhältnismäBigkeitsprinzips kann es damit dazu kommen, dass die Ermittlungspflicht im Sinne von lit. a nicht fruchtet und die Rechteinhaber dann nur über das Verfahren nach lit. c geschützt sind. ${ }^{75}$

Dass die Vorschrift, wenn die Rechteinhaberinnen zu ermitteln sind, auch auf die Bedingungen Einfluss nehmen will, mit denen die Parteien einer möglichen Lizenzierung miteinander verhandeln wollen, ist nicht ohne Weiteres anzunehmen. ${ }^{76}$ Angesichts der möglichen Dauer von Lizenzverhandlungen wäre auch eine rechtliche Erlaubnis des Hochladens während solcher Verhandlungen denkbar. ${ }^{77}$

\section{bb) Branchenübliche Anstrengungen zur Nichtverfügbarkeit von Inhalten, Filterpflichten}

Besonders umstritten und Kernstück der hier geregelten Handlungsanforderungen ist Art. 17 Abs. 4 lit. b DSM-RL und die sich hieraus ergebende rechtliche Erwartung, jeden technisch und finanziell zumutbaren Aufwand einzusetzen, um die Verfügbarkeit geschützter Werke zu verhindern. Nach Auffassung der meisten Beobachterinnen ist dies insbesondere durch Dienste mit einem sehr hohen Aufkommen von hochgeladenen Inhalten wie You-

\footnotetext{
${ }^{72}$ Die Ernsthaftigkeit fordert auch Volkmann, CR 2019, 376 (380).

${ }^{73}$ Vgl. Spindler, GRUR 2020, 253 (255)

${ }^{74}$ Vgl. Hofmann, GRUR 2019, 1219 (1225 f.), dort auch zum Vorschlag der Vereinbarung von Kollektivlizenzen für diesen Fall.

${ }^{75} \mathrm{Vgl}$. auch Erwägungsgrund 66 UAbs. 2 S. 4 DSM-RL.

${ }^{76}$ Das nimmt aber wohl Volkmann, CR 2019, 376 (380), an, die auf den Konflikt zwischen Google und der VG Media verweist, bei dem Google nicht bereit war, für die Darstellung von sog. „Snippets“, also kleinen Ausschnitten des Inhalts referenzierter Webseiten, die durch das neu eingeführte Leistungsschutzrecht erfasst sind, ein Entgelt zu zahlen und drohte, bei Verweigerung einer Zustimmung durch die Presseverlage entsprechende Seiten auszulisten. LG Berlin, Urt. v. 19.02.2016 - 92 O 5/14 Kart, MMR 2016, 620 ff. Snippets, sieht in diesem Verhalten keinen Missbrauch einer marktbeherrschenden Stellung im Sinne von $\S 19$ GWB.

${ }^{77}$ Dreier, GRUR 2019, 771 (776), verweist insoweit zutreffend auf die Dauer der Verhandlungen zwischen der GEMA und YouTube, die eine mehrjährige Sperrung vieler Musikvideos in Deutschland zur Folge hatte.
} 
Tube händisch nicht zu bewerkstelligen, sondern setzt den Einsatz von Filtertechnologien voraus. ${ }^{78}$ Tatsächlich dürfte es rechtlich nur schwer zu begründen sein, bei der Umsetzung auf mitgliedstaatlicher Ebene gänzlich auf solche Filterpflichten zu verzichten, wie aber in rechtspolitischen Stellungnahmen zu vernehmen war. ${ }^{79}$ Allerdings verdeutlicht Art. 17 DSM-RL immer wieder, dass jede dort vorgesehene Anforderung an die Diensteanbieter stets unter dem Vorbehalt der verhältnismäßigen Ausgestaltungsmöglichkeit und Anwendung steht ${ }^{80}$ Dass eine Filterpflicht nicht in jedem Fall das verhältnismäßige Mittel sein wird, gibt die Richtlinie in Erwägungsgrund 66 UAbs. 2 S. 4 wiederum deutlich zu erkennen. Danach ist es gerade nicht auszuschließen, dass die Verfügbarkeit rechtsverletzender Inhalte mitunter nur durch eine Notifizierung des Rechteinhabers verhindert werden kann. Diese Aussage verweist auf Art. 17 Abs. 4 lit. c DSM-RL. ${ }^{81}$ Ferner ist anzumerken, dass sich eine etwaige Pflicht zum Einsatz von Filtern als Einsatz „branchenüblicher Standards" nur auf solche Informationen bezieht, die von den Rechteinhabern bereitgestellt wurden. Ist dies nicht der Fall, liegt es nahe, eine Pflicht zum Einsatz dieser Filter abzulehnen. ${ }^{82}$

Weiterhin ist fraglich, was unter „,hohen branchenüblichen Standards“ zu verstehen ist. Diese jedenfalls im Hinblick auf die deutsche Rechtsordnung untypische Kategorie ${ }^{83}$ wird in den Erwägungsgründen spezifiziert. Danach soll es darauf ankommen, ,ob der Diensteanbieter alle Maßnahmen ergriffen hat, die ein sorgfältiger Betreiber ergreifen würde, um sicherzustellen, dass auf seiner Website keine nicht genehmigten [...] Schutzgegenstände verfügbar sind“" ${ }^{84}$ Nach Maßgabe des Verhältnismäßigkeitsgrundsatzes ${ }^{85}$ sollen dabei insbesondere die Größe des Dienstes und „der sich entwickelnde Stand der Technik bei den bestehenden Mitteln, einschließlich möglicher künftiger Entwicklungen" zu berücksichtigen sein. ${ }^{86}$ Eine gänzliche Ausnahme von der Pflicht nach Art. 17 Abs. 4 lit. b DSM-RL für kleinere Unternehmen liegt jedoch angesichts ihrer expliziten Privilegierung im Rahmen der von Art. 17 Abs. 6 DSM-RL gesetzten Grenzen fern. ${ }^{87}$ Zur Konkretisierung dieser Vor-

\footnotetext{
${ }^{78}$ Vgl. Raue/Steinebach, ZUM 2020, 355 (356); Dreier, GRUR 2019, 771 (776); Gielen/ Tiessen, EuZW 2019, 639 (644); Spindler, CR 2019, 277 (285); Senftleben, ZUM 2019, 369 (371); Volkmann, CR 2019, 376 (380).

${ }^{79}$ Vgl. Volkmann, CR 2019, 376 (380 f.).

${ }^{80}$ Vgl. insbesondere den bereits angesprochenen Art. 17 Abs. 5 DSM-RL.

${ }^{81}$ So auch Gielen/Tiessen, EuZW 2019, 639 (644).

${ }^{82}$ Vgl. auch Spindler, GRUR 2020, 253 (256).

${ }^{83}$ So Spindler, CR 2019, 277 (286), der aber Erwägungsgrund 66 UAbs. 2 S. 3 DSM-RL nicht thematisiert und dessen Schlussfolgerung, es werde insoweit eine „neue Kategorie“ eingeführt, jedenfalls nicht vollends überzeugt.

${ }^{84}$ Erwägungsgrund 66 UAbs. 2 S. 2 DSM-RL.

${ }^{85} \mathrm{Vgl}$. Erwägungsgrund 66 UAbs. 2 S. 2 DSM-RL.

${ }^{86}$ Erwägungsgrund 66 UAbs. 2 S. 3 DSM-RL.

${ }^{87}$ Spindler, GRUR 2020, 253 (256), verweist insoweit zu Recht auf den (politischen) Hintergrund der in Art. 17 Abs. 6 DSM-RL formulierten Privilegierung kleinerer Dienste.
} 
gaben sieht Erwägungsgrund 71 DSM-RL einen Dialog zwischen der Kommission in Zusammenarbeit mit den Mitgliedstaaten und den Interessenvertretern vor, der aktuell im Gang ist. ${ }^{88}$

Die Rechtsnatur der hier vorgesehenen Handlungsanforderungen ist nicht ganz eindeutig. Der Wortlaut selbst spricht zwar einerseits für eine unbedingte Pflicht ${ }^{89}$ Diese Einsicht ist aber rechtstechnisch insoweit zu relativieren, als es sich eben um eine Obliegenheit handelt, deren Verwirklichung im Grundsatz im Belieben des Diensteanbieters steht, sodass die Nichtbefolgung eben nur nachteilige Konsequenzen für diesen hat. Erwägungsgrund 66 UAbs. 4 S. 2 DSM-RL lässt die Annahme einer Pflicht im rechtlichen Sinne noch fernliegender erscheinen und legt stattdessen eher ein Verständnis von Art. 17 Abs. 4 lit. b DSM-RL als Beweislastregelung nahe.

$\mathrm{Zu}$ berücksichtigen ist weiterhin, dass die in lit. $\mathrm{b}$ vorgesehene Verhaltensobliegenheit nur greift, soweit die Rechteinhaber „einschlägige und notwendige Informationen bereitgestellt haben“. Damit kommt es jedenfalls nicht zu einer generellen Filterpflicht aller hochgeladenen Werke, was in der Debatte teilweise aber unterstellt wurde. Stellen die Rechteinhaber die entsprechenden Referenzdateien nicht aktiv bereit, ${ }^{90}$ kann es somit im Grundsatz nicht zu einer Filterung kommen. ${ }^{91}$ „Endlose Recherchen“ kann man den Diensteanbietern allerdings nicht abverlangen, ${ }^{92}$ sodass es auch an dem Verhalten der Rechteinhaber liegen wird, inwieweit es zu Filterpflichten kommen wird. ${ }^{93}$

\section{cc) Notice-and-Stay-Down}

In bekannteren Gefilden bewegt sich dagegen Art. 17 Abs. 4 lit. c DSM-RL, der die Entfernung nach erfolgter Notifizierung und die Pflicht zu redlichen Bemühungen um eine Verhinderung erneuter Rechtsverletzungen vorsieht. Hier ist im Wesentlichen ein „Notice-and-Stay-Down“-Verfahren geregelt. ${ }^{94}$ Insoweit kommt es vor allen Dingen auf das Verhältnis zum Verbot allge-

\footnotetext{
${ }^{88} \mathrm{Vgl}$. ec.europa.eu/digital-single-market/en/stakeholder-dialogue-application-article17-directive-copyright-digital-single-market.

${ }^{89}$ So im Ansatz Spindler, CR 2019, 277 (286).

${ }^{90}$ Spindler, CR 2019, 277 (285 f.), hält es für möglich, dass es insoweit auf eine passive Bereithaltung ankommen könne. Dem spricht aber auch der Wortlaut der englischen Fassung entgegen $(,[\ldots]$ for which the rightholders have provided the service providers with $[\ldots]$ information"; Hervorh. v. Verf.).

${ }^{91}$ Vgl. auch Erwägungsgrund 66 UAbs. 5 DSM-RL.

${ }^{92}$ Spindler, CR 2019, 277 (286).

${ }^{93} \mathrm{Nicht}$ zu leugnen ist allerdings, dass die Rechteinhaber vermittelt durch die DSM-RL einen hohen Anreiz haben werden, die entsprechenden Informationen zur Verfügung zu stellen.
}

${ }^{94}$ Vgl. Hofmann, ZUM 2019, 617 (621). 
meiner Überwachungspflichten an, das Art. 17 Abs. 8 UAbs. 1 DSM-RL trotz Geltung des Art. 15 ECRL statuiert. ${ }^{95}$ Auch hier stellt sich damit in gewisser Weise die Frage nach Filterpflichten, nicht zuletzt weil explizit auf Art. 17 Abs. 4 lit. b DSM-RL Bezug genommen wird.

Das in Art. 17 Abs. 4 lit. c DSM-RL angeordnete Verfahren ist Mindestvoraussetzung, um einer Haftung zu entgehen. Hält der Diensteanbieter dieses nicht ein, dürfte im Regelfall eine Haftung nicht zu vermeiden sein. Das verdeutlicht die Formulierung ,und in jedem Fall“ am Ende von Art. 17 Abs. 4 lit. b DSM-RL. Für die beiden anderen Varianten kann eine Haftung insoweit nur anhand der deutlich weicheren, damit flexibler handzuhabenden Kriterien bestimmt werden, die an die Bemühungen des Diensteanbieters anknüpfen. Eine kumulative Anordnung aller drei Varianten als Voraussetzung der Privilegierung ist damit allerdings nicht gefordert. ${ }^{96}$ Denn eine Haftung ist auch ohne „Notice-and-Stay-Down“-Verfahren möglich, wenn der Diensteanbieter nicht alle Anstrengungen im Sinne von lit. a oder von lit. b übernommen hat. Sie ist aber auch möglich, wenn er diese redlichen Bemühungen unternommen hat, dann aber nicht bzw. nicht unverzüglich auf eine Notifizierung reagiert. ${ }^{97}$ Schließlich wurde bereits dargelegt, dass die Unverfügbarkeit mitunter nur durch Notifizierung sichergestellt werden kann, weil eine Filterung im Sinne von lit. b unverhältnismäßig wäre. ${ }^{98}$

\section{b) Abgestufte Ausgestaltung der Handlungsobliegenheiten}

Die in Art. 17 Abs. 4 DSM-RL vorgesehenen Obliegenheiten sind nach der gesetzgeberischen Konzeption stets am Gebot der Verhältnismäßigkeit auszurichten, wie Art. 17 Abs. 5 explizit festhält. Als Konkretisierung des Verhältnismäßigkeitsgrundsatzes ${ }^{99}$ sind danach insbesondere „die Art, das Publikum und der Umfang der Dienste " ${ }^{\text {"100 }}$ und „die Verfügbarkeit geeigneter und wirksamer Mittel und die Kosten" "101 der Erfüllung der in Art. 17 Abs. 4 DSM-RL statuierten Obliegenheiten zu berücksichtigen. Mit dem Verhältnismäßigkeitsprinzip lassen sich auch weitere Regelungen zur Konkretisierung des Handlungsrahmens für die Diensteanbieter erklären.

Erstens dürfen, wie bereits erwähnt, die den Diensteanbietern auferlegten Obliegenheiten nach Art. 17 Abs. 8 UAbs. 1 DSM-RL nicht zu einer allgemeinen Überwachungspflicht führen. Zweitens darf es nicht dazu kommen,

\footnotetext{
${ }^{95}$ Vgl. Spindler, CR 2019, 277 (287).

${ }^{96}$ So aber Sesing, MMR 2019, 788 (789).

${ }^{97} \mathrm{Vgl}$. auch Erwägungsgrund 66 UAbs. 4 S. 1 DSM-RL.

${ }^{98}$ Vgl. oben Text zu $§ 12$ Fn. 81.

${ }^{99}$ Vgl. auch schon EuGH, Urt. v. 12.11.2011 - C-324/09, MMR 2011, 596 (Rn. 139) -

L'Oréal SA.

${ }^{100}$ Art. 17 Abs. 5 lit. a DSM-RL.

${ }^{101}$ Art. 17 Abs. 5 lit. b DSM-RL.
} 
dass rechtmäßige Inhalte unterdrückt werden, wie Art. 17 Abs. 7 UAbs. 1 DSM-RL feststellt. Damit soll insbesondere auf die Unzulässigkeit des Overblocking hingewiesen werden. Die DSM-RL versucht sich dabei an einem „Spagat zwischen (automatisiertem) Urheberrechtsschutz einerseits und der Gewährleistung von Schranken und Meinungsfreiheit andererseits“ ${ }^{102}$ Drittens dürfen jene Diensteanbieter nicht über Gebühr belastet werden, die aufgrund ihres Alters bzw. ihrer geringeren wirtschaftlichen Handlungsfähigkeit mit den größeren Diensten wie YouTube noch nicht mithalten können. Gemeint sind damit insbesondere Start-ups. Zu deren Schutz hat der Gesetzgeber in Art. 17 Abs. 6 UAbs. 1 DSM-RL eine Beschränkung ihrer Obliegenheiten auf die Bemühung zur Einholung einer Erlaubnis und die unverzügliche Reaktion auf Notifizierungen im Sinne von Art. 17 Abs. 4 lit. a bzw. Art. 17 Abs. 4 lit. c DSM-RL vorgenommen, sodass die Einhaltung der branchenüblichen Standards noch nicht verlangt werden kann. Diese Erleichterung betrifft solche Diensteanbieter, deren Dienste weniger als drei Jahre zur Verfügung stehen und deren Jahresumsatz nicht 10 Millionen Euro übersteigt. ${ }^{103}$ Falls aber ein solcher Dienst in einem Kalenderjahr monatlich im Durchschnitt mehr als fünf Millionen unterschiedliche Nutzer hat, muss auch dieser die in Art. 17 Abs. 4 lit. b erwähnten Anstrengungen zur Verhinderung künftiger Rechtsverletzungen unternehmen. Hier ist allerdings nach dem Gesetzeswortlaut unklar, ob der Maßstab insoweit weniger streng ist als bei Art. 17 Abs. 4 lit. b selbst.

\section{Nutzerrechte}

Die DSM-RL etabliert in zweierlei Hinsicht neue Ansätze zum Schutz der Dienstenutzer. Zum einen formuliert Art. 17 Abs. 7 UAbs. 2 DSM-RL Schranken für den urheberrechtlichen Schutz, wie etwa das Zitatrecht und die im Kontext von „User-generated content" besonders relevanten Nutzungszwecke der Karikaturen, Parodien und Pastiches. Die Besonderheit dieser Regelung ist ihre Ausgestaltung als subjektives Recht auf die Nutzung dieser Schranken. ${ }^{104}$ Zum anderen und im Gegensatz zum NetzDG verpflichtet Art. 17 Abs. 9 DSM-RL die Diensteanbieter explizit, ,,wirksame und zügige Beschwerde- und Rechtsbehelfsverfahren zur Verfügung "zu stellen. Für diese enthält Art. 17 Abs. 9 UAbs. 2 konkrete Vorgaben, u.a. Begründungsobliegenheiten, ${ }^{105}$ Pflichten des Diensteanbieters zur unverzüglichen Bear-

\footnotetext{
${ }^{102}$ Spindler, CR 2019, 277 (288).

${ }^{103}$ Allerdings bleiben auch diese Dienste nicht von rein mitgliedstaatlichen Haftungsinstrumenten verschont, wie sich aus Erwägungsgrund 66 UAbs. 1 S. 3 DSM-RL ergibt. Das betrifft insbesondere die Störerhaftung, vgl. Spindler, CR 2019, 277 (288).

${ }^{104}$ Vgl. Hofmann, GRUR 2019, 1219 (1227); vgl. dort auch Fn. 138 für Nachweise zur Diskussion um die Rechtsnatur von Schranken im Urheberrecht.

${ }^{105}$ Art. 17 Abs. 9 UAbs. 2 S. 1 Erwägungsgrund 70 UAbs. 2 S. 3 DSM-RL.
} 
beitung und Prüfung durch einen Menschen ${ }^{106}$ sowie die Einrichtung von außergerichtlichen Rechtsbehelfsverfahren, die allerdings den Zugang zu den Gerichten der Mitgliedstaaten nicht im Weg stehen dürfen. ${ }^{107}$ Damit greift der Unionsgesetzgeber Vorgaben des EuGH auf, der bereits in der Entscheidung UPC Telekabel die Bedeutung von Rechtsschutzinstrumenten betont hatte. ${ }^{108}$ Der von diesen Nutzerrechten verfolgte Zweck liegt in der Abmilderung der „chilling effects“ bzw. dem vorgeworfenen Overblocking, das durch die Anreizstruktur der Neumodellierung des Haftungsrechts verursacht werde. ${ }^{109}$ Mit Hofmann soll danach eine „Symmetrie zwischen Rechtsdurchsetzung einerseits und Abwehr ungerechtfertigter Rechtsdurchsetzung andererseits hergestellt" werden. ${ }^{110}$

\section{Bewertung vor dem Hintergrund der Verantwortung der Intermediäre}

Die Bewertung des Art. 17 DSM-RL muss angesichts der breiten Diskussion um den Reformbedarf im europäischen Urheberrecht und dessen konkrete Umsetzung in der DSM-RL hier auf einige zentrale Aspekte beschränkt bleiben, die für die Verantwortung der Intermediäre im Kontext dieser Untersuchung von entscheidender Bedeutung sind. Ohnehin verbietet sich eine abschließende Bewertung, solange eine Umsetzung der Vorgaben der Richtlinie sowie deren Konkretisierung durch die Mitgliedstaaten noch nicht erfolgt ist und die konkreten Implikationen der Richtlinie in der Praxis damit noch nicht endgültig absehbar sind.

\section{Differenzierte Positivierung der Verantwortung der Intermediäre}

Wie auch zum NetzDG angemerkt, ist die erstmalige Positivierung der Verantwortung der Diensteanbieter im Kontext des Urheberrechts positiv hervorzuheben. Das lag hier allerdings auch besonders nahe, weil mit der öffentlichen Wiedergabe nach Art. 3 InfoSoc-RL ohnehin eine etablierte rechtliche Kategorie zur Verfügung stand, die bereits aus sich heraus zulässt, den Kreis potenzieller Verletzer von Schutzrechten weiter zu ziehen, als es dies etwa im Persönlichkeitsrecht auf Primärebene möglich wäre. Die Grundaussage des Art. 17 Abs. 1 UAbs. 1 DSM-RL erweist sich damit als konsequente, wenngleich grundlegende Fortentwicklung eines tradierten Konzepts im europä-

${ }^{106}$ Art. 17 Abs. 9 UAbs. 2 S. 2, Erwägungsgrund 70 UAbs. 2 S. 2 DSM-RL.

${ }^{107}$ Art. 17 Abs. 9 UAbs. 2 S. 3-4, Erwägungsgrund 70 UAbs. 2 S. 5-7 DSM-RL.

${ }^{108}$ Vgl. EuGH, Urt. v. 27.03.2014 - C-314/12, GRUR 2014, 468 (Rn. 57) - UPC Telekabel.

${ }^{109}$ Vgl. Hofmann, GRUR 2019, 1219 (1220).

${ }^{110}$ Hofmann, GRUR 2019, 1219 (1220). 
ischen Urheberrecht. ${ }^{111}$ Geradezu paradigmatisch erscheint insoweit auch die deklaratorische Feststellung in Art. 17 Abs. 3 UAbs. 1 DSM-RL, dass die von der Regelung erfassten Diensteanbieter nicht am Privileg des Art. 14 ECRL teilhaben. Das kann man einerseits als Selbstverständlichkeit begreifen. ${ }^{112}$ Andererseits ist aber zu bedenken, dass ohne eine solche Regelung jedenfalls die prinzipielle Differenzierung zwischen neutralen und aktiven Diensten im Sinne der Rechtsprechung des EuGH weiterhin gegolten hätte. ${ }^{113}$ In diesem Sinne erweist sich gerade die Setzung positiver Anreize als richtiger Weg, um einerseits ein Tätigwerden des Diensteanbieters zu veranlassen, andererseits diesen hierfür zu belohnen. In dieser Form wäre das ohne die insoweit vorgenommene Außerkraftsetzung des Art. 14 ECRL und der damit verbundenen Unanwendbarkeit des Neutralitätsprinzips nur schwer zu erreichen gewesen. Dass Art. 14 ECRL weiterhin Wirkungen entfaltet, zeigt sich nicht zuletzt auch an Art. 17 Abs. 3 UAbs. 2 DSM-RL. Der Gesetzgeber geht hier weiterhin von der grundsätzlichen Privilegierung unter der ECRL aus, soweit es um „Zwecke außerhalb des Geltungsbereichs“ der DSM-RL geht.

Nach den bisherigen Überlegungen dieser Untersuchung und den hier geäußerten Forderungen zur differenzierten Herangehensweise an die Frage der Haftung der Intermediäre ist es wenig überraschend, dass die abgestufte Auferlegung von Handlungsobliegenheiten, effektuiert in Art. 17 Abs. 6 DSM-RL, ebenfalls überzeugt. ${ }^{114}$ Vor dem Hintergrund der oben zur Störerhaftung formulierten Forderung, das System der Haftungsprivilegierungen differenzierter auszugestalten, erweist sich der Ansatz in Art. 17 DSM-RL damit als grundsätzlich sinnvoller, positiver Schritt in die Richtung einer funktional differenzierten Betrachtung der Diensteanbieter und einer entsprechenden Abstufung ihrer Sorgfaltspflichten. ${ }^{115}$

\section{Explizite Regelung der Nutzerrechte und Verhältnis zum} Rechtsschutzinstrumentarium der Mitgliedstaaten

Auch die explizite Konturierung von Nutzerrechten fällt - gerade im Vergleich zum NetzDG - positiv auf. Zunächst ist die Ausgestaltung der aus-

${ }^{111}$ Dreier, GRUR 2019, 771 (775), und Wandtke/Hauck, ZUM 2019, 627 (628), sprechen von einem Paradigmenwechsel in Bezug auf die Störerhaftung; zutreffend dagegen Hofmann, GRUR 2019, 1219 (1222); Metzger, ZUM 2018, 233 (238).

${ }^{112}$ In diese Richtung wohl Hofmann, ZUM 2019, 617 (624).

${ }^{113}$ Deren Sinnhaftigkeit wurde hier bereits kritisch erörtert, vgl. oben $\S 8$ B.

${ }^{114}$ Kritisch zu den Einzelheiten allerdings Pravemann, GRUR 2019, 783 (787).

${ }^{115} \mathrm{Ob}$ auch jenseits des in Art. 17 Abs. 6 DSM-RL niedergelegten „Start-Up-Privilegs“ aufgrund des Verhältnismäßigkeitsgrundsatzes die Sorgfaltsanforderungen an kleinere Unternehmen weniger streng anzulegen sind, wie es u.a. Raue/Steinebach, ZUM 2020, 355 (357), fordern, braucht hier nicht vertieft zu werden. 
drücklich erwähnten Schrankenregelungen als subjektive Rechte bemerkenswert und führt zu einem bereits sekundärrechtlich deutlich erhöhten $\mathrm{Maß}$ an Rechtssicherheit. Der Gesetzgeber antizipiert hier zutreffend, dass sich vor allem die außergerichtlichen Streitbeilegungsverfahren als sinnvoller Baustein der Nutzerrechte erweisen könnten. Er geht jedoch nicht so weit, zugunsten dieser den gerichtlichen Rechtsschutz einzuschränken. Inwieweit sich die Nutzerrechte als effektiv erweisen werden ${ }^{116}$ hängt entscheidend von der mitgliedstaatlichen Umsetzung ab, denn eine einfache Statuierung der Schrankenregeln ohne Zuerkennung eines durchsetzbaren Anspruchs dürfte nicht ausreichen. ${ }^{117}$

Vor dem Ziel der Vollharmonisierung erscheint es jedoch zumindest diskussionswürdig, auch jenseits des Regelungsbereichs der DSM-RL Rechtsinstitute der Mitgliedstaaten wie etwa die Störerhaftung unangetastet zu lassen. Zwar wurde oben dargelegt, weshalb diese grundsätzlich ein sinnvolles Instrument darstellt, um kontext- und einzelfallangemessen auf durch Diensteanbieter vermittelte Rechtsverletzungen zu reagieren. Je eher aber das Unionsrecht sich materiell der Regelung des Haftungsgrundes annähert, ${ }^{118}$ desto eher liegt das Risiko einer nur eingeschränkten bzw. stets von den Rechtsordnungen der Mitgliedstaaten bedrohten Harmonisierung nahe. Gerade für ein Institut wie die Störerhaftung, das von der Rechtsprechung im Grundsatz sektorübergreifend eingesetzt wird, birgt dies das Risiko einer dogmatisch unklaren Gemengelage zwischen dem fortwährenden Geltungsanspruch des deutschen bürgerlichen Rechts einerseits und dem sich selbst als Vollharmonisierung begreifenden Unionsrecht. Diese Gemengelage ginge hier wohl eher auf Kosten der Dienstenutzer. Dennoch wird dies aller Voraussicht nach die rechtspraktische Realität der näheren Zukunft darstellen, sodass eine entsprechend pragmatische Vorgehensweise erforderlich sein wird.

\section{Filterpflichten und ihre Konsequenzen}

Einer Erörterung bedarf schließlich die vielfach kritisierte Einführung der Pflicht zum Einsatz technischer Filter. Da ansonsten insbesondere bei sehr beliebten Diensten wie etwa YouTube eine sinnvolle händische Ausführung der in Art. 17 DSM-RL vorgesehenen Schritte kaum möglich wäre, werde hier ein klarer Anreiz zum Einsatz einer Technologie auf Kosten freiheitsrechtlicher Garantien gesetzt.

\footnotetext{
${ }^{116}$ Generell sehr kritisch Spindler, CR 2019, 277 (288 f.).

${ }^{117}$ Vgl. Hofmann, GRUR 2019, 1219 (1227).

${ }^{118}$ Dies war beim Urheberrecht im Gegensatz zum Schutz von Persönlichkeitsrechten ohnehin schon stets der Fall.
} 


\section{a) Vorbemerkung: Die Konkretisierung der Anforderungen an Diensteanbieter in Abhängigkeit der technischen Entwicklung}

Einer besonderen Betonung bedarf allerdings vorab die explizite Inbezugnahme der technischen Entwicklung bei der Ausformung von Sorgfaltsmaßstäben für die Diensteanbieter in der DSM-RL. Es ist zwar klar, dass die von Art. 17 Abs. 4 lit. b DSM-RL geforderten „branchenüblichen Standards“ als eines der künftig zentralen Instrumente der Rechtsdurchsetzung im Urheberrecht eine erhebliche Technizität aufweisen, also maßgeblich auf das Potenzial der Technik zur Verwirklichung normativer Standards abstellt. ${ }^{119} \mathrm{Da}-$ mit stellen sich naturgemä $\beta$ und mit einiger Berechtigung Fragen nach der Transparenz bzw. Opazität technischer Standards, die zunehmend in den Fokus der Diskussion rücken. Wenn allerdings die erhebliche quantitative Steigerung der Risiken für rechtlich geschützte Güter als notwendige Folge eines als wünschenswert und ebenso legitim angesehenen Geschäftsmodells in Kauf genommen werden soll, zugleich aber die Inbezugnahme technischer Lösungen zur Aufarbeitung von Problemlagen, die angesichts ihrer quantitativen Bedeutung kaum mehr allein durch Menschen zu bewältigen wäre, nicht möglich sein soll, entsteht eine Unwucht, die ihrerseits ebenso rechtfertigungsbedürftig erscheint. Genauso wie andernorts die zunehmende und technisch begründete Verfügungsmacht der Intermediäre betont wurde, um daraus Folgerungen für den richtigen rechtlichen Umgang abzuleiten, ${ }^{120}$ geht es auch hier also um die Herstellung eines Gleichgewichts zwischen den normativen Erwartungen an und den technischen Fähigkeiten der Intermediäre. Dies kann und soll natürlich nicht dazu dienen, der Instrumentalisierung opaker Technik zur Lösung rechtlicher, geschweige denn gesellschaftlicher Probleme als Allheilmittel das Wort zu reden. Maßgeblich muss eine stets differenzierte und problembewusste Perspektive bleiben. Auf Skepsis sollten aber Stimmen treffen, die Versuchen der differenzierten und kontrollierten Indienstnahme von Technologie mit dem Vorwurf der „Zensur“ begegnen. ${ }^{121}$

${ }^{119}$ Dies führt neuerdings zur Diskussion eines (wie auch immer zu verstehenden) „Rechts auf Rechtsbruch“, das einer zunehmenden Rechtsdurchsetzung durch Technik entgegenzusetzen sei; vgl. zur Diskussion nur Rademacher, JZ 2019, 702 (707 ff.); Becker, ZUM 2019, 636 (641 ff.). Eine Erörterung dieser Frage würde den Rahmen dieser Untersuchung sprengen. Betont sei hier nur die Einsicht, dass die hinter der Forderung nach einem solchen „Recht“ liegende Problematik im Recht bereits insoweit sinnvoll reflektiert ist, als die Vollzugsebene die Durchsetzung des materiellen Rechts stets einer eigenen Rationalität unterwirft und diese dadurch freiheitsschonend ausgestalten kann; vgl. mit besonderem Augenmerk auf die Durchsetzung subjektiver Rechte Hofmann, in: Fries/Paal, Smart Contracts, S. 125 (137 ff.). Der Forderung nach einem „Recht auf Rechtsbruch“ ist daher mit Skepsis zu begegnen.

${ }^{120} \mathrm{Vgl}$. oben $\S 8 \mathrm{~A}$.

${ }^{121}$ Exemplarisch Senftleben, ZUM 2019, 369 (371). 
Statt eines pragmatischen Umgangs mit den normativen Herausforderungen bedient dieser nicht selten die ,zunehmende Angst der Menschen vor [...] der Computertechnik“"122. Insofern erscheint es auch in diesem Zusammenhang vorzugswürdig, gerade nicht auf das freie Spiel der Kräfte zu vertrauen, sondern die rechtliche Begleitung und Einhegung technischer Risiken als rechtsstaatlichen Normalfall zu betrachten. ${ }^{123}$ Ist die Abkehr von der ubiquitären Nutzung von Informationstechnik in diesem Kontext kaum zu erwarten, spricht daher nicht schon prinzipiell etwas gegen den Versuch, sich der Technik zur Einhegung mit ihr verbundener Risiken zu bedienen, soweit dies auf Grundlage eines hinreichenden Wissensstandes sowie rechtsstaatlich und verhältnismäßig möglich ist. Gerade die mit Art. 17 DSM-RL verbundene Problematik der Filterpflichten bietet hierfür einen Anwendungsfall par excellence, um technische Möglichkeiten zur differenzierten Herangehensweise an ein juristisches Problem auszuloten. ${ }^{124}$ Eine solche Perspektive ist also zwingend auf eine differenzierende Betrachtung angewiesen. Dies vorausgeschickt, ist nun der Blick auf die konkreten rechtlichen Implikationen des in Art. 17 DSM-RL vorgesehenen Einsatzes von Filtertechniken zu richten.

\section{b) Verstoß gegen das Verbot allgemeiner Überwachungspflichten}

Zunächst ist der Blick auf das Verbot allgemeiner Überwachungspflichten zu richten, das Art. 15 ECRL anordnet und das Art. 17 Abs. 8 UAbs. 1 DSMRL wiederholt. Einerseits ist zu berücksichtigen, dass der EuGH in den $S A B A M$-Entscheidungen die Einführung von Filterpflichten noch abgelehnt hatte. Das betraf dort aber sehr weitgehende Filterpflichten, ${ }^{125}$ die hinsichtlich des Differenzierungsgrades kaum mit Art. 17 DSM-RL vergleichbar sind. Wie überdies schon oben ausgeführt wurde, lässt auch die ECRL durchaus Spielraum, in spezifischen Fällen Verhinderungspflichten vorzusehen. ${ }^{126}$ Art. 17 Abs. 4 DSM-RL verpflichtet gerade nicht, sämtliche Inhalte zu überprüfen und damit unterschiedslos zu behandeln, sondern betrifft nur solche Inhalte, die im Sinne von lit. $\mathrm{b}$ bereitgestellt wurden ${ }^{127}$ Bei diesen lässt sich

${ }^{122}$ Bull, Der Staat 58 (2019), 57 (62).

${ }^{123}$ Vgl. Gärditz, Der Staat 54 (2015), 113 (126ff.); ähnlich wie hier auch Wagner, GRUR 2020, 447 (451).

${ }^{124}$ In diesem Kontext sind es insbesondere interdisziplinär angelegte Vorschläge wie etwa der Versuch von Raue/Steinebach, ZUM 2020, 355 (361 ff.), die ein solches Potenzial verwirklichen können.

${ }^{125}$ Vgl. schon oben $\S 4$ Fn. 186 und den zugehörigen Text.

${ }^{126}$ Vgl. oben $\S 4$ C. IV. sowie oben $\S 10$ Fn. 144 und den zugehörigen Text.

${ }^{127}$ So auch Gerpott, MMR 2019, 420 (424f.); Sesing, MMR 2019, 788 (792); letztlich auch Hofmann, ZUM 2019, 617 (625); Gielen/Tiessen, EuZW 2019, 639 (644), sehen dies im Ausgangspunkt auch so, tendieren wohl aber dennoch dazu, einen Verstoß anzunehmen, 
damit ein „spezifischer Fall“ im Sinne von Erwägungsgrund 47 S. 2 annehmen, der zur Prüfung insoweit verpflichten kann. Die Operationalisierung des Einsatzes von Filtertechniken entspricht zudem dem jüngst vom EuGH aufgestellten Grundsatz, dass Art. 15 ECRL einer manuellen Nachprüfung entgegensteht. ${ }^{128}$ Danach soll nur der Einsatz „,automatisierter Techniken“ zulässig sein, um das Hochladen spezifizierter Inhalte zu verhindern. ${ }^{129}$ Der Ausschluss manueller Nachkontrollen wurde aber bereits einer Kritik unterzogen, ${ }^{130}$ die auch in diesem Kontext Geltung beansprucht. Dennoch ergibt sich hier aber de lege lata kein Konflikt mit der ECRL, was entsprechend für Art. 18 Abs. 8 UAbs. 1 DSM-RL zu gelten hat.

\section{c) Verstoß gegen grundrechtliche Garantien}

Wie auch schon bei der Debatte um das NetzDG wird allerdings gegenüber Art. 17 DSM-RL der Vorwurf erhoben, er führe zu einem Overblocking, da die vorgesehene Filterpflicht stets zur Erfassung auch rechtmäßiger Inhalte führen werde. ${ }^{131}$

Zwar ist das Risiko des Overblockings durchaus nicht von der Hand zu weisen. ${ }^{132}$ Die Diensteanbieter sind hiervon in ihrer Berufs- und Unternehmensfreiheit nach Art. 15, 16 GRCh betroffen, ${ }^{133}$ da sie einem erhöhten, finanziell relevanten Compliance-Aufwand unterliegen. Aufseiten der Dienstenutzer sind hier wiederum die Kommunikationsrechte aus Art. 11 GRCh

weil dennoch alle Inhalte zu prüfen seien; dezidiert einen Verstoß annehmend Spindler, CR 2019, 277 (287).

${ }^{128}$ Vgl. EuGH, Urt. v. 03.10.2019 - C-18/18, NJW 2019, 3287 (Rn. 46) - GlawischnigPiesczek.

${ }^{129}$ Vgl. EuGH, Urt. v. 03.10.2019 - C-18/18, NJW 2019, 3287 (Rn. 46) - GlawischnigPiesczek.

${ }^{130} \mathrm{Vgl}$. dazu oben $\S 10$ B. IV. 1. b) und $\S 11$ D. III.

${ }^{131}$ Sehr kurzfristig nach ihrer Verabschiedung hat Polen beim EuGH Nichtigkeitsklage gemäß Art. 263 Abs. 2 AEUV gegen die DSM-RL erhoben, vgl. www.zeit.de/digital/201905/eu-urheberrecht-polen-eugh-klage-zensur-mateusz-morawiecki.

${ }^{132}$ Vgl. Raue/Steinebach, ZUM 2020, 355 (356f.); Specht, GRUR 2019, 253 (258); Senftleben, ZUM 2019, 369 (372).

${ }^{133}$ Es spricht vieles dafür, dass der Charakter der DSM-RL in diesem Punkt - trotz der grundsätzlich nur den Zielen nach verbindlichen Natur der Richtlinie nach Art. 288 Abs. 3 AEUV - zwingend ist und damit eine grundsätzlich unionsweite Vereinheitlichung angestrebt ist, die trotz Erforderlichkeit eines mitgliedstaatlichen Umsetzungsaktes allein die Grundrechte der GRCh zur Anwendung kommen lässt, wie es BVerfG, Beschl. v. 06.11.2019 - 1 BvR 276/17, NVwZ 2020, 63 (Rn. 38) - Recht auf Vergessen II, zum Ausdruck gebracht hat. Denn Art. 17 DSM-RL lässt materiell an dieser Stelle wenig inhaltlichen Spielraum zur Abweichung; vgl. zum eingeschränkten Spielraum Deutschlands auch Peters/Schmidt, GRUR Int 2019, 1006 (1011). Eine ergänzende Anwendbarkeit von Art. 5 Abs. 1 S. 1 GG änderte aber auch nichts an der grundsätzlichen Bewertung. 
berührt, soweit die Anforderungen gegenüber den Diensteanbietern die Entfernung bzw. Sperrung von Inhalten zur Folge hat, die von der Meinungsfreiheit geschützt sind. ${ }^{134}$ Der EuGH hat außerdem schon mehrfach darauf verwiesen, dass er die Erfassung von Falschpositiven für unzulässig hält, ${ }^{135}$ obgleich man aber eine Passage der Entscheidung UPC Telekabel auch so verstehen kann, dass einerseits Overblocking im Grundsatz unzulässig ist, die Pflicht zur Gewährung von Rechtsbehelfen für betroffene Nutzer aber eben nur für solche Fälle gedacht sein kann. ${ }^{136}$ Eine kritische Haltung gegenüber Filterpflichten wird in der Rechtsprechung des EuGH insbesondere aus den $S A B A M$-Entscheidungen offenkundig. ${ }^{137}$ Dort vermisste der Gerichtshof im Kontext der grundrechtlichen Prüfung insbesondere „ein angemessenes Gleichgewicht zwischen dem Schutz des Rechts am geistigen Eigentum, das Inhaber von Urheberrechten genießen, und dem Schutz der unternehmerischen Freiheit, der Wirtschaftsteilnehmern wie den Providern nach Art. 16 der Charta zukommt [...]." ${ }^{“ 138}$ Bekanntlich ging es dort aber um die Überwachung der gesamten elektronischen Kommunikation, ${ }^{139}$ während hier eine solche gerade nicht stattfindet. Vielmehr ist der gesetzgeberische Ansatz erkennbar, immer nur auf einzelne Werke bezogen eine Filterpflicht zu installieren. ${ }^{140}$ Auf dieser Linie liegt auch die Positionierung des EuGH in der Entscheidung Glawischnig-Piesczek, in der die Zulieferung spezifischer Informationen an die Diensteanbieter aus unionsrechtlicher Sicht nicht beanstandet wurde. ${ }^{141}$ Ein pauschaler Verweis auf die frühere Rechtsprechung des EuGH führt hier also nicht zu einem einzigen, zwingenden Ergebnis.

Das Risiko eines relevanten Overblockings lässt sich angesichts des expliziten Verweises auf „branchenübliche Standards“ in Art. 17 Abs. 4 lit. b DSM-RL und den expliziten gesetzlichen Bemühungen relativieren, jede Anwendung der Handlungsobliegenheiten der Diensteanbieter unter den Vorbehalt ihrer Verhältnismäßigkeit im Einzelfall zu stellen. Jede Inanspruch-

${ }^{134}$ Vgl. Spindler, GRUR 2020, 253 (257), der auch auf den Schutz personenbezogener Daten aus Art. 8 GRCh verweist, der hier aber jedenfalls nicht im Schwerpunkt betroffen ist.

${ }^{135}$ Vgl. EuGH, Urt. v. 24.11.2011 - C-70/10, MMR 2012, 174 (Rn. 52) - Scarlet Extended/SABAM; Urt. v. 16.02.2012 - C-360/10, MMR 2012, 334 (Rn. 50) - SABAM/Netlog.

${ }^{136}$ Vgl. EuGH, Urt. v. 27.03.2014 - C-314/12, GRUR 2014, 468 (Rn. 56 f.) - UPC Telekabel; vgl. insoweit Holznagel, ZUM 2018, 350 (356).

${ }^{137} \mathrm{Vgl}$. dazu schon oben $\S 4$ C. IV. 2. a).

${ }^{138}$ EuGH, Urt. v. 24.11.2011 - C-70/10, MMR 2012, 174 (Rn. 46) - Scarlet Extended/SABAM.

${ }^{139}$ EuGH, Urt. v. 24.11.2011 - C-70/10, MMR 2012, 174 (Rn. 47) - Scarlet Extended/SABAM.

${ }^{140}$ Hierauf stellen auch Peters/Schmidt, GRUR Int 2019, 1006 (1015), ab.

${ }^{141}$ Vgl. EuGH, Urt. v. 03.10.2019 - C-18/18, NJW 2019, 3287 (Rn. 45) - GlawischnigPiesczek; vgl. Spindler, GRUR 2020, 253 (258). 
nahme hätte zur Voraussetzung, dass das Gericht die Verhältnismäßigkeit im Rahmen von Art. 17 Abs. 4 DSM-RL vollumfänglich beachtet. Auch die Stärkung der Nutzerrechte mildert den Vorwurf deutlich ab. ${ }^{142}$ Insbesondere die Konkretisierung und subjektiv-rechtliche Aufladung von Schranken des Urheberrechts in Art. 17 Abs. 7 UAbs. 2 DSM-RL bietet Raum, Maßnahmen nur nicht im reaktiven Rechtsschutz vorzusehen, sondern auch ex ante das Erfassen rechtmäßiger Inhalte zu verhindern bzw. dessen Wahrscheinlichkeit zu reduzieren. ${ }^{143}$ Ferner ist nochmals darauf hinzuweisen, dass Intermediäre grundsätzlich ein hohes Interesse an der Verfügbarkeit möglichst vieler Inhalte haben, ${ }^{144}$ sodass im Abstellen auf die Branchenüblichkeit jedenfalls kein besonders erheblicher Anreiz zur ausschließlichen Entfernung von Inhalten gesehen werden kann. ${ }^{145}$

Letztlich wird es zum einen entscheidend darauf ankommen, in welcher Art und Weise die Umsetzung der DSM-RL erfolgt, insbesondere welche konkrete Ausgestaltung die Position der Rechteinhaber und der von der Entfernung von Inhalten Betroffenen erfahren wird. Zum anderen wird die rechtspraktische Anwendung der Haftungsregeln ebenfalls auf die Bewertung des in Art. 17 DSM-RL niedergelegten Haftungsregimes Einfluss nehmen. Theoretisch sind der Rechtspraxis jedenfalls Instrumente an die Hand gegeben, um eine prohibitive oder grundrechtseinschränkende Wirkung zu verhindern. Auf dieses Ziel lassen sich auch die entsprechenden Technologien selbst einrichten, wie Raue und Steinebach in einem interdisziplinären Beitrag darlegen. Insbesondere die bereits jetzt absehbare technische Möglichkeit, für den „Zugriff“ des Filters im konkreten Fall Schwellenwerte sowie Reaktionsalternativen festlegen zu können, ${ }^{146}$ lässt die Perspektive differenzierungsfähiger technischer Lösungen aufscheinen, die mit schematischen Filterpflichten nur noch wenig gemein haben, die der EuGH in den $S A B A M$ Entscheidungen kategorial verworfen hatte.

Insgesamt ist angesichts vieler noch offenere Umsetzungsfragen eine abschließende Bewertung der Vereinbarkeit des von Art. 17 DSM-RL vorgesehenen Einsatzes von Filtern mit grundrechtlichen Vorgaben nicht möglich.

${ }^{142}$ Vgl. Hofmann, GRUR 2019, 1219 (1227).

${ }^{143}$ So schlägt Dreier, GRUR 2019, 771 (777), vor, dass Nutzer beim Hochladen von Inhalten, die unter solche Schranken fallen, diese Inhalte als legitime Verwendung ,ausflaggen" könnten, sodass diese als default nicht zu entfernen wären; dies befürwortet auch Hofmann, GRUR 2019, 1219 (1227 f.).

${ }^{144} \mathrm{Vgl}$. oben $\S 8$ Fn. 37, 87 und den jeweils zugehörigen Text; vgl. ferner Gerpott, MMR 2019, 420 (422); Hofmann, GRUR 2019, 1219 (1227); Eifert, in: Eifert/Gostomzyk, Netzwerkrecht, S. 9 (34); diesen Aspekt lassen aber viele Stimmen unter den Tisch fallen, vgl. etwa Kaesling, JZ 2019, 586 (589); Kastl, GRUR 2016, 671 (678).

${ }^{145}$ So aber ohne weitere Begründung Senftleben, ZUM 2019, 369 (372).

${ }^{146}$ Raue/Steinebach, ZUM 2020, 355 (362 ff.). 
Allerdings sind gerade angesichts der Rechtsprechung des EuGH substanzielle Bemühungen des Unionsgesetzgebers zu erkennen, eine grundrechtsschonende Fortentwicklung des Haftungssystems im Bereich des Urheberrechts zu etablieren. Von einer „Inhaltszensur" zu sprechen liegt aber jedenfalls fern. ${ }^{147}$ Damit wird letztendlich behauptet, die Regelung ermögliche es, missliebige Meinungen über den Hebel des Art. 17 DSM-RL unterdrücken zu können. Der Vorwurf ist, jedenfalls wenn es um die Intention geht, kaum haltbar, soweit er qualitativ mehr meint als die Erfassung von rechtmäßigen Inhalten als Kollateralschaden.

\section{Fazit zur DSM-RL}

Ähnlich wie auch schon zum NetzDG festgestellt, ergibt sich bei der Bewertung des Art.17 DSM-RL ein gemischtes Bild. Die Fortentwicklung des Haftungsrechts für Intermediäre im Bereich des Urheberrechts kann nach dem bisher Gesagten gewiss nicht als völlig unproblematisch bezeichnet werden. Mit Blick auf die Verkürzung der grundrechtlich verbürgten Informations- und Meinungsfreiheit ist ein gewisses Risiko nicht von der Hand zu weisen. Allerdings ist Art. 17 DSM-RL ersichtlich darauf angelegt, eine schonende und kontextsensible Anwendung unter Einsatz moderner, zu Differenzierungen fähiger und nachprüfbarer Technologie zu ermöglichen und fordert diese in weiten Teilen auch selbst ein. Das Potenzial zu einer verhältnismäßigen Anwendung der Vorgaben des Art. 17 DSM-RL in der Praxis ist damit jedenfalls erkennbar.

\section{Fazit}

Inwiefern lassen sich zwischen dem NetzDG und Art. 17 DSM-RL Parallelen ausmachen? Die hier dargestellten neuen Ansätze zur Fortentwicklung der Störerhaftung in spezifischen Konstellationen bzw. Teilrechtsgebieten ähneln sich nicht zufällig in einigen entscheidenden Aspekten. So fällt die ähnliche Herangehensweise bei der genauen Festlegung des Anwendungsbereichs des NetzDG und des Art. 17 DSM-RL auf. Beiden Instrumenten liegt der Versuch und die Überzeugung zugrunde, über die nach Zahl der Nutzerinnen bzw. nach wirtschaftlicher Leistungsfähigkeit differenzierende Anlegung rechtlicher Verhaltensobliegenheiten eine verhältnismäßige Herangehensweise an die Haftung der Diensteanbieter in den jeweiligen Teilrechtsgebieten zu ermöglichen. ${ }^{148}$ Dieser Ansatz reflektiert das hier bereits grund-

${ }^{147}$ So aber Senftleben, ZUM 2019, 369 (371).

${ }^{148}$ Dreier, GRUR 2019, 771 (776), geht sogar so weit, die „große Menge“ im Sinne von 
sätzlich erläuterte Petitum, den schematischen Ansatz der Art. 12 ff. ECRL zu überwinden und stattdessen zu einer stärker funktional differenzierenden Perspektive auf die Diensteanbieter zu gelangen. ${ }^{149}$

Auch die Einsicht, dass durch die Neuformulierung von Sorgfaltsstandards letztlich an Verhaltensweisen angeknüpft wird, die die Unternehmen ohnehin schon aus eigenem Antrieb vornehmen, ist sowohl dem NetzDG als auch Art. 17 DSM-RL immanent. Sowohl die Betreiber sozialer Medien als auch Dienste zum Teilen von Inhalten im Sinne von YouTube haben auch schon vor Erlass der jeweiligen Sonderregeln eigenständige Verfahren zum Umgang mit Nutzerbeschwerden etabliert und in diesem Zusammenhang in nicht unerheblichem Umfang Inhalte entfernt bzw. gesperrt. Selbstredend verändert die gesetzliche Regelung solcher Verfahren die Anreizstruktur, über die sich in den Details trefflich streiten lässt. Fakt ist aber, dass sich jedenfalls für einige von diesen Regulierungsmaßnahmen betroffenen Unternehmen zunächst nicht viel geändert hat. ${ }^{150}$

Abschließend bleibt festzuhalten, dass die netzpolitischen Herausforderungen bei der Entwicklung und Umsetzung von Maßnahmen, um das für die Intermediäre relevante Haftungsrecht fortzuentwickeln, vor enorme Herausforderungen gestellt sind. Von diesen Herausforderungen können sich die hier in den Blick genommenen Regelungen im NetzDG und in Art. 17 DSMRL nicht komplett freimachen. Dennoch haben die Gesetzgeber mit diesen Maßnahmen unter Beweis gestellt, dass der status quo des Haftungsrechts, wie er in der ECRL und der hierzu ergangenen Rechtsprechung auf europäischer und nationaler Ebene zum Ausdruck kommt, nicht in Stein gemeiBelt, sondern Fortentwicklungen zugänglich ist, die die Verantwortung der Intermediäre im Grundsatz reflektieren. Es ist jedoch unzweifelhaft, dass der Fortschritt der technischen Entwicklung ebenso neue Erkenntnisse zeitigen wird wie die ersten Erfahrungen mit den hier in den Blick genommenen Maßnahmen in der Rechtspraxis. Der Handlungs- und Rechtfertigungsdruck für die Gesetzgeber wird damit auf absehbare Zeit kaum abnehmen.

$\S 2$ Nr. 6 DSM-RL in eine Berücksichtigung der Zahl der Nutzer eines Dienstes wie bei § 1 Abs. 2 NetzDG zu ,übersetzen“, da diese einen Anhaltspunkt für jene darstellen könne.

${ }^{149} \mathrm{Vgl}$. oben $\S 11 \mathrm{C}$. I.

${ }^{150}$ Zum NetzDG vgl. oben $\S 12$ Fn. 16; zu Art. 17 DSM-RL exemplarisch Dreier, GRUR 2019, 771 (777). 

Schluss 



\section{$\S 13$}

\section{Abschließende Betrachtung}

In einem vergleichsweise neuen und sich rasant fortentwickelnden Lebensbereich wie dem Internet eignet sich ein historischer Blickwinkel womöglich besonders zur Einordnung und Kontextualisierung des Verhältnisses von Internet und Recht als Instrument hoheitlichen Handelns. Eine solche Perspektive erscheint gerade mit Blick auf das Thema dieser Untersuchung besonders gehaltvoll, denn

„,[d]ie im Cyber-Exzeptionalismus und dominanten Internet Governance-Diskurs angelegte Ablehnung staatlicher Souveränität in digitalen Fragen hatte in einer Stimmung und zu einer Zeit ihren Ursprung, in der die Bedeutung des Staates und mit ihr das Konzept der Souveränität als im Schwinden begriffen wurden. ${ }^{~}{ }^{1}$

Historisch betrachtet passen weitreichende Haftungsprivilegierungen durchaus in diese Zeit, in der die mit der Nutzung des Internets als Technologie der Massen verbundenen Probleme zwar bereits absehbar waren, in der man aber als Reaktion hierauf das „exzeptionelle“ des digitalen Raums bzw. des Cyberspace zur Geltung bringen und dessen gedeihliche Entwicklung unbedingt schützen wollte. Hierzu sah man eine rechtliche Absicherung der im Entstehen begriffenen und teilweise noch nicht einmal absehbaren Geschäftsmodelle als erforderlich an. Eine vollkommene Staats- bzw. Regulierungsfreiheit des digitalen Raums entsprach also schon damals nicht den Tatsachen und unterstreicht den Eindruck, dass die Ideen Barlows von Anfang an unhaltbar waren. ${ }^{2}$

${ }^{1}$ Pohle/Thiel, in: Borucki/Schünemann, Internet und Staat, S. 57 (67).

${ }^{2}$ Vgl. Pohle/Thiel, in: Borucki/Schünemann, Internet und Staat, S. 57 (72). Ein ironischer Ausweis dieser Feststellung liegt in der etymologischen Herkunft des Wortes Cyberspace begründet, das eine Zusammensetzung von Kybernetik (cybernetics) und Raum (space) darstellt, vgl. www.duden.de/rechtschreibung/Cyberspace. Abgeleitet aus dem griechischen $\kappa \mho ß \varepsilon \rho v \eta ́ \tau \eta \varsigma$, das man mit „Steuermann“ übersetzen kann und das übrigens wohl auch den Ursprung des lateinischen Begriffs für Steuerung oder auch Regierung (gubernare) bildet, umfasst das Forschungsgebiet der Kybernetik in den Worten seines Begründers ,die Untersuchung dessen, was einerseits beim Menschen als geistige Funktionen bezeichnet werden kann und andererseits in den Ingenieurwissenschaften Kontroll-, Steuer- und Fernmelde-Verfahren sind“ (Wiener, Physikalische Blätter 5 (1949), 355 (355)). Überspitzt formuliert ist also beim Cyberspace vom Raum der Steuerung die Rede. 
Die damals gefundenen Regelungen prägen die Geschäftsmodelle des Internets und viele Rechtsordnungen bis heute. Die Entwicklung der letzten gut 20 Jahre in diesem Bereich war insgesamt durch einen Prozess permanenter Aushandlung verschiedener, teilweise nur schwer miteinander vereinbarer Interessen geprägt, der zuvörderst die Gerichte beschäftigte. Die Rechtsprechung konnte sich zwar grundsätzlich flexibler Instrumente wie etwa der Störerhaftung bedienen, um zu kontextangemessenen Entscheidungen zu gelangen, war jedoch nicht vor der Herausforderung gefeit, mit der teils rasanten Entwicklung der Technik und der gesellschaftlichen Reaktionen hierauf Schritt zu halten. Die Taktzahl für den Kontext dieser Untersuchung relevanter Entscheidungen ist in den letzten Jahren entsprechend konstant hoch geblieben. Selbst bei unveränderter Fortgeltung des derzeitigen Regelungsregimes ist nicht davon auszugehen, dass sich hieran in näherer Zukunft etwas ändern wird.

Rechtspolitisch betrachtet haben die Entwicklungen der letzten Jahre ebenfalls eine ungeahnte Dynamik in tatsächlicher und rechtlicher Hinsicht in diesen Prozess gebracht. Dies mag weniger an der permanenten Entstehung neuer Geschäftsmodelle, sondern eher an der Gewöhnung der Gesellschaft an die fortschreitende Digitalisierung nahezu aller Lebensbereiche und die Konsolidierung der Stellung einiger weniger Diensteanbieter in ihrem Tätigkeitsfeld liegen, die zu einer immer stärkeren Wahrnehmung der hiermit verbundenen Probleme geführt hat. Dies ist gewiss bedingt durch politische und gesellschaftliche Entwicklungen allgemeinerer Natur. Als Kipppunkt kann hier der Zeitraum der Jahre 2015/2016 bezeichnet werden. Damals erlebte man in den USA einen Präsidentschaftswahlkampf der eher unangenehmeren Sorte, bei dem u.a. Facebook eine unrühmliche Rolle zugeschrieben wurde. ${ }^{3}$ Im selben Zeitraum nahm man - wie bereits angemerkt - die Folgen eines hitzigen Diskurses im Internet mit Blick auf verstärkte Fluchtbzw. Migrationsbewegungen in Europa und in Deutschland immer stärker wahr. Auch hier standen die Intermediäre und ihr Verhalten ebenfalls im Fokus der Aufmerksamkeit.

Seit diesen Ereignissen bzw. einzelnen Phasen steht die politische Großwetterlage in Deutschland und Teilen der EU für eine zunehmend kritische Wahrnehmung der Intermediäre. Die auf den Plan gerufenen Gesetzgeber wollen - trotz bisweilen anzutreffender Schwierigkeiten, das Tempo des technischen Fortschritts mitzugehen - verständlicherweise Handlungsfähigkeit demonstrieren. Die hier dargestellten gesetzlichen Neuerungen wie etwa das NetzDG stellen nur ein Teil der gesetzgeberischen Aktivitäten dar. ${ }^{4}$ Die Lage

${ }^{3}$ Vgl. www.spiegel.de/politik/ausland/russland-und-die-us-wahl-2016-fuer-donald-tru mp-gegen-alle-anderen-a-1244043.html; Ravel, University of Memphis Law Review 49 (2019), 1019 (1049 ff.); vgl. auch die Anklageschrift „USA v. Internet Research Agency et al.", www.justice.gov/file/1035477/download.

${ }^{4}$ Die Bekämpfung von Terrorismus und der Verbreitung terroristischer Inhalte im In- 
im Urheberrecht unterliegt unzweifelhaft anderen Rationalitäten, ist aber strukturell dem skizzierten Befund nicht unähnlich. Die Konfliktlagen sind hier zwar andere, doch ist auch hier in den letzten Jahren mit der Konsolidierung von (nur auf den ersten Blick) kostenlosen Angeboten zum Abruf von Musik im Internet ein steigendes Bewusstsein für deren Konsequenzen festzustellen. Dieses nahm auch die Kommission Juncker wahr und entschloss sich deshalb zu einer Reform des europäischen Urheberrechts, die in der DSM-RL mündete. In beiden Bereichen ist allerdings schon absehbar, dass mit den einmal gefundenen, gesetzlich festgehaltenen Kompromissen die Entwicklung nicht an ihren rechtlichen „Endzustand“ geführt ist, sondern vielmehr weiter Gegenstand rechtlicher wie auch rechtspolitischer Auseinandersetzungen bleiben wird. Es ist davon auszugehen, dass die explizite oder implizite Einbeziehung technischer Lösungsansätze zur Umsetzung von Pflichten oder Verhaltensobliegenheiten angesichts des unaufhaltbar scheinenden technischen Fortschritts weiter zunehmen wird. Dies wird nicht zuletzt im Kontext dieser Untersuchung eine stete Reflexion rechtlich fixierter Erwartungen an die Intermediäre notwendig machen. ${ }^{5}$ Dieser Aufgabe werden sich auch und gerade die Rechtsordnungen und die in diesen tätigen Institutionen und Akteure stellen müssen.

Die damit verbundenen Herausforderungen sind dabei selbstredend nicht ausschließlich auf den europäischen Rechtsraum bezogen, sondern stehen stets im Kontext der internationalen Entwicklung, die aktuell immer noch von den USA dominiert ist. Insoweit ist die Divergenz verschiedener Rechts-, insbesondere Verfassungsordnungen, die sich insbesondere in den Kommunikationsinfrastrukturen des Internets bemerkbar macht, ein nicht zu unterschätzender Faktor. ${ }^{6}$ Die Rolle US-amerikanischer Unternehmen als prägende Akteure vieler Geschäftsmodelle hat dazu geführt, dass verfassungsrechtliche und -kulturelle Spezifika der USA fast weltweite Wirkmacht gerade mit Blick auf die Ausübung kommunikativer Freiheit entfalten konnten. ${ }^{7} \mathrm{Ob}$ die Lösung dieses Problems, soweit man die Annahme eines solchen

ternet stellt hier gewiss einen weiteren Schwerpunkt politischer Aktivitäten der letzten Jahre dar, vgl. dazu oben $\S 4$ Fn. 288.

${ }^{5}$ Rademacher, JZ 2019, 702 (707), meint, dass insoweit eher mit einem verbesserten Potenzial der Angleichung rechtlicher Standards und technischer Möglichkeiten zu rechnen ist.

${ }^{6}$ Vgl. nur Masing, NJW 2012, 2305 (2309): „Nirgends zeigt sich die Schwächung der Ordnungsfunktion des demokratischen Rechtsstaats so deutlich wie in der Internetkommunikation [...]. Indem sich Kommunikation über ein diffuses Netzwerk dezentraler Rechner unter Überwindung von Zeit und Raum in eine virtuelle Welt verlegt, laufen staatliche Regelungen und Sanktionen weithin leer.“

${ }^{7}$, , [A]s if under cover of night, we have now wired these nations with an architecture of communication that builds within their borders a far stronger First Amendment than our ideology ever advanced. [...] We have exported to the world, through the architecture of the 
denn teilt, in der globalen Durchsetzung regionaler Standards liegt, ${ }^{8}$ ist zumindest zweifelhaft, da dies die Pluralität verschiedener Verfassungstraditionen zu überspielen drohte. Demgegenüber ist die politische Wirkung, die man etwa der DS-GVO im internationalen Kontext beimessen kann, womöglich effektiver, ${ }^{9}$ da diese gerade ohne eine weltweite rechtliche Durchsetzbarkeit auskommt. ${ }^{10}$ Insofern spricht viel dafür, gesetzliche Fortschreibungen der Verantwortung der Intermediäre nicht auf nationale Alleingänge zu beschränken, sondern auf der Unionsebene selbstbewusst in Angriff zu nehmen.

Diese Untersuchung hat deutlich werden lassen, dass die Haftung der Intermediäre überaus komplexe Fragen aufwirft. Deren Beantwortung stellt stets eine Gratwanderung dar und muss in besonderem Maße darum bemüht sein, alle betroffenen Interessen gleichermaßen zu berücksichtigen. Eine besondere Herausforderung ergibt sich aus der Kombination mehrerer Faktoren. Zunächst zeichnet sich die maßgebliche Rechtsprechung nicht gerade durch eine einheitliche Linie aus. Außerdem nimmt die Rechtsprechung des EuGH eine zunehmend entscheidende Rolle ein, die aber nicht immer auf die Befindlichkeiten einzelner Mitgliedstaaten eingehen kann, sondern auch die Unionsrechtsordnung im Blick behalten muss. ${ }^{11}$ Schließlich sind in diesem Bereich in jüngerer Zeit immer öfters bereichsspezifische gesetzliche Interventionen festzustellen. In einer immer noch primär durch die Rechtsprechung geprägten Rechtsmaterie stellen solche Interventionen nicht immer nur einen Gewinn an Klarheit dar.

In der rechtswissenschaftlichen Binnenperspektive unterstreicht das Thema dieser Untersuchung den Gedanken der Intradisziplinarität. ${ }^{12}$ Das liegt pragmatisch gesehen schon an der Relevanz einer Regelung wie $§ 10$ TMG für das bürgerliche Recht und das Strafrecht. Beide Teilrechtsordnungen haben dementsprechend seit Anbeginn der Diskussionen um die Haftung

Internet, a First Amendment in code more extreme than our own First Amendment in law", Lessig, Code and other Laws of Cyberspace, S. 167 (Hervorh. im Original).

${ }^{8}$ Vgl. die eher substanzarmen Ausführungen des EuGH, Urt. v. 03.10.2019 - C-18/18, NJW 2019, 3287 (Rn. 48 ff.) - Glawischnig-Piesczek, zur Frage der weltweiten Durchsetzung von Verhinderungspflichten, der diese Frage den Mitgliedstaaten zuweist.

${ }^{9}$ Nicht zuletzt gab die DS-GVO wohl auch den Anstoß zur Erarbeitung des kalifornischen Datenschutzgesetzes (Civil Code of the State of California, Division 3 Part 4 Title 1.81.5. California Consumer Privacy Act of 2018), vgl. Ulloa, Los Angeles Times, 11.03.2019.

${ }^{10}$ Hinsichtlich des „Rechts auf Vergessen“ hat der EuGH, Urt. v. 24.09.2019 C-507/17, NJW 2019, 3499 (Rn. 59 ff.) - Google/CNIL, einer solchen globalen Durchsetzung jüngst auch eine Absage erteilt.

${ }^{11}$ Vgl. Mayer, in: Grabitz/Hilf/Nettesheim, Recht der EU, 71. EL 2020, EUV Art. 19 Rn. 33.

${ }^{12}$ Vgl. auch Hofmann, JZ 2018, 746 (751 f.). 
Impulse geliefert. ${ }^{13}$ Aber auch das klassische öffentliche Recht spielt eine bedeutsame Rolle. So waren grundrechtliche Wertungen schon seit jeher der zivilrechtlichen Haftungsfrage immanent und dementsprechend ein wichtiger Baustein jedes Ansatzes zur Lösung der hiermit verbundenen Konflikte. Mit neuen Regulierungsansätzen wie dem NetzDG, das die etablierten Prinzipien der Störerhaftung ordnungsrechtlich überformt, verstärkt sich die Bedeutung dieses verfassungsrechtlichen Blickwinkels um ein Vielfaches. Die wechselseitigen Einflüsse und Rückbezüge zwischen den einzelnen Teilrechtsordnungen gilt es daher verstärkt in den Blick zu nehmen. Insoweit bietet die Thematik auch einen guten Anlass, sich in kritischer Weise mit der tradierten Säulenstruktur der deutschen Rechtswissenschaft auseinanderzusetzen. ${ }^{14}$ Als besondere Materie des Medienrechts, das wiederum selbst im weiteren Sinne als „Technikrecht" angesehen werden kann, ${ }^{15}$ ist die Haftung der Intermediäre ohnehin eine klassische Querschnittsmaterie. Als solche entzieht sie sich der Bildung eines passgenauen dogmatischen Korsetts und ist vielmehr auf die Impulse vielfältiger rechtswissenschaftlicher Blickwinkel ebenso angewiesen wie auf die Einbeziehung technischer Expertise. ${ }^{16}$

Die Informationsintermediäre haben unzweifelhaft zu einer erheblichen Erweiterung des verfügbaren Wissens auf der ganzen Welt beigetragen. Sie bieten fast grenzenlose Möglichkeiten der Kommunikation und sind damit gewiss Treiber der gesellschaftlichen Vernetzung und des Fortschritts. Dank der fast dauerhaften, preiswerten Verfügbarkeit des mobilen Internets ist dieses unzweifelhaft die Handlungsfähigkeit der Menschheit vergrößernde Potenzial gleichsam allgegenwärtig. Dem stehen jedoch vielfältige kritische Implikationen mit Blick auf die öffentliche Kommunikation gegenüber. ${ }^{17}$ So eröffnen Kommunikationsdienste weitreichende Möglichkeiten und Anreize

${ }^{13}$ Vgl. die oben unter $§ 4$ A. angerissene Debatte zwischen Sieber und Spindler. In der Praxis stellt sich gerade für das Strafrecht, das in dieser Untersuchung nur punktuell thematisiert wurde, erhebliche Herausforderungen bei der Sanktionierung rechtswidrigen Verhaltens im Internet.

${ }^{14}$ Vgl. Lindner, JZ 2016, 697 ff., der die „Zentrifugalität“ der Rechtswissenschaft beklagt, die er insbesondere in deren zunehmender „Versäulung“ ausmacht (697) und dieser eine Idee für die „Einheit der Rechtwissenschaft“ entgegensetzt (698 ff.); nuancierter dagegen Jestaedt, JZ 2014, 1 (11), der eine solche Säulenstruktur pragmatisch als nachvollziehbar erachtet, sich aber an deren „Rechtswesenhaftigkeit“ stört; die Möglichkeit, je nach Säulenwahl einen eigenen „Rechtskosmos“ zu betreten sei bereits aufgrund des horizontal Geltung beanspruchenden Verfassungs- und Unionsrechts kaum plausibel.

${ }^{15} \mathrm{Vgl}$. Eifert/Hoffmann-Riem, in: Schulte/Schröder, Handbuch des Technikrechts, S. $667 \mathrm{ff}$.

${ }^{16}$ Vgl. aber Zech, Entscheidungen digitaler autonomer Systeme, S. 9, der darauf hinweist, dass das Haftungsrecht auch ,eine Antwort auf [...] Informationsasymmetrien darstellen" kann.

${ }^{17}$ Die folgenden Andeutungen stellen selbstredend nur einige Beispiele dar. 
zur Organisation extremistischer Gruppierungen und Strömungen gerade im politischen und damit in einem für demokratische Gesellschaftsordnungen besonders relevanten Bereich. ${ }^{18}$ Sie erleichtern und effektivieren die Koordinierung von Angriffen auf marginalisierte bzw. besonders schutzwürdige Gruppen, Minderheiten oder Personen. ${ }^{19}$ Die Feststellung, wir befänden uns in einer Phase vielfältiger Krisen und Entgrenzungen, ist fast allgegenwärtig. All diese Beobachtungen sind jedoch gewiss keine Besonderheiten des Internets, sondern stellen vielmehr die Normalität menschlichen Zusammenlebens dar. ${ }^{20}$ Nicht selten verschärfen sich jedoch die hierdurch entstehenden gesellschaftlichen Konfliktpotenziale im digitalen Kontext in bedeutender und besonders sichtbarer Weise.

Ist mit Blick auf diese vielfältigen, oftmals auch durch die Tätigkeit der Intermediäre bedingten Herausforderungen nun zu folgern, dass ,,das Internet all das geworden ist, was keiner wollte"? ${ }^{21}$ Eine abschließende Beantwortung dieser Frage fällt schwer, denn die Erreichung eines „digitalen Endpunktes" steht kaum zu erwarten. Realistischer erscheint eher das Bild eines andauernden, sich stetig weiterentwickelnden Interimszustands. Begreift man das Internet und seine Räume der Kommunikation als ein Gebäude, ${ }^{22}$ werden diese wohl kaum je bezugsfertig sein, wie man es bei einer Wohnung oder einem Haus erwarten würde. Dennoch werden wir mit und in ihm leben wollen und müssen. Für die Sicherung der Statik des Gebäudes und die damit verbundene Lebensqualität wird das Recht als Instrument kollektiver Selbstbestimmung im Werkzeugkoffer unverzichtbar bleiben. Das gibt Anlass zum Optimismus.

\footnotetext{
${ }^{18}$ Vgl. Schulze, in: Liebold et al., Demokratie in unruhigen Zeiten, S. 227 ff.; zur Beschreibung und Empirie „rechts motivierter Cyberdelikte“vgl. Wiacek, Strafbarkeit rechts motivierter Cyberkriminalität in sozialen Netzwerken, S. $61 \mathrm{ff}$.

${ }^{19} \mathrm{Vgl}$. dazu die Nachweise oben in $\S 5$ Fn. 15.

${ }^{20} \mathrm{Vgl}$. Hofmann, in: Martinsen, Wissen - Macht - Meinung, S. 14 (18); vgl. auch Egger de Campo, Leviathan 2014, 7 ff., mit einem institutionenbezogenen Blick.

${ }^{21}$ Zitat bei Lovink, Im Bann der Plattformen, S. 46.

${ }^{22}$ Vgl. Kosseff, The Twenty-Six Words that Created the Internet, S. 280.
} 


\section{Zusammenfassung}

\section{Zu Kapitel 1}

1. Mit $\S 230$ CDA schaffte der US-Gesetzgeber zu Beginn der Nutzung des Internets durch die Massen eine rigide Immunisierung der Diensteanbieter, um die technische Entwicklung nicht zu hemmen. Diese Privilegierung erfolgte jedoch nicht zum Selbstzweck. Mit der Überschrift der Privilegierung für ,gute Samariter“ verdeutlicht der Gesetzgeber vielmehr, dass den Diensteanbietern für die eigeninitiative Kontrolle ihres Einflussbereichs kein Risiko drohen soll. Diesen Aspekt vernachlässigen die Gerichte jedoch und schirmen die Intermediäre von fast jedwedem Risiko ab.

2. Eine demgegenüber offenere Regelung der Haftung stellt §512 DMCA dar, der für Urheberrechtsverletzungen ein Notice-and-TakedownRegime installiert. Bei dessen Handhabung erweisen sich jedoch einzelne Kriterien als hohe Hürde für von Rechtsverletzungen Betroffene bzw. werden Kriterien durch die Gerichte teilweise zum Nachteil von Rechteinhabern ausgelegt. Den Diensteanbietern wird auch insoweit kein zwingender Anreiz gesetzt, Beschwerden nachzugehen. Dies gilt insbesondere deswegen, weil die Diensteanbieter nach der gesetzlichen Regelung keine echte materielle Prüfung der Beschwerden vornehmen sollen.

3. Mit $\S 5$ TDG a.F. stellt der deutsche Gesetzgeber bereits frühzeitig die Weichen für das europäische Haftungssystem, das auch der ECRL zugrunde liegt. Im Gegensatz zur Regelung in den USA wird hier eine horizontal geltende Norm geschaffen, die im Grundsatz ebenfalls eine Immunisierung der Diensteanbieter zum Ziel hat. Dies ist einerseits ein begrüßenswertes Anliegen, verkompliziert im Mehrebenensystem der EU sowie im föderalistisch geprägten deutschen Medienrecht jedoch den rechtlichen Zugriff auf die Diensteanbieter.

4. Die Rechtsprechung des EuGH erhebt die „Neutralität“ zum wesentlichen normativen Leitbild der Intermediäre, das über die Anwendbarkeit der Privilegierungen entscheidet. Die Entwicklungslinie mit Blick auf das Verbot allgemeiner Überwachungspflichten deutet auf dessen zunehmende Ergänzung durch die Unionsgrundrechte hin.

5. Der BGH beharrt weiterhin auf der Kategorie des Zueigenmachens fremder Inhalte, obwohl die Vereinbarkeit dieser Figur mit Art. 14 ECRL zweifelhaft ist, und versucht diese mit dem Konzept der Neutralität zu ver- 
knüpfen. Eine Deckungsgleichheit der „Kerntheorie“ der Störerhaftung mit dem Verbot allgemeiner Überwachungspflichten im Sinne des Art. 15 ECRL ist bislang nicht festzustellen.

\section{Zu Kapitel 2}

1. Eine reflektierte begriffliche Herangehensweise erleichtert insbesondere im technisch geprägten Kontext der Kommunikation im Internet den rechtlichen Zugriff. Insoweit ist insbesondere gegenüber dem Begriff „Plattform“ Skepsis angezeigt. Demgegenüber sind Begriffsverwendungen vorzuziehen, die die Akteure unmittelbar adressieren und damit ein Zurechnungsobjekt herstellen können. Hier empfiehlt es sich, von Diensteanbietern bzw. Intermediären zu sprechen. Um von Suchmaschinenbetreibern abzugrenzen, lässt sich auch von Kommunikationsdiensten sprechen.

2. Bereits aus der technischen Struktur netzbasierter Kommunikation lassen sich Schlussfolgerungen für den rechtlichen Zugriff auf die Intermediäre ziehen. Während für den Signalübertragungsweg im weiteren Sinne eine „Netzneutralität“ grundsätzlich sinnvoll erscheint, ist eine solche Vorstellung mit Blick auf die Intermediäre nicht überzeugend.

3. Kommunikationsdiensten und Suchmaschinenbetreibern ist die Kuratierung von Inhalten als wesentliche Eigenschaft gemein.

4. Die Kategorie „Host-Provider“ ist strukturell nicht geeignet, die Tätigkeit der Intermediäre sinnvoll abzubilden. Denn sie verfügen über eine weitreichende Verfügungsmacht beim Umgang mit nutzergenerierten Inhalten, die sich insbesondere auf den Zugang der Nutzer zum Dienst sowie dessen strukturell relevante Kontrolle bezieht. Das Abstellen auf den technischen Vorgang der Speicherung im Rahmen der Haftungsprivilegierung überzeugt daher nicht.

5. Das Paradigma der Neutralität im Sinne der Rechtsprechung des EuGH stellt eine grundsätzlich unangemessene Betrachtungsweise der Rolle der Intermediäre dar. Insoweit überzeugt weder die Herleitung des EuGH im Kontext der ECRL, noch die übergeordnete normative Perspektive.

6. Auch die Suchmaschinenbetreiber verfügen über eine erhebliche Gestaltungsmacht bei der Verfügbarkeit von Informationen im Internet. Die Zuordnung von Suchmaschinendiensten unter den Haftungsprivilegierungen ergibt derzeit allerdings insgesamt eine unklare Rechtslage. Dies erzeugt ein zusätzliches rechtliches Unsicherheitspotenzial.

\section{Kapitel 3}

1. Die Störerhaftung erweist sich grundsätzlich als angemessenes Instrument, um kontext- und einzelfallspezifisch auf die Herausforderungen einzugehen, die insbesondere mit Blick auf Persönlichkeitsrechtsverletzungen entstehen. Das vom BGH entwickelte Prüfprogramm, das eine dia- 
lektische Aufarbeitung äußerungsrechtlicher Konfliktlagen erlaubt, stellt einen grundsätzlich sinnvollen Ansatz zur Prozeduralisierung der Haftung der Intermediäre dar. In Details ist jedoch die Vereinbarkeit der Anforderungen der Störerhaftung mit dem Unionsrecht derzeit nicht gesichert.

2. Der in Zusammenhang mit der Störerhaftung oder anderen Instrumenten, die die Intermediäre zur Entscheidung von Konflikten zwischen ihren Nutzern verpflichten, häufig anzutreffende Vorwurf, es erfolge hier eine „Privatisierung" der Rechtsdurchsetzung, trifft in der Sache nicht zu.

3. Die Anforderungen, die die Störerhaftung an die Substanziierung entsprechender Notifizierungen an die Intermediäre aufstellt, sind im Einzelnen zu hoch angesetzt. Dies stellt einen Anreiz für die Diensteanbieter dar, den jeweils erhobenen Vorwürfen nicht nachzugehen. Insoweit sollten die Anforderungen an die Begründung einer Beschwerde abgesenkt werden. Ausreichen sollte bereits eine hinreichende Wahrscheinlichkeit einer Rechtsverletzung aufgrund des Vortrags der Notifizierenden. Im Urheberrecht ist die Forderung nach der Möglichkeit, bereits aufgrund der ersten Beschwerde an einen Diensteanbieter diesen in Anspruch nehmen zu können, eher zutreffend als im Bereich der Persönlichkeitsrechtsverletzungen.

4. Es bedarf einer Revision der Zwecke des Rechts der Haftungsprivilegierungen, die eine Aufnahme einer „Good Samaritan"-Regel beinhalten sollte. Diese sollte Diensteanbieter für solche rechtswidrigen Inhalte von einem Haftungsrisiko befreien, die sie aufgrund eigener Bemühungen auffinden. Gegenwärtig laufen die Intermediäre Gefahr, aufgrund einer dann anzunehmenden Kenntnis doch für solche Inhalte zu haften. Dergestalt wäre eine solche „Good Samaritan“-Regel mit dem Verbot allgemeiner Überwachungspflichten vereinbar.

5. Der Ansatz der Prozeduralisierung der Anforderungen an den Umgang mit Nutzerbeschwerden sollte im Unionsrecht eine Aufwertung erfahren. Auch im Bereich der Persönlichkeitsrechtsrechtsverletzungen ist dies trotz fehlender Harmonisierung der Regelungen zum Haftungsgrund im materiellen Recht möglich. Die Unzulässigkeit manueller Nachkontrollen im Rahmen erlaubter spezifischer Überwachungs- bzw. Verhinderungspflichten, wie sie vom EuGH vertreten wird, überzeugt nicht.

6. Das NetzDG stellt einen grundsätzlich sinnvollen Versuch dar, die primär durch die Störerhaftung ausgeformte Verantwortung der Intermediäre mit Blick auf ein Durchsetzungsdefizit ordnungsrechtlich zu umrahmen und die Intermediäre dadurch zu einer strikteren Befolgung ihrer Verhaltensobliegenheiten zu motivieren. Mit Blick auf die Nutzerrechte in Folge von Entscheidungen der Intermediäre sind jedoch verfassungsrechtlich relevante Mängel festzustellen. Auch ein mit Blick auf Art. 5 GG relevantes Overblocking kann derzeit nicht gänzlich ausgeschlossen werden. 
7. Die differenzierte Anwendung von Sorgfaltsstandards durch die DSMRL stellt grundsätzlich einen sinnvollen Ansatz dar, um im Bereich von Urheberrechtsverletzungen das Haftungsrecht fortzuentwickeln. Die Konturierung von Nutzerrechten stellt ebenfalls eine positive Neuerung gegenüber dem geltenden Urheberrecht dar. Die nicht zu leugnenden Filterpflichten bergen einerseits das Risiko eines Overblockings. Andererseits versucht die DSM-RL durch eine Betonung von Verhältnismäßigkeitsaspekten und Nutzerrechten dieses Risiko abzuschwächen. Dabei wird der mitgliedstaatlichen Umsetzung eine maßgebliche Rolle zukommen. Zudem ist mit der stetigen Verbesserung entsprechender Technologien zu rechnen, die eine differenzierte Umsetzung von Sorgfaltsanforderungen in der Praxis zunehmend wahrscheinlicher macht. 


\section{Literaturverzeichnis}

American Civil Liberties Union: ACLU Opposes S. 1693, Stop Enabling Sex Traffickers Act of 2017 (,SESTA“) (07.11.2017), abrufbar unter: https://www.aclu.org/letter/aclu-1 etter-opposing-sesta.

Amirmahani, Ali: Digital Apples and Oranges. A Comparative Analysis of Intermediary Copyright Liability in the United States and European Union, Berkeley Technology Law Journal 30 (2015), S. 865-898.

Andreessen, Marc: The Three Kinds of Platforms you meet on the Internet, blog.pmarca.com (16.09.2007), abrufbar unter: https://pmarchive.com/three_kinds_of_platforms you_meet_on_the_internet.html.

Balkin, Jack: Free Speech and Hostile Environments, Columbia Law Review 99 (1999), S. 2295-2320.

-: The Future of Free Expression in the Digital Age, Pepperdine Law Review 36 (2009), S. 427-444.

-: Old-School/New-School Speech Regulation, Harvard Law Review 127 (2014), S. 2296-2342.

-: Free Speech is a Triangle, Columbia Law Review 118 (2018), S. 2011-2055.

Barbrook, Richard/Cameron, Andy: The Californian Ideology, Science as Culture 6 (1996), S. 44-72.

Barlow, John Perry: A Declaration of the Independence of Cyberspace, Electronic Frontier Foundation (08.02.1996), abrufbar unter: https://www.eff.org/de/cyberspace-indep endence.

Beale, Steven: Online Terrorist Speech, Direct Government Regulation, and the Communications Decency Act, Duke Law \& Technology Review 16 (2018), S. 333-350.

Becker, Maximilian: Von der Freiheit, rechtswidrig handeln zu können. „Upload-Filter“ und technische Rechtsdurchsetzung, ZUM 2019, S. 636-648.

Beckmann, Kirsten: Verantwortlichkeit von Online-Diensteanbietern in Europa und den Vereinigten Staaten von Amerika. Ein rechtsvergleichendes Thema am Beispiel der Ehrverletzungsdelikte in Deutschland, den USA und England mit europarechtlichem Ausblick, Münster 2001 (Zugl.: Münster, Univ., Diss., 2000).

BeckOK Datenschutzrecht, herausgegeben von Stefan Brink/Heinrich Amadeus Wolff, 34. Edition, München 2020.

BeckOK Informations- und Medienrecht, herausgegeben von Hubertus Gersdorf/Boris Paal, 30. Edition, München 2020.

Beher, Stefan/Hilgert, Christian/Mämecke, Thorben: Netz-Werke. Funktionale Differenzierung, Selbstdarstellung und Beziehungspflege auf Social Network Platforms, in: Michael Bommes/Veronika Tacke (Hrsg.), Netzwerke in der funktional differenzierten Gesellschaft, Wiesbaden 2010, S. 289-315.

Benjamin, Stuart: Transmitting, Editing, and Communicating. Determining what „The Freedom of Speech" Encompasses, Duke Law Journal 60 (2011), S. 1673-1713.

Benkler, Yochai: The Wealth of Networks. How Social Production Transforms Markets and Freedom, New Haven 2006. 
Bergen, Mark: YouTube Executives Ignored Warnings, Letting Toxic Videos Run Rampant, Bloomberg (02.04.2019), abrufbar unter: https://www.bloomberg.com/news/featu res/2019-04-02/youtube-executives-ignored-warnings-letting-toxic-videos-run-rampan $\mathrm{t}$.

Berger, Arndt/Janal, Ruth: Suchet und Ihr werdet finden? Eine Untersuchung zur Störerhaftung von Online-Auktionshäusern, CR 2004, S. 917-925.

Bhaskar, Michael: Curation. The Power of Selection in a World of Excess, London 2016.

Binder, Reinhart/Vesting, Thomas (Hrsg.): Beck'scher Kommentar zum Rundfunkrecht. 4. Aufl., München 2018.

Black, Julia: Decentring Regulation. Understanding the Role of Regulation and SelfRegulation in a ,Post-Regulatory“ World, Current Legal Problems 54 (2001), S. $103-146$.

Bleisteiner, Stephan: Rechtliche Verantwortlichkeit im Internet. Unter besonderer Berücksichtigung des Teledienstegesetzes und des Mediendienste-Staatsvertrags, Köln 1999 (Zugl.: Würzburg, Univ., Diss., 1998).

Bozdag, Engin: Bias in Algorithmic Filtering and Personalization, Ethics and Information Technology 15 (2013), S. 209-227.

Braun, Johanna: Leitbilder im Recht, Tübingen 2015 (Zugl.: Freiburg, Univ., Diss., 2014).

Bräutigam, Peter/Sonnleithner, Bernhard von: Vertragliche Aspekte der Social Media, in: Gerrit Hornung/Ralf Müller-Terpitz (Hrsg.), Rechtshandbuch Social Media, Heidelberg 2015, S. 35-77.

Bridy, Annemarie: Remediating Social Media. A Layer-Conscious Approach, Boston University Journal of Science \& Technology Law 24 (2018), S. 193-228.

Britz, Gabriele: Prozedurale Lösungen von Grundrechtskollisionen ,grundrechtliches Vorverfahren“, Der Staat 42 (2003), S. 35-59.

Brock, Marc/Schmittmann, Michael: Kapitel 10. Telemedien, in: Rolf Schwartmann (Hrsg.), Praxishandbuch Medien-, IT- und Urheberrecht, 4. Aufl., Heidelberg 2017.

Bröhl, Georg: EGG - Gesetz über rechtliche Rahmenbedingungen des elektronischen Geschäftsverkehrs. Erläuterungen zum Referentenentwurf, MMR 2001, S. 67-71.

Brunn, Inka Frederike: Cache me if you can. Verfassungsrechtliche Aspekte der urheberrechtlichen Einordnung von Suchmaschinen, Baden-Baden 2013 (Zugl.: Hamburg, Univ., Diss., 2012).

Bruns, Axel: Vom Gatekeeping zum Gatewatching. Modelle der journalistischen Vermittlung im Internet, in: Christoph Neuberger/Christian Nuernbergk/Melanie Rischke (Hrsg.), Journalismus im Internet. Profession, Partizipation, Technisierung, Wiesbaden 2009, S. 107-128.

Buchheim, Johannes: Das Vorrecht des Ersten Zugriffs auf das Verwaltungsrechtsverhältnis. Das französische „,privilège du préalable“ als leistungsfähiger Begriff deutscher Verwaltungsrechtsdogmatik?, Die Verwaltung 49 (2016), S. 55-79.

-: Anfängerhausarbeit - Öffentliches Recht. Grundrechte - Zensor wider Willen?, JuS 2018, S. 548-556.

Bull, Hans Peter: Über die rechtliche Einbindung der Technik. Juristische Antworten auf Fragen der Technikentwicklung, Der Staat 58 (2019), S. 57-100.

Bundesamt für Justiz: Pressemitteilung. Bundesamt für Justiz erlässt Bußgeldbescheid gegen Facebook, Bonn 03.07.2019, abrufbar unter: https://www.bundesjustizamt.de/DE/ Presse/Archiv/2019/20190702.html?nn=3451904.

Bundeskartellamt: Pressemitteilung. Bundeskartellamt untersagt Facebook die Zusammenführung von Nutzerdaten aus verschiedenen Quellen, Bonn 07.02.2019, abrufbar unter: https://www.bundeskartellamt.de/SharedDocs/Meldung/DE/Pressemitteilunge n/2019/07_02_2019_Facebook.html. 
Burgess, Jean/Green, Joshua/Rebane, Gala: Agency and Controversy in the YouTube Community, in: Heidrun Friese/Gala Rebane/Marcus Nolden/Miriam Schreiter (Hrsg.), Handbuch Soziale Praktiken und Digitale Alltagswelten, Wiesbaden 2016, S. 1-12.

Burkhardt, Emanuel: Kapitel 4. Wortberichterstattung - die Äußerung, in: Egbert Wenzel (Hrsg.), Das Recht der Wort- und Bildberichterstattung. Handbuch des Äußerungsrechts, 6. Aufl., Köln 2018.

-: Kapitel 12. Unterlassungsanspruch, in: Egbert Wenzel (Hrsg.), Das Recht der Wort- und Bildberichterstattung. Handbuch des Äußerungsrechts, 6. Aufl., Köln 2018.

Burkhardt, Emanuel/Peifer, Karl-Nikolaus: Kapitel 10. Sonderfragen, in: Egbert Wenzel (Hrsg.), Das Recht der Wort- und Bildberichterstattung. Handbuch des Äußerungsrechts, 6. Aufl., Köln 2018.

Calabresi, Guido: The Costs of Accidents. A Legal and Economic Analysis, New Haven 1970.

Calliess, Christian/Ruffert, Matthias (Hrsg.): EUV/AEUV. Das Verfassungsrecht der Europäischen Union mit Europäischer Grundrechtecharta. 5. Aufl., München 2016.

Center for Democracy \& Technology: Joint Letter on Free Speech Concerns with S. 1693, the Stop Enabling Sex Traffickers Act (04.08.2017), abrufbar unter: https://cdt.org/insig hts/joint-letter-on-free-speech-concerns-with-s-1693-sesta.

Chamberlain, Lura: FOSTA. A Hostile Law With A Human Cost, Fordham Law Review 87 (2019), S. 2171-2211.

Chang, Liliana: The Red Flag Test for Apparent Knowledge under the DMCA Sec. 512(c) Safe Harbor, Cardozo Arts \& Entertainment Law Journal 28 (2010), S. 195-222.

Chen, DenalDurkee, Musetta/Friend, Jared/Urban, Jennifer: Updating 17 U.S.C. §512's Notice and Takedown Procedure for Innovators, Creators, and Consumers, herausgegeben von Samuelson Law, Technology \& Public Policy Clinic, Berkeley 2011, abrufbar unter: https://www.law.berkeley.edu/wp-content/uploads/2015/04/cranoticetakedown. pdf.

Chmelík, Tomáš: Social Network Sites - Soziale Netzwerke, Baden-Baden 2016 (Zugl.: Freiburg, Univ., Diss., 2015).

Citron, Danielle: Hate Crimes in Cyberspace, Cambridge 2014.

Citron, Danielle/Jurecic, Quinta: FOSTA: The New Anti-Sex-Trafficking Legislation May Not End the Internet, But It's Not Good Law Either, Lawfare (28.03.2018), abrufbar unter: https://www.lawfareblog.com/fosta-new-anti-sex-trafficking-legislation-may-n ot-end-internet-its-not-good-law-either.

Citron, Danielle/Wittes, Benjamin: The Internet Will Not Break. Denying Bad Samaritans Sec. 230 Immunity, Fordham Law Review 86 (2017), S. 401-423.

Cohen, Noam: U.C.L.A. Professor Makes a Case for Google as Publisher, New York Times, 20.05.2012, abrufbar unter: https://www.nytimes.com/2012/05/21/business/med ia/eugene-volokh-ucla-professor-makes-a-case-for-google-as-publisher.html.

Cordes, Christoph: Google darf Presseartikel mit Gesundheitsdaten wiedergeben, GRURPrax 2018, S. 475.

Corra, Mamadi/Willer, David: The Gatekeeper, Sociological Theory 20 (2002), S. 180-207.

Coy, Wolfgang: Kulturen - nicht betreten? Anmerkungen zur „Kulturtechnik Informatik“, Informatik Spektrum 31 (2008), S. 30-34.

Czychowski, Christian/Nordemann, Jan Bernd: Grenzenloses Internet - entgrenzte Haftung? Leitlinien für ein Haftungsmodell der Vermittler, GRUR 2013, S. 986-996.

Danckwerts, Rolf: Neues vom Störer: Was ist ein ,von der Rechtsordnung gebilligtes Geschäftsmodell"?, GRUR-Prax 2011, S. 260-262.

Dankert, Kevin: Normative Technologie in sozialen Netzwerkdiensten. NeueMachtstrukturen als Anreiz für einen Paradigmenwechsel der Kommunikationsregulierung?, KritV 2015, S. 49-73. 
Davis, Bryan: Untangling the Publisher versus Information Content Provider Paradox of 47 U.S.C. 230. Toward a Rational Application of the Communications Decency Act in Defamation Suits against Internet Service Providers, New Mexico Law Review 32 (2002), S. 75-97.

Denninger, Erhard/Schneider, Hans-Peter/Stein, Ekkehart/Hoffmann-Riem, Wolfgang (Hrsg.): Kommentar zum Grundgesetz für die Bundesrepublik Deutschland. 3. Aufl., Neuwied 2001.

Deutscher Anwaltverein: Stellungnahme zum Entwurf eines Gesetzes zur Verbesserung der Rechtsdurchsetzung in sozialen Netzwerken, Stellungnahme 41/2017, Berlin 2017, abrufbar unter: https://anwaltverein.de/de/newsroom/sn-41-17-netzwerkdurchsetzungsge setz-netzdg.

Di Fabio, Udo: Grundrechtsgeltung in digitalen Systemen. Selbstbestimmung und Wettbewerb im Netz, München 2016.

die medienanstalten: Vielfaltsbericht der Medienanstalten, Berlin 2019, abrufbar unter: ht tps://www.die-medienanstalten.de/fileadmin/user_upload/die_medienanstalten/Publi kationen/Vielfaltsbericht/Vielfaltsbericht_2019.pdf.

Diesterhöft, Martin: Das Recht auf medialen Neubeginn. Die „Unfähigkeit des Internets zu vergessen" als Herausforderung für das allgemeine Persönlichkeitsrecht, Berlin 2014 (Zugl.: Freiburg, Univ., Diss., 2013).

Directorate General of Human Rights and Legal Affair of the Council of Europe: Human rights Guidelines for Internet Service Providers, abrufbar unter: https://rm.coe.int/168 05a39d5.

Dorn, Jenny Katharina: Private und administrative Rechtsdurchsetzung im europäischen Beihilfenrecht. Vom indirekten Vollzug zum Kooperationsprinzip, Baden-Baden 2017 (Zugl.: Berlin, Humboldt-Univ., Diss., 2017).

Dörr, Dieter/Natt, Alexander: Suchmaschinen und Meinungsvielfalt, ZUM 2014, S. 829-847.

Dörr, Dieter/Schuster, Simon: Suchmaschinen im Spannungsfeld zwischen Nutzung und Regulierung. Rechtliche Bestandsaufnahme und Grundstrukturen einer Neuregelung, in: Birgit Stark/Dieter Dörr/Stefan Aufenanger (Hrsg.), Die Googleisierung der Informationssuche. Suchmaschinen zwischen Nutzung und Regulierung, Berlin 2014, S. 262-323.

Dreier, Horst (Hrsg.): Grundgesetz. Band III: Art. 83-146. 3. Aufl., Tübingen 2018.

Dreier, Thomas: Die Schlacht ist geschlagen - ein Überblick. Zum Ergebnis des Copyright Package der EU-Kommission, GRUR 2019, S. 771-779.

Drexl, Josef: Bedrohung der Meinungsvielfalt durch Algorithmen, ZUM 2017, S. 529-543.

Edwards, Benj: The Lost Civilization of Dial-Up Bulletin Board Systems, The Atlantic (04.11.2016), abrufbar unter: https://www.theatlantic.com/technology/archive/2016/11/ the-lost-civilization-of-dial-up-bulletin-board-systems/506465.

Egger de Campo, Marianne: Neue Medien - alte Greedy Institutions, Leviathan 2014, S. 7-28.

Ehmann, Eugen/Selmayr, Martin (Hrsg.): Datenschutz-Grundverordnung. Kommentar. 2. Aufl., München 2018.

Ehret, Susanne: Internet-Auktionshäuser auf dem haftungsrechtlichen Prüfstand. Ein Beitrag zur zivilrechtlichen Haftung von Internet-Auktionshäusern für rechtswidrige Auktionsangebote, CR 2003, S. 754-761.

Ehrlich, Paul: Communications Decency Act $§ 230$, Berkeley Technology Law Journal 17 (2002), S. 401-419.

Eidenmüller, Horst: Effizienz als Rechtsprinzip. Möglichkeiten und Grenzen der ökonomischen Analyse des Rechts. 4. Aufl., Tübingen 2015 (Zugl.: München, LudwigsMaximilians-Univ., Diss., 1994). 
Eifert, Martin: Innovationsverantwortung im Netz. Die rechtliche Konturierung angemessener Verhaltensstandards im Netz, in: Martin Eifert/Wolfgang Hoffmann-Riem (Hrsg.), Innovation, Recht und öffentliche Kommunikation. Innovation und Recht IV, Berlin 2011, S. 255-277.

-: Rechenschaftspflichten für soziale Netzwerke und Suchmaschinen. Zur Veränderung des Umgangs von Recht und Politik mit dem Internet, NJW 2017, S. 1450-1454.

-: Das Netzwerkdurchsetzungsgesetz und Plattformregulierung, in: Martin Eifert/Tobias Gostomzyk (Hrsg.), Netzwerkrecht. Die Zukunft des NetzDG und seine Folgen für die Netzwerkkommunikation, Baden-Baden 2018, S. 9-43.

-: Medienföderalismus. Verfassungsrechtliche Probleme der Zuständigkeitsparzellierung im Kommunikationsraum, in: Martin Eifert/Tobias Gostomzyk (Hrsg.), Medienföderalismus. Föderale Spannungslagen und Lösungsansätze in der Medienregulierung, Baden-Baden 2018, S. 7-22.

-: Regulierung von Dynamik und dynamische Regulierung als netzwerkgerechtes Recht. Eine Skizze am Beispiel von Hate Speech in sozialen Netzwerken, in: Yoan Hermstrüwer/Jörn Lüdemann (Hrsg.), Der Schutz der Meinungsbildung im digitalen Zeitalter. Instrumente und Instrumentenvergleich, Tübingen 2021, S. 189-203.

Eifert, Martin/Hoffmann-Riem, Wolfgang: Telekommunikations- und Medienrecht als Technikrecht, in: Martin Schulte/Rainer Schröder (Hrsg.), Handbuch des Technikrechts. Allgemeine Grundlagen - Umweltrecht - Gentechnikrecht - Energierecht - Telekommunikations- und Medienrecht Patentrecht - Computerrecht, 2. Aufl., Heidelberg 2011, S. 667-719.

Eifert, Martin/Landenberg-Roberg, Michael von/Theß, Sebastian/Wienfort, Nora: Netzwerkdurchsetzungsgesetz in der Bewährung. Juristische Evaluation und Optimierungspotenzial, Baden-Baden 2020.

Elixmann, Robert: Datenschutz und Suchmaschinen. Neue Impulse für einen Datenschutz im Internet, Berlin 2012 (Zugl.: Freiburg, Univ., Diss., 2011).

Engel-Flechsig, Stefan: Das Informations- und Kommunikationsdienstegesetz des Bundes und der Mediendienstestaatsvertrag der Bundesländer, ZUM 1997, S. 231-239.

Engel-Flechsig, Stefan/Maennel, Frithjof/Tettenborn, Alexander: Das neue Informationsund Kommunikationsdienste-Gesetz, NJW 1997, S. 2981-2992.

Ensthaler, Jürgen/Heinemann, Mirko: Die Fortentwicklung der Providerhaftung durch die Rechtsprechung, GRUR 2012, S. 433-439.

Esser, Josef: Vorverständnis und Methodenwahl in der Rechtsfindung. Rationalitätsgarantien der richterlichen Entscheidungspraxis, Frankfurt am Main 1970.

Europäische Kommission: Umgang mit illegalen Online-Inhalten. Mehr Verantwortung für Online-Plattformen, COM(2017) 555 final, Brüssel 2017, abrufbar unter: https://eur-le x.europa.eu/legal-content/DEU/TXT/PDF/?uri=celex:52017DC0555.

Fawzi, Nayla: Cyber-Mobbing. Ursachen und Auswirkungen von Mobbing im Internet. 2. Aufl., Baden-Baden 2015.

Felder, Ekkehard: Semantische Kämpfe außerhalb und innerhalb des Rechts, Der Staat 2010, S. 543-571.

Fischer, Linn-Karen: Die Einbindung von Providern in die Durchsetzung von Urheberrechten. Eine rechtsvergleichende Studie zum deutschen und französischen Recht, Tübingen 2020 (Zugl.: Düsseldorf, Univ., Diss., 2019).

-: Die Richtlinie über das Urheberrecht im digitalen Binnenmarkt. Ein guter Anlass zur Schaffung eines kohärenten Providerrechts, ZGE 2020, S. 272-305.

Fitzner, Julia: Sind Haftungsbeschränkungen für Host-Provider noch zeitgemäß? Der „Safe Harbor“ gem. §512 (c) Copyright Act und die Haftungsbeschränkungen gem. Art. 14 E-Commerce-Richtlinie bzw. §10 TMG, GRUR Int 2012, S. 109-117. 
Förster, Achim: Fair Use. Ein Systemvergleich der Schrankengeneralklausel des USamerikanischen Copyright Act mit dem Schrankenkatalog des deutschen Urheberrechtsgesetzes, Tübingen 2008 (Zugl.: Bayreuth, Univ., Diss., 2008).

Franks, Mary Anne: The Lawless Internet? Myths and Misconceptions About CDA Section 230, Huffington Post (18.12.2013), abrufbar unter: https://www.huffingtonpost.co $\mathrm{m} /$ mary-anne-franks/section-230-the-lawless-internet_b_4455090.html.

Franzius, Claudio: Grundrechtsschutz in Europa. Zwischen Selbstbehauptungen und Selbstbeschränkungen der Rechtsordnungen und ihrer Gerichte, ZaöRV 75 (2015), S. 383-412.

-: Das Internet und die Grundrechte, JZ 2016, S. 650-659.

Frey, Harald: Haftungsprivilegierung der Access-Provider nach $\S 8$ TMG? Auflösung eines Normwiderspruchs innerhalb des TMG, MMR 2014, S. 650-654.

-: Die Haftung von Host-Providern für Immaterialgüterrechtsverletzungen, Baden-Baden 2018 (Zugl.: Konstanz, Univ., Diss., 2017).

-: Grundsätze und Fortentwicklung des europäischen Haftungssystems für Host-Provider, ZUM 2019, S. 40-48.

Freytag, Stefan: Haftung im Netz. Verantwortlichkeit für Urheber-, Marken- und Wettbewerbsrechtsverletzungen nach $\S 5$ TDG und $§ 5$ MDStV, München 1999 (Zugl.: München, Ludwigs-Maximilians-Univ., Diss., 1998).

-: Urheberrechtliche Haftung im Netz. Zur dogmatischen Einordnung und praktischen Umsetzung von $\S 5$ TDG und $\S 5$ MDStV bei Urheberrechtsverletzungen im Internet, ZUM 1999, S. 185-195.

-: (K)ein deutscher Sonderweg. Haftung für ,zueigengemachte“ Inhalte nach deutschem und europäischem Recht, GRUR-Prax 2010, S. 355-358.

Galloway, Scott: The Four. The Hidden DNA of Amazon, Apple, Facebook and Google, London 2018.

Gärditz, Klaus Ferdinand: Der digitalisierte Raum des Netzes als emergente Ordnung und die repräsentativ-demokratische Herrschaftsform, Der Staat 54 (2015), S. 113-139.

Geiß, Stefan: Die Aufmerksamkeitsspanne der Öffentlichkeit. Eine Studie zur Dauer und Intensität von Meinungsbildungsprozessen, Baden-Baden 2015 (Zugl.: Mainz, Univ., Diss., 2014).

Geppert, Martin/Schütz, Raimund (Hrsg.): Beck'scher TKG-Kommentar. 4. Aufl., München 2013.

Gerpott, Torsten: Artikel 17 der neuen EU-Urheberrechtsrichtlinie: Fluch oder Segen? Einordnung des Streits um „Upload-Filter“ auf Online-Sharing-Plattformen, MMR 2019, S. $420-426$.

Gersdorf, Hubertus: Hate Speech in sozialen Netzwerken. Verfassungswidrigkeit des NetzDG-Entwurfs und grundrechtliche Einordnung der Anbieter sozialer Netzwerke, MMR 2017, S. 439-447.

Gielen, Nico/Tiessen, Marten: Die neue Plattformhaftung nach der Richtlinie über das Urheberrecht im digitalen Binnenmarkt, EuZW 2019, S. 639-646.

Gieseler, Christoph: Öffentliche Kommunikation auf sozialen Netzwerkplattformen. Eine Rekonstruktion des grundrechtlichen Persönlichkeits- und Datenschutzes im Zeitalter sozialer Medien, Berlin 2018 (Zugl.: Münster, Univ., Diss., 2017).

Gillespie, Tarleton: The Politics of ,Platforms', New Media \& Society 12 (2010), S. 347-364.

-: Die Plattform Metapher - Revisited, Alexander von Humboldt Institut für Internet und Gesellschaft (24.08.2017), abrufbar unter: https://www.hiig.de/die-plattform-metaphe r-revisited.

-: Custodians of the Internet. Platforms, Content Moderation, and the Hidden Decisions that Shape Social Media, New Haven 2018. 
-: Platforms are not Intermediaries, Georgetown Law Technology Review 2 (2018), S. 198-216.

Goldberg, Carrie: Herrick v. Grindr: Why Section 230 of the Communications Decency Act Must be Fixed, Lawfare (14.08.2019), abrufbar unter: https://www.lawfareblog.c om/herrick-v-grindr-why-section-230-communications-decency-act-must-be-fixed.

Goldman, Eric: The Ten Most Important Section 230 Rulings, Tulane Journal of Technology and Intellectual Property 20 (2017), S. 1-10.

-: The Complicated Story of FOSTA and Section 230, First Amendment Law Review 17 (2019), S. 279-293.

Gounalakis, Georgios: Rechtliche Grenzen der Autocomplete-Funktion von Google, NJW 2013, S. 2321-2324.

Gounalakis, Georgios/Muer, Benedikt: Reaktive Prüfpflichten für Google ab Kenntnis einer offensichtlichen Rechtsverletzung, NJW 2018, S. 2299-2301.

Grabitz, EberhardlHilf, Meinhard/Nettesheim, Martin (Hrsg.): Das Recht der Europäischen Union. 40. EL, München 2009.

-: Das Recht der Europäischen Union. 71. EL, München 2020.

Grimm, Dieter: Grundrechte und soziale Wirklichkeit. Zum Problem eines interdisziplinären Grundrechtsverständnisses, in: Winfried Hassemer/Wolfgang Hoffmann-Riem/ Jutta Limbach (Hrsg.), Grundrechte und soziale Wirklichkeit, Baden-Baden 1982, S. 39-76.

Grimmelmann, James: The Virtues of Moderation, Yale Journal of Law \& Technology 17 (2015), S. 42-109.

Grind, Kirsten/Schechner, Sam/McMillan, Robert/West, John: How Google Interferes With Its Search Algorithms and Changes Your Results. The internet giant uses blacklists, algorithm tweaks and an army of contractors to shape what you see, The Wall Street Journal, 15.11.2019, abrufbar unter: https://www.wsj.com/articles/how-google-interfer es-with-its-search-algorithms-and-changes-your-results-11573823753.

Grothe, Nela: Datenmacht in der kartellrechtlichen Missbrauchskontrolle, Baden-Baden 2019 (Zugl.: Freiburg, Univ., Diss., 2019).

Groys, Boris: Google, Words beyond Grammar, Ostfildern 2011.

Grünberger, Michael: Verträge über digitale Güter, AcP 218 (2018), S. 213-296.

Guggenberger, Nikolaus: Das Netzwerkdurchsetzungsgesetz - schön gedacht, schlecht gemacht, ZRP 2017, S. 98-101.

Habermas, Jürgen: Ach, Europa, Frankfurt am Main 2008.

Hacker, Philipp: Mehrstufige Informationsanbieterverhältnisse zwischen Datenschutz und Störerhaftung. Gestufte Kontrolle - gemeinsame Verantwortung?, MMR 2018, S. 779-784.

Halpern, Steven: New Protections for Internet Service Providers. An Analysis of the Online Copyright Infringement Liability Limitation Act, Seton Hall Legislative Journal 23 (1999), S. 359-408.

Härting, Niko: Internetrecht. 6. Aufl., Köln 2017.

Hartl, Korbinian: Suchmaschinen, Algorithmen und Meinungsmacht. Eine verfassungsund einfachrechtliche Betrachtung, Wiesbaden 2017 (Zugl.: Passau, Univ., Diss., 2016).

Hartung, Helmut: Der Vielfalt verpflichtet, FAZ, 09.08.2019, abrufbar unter: https://www. faz.net/aktuell/feuilleton/medien/medienstaatsvertrag-der-vielfalt-verpflichtet-1632435 4.html.

Hassanabadi, Amir: Viacom v. YouTube - All Eyes Blind. The Limits of the DMCA in a Web 2.0 World, Berkeley Technology Law Journal 26 (2011), S. 405-439.

Hassemer, Winfried: Juristische Hermeneutik, ARSP 72 (1986), S. 195-212.

Hegel, Georg Wilhelm Friedrich: Phänomenologie des Geistes, herausgegeben von Wolfgang Bonsiepen/Reinhard Heede, Georg Wilhelm Friedrich Hegel, Gesammelte Werke Band 9, Hamburg 1980. 
Hegemann, Jan/Amelung, Ulrich: Rechtsfolgen unzulässiger Medienberichterstattung, in: Peter Raue/Jan Hegemann (Hrsg.), Münchener Anwalts-Handbuch Urheber- und Medienrecht, 2. Aufl., München 2017.

Helbig, Karoline: Der Einfluss von Algorithmen auf demokratische Deliberation, in: Resa Mohabbat Kar/Basanta Thapa/Peter Parycek (Hrsg.), (Un)berechenbar? Algorithmen und Automatisierung in Staat und Gesellschaft, Berlin 2018, S. 339-363.

Hellgardt, Alexander: Wer hat Angst vor der unmittelbaren Drittwirkung? Die Konsequenzen der Stadionverbot-Entscheidung des BVerfG für die deutsche Grundrechtsdogmatik, JZ 2018, S. 901-910.

Herberger, Maximilian: „Künstliche Intelligenz“ und Recht. Ein Orientierungsversuch, NJW 2018, S. 2825-2829.

Higgins, Parker: The Web's First Blackout Protest, Electronic Frontier Foundation (23.02.2016), abrufbar unter: https://www.eff.org/de/deeplinks/2016/02/webs-first-blac kout-protest-cda-20-years-later.

Hildebrandt, Mireille: Smart Technologies and the End(s) of Law. Novel Entanglements of Law and Technology, Cheltenham 2016.

Hindelang, Steffen: Freiheit und Kommunikation. Zur verfassungsrechtlichen Sicherung kommunikativer Selbstbestimmung in einer vernetzten Gesellschaft, Berlin 2019.

Höch, Dominik: Nachbessern: ja, verteufeln: nein. Das NetzDG ist besser als sein Ruf, K\&R 2017, S. 289-292.

Hoeren, Thomas: Vorschlag für eine EU-Richtlinie über E-Commerce. Eine erste kritische Analyse, MMR 1999, S. 192-199.

-: E-Commerce-Verträge, in: Friedrich Graf von Westphalen/Gregor Thüsing (Hrsg.), Vertragsrecht und AGB-Klauselwerke, 35. EL, München 2014.

-: Internetrecht, Münster 2019.

Hoffer, Raoul/Lehr, Leo Alexander: Onlineplattformen und Big Data auf dem Prüfstand. Gemeinsame Betrachtung der Fälle Amazon, Google und Facebook, NZKart 2019, S. $10-20$.

Hoffmann-Riem, Wolfgang: Wissen, Recht und Innovation, in: Hans Christian Röhl (Hrsg.), Wissen. Zur kognitiven Dimension des Rechts, Berlin 2010, S. 160-211.

-: Innovation, Recht und öffentliche Kommunikation - zur Einführung, in: Martin Eifert/ Wolfgang Hoffmann-Riem (Hrsg.), Innovation, Recht und öffentliche Kommunikation. Innovation und Recht IV, Berlin 2011, S. 9-24.

-: Regelungsstrukturen für öffentliche Kommunikation im Internet, AöR 137 (2012), S. 509-544.

-: Selbstregelung, Selbstregulierung und regulierte Selbstregulierung im digitalen Kontext, in: Michael Fehling/Utz Schliesky (Hrsg.), Neue Macht- und Verantwortungsstrukturen in der digitalen Welt, Baden-Baden 2016, S. 27-51.

-: Re:claim Autonomy. Die Macht digitaler Konzerne, in: Jakob Augstein (Hrsg.), Reclaim Autonomy. Selbstermächtigung in der digitalen Weltordnung, Berlin 2017, S. 121-139.

-: Verhaltenssteuerung durch Algorithmen. Eine Herausforderung für das Recht, AöR 142 (2017), S. 1-42.

-: Wirkungsorientierte Rechtswissenschaft, Zeitschrift für Rechtssoziologie 2018, S. 20-41.

-: Die digitale Transformation als Herausforderung für die Legitimation rechtlicher Entscheidungen, in: Sebastian Unger/Antje von Ungern-Sternberg (Hrsg.), Demokratie und künstliche Intelligenz, Tübingen 2019, S. 129-159.

Hofmann, Franz: Anmerkung zu EuGH, Urt. v. 07.07.2016 - C-494/15 - Tommy Hilfiger, GRUR 2016, S. 1064-1065. 
-: Mittelbare Verantwortlichkeit im Internet, JuS 2017, S. 713-720.

-: Prozeduralisierung der Haftungsvoraussetzungen im Medienrecht. Vorbild für die Intermediärshaftung im Allgemeinen?, ZUM 2017, S. 102-109.

-: Rechtsdurchsetzung im Internet - Aufregung um das NetzDG, WRP 9/2017, Editorial.

-: Disziplinarität, Intradisziplinarität und Interdisziplinarität am Beispiel der Grundsätze „mittelbarer Verantwortlichkeit“. Vergewisserungen aus Sicht der Privatrechtswissenschaft, JZ 2018, S. 746-754.

-: Die Plattformverantwortlichkeit nach dem neuen europäischen Urheberrecht - „Much Ado About Nothing"'?, ZUM 2019, S. 617-627.

-: Fünfzehn Thesen zur Plattformhaftung nach Art.17 DSM-RL, GRUR 2019, S. 1219-1229.

-: Smart contracts und Overenforcement. Analytische Überlegungen zum Verhältnis von Rechtszuweisung und Rechtsdurchsetzung, in: Martin Fries/Boris Paal (Hrsg.), Smart Contracts, Tübingen 2019, S. 125-140.

Hofmann, Jeanette: Digitalisierung und demokratischer Wandel als Spiegelbilder?, in: Franziska Martinsen (Hrsg.), Wissen - Macht - Meinung. Demokratie und Digitalisierung, Weilerswist 2018, S. 14-21.

Hohlfeld, RalfiGodulla, Alexander: Das Phänomen der Sozialen Medien, in: Gerrit Hornung/Ralf Müller-Terpitz (Hrsg.), Rechtshandbuch Social Media, Heidelberg 2015, S. 11-33.

Hollenders, Anna-Sophie: Mittelbare Verantwortlichkeit von Intermediären im Netz, Baden-Baden 2012 (Zugl.: Münster, Univ., Diss., 2011).

Holznagel, Daniel: Zur Providerhaftung - Notice and Take-Down in $\$ 512$ U.S. Copyright Act, GRUR Int 2007, S. 971-986.

-: Notice and Take-Down-Verfahren als Teil der Providerhaftung. Untersuchung des rechtlichen Rahmens von Verfahren zur Beanstandung und Verteidigung von Inhalten im Internet, insbesondere auf „User Generated Content“-Plattformen, Tübingen 2013 (Zugl.: Göttingen, Univ., Diss., 2012).

-: Melde- und Abhilfeverfahren zur Beanstandung rechtswidrig gehosteter Inhalte nach europäischem und deutschem Recht im Vergleich zu gesetzlich geregelten notice and take-down-Verfahren, GRUR Int 2014, S. 105-113.

-: Unterlassungsanordnungen gegen Betreiber unverschlüsselter WLANs („Mc Fadden vs. Sony“), jurisPR-WettbR 10/2016, Anm. 1.

-: Der Konflikt zwischen Art. 15 E-Commerce-RL und pro-aktiven Verhinderungspflichten von Host-Providern, ZUM 2018, S. 350-357.

-: Overblocking durch User Generated Content (UGC) - Plattformen. Ansprüche der Nutzer auf Wiederherstellung oder Schadensersatz?, CR 2018, S. 369-378.

-: Verpflichtung von Host-Providern zum Auffinden und Löschen wort- und sinngleicher Verletzungen. Anmerkung zu EuGH, Urt. v. 03.10.2019 - C-18/18 - Glawischnig-Piesczek/Facebook Ireland, ZUM 2019, S. 910-913.

Hong, Mathias: Das NetzDG und die Vermutung für die Freiheit der Rede, Verfassungsblog (09.01.2018), abrufbar unter: https://verfassungsblog.de/das-netzdg-und-die-verm utung-fuer-die-freiheit-der-rede.

-: Hate Speech im Internet. Grundrechtliche Rahmenbedingungen ihrer Regulierung, in: Marion Albers/Ioannis Katsivelas (Hrsg.), Recht \& Netz, Baden-Baden 2018, S. 59-87.

Hörnle, Tatjana: Plädoyer für die Aufgabe der Kategorie „,bedingter Vorsatz“, JZ 2019, S. 440-449.

Hornschuh, Matthias: In Ketten tanzen. Warum es bei der EU-Urheberrechtsrichtlinie um sehr viel mehr geht als um Urhebervergütung, ZUM 2019, S. 222-226.

Hurtz, Simon: Was genau sind eigentlich diese Upload-Filter?, Süddeutsche Zeitung, 07.03.2019, abrufbar unter: https://www.sueddeutsche.de/digital/urheberrecht-upload-f ilter-1.4358094. 
Husovec, Martin: Injunctions against Intermediaries in the European Union. Accountable but not liable?, Cambridge 2017.

Information Infrastructure Task Force: Intellectual Property and the National Information Infrastructure. Report of the Working Group on Intellectual Property Rights, Washington, D.C. 1995, abrufbar unter: https://hdl.handle.net/2027/mdp.39015040596903.

Ingendaay, Dominik: Zur Verbreiterhaftung des Buchhandels. Unterlassungspflicht des Buchhändlers bei der Verbreitung urheberrechtsverletzender Inhalte - Täter oder Störer?, AfP 2011, S. 126-134.

Ingold, Albert: Digitalisierung demokratischer Öffentlichkeiten, Der Staat 56 (2017), S. 491-533.

-: Governance of Algorithms. Kommunikationskontrolle durch „Content Curation“ in sozialen Netzwerken, in: Sebastian Unger/Antje von Ungern-Sternberg (Hrsg.), Demokratie und künstliche Intelligenz, Tübingen 2019, S. 184-213.

Ingram, Mathew: The Myth of Social Media Anti-Conservative Bias Refuses to Die, Columbia Journalism Review (08.08.2019), abrufbar unter: https://www.cjr.org/the_media today/platform-bias.php.

International Telecommunication Union: Open Systems Interconnection Basic Reference Model. ITU-T Recommendation X.200 (07/94), abrufbar unter: http://handle.itu.int/1 $1.1002 / 1000 / 2820$.

Internet Association: Pressemitteilung. Statement In Support Of The Bipartisan Compromise To The Stop Enabling Sex Traffickers Act, Washington, D.C. 03.11.2017, abrufbar unter: https://internetassociation.org/statement-in-support-of-the-bipartisan-co mpromise-to-stop-enabling-sex-trafficking-act-sesta.

Jaeschke, Lars: Anmerkung zu EuGH, Urt. v. 23.03.20120 - C-236/08 - Google France und Google, EuZW 2010, S. 426-427.

Jaworski, Stanislaus/Nordemann, Jan Bernd: Gehilfenhaftung von Intermediären bei Rechtsverletzungen im Internet. BGH-Rechtsprechung und neueste Entwicklungen in den Instanzen, GRUR 2017, S. 567-572.

Jeong, Sarah: A New Bill to fight Sex Trafficking would destroy a Core Pillar of Internet Freedom, The Verge (01.08.2017), abrufbar unter: https://www.theverge.com/2017/8/1/1 6072680/cda-230-stop-enabling-sex-traffickers-act-liability-shield-senate-backpage.

Jestaedt, Matthias: Wissenschaft im Recht. Rechtsdogmatik im Wissenschaftsvergleich, JZ 2014, S. 1-12.

Johnson, David/Post, David: Law And Borders. The Rise of Law in Cyberspace, Stanford Law Review 48 (1996), S. 1367-1402.

Jülicher, Tim/Röttgen, Charlotte/Schönfeld, Max von: Das Recht auf Datenübertragbarkeit. Ein datenschutzrechtliches Novum, ZD 2016, S. 358-362.

Jürgens, PascallStark, Birgit/Magin, Melanie: Gefangen in der Filter Bubble? Search Engine Bias und Personalisierungsprozesse bei Suchmaschinen, in: Birgit Stark/Dieter Dörr/Stefan Aufenanger (Hrsg.), Die Googleisierung der Informationssuche. Suchmaschinen zwischen Nutzung und Regulierung, Berlin 2014, S. 98-135.

Just, Natascha/Latzer, Michael: Governance by Algorithms. Reality Construction by Algorithmic Selection on the Internet, Media, Culture \& Society 39 (2017), S. 238-258.

Kaesling, Katharina: Die EU-Urheberrechtnovelle - der Untergang des Internets?, JZ 2019, S. 586-591.

Kähler, Harro Dietrich: Das Konzept des sozialen Netzwerks. Eine Einführung in die Literatur, Zeitschrift für Soziologie 1975, S. 283-290.

Kaiser, Anna-BettinalReiling, Ines: Meinungsfilter durch soziale Medien - und das demokratische Ideal der Meinungsvielfalt?, in: Sebastian Unger/Antje von Ungern-Sternberg (Hrsg.), Demokratie und künstliche Intelligenz, Tübingen 2019, S. 85-110. 
Kalscheuer, Fiete/Hornung, Christian: Das Netzwerkdurchsetzungsgesetz - Ein verfassungswidriger Schnellschuss, NVwZ 2017, S. 1721-1725.

Kant, Immanuel: Metaphysische Anfangsgründe der Rechtslehre. Metaphysik der Sitten Erster Teil, herausgegeben von Bernd Ludwig, Philosophische Bibliothek Band 360, Hamburg 2009.

Kartal-Aydemir, Aliye/Krieg, Rebecca: Haftung von Anbietern kollaborativer Internetplattformen. Störerhaftung für User Generated Content?, MMR 2012, S. 647-652.

Kastl, Graziana: Automatisierung im Internet. Urheber- und äußerungsrechtliche Implikationen, Baden-Baden 2016 (Zugl.: Freiburg, Univ., Diss., 2015).

-: Filter - Fluch oder Segen?, GRUR 2016, S. 671-678.

Katz, Michael/Shapiro, Carl: System Competition and Network Effects, Journal of Economic Perspectives 8 (1994), S. 93-115.

Katzenbach, Christian: Die Regeln digitaler Kommunikation. Governance zwischen Norm, Diskurs und Technik, Wiesbaden 2018 (Zugl.: Berlin, Freie Univ., Diss., 2016).

Kellner, Anna: Die Regulierung der Meinungsmacht von Internetintermediären, BadenBaden 2019 (Zugl.: Regensburg, Univ., Diss., 2019).

Kersten, Jens: Anonymität in der liberalen Demokratie, JuS 2017, S. 193-203.

-: Schwarmdemokratie. Der digitale Wandel des liberalen Verfassungsstaats, Tübingen 2017.

Khanna, Derek: The Law that Gave Us the Modern Internet - and the Campaign to Kill It, The Atlantic (12.09.2013), abrufbar unter: https://www.theatlantic.com/business/archi ve/2013/09/the-law-that-gave-us-the-modern-internet-and-the-campaign-to-kill-it/279 588.

Klatt, Heiko: Die Kerngleichheit als Grenze der Prüfungspflichten und der Haftung des Hostproviders, ZUM 2009, S. 265-274.

Klein, Catherine Jelena: Haftung von Social-Sharing-Plattformen. Diensteanbieter zwischen Content- und Host-Providing, München 2012 (Zugl.: Marburg, Univ., Diss., 2012).

Kleiner, Krystyna: Die urheberrechtliche Wirksamkeit von Nutzungsbedingungen sozialer Netzwerke. Eine Analyse anhand des Praxisbeispiels Facebook, Baden-Baden 2019.

Klonick, Kate: The New Governors. The People, Rules, and Processes Governing Online Speech, Harvard Law Review 131 (2018), S. 1598-1670.

Körber, Torsten: Die Facebook-Entscheidung des Bundeskartellamtes. Machtmissbrauch durch Verletzung des Datenschutzrechts?, NZKart 2019, S. 187-195.

Koreng, Ansgar/Feldmann, Thorsten: Blogs, Foren und Bewertungsportale, in: Thomas Hoeren/Viola Bensinger (Hrsg.), Haftung im Internet. Die neue Rechtslage, Berlin 2014, S. 461-498.

Kosseff, Jeff: Correcting a Persistent Myth About the Law that Created the Internet, The Regulatory Review (15.07.2019), abrufbar unter: https://www.theregreview.org/2019/0 7/15/kosseff-correcting-persistent-myth-about-law-that-created-the-internet.

-: The Twenty-Six Words that Created the Internet, Ithaca (New York) 2019.

Kovacs, Andrea: Die Haftung der Host-Provider für persönlichkeitsrechtsverletzende Internetäußerungen, Baden-Baden 2018 (Zugl.: Augsburg, Univ., Diss., 2018).

Kraakman, Reinier: Gatekeepers. The Anatomy of a Third-Party Enforcement Strategy, Journal of Law, Economics, and Organization 2 (1986), S. 53-104.

Kriegesmann, Torben: Anmerkung zu BGH, Urt. v. 01.03.2016 - VI ZR 34/15 - Ärztebewertungsportal III, CR 2016, S. 394-396.

Krüger, Stefan/Apel, Simon: Haftung von Plattformbetreibern für urheberrechtlich geschützte Inhalte. Wie weit geht die Haftung und wann droht Schadensersatz?, MMR 2012, S. 144-151. 
Kube, Hanno: §91. Neue Medien - Internet, in: Josef Isensee/Paul Kirchhof (Hrsg.), Handbuch des Staatsrechts. Band IV, 3. Aufl., Heidelberg 2006.

Kubiciel, Michael: Neuartige Sanktionen für soziale Netzwerke? Der Regierungsentwurf zur Verbesserung der Rechtsdurchsetzung in sozialen Netzwerken, jurisPR-StrafR 7/2017, Anm. 1.

Kuczerawy, Aleksandra: Intermediary Liability and Freedom of Expression in the EU. From Concepts to Safeguards, Cambridge 2018.

Kühling, Jürgen: Der Fall der Vorratsdatenspeicherungsrichtlinie und der Aufstieg des EuGH zum Grundrechtsgericht, NVwZ 2014, S. 681-685.

Kühling, Jürgen/Buchner, Benedikt (Hrsg.): Datenschutz-Grundverordnung/Bundesdatenschutzgesetz. Kommentar. 2. Aufl., München 2018.

Kumkar, Lea Katharina: Online-Märkte und Wettbewerbsrecht. Implikationen der Platform Revolution für das EU-Vertriebskartellrecht, Baden-Baden 2017 (Zugl.: Freiburg, Univ., Diss, 2017).

Kurz, Constanze: Die Meinungsfreiheit und das NetzDG, Netzpolitik.org (18.02.2018), abrufbar unter: https://netzpolitik.org/2018/die-meinungsfreiheit-und-das-netzdg-schw erwiegender-verstoss-gegen-grundrecht.

Ladeur, Karl-Heinz: Die Netzwerke des Rechts, in: Michael Bommes/Veronika Tacke (Hrsg.), Netzwerke in der funktional differenzierten Gesellschaft, Wiesbaden 2010, S. $143-171$.

-: Anmerkung zu BGH, Urt. v. 14.05.2013 - VI ZR 269/12 - Autocomplete, JZ 2013, S. 792-794.

Ladeur, Karl-Heinz/Gostomzyk, Tobias: Das Medienrecht und die Herausforderung der technologischen Hybridisierung. Eine Kommentierung der Regelungen zu Medienintermediären im Entwurf des Medienstaatsvertrags der Länder, K\&R 2018, S. 686-693.

Laidlaw, Emily: Regulating Speech in Cyberspace. Gatekeepers, Human Rights and Corporate Responsibility, Cambridge 2015 (Zugl.: London, London School of Economics and Political Science, Diss., 2012).

Lang, Andrej: Netzwerkdurchsetzungsgesetz und Meinungsfreiheit. Zur Regulierung privater Internet-Intermediäre bei der Bekämpfung von Hassrede, AöR 143 (2018), S. 220-250.

Langville, Amy/Meyer, Carl Dean: Google‘s PageRank and Beyond. The Science of Search Engine Rankings, Princeton 2006.

Laslo, Matt: The Fight Over Section 230 - and the Internet as We Know It, Wired (13.08.2019), abrufbar unter: https://www.wired.com/story/fight-over-section-230-inter net-as-we-know-it.

Leary, Mary Graw: The Indecency and Injustice of Section 230 of the Communications Decency Act, Harvard Journal of Law \& Public Policy 41 (2018), S. 553-622.

Lee, Edward: Decoding the DMCA Safe Harbors, Columbia Journal of Law \& the Arts 32 (2008), S. 233-269.

Lehmann, Michael: Rechtsgeschäfte und Verantwortlichkeit im Netz. Der Richtlinienvorschlag der EU-Kommission, ZUM 1999, S. 180-184.

Lehment, Cornelis: Neuordnung der Täter- und Störerhaftung, WRP 2012, S. 149-159.

Leistner, Matthias: Störerhaftung und mittelbare Schutzrechtsverletzung, GRUR-Beilage 2010, S. 1-32.

-: Grundlagen und Perspektiven der Haftung für Urheberrechtsverletzungen im Internet, ZUM 2012, S. 722-740.

-: Reformbedarf im materiellen Urheberrecht: Online-Plattformen und Aggregatoren, ZUM 2016, S. 580-594.

Leistner, Matthias/Metzger, Axel: Wie sich das Problem illegaler Musiknutzung lösen lässt, FAZ, 04.01.2017, abrufbar unter: http://www.faz.net/aktuell/feuilleton/medien/g ema-youtube-wie-sich-urheberrechts-streit-schlichten-liesse-14601949.html. 
Lembke, Ulrike: Kollektive Rechtsmobilisierung gegen Digitale Gewalt, herausgegeben von Heinrich-Böll-Stiftung, Berlin 2017, abrufbar unter: https://www.gwi-boell.de/sites/ default/files/e-paper_43_kollektive_rechtsmobi.pdf.

Lemley, Mark/Lessig, Lawrence: The End of End-to-End, UCLA Law Review 48 (2001), S. 925-972.

Lessig, Lawrence: Code and other Laws of Cyberspace, New York 1999.

-: Code. Version 2.0, New York 2006.

Lewandowski, Dirk: Suchmaschinen verstehen, Berlin 2015.

-: Is Google Responsible for Providing Fair and Unbiased Results?, in: Mariarosaria Taddeo/Luciano Floridi (Hrsg.), The Responsibilities of Online Service Providers, Cham 2017, S. 61-77.

Lewin, Kurt: Frontiers in Group Dynamics. II. Channels of Group Life; Social Planning and Action Research, Human Relations 1, 2 (1947), S. 143-153.

Lindner, Josef Franz: Einheit der Rechtswissenschaft als Aufgabe, JZ 2016, S. 697-752.

Lischka, Konrad: Wie Algorithmen Öffentlichkeit strukturieren. Grundlagen, Folgen, Lösungsansätze, itrb 2018, S. 235-239.

Lischka, Konrad/Stöcker, Christan: Digitale Öffentlichkeit. Wie algorithmische Prozesse den gesellschaftlichen Diskurs beeinflussen, herausgegeben von Bertelsmann Stiftung, Gütersloh 2017, abrufbar unter: https://www.bertelsmann-stiftung.de/fileadmin/files/B St/Publikationen/GrauePublikationen/Digitale_Oeffentlichkeit_final.pdf.

Löber, Lena/Roßnagel, Alexander: Das Netzwerkdurchsetzungsgesetz in der Umsetzung. Bilanz nach den ersten Transparenzberichten, MMR 2019, S. 71-76.

Lobigs, Frank/Neuberger, Christoph: Meinungsmacht im Internet und die Digitalstrategien von Medienunternehmen. Neue Machtverhältnisse trotz expandierender InternetGeschäfte der traditionellen Massenmedien-Konzerne, Gutachten für die Kommission zur Ermittlung der Konzentration im Medienbereich (KEK), herausgegeben von die medienanstalten, Berlin 2018, abrufbar unter: https://www.kek-online.de/fileadmin/use r_upload/KEK/Publikationen/Gutachten/Meinungsmacht_im_Internet_ALM51_web 2018.pdf.

Lodder, Arno: Directive 2000/31/EC on certain legal aspects of Information Society Services, in particular Electronic Commerce, in the Internal Market, in: Arno Lodder/ Andrew Murray (Hrsg.), EU Regulation of E-Commerce. A Commentary, Cheltenham 2017, S. 15-58.

Lombardi, Claudio: The Illusion of a "Marketplace of Ideas“ and the Right to Truth, American Affairs 3, 1 (2019), S. 198-209.

Lovink, Geert: Im Bann der Plattformen. Die nächste Runde der Netzkritik, Bielefeld 2017.

Luckerson, Victor: Here's Why Facebook Won't Put Your News Feed in Chronological Order, Time (09.07.2015), abrufbar unter: https://time.com/3951337/facebook-chronol ogical-order.

Lüdemann, Jörn: Privatisierung der Rechtsdurchsetzung in sozialen Netzwerken?, in: Martin Eifert/Tobias Gostomzyk (Hrsg.), Netzwerkrecht. Die Zukunft des NetzDG und seine Folgen für die Netzwerkkommunikation, Baden-Baden 2018, S. 153-168.

-: Grundrechtliche Vorgaben für die Löschung von Beiträgen in sozialen Netzwerken. Private Ordnung digitaler Kommunikation unter dem Grundgesetz, MMR 2019, S. 279-284.

Lukmire, David: Can the Courts Tame the Communications Decency Act. The Reverberations of Zeran v. American Online, NYU Annual Survey of American Law 66 (2010), S. 371-411.

Maennel, Frithjof: Elektronischer Geschäftsverkehr ohne Grenzen. Der Richtlinienvorschlag der Europäischen Kommission, MMR 1999, S. 187-192. 
Maier, Henrike: Remixe auf Hosting-Plattformen. Eine urheberrechtliche Untersuchung filmischer Remixe zwischen grundrechtsrelevanten Schranken und Inhaltefiltern, Tübingen 2018 (Zugl.: Berlin, Humboldt-Univ., Diss., 2017).

Mandl, Peter: Internet Internals. Vermittlungsschicht, Aufbau und Protokolle, Wiesbaden 2019.

Masing, Johannes: Herausforderungen des Datenschutzes, NJW 2012, S. 2305-2311.

Matthies, Ulf: Providerhaftung für Online-Inhalte. Eine vergleichende Untersuchung zur Rechtslage in Deutschland, Österreich und England, Baden-Baden 2004 (Zugl.: Hamburg, Univ., Diss., 2003).

Mayen, Thomas: Über die mittelbare Grundrechtsbindung Privater in Zeiten des Einflusses sozialer Netzwerke auf die öffentliche Kommunikation, ZHR 2018, S. 1-7.

Medeiros, Ben: Platform (Non-)Intervention and the „Marketplace“ Paradigm for Speech Regulation, Social Media + Society 3 (2017), S. 1-10.

Mengden, Martin: Zugangsfreiheit und Aufmerksamkeitsregulierung. Zur Reichweite des Gebots der Gewährleistung freier Meinungsbildung am Beispiel algorithmengestützter Zugangsdienste im Internet, Tübingen 2018 (Zugl.: Mainz, Univ., Diss., 2017).

Metzger, Axel: Extra legem, intra ius. Allgemeine Rechtsgrundsätze im Europäischen Privatrecht, Tübingen 2009 (Zugl.: Hamburg, Univ., Habil., 2008).

-: Anmerkung zu EuGH, Urt. v. 12.02.2012 - C-360/10 - SABAM/Netlog, GRUR 2012, S. 384-385.

-: Dienst gegen Daten. Ein synallagmatischer Vertrag, AcP 216 (2016), S. 817-865.

-: Regulierung im Urheberrecht. Herausforderungen und Perspektiven, ZUM 2018, S. 233-242.

Michl, Fabian: Situativ staatsgleiche Grundrechtsbindung privater Akteure. Zugleich Besprechung von BVerfG, Beschluss vom 11. 4. 2018 - 1 BvR 3080/09, JZ 2018, S. 910-918.

Milker, Jens: Die Umsetzung des „Rechts auf Vergessenwerden“ im deutschen Recht. Der Datenschutz als Taktgeber für das Äußerungsrecht, Wiesbaden 2019 (Zugl:: Mainz, Univ., Diss., 2019).

Milstein, Alexander/Lippold, Matthias: Suchmaschinenergebnisse im Lichte der Meinungsfreiheit der nationalen und europäischen Grund- und Menschenrechte, NVwZ 2013, S. 182-187.

Mischau, Lena: Daten als „Gegenleistung“ im neuen Verbrauchervertragsrecht, ZEuP 2020, S. 335-365.

Morozov, Evgeny: The Meme Hustler, The Baffler 22 (2013), S. 125-147.

Mörsdorf, Oliver: Neues zum Auslistungsanspruch gegen Suchmaschinenbetreiber, NJW 2020, S. 3417-3420.

Müller, Willem: Die unmittelbare Inanspruchnahme des Access-Providers. Aktuelle Voraussetzungen einer Internetsperre bei Urheberrechtsverletzungen, MMR 2019, S. 426-431.

Müller-Terpitz, Ralf: Soziale Netzwerke als Gegenstand des geltenden Rechts. Eine rechtssystematische Einordnung, in: Martin Eifert/Tobias Gostomzyk (Hrsg.), Netzwerkrecht. Die Zukunft des NetzDG und seine Folgen für die Netzwerkkommunikation, Baden-Baden 2018, S. 45-60.

Münchener Kommentar zum Bürgerlichen Gesetzbuch. Band 2: Schuldrecht - Allgemeiner Teil I, herausgegeben von Franz Jürgen Säcker/Roland Rixecker/Hartmut Oetker/Bettina Limperg, 8. Aufl., München 2019.

Münchener Kommentar zum Bürgerlichen Gesetzbuch. Band 7: Schuldrecht - Besonderer Teil IV, $\S$ 705-853, Partnerschaftsgesellschaftsgesetz, Produkthaftungsgesetz, herausgegeben von Franz Jürgen Säcker/Roland Rixecker/Hartmut Oetker/Bettina Limperg, 8. Aufl., München 2020. 
Münchener Kommentar zum Bürgerlichen Gesetzbuch. Band 8: Sachenrecht - §§ 854-1296, WEG, ErbbauRG, herausgegeben von Franz Jürgen Säcker/Roland Rixecker/Hartmut Oetker/Bettina Limperg, 8. Aufl., München 2020.

Münchener Kommentar zum Strafgesetzbuch. Band 7: Nebenstrafrecht, herausgegeben von Wolfgang Joecks/Klaus Miebach, 3. Aufl., München 2019.

Münchener Kommentar zur Zivilprozessordnung. Mit Gerichtsverfassungsgesetz und Nebengesetzen, herausgegeben von Wolfgang Krüger/Thomas Rauscher, 6. Aufl., München 2020.

Münkler, Laura: Metaphern im Recht. Zur Bedeutung organischer Vorstellungen von Staat und Recht, Der Staat 55 (2016), S. 181-211.

Neuberger, Christoph: Internet, Journalismus und Öffentlichkeit. Analyse des Medienumbruchs, in: Christoph Neuberger/Christian Nuernbergk/Melanie Rischke (Hrsg.), Journalismus im Internet. Profession, Partizipation, Technisierung, Wiesbaden 2009, S. $19-105$.

Neumann, Sophie: Die Haftung der Intermediäre im Internationalen Immaterialgüterrecht. Vorgaben und Grenzen von Territorialitätsprinzip und Schutzlandanknüpfung, Baden-Baden 2014 (Zugl.: München, Ludwigs-Maximilians-Univ., Diss., 2014).

Newman, Nic: Reuters Institute Digital News Report 2019, herausgegeben von Reuters Institute for the Study of Journalism, Oxford 2019, abrufbar unter: https://reutersinstit ute.politics.ox.ac.uk/sites/default/files/inline-files/DNR_2019_FINAL.pdf.

Nölscher, Patrick: Das Netzwerkdurchsetzungsgesetz und seine Vereinbarkeit mit dem Unionsrecht, ZUM 2020, S. 301-312.

Nolte, Georg: Hate-Speech, Fake-News, das „Netzwerkdurchsetzungsgesetz“ und Vielfaltsicherung durch Suchmaschinen, ZUM 2017, S. 552-565.

Nolte, Georg/Wimmers, Jörg: Wer stört? Gedanken zur Haftung von Intermediären im Internet - von praktischer Konkordanz, richtigen Anreizen und offenen Fragen, GRUR 2014, S. 16-27.

Nordemann, Jan Bernd: Haftung von Providern im Urheberrecht. Der aktuelle Stand nach dem EuGH-Urt. v. 12.07.2011 - C-324/09 - L`Oréal/eBay, GRUR 2011, S. 977-981.

o. A.: Section 230 as First Amendment Rule, Harvard Law Review 131 (2018), S. 2027-2048.

Oermann, Markus: Gewährleistung der Möglichkeit internetbasierter Kommunikation. Eine Vermessung des grundgesetzlichen Schutzkonzepts, Baden-Baden 2018 (Zugl.: Hamburg, Univ., Diss., 2018).

Ohly, Ansgar: Die Verantwortlichkeit von Intermediären, ZUM 2015, S. 308-318.

-: Der weite Täterbegriff des EuGH in den Urteilen „GS Media“, „Filmspeler“ und „The Pirate Bay“. Abenddämmerung für die Störerhaftung?, ZUM 2017, S. 793-802.

Oremus, Will: Who Controls Your Facebook Feed, Slate (03.01.2016), abrufbar unter: ht tp://www.slate.com/articles/technology/cover_story/2016/01/how_facebook_s_news_f eed_algorithm_works.html.

Ott, Stephan: Haftung für Hyperlinks. Eine Bestandsaufnahme nach 10 Jahren, WRP 2006, S. 691-703.

-: Haftung für verlinkte urheberrechtswidrige Inhalte in Deutschland, Österreich und den USA, GRUR Int 2007, S. 14-28.

-: Die Haftung von YouTube für urheberrechtsverletzende Uploads seiner Nutzer nach US-amerikanischem Recht, GRUR Int 2008, S. 563-569.

-: Das Neutralitätsgebot als Voraussetzung der Haftungsprivilegierung des Host-Providers. Eine kritische Auseinandersetzung mit den EuGH-Entscheidungen Google France und L'Oréal/eBay, K\&R 2012, S. 387-393.

Paal, Boris: Online-Suchmaschinen - Persönlichkeitsrechts- und Datenschutz. Internationale Zuständigkeit, anwendbares Recht und sachrechtliche Fragen, ZEuP 2016, S. 591-628. 
-: Intermediäre. Regulierung und Vielfaltssicherung, Rechtsgutachten im Auftrag der Landesanstalt für Medien Nordrhein-Westfalen, herausgegeben von Landesanstalt für Medien Nordrhein-Westfalen, Düsseldorf 2018, abrufbar unter: https://www.medienan stalt-nrw.de/fileadmin/user_upload/lfm-nrw/Foerderung/Forschung/Dateien_Forschu ng/Paal_Intermediaere_Regulierung-und-Vielfaltssicherung_Gutachten-2018.pdf.

-: Anmerkung zu EuGH, Urt. v. 03.10.2019 - C-18/18 - Eva Glawischnig-Piesczek ./. Facebook Ireland Limited, JZ 2020, S. 92-96.

Paal, Boris/Hennemann, Moritz: Meinungsbildung im digitalen Zeitalter. Regulierungsinstrumente für einen gefährdungsadäquaten Rechtsrahmen, JZ 2017, S. 641-652.

Pankoke, Stefan: Von der Presse- zur Providerhaftung. Eine rechtspolitische und rechtsvergleichende Untersuchung zur Inhaltsverantwortlichkeit im Netz, München 2000 (Zugl.: Tübingen, Univ., Diss., 1999/2000).

Pariser, Eli: The Filter Bubble. What the Internet is hiding from you, New York 2011.

Pasquale, Frank: The Black Box Society. The Secret Algorithms that control Money and Information, Cambridge, Massachusetts 2015.

-: Platform Neutrality. Enhancing Freedom of Expression in Spheres of Private Power, Theoretical Inquiries in Law 17 (2016), S. 487-513.

-: The Automated Public Sphere, in: Ann Rudinow Saetnan/Ingrid Schneider/Nicola Green (Hrsg.), The Politics of Big Data. Big Data, Big Brother?, London, New York 2018, S. 110-128.

Peifer, Karl-Nikolaus: Die zivilrechtliche Verteidigung gegen Äußerungen im Internet, AfP 2015, S. 193-201.

-: Sharing-Plattformbetreiberhaftung im Urheberrecht (Art. 17 DSM-RL), GRUR-Prax 2019, S. 403-405.

Pelz, Christian: Die strafrechtliche Verantwortlichkeit von Internet-Providern, ZUM 1998, S. 530-534.

Pentz, Vera von: Ausgewählte Fragen des Medien- und Persönlichkeitsrechts im Lichte der aktuellen Rechtsprechung des VI. Zivilsenats, AfP 2014, S. 8-18.

-: Ausgewählte Fragen des Medien- und Persönlichkeitsrechts im Lichte der aktuellen Rechtsprechung des VI. Zivilsenats, AfP 2017, S. 102-118.

-: Ausgewählte Fragen des Medien- und Persönlichkeitsrechts im Lichte der aktuellen Rechtsprechung des VI. Zivilsenats, AfP 2019, S. 113-128.

Perel, Maayan/Elkin-Koren, Niva: Accountability in Algorithmic Copyright Enforcement, Stanford Technology Law Review 19 (2016), S. 473-532.

Peters, Nils/Schmidt, Jan Hendrik: Das Ringen um Upload-Filter geht in die 2. Runde, GRUR Int 2019, S. 1006-1016.

Peukert, Alexander: Gewährleistung der Meinungs- und Informationsfreiheit in sozialen Netzwerken. Vorschlag für eine Ergänzung des NetzDG um sog. Put-back-Verfahren, MMR 2018, S. 572-578.

Pille, Jens-Ullrich: Meinungsmacht sozialer Netzwerke, Baden-Baden 2016 (Zugl.: Hamburg, Univ., Diss., 2015).

-: Der Grundsatz der Eigenverantwortlichkeit im Internet, NJW 2018, S. 3545-3550.

Podszun, Rupprecht/Schwalbe, Ulrich: Digitale Plattformen und GWB-Novelle: Überzeugende Regeln für die Internetökonomie?, NZKart 2017, S. 98-106.

Pohle, Julia/Thiel, Thorsten: Digitale Vernetzung und Souveränität. Genealogie eines Spannungsverhältnisses, in: Isabelle Borucki/Wolf Schünemann (Hrsg.), Internet und Staat. Perspektiven auf eine komplizierte Beziehung, Baden-Baden 2019, S. 57-79.

Poscher, Ralf: Wozu Juristen streiten. Eine agonistische Theorie juristischer Meinungsverschiedenheiten, JZ 2013, S. 1-11.

-: The Common Error in Theories of Adjudication. An Inferentialist Argument for a Doctrinal Conception, in: Janet Giltrow/Dieter Stein (Hrsg.), The Pragmatic Turn in Law. Inference and Interpretation in Legal Discourse, Boston 2017, S. 307-334. 
Postema, Gerald: Sweet Dissonance. Conflict, Consensus, and the Rule of Law, The Harvard Review of Philosophy 17 (2010), S. 36-55.

Pravemann, Timm: Art. 17 der Richtlinie zum Urheberrecht im digitalen Binnenmarkt. Eine Analyse der neuen europäischen Haftungsregelung für Diensteanbieter für das Teilen von Online-Inhalten, GRUR 2019, S. 783-788.

Rademacher, Timo: Wenn neue Technologien altes Recht durchsetzen: Dürfen wir es unmöglich machen, rechtswidrig zu handeln?, JZ 2019, S. 702-710.

Raue, Benjamin: Meinungsfreiheit in sozialen Netzwerken. Ansprüche von Nutzern sozialer Netzwerke gegen die Löschung ihrer Beiträge, JZ 2019, S. 961-970.

Raue, Benjamin/Steinebach, Martin: Uploadfilter. Funktionsweisen, Einsatzmöglichkeiten und Parametrisierung, ZUM 2020, S. 355-364.

Ravel, Ann: A New Kind of Voter Suppression in Modern Elections, University of Memphis Law Review 49 (2019), S. 1019-1063.

Ravn, Michelle: Navigating Terra Incognita: Why the Digital Millennium Copyright Act was Needed to Chart the Course of Online Service Provider Liability for Copyright Infringement, Ohio State Law Journal 60 (1999), S. 755-798.

Reidenberg, Joel: Lex Informatica. The Formulation of Information Policy Rules through Technology, Texas Law Review 76 (1998), S. 553-593.

Rhode, Lars: Publizistische Sorgfalt und redaktionelle Rechtspflichten. Eine Inhalts- und Strukturanalyse unter Einbezug der neuen Medien, München 2004 (Zugl.: Marburg, Univ., Diss., 2004).

Riordan, Jaani: The Liability of Internet Intermediaries, Oxford 2016.

Roberts, Sarah: Behind the Screen. Content Moderation in the Shadows of Social Media, New Haven 2019.

Röhricht, Volker/Westphalen, Friedrich Graf von/Haas, Ulrich (Hrsg.): HGB. Kommentar zu Handelsstand, Handelsgesellschaften, Handelsgeschäften, besonderen Handelsverträgen und internationalem Vertragsrecht (ohne Bilanz-, Transport- und Seerecht). 5. Aufl., Köln 2019.

Rosenbaum, Steven: Curation Nation. How to Win in a World where Consumers are Creators, New York 2011.

Schantz, Peter: Die zivilrechtliche Verantwortlichkeit von Ratingagenturen gegenüber Investoren, Berlin 2015 (Zugl.: Berlin, Humboldt-Univ., Diss., 2013).

Schapiro, Leo: Unterlassungsansprüche gegen die Betreiber von Internet-Auktionshäusern und Internet-Meinungsforen. Zugleich ein Beitrag zugunsten einer Aufgabe der Störerhaftung im Urheber-, Marken- und Wettbewerbsrecht, Tübingen 2011 (Zugl.: Berlin, Freie Univ., Diss., 2010).

-: Anhaltende Rechtsunsicherheit für die Betreiber von Internetmeinungsportalen?, ZUM 2014, S. 201-210.

Scharpf, Fritz: The Asymmetry of European Integration, or why the EU cannot be a ,Social Market Economy', Socio-Economic Review 8 (2010), S. 211-250.

Schauer, Frederick: The Politics and Incentives of First Amendment Coverage, William \& Mary Law Review 56 (2015), S. 1613-1636.

Schiff, Alexander: Anmerkung zu BGH, Urt. v. 20.02.2018 - VI ZR 30/17 - „Ärztebewertungsportal IV“, ZD 2018, S. 534-536.

-: Meinungsfreiheit in mediatisierten digitalen Räumen. Das NetzDG auf dem Prüfstand des Verfassungsrechts, MMR 2018, S. 366-371.

Schirmer, Jan-Erik: Robotik und Verkehr. Was bleibt von der Halterhaftung?, RW 9 (2018), S. 453-476.

Schmidt, Jan-Hinrik: Social Media. 2. Aufl., Wiesbaden 2018.

Schmidt, Jan-Hinrik/Taddicken, Monika: Soziale Medien. Funktionen, Praktiken, Formationen, in: Jan-Hinrik Schmidt/Monika Taddicken (Hrsg.), Handbuch Soziale Medien, Wiesbaden 2017, S. 23-37. 
Schmidt, Markus/Pruß, Michael: Technische Grundlagen des Internets, in: Astrid AuerReinsdorff/Isabell Conrad (Hrsg.), Handbuch IT- und Datenschutzrecht, 3. Aufl., München 2019.

Schmidt-Aßmann, Eberhard: Due Process und Grundrechtsschutz durch Verfahren. Eine vergleichende Untersuchung zum amerikanischen und deutschen Verwaltungsverfahrensrecht, AöR 142 (2017), S. 325-365.

Schmoll, Andrea: Die deliktische Haftung der Internet-Service-Provider. Eine rechtsvergleichende Untersuchung zu Deutschland, Frankreich, England und den USA, Frankfurt am Main 2001 (Zugl.: Jena, Univ., Diss., 2000).

Schneider, Ingrid: Bringing the State Back In. Big Data-Based Capitalism, Disruption, and Novel Regulatory Approaches In Europe, in: Ann Rudinow Saetnan/Ingrid Schneider/ Nicola Green (Hrsg.), The Politics of Big Data. Big Data, Big Brother?, London, New York 2018, S. 129-175.

Schoch, Friedrich: Gewährleistungsverwaltung: Stärkung der Privatrechtsgesellschaft?, NVwZ 2008, S. 241-247.

Schröder, Meinhard: Persönlichkeitsrechtsschutz bei Bewertungsportalen im Internet, Verwaltungsarchiv 101 (2010), S. 205-230.

Schulz, Wolfgang: Verfassungsrechtliche Eckpunkte eines Umganges mit sozialen Medien, Rechtsstandort Hamburg (16.06.2016), abrufbar unter: http://www.rechtsstandort-ha mburg.de/fileadmin/rechtsstandort-hamburg/docs/2017-06-16-rede-schulz-wolfgang.p df.

-: Kontrolle vorherrschender Meinungsmacht. Dekonstruktion eines medienrechtlichen Schlüsselbegriffs, AfP 2017, S. 373-379.

Schulz, Wolfgang/Dankert, Kevin: Die Macht der Informationsintermediäre. Erscheinungsformen, Strukturen und Regulierungsoptionen, herausgegeben von FriedrichEbert-Stiftung, Bonn 2016.

Schulz, Wolfgang/Held, Thorsten/Laudien, Arne: Suchmaschinen als Gatekeeper in der öffentlichen Kommunikation. Rechtliche Anforderungen an Zugangsoffenheit und Transparenz bei Suchmaschinen im WWW, Berlin 2005.

Schulze, Andreas: Rechtsextremismus in sozialen Netzwerken. Ein kursorischer Blick auf Facebook-Seiten im „Wahlherbst“ 2017, in: Sebastian Liebold/Tom Mannewitz/Madeleine Petschke/Tom Thieme (Hrsg.), Demokratie in unruhigen Zeiten. Festschrift für Eckhard Jesse, Baden-Baden 2018, S. 227-240.

Schütz, Raimund: Regulierung in der digitalen Medienwelt. Fünf aktuelle Herausforderungen, MMR 2018, S. 36-39.

Schwartmann, RolflHermann, Maximilian/Mühlenbeck, Robin: Eine Medienordnung für Intermediäre. Das Zwei-Säulen-Modell zur Sicherung der Vielfalt im Netz, MMR 2019, S. 498-503.

Schweiger, Wolfgang: Der (des)informierte Bürger im Netz. Wie soziale Medien die Meinungsbildung verändern, Wiesbaden 2017.

Schweitzer, Heike: Digitale Plattformen als private Gesetzgeber. Ein Perspektivwechsel für die europäische „Plattform-Regulierung“, ZEuP 2019, S. 1-12.

Secretary-General of the OECD: The Economic and Social Role of Internet Intermediaries, Paris 2010, abrufbar unter: http://www.oecd.org/internet/ieconomy/44949023.pdf.

-: The Role of Internet Intermediaries in Advancing Public Policy Objectives, Paris 2011.

Seiler, Frank: Plattform - Text - Ideologie, in: Coline Baechler/Eva Martha Eckkrammer/ Johannes Müller-Lancé/Verena Thaler (Hrsg.), Medienlinguistik 3.0. Formen und Wirkung von Textsorten im Zeitalter des Social Web, Berlin 2016, S. 63-80.

Senftleben, Martin: Filterverpflichtungen nach der Reform des europäischen Urheberrechts. Das Ende der freien Netzkultur?, ZUM 2019, S. 369-374. 
Sesing, Andreas: Digitaler Binnenmarkt zwischen Urheber- und Telemedienrecht. Vorschlag zur Umsetzung von Art. 17 Urh-RL in deutsches Recht, MMR 2019, S. 788-793.

Sieber, Ulrich: Strafrechtliche Verantwortlichkeit für den Datenverkehr in internationalen Computernetzen (1). Neue Herausforderungen des Internet, JZ 1996, S. 429-442.

-: Strafrechtliche Verantwortlichkeit für den Datenverkehr in internationalen Computernetzen (2). Neue Herausforderungen des Internet - Fortsetzung und Schluß, JZ 1996, S. 494-507.

-: Verantwortlichkeit im Internet. Technische Kontrollmöglichkeiten und multimediarechtliche Regelungen; zugleich eine Kommentierung von $\S 5$ TDG und $\S 5 \mathrm{MDStV}$, München 1999.

Sieber, Ulrich/Höfinger, Frank: Teil 18.1. Allgemeine Grundsätze der Haftung, in: Thomas Hoeren/Ulrich Sieber/Bernd Holznagel (Hrsg.), Handbuch Multimedia-Recht. Rechtsfragen des elektronischen Geschäftsverkehrs, 53. EL, München 2020.

Sieber, Ulrich/Liesching, Marc: Die Verantwortlichkeit der Suchmaschinenbetreiber nach dem Telemediengesetz, MMR-Beilage 8/2007, S. 1-30.

Siegmund, Julian: Suchmaschinenalgorithmen im Kartellrecht der EU und der USA. Untersuchung der Möglichkeiten einer Ausgestaltungskontrolle im Spannungsfeld der Implikationen wesentlicher Einrichtungen und bestehender Schutzdimensionen, BadenBaden 2017 (Zugl.: Freiburg, Univ., Diss., 2017).

Sobola, Sabine: Verantwortung und Haftung für Inhalte im Internet, in: Astrid AuerReinsdorff/Isabell Conrad (Hrsg.), Handbuch IT- und Datenschutzrecht, 3. Aufl., München 2019.

Soehring, Jörg: §16. Verbreiterhaftung, in: Jörg Soehring/Verena Hoene (Hrsg.), Presserecht. Recherche, Darstellung, Haftung im Recht der Presse, des Rundfunks und der neuen Medien, 6. Aufl., Köln 2019.

Sokol, Ljuba: Die Bestimmung der Verantwortlichkeit für die Abwehr und Beseitigung von Störungen im öffentlichen und privaten Recht, Tübingen 2016 (Zugl.: Heidelberg, Univ., Diss., 2015).

Solum, Lawrence/Chung, Minn: The Layers Principle. Internet Architecture and the Law, Notre Dame Law Review 79 (2004), S. 815-948.

Spano, Robert: Intermediary Liability for Online User Comments under the European Convention on Human Rights, Human Rights Law Review 2017, S. 665-679.

Specht, Louisa: Ausgestaltung der Verantwortlichkeit von Plattformbetreibern zwischen Vollharmonisierung und nationalem Recht, ZUM 2017, S. 114-122.

-: Anmerkung zu EuGH, Urt. v. 03.10.2019 - C-18/18 - Glawischnig-Piesczek, MMR 2019, S. 801-802.

-: Zum Verhältnis von (Urheber-)Recht und Technik. Erfordernis eines Dualismus von techniksensitivem Recht und rechtssensitiven technischen Durchsetzungsbefugnissen, GRUR 2019, S. 253-259.

Specht, LouisalEickhoff, Vera: Ein reformiertes Haftungskonzept für rechtswidrige ÄuBerungen auf Bewertungsportalen? Überlegungen zu einem auf das Äußerungsrecht beschränkten sektorspezifischen Haftungskonzept, CR 2016, S. 740-746.

Spiecker gen. Döhmann, Indra: Rechtliche Begleitung der Technikentwicklung im Bereich moderner Infrastrukturen und Informationstechnologien, in: Hermann Hill/Utz Schliesky (Hrsg.), Die Vermessung des virtuellen Raums. E-Volution des Rechts- und Verwaltungssystems III, Baden-Baden 2012, S. 137-161.

Spindler, Gerald: Deliktsrechtliche Haftung im Internet - nationale und internationale Rechtsprobleme, ZUM 1996, S. 533-563.

-: Haftungsrechtliche Grundprobleme der neuen Medien, NJW 1997, S. 3193-3199.

-: Das Gesetz zum elektronischen Geschäftsverkehr. Verantwortlichkeit der Diensteanbieter und Herkunftslandprinzip, NJW 2002, S. 921-927. 
-: „Die Tür ist auf“ - Europarechtliche Zulässigkeit von Auskunftsansprüchen gegenüber Providern. Urteilsanmerkung zu EuGH „Promusicae/Telefónica“, GRUR 2008, S. 574-577.

-: Präzisierungen der Störerhaftung im Internet. Besprechung des BGH-Urteils „Kinderhochstühle im Internet", GRUR 2011, S. 101-108.

-: Rechtliche Verantwortlichkeit nach Maßgabe technischer Kontrollmöglichkeiten? Das Beispiel der Verantwortlichkeit von Internet-Providern, in: Martin Eifert/Wolfgang Hoffmann-Riem (Hrsg.), Innovation, Recht und öffentliche Kommunikation. Innovation und Recht IV, Berlin 2011, S. 67-94.

-: Der Regierungsentwurf zum Netzwerkdurchsetzungsgesetz - europarechtswidrig?, ZUM 2017, S. 473-487.

-: Haftung ohne Ende? Über Stand und Zukunft der Haftung von Providern, MMR 2018, S. 48-52.

-: Rechtsdurchsetzung von Persönlichkeitsrechten. Bußgelder gegen Provider als Enforcement?, GRUR 2018, S. 365-373.

-: Die neue Urheberrechts-Richtlinie der EU, insbesondere „Upload-Filter“ - Bittersweet?, CR 2019, S. 277-291.

-: Weltweite Löschungspflichten bei Persönlichkeitsrechtsverletzungen im Internet, NJW 2019, S. 3274-3277.

-: Art. 17 DSM-RL und dessen Vereinbarkeit mit primärem Europarecht. Zugleich ein Beitrag zu Umsetzungsmöglichkeiten, GRUR 2020, S. 253-261.

Spindler, Gerald/Schmitz, Peter (Hrsg.): Telemediengesetz mit Netzwerkdurchsetzungsgesetz. 2. Aufl., München 2018.

Spindler, Gerald/Schuster, Fabian (Hrsg.): Recht der elektronischen Medien. Kommentar. 4. Aufl., München 2019.

Spindler, Gerald/Volkmann, Christian: Die zivilrechtliche Störerhaftung der Internet-Provider, WRP 2003, S. 1-15.

Staab, Philipp: Digitaler Kapitalismus. Markt und Herrschaft in der Ökonomie der Unknappheit, Berlin 2019.

Stalla-Bourdillon, Sophie: Internet Intermediaries as Responsible Actors? Why It Is Time to Rethink the E-Commerce Directive as Well, in: Mariarosaria Taddeo/Luciano Floridi (Hrsg.), The Responsibilities of Online Service Providers, Cham 2017, S. 275-293.

Stark, Birgit: „Don't be evil“. Die Macht von Google und die Ohnmacht der Nutzer und Regulierer, in: Birgit Stark/Dieter Dörr/Stefan Aufenanger (Hrsg.), Die Googleisierung der Informationssuche. Suchmaschinen zwischen Nutzung und Regulierung, Berlin 2014, S. 1-19.

Stark, Birgit/Magin, Melanie/Jürgens, Pascal: Ganz meine Meinung? Informationsintermediäre und Meinungsbildung - Eine Mehrmethodenstudie am Beispiel von Facebook, herausgegeben von Landesanstalt für Medien Nordrhein-Westfalen, Düsseldorf 2017, abrufbar unter: http://lfmpublikationen.lfm-nrw.de/index.php?view=product_detai 1\&product_id $=492$.

Steinbach, Armin: Meinungsfreiheit im postfaktischen Umfeld, JZ 2017, S. 653-661.

Steiner, Miriam/Magin, Melanie/Stark, Birgit/Jürgens, Pascal: Aus Versehen informiert? Facebooks Bedeutung für die Nachrichtennutzung nebenbei - eine MehrmethodenAnalyse, in: Patrick Weber/Frank Mangold/Matthias Hofer (Hrsg.), Meinungsbildung in der Netzöffentlichkeit. Aktuelle Studien zu Nachrichtennutzung, Meinungsaustausch und Meinungsbeeinflussung in Social Media, Baden-Baden 2019, S. 17-36.

Stieper, Malte: Ausschließlichkeitsrecht oder Vergütungsanspruch: Vergütungsmodelle bei Aufmerksamkeitsplattformen, ZUM 2017, S. 132-140.

Stöcker, Christan/Lischka, Konrad: Wie algorithmische Prozesse Öffentlichkeit strukturieren, in: Resa Mohabbat Kar/Basanta Thapa/Peter Parycek (Hrsg.), (Un)berechenbar? Algorithmen und Automatisierung in Staat und Gesellschaft, Berlin 2018, S. 364-391. 
Sunstein, Cass: Deliberative Trouble? Why Groups go to Extremes, Yale Law Journal 110 (2000), S. 71-119.

-: \#republic. Divided Democracy in the Age of Social Media, Princeton 2017.

Sydow, Gernot (Hrsg.): Europäische Datenschutzgrundverordnung. Handkommentar. 2. Aufl., Baden-Baden 2018.

Sylvain, Olivier: Intermediary Design Duties, Connecticut Law Review 50 (2018), S. 203-277.

Taddicken, Monika/Schmidt, Jan-Hinrik: Entwicklung und Verbreitung sozialer Medien, in: Jan-Hinrik Schmidt/Monika Taddicken (Hrsg.), Handbuch Soziale Medien, Wiesbaden 2017, S. 3-22.

Teubner, Gunther: Globale Zivilverfassungen. Alternativen zur staatszentrierten Verfassungstheorie, ZaöRV 2003, S. 1-28.

Thiel, Thorsten: Anonymität und der digitale Strukturwandel der Öffentlichkeit, Zeitschrift für Menschenrechte 10 (2016), S. 9-24.

Thorson, Kjerstin/Wells, Chris: Curated Flows. A Framework for Mapping Media Exposure in the Digital Age, Communication Theory 26 (2016), S. 309-328.

Tremble, Catherine: Wild Westworld. Section 230 of the CDA and Social Networks' Use of Machine-Learning Algorithms, Fordham Law Review 86 (2017), S. 825-869.

Tushnet, Rebecca: Power without Responsibility. Intermediaries and the First Amendment, George Washington Law Review 76 (2008), S. 986-1016.

U.S. Department of Justice: Views on H.R. 1865. Letter to the Committee on the Judiciary of the U.S. House of Representatives, Washington, D.C. 27.02.2018, abrufbar unter: ht tps://www.justice.gov/ola/page/file/1042721/download.

Ulloa, Jazmine: California has become a Battleground for the Protection of Consumer Privacy Rules, Los Angeles Times, 11.03.2019, abrufbar unter: https://www.latimes.c om/politics/la-pol-ca-california-privacy-law-battles-20190311-story.html.

Ungern-Sternberg, Antje von: Demokratische Meinungsbildung und künstliche Intelligenz, in: Sebastian Unger/Antje von Ungern-Sternberg (Hrsg.), Demokratie und künstliche Intelligenz, Tübingen 2019, S. 3-31.

Unkel, Julian: Informationsselektion mit Suchmaschinen. Wahrnehmung und Auswahl von Suchresultaten, Baden-Baden 2019 (Zugl.: München, Ludwigs-Maximilians-Univ., Diss., 2019).

Urban, Jennifer/Quilter, Laura: Efficient Process or Chilling Effects - Takedown Notices under Section 512 of the Digital Millennium Copyright Act, Santa Clara Computer \& High Technology Law Journal 22 (2006), S. 621-693.

Valcke, Peggy/Kuczerawy, AleksandralOmbelet, Pieter-Jan: Did the Romans get it right? What Delfi, Google, eBay, and UPC TeleKabel Wien have in common, in: Mariarosaria Taddeo/Luciano Floridi (Hrsg.), The Responsibilities of Online Service Providers, Cham 2017, S. 101-116.

Van Eecke, Patrick: Online Service Providers and Liability. A Plea for a Balanced Approach, Common Market Law Review 48 (2011), S. 1455-1502.

Vesting, Thomas: Die Medien des Rechts: Computernetzwerke, Weilerswist 2015.

-: Die Veränderung der Öffentlichkeit durch künstliche Intelligenz, in: Sebastian Unger/ Antje von Ungern-Sternberg (Hrsg.), Demokratie und künstliche Intelligenz, Tübingen 2019, S. 33-49.

Volkmann, Caroline: Art. 17 Urh-RL und die Upload-Filter: verschärfte Störerhaftung oder das Ende der Freiheit im Internet?, CR 2019, S. 376-384.

Volkmann, Christian: Der Störer im Internet. Zur Verantwortlichkeit der Internet-Provider im allgemeinen Zivil-, Wettbewerbs-, Marken- und öffentlichen Recht, München 2005 (Zugl.: Göttingen, Univ., Diss., 2004). 
Volmar, Maximilian: Digitale Marktmacht, Baden-Baden 2019 (Zugl.: Hamburg, Univ., Diss., 2019).

Volokh, Eugene/Falk, Donald: Google. First Amendment Protection for Search Engine Results, Journal of Law, Economics \& Policy 8 (2012), S. 883-899.

Wagner, Bernd: Disruption der Verantwortlichkeit. Private Nutzer als datenschutzrechtliche Verantwortliche im Internet of Things, ZD 2018, S. 307-312.

Wagner, Gerhard: Die Voraussetzungen negatorischen Rechtsschutzes. Versuch einer Neubegründung, in: Volker Beuthien/Maximilian Fuchs/Herbert Roth/Gottfried Schiemann/Andreas Wacke (Hrsg.), Perspektiven des Privatrechts am Anfang des 21. Jahrhunderts. Festschrift für Dieter Medicus zum 80. Geburtstag am 9. Mai 2009, Köln 2009, S. 589-610.

-: Haftung von Plattformen für Rechtsverletzungen (Teil 1), GRUR 2020, S. 329-338.

-: Haftung von Plattformen für Rechtsverletzungen (Teil 2), GRUR 2020, S. 447-457.

Wagner, GerhardlEidenmüller, Horst: In der Falle der Algorithmen? Abschöpfen von Konsumentenrente, Ausnutzen von Verhaltensanomalien und Manipulation von Präferenzen. Die Regulierung der dunklen Seite personalisierter Transaktionen, ZfPW 2019, S. 220-246.

Waldenberger, Arthur: Zur zivilrechtlichen Verantwortlichkeit für Urheberrechtsverletzungen im Internet, ZUM 1997, S. 176-188.

Wandtke, Artur/Hauck, Ronny: Art. 17 DSM-Richtlinie - Ein neues Haftungssystem im Urheberrecht, ZUM 2019, S. 627-636.

Wang, Edmund: The Line between Copyright and the First Amendment and Why Its Vagueness May Further Free Speech Interests, University of Pennsylvania Journal of Constitutional Law 13 (2011), S. 1471-1498.

Whitney, Heather: Search Engines, Social Media, and the Editorial Analogy, Knight First Amendment Institute (27.02.2018), abrufbar unter: https://knightcolumbia.org/content/ search-engines-social-media-and-editorial-analogy.

Wiacek, Martin: Strafbarkeit rechts motivierter Cyberkriminalität in sozialen Netzwerken, Baden-Baden 2019 (Zugl.: Trier, Univ., Diss., 2019).

Wiebe, Andreas: Providerhaftung in Europa. Neue Denkanstöße durch den EuGH (Teil 1), WRP 2012, S. 1182-1189.

Wielsch, Dan: Die Ordnung der Netzwerke. AGB - Code - Community Standards, in: Martin Eifert/Tobias Gostomzyk (Hrsg.), Netzwerkrecht. Die Zukunft des NetzDG und seine Folgen für die Netzwerkkommunikation, Baden-Baden 2018, S. 61-94.

-: Verantwortung von digitalen Intermediären für Rechtsverletzungen Dritter, ZGE 2018, S. 1-34.

-: Funktion und Verantwortung. Zur Haftung im Netzwerk, RW 10 (2019), S. 84-108.

Wiener, Norbert: Kybernetik, Physikalische Blätter 5 (1949), S. 355-362.

Wilmer, Thomas: Überspannte Prüfpflichten für Host-Provider? Vorschlag für eine Haftungsmatrix, NJW 2008, S. 1845-1851.

Wimmers, Jörg/Barudi, Malek: Der Mythos vom Value Gap. Kritik zur behaupteten Wertschöpfungslücke bei der Nutzung urheberrechtlich geschützter Inhalte auf HostingDiensten, GRUR 2017, S. 327-338.

Wimmers, Jörg/Heymann, Britta: Zum Referentenentwurf eines Netzwerkdurchsetzungsgesetzes (NetzDG). Eine kritische Stellungnahme, AfP 2017, S. 93-102.

Winner, Langdon: Do Artifacts Have Politics?, Daedalus 109 (1980), S. 121-136.

Wischmeyer, Thomas: Regulierung intelligenter Systeme, AöR 143 (2018), S. 1-66.

Wissenschaftlicher Dienst des Deutschen Bundestages: Entwurf eines Netzwerkdurchsetzungsgesetzes. Vereinbarkeit mit der Meinungsfreiheit, WD 10-3000-037/17, Berlin 2017, abrufbar unter: https://www.bundestag.de/resource/blob/510514/eefb7cf92dee88e c74ce8e796e9bc25c/wd-10-037-17-pdf-data.pdf. 
Wu, Tim: Will Artificial Intelligence Eat The Law? The Rise of Hybrid Social-Ordering Systems, Columbia Law Review 119 (2019), S. 2001-2028.

Wyden, Ron: Pressemitteilung. Wyden Issues Warning About SESTA, Washington, D.C. 08.11.2017, abrufbar unter: https://www.wyden.senate.gov/news/press-releases/wydenissues-warning-about-sesta.

Yoo, Christopher: Free Speech and the Myth of the Internet as an Unintermediated Experience, George Washington Law Review 78 (2010), S. 697-773.

Zara, Christopher: The Most Important Law in Tech Has a Problem, Wired (01.03.2017), abrufbar unter: https://www.wired.com/2017/01/the-most-important-law-in-tech-has-aproblem.

Zech, Herbert: Zivilrechtliche Haftung für den Einsatz von Robotern. Zuweisung von Automatisierungs- und Autonomierisiken, in: Sabine Gless/Kurt Seelmann (Hrsg.), Intelligente Agenten und das Recht, Baden-Baden 2016, S. 163-204.

-: Entscheidungen digitaler autonomer Systeme. Empfehlen sich Regelungen zu Verantwortung und Haftung?, Gutachten A zum 73. Deutschen Juristentag (Manuskript), Berlin 2020.

Ziegler, Katharina: Urheberrechtsverletzungen durch Social Sharing. Urheber- und haftungsrechtliche Aspekte sozialer Netzwerke am Beispiel der Plattform Facebook, Tübingen 2016 (Zugl.: Regensburg, Univ., Diss., 2016).

Zimmer, AnjalKunow, Kristian: Intermediäre und Meinungsbildung, in: die medienanstalten (Hrsg.), Vielfaltsbericht der Medienanstalten, Berlin 2019, abrufbar unter: https://w ww.die-medienanstalten.de/fileadmin/user_upload/die_medienanstalten/Publikation en/Vielfaltsbericht/Vielfaltsbericht_2019.pdf.

Zittrain, Jonathan: A History of Online Gatekeeping, Harvard Journal of Law \& Technology 19 (2006), S. 253-298.

Zuboff, Shoshana: You Are Now Remotely Controlled. Surveillance Capitalists Control the Science and the Scientists, the Secrets and the Truth, The New York Times, 26.01.2020, abrufbar unter: https://www.nytimes.com/2020/01/24/opinion/sunday/sur veillance-capitalism.html. 



\section{Register}

AGB

- -kontrolle 160

- Gestaltungsmacht der Intermediäre 159-160

- Kontrolle der Intermediäre über den Zugang zu Informationen 154-156

Aktive Rolle der Diensteanbieter Siehe Neutrale Rolle der Diensteanbieter

Allgemeine Überwachungspflicht Siehe Verbot allgemeiner Überwachungspflichten

Anreizstrukturen

- im Communications Decency Act 16-17, 30

- im Digital Millennium Copyright Act 38, 48-49

- Kritik der im Rahmen der Störerhaftung 244-245

- Verbot allgemeiner Überwachungspflichten 274-275

- Vergleich der US-amerikanischen und unionsrechtlichen Regelungen 109-110

- Vorschlag zur Fortentwicklung im Kontext der Störerhaftung 267-269

Auslistungsanspruch 212-216

Autocomplete 87-88, 176, 199

Begründungslast Siehe Substanziierungslast

Caching

- Abgrenzung vom Hosting 66

- Bedeutung 123

- Zuordnung zu Haftungsprivilegierungen 181-182

CDA Siehe Communications Decency Act

Cheapest cost avoider 196, 251, 268
Communications Decency Act

- Auslegung durch die US-Gerichte $17-25$

- Entstehungsgeschichte 4, 14-17

- Reformbemühungen 27-30

- US-verfassungsrechtlicher Kontext 25-27

- Verhältnis zum Common Law 14 15,18

- Wortlaut von $\S 23013$

Content Moderation 48, 159, 170, 274

Counter-notice-Verfahren, Siehe auch Wiederherstellungsanspruch

- Forderung einer Etablierung im deutschen Recht 240-241

- im Digital Millennium Copyright Act 41-42, 45, 109

Datenschutz-Grundverordnung

- Verhältnis zur E-CommerceRichtlinie 73-74

Diensteanbieter

- Begriff 143-144

- Funktionen 119-129

Digital Millennium Copyright Act

- Entstehungsgeschichte 33-35

- Notice-And-Takedown-Verfahren

- Auslegung durch die Gerichte 35-42

- Kritik an der US-amerikanischen Regelung 42-49

- Wortlaut von §512(c) 32

DMCA Siehe Digital Millennium Copyright Act

DSM-Richtlinie

- Bewertung 302-310

- Filterpflicht 297-299, 304-310

- Hintergrund 294-295

- Öffentliche Wiedergabe 295-296 
- Overblocking 307-310

- Pflichten der Diensteanbieter 296301

- sachlicher Anwendungsbereich 143144

- Verbot allgemeiner Überwachungspflichten 306-307

E-Commerce-Richtlinie

- Anwendbarkeit der Haftungsprivilegierungen auf Suchmaschinen 61-63, 178-181

- Hintergrund 58-59

- neutrale Rolle Siehe Neutrale Rolle der Diensteanbieter

- Persönlicher Anwendungsbereich 60-63

- Verhältnis zur Datenschutz-Grundverordnung 73-74

- Voraussetzungen des Haftungsprivilegs nach Art. 14 65-73

ECRL Siehe E-Commerce-Richtlinie

Eigene Inhalte, Haftung für 85

Eigenverantwortung, Prinzip der 163, 265, 268

Ende-zu-Ende-Prinzip 117

Entgeltlichkeit als Anwendungsvoraussetzung der E-Commerce-Richtlinie 61,65

Filterpflichten, Siehe auch Verbot allgemeiner Überwachungspflichten

- DSM-Richtlinie 297, 304

- im Kontext der Störerhaftung 224

- im Kontext des Verbots allgemeiner Überwachungspflichten 77, 275

- manuelle Kontrolle 79, 225, 244, 276, 307

Gatekeeper

- Begriff 187-188

- Inpflichtnahme der Intermediäre als 188-189

- Modell nach Kraakman 189-194

Grundrechte, Siehe auch Meinungsfreiheit

- Bedeutung für die Haftungsprivilegierungen der E-CommerceRichtlinie 257-259
- DSM-Richtlinie 307-310

- Netzwerkdurchsetzungsgesetz 288292

- Stellungnahmeverfahren im Kontext der Störerhaftung 236-237

- Verhaltenspflichten der Diensteanbieter 79-80

Gute Samariter-Regelungen

- Bedürfnis einer Regelung in der EU 260-262, 274-275

- in $\S 230$ Communications Decency Act 16-17, 31, 109

Haftungsprivileg

- Allgemeine Überwachungspflichten Siehe Verbot allgemeiner Überwachungspflichten

- Anwendbarkeit auf Suchmaschinendienste 61-63, 178-182

- konzeptionelle Kritik 152-164

- praktische Bedeutung 257-259

- Verhältnis zum Grundrechtsschutz 79-80, 257-259

- Verhältnis zur Störerhaftung 226-229

- Voraussetzungen nach § 10 TMG 93-97

- Voraussetzungen nach Art. 14 E-Commerce-Richtlinie 65-73

- Vorschlag zur Neukonzeption 262-273

- Zwecke 105-107

Host-Provider

- Kritik des Konzepts 151-164

- Voraussetzungen des Haftungsprivilegs nach § 10 TMG 93-97

- Voraussetzungen des Haftungsprivilegs nach Art. 14 E-CommerceRichtlinie 65-73

Intermediäre

- Begriff 145-148

- Funktionen 119-129

- Geschäftsmodell 157-159, 169, 174-175

- Meinungsfreiheit 252-254

- Technische Gestaltungsmacht der 156-161 
Internet

- Schutz des durch Haftungsprivilegierungen 107-108

- Struktur 115-119

- und staatliche Regulierung 3-6, 315-320

Kenntnisnahme

- bei $\$ 10$ TMG 94-96

- bei $\S 512$ Digital Millennium Copyright Act 39-40, 46-47

- bei Art. 14 E-Commerce-Richtlinie 68-73

- durch automatisierte Systeme 95-96

- im Modell nach Kraakman 191-192

- im Stellungnahmeverfahren der Störerhaftung 207-212, 242-246

- Vorschlag zur Fortentwicklung 267-271

Kerngleichheit

- im Kontext der Störerhaftung 221-224

- $\quad$ in der Rechtsprechung des EuGH 78-79

Kollateralzensur 291-292

Kommunikationsdienste 120-122

Kuratierung von Inhalten

- Abgrenzung zu von Nutzern eingegebenen Informationen 66-67

- als wesentliche Funktion der Intermediäre 127-128, 173-174

- Verhältnis von Intermediären und Nutzern 163-164

Marketplace of Ideas 25-26

Meinungsfreiheit

- der Intermediäre 252-254

- und Netzwerkdurchsetzungsgesetz 288-292

Netzwerk, soziales

- Begriff 139-142

- Rezeption im Netzwerkdurchsetzungsgesetz 141

Netzwerkdurchsetzungsgesetz

- Argument der Privatisierung der Rechtsdurchsetzung 286

- Begriff des sozialen Netzwerks 141

- Bewertung 284-292
- Hintergrund 281-282

- Overblocking 289-292

- Pflichten der Diensteanbieter 283

- Verhältnismäßigkeit 288-292

Neutrale Rolle der Diensteanbieter

- Abgrenzung zur aktiven Rolle 64-65

- Herleitung durch den EuGH 63-65

- Kritik des Konzepts 164-173

- Rechtsprechung des BGH 96-97

- Suchmaschinendienste 61-63

- Vorschlag zur Aufgabe des Konzepts 265-267

- Zueigenmachen fremder Inhalte 91-93

Notice-and-Takedown-Verfahren

- Handhabung durch die US-Gerichte 35-42

- im Rahmen der Störerhaftung 208-212

- Regelung in Art. 14 E-CommerceRichtlinie 105, 272

- Wortlaut von $\S 512$ Digital Millennium Copyright Act 32-33

Notifizierung

- Anforderungen im Digital Millennium Copyright Act 41-42

- im Kontext des Art. 17 DSMRichtlinie 298

- im Rahmen der Störerhaftung 208-212

- $\quad$ in der Rechtsprechung des EuGH 70

- Umgang mit einer im Kontext des Netzwerkdurchsetzungsgesetzes 283

- Vorschlag zur Fortentwicklung 271273

Overblocking

- DSM-Richtlinie 301, 307-310

- im Modell nach Kraakman 191

- Netzwerkdurchsetzungsgesetz 289-292

Passive Rolle der Diensteanbieter Siehe Neutrale Rolle

Plattform

- Begriff 132-135

- Kritik des Begriffs 137-139

Privatisierung der Rechtsdurchsetzung, Argument der 231-234, 286 
Prüfpflichten

- Bewertung 235-246

- Grundlagen 203-207

- Suchmaschinendienste 212-217

- Verfahren bei Persönlichkeitsrechtsverletzungen 207-212

Put-back-Anspruch Siehe Counternotice-Verfahren; Wiederherstellungsanspruch

Relevanz

- Erzeugung von als zentrales Ziel der Intermediäre 157-159, 174-175

Schadensersatzansprüche

- Privilegierung nach Art. 14 E-Commerce-Richtlinie 72

- Störerhaftung 249-252

Speichern von Informationen im Auftrag des Nutzers

- als Anwendungsvoraussetzung von Art. 14 E-Commerce-Richtlinie 67-68

- Kritik des Konzepts 161-164

Stellungnahmeverfahren

- Bewertung 235-246

- Fehlende Umsetzung im Netzwerkdurchsetzungsgesetz 286-288

- Grundlagen 207-212

Störerhaftung

- Anwendungsbereich 198-202

- Dogmatische Kritik 229-231

- Gefahr der Erstbegehung u. Wiederholung 219-220, 246-249

- Grundlagen 195-229

- Kosten der Inanspruchnahme 225-226

- Notice-and-Takedown-Verfahren 208

- Problematische Aspekte 241-254

- Prüfpflichten 203-207

- Rechtsfolgen 216-226

- Schadensersatzansprüche 249-252

- Subsidiarität 200-202

- Suchmaschinendienste 212-216

- Voraussetzungen bei Persönlichkeitsrechtsverletzungen 207-212
- Voraussetzungen 202-212

- Vorzüge 235-241

- Zweck 196-197

Substanziierungslast

- im Rahmen der Störerhaftung 208-212, 242-246

- $\quad$ im TMG 97

- in der Rechtsprechung des EuGH 70

- Vorschlag zur Fortentwicklung 270-271

Suchmaschinendienste

- Anwendbarkeit der Art. 12 ff. E-Commerce-Richtlinie 61-63, 178-182

- Funktionen 122-124, 174-175

- Haftung für AutocompleteSuchvorschläge 87-88

- Störerhaftung 212-216

Teledienstegesetz 54-58

Überwachungspflicht Siehe Verbot allgemeiner Überwachungspflichten

Uploadfilter Siehe Filterpflichten

Verbot allgemeiner Überwachungspflichten

- Abgrenzung von „spezifischen“ Überwachungspflichten nach Art. 15 E-Commerce-Richtlinie 76-80

- Art. 15 E-Commerce-Richtlinie 75-83

- DSM-Richtlinie 306-307

- Störerhaftung 220-225

- US-amerikanisches Recht 40

- Vorschlag zur Fortentwicklung 273-278

Verkehrspflichten

- Störerhaftung 230, 250-252

Wiederherstellungsanspruch 240-241, 261, 271, 274, 287

Zueigenmachen fremder Inhalte

- Konzept 88-91

- Vereinbarkeit mit dem Unionsrecht 91-93 